\title{
A theoretical study on the assembly and stabilization of a silicon-doped all-metal aromatic unit $\mathrm{SiAl}_{3}{ }^{-}$
}

\section{Supporting Information.}

Li-ming Yang, Jian Wang, Yi-hong Ding*, Chia-chung Sun

State Key Laboratory of Theoretical and Computational Chemistry, Institute of Theoretical Chemistry, Jilin University, Changchun 130023, People's Republic of China

(E-mail: yhdd@mail.jlu.edu.cn; Fax: +86-431-8498026)

\section{Full citations for ref 23:}

(23) Gaussian03 (RevisionA.1), M. J. Frisch, G. W. Trucks, H. B. Schlegel, G. E. Scuseria, M. A. Robb, J. R. Cheeseman, J. A. Montgomery, Jr., T. Vreven, K. N. Kudin, J. C. Burant, J. M. Millam, S. S. Iyengar, J. Tomasi, V. Barone, B. Mennucci, M. Cossi, G. Scalmani, N. Rega, G. A. Petersson, H. Nakatsuji, M. Hada, M. Ehara, K. Toyota, R. Fukuda, J. Hasegawa, M. Ishida, T. Nakajima, Y. Honda,O. Kitao, H. Nakai, M. Klene, X. Li, J. E. Knox, H. P. Hratchian, J. B. Cross, C. Adamo, J. Jaramillo, R. Gomperts, R. E. Stratmann, O. Yazyev, A. J. Austin, R. Cammi, C. Pomelli, J. W. Ochterski, P. Y. Ayala, K. Morokuma, G. A. Voth, P. Salvador, J. J. Dannenberg, V. G. Zakrzewski, S. Dapprich, A. D. Daniels, M. C. Strain, O. Farkas, D. K. Malick, A. D. Rabuck, K. Raghavachari, J. B. Foresman, J. V. Ortiz, Q. Cui, A. G. Baboul, S. Clifford, J. Cioslowski, B. B. Stefanov, G. Liu, A. Liashenko, P. Piskorz, I. Komaromi, R. L. Martin, D. J. Fox, T. Keith, M. A. Al-Laham, C. Y. Peng, A. Nanayakkara, M. Challacombe, P. M.W. Gill, B. Johnson, W. Chen, M.W. Wong, C. Gonzalez, J. A. Pople, Gaussian, Inc., Pittsburgh, PA, 2003. 
Note1. An imaginary frequency indicates the existence of a vibrational mode that is dynamically unstable and leads to a more stable structure. Transition states of a chemical reaction are saddle points exhibiting only one imaginary frequency. Saddle points with more dynamical systems with sufficiently high vibrational energy but are generally not of chemical significance.

In this report, NIMAG means the number of imaginary frequency of saddle point. ${ }^{\text {[Note1] }}$ ${ }^{\mathbf{m}} \mathbf{N}^{\mathbf{q}}$ : “ $\mathbf{m}$ ” means spin electron state (singlet, triplet), “ $\mathbf{N}$ ” means the sequence number of various isomers, superscript “q-" means the charge of the total system.

Figure 1. Schematic energy profile for $\left.\left.\left.\left[\mathrm{Si}_{2} \mathrm{Al}_{6} \mathrm{M}\right]^{\mathrm{q}-}: \mathrm{a}\right)(\mathrm{M}, \mathrm{q})=(\mathrm{Li}, 1) ; \mathrm{b}\right)(\mathrm{M}, \mathrm{q})=(\mathrm{Na}, 1) ; \mathrm{c}\right)(\mathrm{M}, \mathrm{q})=(\mathrm{K}, 1)$; d) $(\mathrm{M}, \mathrm{q})=(\mathrm{Be}, 0)$; e) $(\mathrm{M}, \mathrm{q})=(\mathrm{Mg}, 0)$; f $(\mathrm{M}, \mathrm{q})=(\mathrm{Ca}, 0)$ calculated at the B3LYP/6-311+G(d) level. Energy values are in kcal/mol. "NIMAG” means the number of imaginary frequency of saddle point. ${ }^{\mathbf{m}} \mathbf{N}^{\mathbf{q}}$ : “ $\mathbf{m}$ ” means spin electron state, “ $\mathbf{N}$ ” represents the sequence number of various isomers, superscript "q-" means the charge of the total system. For simplicity, the notations "Ho-Li", “Ho-Na” "Ho-K” “Ho-Be” "Ho-Mg” and “Ho-Ca” for isomers are omitted. 


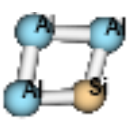

di

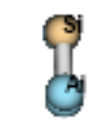

1(s-c-1) $)^{-}$

0.00

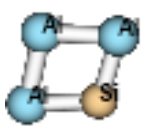

di

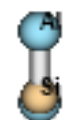

1(s-c-2)

3.26

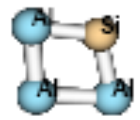

di

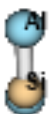

$1(\mathrm{~s}-\mathrm{s})^{-}$

6.56

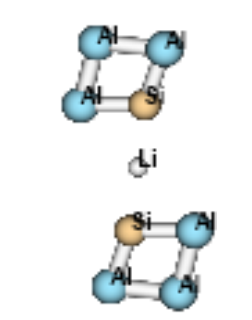

1(c-c-1)

(NIMAG=1)

0.00

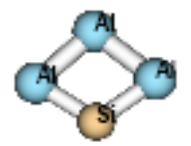

bi

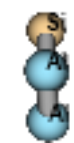

1(c-c-90) ${ }^{-}$

$(\mathrm{NIMAG}=3)$

0.61

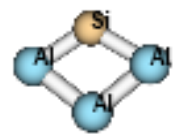

thi

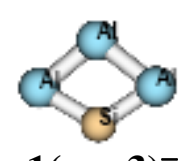

1(c-c-3)

(NIMAG=3)

14.06

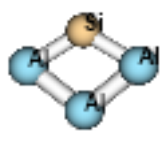

ti

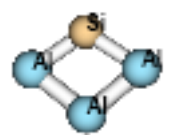

1(c-c-2)

(NIMAG=3)

7.19

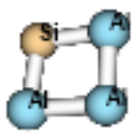

bi

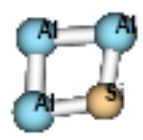

1(s-s-1) ${ }^{-}$

(NIMAG=1)

6.78

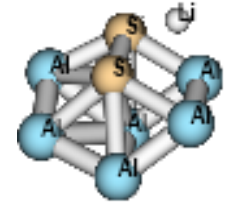

$-50.26$

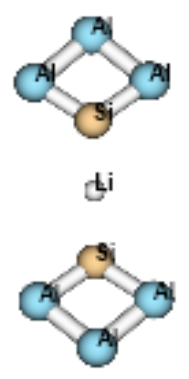

1(c-c-3)

$(\mathrm{NIMAG}=3)$

0.69

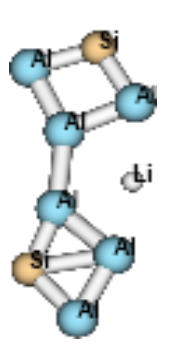

5.68

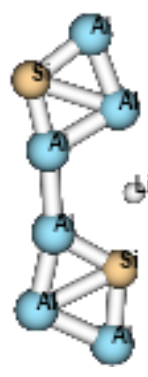

$-3.68$

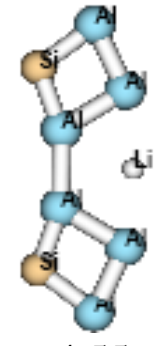

4.55

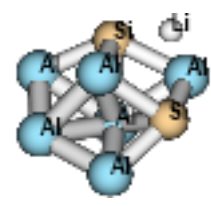

$-48.72$

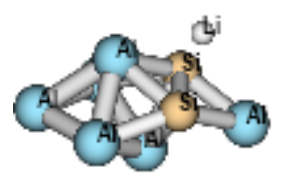

$-35.39$

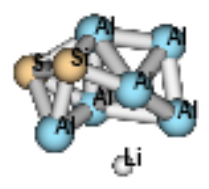

$-34.85$

a) $\left[\mathrm{Si}_{2} \mathrm{Al}_{6} \mathrm{Li}\right]^{-}$ 


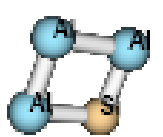

Na

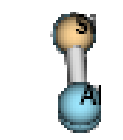

1(c-c-90)

0.00

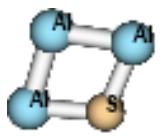

(4)

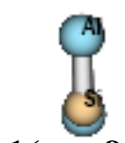

1(s-c-90)

2.55

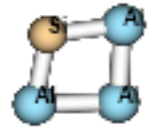

तs

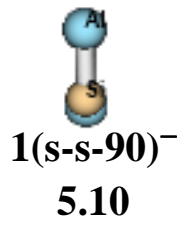

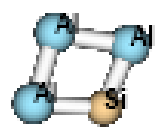

Ha

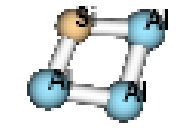

1(c-c-0-1) $)^{-}$

(NIMAG=1)

0.01
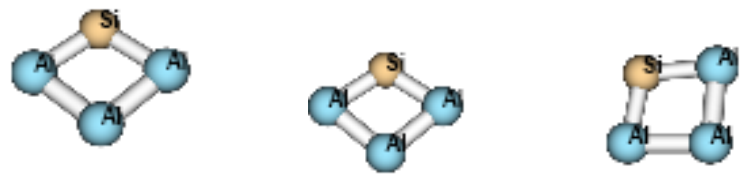

Cis

Na

Cla

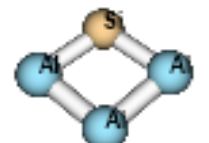

1(c-c-0-3) $\quad$ 1(c-c-0-2)

(NIMAG=3)

5.73
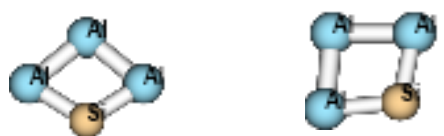

1(s-s-1) ${ }^{-}$

(NIMAG=3) $\quad($ NIMAG=1)

11.23

5.32
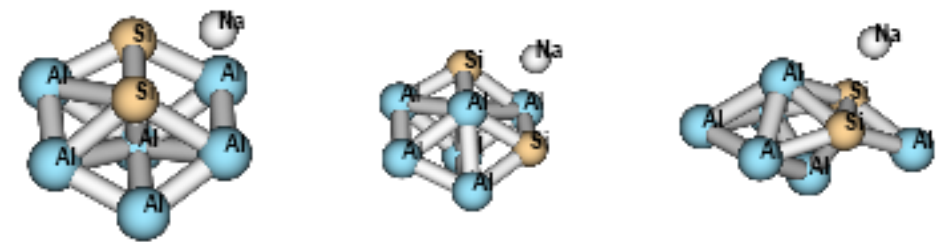

$-48.89$

$-46.15$

$-33.90$

b) $\left[\mathrm{Si}_{2} \mathrm{Al}_{6} \mathrm{Na}\right]^{-}$ 


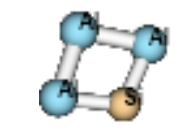

0

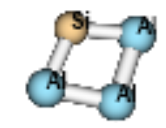

1(c-c-1)

0.00

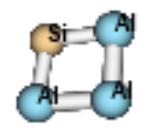

O

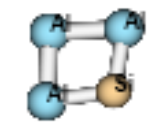

$$
\text { 1(s-s-1) }
$$

(NIMAG=1)

5.31

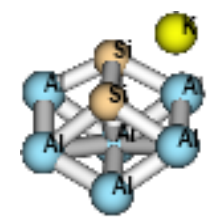

$-48.91$

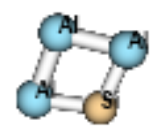

0

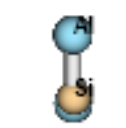

1(s-c-2)

2.55

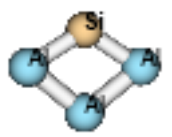

O

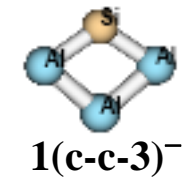

(NIMAG=3)

5.51

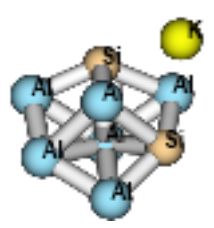

$-47.21$

c) $\left[\mathrm{Si}_{2} \mathrm{Al}_{6} \mathrm{~K}\right]^{-}$

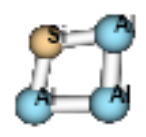

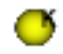

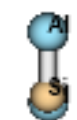

1(s-s)

5.17

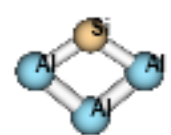

O

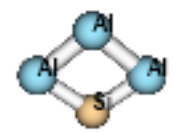

1(c-c-1)

(NIMAG=3)

10.96

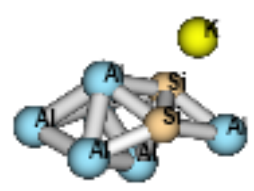

$-34.56$ 

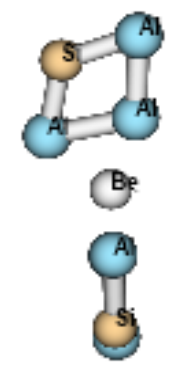

$$
\begin{gathered}
\text { 1(s-s-90) } \\
0.00
\end{gathered}
$$

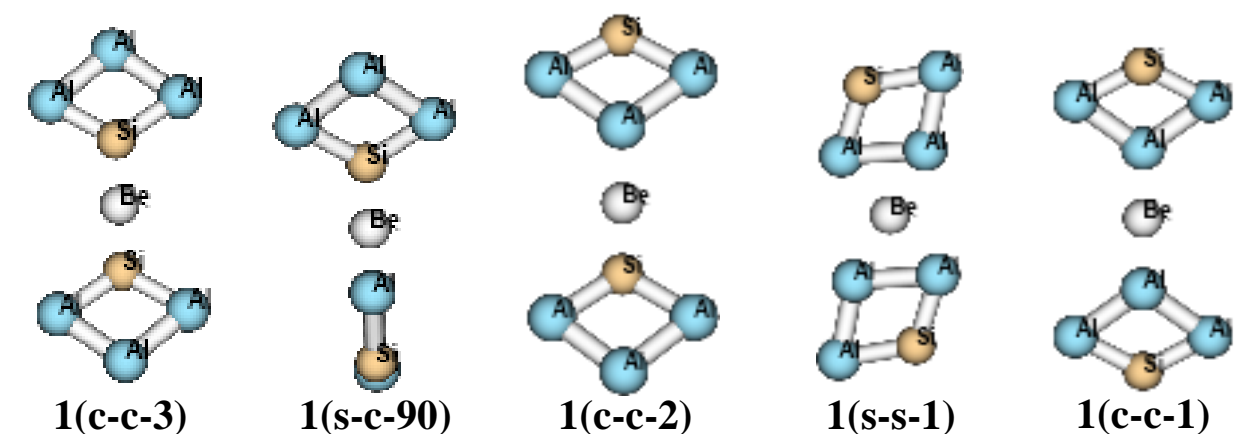

$(\mathrm{NIMAG}=4) \quad(\mathrm{NIMAG}=1) \quad(\mathrm{NIMAG}=3) \quad(\mathrm{NIMAG}=1) \quad(\mathrm{NIMAG}=3)$ $-9.41$

$-4.04$

2.53

3.34

15.85

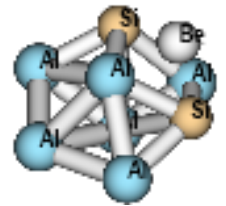

$-56.00$

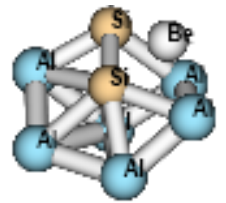

$-54.66$

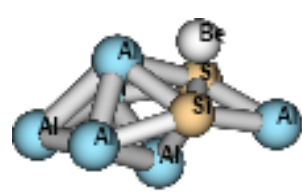

$-34.52$

d) $\left[\mathrm{Si}_{2} \mathrm{Al}_{6} \mathrm{Be}\right]$ 

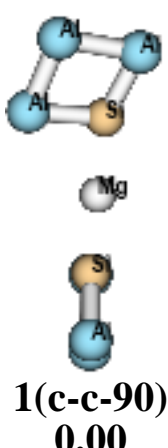
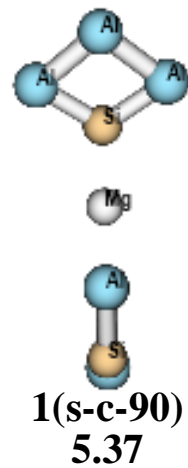

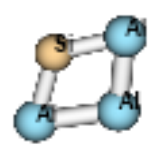

OIg

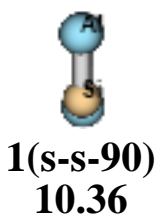

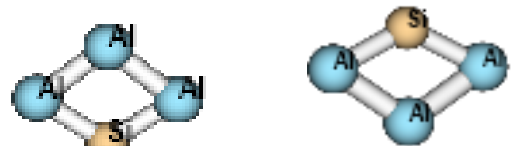
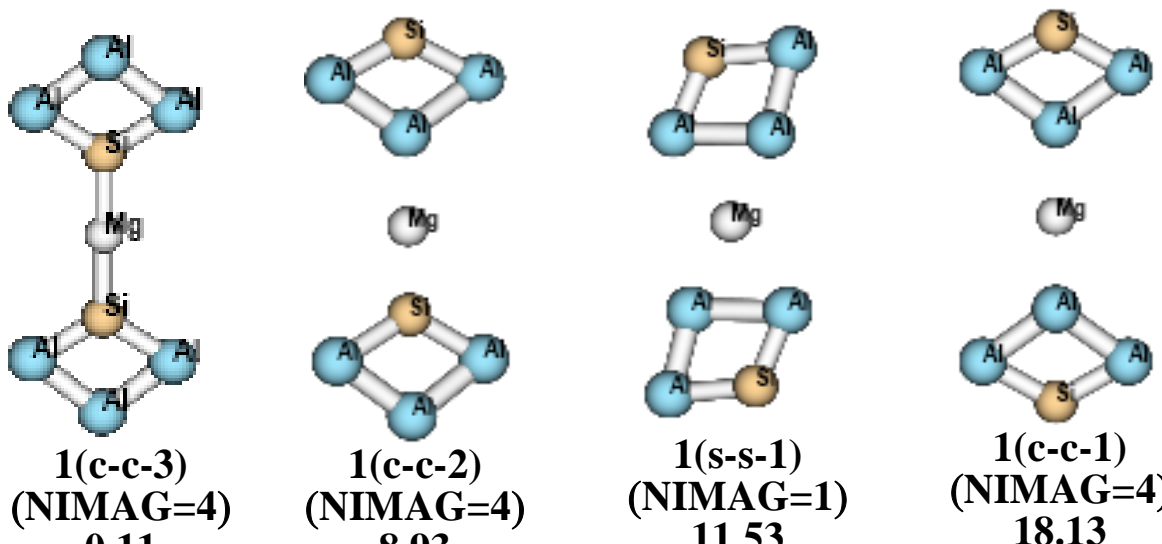

(1)
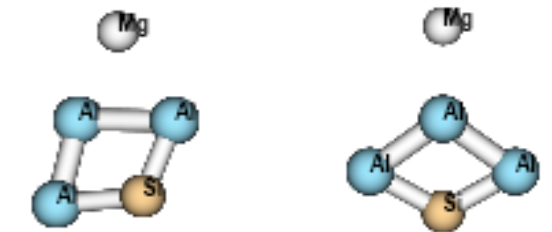

1(s-s-1)

(NIMAG=1)

11.53

(NIMAG=4)

8.93
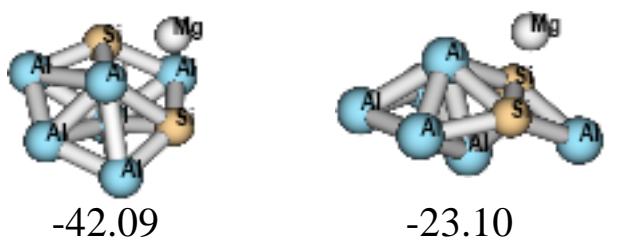

$-23.10$

e) $\left[\mathrm{Si}_{2} \mathrm{Al}_{6} \mathrm{Mg}\right]$ 


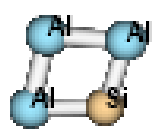

$\sigma^{\mathrm{a}}$

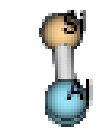

1(s-s-90-1)

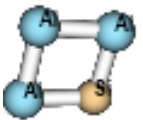

(3)

1(s-s-90-2)

6.20

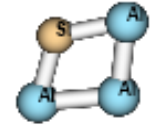

(3)

1(s-s-90-3)

12.68

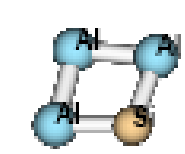

6a

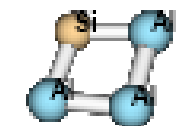

1(c-c-0)

(NIMAG=1)

0.22

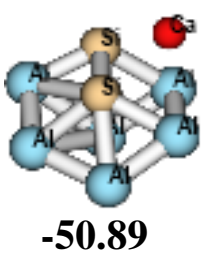

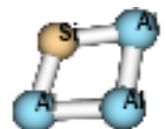
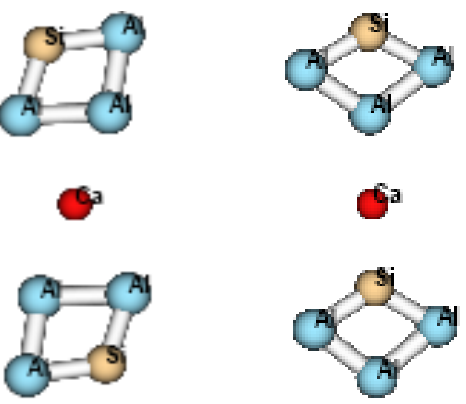

1(s-s-1)

$\underset{13.13}{(\mathrm{NIMAG}}=1)$
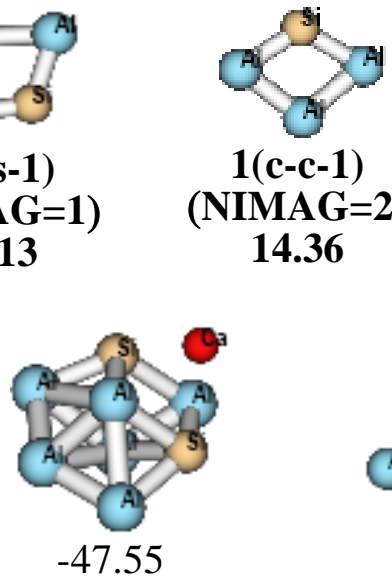

1(c-c-1)

$\underset{14.36}{\text { NIMAG }}=2)$

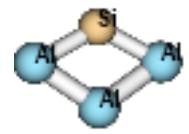

ga

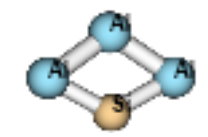

1(c-c-3)

(NIMAG=4)

26.31

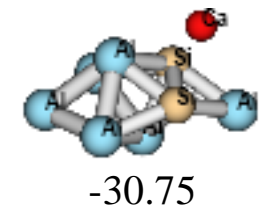

f) $\left[\mathrm{Si}_{2} \mathrm{Al}_{6} \mathrm{Ca}\right]$ 
Figure 2. Schematic energy profile for singlet hetero-decked compounds ${ }^{1}\left[\mathrm{CpM}\left(\mathrm{SiAl}_{3}\right)\right]^{q-}$ : a) $(M, q)=(L i, 1) ; b)(M, q)=(N a, 1) ; c)(M, q)=(K, 1) ; d)(M, q)=(B e, 0) ;$ e) $(M, q)=(M g, 0) ; f)(M, q)=(C a, 0)$ calculated at the B3LYP/6-311+G(d) level. Energy values are in kcal/mol. "f-f" stands for face-to-face sandwich form, "f-s" stands for face-to-side sandwich form, "f-c" stands for face-to-corner sandwich form. For simplicity, the notations "Het-Li", "Het-Na” "Het-K” "Het-Be" "Het-Mg" and "Het-Ca" for isomers are omitted.

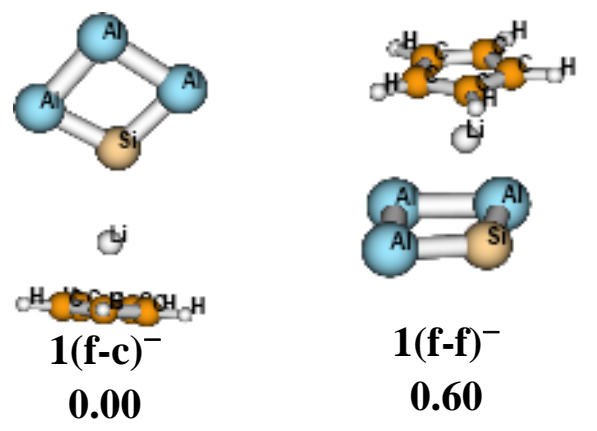

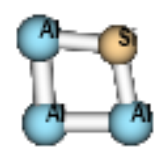

bi

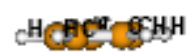
1(f-s) ${ }^{-}$

2.33
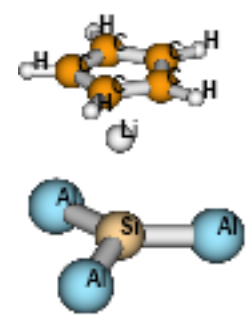

6.37

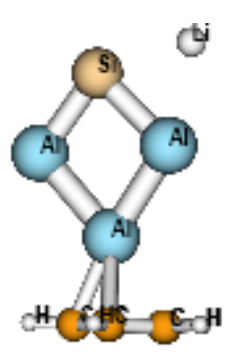

22.42

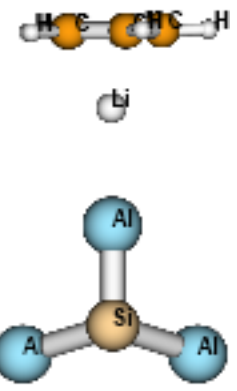

7.34

Hifregey $-\mathrm{H}$

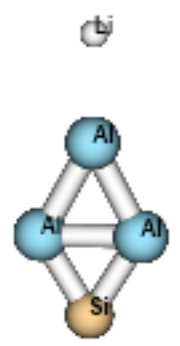

22.85

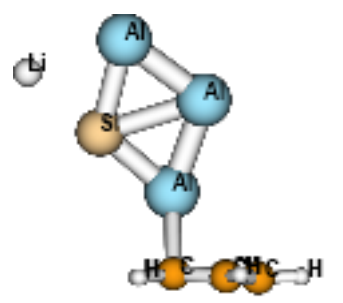

21.12

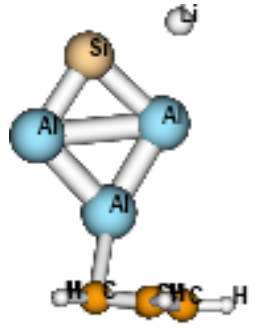

22.35

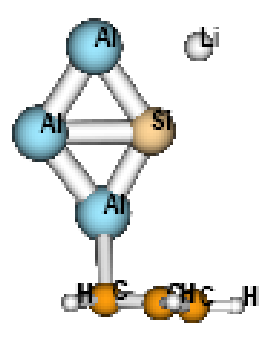

23.16

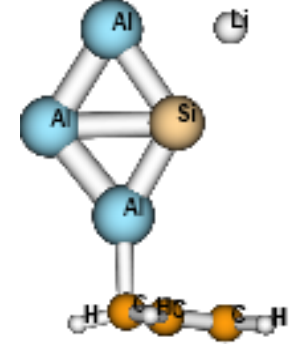

23.38

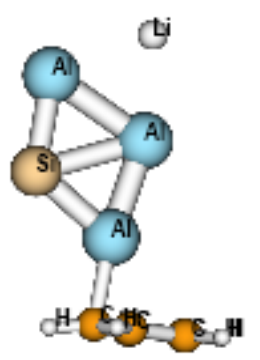

23.48

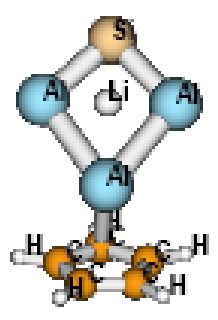

26.65

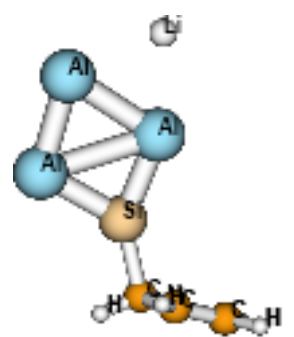

44.96

\section{a) $\left[\mathrm{CpLi}\left(\mathrm{SiAl}_{3}\right)\right]^{-}$}




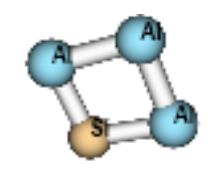

\%

arestas-H

$1(\mathbf{f}-\mathrm{c})^{-}$

0.00
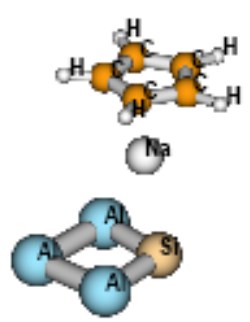

1(f-f)

0.48
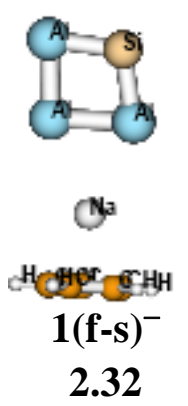

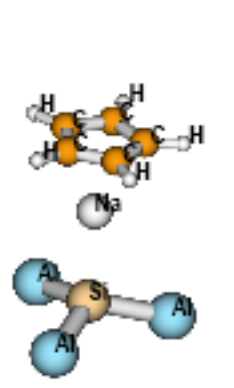

6.24

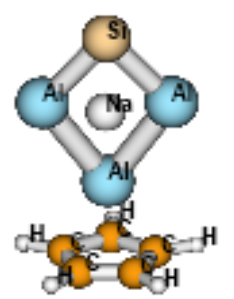

14.37

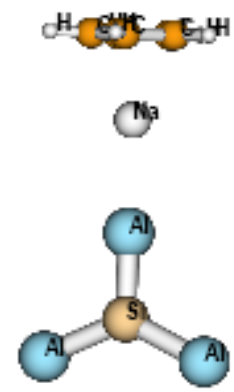

8.98

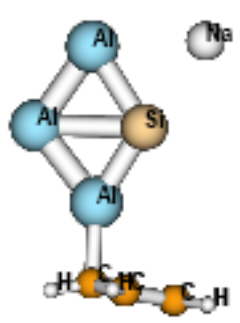

14.56

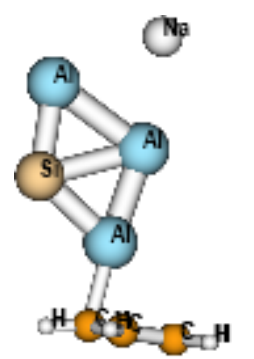

12.93

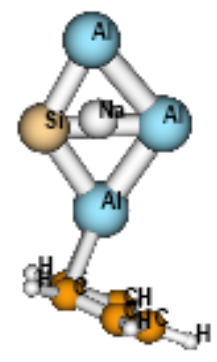

14.60

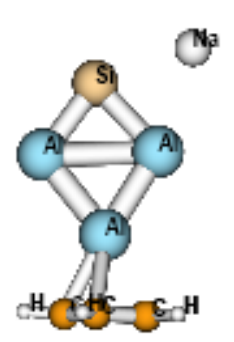

13.98

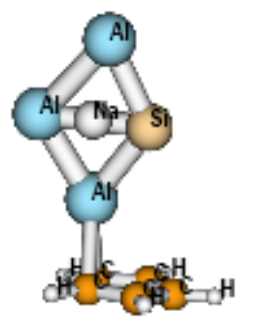

17.50

H.

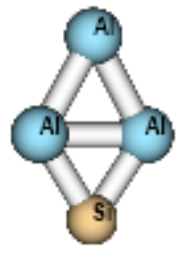

23.54

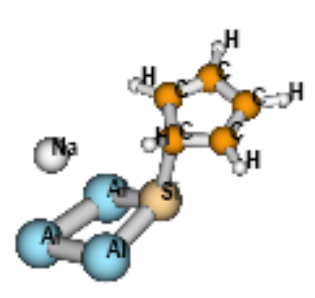

32.43

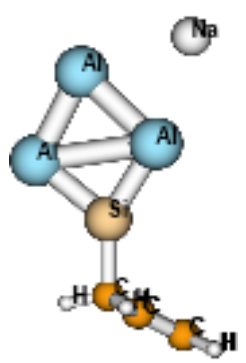

33.13

b) $\left[\mathrm{CpNa}\left(\mathrm{SiAl}_{3}\right)\right]^{-}$ 


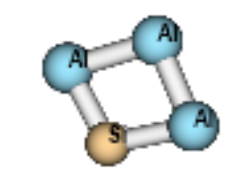

C

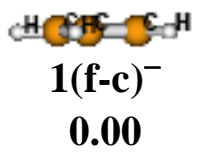

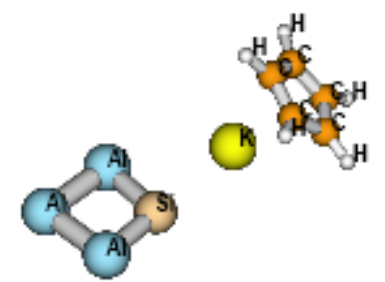

1(f-f-slope) ${ }^{-}$

0.11
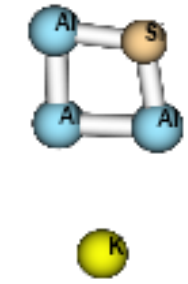

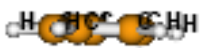

1(f-s)

2.12

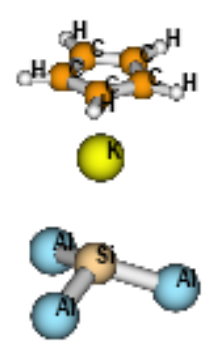

6.60

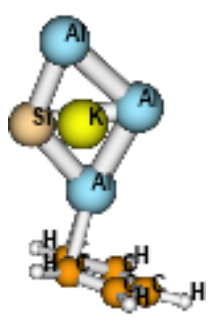

17.36

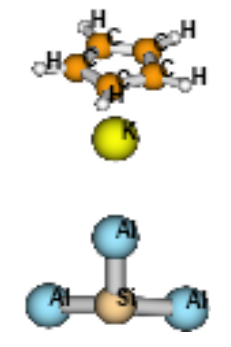

9.69

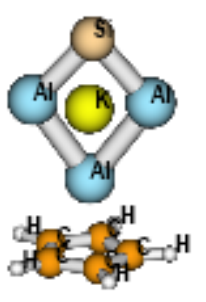

17.98

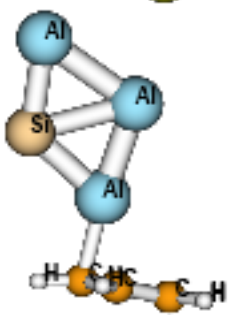

17.22

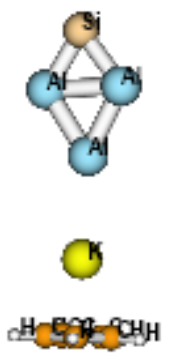

24.02

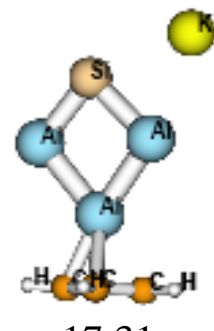

17.31
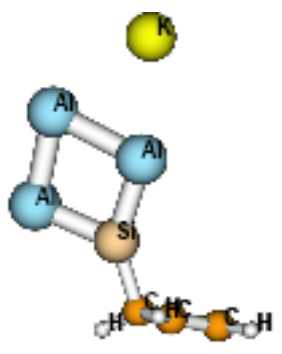

36.30

c) $\left[\mathrm{CpK}\left(\mathrm{SiAl}_{3}\right)\right]^{-}$ 


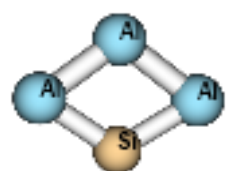

(B)

HCAtS-C-H

1(f-c)

0.00

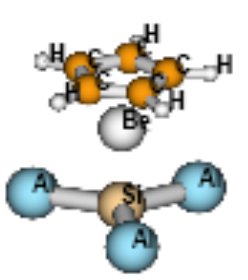

6.43

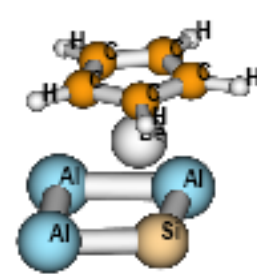

1(f-f)

2.82

HACOPGH'

(B)

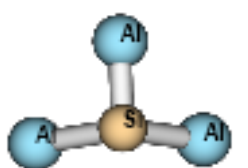

9.28

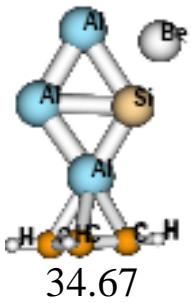

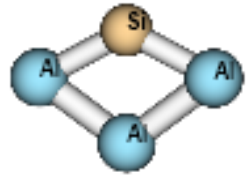

(B)

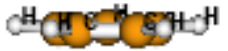

1(f-c)

7.93

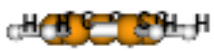

(B)

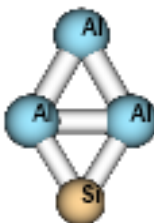

23.21
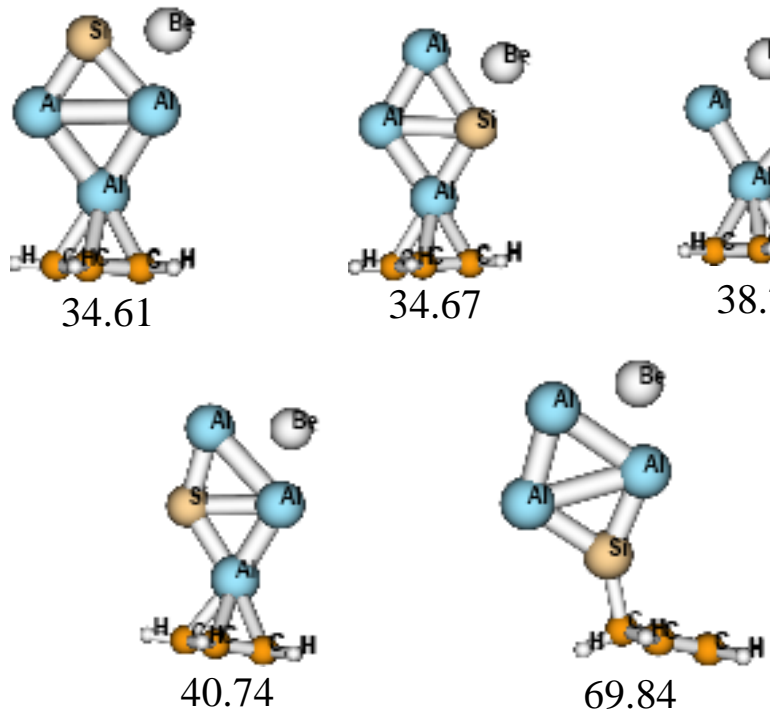

69.84

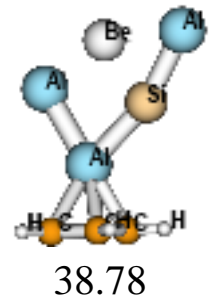

d) $\left[\mathrm{CpBe}\left(\mathrm{SiAl}_{3}\right)\right]$ 
Figure 3. Schematic energy profile for singlet saturated hetero-decked compounds ${ }^{1}(\mathrm{M})^{+}\left[\mathrm{CpM}\left(\mathrm{Al}_{3} \mathrm{Si}\right)\right]^{-}(\mathrm{M}=\mathrm{Li}, \mathrm{Na}, \mathrm{K})$ calculated at the B3LYP/6-311+G(d) level. Energy values are in kcal/mol. For simplicity, the notations "Het-Li", "Het-Na" "Het-K" for isomers are omitted.

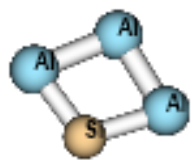

tbi bi

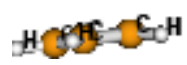

0.00

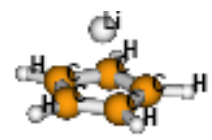

bi

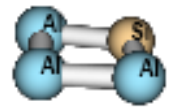

0.64

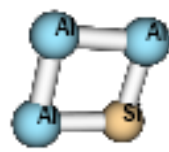

bi

Hitegerent

di

0.89

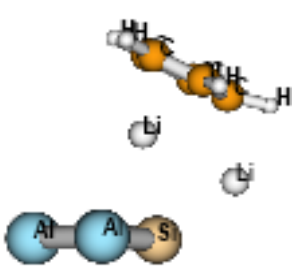

1.44

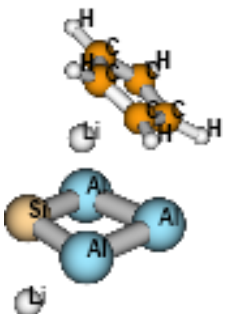

12.21

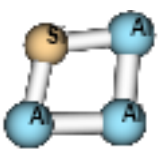

bi

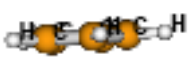

bi

5.47

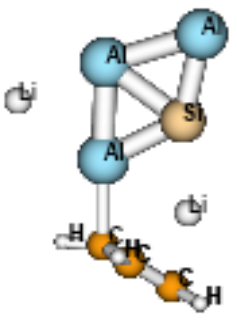

12.98

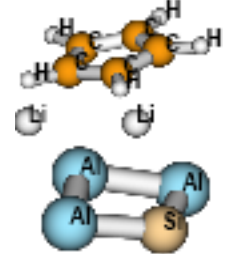

6.61

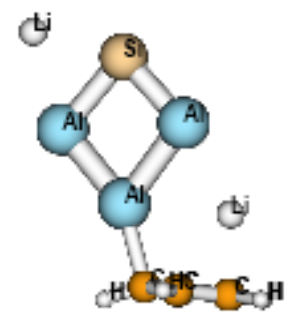

16.31

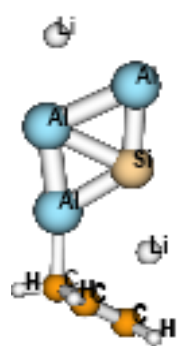

9.79

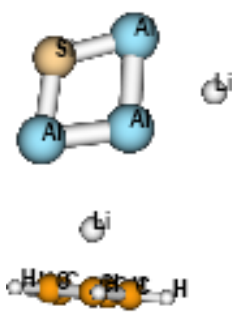

19.79

a) $(\mathrm{Li})^{+}\left[\mathrm{CpLi}\left(\mathrm{SiAl}_{3}\right)\right]^{-}$ 


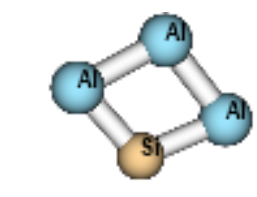

(⿻)

H H

0.00

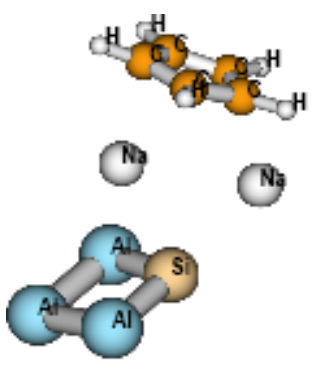

0.27

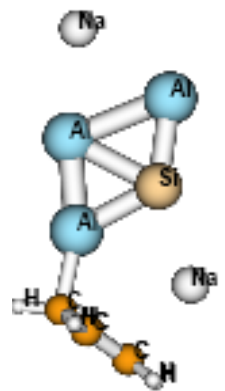

1.05

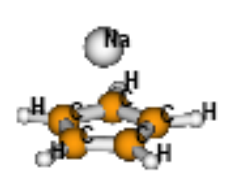

(1)

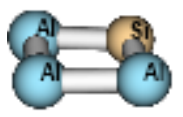

3.07
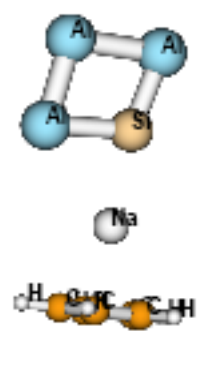

CHa

4.25

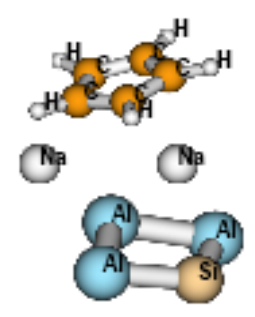

4.49

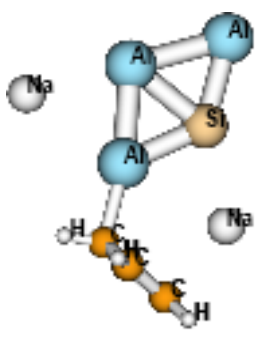

5.06

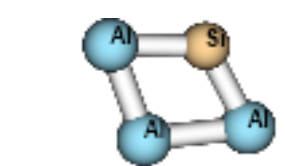

(1) HCOAS - C $-\mathrm{H}$

6.47

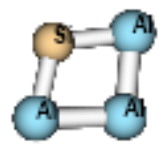

(1)

Hic ofte ${ }^{H}$

$\begin{array}{r}7.59 \\ \hline\end{array}$
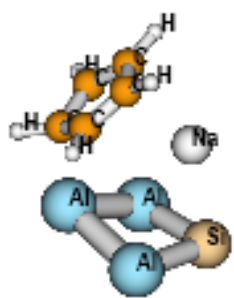

(N)

7.95

b) $(\mathrm{Na})^{+}\left[\mathrm{CpNa}\left(\mathrm{SiAl}_{3}\right)\right]^{-}$ 


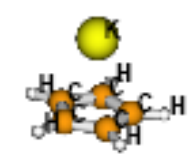

(1)

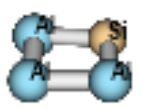

0.00

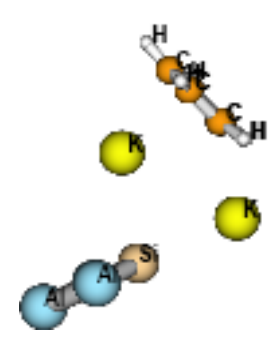

1.33

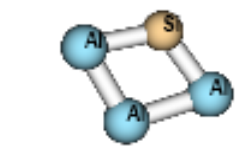

(b) 0

${ }_{4}\left(C^{3} C^{H}\right.$

7.61

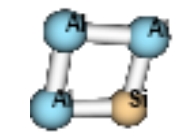

0

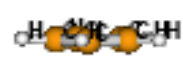

(

0.47
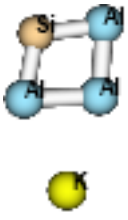

HCAt $\mathrm{H}$

$\bigcirc$
3.78

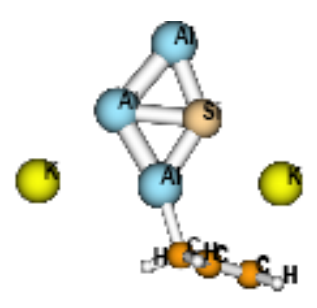

9.17
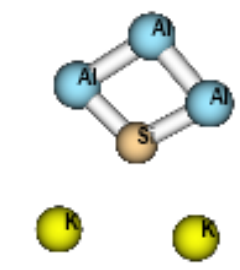

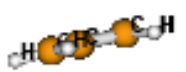

0.85

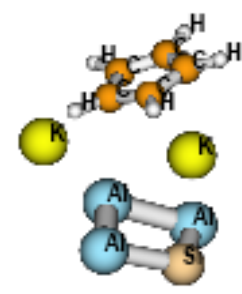

5.66

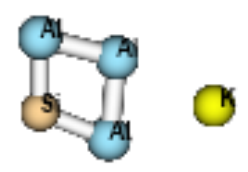

0

CHSf

15.34

c) $(\mathrm{K})^{+}\left[\mathrm{CpK}\left(\mathrm{SiAl}_{3}\right)\right]^{-}$ 
Figure 4. Various extend species $(\mathrm{CpLi})_{\mathrm{m}}\left(\mathrm{SiAl}_{3}{ }^{-}\right)_{\mathrm{n}}$ were designed and obtained as energy minima, they were calculated at the B3LYP/6-31+G(d) level. Energy values are in kcal/mol.

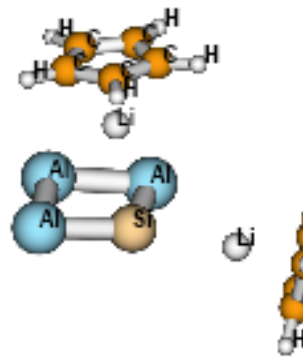

0.00

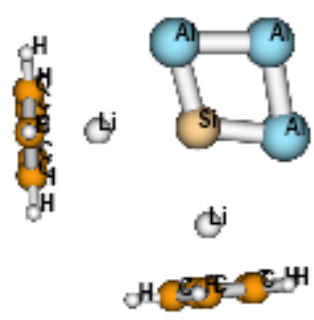

0.23

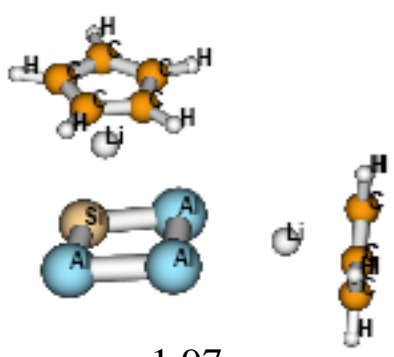

1.97

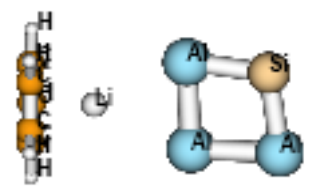

b) atedert ${ }^{H}$

4.15

a) $\left[C p_{2} \mathrm{Li}_{2}\left(\mathrm{SiAl}_{3}{ }^{-}\right)\right]$

$\sum_{0}^{H}$<smiles>c1ccoc1</smiles>

di

ch

bi Heresteger

b) $\left[\mathrm{Cp}_{3} \mathrm{Li}_{3}\left(\mathrm{SiAl}_{3}{ }^{-}\right)\right]$

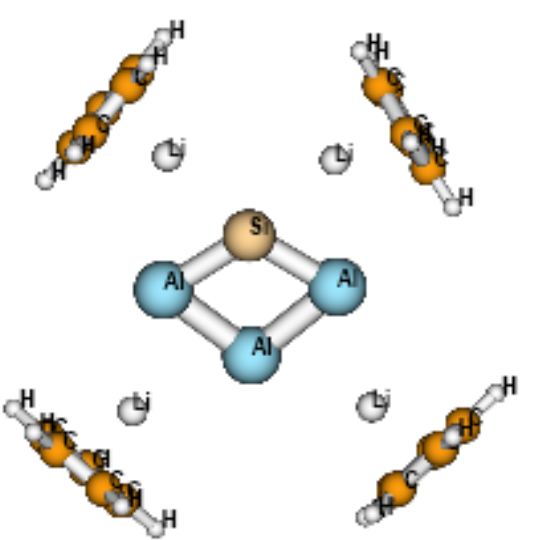

c) $\left[\mathrm{Cp}_{4} \mathrm{Li}_{4}\left(\mathrm{SiAl}_{3}{ }^{-}\right)\right]$ 


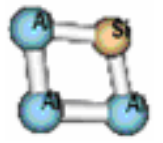

Summary of Natural Population Analysis:

$\wedge \mathrm{M}$

Natural Population $\wedge \mathrm{M}$

\begin{tabular}{|c|c|c|c|c|c|c|}
\hline Atom & No & $\begin{array}{l}\text { Natural } \\
\text { Charge }\end{array}$ & Core & Valence & Rydberg & Total $\wedge \mathrm{M}$ \\
\hline $\mathrm{Al}$ & 1 & 0.02708 & 999785 & 2.93819 & 0.03688 & $12.97292^{\wedge} \mathrm{M}$ \\
\hline $\mathrm{Al}$ & 2 & -0.17974 & 9.99828 & 3.15043 & 0.03102 & $13.17974 \wedge \mathrm{M}$ \\
\hline $\mathrm{Al}$ & 3 & 0.02708 & 9.99785 & 2.93819 & 0.03688 & $12.97292 \wedge \mathrm{M}$ \\
\hline $\mathrm{Si}$ & 4 & -0.87442 & 9.99864 & 4.84895 & 0.02683 & $14.87442^{\wedge} \mathrm{M}$ \\
\hline
\end{tabular}

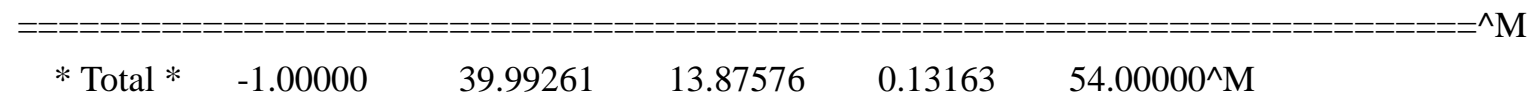

\section{SiAl3-NICS-b36311+gx.log}

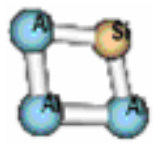

\begin{tabular}{|c|c|c|c|c|c|}
\hline $\mathrm{Bq}$ & Isotropic $=$ & 34.2975 & \multicolumn{2}{|c|}{ Anisotropy = } & $47.7026 \wedge \mathrm{M}$ \\
\hline $\mathrm{XX}=$ & 66.0993 & $\mathrm{YX}=$ & 0.0000 & $\mathrm{ZX}=$ & $0.0000^{\wedge} \mathrm{M}$ \\
\hline$X Y=$ & 0.0000 & $Y Y=$ & 18.2307 & $\mathrm{ZY}=$ & $-0.0001^{\wedge} \mathrm{M}$ \\
\hline$X Z=$ & 0.0000 & $\mathrm{YZ}=$ & 0.0000 & $\mathrm{ZZ}=$ & $18.562 \wedge^{\wedge} \mathrm{M}$ \\
\hline
\end{tabular}

Eigenvalues: $\quad 18.2307 \quad 18.5626 \quad 66.0993^{\wedge} \mathrm{M}$

$6 \mathrm{~Bq} \quad$ Isotropic $=26.5645$ Anisotropy $=39.4017 \wedge \mathrm{M}$

\section{CpLiAl3Si-f-s-Al-Al-b36311+gx-NBO.log}

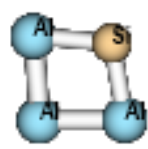

bi

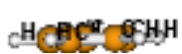

Summary of Natural Population Analysis:

Natural Population

\begin{tabular}{rrrrrrr} 
& \multicolumn{7}{c}{ Natural } & - & & \\
Atom & No & Charge & Core & Valence & Rydberg & Total \\
\hline Al & 1 & 0.00689 & 9.99763 & 2.95867 & 0.03681 & 12.99311 \\
Al & 2 & 0.12145 & 9.99757 & 2.84986 & 0.03112 & 12.87855 \\
C & 3 & -0.37684 & 1.99916 & 4.35137 & 0.02631 & 6.37684 \\
C & 4 & -0.37853 & 1.99916 & 4.35286 & 0.02651 & 6.37853 \\
C & 5 & -0.38291 & 1.99916 & 4.35688 & 0.02687 & 6.38291 \\
H & 6 & 0.19988 & 0.00000 & 0.79927 & 0.00085 & 0.80012 \\
C & 7 & -0.38202 & 1.99916 & 4.35609 & 0.02677 & 6.38202
\end{tabular}




$\begin{array}{ccccccc}\mathrm{H} & 8 & 0.19973 & 0.00000 & 0.79945 & 0.00082 & 0.80027 \\ \mathrm{C} & 9 & -0.37878 & 1.99916 & 4.35326 & 0.02636 & 6.37878 \\ \mathrm{H} & 10 & 0.19962 & 0.00000 & 0.79960 & 0.00079 & 0.80038 \\ \mathrm{H} & 11 & 0.20009 & 0.00000 & 0.79912 & 0.00079 & 0.79991 \\ \mathrm{H} & 12 & 0.20147 & 0.00000 & 0.79767 & 0.00085 & 0.79853 \\ \mathrm{Li} & 13 & 0.80626 & 1.99665 & 0.16333 & 0.03376 & 2.19374 \\ \mathrm{Al} & 14 & -0.20911 & 9.99808 & 3.17675 & 0.03427 & 13.20911 \\ \mathrm{Si} & 15 & -0.82720 & 9.99848 & 4.80603 & 0.02269 & 14.82720 \\ ======================================================= \\ \text { * Total * } & -1.00000 & 51.98421 & 39.72021 & 0.29558 & 92.00000\end{array}$

The charge of SiAl3 unit is $=0.00689+0.12145-0.20911-0.82720=-0.90797$

The charge of Cp unit is

$=-0.37684-0.37853-0.38291+0.19988-0.38202+0.19973-0.37878+0.19962+0.20009+0.20147=-0.89829$

CpLiAl3Si-f-s-Al-Al-b36311+gx-NICS.log

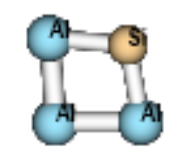

\section{di}

$\operatorname{NICS}(0)=-37.0816$

$\operatorname{NICS}(1)=-28.1654$

\begin{tabular}{cccccc}
16 & $\mathrm{~Bq}$ & Isotropic $=$ & \multicolumn{2}{c}{37.0816} & \multicolumn{2}{c}{ Anisotropy $=$} & 47.0121 \\
$\mathrm{XX}=$ & 21.2567 & $\mathrm{YX}=$ & -1.1949 & $\mathrm{ZX}=$ & 0.2680 \\
$\mathrm{XY}=$ & -1.0516 & $\mathrm{YY}=$ & 21.5669 & $\mathrm{ZY}=$ & -0.0424 \\
$\mathrm{XZ}=$ & 0.3205 & $\mathrm{YZ}=$ & -0.0504 & $\mathrm{ZZ}=$ & 68.4211 \\
Eigenvalues: & 20.2772 & 22.5446 & \multicolumn{2}{c}{68.4230}
\end{tabular}

\begin{tabular}{|c|c|c|c|c|c|}
\hline $7 \quad \mathrm{~Bq}$ & Isotropic = & 28.1654 & \multicolumn{2}{|c|}{ Anisotropy = } & 39.4692 \\
\hline $\mathrm{XX}=$ & 15.8046 & $Y X=$ & 1.0803 & $\mathrm{ZX}=$ & -0.0918 \\
\hline$X Y=$ & 0.7494 & $Y Y=$ & 14.2500 & $\mathrm{ZY}=$ & -0.4673 \\
\hline $\mathrm{XZ}=$ & -1.7551 & $\mathrm{YZ}=$ & 2.0447 & $\mathrm{ZZ}=$ & 54.4415 \\
\hline Eige & & .7968 & 16.2212 & 54.478 & \\
\hline
\end{tabular}

$18 \mathrm{~Bq} \quad$ Isotropic $=28.1026 \quad$ Anisotropy $=39.4170$

CpNaAl3Si-f-c-Si-b36311+gx-NICS.log

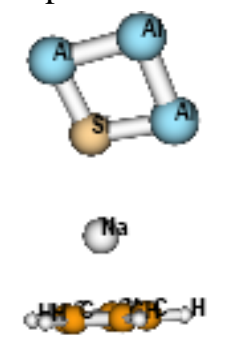

$\operatorname{NICS}(0)=-35.4754$

$\operatorname{NICS}(1)=-26.8889$ 


\begin{tabular}{|c|c|c|c|c|c|}
\hline $5 \quad \mathrm{~Bq}$ & Isotropic = & 35.4754 & \multicolumn{2}{|c|}{ Anisotropy = } & 44.3896 \\
\hline $\mathrm{XX}=$ & 21.7339 & $\mathrm{YX}=$ & -2.1545 & $\mathrm{ZX}=$ & 0.0325 \\
\hline$X Y=$ & -3.2963 & $Y Y=$ & 19.6239 & $\mathrm{ZY}=$ & 0.0022 \\
\hline $\mathrm{XZ}=$ & 0.0328 & $\mathrm{YZ}=$ & -0.0011 & $\mathrm{ZZ}=$ & 65.0684 \\
\hline Eige & & 564 & 23.6013 & 65. & \\
\hline
\end{tabular}

$17 \mathrm{~Bq} \quad$ Isotropic $=26.8889 \quad$ Anisotropy $=38.4127$ CpNaAl3Si-f-f-to-f-f-c-Si-b36311+gx-NBO.log

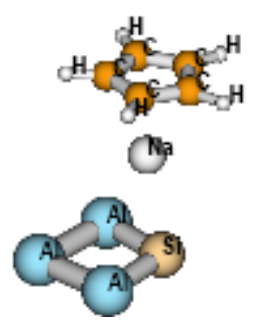

Summary of Natural Population Analysis:

\begin{tabular}{|c|c|c|c|c|c|c|}
\hline \multirow[b]{2}{*}{ Atom } & \multirow[b]{2}{*}{ No } & \multirow{2}{*}{$\begin{array}{l}\text { Natural } \\
\text { Charge }\end{array}$} & \multicolumn{3}{|c|}{ Natural Population } & \multirow[b]{2}{*}{ Total } \\
\hline & & & Core & Valence & Rydberg & \\
\hline $\mathrm{C}$ & 1 & -0.37971 & 1.99917 & 4.35557 & 0.02496 & 6.37971 \\
\hline $\mathrm{C}$ & 2 & -0.37821 & 1.99917 & 4.35454 & 0.02450 & 6.37821 \\
\hline $\mathrm{C}$ & 3 & -0.37820 & 1.99917 & 4.35446 & 0.02457 & 6.37820 \\
\hline $\mathrm{C}$ & 4 & -0.37878 & 1.99917 & 4.35511 & 0.02450 & 6.37878 \\
\hline $\mathrm{C}$ & 5 & -0.38003 & 1.99917 & 4.35589 & 0.02496 & 6.38003 \\
\hline $\mathrm{H}$ & 6 & 0.19148 & 0.00000 & 0.80773 & 0.00079 & 0.80852 \\
\hline $\mathrm{H}$ & 7 & 0.19203 & 0.00000 & 0.80718 & 0.00078 & 0.80797 \\
\hline $\mathrm{H}$ & 8 & 0.19238 & 0.00000 & 0.80683 & 0.00079 & 0.80762 \\
\hline $\mathrm{H}$ & 9 & 0.19203 & 0.00000 & 0.80719 & 0.00078 & 0.80797 \\
\hline $\mathrm{H}$ & 10 & 0.19150 & 0.00000 & 0.80771 & 0.00079 & 0.80850 \\
\hline $\mathrm{Al}$ & 11 & -0.06167 & 9.99828 & 3.03835 & 0.02503 & 13.06167 \\
\hline $\mathrm{Al}$ & 12 & 0.12159 & 9.99779 & 2.84795 & 0.03266 & 12.87841 \\
\hline $\mathrm{Al}$ & 13 & 0.09446 & 9.99781 & 2.87501 & 0.03272 & 12.90554 \\
\hline $\mathrm{Na}$ & 14 & 0.83911 & 9.99749 & 0.13343 & 0.02997 & 10.16089 \\
\hline $\mathrm{Si}$ & 15 & -1.05799 & 9.99872 & 5.03050 & 0.02876 & 15.05799 \\
\hline
\end{tabular}

$\begin{array}{llllll}* \text { Total * } & -1.00000 & 59.98597 & 39.73745 & 0.27658 & 100.00000\end{array}$

The charge of SiAl3 unit is $=-0.06167+0.12159+0.09446-1.05799=-0.90361$

The charge of Cp unit is

$=-0.37971-0.37821-0.37820-0.37878-0.38003+0.19148+0.19203+0.19238+0.19203+0.19150=-0.93551$

CpNaAl3Si-f-f-to-f-f-c-Si-b36311+gx-NICS.log 


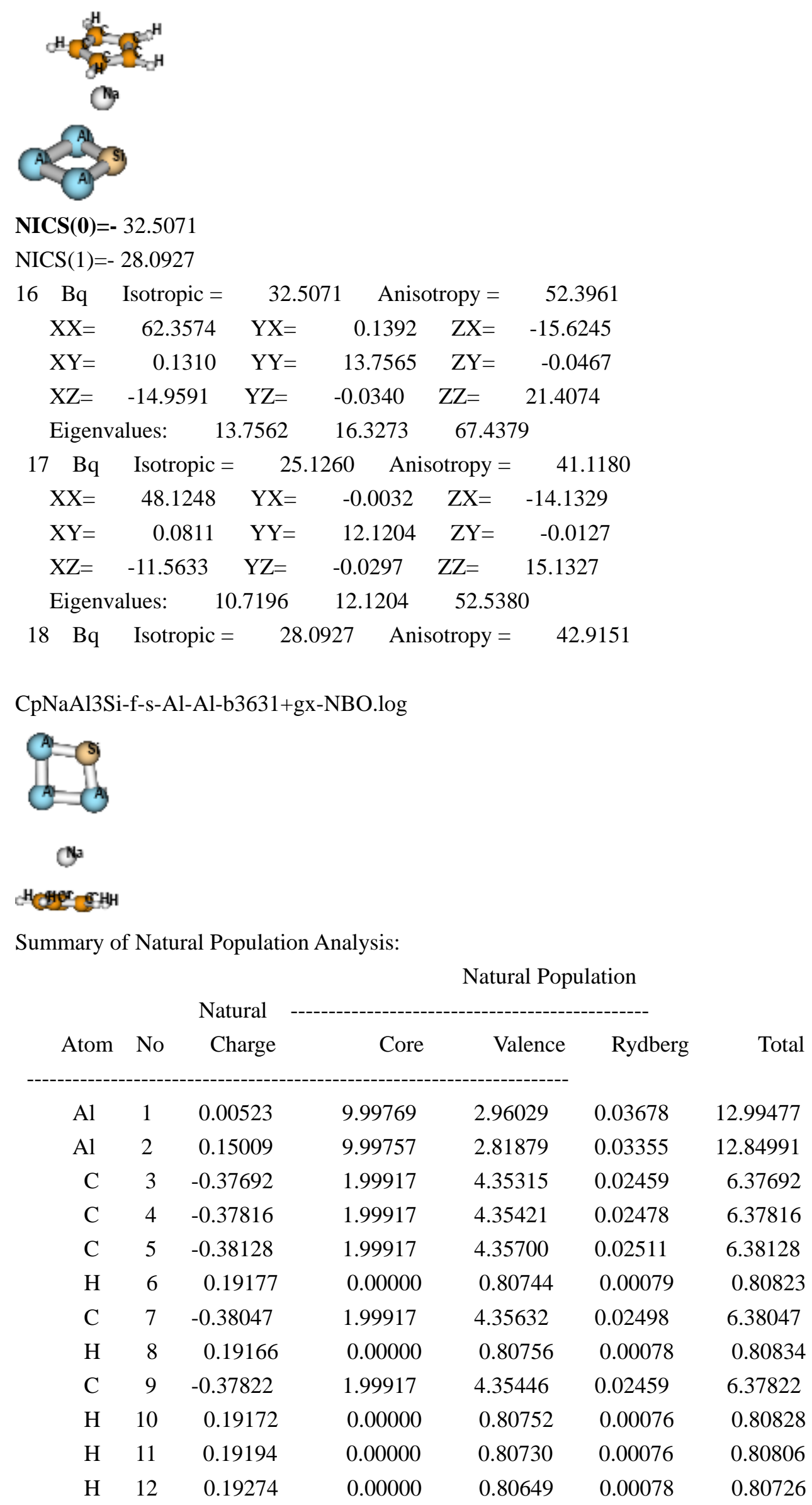




$\begin{array}{ccccccc}\mathrm{Na} & 13 & 0.85296 & 9.99574 & 0.12573 & 0.02558 & 10.14704 \\ \mathrm{Al} & 14 & -0.25158 & 9.99810 & 3.21969 & 0.03379 & 13.25158 \\ \mathrm{Si} & 15 & -0.82148 & 9.99849 & 4.79961 & 0.02338 & 14.82148\end{array}$

$\begin{array}{llllll}* \text { Total * } & -1.00000 & 59.98346 & 39.73555 & 0.28099 & 100.00000\end{array}$

The charge of SiAl3 $=0.00523+0.15009-0.25158-0.82148=-0.91774$

The charge of

$\mathrm{CP}=-0.37692-0.37816-0.38128+0.19177-0.38047+0.19166-0.37822+0.19172+0.19194+0.19274=-0.93522$

CpNaAl3Si-f-s-Al-Al-b3631+gx-NICS.log

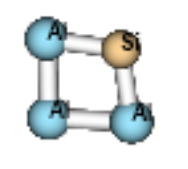

Oya

새er- $\mathrm{CHH}$

$\operatorname{NICS}(\mathbf{0})=-36.8983$

$\operatorname{NICS(1)=-27.9799}$

$16 \mathrm{~Bq}$ Isotropic $=36.8983$ Anisotropy $=46.3885$

$\mathrm{XX}=21.0218 \quad \mathrm{YX}=\quad-1.6138 \quad \mathrm{ZX}=0.0365$

$\mathrm{XY}=-1.3760 \quad \mathrm{YY}=21.8491 \quad \mathrm{ZY}=\quad-0.0059$

$\mathrm{XZ}=\quad 0.0453 \quad \mathrm{YZ}=\quad-0.0042 \quad \mathrm{ZZ}=67.8239$

Eigenvalues: $\quad 19.8843 \quad 22.9865 \quad 67.8240$

$17 \mathrm{~Bq} \quad$ Isotropic $=27.9799 \quad$ Anisotropy $=39.0805$ CpNa2Al3Si-f-s-s-2Si-Al-b36311+gx-NICS.log

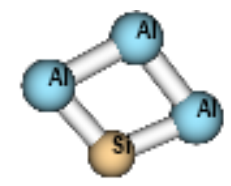

N

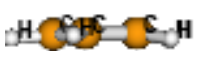

$\operatorname{NICS}(0)=-37.0323$

$\operatorname{NICS}(1)=-26.9994$

$17 \mathrm{~Bq}$ Isotropic $=37.0323$ Anisotropy $=42.7379$

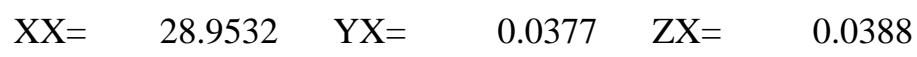

$\mathrm{XY}=\quad-0.1725 \quad \mathrm{YY}=16.6195 \quad \mathrm{ZY}=0.0685$

$\mathrm{XZ}=0.0248 \quad \mathrm{YZ}=\quad 0.0654 \quad \mathrm{ZZ}=65.5241$

Eigenvalues: $\quad 16.6191 \quad 28.9536 \quad 65.5242$

$18 \mathrm{~Bq}$ Isotropic $=26.9994 \quad$ Anisotropy $=39.4752$

KCpKAl3Si-f-f-f-b36311+gx-NBO.log 


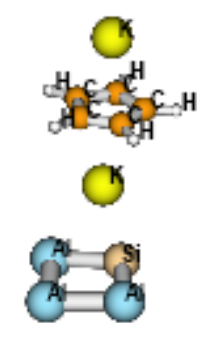

Summary of Natural Population Analysis:

Natural Population

\begin{tabular}{|c|c|c|c|c|c|c|}
\hline Atom & No & $\begin{array}{c}\text { Natural } \\
\text { Charge }\end{array}$ & Core & Valence & Rydberg & Total \\
\hline $\mathrm{C}$ & 1 & -0.40378 & 1.99917 & 4.37708 & 0.02753 & 6.40378 \\
\hline $\mathrm{C}$ & 2 & -0.40316 & 1.99917 & 4.37686 & 0.02713 & 6.40316 \\
\hline $\mathrm{C}$ & 3 & -0.40288 & 1.99917 & 4.37668 & 0.02703 & 6.40288 \\
\hline $\mathrm{C}$ & 4 & -0.40314 & 1.99917 & 4.37684 & 0.02713 & 6.40314 \\
\hline $\mathrm{C}$ & 5 & -0.40378 & 1.99917 & 4.37707 & 0.02753 & 6.40378 \\
\hline $\mathrm{H}$ & 6 & 0.21301 & 0.00000 & 0.78635 & 0.00064 & 0.78699 \\
\hline $\mathrm{H}$ & 7 & 0.21254 & 0.00000 & 0.78680 & 0.00066 & 0.78746 \\
\hline $\mathrm{H}$ & 8 & 0.21230 & 0.00000 & 0.78701 & 0.00069 & 0.78770 \\
\hline $\mathrm{H}$ & 9 & 0.21254 & 0.00000 & 0.78680 & 0.00066 & 0.78746 \\
\hline $\mathrm{H}$ & 10 & 0.21301 & 0.00000 & 0.78635 & 0.00064 & 0.78699 \\
\hline $\mathrm{Al}$ & 11 & -0.05616 & 9.99828 & 3.03313 & 0.02475 & 13.05616 \\
\hline $\mathrm{Al}$ & 12 & 0.09790 & 9.99780 & 2.87211 & 0.03219 & 12.90210 \\
\hline $\mathrm{Al}$ & 13 & 0.09997 & 9.99780 & 2.87003 & 0.03219 & 12.90003 \\
\hline K & 14 & 0.85752 & 17.99271 & 0.12646 & 0.02331 & 18.14248 \\
\hline $\mathrm{Si}$ & 15 & -1.01811 & 9.99870 & 4.99271 & 0.02669 & 15.01811 \\
\hline K & 16 & 0.97221 & 17.99675 & 0.00777 & 0.02328 & 18.02779 \\
\hline
\end{tabular}

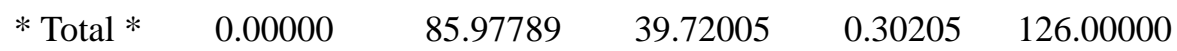

The charge of SiAl3=-0.05616 $+0.09790+0.09997-1.01811=-0.8764$

The

charge

of

$\mathrm{CP}=-0.40378-0.40316-0.40288-0.40314-0.40378+0.21301+0.21254+0.21230+0.21254+0.21301=-0.95334$

CpMgAl3Si-f-f-b36311+gx-NICS.log

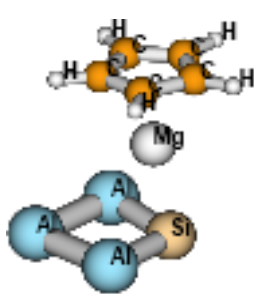

$\begin{array}{lrrrr}\text { NICS }(0)=-27.4414 & & & \\ \text { NICS }(1)=- & 20.9355 & & & \\ 16 \quad \mathrm{~Bq} & \text { Isotropic }= & 27.4414 & \text { Anisotropy }= & 58.5732 \\ \text { XX }= & 61.2704 & \text { YX }= & -0.1702 \quad \text { ZX }= & 16.7649\end{array}$ 


$$
\begin{array}{lrrrrr}
\mathrm{XY}= & -0.1468 & \mathrm{YY}= & 4.3598 & \mathrm{ZY}= & -0.0740 \\
\mathrm{XZ}= & 15.4780 & \mathrm{YZ}= & -0.0236 & \mathrm{ZZ}= & 16.6939 \\
\text { Eigenvalues: } & 4.3594 & 11.4746 & 66.4901
\end{array}
$$

$17 \mathrm{~Bq}$ Isotropic $=20.9355$ Anisotropy $=43.2945$

Cp2Li2Al3Si-f-f-c-f-Si-b3631+gx-NBO.log

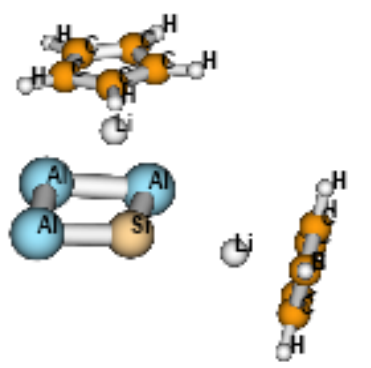

\begin{tabular}{|c|c|c|c|c|c|c|}
\hline Atom & No & $\begin{array}{c}\text { Natural } \\
\text { Charge }\end{array}$ & Core & Valence & Rydberg & Total \\
\hline $\mathrm{Al}$ & 1 & 0.20045 & 9.99784 & 2.76936 & 0.03235 & 12.79955 \\
\hline $\mathrm{Al}$ & 2 & -0.02288 & 9.99814 & 3.00281 & 0.02193 & 13.02288 \\
\hline $\mathrm{Al}$ & 3 & 0.14805 & 9.99768 & 2.82356 & 0.03071 & 12.85195 \\
\hline $\mathrm{C}$ & 4 & -0.41573 & 1.99910 & 4.39249 & 0.02413 & 6.41573 \\
\hline C & 5 & -0.41730 & 1.99910 & 4.39357 & 0.02463 & 6.41730 \\
\hline $\mathrm{C}$ & 6 & -0.41922 & 1.99910 & 4.39553 & 0.02460 & 6.41922 \\
\hline $\mathrm{H}$ & 7 & 0.23725 & 0.00000 & 0.76202 & 0.00073 & 0.76275 \\
\hline $\mathrm{C}$ & 8 & -0.41671 & 1.99910 & 4.39333 & 0.02429 & 6.41671 \\
\hline $\mathrm{H}$ & 9 & 0.23722 & 0.00000 & 0.76196 & 0.00081 & 0.76278 \\
\hline $\mathrm{C}$ & 10 & -0.41828 & 1.99910 & 4.39492 & 0.02426 & 6.41828 \\
\hline $\mathrm{H}$ & 11 & 0.23544 & 0.00000 & 0.76371 & 0.00086 & 0.76456 \\
\hline $\mathrm{H}$ & 12 & 0.23639 & 0.00000 & 0.76271 & 0.00091 & 0.76361 \\
\hline $\mathrm{H}$ & 13 & 0.23558 & 0.00000 & 0.76348 & 0.00094 & 0.76442 \\
\hline $\mathrm{C}$ & 14 & -0.41029 & 1.99909 & 4.38673 & 0.02446 & 6.41029 \\
\hline $\mathrm{C}$ & 15 & -0.41178 & 1.99910 & 4.38850 & 0.02418 & 6.41178 \\
\hline $\mathrm{C}$ & 16 & -0.41160 & 1.99910 & 4.38835 & 0.02416 & 6.41160 \\
\hline $\mathrm{H}$ & 17 & 0.24013 & 0.00000 & 0.75915 & 0.00072 & 0.75987 \\
\hline $\mathrm{C}$ & 18 & -0.41363 & 1.99910 & 4.39019 & 0.02434 & 6.41363 \\
\hline $\mathrm{H}$ & 19 & 0.23967 & 0.00000 & 0.75961 & 0.00072 & 0.76033 \\
\hline $\mathrm{C}$ & 20 & -0.41323 & 1.99910 & 4.38979 & 0.02434 & 6.41323 \\
\hline $\mathrm{H}$ & 21 & 0.24003 & 0.00000 & 0.75922 & 0.00075 & 0.75997 \\
\hline $\mathrm{H}$ & 22 & 0.23868 & 0.00000 & 0.76034 & 0.00099 & 0.76132 \\
\hline $\mathrm{H}$ & 23 & 0.24028 & 0.00000 & 0.75879 & 0.00093 & 0.75972 \\
\hline $\mathrm{Li}$ & 24 & 0.87274 & 1.99739 & 0.09305 & 0.03682 & 2.12726 \\
\hline $\mathrm{Li}$ & 25 & 0.79844 & 1.99851 & 0.16045 & 0.04261 & 2.20156 \\
\hline $\mathrm{Si}$ & 26 & -1.22969 & 9.99837 & 5.20019 & 0.03113 & 15.22969 \\
\hline
\end{tabular}

Summary of Natural Population Analysis:

Natural Population 


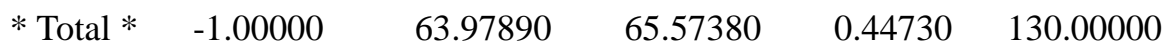

The charge of SiAl3=0.20045-0.02288 $+0.14805-1.22969=-0.90407$

CpLi2Al3Si-f-s-Si-Al-b36311+gx-NBO.log

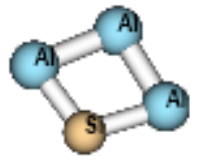

$$
\text { di bi }
$$

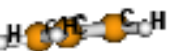

\begin{tabular}{|c|c|c|c|c|c|c|}
\hline \multirow[b]{2}{*}{ Atom } & \multirow[b]{2}{*}{ No } & \multirow{2}{*}{$\begin{array}{l}\text { Natural } \\
\text { Charge }\end{array}$} & \multicolumn{4}{|c|}{ Natural Population } \\
\hline & & & Core & Valence & Rydberg & Total \\
\hline $\mathrm{Al}$ & 1 & 0.12685 & 9.99783 & 2.85060 & 0.02472 & 12.87315 \\
\hline $\mathrm{Al}$ & 2 & 0.38349 & 9.99756 & 2.58823 & 0.03072 & 12.61651 \\
\hline $\mathrm{Al}$ & 3 & 0.13596 & 9.99761 & 2.84017 & 0.02626 & 12.86404 \\
\hline $\mathrm{C}$ & 4 & -0.37005 & 1.99916 & 4.34549 & 0.02540 & 6.37005 \\
\hline C & 5 & -0.34495 & 1.99917 & 4.31905 & 0.02673 & 6.34495 \\
\hline $\mathrm{C}$ & 6 & -0.34467 & 1.99917 & 4.31876 & 0.02674 & 6.34467 \\
\hline $\mathrm{H}$ & 7 & 0.21874 & 0.00000 & 0.78057 & 0.00068 & 0.78126 \\
\hline $\mathrm{C}$ & 8 & -0.44166 & 1.99919 & 4.41242 & 0.03005 & 6.44166 \\
\hline $\mathrm{H}$ & 9 & 0.21863 & 0.00000 & 0.78076 & 0.00060 & 0.78137 \\
\hline $\mathrm{C}$ & 10 & -0.44338 & 1.99919 & 4.41404 & 0.03015 & 6.44338 \\
\hline $\mathrm{H}$ & 11 & 0.21863 & 0.00000 & 0.78077 & 0.00060 & 0.78137 \\
\hline $\mathrm{H}$ & 12 & 0.21146 & 0.00000 & 0.78762 & 0.00092 & 0.78854 \\
\hline $\mathrm{H}$ & 13 & 0.21133 & 0.00000 & 0.78774 & 0.00093 & 0.78867 \\
\hline $\mathrm{Li}$ & 14 & 0.85850 & 1.99664 & 0.10759 & 0.03727 & 2.14150 \\
\hline $\mathrm{Si}$ & 15 & -1.49147 & 9.99838 & 5.47098 & 0.02211 & 15.49147 \\
\hline $\mathrm{Li}$ & 16 & 0.85256 & 1.99642 & 0.12225 & 0.02876 & 2.14744 \\
\hline
\end{tabular}

Summary of Natural Population Analysis:

\begin{tabular}{|c|c|c|}
\hline * Total * & 0.00000 & 53.98032 \\
\hline
\end{tabular}

The charge of SiAl3=0.12685 $+0.38349+0.13596-1.49147=-0.84517$

The

charge

of

$\mathrm{Cp}=-0.37005-0.34495-0.34467+0.21874-0.44166+0.21863-0.44338+0.21863+0.21146+0.21133=-0.86592$ CpLiAl3Si-f-c-Si-b36311+gx-NICS.log

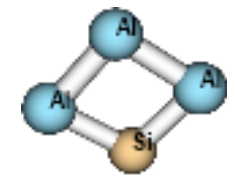

방

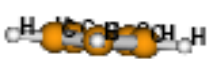

$\operatorname{NICS}(0)=-35.1748$ 


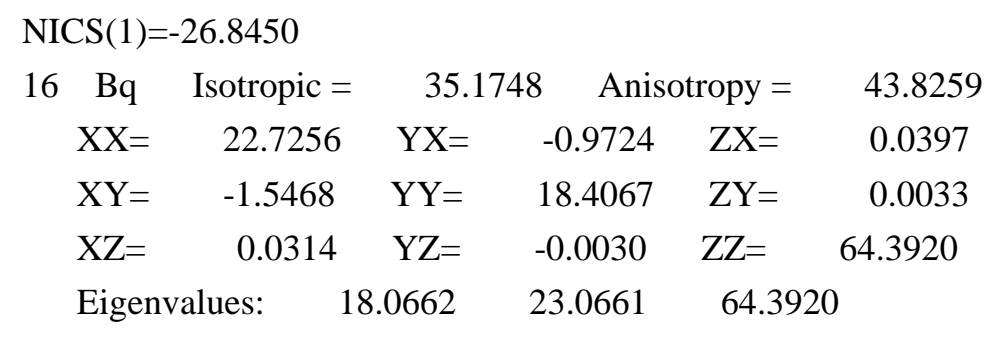

$17 \mathrm{~Bq} \quad$ Isotropic $=26.8450 \quad$ Anisotropy $=37.8533$ CpBeAl3Si-Al-Si-f-s-to-f-c-Si-b36311+gx-NICS.log

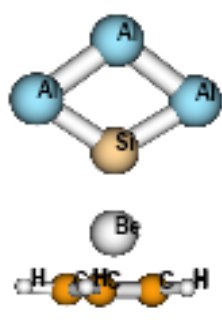

\begin{tabular}{rccccc}
16 & Iq & Isotropic $=$ & \multicolumn{2}{c}{33.4322} & \multicolumn{2}{c}{ Anisotropy $=$} & 34.7959 \\
$\mathrm{XX}=$ & 26.8409 & $\mathrm{YX}=$ & 0.0859 & $\mathrm{ZX}=$ & -0.0059 \\
$\mathrm{XY}=$ & 0.1573 & $\mathrm{YY}=$ & 16.8263 & $\mathrm{ZY}=$ & 0.0000 \\
$\mathrm{XZ}=$ & -0.0255 & $\mathrm{YZ}=$ & 0.0000 & $\mathrm{ZZ}=$ & 56.6294
\end{tabular}

Eigenvalues: $\quad 16.8248 \quad 26.8423 \quad 56.6294$

$17 \mathrm{~Bq} \quad$ Isotropic $=24.3553 \quad$ Anisotropy $=34.1372$ CpMgAl3Si-Al-Si-f-s-to-f-c-Si-b36311+gx-NICS.log

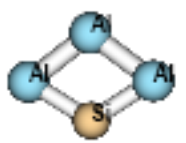

$\mathrm{CH}_{9}$

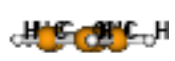

$16 \mathrm{~Bq}$ Isotropic $=34.0034$ Anisotropy $=36.7062$

$\mathrm{XX}=27.0694 \quad \mathrm{YX}=-0.1710 \quad \mathrm{ZX}=0.0298$

$\mathrm{XY}=\quad-0.2625 \quad \mathrm{YY}=\quad 16.4666 \quad \mathrm{ZY}=\quad-0.0019$

$\mathrm{XZ}=\quad 0.0124 \quad \mathrm{YZ}=\quad-0.0073 \quad \mathrm{ZZ}=58.4742$

Eigenvalues: $\quad 16.4622 \quad 27.0738 \quad 58.4742$

$17 \mathrm{~Bq} \quad$ Isotropic $=24.6535$ Anisotropy $=35.8660$

CpBeAl3Si-Al-Al-f-s-to-f-c-Al-b36311+gx-NBO.log

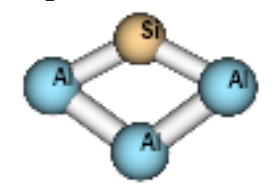

(B)

Herestoget

Summary of Natural Population Analysis:

Natural Population

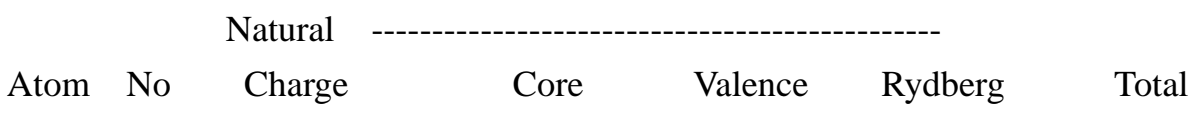




$\begin{array}{ccccccc}\mathrm{Al} & 1 & 0.44796 & 9.99698 & 2.52858 & 0.02647 & 12.55204 \\ \mathrm{Al} & 2 & 0.44983 & 9.99698 & 2.52670 & 0.02648 & 12.55017 \\ \mathrm{C} & 3 & -0.39393 & 1.99913 & 4.36224 & 0.03255 & 6.39393 \\ \mathrm{C} & 4 & -0.39554 & 1.99913 & 4.36356 & 0.03284 & 6.39554 \\ \mathrm{C} & 5 & -0.39684 & 1.99914 & 4.36458 & 0.03312 & 6.39684 \\ \mathrm{H} & 6 & 0.23975 & 0.00000 & 0.75947 & 0.00078 & 0.76025 \\ \mathrm{C} & 7 & -0.39586 & 1.99913 & 4.36379 & 0.03293 & 6.39586 \\ \mathrm{H} & 8 & 0.23930 & 0.00000 & 0.75989 & 0.00081 & 0.76070 \\ \mathrm{C} & 9 & -0.39330 & 1.99913 & 4.36168 & 0.03249 & 6.39330 \\ \mathrm{H} & 10 & 0.23895 & 0.00000 & 0.76021 & 0.00084 & 0.76105 \\ \mathrm{H} & 11 & 0.23918 & 0.00000 & 0.76000 & 0.00082 & 0.76082 \\ \mathrm{H} & 12 & 0.23983 & 0.00000 & 0.75940 & 0.00077 & 0.76017 \\ \mathrm{Be} & 13 & 1.27994 & 1.99796 & 0.54780 & 0.17429 & 2.72006 \\ \mathrm{Al} & 14 & -0.55759 & 9.99629 & 3.53246 & 0.02884 & 13.55759 \\ \mathrm{Si} & 15 & -0.84169 & 9.99823 & 4.82604 & 0.01742 & 14.84169 \\ ============================================================ \\ \text { * Total * } & 0.00000 & 51.98211 & 39.57641 & 0.44148 & 92.00000\end{array}$

The charge of SiAl3=0.44796 $+0.44983-0.55759-0.84169=-0.50149$

The

charge

of

$C p=-0.39393-0.39554-0.39684+0.23975-0.39586+0.23930-0.39330+0.23895+0.23918+0.23983=-0.77846$

CpBeAl3Si-Al-Al-f-s-to-f-c-Al-b36311+gx-NICS.log

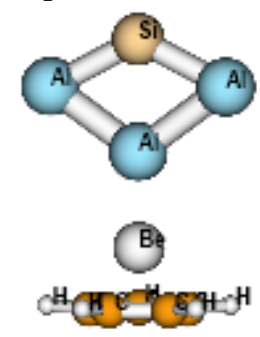

$16 \mathrm{~Bq}$ Isotropic $=34.9233$ Anisotropy $=27.3995$

$\mathrm{XX}=31.5268 \quad \mathrm{YX}=0.0036 \quad \mathrm{ZX}=-0.0143$

$\mathrm{XY}=-0.0050 \quad \mathrm{YY}=20.0534 \quad \mathrm{ZY}=-0.0002$

$\mathrm{XZ}=\quad 0.0070 \quad \mathrm{YZ}=\quad 0.0011 \quad \mathrm{ZZ}=53.1896$

Eigenvalues: $\quad 20.0534 \quad 31.5268 \quad 53.1896$

$17 \mathrm{~Bq}$ Isotropic $=23.7283$ Anisotropy $=26.8954$

CpBeAl3Si-f-f-b36311+gx-NBO.log

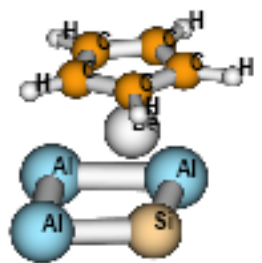

Summary of Natural Population Analysis:

Natural Population 


\begin{tabular}{|c|c|c|c|c|c|c|}
\hline Atom & No & $\begin{array}{l}\text { Natural } \\
\text { Charge }\end{array}$ & Core & Valence & Rydberg & Total \\
\hline $\mathrm{C}$ & 1 & -0.39103 & 1.99911 & 4.35897 & 0.03295 & 6.39103 \\
\hline $\mathrm{C}$ & 2 & -0.39153 & 1.99912 & 4.36076 & 0.03164 & 6.39153 \\
\hline $\mathrm{C}$ & 3 & -0.38604 & 1.99912 & 4.35486 & 0.03206 & 6.38604 \\
\hline $\mathrm{C}$ & 4 & -0.39155 & 1.99912 & 4.36078 & 0.03165 & 6.39155 \\
\hline $\mathrm{C}$ & 5 & -0.39103 & 1.99911 & 4.35896 & 0.03296 & 6.39103 \\
\hline $\mathrm{H}$ & 6 & 0.24085 & 0.00000 & 0.75757 & 0.00158 & 0.75915 \\
\hline $\mathrm{H}$ & 7 & 0.24257 & 0.00000 & 0.75652 & 0.00091 & 0.75743 \\
\hline $\mathrm{H}$ & 8 & 0.24071 & 0.00000 & 0.75817 & 0.00112 & 0.75929 \\
\hline $\mathrm{H}$ & 9 & 0.24257 & 0.00000 & 0.75652 & 0.00091 & 0.75743 \\
\hline $\mathrm{H}$ & 10 & 0.24086 & 0.00000 & 0.75756 & 0.00158 & 0.75914 \\
\hline $\mathrm{Al}$ & 11 & 0.11349 & 9.99836 & 2.86655 & 0.02160 & 12.88651 \\
\hline $\mathrm{Al}$ & 12 & 0.23822 & 9.99769 & 2.73253 & 0.03155 & 12.76178 \\
\hline $\mathrm{Al}$ & 13 & 0.23852 & 9.99769 & 2.73223 & 0.03155 & 12.76148 \\
\hline $\mathrm{Be}$ & 14 & 1.27681 & 1.99868 & 0.51275 & 0.21177 & 2.72319 \\
\hline $\mathrm{Si}$ & 15 & -1.12342 & 9.99841 & 5.08985 & 0.03515 & 15.12342 \\
\hline
\end{tabular}

$\begin{array}{llrrrr}* \text { Total } & 0.00000 & 51.98643 & 39.51458 & 0.49899 & 92.00000\end{array}$

The charge of SiAl3=0.11349+0.23822 $+0.23852-1.12342=-0.53319$

The

charge

of

$C p=-0.39103-0.39153-0.38604-0.39155-0.39103+0.24085+0.24257+0.24071+0.24257+0.24086=-0.74362$

CpBeAl3Si-f-f-b36311+gx-NICS.log

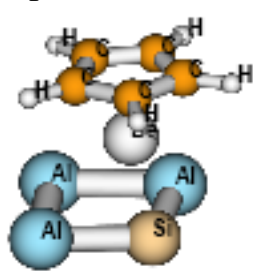

$16 \mathrm{~Bq}$ Isotropic $=31.8654 \quad$ Anisotropy $=54.8194$

$X X=64.7406 \quad Y X=-0.0006 \quad Z X=-13.1640$

$\mathrm{XY}=\quad-0.0018 \quad \mathrm{YY}=\quad 10.8505 \quad \mathrm{ZY}=\quad-0.0041$

$\mathrm{XZ}=-13.4972 \quad \mathrm{YZ}=0.0020 \quad \mathrm{ZZ}=20.0052$

Eigenvalues: $\quad \begin{array}{lll}10.8505 & 16.3341 & 68.4117\end{array}$

$17 \mathrm{~Bq} \quad$ Isotropic $=21.5574 \quad$ Anisotropy $=45.0943$

CpLiAl3Si-f-f-b36311+gx-NICS.log

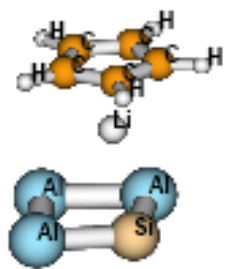

$16 \mathrm{~Bq}$ Isotropic $=33.9133$ Anisotropy $=51.4362$

$X X=66.0799 \quad Y X=-0.0541 \quad Z X=10.1112$ 


$\begin{array}{llrlll}\mathrm{XY}= & -0.0470 & \mathrm{YY}= & 15.7085 & \mathrm{ZY}= & -0.0207 \\ \mathrm{XZ}= & 10.1366 & \mathrm{YZ}= & -0.0075 & \mathrm{ZZ}= & 19.9513 \\ \text { Eigenvalues: } & 15.7084 & 17.8273 & 68.2041\end{array}$

$17 \mathrm{~Bq}$ Isotropic $=25.2241$ Anisotropy $=41.9520$

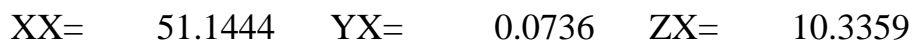

$\mathrm{XY}=-0.0098 \quad \mathrm{YY}=11.7983 \quad \mathrm{ZY}=0.0163$

$\mathrm{XZ}=\quad 7.8687 \quad \mathrm{YZ}=\quad-0.0114 \quad \mathrm{ZZ}=12.7296$

Eigenvalues: $\quad 10.6819 \quad 11.7983 \quad 53.1921$

$18 \mathrm{~Bq} \quad$ Isotropic $=30.2569$ Anisotropy $=42.5503$ CpMgAl3Si-Al-Si-f-s-to-f-c-Si-b36311+gx-NBO.log

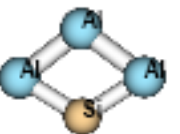

O99

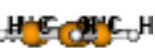

Summary of Natural Population Analysis:

Natural Population

\begin{tabular}{|c|c|c|c|c|c|c|}
\hline Atom & \multicolumn{2}{|r|}{ Natural } & Core & Valence & Rydberg & Total \\
\hline $\mathrm{Al}$ & 1 & 0.10565 & 9.99796 & 2.87045 & 0.02593 & 12.89435 \\
\hline $\mathrm{Al}$ & 2 & 0.39705 & 9.99744 & 2.57895 & 0.02656 & 12.60295 \\
\hline $\mathrm{Al}$ & 3 & 0.37747 & 9.99746 & 2.59844 & 0.02663 & 12.62253 \\
\hline $\mathrm{C}$ & 4 & -0.39973 & 1.99919 & 4.37037 & 0.03017 & 6.39973 \\
\hline $\mathrm{C}$ & 5 & -0.40012 & 1.99919 & 4.37066 & 0.03027 & 6.40012 \\
\hline $\mathrm{C}$ & 6 & -0.39997 & 1.99919 & 4.37067 & 0.03011 & 6.39997 \\
\hline $\mathrm{H}$ & 7 & 0.22620 & 0.00000 & 0.77318 & 0.00062 & 0.77380 \\
\hline $\mathrm{C}$ & 8 & -0.40052 & 1.99919 & 4.37130 & 0.03003 & 6.40052 \\
\hline $\mathrm{H}$ & 9 & 0.22630 & 0.00000 & 0.77308 & 0.00062 & 0.77370 \\
\hline $\mathrm{C}$ & 10 & -0.40026 & 1.99919 & 4.37072 & 0.03035 & 6.40026 \\
\hline $\mathrm{H}$ & 11 & 0.22614 & 0.00000 & 0.77323 & 0.00062 & 0.77386 \\
\hline $\mathrm{H}$ & 12 & 0.22608 & 0.00000 & 0.77329 & 0.00062 & 0.77392 \\
\hline $\mathrm{H}$ & 13 & 0.22639 & 0.00000 & 0.77300 & 0.00062 & 0.77361 \\
\hline Mg & 14 & 1.63372 & 9.99725 & 0.30871 & 0.06032 & 10.36628 \\
\hline $\mathrm{Si}$ & 15 & -1.64440 & 9.99820 & 5.62301 & 0.02319 & 15.64440 \\
\hline * Total & & 0.00000 & 59.98426 & 39.69908 & 0.31666 & 00.00000 \\
\hline
\end{tabular}

The charge of SiAl3 $=0.10565+0.39705+0.37747-1.64440=-0.76423$

The

charge

of

$C p=-0.39973-0.40012-0.39997+0.22620-0.40052+0.22630-0.40026+0.22614+0.22608+0.22639=-0.86949$

CpMgAl3Si-Al-Al-f-s-b36311+gx-NBO.log 


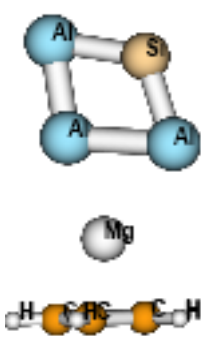

Summary of Natural Population Analysis:

\begin{tabular}{ccccccc} 
& & & \multicolumn{5}{c}{ Natural Population } \\
Atom & No & Charge & Core & Valence & Rydberg & Total \\
& & & & & & \\
Al & 1 & 0.18692 & 9.99728 & 2.78157 & 0.03423 & 12.81308 \\
$\mathrm{Al}$ & 2 & 0.46393 & 9.99672 & 2.50784 & 0.03151 & 12.53607 \\
$\mathrm{C}$ & 3 & -0.39596 & 1.99918 & 4.36772 & 0.02907 & 6.39596 \\
$\mathrm{C}$ & 4 & -0.39406 & 1.99918 & 4.36617 & 0.02872 & 6.39406 \\
$\mathrm{C}$ & 5 & -0.39905 & 1.99918 & 4.37004 & 0.02982 & 6.39905 \\
$\mathrm{H}$ & 6 & 0.22560 & 0.00000 & 0.77375 & 0.00064 & 0.77440 \\
$\mathrm{C}$ & 7 & -0.40154 & 1.99918 & 4.37232 & 0.03005 & 6.40154 \\
$\mathrm{H}$ & 8 & 0.22554 & 0.00000 & 0.77382 & 0.00064 & 0.77446 \\
$\mathrm{C}$ & 9 & -0.38930 & 1.99918 & 4.36138 & 0.02875 & 6.38930 \\
$\mathrm{H}$ & 10 & 0.22626 & 0.00000 & 0.77308 & 0.00066 & 0.77374 \\
$\mathrm{H}$ & 11 & 0.22623 & 0.00000 & 0.77311 & 0.00065 & 0.77377 \\
$\mathrm{H}$ & 12 & 0.22652 & 0.00000 & 0.77278 & 0.00070 & 0.77348 \\
$\mathrm{Mg}$ & 13 & 1.49413 & 9.99620 & 0.43704 & 0.07263 & 10.50587 \\
$\mathrm{Al}$ & 14 & -0.53322 & 9.99714 & 3.49977 & 0.03632 & 13.53322 \\
$\mathrm{Si}$ & 15 & -0.76200 & 9.99819 & 4.74361 & 0.02021 & 14.76200
\end{tabular}

$\begin{array}{lrrrrr}* \text { Total * } & 0.00000 & 59.98141 & 39.67400 & 0.34459 & 100.00000\end{array}$

The charge of SiAl3 $=0.18692+0.46393-0.53322-0.76200=-0.64437$

The charge of

$C p=-0.39596-0.39406-0.39905+0.22560-0.40154+0.22554-0.38930+0.22626+0.22623+0.22652=-0.84976$

KCpKAl3Si-f-f-f-b36311+gx-NICS.log

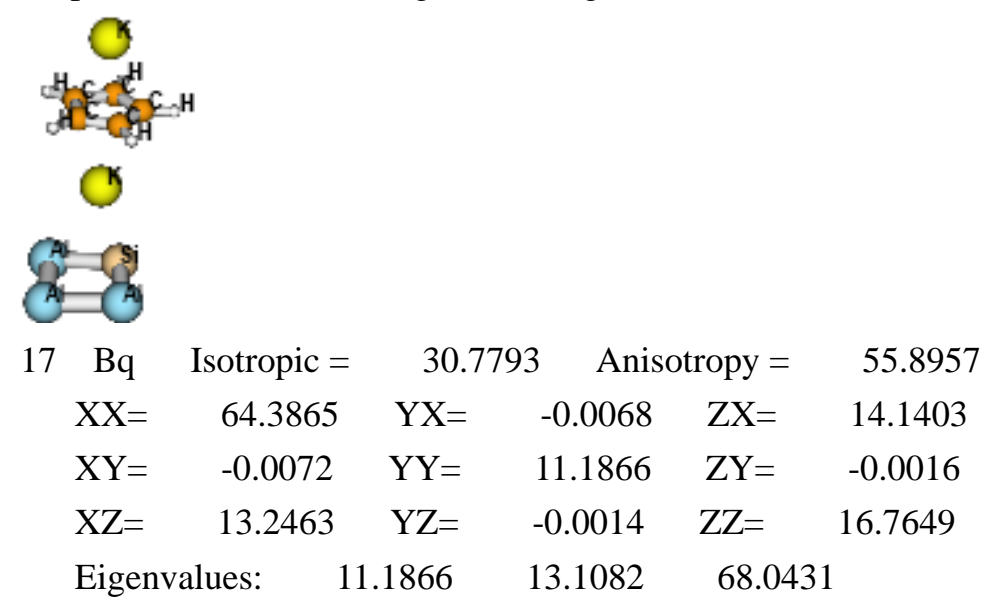




\begin{tabular}{cccccc}
$18 \quad \mathrm{~Bq}$ & Isotropic $=$ & \multicolumn{2}{c}{25.0982} & Anisotropy $=$ & 41.8995 \\
$\mathrm{XX}=$ & 49.8487 & $\mathrm{YX}=$ & -0.0178 & $\mathrm{ZX}=$ & 12.2764 \\
$\mathrm{XY}=$ & -0.0068 & $\mathrm{YY}=$ & 12.1841 & $\mathrm{ZY}=$ & -0.0070 \\
$\mathrm{XZ}=$ & 10.2241 & $\mathrm{YZ}=$ & -0.0034 & $\mathrm{ZZ}=$ & 13.2618 \\
\multicolumn{2}{l}{ Eigenvalues: } & 10.0793 & 12.1841 & 53.0312
\end{tabular}

$19 \mathrm{~Bq} \quad$ Isotropic $=26.4258 \quad$ Anisotropy $=47.5161$ CpKAl3Si-Al-Si-f-s-b36311+gx-NICS.log

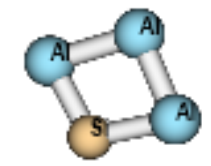

(6)

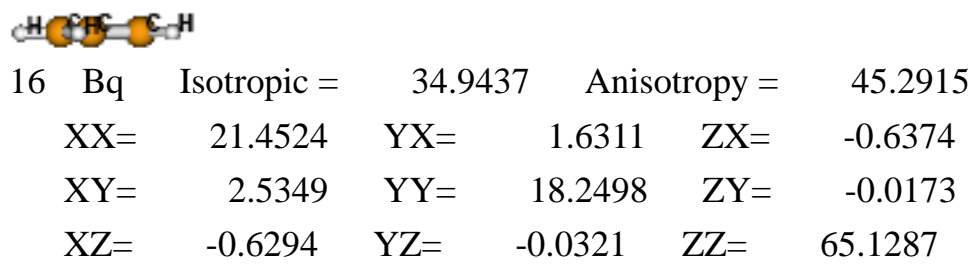

$17 \mathrm{~Bq} \quad$ Isotropic $=26.6504 \quad$ Anisotropy $=38.5869$ CpKAl3Si-Al-Al-f-s-b36311+gx-NBO.log

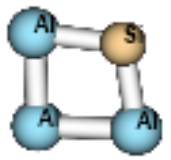

\begin{tabular}{|c|c|c|c|c|c|c|}
\hline Atom & No & $\begin{array}{l}\text { Natural } \\
\text { Charge }\end{array}$ & Core & Valence & Rydberg & Total \\
\hline $\mathrm{Al}$ & 1 & -0.00055 & 9.99774 & 2.96311 & 0.03971 & 13.00055 \\
\hline $\mathrm{Al}$ & 2 & 0.13906 & 9.99760 & 2.82791 & 0.03543 & 12.86094 \\
\hline $\mathrm{C}$ & 3 & -0.38053 & 1.99917 & 4.35367 & 0.02769 & 6.38053 \\
\hline $\mathrm{C}$ & 4 & -0.38161 & 1.99917 & 4.35460 & 0.02785 & 6.38161 \\
\hline $\mathrm{C}$ & 5 & -0.38063 & 1.99917 & 4.35334 & 0.02811 & 6.38063 \\
\hline $\mathrm{H}$ & 6 & 0.18818 & 0.00000 & 0.81100 & 0.00082 & 0.81182 \\
\hline C & 7 & -0.38143 & 1.99917 & 4.35425 & 0.02801 & 6.38143 \\
\hline $\mathrm{H}$ & 8 & 0.18833 & 0.00000 & 0.81085 & 0.00082 & 0.81167 \\
\hline $\mathrm{C}$ & 9 & -0.37791 & 1.99917 & 4.35110 & 0.02765 & 6.37791 \\
\hline $\mathrm{H}$ & 10 & 0.18761 & 0.00000 & 0.81158 & 0.00082 & 0.81239 \\
\hline $\mathrm{H}$ & 11 & 0.18813 & 0.00000 & 0.81105 & 0.00082 & 0.81187 \\
\hline
\end{tabular}

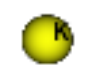

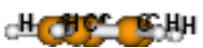

Summary of Natural Population Analysis:

Natural Population 


$\begin{array}{ccccccc}\mathrm{H} & 12 & 0.18805 & 0.00000 & 0.81113 & 0.00082 & 0.81195 \\ \mathrm{~K} & 13 & 0.91377 & 17.99003 & 0.06981 & 0.02639 & 18.08623 \\ \mathrm{Al} & 14 & -0.27358 & 9.99816 & 3.23983 & 0.03559 & 13.27358 \\ \mathrm{Si} & 15 & -0.81688 & 9.99853 & 4.80078 & 0.01757 & 14.81688\end{array}$

$\begin{array}{lrrrrr}* \text { Total * } & -1.00000 & 67.97790 & 39.72400 & 0.29810 & 108.00000\end{array}$

The charge of SiAl3=-0.00055+0.13906-0.27358-0.81688=-0.95195

The charge of

$\mathrm{C}=-0.38053-0.38161-0.38063+0.18818-0.38143+0.18833-0.37791+0.18761+0.18813+0.18805=-0.96181$

CpKAl3Si-Al-Al-f-s-b36311+gx-NICS.log
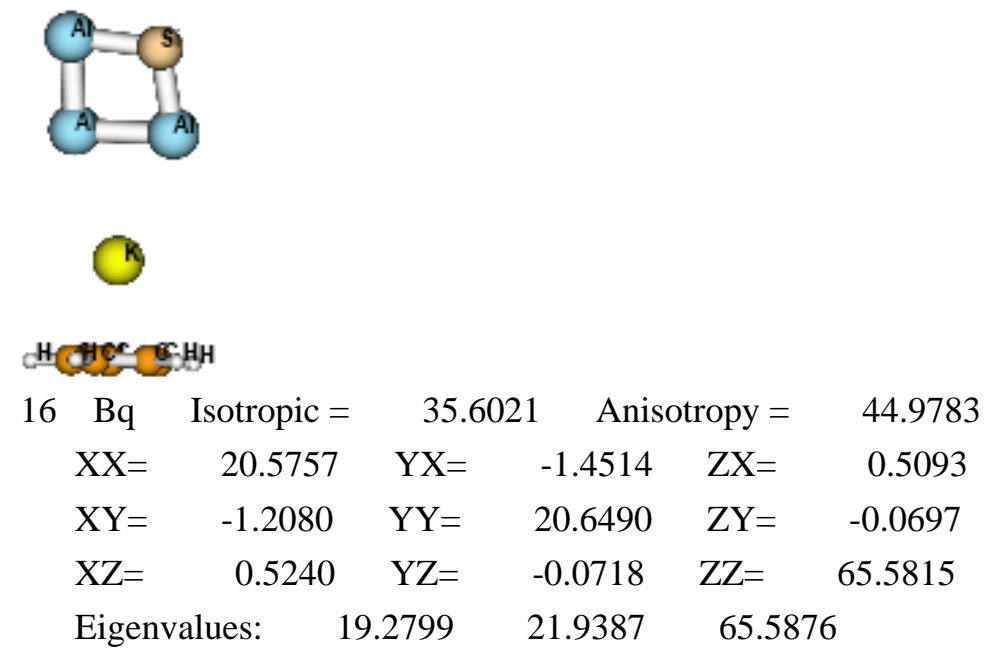

$17 \mathrm{~Bq} \quad$ Isotropic $=27.1856 \quad$ Anisotropy $=37.7040$

\section{CpKAI3Si-f-f-to-f-f-c-slope-b36311+gx-NBO.log}

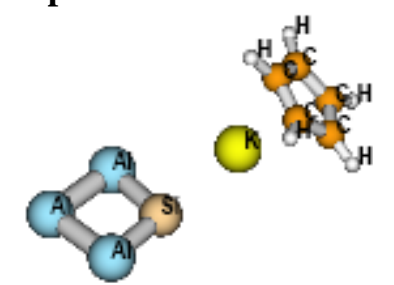

Summary of Natural Population Analysis:

\begin{tabular}{|c|c|c|c|c|c|c|}
\hline \multirow[b]{2}{*}{ Atom } & \multirow[b]{2}{*}{ No } & \multirow{2}{*}{$\begin{array}{l}\text { Natural } \\
\text { Charge }\end{array}$} & \multicolumn{4}{|c|}{ Natural Population } \\
\hline & & & Core & Valence & Rydberg & Total \\
\hline $\mathrm{C}$ & 1 & -0.37850 & 1.99917 & 4.35338 & 0.02595 & 6.37850 \\
\hline $\mathrm{C}$ & 2 & -0.37823 & 1.99917 & 4.35303 & 0.02603 & 6.37823 \\
\hline $\mathrm{C}$ & 3 & -0.37793 & 1.99917 & 4.35273 & 0.02603 & 6.37793 \\
\hline $\mathrm{C}$ & 4 & -0.37828 & 1.99917 & 4.35309 & 0.02602 & 6.37828 \\
\hline $\mathrm{C}$ & 5 & -0.37857 & 1.99917 & 4.35346 & 0.02595 & 6.37857 \\
\hline $\mathrm{H}$ & 6 & 0.18727 & 0.00000 & 0.81195 & 0.00077 & 0.81273 \\
\hline $\mathrm{H}$ & 7 & 0.18746 & 0.00000 & 0.81177 & 0.00077 & 0.81254 \\
\hline $\mathrm{H}$ & 8 & 0.18760 & 0.00000 & 0.81162 & 0.00078 & 0.81240 \\
\hline
\end{tabular}




$\begin{array}{ccccccc}\mathrm{H} & 9 & 0.18757 & 0.00000 & 0.81166 & 0.00077 & 0.81243 \\ \mathrm{H} & 10 & 0.18736 & 0.00000 & 0.81186 & 0.00077 & 0.81264 \\ \mathrm{Al} & 11 & -0.08654 & 9.99824 & 3.06279 & 0.02551 & 13.08654 \\ \mathrm{Al} & 12 & 0.14249 & 9.99777 & 2.82715 & 0.03259 & 12.85751 \\ \mathrm{Al} & 13 & 0.12147 & 9.99778 & 2.84811 & 0.03264 & 12.87853 \\ \mathrm{~K} & 14 & 0.92089 & 17.99067 & 0.04853 & 0.03991 & 18.07911 \\ \mathrm{Si} & 15 & -1.14406 & 9.99866 & 5.11851 & 0.02688 & 15.14406\end{array}$

$\begin{array}{llllll}* \text { Total * } & -1.00000 & 67.97897 & 39.72964 & 0.29139 & 108.00000\end{array}$

The charge of SiAl3=-0.08654+0.14249+0.12147-1.14406=-0.96664

The charge of

$\mathrm{Cp}=-0.37850-0.37823-0.37793-0.37828-0.37857+0.18727+0.18746+0.18760+0.18757+0.18736=-0.95425$

CpKAl3Si-f-f-to-f-f-c-slope-b36311+gx-NICS.log

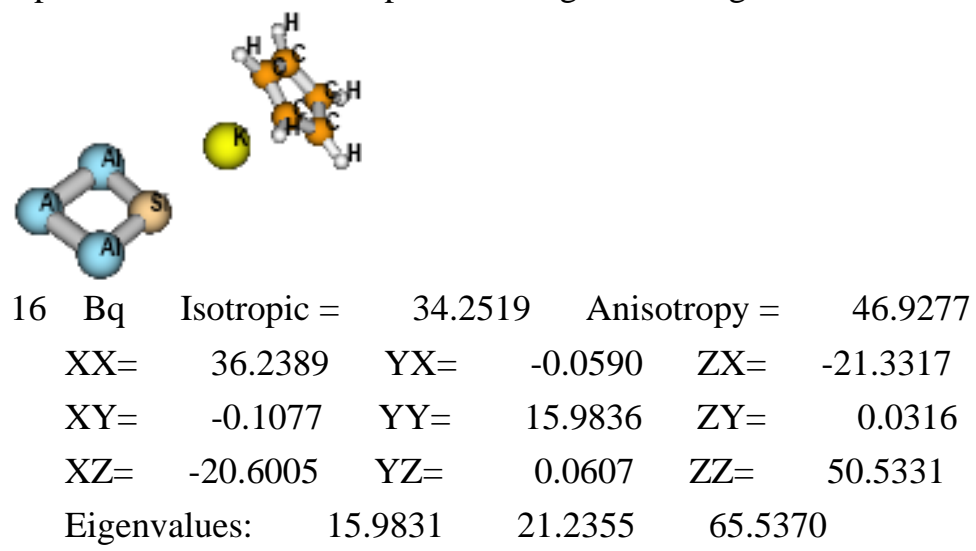

$17 \mathrm{~Bq}$ Isotropic $=26.5313$ Anisotropy $=39.6502$

$\mathrm{XX}=28.1306 \quad \mathrm{YX}=-0.0605 \quad \mathrm{ZX}=-17.7582$

$\mathrm{XY}=\quad-0.0958 \quad \mathrm{YY}=\quad 12.8711 \quad \mathrm{ZY}=0.0593$

$\mathrm{XZ}=\quad-20.0260 \quad \mathrm{YZ}=\quad 0.1842 \quad \mathrm{ZZ}=38.5922$

Eigenvalues: $\quad 12.8704 \quad 13.7587 \quad 52.9648$

$18 \mathrm{~Bq} \quad$ Isotropic $=26.5412 \quad$ Anisotropy $=38.3784$

\section{CpMgAl3Si-Al-Al-f-s-b36311+gx-NICS.log}

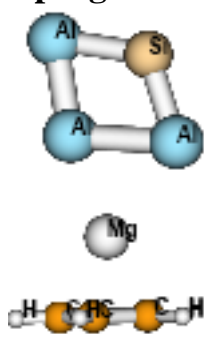

$16 \mathrm{~Bq}$ Isotropic $=41.3196 \quad$ Anisotropy $=44.5772$

$\mathrm{XX}=25.8625 \quad \mathrm{YX}=\quad 7.8430 \quad \mathrm{ZX}=\quad-0.1446$

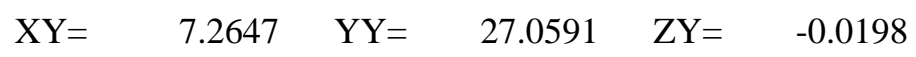

$\mathrm{XZ}=\quad-0.1550 \quad \mathrm{YZ}=\quad 0.0089 \quad \mathrm{ZZ}=\quad 71.0372$

Eigenvalues: $\quad 18.8831 \quad 34.0380 \quad 71.0378$

$17 \mathrm{~Bq} \quad$ Isotropic $=29.8824 \quad$ Anisotropy $=39.7166$ 
CpCaAl3Si-f-f-to-f-f-c-Si-b36311+gx-NICS.log

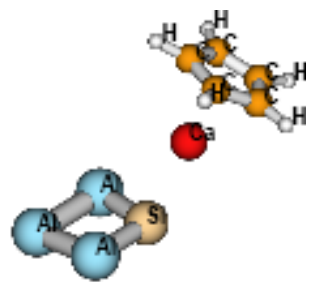

$16 \mathrm{~Bq}$ Isotropic $=$

31.7086 Anisotropy $=\quad 47.8757$

$\mathrm{XX}=\quad 38.3467$

$\mathrm{YX}=0.0069 \quad \mathrm{ZX}=$

21.5965

$\mathrm{XY}=0.0086 \quad \mathrm{YY}=\quad 9.7937 \quad \mathrm{ZY}=0.0010$

$\mathrm{XZ}=19.4231 \quad \mathrm{YZ}=\quad 0.0008 \quad \mathrm{ZZ}=46.9855$

Eigenvalues: $\quad 9.7937 \quad 21.7064 \quad 63.6258$

$17 \mathrm{~Bq} \quad$ Isotropic $=25.0604 \quad$ Anisotropy $=36.3552$

CpCaAl3Si-f-f-to-f-f-c-Si-b36311+gx-NBO.log

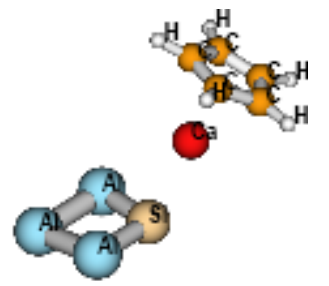

Summary of Natural Population Analysis:

$\wedge \mathrm{M}$

\begin{tabular}{|c|c|c|c|c|c|c|}
\hline \multirow[b]{2}{*}{ Atom } & \multirow[b]{2}{*}{ No } & \multirow[b]{2}{*}{$\begin{array}{l}\text { Natural } \\
\text { Charge }\end{array}$} & \multicolumn{4}{|c|}{ Natural Population $\wedge \mathrm{M}$} \\
\hline & & & Core & Valence & Rydberg & $\operatorname{Total}^{\wedge} \wedge \mathrm{M}$ \\
\hline $\mathrm{C}$ & 1 & -0.39118 & 1.99919 & 4.36123 & 0.03076 & $6.39118^{\wedge} \mathrm{M}$ \\
\hline $\mathrm{C}$ & 2 & -0.39272 & 1.99919 & 4.36265 & 0.03088 & $6.39272^{\wedge} \mathrm{M}$ \\
\hline $\mathrm{C}$ & 3 & -0.39312 & 1.99919 & 4.36284 & 0.03109 & $6.39312^{\wedge} \mathrm{M}$ \\
\hline $\mathrm{C}$ & 4 & -0.39278 & 1.99919 & 4.36271 & 0.03088 & $6.39278 \wedge \mathrm{M}$ \\
\hline $\mathrm{C}$ & 5 & -0.39121 & 1.99919 & 4.36126 & 0.03077 & $6.39121^{\wedge} \mathrm{M}$ \\
\hline $\mathrm{H}$ & 6 & 0.22051 & 0.00000 & 0.77887 & 0.00061 & $0.77949 \wedge \mathrm{M}$ \\
\hline $\mathrm{H}$ & 7 & 0.22042 & 0.00000 & 0.77895 & 0.00063 & $0.77958 \wedge \mathrm{M}$ \\
\hline $\mathrm{H}$ & 8 & 0.22050 & 0.00000 & 0.77882 & 0.00068 & $0.77950^{\wedge} \mathrm{M}$ \\
\hline $\mathrm{H}$ & 9 & 0.22041 & 0.00000 & 0.77896 & 0.00063 & $0.77959 \wedge \mathrm{M}$ \\
\hline $\mathrm{H}$ & 10 & 0.22051 & 0.00000 & 0.77887 & 0.00061 & $0.77949 \wedge \mathrm{M}$ \\
\hline $\mathrm{Al}$ & 11 & 0.11548 & 9.99817 & 2.86234 & 0.02400 & $12.88452^{\wedge} \mathrm{M}$ \\
\hline $\mathrm{Al}$ & 12 & 0.27374 & 9.99769 & 2.69876 & 0.02980 & $12.72626^{\wedge} \mathrm{M}$ \\
\hline $\mathrm{Al}$ & 13 & 0.27207 & 9.99770 & 2.70043 & 0.02980 & $12.72793^{\wedge} \mathrm{M}$ \\
\hline $\mathrm{Ca}$ & 14 & 1.70247 & 17.98978 & 0.14362 & 0.16413 & $18.29753^{\wedge} \mathrm{M}$ \\
\hline $\mathrm{Si}$ & 15 & -1.50512 & 9.99851 & 5.48246 & 0.02415 & $15.50512^{\wedge} \mathrm{M}$ \\
\hline
\end{tabular}

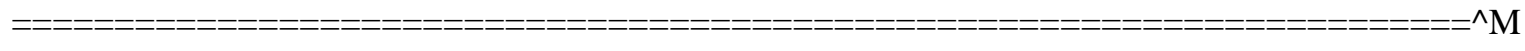
$\begin{array}{llrrrr}* \text { Total * } & 0.00000 & 67.97779 & 39.59278 & 0.42943 & 108.00000 \wedge \mathrm{M}\end{array}$

The charge of SiAl3 $=0.11548+0.27374+0.27207-1.50512=-0.84383$

The

charge

of 


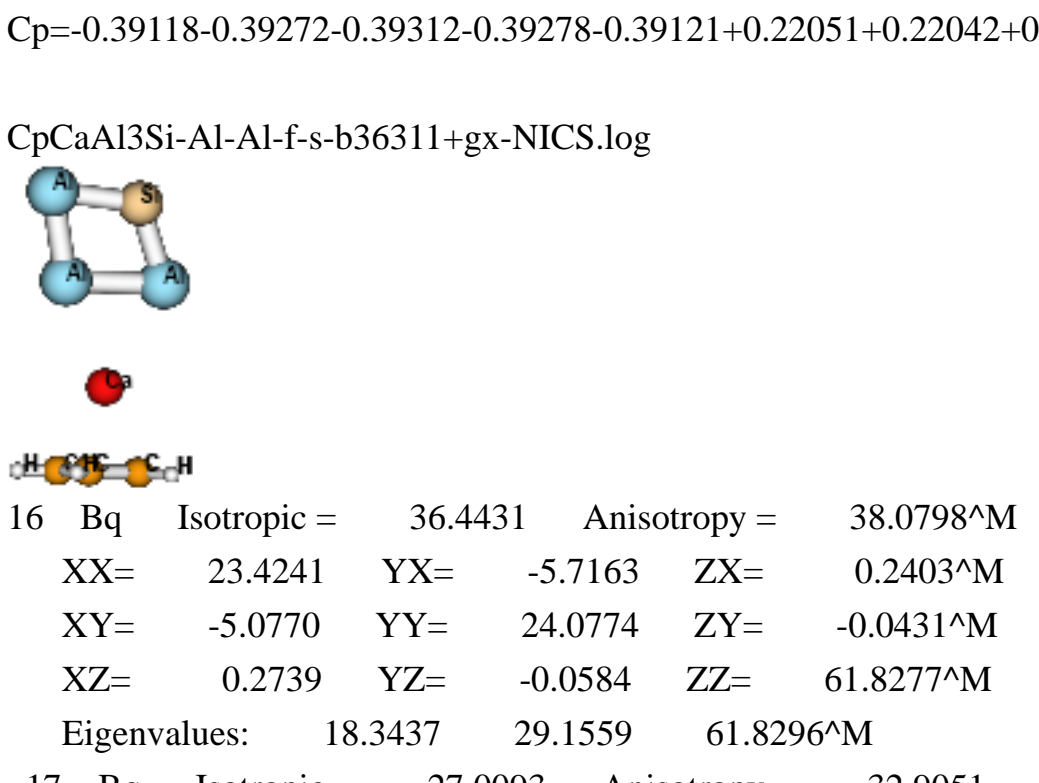

$17 \mathrm{~Bq}$ Isotropic $=27.0093 \quad$ Anisotropy $=32.9051$

CpCaAl3Si-Al-Si-f-s-b36311+gx-NICS.log

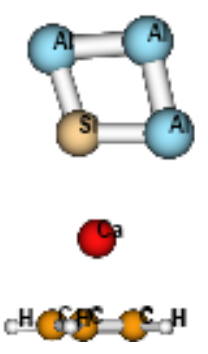

\begin{tabular}{|c|c|c|c|c|c|}
\hline $\mathrm{Bq}$ & Isotropic = & 34.565 & \multicolumn{2}{|c|}{ Anisotropy = } & $42.4843 \wedge \mathrm{M}$ \\
\hline $\mathrm{XX}=$ & 21.4247 & $\mathrm{YX}=$ & 3.9997 & $\mathrm{ZX}=$ & $-0.3351^{\wedge} \mathrm{M}$ \\
\hline $\mathrm{XY}=$ & 6.3345 & $\mathrm{YY}=$ & 19.3868 & $\mathrm{ZY}=$ & $-0.0053^{\wedge} \mathrm{M}$ \\
\hline $\mathrm{XZ}=$ & -0.2955 & $\mathrm{YZ}=$ & -0.0188 & $\mathrm{ZZ}=$ & $62.8863^{\wedge} \mathrm{M}$ \\
\hline Eige & lues: & 1383 & 25.6707 & 62.8 & ${ }^{\wedge} \wedge \mathrm{M}$ \\
\hline
\end{tabular}

$17 \mathrm{~Bq} \quad$ Isotropic $=25.6747 \quad$ Anisotropy $=\quad 37.6230^{\wedge} \mathrm{M}$ Cp4Li4Al3Si-extend4-b3631+gx-NBO.log

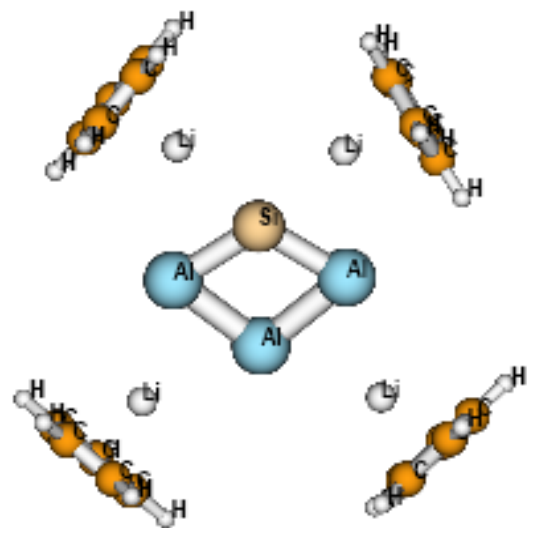

Summary of Natural Population Analysis:

$\wedge \mathrm{M}$

Natural Population $\wedge \mathrm{M}$

Natural $\wedge \mathrm{M}$ 
Atom No Charge $\quad$ Core $\quad$ Valence Rydberg $\quad$ Total $\wedge \mathrm{M}$

\begin{tabular}{|c|c|c|c|c|c|c|}
\hline $\mathrm{Al}$ & 1 & 0.26865 & 9.99745 & 2.69622 & 0.03769 & $12.73135^{\wedge} \mathrm{M}$ \\
\hline $\mathrm{Al}$ & 2 & -0.16415 & 9.99744 & 3.12784 & 0.03888 & $13.16415^{\wedge} \mathrm{M}$ \\
\hline $\mathrm{Li}$ & 3 & 0.83698 & 1.99646 & 0.13115 & 0.03541 & $2.16302^{\wedge} \mathrm{M}$ \\
\hline $\mathrm{Li}$ & 4 & 0.83811 & 1.99631 & 0.13015 & 0.03543 & $2.16189 \wedge \mathrm{M}$ \\
\hline $\mathrm{C}$ & 5 & -0.41474 & 1.99909 & 4.39090 & 0.02474 & $6.41474 \wedge \mathrm{M}$ \\
\hline $\mathrm{C}$ & 6 & -0.41983 & 1.99909 & 4.39511 & 0.02563 & $6.41983^{\wedge} \mathrm{M}$ \\
\hline $\mathrm{C}$ & 7 & -0.41732 & 1.99909 & 4.39309 & 0.02514 & $6.41732^{\wedge} \mathrm{M}$ \\
\hline $\mathrm{H}$ & 8 & 0.24084 & 0.00000 & 0.75836 & 0.00080 & $0.75916^{\wedge} \mathrm{M}$ \\
\hline $\mathrm{C}$ & 9 & -0.41384 & 1.99909 & 4.38977 & 0.02498 & $6.41384 \wedge \mathrm{M}$ \\
\hline $\mathrm{H}$ & 10 & 0.23996 & 0.00000 & 0.75914 & 0.00089 & $0.76004^{\wedge} \mathrm{M}$ \\
\hline $\mathrm{C}$ & 11 & -0.41807 & 1.99909 & 4.39361 & 0.02537 & $6.41807^{\wedge} \mathrm{M}$ \\
\hline $\mathrm{H}$ & 12 & 0.24028 & 0.00000 & 0.75887 & 0.00085 & $0.75972^{\wedge} \mathrm{M}$ \\
\hline $\mathrm{H}$ & 13 & 0.24288 & 0.00000 & 0.75631 & 0.00081 & $0.75712^{\wedge} \mathrm{M}$ \\
\hline $\mathrm{H}$ & 14 & 0.24095 & 0.00000 & 0.75818 & 0.00087 & $0.75905^{\wedge} \mathrm{M}$ \\
\hline C & 15 & -0.41771 & 1.99909 & 4.39327 & 0.02535 & $6.41771^{\wedge} \mathrm{M}$ \\
\hline C & 16 & -0.41610 & 1.99909 & 4.39187 & 0.02514 & $6.41610^{\wedge} \mathrm{M}$ \\
\hline $\mathrm{C}$ & 17 & -0.41291 & 1.99910 & 4.38917 & 0.02465 & $6.41291^{\wedge} \mathrm{M}$ \\
\hline $\mathrm{H}$ & 18 & 0.24017 & 0.00000 & 0.75895 & 0.00087 & $0.75983 \wedge \mathrm{M}$ \\
\hline C & 19 & -0.41488 & 1.99909 & 4.39078 & 0.02501 & $6.41488^{\wedge} \mathrm{M}$ \\
\hline $\mathrm{H}$ & 20 & 0.24187 & 0.00000 & 0.75727 & 0.00086 & $0.75813^{\wedge} \mathrm{M}$ \\
\hline $\mathrm{C}$ & 21 & -0.41913 & 1.99909 & 4.39451 & 0.02553 & $6.41913^{\wedge} \mathrm{M}$ \\
\hline $\mathrm{H}$ & 22 & 0.24131 & 0.00000 & 0.75791 & 0.00078 & $0.75869 \wedge \mathrm{M}$ \\
\hline $\mathrm{H}$ & 23 & 0.24244 & 0.00000 & 0.75672 & 0.00084 & $0.75756^{\wedge} \mathrm{M}$ \\
\hline $\mathrm{H}$ & 24 & 0.24012 & 0.00000 & 0.75898 & 0.00090 & $0.75988 \wedge \mathrm{M}$ \\
\hline $\mathrm{Si}$ & 25 & -1.31533 & 9.99772 & 5.29208 & 0.02553 & $15.31533^{\wedge} \mathrm{M}$ \\
\hline $\mathrm{Al}$ & 26 & 0.25743 & 9.99722 & 2.70496 & 0.04039 & $12.74257^{\wedge} \mathrm{M}$ \\
\hline $\mathrm{Li}$ & 27 & 0.87009 & 1.99716 & 0.09645 & 0.03631 & $2.12991^{\wedge} \mathrm{M}$ \\
\hline $\mathrm{C}$ & 28 & -0.41279 & 1.99910 & 4.38928 & 0.02441 & $6.41279 \wedge \mathrm{M}$ \\
\hline $\mathrm{C}$ & 29 & -0.41745 & 1.99910 & 4.39396 & 0.02439 & $6.41745^{\wedge} \mathrm{M}$ \\
\hline $\mathrm{C}$ & 30 & -0.41223 & 1.99911 & 4.38894 & 0.02418 & $6.41223^{\wedge} \mathrm{M}$ \\
\hline $\mathrm{H}$ & 31 & 0.24049 & 0.00000 & 0.75871 & 0.00081 & $0.75951 \wedge \mathrm{M}$ \\
\hline C & 32 & -0.41531 & 1.99910 & 4.39198 & 0.02424 & $6.41531^{\wedge} \mathrm{M}$ \\
\hline $\mathrm{H}$ & 33 & 0.24052 & 0.00000 & 0.75862 & 0.00086 & $0.75948 \wedge \mathrm{M}$ \\
\hline C & 34 & -0.41579 & 1.99909 & 4.39199 & 0.02471 & $6.41579 \wedge \mathrm{M}$ \\
\hline $\mathrm{H}$ & 35 & 0.24167 & 0.00000 & 0.75763 & 0.00070 & $0.75833^{\wedge} \mathrm{M}$ \\
\hline $\mathrm{H}$ & 36 & 0.24102 & 0.00000 & 0.75815 & 0.00083 & $0.75898 \wedge \mathrm{M}$ \\
\hline $\mathrm{H}$ & 37 & 0.24003 & 0.00000 & 0.75909 & 0.00088 & $0.75997 \wedge \mathrm{M}$ \\
\hline $\mathrm{Li}$ & 38 & 0.86702 & 1.99680 & 0.10163 & 0.03455 & $2.13298 \wedge \mathrm{M}$ \\
\hline $\mathrm{C}$ & 39 & -0.41450 & 1.99910 & 4.39137 & 0.02403 & $6.41450^{\wedge} \mathrm{M}$ \\
\hline $\mathrm{C}$ & 40 & -0.41224 & 1.99910 & 4.38926 & 0.02388 & $6.41224 \wedge \mathrm{M}$ \\
\hline $\mathrm{C}$ & 41 & -0.40399 & 1.99910 & 4.38117 & 0.02373 & $6.40399 \wedge \mathrm{M}$ \\
\hline $\mathrm{H}$ & 42 & 0.24255 & 0.00000 & 0.75663 & 0.00082 & $0.75745^{\wedge} \mathrm{M}$ \\
\hline
\end{tabular}




$\begin{array}{ccccccc}\mathrm{C} & 43 & -0.40786 & 1.99909 & 4.38474 & 0.02403 & 6.40786^{\wedge} \mathrm{M} \\ \mathrm{H} & 44 & 0.24295 & 0.00000 & 0.75625 & 0.00081 & 0.75705^{\wedge} \mathrm{M} \\ \mathrm{C} & 45 & -0.41115 & 1.99912 & 4.38820 & 0.02383 & 6.41115^{\wedge} \mathrm{M} \\ \mathrm{H} & 46 & 0.24251 & 0.00000 & 0.75672 & 0.00078 & 0.75749 \wedge \mathrm{M} \\ \mathrm{H} & 47 & 0.24223 & 0.00000 & 0.75696 & 0.00081 & 0.75777 \wedge \mathrm{M} \\ \mathrm{H} & 48 & 0.24427 & 0.00000 & 0.75499 & 0.00074 & 0.75573^{\wedge} \mathrm{M} \\ =============================================================\wedge \mathrm{M} \\ \text { * Total * } \quad-1.00000 \quad 87.95846 & 117.24789 & 0.79365 & 206.00000 \wedge \mathrm{M}\end{array}$

The charge of SiAl3=0.26865-0.16415-1.31533+0.25743=-0.9534

CpBeAl3Si-Al-Si-f-s-to-f-c-Si-NBO.log

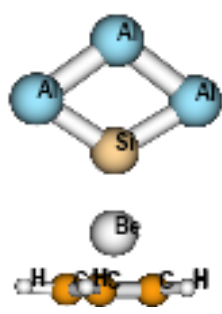

Summary of Natural Population Analysis:

$\wedge \mathrm{M}$

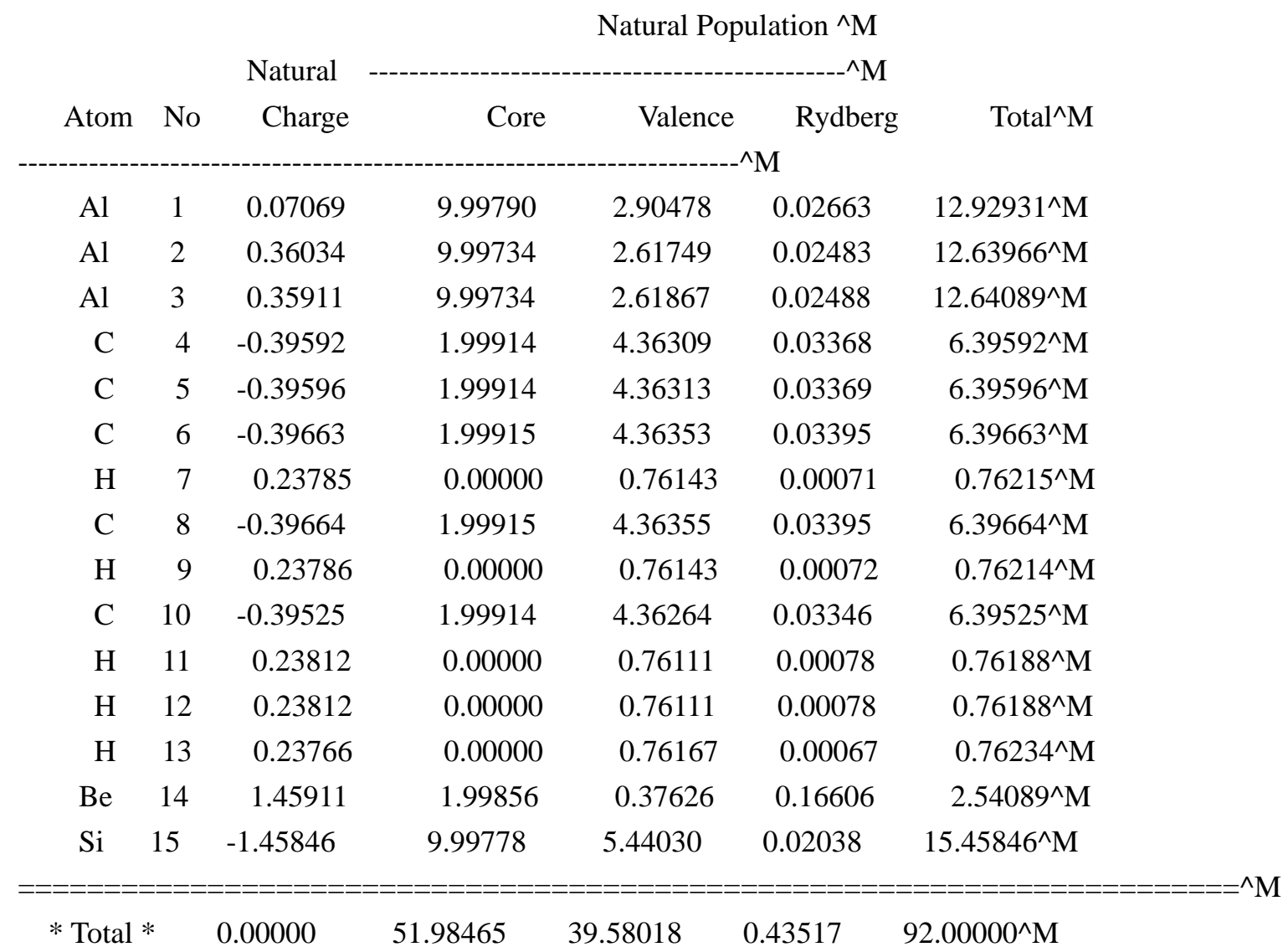

The charge of SiAl3=0.07069+0.36034 $+0.35911-1.45846=-0.66832$

The

charge

of

$C p=-0.39592-0.39596-0.39663+0.23785-0.39664+0.23786-0.39525+0.23812+0.23812+0.23766=-0.79079$

CpCaAl3Si-Al-Si-f-s-b36311+gx-NBO.log 


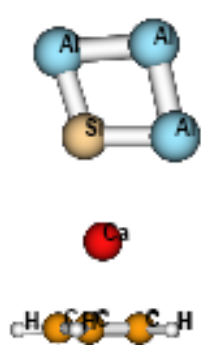

Summary of Natural Population Analysis:

$\wedge \mathrm{M}$

Natural Population $\wedge \mathrm{M}$

\begin{tabular}{|c|c|c|c|c|c|c|}
\hline Atom & No & $\begin{array}{l}\text { Natural } \\
\text { Charge }\end{array}$ & Core & Valence & Rydberg & Total $^{\wedge} \mathrm{M}$ \\
\hline$\Delta 1$ & 1 & 17071 & 000700 & 205030 & בזכרט 0 & 12) $87179 \wedge \mathrm{M}$ \\
\hline $\mathrm{Al}$ & 2 & 0.02102 & 9.99763 & 2.95065 & 0.03071 & $12.97898^{\wedge} \mathrm{M}$ \\
\hline $\mathrm{Al}$ & 3 & 0.38278 & 9.99752 & 2.58471 & 0.03499 & $12.61722^{\wedge} \mathrm{M}$ \\
\hline $\mathrm{C}$ & 4 & -0.39177 & 1.99919 & 4.36159 & 0.03099 & $6.39177 \wedge \mathrm{M}$ \\
\hline C & 5 & -0.39167 & 1.99919 & 4.36148 & 0.03100 & $6.39167 \wedge \mathrm{M}$ \\
\hline $\mathrm{C}$ & 6 & -0.39290 & 1.99919 & 4.36275 & 0.03096 & $6.39290 \wedge \mathrm{M}$ \\
\hline $\mathrm{H}$ & 7 & 0.22003 & 0.00000 & 0.77929 & 0.00068 & $0.77997 \wedge \mathrm{M}$ \\
\hline $\mathrm{C}$ & 8 & -0.39313 & 1.99919 & 4.36295 & 0.03099 & 6.39313^M \\
\hline $\mathrm{H}$ & 9 & 0.22005 & 0.00000 & 0.77927 & 0.00068 & $0.77995^{\wedge} \mathrm{M}$ \\
\hline $\mathrm{C}$ & 10 & -0.38964 & 1.99919 & 4.35953 & 0.03092 & $6.38964 \wedge \mathrm{M}$ \\
\hline $\mathrm{H}$ & 11 & 0.21985 & 0.00000 & 0.77949 & 0.00066 & $0.78015^{\wedge} \mathrm{M}$ \\
\hline $\mathrm{H}$ & 12 & 0.21982 & 0.00000 & 0.77952 & 0.00066 & $0.78018^{\wedge} \mathrm{M}$ \\
\hline $\mathrm{H}$ & 13 & 0.22027 & 0.00000 & 0.77910 & 0.00063 & $0.77973 \wedge \mathrm{M}$ \\
\hline $\mathrm{Ca}$ & 14 & 1.72221 & 17.98979 & 0.12939 & 0.15861 & $18.27779^{\wedge} \mathrm{M}$ \\
\hline $\mathrm{Si}$ & 15 & -1.39512 & 9.99838 & 5.37275 & 0.02398 & $15.39512^{\wedge} \mathrm{M}$ \\
\hline
\end{tabular}

The charge of SiAl3=0.12821 $+0.02102+0.38278-1.39512=-0.86311$

The

charge

of

$C p=-0.39177-0.39167-0.39290+0.22003-0.39313+0.22005-0.38964+0.21985+0.21982+0.22027=-0.85909$

CpKAl3Si-Al-Si-f-s-b36311+gx-NBO.log
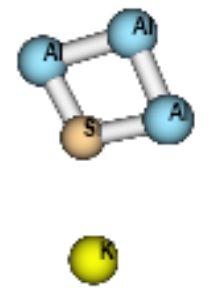

CHCABC-C.

Summary of Natural Population Analysis:

$\wedge \mathrm{M}$

Natural Population $\wedge \mathrm{M}$

Natural $\wedge \mathrm{M}$ 


\begin{tabular}{|c|c|c|c|c|c|c|}
\hline Atom & No & Charge & Core & Valence & Rydberg & Total $\wedge \mathrm{M}$ \\
\hline $\mathrm{Al}$ & 1 & -0.09103 & 9.99821 & 3.06689 & 0.02593 & $13.09103^{\wedge} \mathrm{M}$ \\
\hline $\mathrm{Al}$ & 2 & 0.09081 & 9.99779 & 2.87986 & 0.03154 & $12.90919 \wedge \mathrm{M}$ \\
\hline $\mathrm{Al}$ & 3 & 0.15515 & 9.99778 & 2.81208 & 0.03499 & $12.84485^{\wedge} \mathrm{M}$ \\
\hline $\mathrm{C}$ & 4 & -0.37775 & 1.99917 & 4.35237 & 0.02622 & $6.37775^{\wedge} \mathrm{M}$ \\
\hline $\mathrm{C}$ & 5 & -0.37748 & 1.99917 & 4.35217 & 0.02614 & $6.37748 \wedge \mathrm{M}$ \\
\hline $\mathrm{C}$ & 6 & -0.37870 & 1.99917 & 4.35322 & 0.02632 & $6.37870^{\wedge} \mathrm{M}$ \\
\hline $\mathrm{H}$ & 7 & 0.18727 & 0.00000 & 0.81194 & 0.00079 & $0.81273^{\wedge} \mathrm{M}$ \\
\hline $\mathrm{C}$ & 8 & -0.37895 & 1.99917 & 4.35341 & 0.02638 & $6.37895^{\wedge} \mathrm{M}$ \\
\hline $\mathrm{H}$ & 9 & 0.18726 & 0.00000 & 0.81195 & 0.00079 & $0.81274 \wedge \mathrm{M}$ \\
\hline $\mathrm{C}$ & 10 & -0.37858 & 1.99917 & 4.35331 & 0.02610 & $6.37858^{\wedge} \mathrm{M}$ \\
\hline $\mathrm{H}$ & 11 & 0.18721 & 0.00000 & 0.81200 & 0.00079 & $0.81279 \wedge \mathrm{M}$ \\
\hline $\mathrm{H}$ & 12 & 0.18708 & 0.00000 & 0.81213 & 0.00079 & $0.81292^{\wedge} \mathrm{M}$ \\
\hline $\mathrm{H}$ & 13 & 0.18732 & 0.00000 & 0.81190 & 0.00078 & $0.81268 \wedge \mathrm{M}$ \\
\hline K & 14 & 0.92771 & 17.99148 & 0.04214 & 0.03867 & $18.07229 \wedge \mathrm{M}$ \\
\hline $\mathrm{Si}$ & 15 & -1.12731 & 9.99863 & 5.10205 & 0.02664 & $15.12731^{\wedge} \mathrm{M}$ \\
\hline
\end{tabular}

\begin{tabular}{|c|c|c|c|c|c|}
\hline$*$ Total * & -1.00000 & 67.97973 & 39.72741 & 0.29287 & $108.00000^{\wedge} \mathrm{M}$ \\
\hline
\end{tabular}

The charge of SiAl3=-0.09103+0.09081+0.15515-1.12731=-0.97238

The

charge

of

$\mathrm{CP}=-0.37775-0.37748-0.37870+0.18727-0.37895+0.18726-0.37858+0.18721+0.18708+0.18732=-0.95532$

CpLiAl3Si-f-c-Si-b36311+gx-NBO.log
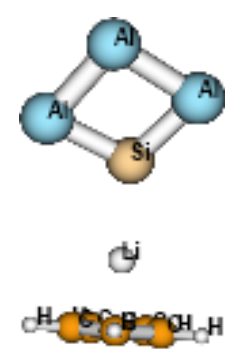

Summary of Natural Population Analysis:

$\wedge \mathrm{M}$

Natural Population $\wedge \mathrm{M}$

\begin{tabular}{|c|c|c|c|c|c|c|}
\hline \multirow[b]{2}{*}{ Atom } & & \multicolumn{4}{|c|}{ ^ی } \\
\hline & No & $\begin{array}{l}\text { Natural } \\
\text { Charge }\end{array}$ & Core & Valence & Rydberg & Total^M \\
\hline $\mathrm{C}$ & 1 & -0.38349 & 1.99916 & 4.35842 & 0.02592 & $6.38349^{\wedge} \mathrm{M}$ \\
\hline $\mathrm{C}$ & 2 & -0.38409 & 1.99916 & 4.35866 & 0.02627 & $6.38409 \wedge \mathrm{M}$ \\
\hline $\mathrm{C}$ & 3 & -0.38334 & 1.99916 & 4.35816 & 0.02602 & $6.38334 \wedge \mathrm{M}$ \\
\hline $\mathrm{H}$ & 4 & 0.19892 & 0.00000 & 0.80033 & 0.00075 & $0.80108^{\wedge} \mathrm{M}$ \\
\hline $\mathrm{C}$ & 5 & -0.38316 & 1.99916 & 4.35791 & 0.02609 & $6.38316^{\wedge} \mathrm{M}$ \\
\hline $\mathrm{H}$ & 6 & 0.19873 & 0.00000 & 0.80047 & 0.00080 & $0.80127 \wedge \mathrm{M}$ \\
\hline $\mathrm{C}$ & 7 & -0.38335 & 1.99916 & 4.35797 & 0.02622 & $6.38335^{\wedge} \mathrm{M}$ \\
\hline $\mathrm{H}$ & 8 & 0.19874 & 0.00000 & 0.80049 & 0.00077 & $0.80126^{\wedge} \mathrm{M}$ \\
\hline
\end{tabular}




$\begin{array}{ccccccc}\mathrm{H} & 9 & 0.19906 & 0.00000 & 0.80016 & 0.00079 & 0.80094 \wedge \mathrm{M} \\ \mathrm{H} & 10 & 0.19883 & 0.00000 & 0.80038 & 0.00079 & 0.80117 \wedge \mathrm{M} \\ \mathrm{Al} & 11 & -0.10991 & 9.99817 & 3.08618 & 0.02556 & 13.10991^{\wedge} \mathrm{M} \\ \mathrm{Al} & 12 & 0.16743 & 9.99773 & 2.80140 & 0.03344 & 12.83257 \wedge \mathrm{M} \\ \mathrm{Al} & 13 & 0.16676 & 9.99769 & 2.80473 & 0.03082 & 12.83324 \wedge \mathrm{M} \\ \mathrm{Li} & 14 & 0.86418 & 1.99687 & 0.09906 & 0.03989 & 2.13582 \wedge \mathrm{M} \\ \mathrm{Si} & 15 & -1.16529 & 9.99851 & 5.12940 & 0.03738 & 15.16529 \wedge \mathrm{M} \\ ===============================================================\wedge \mathrm{M} \\ \text { * Total * } \quad-1.00000 \quad 51.98477 \quad 39.71372 & 0.30151 & 92.00000 \wedge \mathrm{M}\end{array}$

The charge of SiAl3=-0.10991+0.16743+0.16676-1.16529=-0.94101

The charge of $C p=-0.38349-0.38409-0.38334+0.19892-0.38316+0.19873-0.38335+0.19874+0.19906+0.19883=-0.92315$

CpLiAl3Si-f-f-b36311+gx-NBO.log

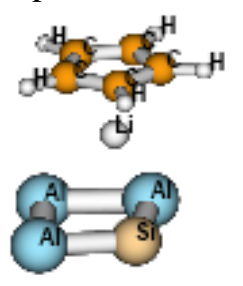

Summary of Natural Population Analysis:

$\wedge \mathrm{M}$

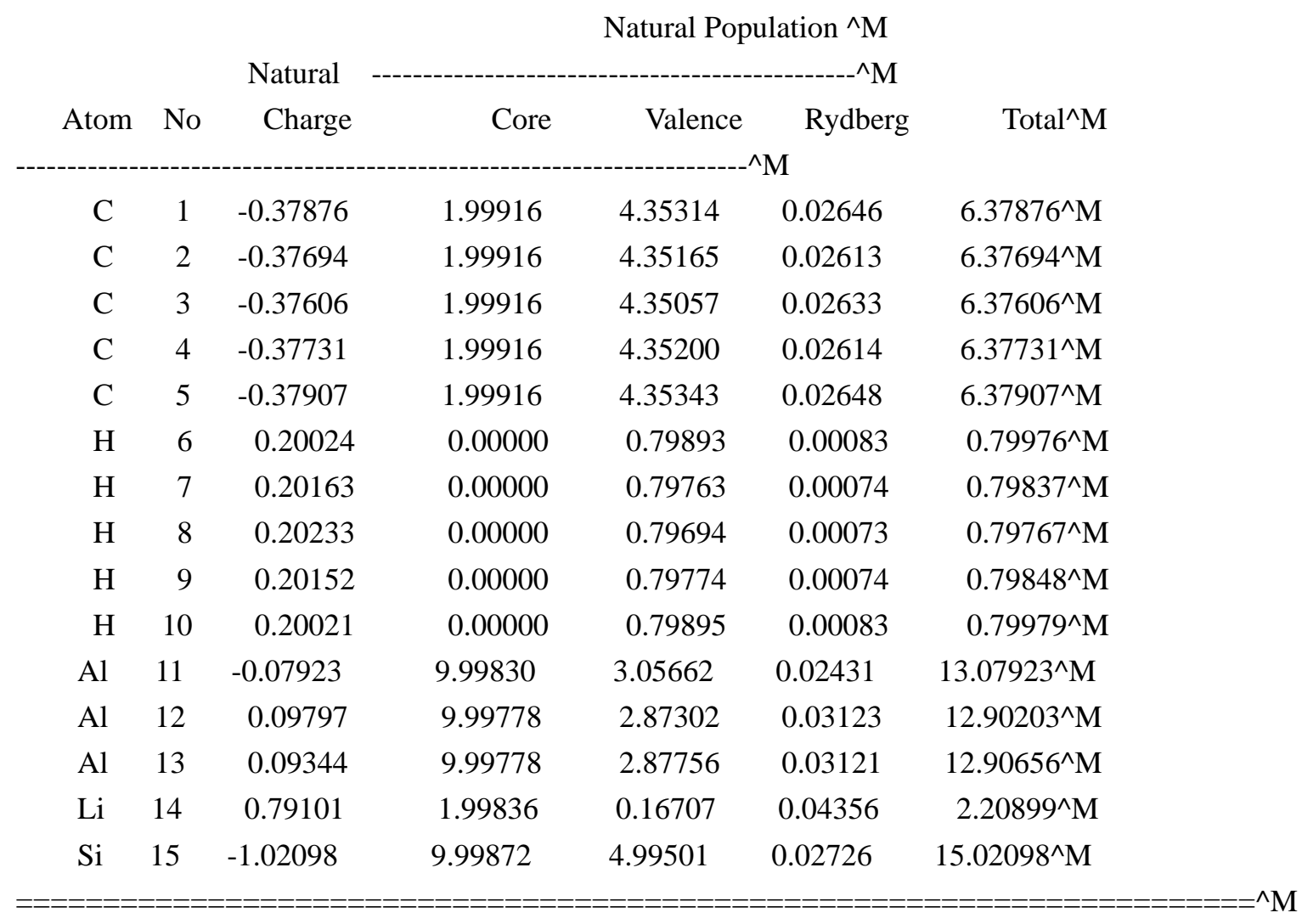

$\begin{array}{llllll}* \text { Total * } & -1.00000 & 51.98676 & 39.72027 & 0.29298 & 92.00000^{\wedge} \mathrm{M}\end{array}$

The charge of SiAl3 $=-0.07923+0.09797+0.09344-1.02098=-0.9088$

The charge of $C p=-0.37876-0.37694-0.37606-0.37731-0.37907+0.20024+0.20163+0.20233+0.20152+0.20021=-0.88221$ 
CpMgAl3Si-f-f-b36311+gx-NBO.log

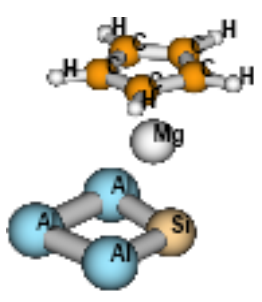

Summary of Natural Population Analysis:

$\wedge \mathrm{M}$

\begin{tabular}{|c|c|c|c|c|c|c|}
\hline \multirow[b]{2}{*}{ Atom } & \multirow[b]{2}{*}{ No } & \multirow[b]{2}{*}{$\begin{array}{c}\text { Natural } \\
\text { Charge }\end{array}$} & \multicolumn{4}{|c|}{ Natural Population $\wedge^{\wedge} \mathrm{M}$} \\
\hline & & & Core & Valence & Rydberg & $\operatorname{Total}^{\wedge} \mathrm{M}$ \\
\hline C & 1 & -0.39654 & 1.99918 & 4.36746 & 0.02989 & $6.39654 \wedge \mathrm{M}$ \\
\hline $\mathrm{C}$ & 2 & -0.39252 & 1.99918 & 4.36411 & 0.02922 & $6.39252^{\wedge} \mathrm{M}$ \\
\hline $\mathrm{C}$ & 3 & -0.39148 & 1.99918 & 4.36299 & 0.02931 & $6.39148 \wedge \mathrm{M}$ \\
\hline $\mathrm{C}$ & 4 & -0.39355 & 1.99918 & 4.36497 & 0.02939 & $6.39355^{\wedge} \mathrm{M}$ \\
\hline $\mathrm{C}$ & 5 & -0.39863 & 1.99918 & 4.36932 & 0.03013 & $6.39863 \wedge \mathrm{M}$ \\
\hline $\mathrm{H}$ & 6 & 0.22770 & 0.00000 & 0.77168 & 0.00062 & $0.77230^{\wedge} \mathrm{M}$ \\
\hline $\mathrm{H}$ & 7 & 0.22664 & 0.00000 & 0.77276 & 0.00060 & $0.77336^{\wedge} \mathrm{M}$ \\
\hline $\mathrm{H}$ & 8 & 0.22542 & 0.00000 & 0.77396 & 0.00062 & $0.77458^{\wedge} \mathrm{M}$ \\
\hline $\mathrm{H}$ & 9 & 0.22693 & 0.00000 & 0.77246 & 0.00060 & $0.77307 \wedge \mathrm{M}$ \\
\hline $\mathrm{H}$ & 10 & 0.22809 & 0.00000 & 0.77128 & 0.00062 & $0.77191^{\wedge} \mathrm{M}$ \\
\hline $\mathrm{Al}$ & 11 & 0.13859 & 9.99838 & 2.83829 & 0.02475 & $12.86141 \wedge \mathrm{M}$ \\
\hline $\mathrm{Al}$ & 12 & 0.22098 & 9.99779 & 2.74850 & 0.03273 & $12.77902^{\wedge} \mathrm{M}$ \\
\hline $\mathrm{Al}$ & 13 & 0.22836 & 9.99778 & 2.74128 & 0.03259 & $12.77164 \wedge \mathrm{M}$ \\
\hline Mg & 14 & 1.48724 & 9.99755 & 0.43116 & 0.08406 & $10.51276^{\wedge} \mathrm{M}$ \\
\hline $\mathrm{Si}$ & 15 & -1.23724 & 9.99866 & 5.20030 & 0.03828 & $15.23724 \wedge \mathrm{M}$ \\
\hline
\end{tabular}

$==$

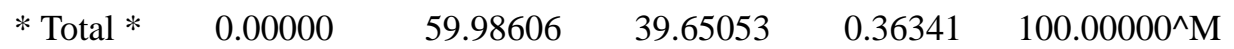

The charge of SiAl3=0.13859+0.22098+0.22836-1.23724=-0.64931

The

charge

of

$\mathrm{C} p=-0.39654-0.39252-0.39148-0.39355-0.39863+0.22770+0.22664+0.22542+0.22693+0.22809=-0.83794$

CpNa2Al3Si-f-s-s-2Si-Al-b36311+gx-NBO.log

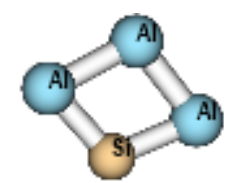

(1)

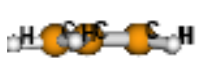

Summary of Natural Population Analysis:

$\wedge \mathrm{M}$

Natural Population $\wedge \mathrm{M}$ 


\begin{tabular}{|c|c|c|c|c|c|c|}
\hline Atom & & $\begin{array}{c}\text { Natural } \\
\text { Charge }\end{array}$ & Core & \multicolumn{2}{|r|}{ M } & Total $\wedge \mathrm{M}$ \\
\hline & & & & & & \\
\hline $\mathrm{Al}$ & 1 & 0.12762 & 9.99800 & 2.84994 & 0.02444 & $12.87238^{\wedge} \mathrm{M}$ \\
\hline $\mathrm{Al}$ & 2 & 0.29825 & 9.99767 & 2.67462 & 0.02946 & $12.70175^{\wedge} \mathrm{M}$ \\
\hline $\mathrm{Al}$ & 3 & 0.20186 & 9.99773 & 2.77173 & 0.02867 & $12.79814 \wedge \mathrm{M}$ \\
\hline $\mathrm{C}$ & 4 & -0.39748 & 1.99918 & 4.37446 & 0.02385 & $6.39748^{\wedge} \mathrm{M}$ \\
\hline $\mathrm{C}$ & 5 & -0.34776 & 1.99917 & 4.32453 & 0.02405 & $6.34776^{\wedge} \mathrm{M}$ \\
\hline $\mathrm{C}$ & 6 & -0.35021 & 1.99917 & 4.32692 & 0.02411 & $6.35021^{\wedge} \mathrm{M}$ \\
\hline $\mathrm{H}$ & 7 & 0.20885 & 0.00000 & 0.79051 & 0.00064 & $0.79115^{\wedge} \mathrm{M}$ \\
\hline C & 8 & -0.40769 & 1.99918 & 4.38382 & 0.02469 & $6.40769 \wedge \mathrm{M}$ \\
\hline $\mathrm{H}$ & 9 & 0.20783 & 0.00000 & 0.79158 & 0.00059 & $0.79217 \wedge \mathrm{M}$ \\
\hline $\mathrm{C}$ & 10 & -0.40476 & 1.99918 & 4.38096 & 0.02462 & $6.40476^{\wedge} \mathrm{M}$ \\
\hline $\mathrm{H}$ & 11 & 0.20790 & 0.00000 & 0.79151 & 0.00059 & $0.79210^{\wedge} \mathrm{M}$ \\
\hline $\mathrm{H}$ & 12 & 0.19723 & 0.00000 & 0.80197 & 0.00079 & $0.80277^{\wedge} \mathrm{M}$ \\
\hline $\mathrm{H}$ & 13 & 0.19769 & 0.00000 & 0.80153 & 0.00078 & $0.80231^{\wedge} \mathrm{M}$ \\
\hline $\mathrm{Na}$ & 14 & 0.89194 & 9.99569 & 0.09221 & 0.02016 & $10.10806^{\wedge} \mathrm{M}$ \\
\hline $\mathrm{Si}$ & 15 & -1.50393 & 9.99855 & 5.48269 & 0.02268 & $15.50393 \wedge \mathrm{M}$ \\
\hline $\mathrm{Na}$ & 16 & 0.87264 & 9.99508 & 0.11839 & 0.01389 & $10.12736^{\wedge} \mathrm{M}$ \\
\hline$====$ & 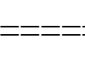 & $=========$ & $========$ & $=========$ & $=========$ & $=========$ \\
\hline * Total & & 0.00000 & 69.97862 & 39.75738 & 0.26400 & $10.00000^{\wedge} \mathrm{M}$ \\
\hline
\end{tabular}

The charge of SiAl3 $=0.12762+0.29825+0.20186-1.50393=-0.8762$

The

charge

of

$\mathrm{Cp}=-0.39748-0.34776-0.35021+0.20885-0.40769+0.20783-0.40476+0.20790+0.19723+0.19769=-0.8884$

CpNaAl3Si-f-c-Si-b36311+gx-NBO.log

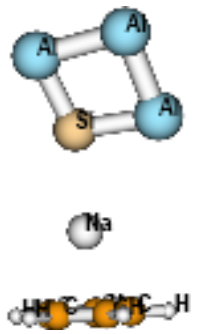

Summary of Natural Population Analysis:

$\wedge \mathrm{M}$

Natural Population $\wedge \mathrm{M}$

\begin{tabular}{|c|c|c|c|c|c|c|}
\hline Atom & No & $\begin{array}{l}\text { Natural } \\
\text { Charge }\end{array}$ & Core & Valence & $\begin{array}{l}-----\wedge M \\
\text { Rydberg }\end{array}$ & Total $\wedge \mathrm{M}$ \\
\hline $\mathrm{Al}$ & 1 & -0.08707 & 9.99818 & 3.06382 & 0.02507 & $13.08707 \wedge \mathrm{M}$ \\
\hline $\mathrm{Al}$ & 2 & 0.12861 & 9.99774 & 2.84289 & 0.03075 & $12.87139 \wedge \mathrm{M}$ \\
\hline $\mathrm{Al}$ & 3 & 0.17641 & 9.99776 & 2.79133 & 0.03450 & $12.82359 \wedge \mathrm{M}$ \\
\hline $\mathrm{C}$ & 4 & -0.37896 & 1.99917 & 4.35517 & 0.02462 & $6.37896 \wedge \mathrm{M}$ \\
\hline C & 5 & -0.38028 & 1.99917 & 4.35635 & 0.02475 & $6.38028 \wedge \mathrm{M}$ \\
\hline C & 6 & -0.38089 & 1.99917 & 4.35697 & 0.02474 & $6.38089 \wedge \mathrm{M}$ \\
\hline
\end{tabular}




$\begin{array}{ccccccc}\mathrm{H} & 7 & 0.19163 & 0.00000 & 0.80756 & 0.00081 & 0.80837 \wedge \mathrm{M} \\ \mathrm{C} & 8 & -0.38067 & 1.99917 & 4.35705 & 0.02444 & 6.38067 \wedge \mathrm{M} \\ \mathrm{H} & 9 & 0.19132 & 0.00000 & 0.80789 & 0.00080 & 0.80868^{\wedge} \mathrm{M} \\ \mathrm{C} & 10 & -0.38053 & 1.99917 & 4.35697 & 0.02439 & 6.38053^{\wedge} \mathrm{M} \\ \mathrm{H} & 11 & 0.19127 & 0.00000 & 0.80794 & 0.00079 & 0.80873^{\wedge} \mathrm{M} \\ \mathrm{H} & 12 & 0.19132 & 0.00000 & 0.80790 & 0.00078 & 0.80868 \wedge \mathrm{M} \\ \mathrm{H} & 13 & 0.19137 & 0.00000 & 0.80786 & 0.00077 & 0.80863^{\wedge} \mathrm{M} \\ \mathrm{Na} & 14 & 0.89503 & 9.99637 & 0.07956 & 0.02904 & 10.10497 \wedge \mathrm{M} \\ \mathrm{Si} & 15 & -1.16858 & 9.99856 & 5.14083 & 0.02918 & 15.16858^{\wedge} \mathrm{M} \\ ===============================================================\wedge \mathrm{M} \\ \text { * Total * }\end{array}$

The charge of SiAl3 $=-0.08707+0.12861+0.17641-1.16858=-0.95063$

The

charge

of

$\mathrm{Cp}=-0.37896-0.38028-0.38089+0.19163-0.38067+0.19132-0.38053+0.19127+0.19132+0.19137=-0.94442$

CpCaAl3Si-Al-Al-f-s-b36311+gx-NBO.log

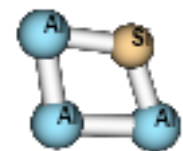

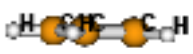

Summary of Natural Population Analysis:

$\wedge \mathrm{M}$

Natural Population $\wedge \mathrm{M}$

\begin{tabular}{|c|c|c|c|c|c|c|}
\hline Atom & No & $\begin{array}{l}\text { Natural } \\
\text { Charge }\end{array}$ & 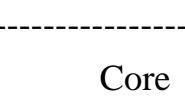 & Valence & Rydberg & Total $\wedge \mathrm{M}$ \\
\hline & & & & & & \\
\hline $\mathrm{Al}$ & 1 & 0.02124 & 9.99721 & 2.94352 & 0.03803 & $12.97876^{\wedge} \mathrm{M}$ \\
\hline $\mathrm{Al}$ & 2 & 0.42362 & 9.99694 & 2.54532 & 0.03412 & $12.57638^{\wedge} \mathrm{M}$ \\
\hline C & 3 & -0.39242 & 1.99919 & 4.36123 & 0.03201 & $6.39242^{\wedge} \mathrm{M}$ \\
\hline $\mathrm{C}$ & 4 & -0.39262 & 1.99918 & 4.36138 & 0.03206 & $6.39262^{\wedge} \mathrm{M}$ \\
\hline $\mathrm{C}$ & 5 & -0.39574 & 1.99918 & 4.36377 & 0.03278 & $6.39574 \wedge \mathrm{M}$ \\
\hline $\mathrm{H}$ & 6 & 0.22065 & 0.00000 & 0.77865 & 0.00070 & $0.77935^{\wedge} \mathrm{M}$ \\
\hline $\mathrm{C}$ & 7 & -0.39521 & 1.99918 & 4.36330 & 0.03273 & $6.39521^{\wedge} \mathrm{M}$ \\
\hline $\mathrm{H}$ & 8 & 0.22067 & 0.00000 & 0.77863 & 0.00070 & $0.77933^{\wedge} \mathrm{M}$ \\
\hline $\mathrm{C}$ & 9 & -0.38740 & 1.99919 & 4.35684 & 0.03137 & $6.38740^{\wedge} \mathrm{M}$ \\
\hline $\mathrm{H}$ & 10 & 0.22055 & 0.00000 & 0.77873 & 0.00072 & $0.77945^{\wedge} \mathrm{M}$ \\
\hline $\mathrm{H}$ & 11 & 0.22064 & 0.00000 & 0.77864 & 0.00072 & $0.77936^{\wedge} \mathrm{M}$ \\
\hline $\mathrm{H}$ & 12 & 0.22145 & 0.00000 & 0.77785 & 0.00070 & $0.77855^{\wedge} \mathrm{M}$ \\
\hline $\mathrm{Ca}$ & 13 & 1.67256 & 17.98799 & 0.19805 & 0.14140 & $18.32744 \wedge \mathrm{M}$ \\
\hline $\mathrm{Al}$ & 14 & -0.52275 & 9.99746 & 3.48832 & 0.03698 & $13.52275^{\wedge} \mathrm{M}$ \\
\hline $\mathrm{Si}$ & 15 & -0.73524 & 9.99821 & 4.71743 & 0.01960 & $14.73524 \wedge \mathrm{M}$ \\
\hline
\end{tabular}

The charge of SiAl3 $=0.02124+0.42362-0.52275-0.73524=-0.81313$ 
The

charge

of

$C p=-0.39242-0.39262-0.39574+0.22065-0.39521+0.22067-0.38740+0.22055+0.22064+0.22145=-0.85943$ 
Cp3Li3Al3Si-extend3-b3631+gx-NBO.log
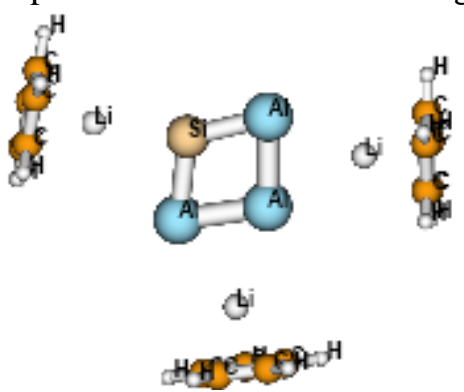

Summary of Natural Population Analysis:

Natural Population

\begin{tabular}{|c|c|c|c|c|c|c|}
\hline \multirow[b]{2}{*}{ Atom } & \multicolumn{2}{|r|}{ Natural } & \multirow{2}{*}{\multicolumn{3}{|c|}{ Rydberg }} & \multirow[b]{2}{*}{ Total } \\
\hline & No & Charge & & & & \\
\hline $\mathrm{Al}$ & 1 & 0.21628 & 9.99744 & 2.75101 & 0.03526 & 12.78372 \\
\hline $\mathrm{Al}$ & 2 & -0.23955 & 9.99766 & 3.20268 & 0.03922 & 13.23955 \\
\hline $\mathrm{Li}$ & 3 & 0.83077 & 1.99668 & 0.13681 & 0.03574 & 2.16923 \\
\hline $\mathrm{Li}$ & 4 & 0.82747 & 1.99666 & 0.13942 & 0.03645 & 2.17253 \\
\hline $\mathrm{C}$ & 5 & -0.41568 & 1.99910 & 4.39179 & 0.02480 & 6.41568 \\
\hline $\mathrm{C}$ & 6 & -0.41977 & 1.99909 & 4.39503 & 0.02565 & 6.41977 \\
\hline $\mathrm{C}$ & 7 & -0.41797 & 1.99909 & 4.39359 & 0.02529 & 6.41797 \\
\hline $\mathrm{H}$ & 8 & 0.23979 & 0.00000 & 0.75937 & 0.00084 & 0.76021 \\
\hline $\mathrm{C}$ & 9 & -0.41333 & 1.99910 & 4.38936 & 0.02488 & 6.41333 \\
\hline $\mathrm{H}$ & 10 & 0.23894 & 0.00000 & 0.76013 & 0.00094 & 0.76106 \\
\hline $\mathrm{C}$ & 11 & -0.41714 & 1.99909 & 4.39278 & 0.02526 & 6.41714 \\
\hline $\mathrm{H}$ & 12 & 0.23925 & 0.00000 & 0.75984 & 0.00090 & 0.76075 \\
\hline $\mathrm{H}$ & 13 & 0.24175 & 0.00000 & 0.75743 & 0.00082 & 0.75825 \\
\hline $\mathrm{H}$ & 14 & 0.24011 & 0.00000 & 0.75901 & 0.00088 & 0.75989 \\
\hline $\mathrm{C}$ & 15 & -0.41908 & 1.99909 & 4.39453 & 0.02547 & 6.41908 \\
\hline $\mathrm{C}$ & 16 & -0.41459 & 1.99909 & 4.39048 & 0.02502 & 6.41459 \\
\hline $\mathrm{C}$ & 17 & -0.41546 & 1.99909 & 4.39162 & 0.02474 & 6.41546 \\
\hline $\mathrm{H}$ & 18 & 0.23910 & 0.00000 & 0.76002 & 0.00088 & 0.76090 \\
\hline $\mathrm{C}$ & 19 & -0.41454 & 1.99909 & 4.39046 & 0.02498 & 6.41454 \\
\hline $\mathrm{H}$ & 20 & 0.24187 & 0.00000 & 0.75734 & 0.00079 & 0.75813 \\
\hline $\mathrm{C}$ & 21 & -0.41983 & 1.99909 & 4.39528 & 0.02546 & 6.41983 \\
\hline $\mathrm{H}$ & 22 & 0.23925 & 0.00000 & 0.75993 & 0.00082 & 0.76075 \\
\hline $\mathrm{H}$ & 23 & 0.24185 & 0.00000 & 0.75736 & 0.00079 & 0.75815 \\
\hline $\mathrm{H}$ & 24 & 0.23893 & 0.00000 & 0.76018 & 0.00089 & 0.76107 \\
\hline $\mathrm{Si}$ & 25 & -1.05354 & 9.99800 & 5.02377 & 0.03178 & 15.05354 \\
\hline $\mathrm{Al}$ & 26 & 0.22065 & 9.99751 & 2.74623 & 0.03560 & 12.77935 \\
\hline $\mathrm{Li}$ & 27 & 0.87463 & 1.99729 & 0.08790 & 0.04018 & 2.12537 \\
\hline $\mathrm{C}$ & 28 & -0.41902 & 1.99910 & 4.39552 & 0.02439 & 6.41902 \\
\hline $\mathrm{C}$ & 29 & -0.41943 & 1.99910 & 4.39599 & 0.02434 & 6.41943 \\
\hline $\mathrm{C}$ & 30 & -0.41914 & 1.99910 & 4.39568 & 0.02436 & 6.41914 \\
\hline
\end{tabular}




$\begin{array}{ccccccc}\mathrm{H} & 31 & 0.23742 & 0.00000 & 0.76179 & 0.00079 & 0.76258 \\ \mathrm{C} & 32 & -0.41884 & 1.99910 & 4.39550 & 0.02424 & 6.41884 \\ \mathrm{H} & 33 & 0.23708 & 0.00000 & 0.76212 & 0.00080 & 0.76292 \\ \mathrm{C} & 34 & -0.41967 & 1.99910 & 4.39606 & 0.02451 & 6.41967 \\ \mathrm{H} & 35 & 0.23742 & 0.00000 & 0.76181 & 0.00078 & 0.76258 \\ \mathrm{H} & 36 & 0.23709 & 0.00000 & 0.76212 & 0.00080 & 0.76291 \\ \mathrm{H} & 37 & 0.23694 & 0.00000 & 0.76225 & 0.00081 & 0.76306\end{array}$

$\begin{array}{llllll}* \text { Total * } & -1.00000 & 75.96766 & 91.39220 & 0.64014 & 168.00000\end{array}$

The charge of SiAl3=0.21628-0.23955-1.05354+0.22065=-0.85616

Cp3Li3Al3Si-extend3-b3631+gx-NICS.log
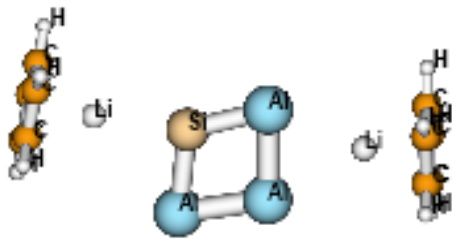

bi

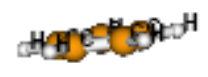

$38 \mathrm{~Bq}$ Isotropic $=41.3374 \quad$ Anisotropy $=42.7665$

$\mathrm{XX}=29.6657 \quad \mathrm{YX}=\quad-4.1409 \quad \mathrm{ZX}=0.4409$

$\mathrm{XY}=\quad-4.9349 \quad \mathrm{YY}=\quad 24.5143 \quad \mathrm{ZY}=\quad-0.6847$

$\mathrm{XZ}=\quad 0.3857 \quad \mathrm{YZ}=\quad-0.6870 \quad \mathrm{ZZ}=69.8322$

Eigenvalues: $\quad 21.8690 \quad 32.2948 \quad 69.8484$

$39 \mathrm{~Bq}$ Isotropic $=29.8134$ Anisotropy $=38.2166$ 
Cp2Li2Al3Si-f-f-c-f-Si-b3631+gx-NICS.log

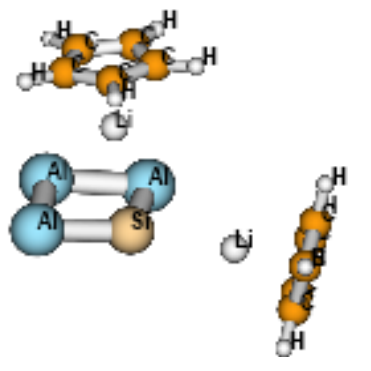

$27 \mathrm{~Bq}$ Isotropic $=\quad 35.9552 \quad$ Anisotropy $=47.9561$

\begin{tabular}{lrrrlr}
$\mathrm{XX}=$ & 33.2537 & $\mathrm{YX}=$ & 21.3615 & $\mathrm{ZX}=$ & 3.2934 \\
$\mathrm{XY}=$ & 20.9788 & $\mathrm{YY}=$ & 54.8267 & $\mathrm{ZY}=$ & 0.7805 \\
$\mathrm{XZ}=$ & 4.5525 & $\mathrm{YZ}=$ & $\mathbf{0 . 1 7 7 0}$ & $\mathrm{ZZ}=$ & 19.7853 \\
\multicolumn{2}{l}{ Eigenvalues: } & 16.8637 & 23.0761 & $\mathbf{6 7 . 9 2 6 0}$
\end{tabular}

$28 \mathrm{~Bq}$ Isotropic $=\quad 25.5825 \quad$ Anisotropy $=\quad 41.4305$ /gpfs1/u/doctor/jwang/ylm/ylm

Cp4Li4Al3Si-extend4-b3631+gx-NICS.log

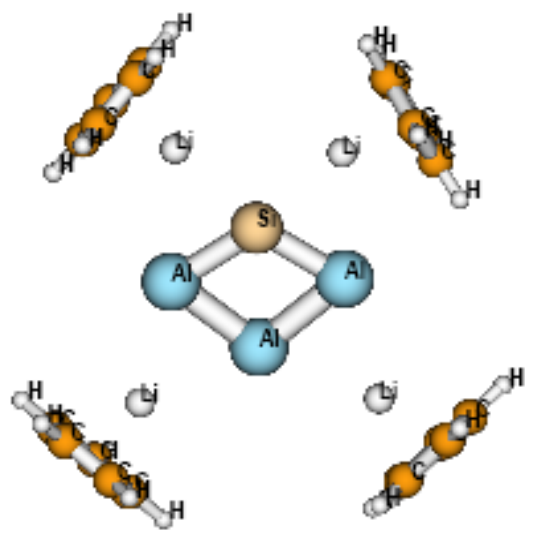

$49 \mathrm{~Bq}$ Isotropic $=41.7803$ Anisotropy $=36.7139$

$\mathrm{XX}=36.7690 \quad \mathrm{YX}=\quad 0.0150 \quad \mathrm{ZX}=0.0300$

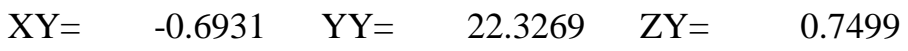

$\mathrm{XZ}=0.0201 \quad \mathrm{YZ}=0.6473 \quad \mathrm{ZZ}=66.2451$

Eigenvalues: $\quad 22.3078 \quad 36.7769 \quad 66.2563$

$50 \mathrm{~Bq} \quad$ Isotropic $=29.6616$ Anisotropy $=34.9654$ 
Al3SiLiAl3Si-c-c--90-Si-Si-b36311+gx-frq.frq6.8 $\quad 0.00$

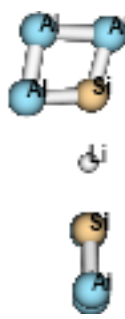

$\begin{array}{rrrrc}13 & 0 & -2.636483 & 1.327921 & 1.043641 \\ 3 & 0 & -0.000066 & -0.000129 & -0.476680 \\ 13 & 0 & 2.636790 & -1.328334 & 1.043377 \\ 13 & 0 & 5.085874 & -0.669042 & 0.678496 \\ 13 & 0 & -5.085706 & 0.669333 & 0.678662 \\ 14 & 0 & -2.502332 & -0.452143 & -0.569941 \\ 13 & 0 & -4.692513 & -1.211759 & -1.053346 \\ 13 & 0 & 4.692169 & 1.212252 & -1.053158 \\ 14 & 0 & 2.502225 & 0.451827 & -0.570038\end{array}$

State $=1-A \backslash H F=-2041.3111486$

1

A

Frequencies -- $\quad 6.7648$

Red. masses -- 26.4045

Frc consts -- 0.0007

IR Inten $\quad--\quad 0.0328$

Atom AN $\mathrm{X} \quad \mathrm{Y}$

113

$0.12-0.29$

23

$0.00 \quad 0.00$

313

$-0.13$

0.29

$4 \quad 13$

$-0.09$

0.20

$0.09 \quad-0.20$

$-0.02$

0.13

$6 \quad 14$

$7 \quad 13$

$-0.07$

0.2

0.07

$\begin{array}{ll}8 & 13\end{array}$
0.02

95

$$
\begin{array}{lll}
0.02 & -0.13 & -0.25
\end{array}
$$

4

A

Frequencies -- $\quad 25.4171$

Red. masses -- $\quad 27.2365$

Frc consts -- 0.0104

IR Inten $\quad$-- 0.1051

Atom AN $\mathrm{X} \quad \mathrm{Y}$

$\begin{array}{lllll}2 & 3 & 0.00 & 0.00 & 0.07\end{array}$

$\begin{array}{lllll}3 & 13 & 0.37 & -0.27 & 0.08\end{array}$

$4 \quad 13$

0.25

$0.19 \quad 0.04$

$\begin{array}{lll}\text { Z } & \mathrm{X} & \mathrm{Y}\end{array}$

2

9.5300

25.7566

0.0014

0.0470

$\mathrm{X}$

$-0.16$

$-0.17$

$\begin{array}{lll}0.00 & 0.00 & 0.26\end{array}$

$\begin{array}{lll}0.16 & 0.18 & 0.30\end{array}$

$\begin{array}{llll}0.14 & -0.09 & -0.29\end{array}$

$\begin{array}{lll}-0.14 & 0.08 & -0.29\end{array}$

$\begin{array}{lll}0.03 & -0.12 & 0.26\end{array}$

$\begin{array}{lll}0.06 & 0.15 & -0.32\end{array}$

$\begin{array}{lll}-0.06 & -0.16 & -0.32\end{array}$

$-0.02$

0.12

5

A

25.5699

26.8503

0.0103

0.1152

X Y

$\begin{array}{lll}-0.26 & 0.09 & 0.31\end{array}$

$\begin{array}{lll}0.10 & 0.12 & 0.00\end{array}$

$\begin{array}{lll}-0.23 & 0.07 & -0.30\end{array}$

$\begin{array}{ll}-0.15 & -0.02\end{array}$
3

A

12.5478

25.2189

0.0023

0.0480

$\begin{array}{lll}\mathrm{X} & \mathrm{Y} & \mathrm{Z}\end{array}$

$\begin{array}{lll}-0.16 & 0.20 & -0.10\end{array}$

$\begin{array}{lll}0.03 & 0.32 & 0.00\end{array}$

$\begin{array}{lll}-0.17 & 0.20 & 0.09\end{array}$

$\begin{array}{lll}-0.06 & -0.35 & -0.17\end{array}$

$\begin{array}{lll}-0.06 & -0.35 & 0.17\end{array}$

$\begin{array}{lll}0.05 & 0.31 & -0.20\end{array}$

$\begin{array}{lll}0.17 & -0.21 & 0.07\end{array}$

$\begin{array}{lll}0.17 & -0.21 & -0.07\end{array}$

$\begin{array}{lll}0.05 & 0.31 & 0.20\end{array}$

6

A

75.7676

27.0242

0.0914

0.3817

$\begin{array}{lllll}Z & X & Y & \text { Z }\end{array}$

$\begin{array}{lll}0.22 & 0.17 & 0.11\end{array}$

$\begin{array}{lll}0.00 & 0.00 & -0.09\end{array}$

$\begin{array}{lll}-0.22 & -0.17 & 0.11\end{array}$

$\begin{array}{lll}-0.31 & 0.09 & -0.09\end{array}$ 


$$
\begin{array}{rrrrr}
5 & 13 & -0.24 & -0.19 & 0.05 \\
6 & 14 & -0.07 & 0.41 & -0.07 \\
7 & 13 & 0.08 & -0.01 & -0.07 \\
8 & 13 & -0.10 & 0.02 & -0.08 \\
9 & 14 & 0.06 & -0.41 & -0.04
\end{array}
$$

A

Frequencies -- 104.0395

Red. masses -- $\quad 7.8931$

Frc consts -- 0.0503

IR Inten -- 18.3090
Atom AN $\mathrm{X} \quad \mathrm{Y}$

$\begin{array}{lllll}1 & 13 & -0.01 & -0.09 & -0.02\end{array}$

$\begin{array}{lllll}2 & 3 & -0.11 & 0.97 & 0.00\end{array}$

$\begin{array}{lllll}3 & 13 & -0.01 & -0.09 & 0.02\end{array}$

$\begin{array}{lllll}4 & 13 & -0.03 & 0.04 & 0.01\end{array}$

$\begin{array}{llllll}5 & 13 & -0.03 & 0.04 & -0.01\end{array}$

$\begin{array}{llllll}6 & 14 & 0.04 & -0.08 & -0.03\end{array}$

$$
\begin{array}{rcc}
-0.16 & -0.03 & -0.19 \\
0.12 & 0.03 & 0.42 \\
0.26 & -0.08 & -0.05 \\
0.25 & -0.08 & 0.04 \\
0.13 & 0.00 & -0.42 \\
\multicolumn{3}{c}{8} \\
\multicolumn{4}{c}{8}
\end{array}
$$

\section{8}

A

108.9465

7.7252

0.0540

9.2100

X $\quad \mathrm{Y}$

$\begin{array}{lll}0.01 & 0.00 & -0.08\end{array}$

$\begin{array}{lll}0.00 & 0.00 & 0.98\end{array}$

$\begin{array}{lll}-0.01 & 0.00 & -0.08\end{array}$

$\begin{array}{lll}-0.01 & 0.02 & 0.04\end{array}$

$\begin{array}{lll}0.01 & -0.02 & 0.04\end{array}$

$\begin{array}{lll}0.05 & -0.02 & -0.06\end{array}$

$\begin{array}{lll}0.03 & 0.04 & -0.03\end{array}$

$\begin{array}{lll}-0.03 & -0.04 & -0.03\end{array}$

$\begin{array}{lll}-0.05 & 0.02 & -0.06\end{array}$

11

A

Frequencies -- 116.2685

Red. masses -- 25.5184

Frc consts -- 0.2032

IR Inten -- 3.4603

Atom AN $\quad \mathrm{X} \quad \mathrm{Y} \quad \mathrm{Z}$

$\begin{array}{lllll}1 & 13 & -0.03 & 0.24 & -0.25\end{array}$

$\begin{array}{llllll}2 & 3 & 0.00 & 0.00 & -0.29\end{array}$

$\begin{array}{llllll}3 & 13 & 0.03 & -0.24 & -0.25\end{array}$

$\begin{array}{llllll}4 & 13 & -0.01 & 0.21 & 0.24\end{array}$

$\begin{array}{llllll}5 & 13 & 0.01 & -0.21 & 0.24\end{array}$

$\begin{array}{llllll}6 & 14 & 0.01 & -0.23 & 0.28\end{array}$

$\begin{array}{lllll}7 & 13 & -0.04 & 0.22 & -0.24\end{array}$

$\begin{array}{llllll}8 & 13 & 0.04 & -0.22 & -0.24\end{array}$

$\begin{array}{lllll}9 & 14 & -0.01 & 0.23 & 0.28\end{array}$

13

A

Frequencies -- $\quad 263.3210$

Red. masses -- 26.5892

Frc consts -- 1.0862

IR Inten -- $\quad 1.2857$

Atom AN X Y
A

156.7408

20.6623

0.2991

2.7741

X Y

$\begin{array}{lll}0.15 & 0.16 & 0.13\end{array}$

$\begin{array}{lll}-0.39 & 0.42 & 0.00\end{array}$

$\begin{array}{lll}0.15 & 0.16 & -0.13\end{array}$

$\begin{array}{lll}0.30 & -0.11 & 0.13\end{array}$

$\begin{array}{lll}0.30 & -0.11 & -0.13\end{array}$

$\begin{array}{lll}-0.26 & 0.07 & 0.12\end{array}$

$\begin{array}{lll}-0.14 & -0.17 & -0.14\end{array}$

$\begin{array}{lll}-0.14 & -0.17 & 0.14\end{array}$

$\begin{array}{lll}-0.26 & 0.07 & -0.12\end{array}$

14

A

263.8500

26.9115

1.1038

X Y
0.5303 $\begin{array}{lll}0.31 & -0.09 & -0.09\end{array}$

$\begin{array}{lll}0.26 & 0.17 & 0.10\end{array}$

$\begin{array}{lll}0.42 & -0.06 & -0.11\end{array}$

$\begin{array}{lll}-0.42 & 0.06 & -0.11\end{array}$

$\begin{array}{lll}-0.26 & -0.17 & 0.10\end{array}$

9

A

115.2930

26.6601

0.2088

2.6745

$\begin{array}{llllll}\mathrm{Z} & \mathrm{X} & \mathrm{Y} & \mathrm{Z}\end{array}$

$\begin{array}{lll}0.03 & -0.22 & 0.28\end{array}$

$\begin{array}{lll}0.02 & -0.17 & 0.00\end{array}$

$\begin{array}{lll}0.03 & -0.22 & -0.28\end{array}$

$\begin{array}{lll}-0.01 & 0.22 & 0.25\end{array}$

$\begin{array}{lll}-0.01 & 0.22 & -0.25\end{array}$

$\begin{array}{lll}-0.04 & 0.25 & -0.26\end{array}$

$\begin{array}{lll}0.02 & -0.24 & 0.25\end{array}$

$\begin{array}{llll}0.02 & -0.24 & -0.25\end{array}$

$\begin{array}{lll}-0.04 & 0.25 & 0.26\end{array}$

12

A

177.4219

24.8343

0.4606

2.8167

$\begin{array}{lllll}Z & X & Y & Z\end{array}$

$\begin{array}{lll}-0.10 & -0.16 & -0.13\end{array}$

$\begin{array}{lll}0.00 & 0.00 & -0.35\end{array}$

$\begin{array}{lll}0.10 & 0.16 & -0.13\end{array}$

$\begin{array}{lll}0.28 & -0.12 & 0.13\end{array}$

$\begin{array}{lll}-0.28 & 0.12 & 0.13\end{array}$

$\begin{array}{lll}0.34 & -0.07 & -0.09\end{array}$

$\begin{array}{lll}0.31 & 0.18 & 0.14\end{array}$

$\begin{array}{lll}-0.31 & -0.18 & 0.14\end{array}$

$\begin{array}{lll}-0.34 & 0.07 & -0.09\end{array}$

15

A

308.6112

26.8137

1.5046

3.9298 


$\begin{array}{rrrrr}1 & 13 & 0.32 & -0.04 & -0.06 \\ 2 & 3 & -0.14 & 0.06 & 0.00 \\ 3 & 13 & 0.31 & -0.04 & 0.06 \\ 4 & 13 & -0.32 & 0.20 & -0.21 \\ 5 & 13 & -0.33 & 0.21 & 0.22 \\ 6 & 14 & -0.14 & 0.08 & 0.09 \\ 7 & 13 & 0.17 & -0.26 & -0.25 \\ 8 & 13 & 0.17 & -0.26 & 0.25 \\ 9 & 14 & -0.13 & 0.08 & -0.08 \\ & & & 16 & \end{array}$

A

Frequencies -- 308.9945

Red. masses -- 26.9375

Frc consts -- 1.5153

IR Inten -- 2.0346

Atom AN $\quad \mathrm{X} \quad \mathrm{Y} \quad \mathrm{Z}$

$$
\begin{array}{ccc}
0.31 & -0.03 & -0.05 \\
0.00 & 0.00 & 0.08 \\
-0.32 & 0.03 & -0.06 \\
0.33 & -0.21 & 0.22 \\
-0.32 & 0.20 & 0.21 \\
-0.15 & 0.08 & 0.09 \\
0.12 & -0.28 & -0.26 \\
-0.13 & 0.29 & -0.27 \\
0.15 & -0.09 & 0.09 \\
\multicolumn{3}{c}{17} \\
\hline
\end{array}
$$

A

357.8258

18.4237

$$
1.3899
$$

9.8667

$$
\begin{array}{ccc}
0.37 & 0.10 & 0.05 \\
-0.09 & 0.04 & 0.00 \\
0.38 & 0.10 & -0.06 \\
-0.32 & -0.31 & 0.25 \\
-0.31 & -0.30 & -0.24 \\
-0.05 & -0.02 & -0.01 \\
0.01 & 0.22 & 0.19 \\
0.01 & 0.22 & -0.20 \\
-0.05 & -0.02 & 0.01 \\
& \multicolumn{2}{c}{18}
\end{array}
$$

A

368.3693

27.4068

2.1912

7.8692

$\begin{array}{lllllllllll}1 & 13 & -0.39 & -0.11 & -0.06 & 0.06 & 0.22 & 0.19 & 0.01 & 0.35 & 0.32\end{array}$

$\begin{array}{llllllllllll}2 & 3 & 0.00 & 0.00 & -0.05 & 0.65 & 0.11 & 0.00 & 0.00 & 0.00 & -0.01\end{array}$

$\begin{array}{llllllllllll}3 & 13 & 0.38 & 0.11 & -0.06 & 0.06 & 0.22 & -0.19 & -0.01 & -0.35 & 0.32\end{array}$

$\begin{array}{llllllllllll}4 & 13 & -0.32 & -0.30 & 0.24 & -0.10 & 0.03 & -0.04 & 0.08 & -0.08 & 0.08\end{array}$

$\begin{array}{lllllllllll}5 & 13 & 0.33 & 0.31 & 0.24 & -0.10 & 0.03 & 0.04 & -0.08 & 0.08 & 0.08\end{array}$

$\begin{array}{lllllllllll}6 & 14 & 0.05 & 0.02 & 0.01 & 0.22 & -0.11 & -0.12 & 0.21 & -0.30 & -0.29\end{array}$

$\begin{array}{lllllllllll}7 & 13 & 0.02 & -0.21 & -0.19 & -0.28 & -0.15 & -0.11 & -0.11 & -0.12 & -0.10\end{array}$

$\begin{array}{lllllllllll}8 & 13 & -0.02 & 0.20 & -0.18 & -0.28 & -0.15 & 0.11 & 0.11 & 0.12 & -0.10\end{array}$

$\begin{array}{lllllllllll}9 & 14 & -0.05 & -0.02 & 0.01 & 0.22 & -0.11 & 0.12 & -0.21 & 0.30 & -0.29\end{array}$

$19 \quad 20$

A

A

Frequencies -- $\quad 380.4981$

Red. masses -- 18.4819

415.8613

A

Frc consts -- 1.5765

IR Inten -- $\quad 45.4505$

27.5588

479.7616

10.1384

2.8081

1.3749

0.2079

378.1344

$\begin{array}{lllllllllll}\text { Atom AN } & \text { X } & \text { Y } & \text { Z } & \text { X } & \text { Y } & \text { Z } & \text { X } & \text { Y } & \text { Z }\end{array}$

$\begin{array}{lllllllllll}1 & 13 & 0.05 & -0.21 & -0.19 & 0.07 & -0.07 & -0.07 & -0.02 & 0.01 & 0.01\end{array}$

$\begin{array}{lllllllllll}2 & 3 & 0.65 & 0.12 & 0.00 & 0.00 & 0.00 & 0.01 & 0.91 & 0.14 & 0.00\end{array}$

$\begin{array}{lllllllllll}3 & 13 & 0.05 & -0.21 & 0.19 & -0.07 & 0.07 & -0.07 & -0.02 & 0.01 & -0.01\end{array}$

$\begin{array}{lllllllllll}4 & 13 & -0.01 & -0.07 & 0.06 & 0.07 & 0.04 & -0.03 & 0.03 & 0.00 & 0.00\end{array}$

$\begin{array}{lllllllllll}5 & 13 & -0.01 & -0.07 & -0.06 & -0.07 & -0.04 & -0.03 & 0.03 & 0.00 & 0.00\end{array}$

$\begin{array}{lllllllllll}6 & 14 & 0.09 & 0.27 & 0.24 & 0.46 & 0.23 & 0.16 & -0.23 & -0.06 & -0.03\end{array}$

$\begin{array}{lllllllllll}7 & 13 & -0.22 & -0.02 & 0.00 & -0.41 & -0.11 & -0.06 & 0.11 & 0.03 & 0.02\end{array}$

$\begin{array}{llllllllllll}8 & 13 & -0.22 & -0.02 & 0.00 & 0.41 & 0.11 & -0.06 & 0.11 & 0.03 & -0.02\end{array}$

$\begin{array}{lllllllllll}9 & 14 & 0.09 & 0.27 & -0.24 & -0.46 & -0.23 & 0.16 & -0.23 & -0.06 & 0.03\end{array}$

Al3SiLiAl3Si-s-Al-Al-c-Si-90-b36311+gx-frq.frq7.2 


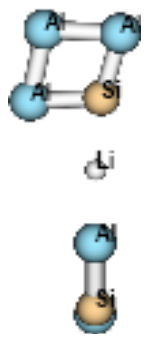

13

13

3

13

13

13

14

14

13

$\begin{array}{rrr}2.632384 & 1.634799 & 0.282372 \\ 5.102794 & 0.977601 & 0.144789 \\ 0.047469 & -0.356258 & -0.062844 \\ -2.573719 & 0.082467 & -1.168133 \\ -5.104810 & 0.311119 & -1.249060 \\ 4.773936 & -1.541093 & -0.332228 \\ 2.556131 & -0.729178 & -0.168098 \\ -4.772732 & -0.164405 & 1.055660 \\ -2.454432 & -0.420359 & 1.380927\end{array}$

State $=1-A \backslash H F=-2041.305955$

A

A

A

Frequencies -- $\quad 7.2091$

15.1090

17.8286

23.6045

23.6108

0.0032

0.0044

Frc consts -- 0.0008

0.0134

0.3422

$\begin{array}{llrr}\text { Atom AN } & & \mathrm{X} & \mathrm{Y} \\ \end{array}$

$\begin{array}{llll}1 & 13 & 0.02 & -0.08\end{array}$

$\mathrm{Z}$

$\mathrm{X} \quad \mathrm{Y}$

$\mathrm{Z}$

$\begin{array}{ll}\mathrm{X} & \mathrm{Y}\end{array}$

Z

$\begin{array}{llll}2 & 13 & 0.01 & -0.07\end{array}$

0.45

$\begin{array}{lll}0.04 & 0.08 & -0.28\end{array}$

0.40

$0.26 \quad 0.06$

$\begin{array}{lllll}3 & 3 & 0.00 & 0.03 & -0.06\end{array}$

$\begin{array}{lll}0.03 & -0.12 & 0.49\end{array}$

$\begin{array}{lll}0.27 & -0.23 & -0.10\end{array}$

$\begin{array}{llllll}4 & 13 & -0.05 & -0.31 & -0.10\end{array}$

$\begin{array}{lll}-0.01 & 0.12 & -0.41\end{array}$

$\begin{array}{lll}-0.04 & 0.40 & 0.12\end{array}$

$\begin{array}{llllll}5 & 13 & -0.05 & -0.37 & -0.08\end{array}$

$\begin{array}{lll}-0.15 & 0.03 & -0.10\end{array}$

$\begin{array}{lll}-0.05 & 0.23 & 0.06\end{array}$

$\begin{array}{lllll}6 & 13 & -0.02 & 0.06 & -0.42\end{array}$

$\begin{array}{lll}-0.17 & -0.07 & 0.18\end{array}$

$\begin{array}{lll}-0.09 & -0.27 & -0.07\end{array}$

$\begin{array}{lll}-0.03 & -0.07 & 0.27\end{array}$

$\begin{array}{lll}-0.24 & -0.18 & -0.04\end{array}$

$\begin{array}{llllll}7 & 14 & -0.01 & 0.03 & -0.16\end{array}$

$\begin{array}{lll}-0.01 & 0.12 & -0.46\end{array}$

$\begin{array}{lll}-0.07 & 0.27 & 0.10\end{array}$

$8 \quad 14$

$\begin{array}{lll}0.03 & 0.30 & 0.05\end{array}$

$0.12-0.05$

0.14

$\begin{array}{lll}-0.12 & -0.30 & -0.07\end{array}$

$9 \quad 13$

$\begin{array}{lll}0.05 & 0.42 & 0.04\end{array}$

4

$\begin{array}{lll}0.17 & 0.04 & -0.12\end{array}$

$-0.09$

$0.12 \quad 0.04$

5

A

A

Frequencies -- $\quad 37.9265$

45.3037

70.8212

23.5927

26.7967

0.0285

0.0792

Frc consts -- 0.0212

1.6790

1.0786

IR Inten -- 1.0825

$\begin{array}{llll}\text { Atom AN } & \text { X } & \text { Y } & \text { Z }\end{array}$

$\begin{array}{lllll}1 & 13 & -0.25 & -0.27 & -0.05\end{array}$

$\mathrm{X} \quad \mathrm{Y}$

$\mathrm{X} \quad \mathrm{Y}$

Z

$\begin{array}{lllll}2 & 13 & -0.18 & 0.06 & 0.02\end{array}$

$\begin{array}{lll}0.01 & -0.05 & 0.21\end{array}$

$\mathrm{Z}$

$\begin{array}{lll}0.23 & -0.18 & -0.06\end{array}$

$\begin{array}{lllll}3 & 3 & 0.07 & 0.31 & 0.06\end{array}$

$\begin{array}{lll}0.01 & 0.04 & -0.19\end{array}$

$\begin{array}{lll}0.31 & 0.13 & 0.04\end{array}$

413

$\begin{array}{lll}0.11 & 0.54 & 0.10\end{array}$

$\begin{array}{lll}0.01 & 0.08 & -0.42\end{array}$

$\begin{array}{lll}0.07 & -0.04 & 0.13\end{array}$

$\begin{array}{lll}-0.23 & 0.09 & -0.35\end{array}$

$\begin{array}{lll}-0.28 & 0.04 & 0.01\end{array}$ 


\begin{tabular}{|c|c|c|c|c|c|c|c|c|c|c|}
\hline 5 & 13 & 0.04 & -0.26 & -0.05 & -0.28 & -0.02 & 0.20 & -0.32 & 0.02 & 0.01 \\
\hline 6 & 13 & 0.12 & 0.03 & 0.01 & 0.02 & -0.01 & 0.07 & 0.45 & 0.12 & 0.01 \\
\hline 7 & 14 & 0.00 & -0.29 & -0.06 & 0.02 & -0.08 & 0.40 & 0.30 & -0.18 & -0.07 \\
\hline 8 & 14 & 0.04 & -0.26 & -0.05 & 0.18 & -0.04 & 0.13 & -0.36 & 0.02 & 0.02 \\
\hline 9 & 13 & 0.10 & 0.39 & 0.07 & 0.25 & 0.05 & -0.38 & -0.34 & 0.04 & 0.00 \\
\hline & & & 7 & & & 8 & & & & 9 \\
\hline
\end{tabular}

A

A

A

Frequencies -- 103.9297

Red. masses -- 12.7916

109.9229

23.9254

115.5174

Frc consts -- 0.0814

0.1703

27.1680

3.4731

0.2136

\begin{tabular}{llll} 
IR Inten & -- & & 3.7535 \\
\hline
\end{tabular}

X Y

1.4998

$\begin{array}{rrrrr}\text { Atom AN } & \text { X } & \text { Y } & \text { Z } \\ 1 & 13 & -0.02 & 0.03 & -0.04 \\ 2 & 13 & -0.03 & -0.02 & 0.04 \\ 3 & 3 & 0.05 & -0.17 & 0.83 \\ 4 & 13 & 0.17 & 0.04 & -0.28 \\ 5 & 13 & 0.12 & -0.04 & 0.16 \\ 6 & 13 & -0.02 & -0.01 & -0.02 \\ 7 & 14 & 0.00 & 0.03 & -0.05 \\ 8 & 14 & -0.13 & -0.03 & 0.20 \\ 9 & 13 & -0.10 & 0.06 & -0.23\end{array}$

A

$\begin{array}{lll}0.00 & 0.04 & 0.05\end{array}$

$\begin{array}{lll}\mathrm{Z} & \mathrm{X} & \mathrm{Y}\end{array}$

Z

$0.00-0.09-0.46$

$\begin{array}{rrrrrr}-0.02 & -0.40 & -0.07 & 0.01 & 0.06 & 0.03\end{array}$

$\begin{array}{lllllll}0.04 & 0.43 & 0.08 & 0.02 & -0.04 & -0.02\end{array}$

$\begin{array}{llllll}-0.04 & -0.45 & -0.09 & 0.03 & 0.04 & 0.01\end{array}$

$\begin{array}{lllllll}0.01 & -0.03 & 0.04 & 0.00 & -0.09 & 0.48\end{array}$

$\begin{array}{lllllll}0.03 & 0.05 & -0.04 & -0.01 & 0.09 & -0.51\end{array}$

$\begin{array}{llllll}0.03 & 0.47 & 0.09 & -0.03 & -0.04 & 0.00\end{array}$

$\begin{array}{llllll}-0.04 & -0.42 & -0.08 & -0.02 & 0.04 & 0.00\end{array}$

11

A

12

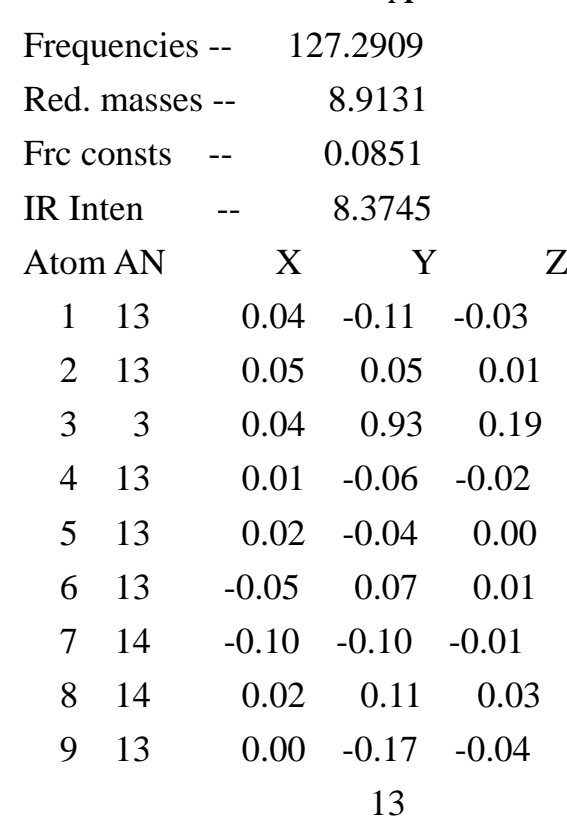

A

Frequencies -- $\quad 264.1187$

Red. masses -- 26.5192

Frc consts -- 1.0900

166.2062

18.9191

0.3079

1.9707

Z $\quad \mathrm{X} \quad \mathrm{Y}$

$\begin{array}{lll}0.17 & -0.24 & -0.05\end{array}$

$\begin{array}{lrr}Z & X & Y\end{array}$

175.8120

11.9026

0.2168

12.2077

A

$\begin{array}{llllll}0.37 & 0.22 & 0.04 & 0.02 & 0.01 & 0.01\end{array}$

$\begin{array}{lll}-0.33 & -0.53 & -0.15\end{array}$

$\begin{array}{lll}0.00 & -0.19 & 0.85\end{array}$

$\begin{array}{lll}0.05 & 0.04 & -0.02\end{array}$

$\begin{array}{lll}-0.16 & 0.01 & 0.06\end{array}$

$\begin{array}{lll}0.09 & -0.01 & 0.02\end{array}$

$\begin{array}{lll}-0.26 & 0.04 & -0.10\end{array}$

$\begin{array}{llllll}-0.27 & 0.26 & 0.05 & -0.02 & 0.01 & 0.01\end{array}$

$\begin{array}{llllll}-0.36 & -0.15 & -0.02 & -0.02 & 0.00 & -0.04\end{array}$
$\begin{array}{llllll}0.02 & -0.03 & 0.01 & 0.26 & 0.01 & -0.17\end{array}$
$\begin{array}{lll}0.02 & 0.05 & 0.00\end{array}$
14
$\begin{array}{lll}0.17 & -0.02 & 0.05\end{array}$
15

A

A

274.4483
26.5758
1.1794
1.1438
$X \quad Y$

310.2863

26.0721

1.4789

7.0455

$\begin{array}{lrrr}\text { IR Inten } & -- & & 0.5886 \\ \text { Atom AN } & & X & \mathrm{Y}\end{array}$

Z

$\begin{array}{lll}\mathrm{X} & \mathrm{Y}\end{array}$

$\mathrm{X} \quad \mathrm{Y}$

Z 


$\begin{array}{rrrrrrrrrrr}1 & 13 & -0.43 & -0.11 & -0.02 & 0.02 & 0.00 & 0.00 & 0.50 & -0.13 & -0.03 \\ 2 & 13 & 0.44 & 0.43 & 0.08 & -0.02 & -0.02 & 0.00 & -0.44 & 0.49 & 0.10 \\ 3 & 3 & 0.14 & 0.08 & 0.01 & 0.07 & -0.04 & 0.13 & -0.21 & -0.04 & 0.05 \\ 4 & 13 & 0.00 & -0.01 & 0.03 & -0.24 & -0.09 & 0.60 & -0.18 & 0.03 & -0.06 \\ 5 & 13 & -0.01 & 0.00 & -0.01 & 0.22 & 0.02 & -0.19 & 0.17 & -0.01 & 0.00 \\ 6 & 13 & -0.21 & -0.52 & -0.10 & -0.01 & 0.03 & 0.01 & 0.05 & -0.39 & -0.07 \\ 7 & 14 & 0.19 & 0.17 & 0.03 & -0.01 & -0.01 & 0.00 & -0.08 & 0.04 & 0.01 \\ 8 & 14 & -0.02 & 0.00 & 0.01 & -0.11 & -0.03 & 0.17 & 0.00 & 0.00 & -0.01 \\ 9 & 13 & 0.00 & 0.00 & -0.03 & 0.14 & 0.11 & -0.62 & 0.03 & -0.01 & 0.06\end{array}$

A

Frequencies -- 313.4981

Red. masses -- 23.5901

Frc consts -- 1.3660

IR Inten -- 17.5588

$\begin{array}{llll}\text { Atom AN } & \mathrm{X} & \mathrm{Y} & \mathrm{Z}\end{array}$

$\begin{array}{lllll}1 & 13 & 0.18 & -0.07 & -0.01\end{array}$

$\begin{array}{lllll}2 & 13 & -0.15 & 0.14 & 0.03\end{array}$

$\begin{array}{lllll}3 & 3 & 0.38 & -0.04 & -0.16\end{array}$

$\begin{array}{llllll}4 & 13 & 0.59 & -0.09 & 0.22\end{array}$

$\begin{array}{rrrrr}5 & 13 & -0.52 & 0.04 & 0.00\end{array}$

$\begin{array}{llllll}6 & 13 & -0.11 & -0.04 & -0.01\end{array}$

$\begin{array}{lllll}7 & 14 & -0.01 & -0.01 & 0.00\end{array}$

$\begin{array}{lllll}8 & 14 & 0.03 & 0.00 & 0.01\end{array}$

$9 \quad 13$ $\begin{array}{lll}-0.11 & 0.05 & -0.20\end{array}$

19
A

356.6220

15.6337

1.1715

47.5788

A

371.1908

23.9413

1.9435

41.6420

$\begin{array}{lll}\mathrm{X} & \mathrm{Y}\end{array}$

$\begin{array}{lll}0.08 & -0.28 & -0.05\end{array}$

$-0.10 \quad-0.04 \quad-0.01$

$\begin{array}{llll}0.75 & -0.09 & -0.01\end{array}$

$\begin{array}{lll}-0.10 & 0.03 & -0.10\end{array}$

$\begin{array}{lll}0.02 & 0.02 & -0.13\end{array}$

$\begin{array}{lll}-0.31 & 0.18 & 0.04\end{array}$

$\begin{array}{lll}0.23 & 0.16 & 0.03\end{array}$

$\begin{array}{lll}-0.16 & -0.01 & 0.10\end{array}$

$\begin{array}{lll}0.15 & -0.03 & 0.12\end{array}$

20

A

426.5149

26.6651

2.8580

31.9155

IR Inten -- 23.9752

$\begin{array}{lllll}\text { Atom AN } & X & \text { Y } & \text { Z }\end{array}$

$\begin{array}{llllllll}1 & 13 & 0.05 & 0.21 & 0.04 & 0.03 & 0.04 & 0.01\end{array}$

$\begin{array}{llllllll}2 & 13 & -0.02 & 0.08 & 0.02 & -0.04 & 0.02 & 0.00\end{array}$

$\begin{array}{lllllllll}3 & 3 & 0.62 & -0.07 & -0.02 & -0.19 & 0.03 & -0.06\end{array}$

$\begin{array}{llllllll}4 & 13 & -0.13 & 0.00 & 0.07 & -0.09 & 0.02 & -0.05\end{array}$

$\begin{array}{lllll}5 & 13 & 0.13 & -0.08 & 0.38\end{array}$

$\begin{array}{lll}0.05 & 0.06 & -0.33\end{array}$

$\begin{array}{lll}-0.19 & 0.05 & 0.01\end{array}$

$\begin{array}{lll}0.25 & -0.11 & -0.02\end{array}$

$\begin{array}{lll}0.53 & -0.12 & 0.38\end{array}$

$\begin{array}{lll}-0.52 & 0.04 & 0.00\end{array}$

$\begin{array}{rrrrr}6 & 13 & -0.26 & 0.03 & 0.01\end{array}$

$\begin{array}{lllll}7 & 14 & 0.15 & -0.30 & -0.06\end{array}$

$\begin{array}{lllll}8 & 14 & 0.09 & 0.06 & -0.35\end{array}$

$\begin{array}{lllll}9 & 13 & -0.19 & 0.03 & -0.08\end{array}$

Al3SiLiAl3Si-s-s-90-Al-Al-b36311+gx-frq.frq11.

$\begin{array}{lll}0.01 & 0.47 & 0.09\end{array}$

$\begin{array}{lll}0.05 & 0.13 & 0.03\end{array}$

$\begin{array}{lll}0.41 & -0.06 & 0.00\end{array}$

$\begin{array}{lll}0.02 & 0.02 & -0.11\end{array}$

$\begin{array}{lll}-0.08 & 0.07 & -0.32\end{array}$

$\begin{array}{lll}-0.04 & -0.10 & -0.02\end{array}$

$\begin{array}{lll}-0.10 & -0.47 & -0.09\end{array}$

$\begin{array}{lll}-0.21 & -0.04 & 0.27\end{array}$

$\begin{array}{lll}0.26 & -0.05 & 0.14\end{array}$

21

A

453.2337

12.4533

1.5072

197.0367

$\begin{array}{llllll}\mathrm{Z} & \mathrm{X} & \mathrm{Y} & \mathrm{Z}\end{array}$

$\begin{array}{lll}-0.04 & -0.04 & -0.01\end{array}$

$\begin{array}{lll}0.05 & -0.01 & 0.00\end{array}$

$\begin{array}{lll}0.85 & -0.08 & -0.05\end{array}$

$\begin{array}{lll}-0.08 & 0.01 & -0.02\end{array}$

$\begin{array}{lll}0.04 & 0.00 & -0.03\end{array}$

$\begin{array}{lll}0.22 & -0.06 & -0.01\end{array}$

$\begin{array}{lll}-0.37 & 0.12 & 0.03\end{array}$

$\begin{array}{lll}0.13 & -0.02 & 0.05\end{array}$

$\begin{array}{lll}-0.17 & 0.01 & 0.01\end{array}$ 


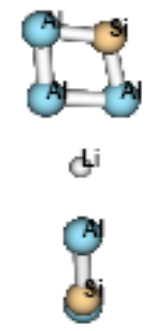

$\begin{array}{rrrrc}13 & 0 & -2.616757 & 0.798826 & 0.833842 \\ 3 & 0 & -0.000016 & 0.000352 & 0.006686 \\ 13 & 0 & 2.497672 & 1.042233 & -0.997905 \\ 13 & 0 & 2.616598 & -0.802341 & 0.830438 \\ 13 & 0 & -2.497497 & -1.037316 & -1.002944 \\ 13 & 0 & -5.154186 & 0.881598 & 0.943598 \\ 13 & 0 & 5.154033 & -0.886362 & 0.939395 \\ 14 & 0 & -4.820774 & -0.779357 & -0.720484 \\ 14 & 0 & 4.820905 & 0.782402 & -0.716913\end{array}$

State $=1-A \backslash H F=-2041.3007014$

\section{1}

A

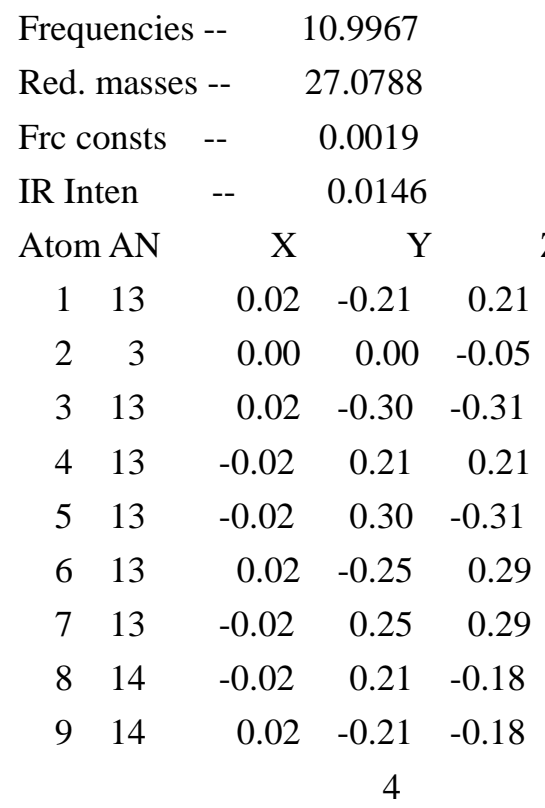

A

Frequencies -- $\quad 56.1739$

Red. masses -- 26.5491

Frc consts -- 0.0494

IR Inten $\quad$-- 0.4614

Atom AN $\quad \mathrm{X} \quad \mathrm{Y} \quad \mathrm{Z}$

$\begin{array}{lllll}1 & 13 & 0.09 & 0.11 & -0.46\end{array}$

$\begin{array}{lllll}2 & 3 & -0.02 & -0.16 & 0.02\end{array}$

$\begin{array}{lllll}3 & 13 & -0.17 & -0.06 & 0.38\end{array}$

$\begin{array}{lllll}4 & 13 & 0.13 & 0.01 & 0.45\end{array}$

$5 \quad 13$

$\begin{array}{lll}-0.15 & 0.02 & -0.38\end{array}$
2

A

20.2518

21.1065

0.0051

1.2098

$\mathrm{X} \quad \mathrm{Y}$

$\begin{array}{lll}-0.14 & 0.25 & -0.07\end{array}$

$\begin{array}{lll}-0.01 & 0.55 & 0.03\end{array}$

0.15

$-0.13$

0.16

$-0.15$

$-0.14$

$\begin{array}{lll}0.12 & -0.25 & 0.08\end{array}$

$\begin{array}{lll}0.11 & -0.26 & -0.11\end{array}$

5

A

56.2650

26.6065

0.0496

0.1381

X Y

$\begin{array}{lll}-0.18 & 0.44 & -0.01\end{array}$

$\begin{array}{lll}0.00 & 0.02 & 0.14\end{array}$

$\begin{array}{lll}-0.08 & -0.37 & -0.02\end{array}$

$\begin{array}{lll}0.16 & -0.45 & -0.11\end{array}$

$\begin{array}{lll}0.11 & 0.38 & 0.06\end{array}$

\section{3}

A

20.3103

21.1636

0.0051

1.2379

$\begin{array}{lllll}Z & X & Y & \text { Z }\end{array}$

$\begin{array}{lll}-0.12 & -0.12 & 0.28\end{array}$

$\begin{array}{lll}0.00 & -0.03 & 0.55\end{array}$

$\begin{array}{lll}-0.16 & -0.01 & 0.19\end{array}$

$\begin{array}{lll}0.13 & 0.09 & 0.27\end{array}$

$\begin{array}{lll}0.15 & -0.01 & 0.19\end{array}$

$\begin{array}{lll}-0.14 & 0.05 & -0.26\end{array}$

$\begin{array}{lll}0.15 & -0.03 & -0.25\end{array}$

$\begin{array}{lll}0.11 & 0.12 & -0.27\end{array}$

$\begin{array}{lll}-0.12 & -0.10 & -0.27\end{array}$

\section{6}

A

65.8709

26.9967

0.0690

0.0030

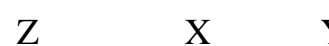

Z 


\begin{tabular}{rrrrrrrrrrc}
6 & 13 & 0.14 & -0.02 & 0.19 & -0.23 & -0.18 & -0.02 & 0.33 & -0.03 & 0.01 \\
7 & 13 & 0.18 & 0.02 & -0.18 & 0.19 & 0.18 & 0.02 & -0.33 & 0.03 & 0.01 \\
8 & 14 & -0.10 & -0.03 & 0.14 & 0.07 & -0.14 & 0.00 & 0.38 & -0.01 & 0.01 \\
9 & 14 & -0.11 & 0.00 & -0.14 & -0.04 & 0.14 & 0.03 & -0.38 & 0.01 & 0.01 \\
& & & 7 & & \multicolumn{2}{c}{8} & & & &
\end{tabular}

A

A

IR Inten $\quad$-- $\quad 5.7150$

$\begin{array}{rrrrc}\text { Atom AN } & \mathrm{X} & \mathrm{Y} & \mathrm{Z} \\ 1 & 13 & 0.10 & 0.27 & -0.04 \\ 2 & 3 & 0.06 & -0.71 & 0.00 \\ 3 & 13 & -0.08 & 0.00 & -0.20 \\ 4 & 13 & 0.10 & 0.27 & 0.04 \\ 5 & 13 & -0.08 & 0.00 & 0.20 \\ 6 & 13 & 0.08 & -0.22 & 0.07 \\ 7 & 13 & 0.08 & -0.22 & -0.07 \\ 8 & 14 & -0.10 & 0.04 & -0.22 \\ 9 & 14 & -0.10 & 0.04 & 0.22 \\ & & & 10 & \end{array}$

A

Frequencies -- $\quad 113.3762$

Red. masses -- 18.5088

Frc consts -- 0.1402

IR Inten -- $\quad 2.4717$

$\begin{array}{ccccc}\text { Atom AN } & \mathrm{X} & \mathrm{Y} & \mathrm{Z} \\ 1 & 13 & 0.12 & 0.18 & 0.03 \\ 2 & 3 & 0.00 & 0.00 & -0.66 \\ 3 & 13 & 0.08 & 0.10 & 0.28 \\ 4 & 13 & -0.12 & -0.18 & 0.03 \\ 5 & 13 & -0.08 & -0.10 & 0.28 \\ 6 & 13 & 0.11 & -0.20 & 0.05 \\ 7 & 13 & -0.11 & 0.20 & 0.06 \\ 8 & 14 & -0.10 & 0.09 & -0.26 \\ 9 & 14 & 0.10 & -0.08 & -0.26\end{array}$

13

A

Frequencies -- 273.2055

Red. masses -- 25.7620

Frc consts --- 1.1329

IR Inten -- 5.8696

$\begin{array}{lllll}\text { Atom AN } & \mathrm{X} & \mathrm{Y} & \mathrm{Z}\end{array}$

$\begin{array}{lllll}1 & 13 & 0.15 & 0.31 & 0.31\end{array}$

\begin{tabular}{ccc} 
X & $Y$ \\
-0.11 & 0.05 & -0.28 \\
0.00 & 0.00 & 0.68 \\
-0.06 & 0.21 & 0.02 \\
0.11 & -0.05 & -0.28 \\
0.06 & -0.21 & 0.02 \\
-0.09 & -0.09 & 0.23 \\
0.09 & 0.08 & 0.23 \\
0.08 & 0.23 & -0.06 \\
-0.08 & -0.23 & -0.06 \\
\multicolumn{3}{c}{11} \\
\hline
\end{tabular}

A

179.8775

11.1419

0.2124

13.1517
108.2538

17.9043

0.1236

0.1585

\subsection{1}

19.5306

0.1439

7.1099

$\begin{array}{llll}\mathrm{Z} & \mathrm{X} & \mathrm{Y}\end{array}$

$\begin{array}{lll}0.11 & 0.01 & 0.19\end{array}$

$\begin{array}{lll}0.05 & -0.62 & 0.00\end{array}$

$\begin{array}{lll}-0.09 & 0.28 & 0.12\end{array}$

$\begin{array}{lll}0.11 & 0.01 & -0.19\end{array}$

$\begin{array}{lll}-0.09 & 0.28 & -0.12\end{array}$

$\begin{array}{lll}0.09 & 0.07 & -0.21\end{array}$

$\begin{array}{lll}0.09 & 0.07 & 0.21\end{array}$

$\begin{array}{lll}-0.11 & -0.28 & 0.11\end{array}$

$\begin{array}{lll}-0.11 & -0.28 & -0.11\end{array}$

12

A

180.1704

11.1652

0.2135

5.1019

$\begin{array}{llllll}X & Y & Z & X & Y\end{array}$

$\begin{array}{lll}0.10 & 0.00 & 0.04\end{array}$

0.10

$0.04 \quad 0.00$

$\begin{array}{lll}-0.02 & 0.89 & 0.00\end{array}$

$\begin{array}{lll}0.00 & 0.00 & 0.89\end{array}$

$\begin{array}{lll}-0.11 & 0.00 & -0.05\end{array}$

$\begin{array}{lll}0.11 & -0.05 & 0.00\end{array}$

$\begin{array}{llll}0.10 & 0.00 & -0.04\end{array}$

$\begin{array}{lll}-0.10 & -0.04 & 0.00\end{array}$

$\begin{array}{lll}-0.11 & 0.00 & 0.05\end{array}$

$\begin{array}{lll}-0.11 & 0.05 & 0.00\end{array}$

$\begin{array}{lll}0.18 & -0.05 & -0.05\end{array}$

$\begin{array}{lll}0.18 & -0.05 & 0.05\end{array}$

$\begin{array}{lll}0.17 & -0.05 & -0.05\end{array}$

$\begin{array}{lll}-0.17 & 0.05 & -0.05\end{array}$

$\begin{array}{lll}-0.16 & -0.07 & -0.09\end{array}$

$\begin{array}{lll}-0.16 & -0.07 & 0.09\end{array}$

14

A

274.8532

26.5890

1.1835

0.1447

$\begin{array}{rrr}-0.17 & -0.09 & -0.06\end{array}$

$\begin{array}{lll}0.17 & 0.09 & -0.06\end{array}$

15

A

301.8989

16.3005

0.8753

78.1598
$\begin{array}{lllllll}X & Y & Z & X & Y & \text { Z }\end{array}$ $\begin{array}{llllll}0.19 & 0.30 & 0.30 & 0.32 & -0.06 & -0.07\end{array}$ 


$\begin{array}{cccccccccccc}2 & 3 & -0.22 & 0.12 & 0.00 & 0.00 & 0.00 & 0.15 & 0.71 & 0.16 & 0.00 \\ 3 & 13 & -0.10 & -0.32 & 0.31 & 0.11 & 0.31 & -0.31 & -0.05 & 0.04 & -0.04 \\ 4 & 13 & 0.15 & 0.31 & -0.30 & -0.19 & -0.30 & 0.30 & 0.32 & -0.07 & 0.07 \\ 5 & 13 & -0.10 & -0.31 & -0.31 & -0.11 & -0.31 & -0.31 & -0.05 & 0.04 & 0.04 \\ 6 & 13 & -0.11 & -0.10 & -0.10 & -0.17 & -0.10 & -0.09 & -0.33 & 0.01 & 0.01 \\ 7 & 13 & -0.11 & -0.10 & 0.10 & 0.17 & 0.10 & -0.09 & -0.33 & 0.01 & -0.01 \\ 8 & 14 & 0.09 & 0.09 & 0.08 & 0.08 & 0.09 & 0.09 & -0.03 & 0.00 & 0.00 \\ 9 & 14 & 0.09 & 0.09 & -0.08 & -0.08 & -0.09 & 0.09 & -0.03 & 0.00 & 0.00\end{array}$

\section{A}

Frequencies -- 318.8731

Red. masses -- 26.4663

Frc consts -- 1.5855

IR Inten $\quad--\quad 1.5880$

$\begin{array}{llll}\text { Atom AN } & X & Y & \text { Z }\end{array}$

$\begin{array}{rrrrr}1 & 13 & 0.48 & -0.14 & -0.15 \\ 2 & 3 & 0.00 & 0.00 & 0.16\end{array}$

$\begin{array}{lllll}3 & 13 & 0.10 & -0.14 & 0.14\end{array}$

$\begin{array}{lllll}4 & 13 & -0.48 & 0.15 & -0.15\end{array}$

$\begin{array}{lllll}5 & 13 & -0.10 & 0.14 & 0.14\end{array}$

$\begin{array}{lllll}6 & 13 & -0.40 & -0.01 & 0.00\end{array}$

$\begin{array}{lllll}7 & 13 & 0.40 & 0.01 & 0.00\end{array}$

$\begin{array}{lllll}8 & 14 & 0.06 & 0.00 & 0.00\end{array}$

$\begin{array}{lllll}9 & 14 & -0.06 & 0.00 & 0.00\end{array}$

19

A

Frequencies -- 390.4238

Red. masses -- 15.3180

Frc consts -- $\quad 1.3757$

IR Inten $\quad$-- 60.2451

Atom AN $\quad \mathrm{X} \quad \mathrm{Y}$

$\begin{array}{lllll}2 & 3 & -0.77 & 0.00 & 0.00\end{array}$

$\begin{array}{lllll}3 & 13 & 0.10 & -0.03 & 0.03\end{array}$

$\begin{array}{llllll}4 & 13 & 0.13 & 0.03 & -0.03\end{array}$

$\begin{array}{llllll}5 & 13 & 0.10 & -0.03 & -0.03\end{array}$

$\begin{array}{lllll}6 & 13 & -0.11 & 0.21 & 0.21\end{array}$

$\begin{array}{lllll}7 & 13 & -0.11 & 0.21 & -0.21\end{array}$

$\begin{array}{lllll}8 & 14 & -0.02 & -0.20 & -0.20\end{array}$

$\begin{array}{lllll}9 & 14 & -0.02 & -0.20 & 0.20\end{array}$
A

355.4248

13.0195

0.9690

134.3579
A

376.6080

27.3322

2.2840

3.1624

$\begin{array}{ccccccc}X & Y & Z & X & Y & Z\end{array}$

$\begin{array}{rrrrrr}-0.14 & 0.08 & 0.08 & -0.09 & -0.08 & -0.08\end{array}$

$\begin{array}{llllll}0.84 & -0.03 & 0.00 & 0.00 & 0.00 & 0.01\end{array}$

$\begin{array}{llllll}0.17 & -0.10 & 0.10 & 0.29 & -0.10 & 0.11\end{array}$

$\begin{array}{llllll}-0.14 & 0.08 & -0.08 & 0.09 & 0.08 & -0.08\end{array}$

$\begin{array}{llllll}0.17 & -0.10 & -0.10 & -0.29 & 0.10 & 0.11\end{array}$

$\begin{array}{llllll}0.04 & 0.10 & 0.10 & 0.13 & -0.30 & -0.30\end{array}$

$\begin{array}{lllllll}0.04 & 0.10 & -0.10 & -0.13 & 0.30 & -0.30\end{array}$

$\begin{array}{llllll}-0.17 & -0.08 & -0.08 & 0.21 & 0.26 & 0.26\end{array}$

$\begin{array}{lll}-0.17 & -0.08 & 0.08\end{array}$

20

A

429.1142

27.4020

2.9729

1.0781

$\begin{array}{lll}-0.21 & -0.26 & 0.26\end{array}$

21

A

434.2427

22.0044

2.4447

2.8717

$\mathrm{X} \quad \mathrm{Y}$

$\begin{array}{llll}\mathrm{Z} & \mathrm{X} & \mathrm{Y}\end{array}$

Z

$\begin{array}{llllll}-0.08 & 0.03 & 0.03 & 0.12 & -0.03 & -0.03\end{array}$

$\begin{array}{llllll}0.00 & 0.00 & 0.07 & -0.51 & -0.07 & 0.00\end{array}$

$\begin{array}{llllll}0.41 & 0.00 & 0.01 & 0.38 & 0.00 & 0.00\end{array}$

$\begin{array}{llllll}0.08 & -0.03 & 0.03 & 0.12 & -0.03 & 0.03\end{array}$

$\begin{array}{llllll}-0.41 & 0.00 & 0.01 & 0.38 & 0.00 & 0.00\end{array}$

$\begin{array}{lllllll}0.04 & 0.17 & 0.17 & -0.06 & -0.12 & -0.12\end{array}$

$\begin{array}{llllll}-0.04 & -0.17 & 0.17 & -0.06 & -0.12 & 0.12\end{array}$

$\begin{array}{llllll}0.42 & -0.21 & -0.21 & -0.36 & 0.15 & 0.16\end{array}$

$\begin{array}{llllll}-0.41 & 0.21 & -0.21 & -0.36 & 0.16 & -0.16\end{array}$

Al3SiLiAl3Si-s-s-to-c-c-0-C2h-b36311+gx-frq.frq-9.9 


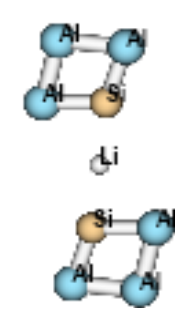

$\begin{array}{rrrrc}13 & 0 & -0.521973 & 4.854711 & 0.000000 \\ 13 & 0 & 2.393150 & 2.290933 & 0.000000 \\ 13 & 0 & 2.064874 & 4.832309 & 0.000000 \\ 3 & 0 & 0.000000 & 0.000000 & 0.000000 \\ 13 & 0 & -2.393150 & -2.290933 & 0.000000 \\ 13 & 0 & -2.064874 & -4.832309 & 0.000000 \\ 13 & 0 & 0.521973 & -4.854711 & 0.000000 \\ 14 & 0 & 0.000000 & 2.545095 & 0.000000 \\ 14 & 0 & 0.000000 & -2.545095 & 0.000000\end{array}$

State $=1-A G \backslash H F=-2041.3111411$

\section{1}

AU

Frequencies -- $\quad$-9.8992

Red. masses -- 26.2932

Frc consts -- 0.0015

IR Inten $\quad$-- $\quad 0.3680$

Atom AN $\mathrm{X} \quad \mathrm{Y}$

$\begin{array}{lllll}1 & 13 & 0.00 & 0.00 & 0.33\end{array}$

$\begin{array}{llllll}2 & 13 & 0.00 & 0.00 & -0.31\end{array}$

$\begin{array}{lllll}3 & 13 & 0.00 & 0.00 & 0.38\end{array}$

$\begin{array}{lllll}4 & 3 & 0.00 & 0.00 & -0.22\end{array}$

$\begin{array}{lllll}5 & 13 & 0.00 & 0.00 & -0.31\end{array}$

$\begin{array}{llllll}6 & 13 & 0.00 & 0.00 & 0.38\end{array}$

$\begin{array}{lllll}7 & 13 & 0.00 & 0.00 & 0.33\end{array}$

$8 \quad 14$

$\begin{array}{ll}9 & 14\end{array}$

$$
\begin{array}{lll}
0.00 & 0.00 & -0.35
\end{array}
$$$$
\begin{array}{lll}
0.00 & 0.00 & -0.35
\end{array}
$$

4

BG

Frequencies -- $\quad 17.0440$

Red. masses -- 27.5572

Frc consts -- 0.0047

IR Inten --- $\quad 0.0000$

$\begin{array}{rrrrr}\text { Atom AN } & \text { X } & \text { Y } & \text { Z } \\ 1 & 13 & 0.00 & 0.00 & -0.08 \\ 2 & 13 & 0.00 & 0.00 & 0.29 \\ 3 & 13 & 0.00 & 0.00 & -0.35 \\ 4 & 3 & 0.00 & 0.00 & 0.00 \\ 5 & 13 & 0.00 & 0.00 & -0.29\end{array}$

$\begin{array}{llllll}5 & 13 & 0.00 & 0.00 & -0.29\end{array}$

$\begin{array}{lll}-0.39 & -0.34 & 0.00\end{array}$

AU

3

$\mathrm{BU}$

4.2184

17.0410

26.2366

24.3103

0.0003

0.0042

0.0289

0.0130

$\mathrm{X} \quad \mathrm{Y}$

Z

$\mathrm{X} \quad \mathrm{Y}$

Z

$\begin{array}{llllll}0.00 & 0.00 & -0.38 & 0.25 & 0.27 & 0.00\end{array}$

$\begin{array}{llllll}0.00 & 0.00 & 0.37 & -0.24 & -0.26 & 0.00\end{array}$

$\begin{array}{lll}0.00 & 0.00 & 0.34\end{array}$

$\begin{array}{lll}0.24 & -0.21 & 0.00\end{array}$

$\begin{array}{lll}0.00 & 0.00 & -0.21\end{array}$

$\begin{array}{lll}-0.34 & 0.16 & 0.00\end{array}$

$\begin{array}{lll}0.00 & 0.00 & 0.37\end{array}$

$\begin{array}{lll}-0.24 & -0.26 & 0.00\end{array}$

$\begin{array}{llllll}0.00 & 0.00 & 0.34 & 0.24 & -0.21 & 0.00\end{array}$

$\begin{array}{lll}0.00 & 0.00 & -0.38\end{array}$

$\begin{array}{lll}0.25 & 0.27 & 0.00\end{array}$

$\begin{array}{llll}0.00 & 0.00 & -0.29\end{array}$

$-0.20$

$\begin{array}{ll}0.17 & 0.00\end{array}$

0.00

$\begin{array}{ll}0.00 & -0.29\end{array}$

5

AG

$-0.20$

$0.17 \quad 0.00$

\section{6}

AG

28.1958

75.9047

27.2387

27.1908

0.0128

0.0923

0.0000

0.0000

$\begin{array}{ll}\mathrm{X} & \mathrm{Y}\end{array}$

Z

$\begin{array}{ll}\mathrm{X} & \mathrm{Y}\end{array}$

Z

$\begin{array}{lll}-0.04 & -0.10 & 0.00\end{array}$

$\begin{array}{lll}0.18 & 0.40 & 0.00\end{array}$

$\begin{array}{lll}0.39 & 0.34 & 0.00\end{array}$

$\begin{array}{lll}-0.16 & 0.25 & 0.00\end{array}$

$\begin{array}{lll}-0.03 & 0.30 & 0.00\end{array}$

$\begin{array}{lll}0.18 & 0.29 & 0.00\end{array}$

$\begin{array}{lll}0.00 & 0.00 & 0.00\end{array}$

$\begin{array}{lll}0.00 & 0.00 & 0.00\end{array}$

$\begin{array}{lll}0.16 & -0.25 & 0.00\end{array}$ 


$\begin{array}{rrrrrrrrrrr}6 & 13 & 0.00 & 0.00 & 0.35 & 0.03 & -0.30 & 0.00 & -0.18 & -0.29 & 0.00 \\ 7 & 13 & 0.00 & 0.00 & 0.08 & 0.04 & 0.10 & 0.00 & -0.18 & -0.40 & 0.00 \\ 8 & 14 & 0.00 & 0.00 & 0.54 & 0.36 & -0.01 & 0.00 & -0.15 & 0.29 & 0.00 \\ 9 & 14 & 0.00 & 0.00 & -0.54 & -0.36 & 0.01 & 0.00 & 0.15 & -0.29 & 0.00 \\ & & & 7 & & & 8 & & & & 9\end{array}$

AU BU B

\begin{tabular}{|c|c|c|c|c|c|c|c|c|c|}
\hline Frequencies -- & \multicolumn{3}{|c|}{97.9839} & \multicolumn{3}{|c|}{106.0939} & & \multicolumn{2}{|c|}{114.8857} \\
\hline Red. masses & \multicolumn{3}{|c|}{7.3248} & \multicolumn{3}{|c|}{8.3447} & & \multicolumn{2}{|c|}{27.2288} \\
\hline Frc consts & \multicolumn{3}{|c|}{0.0414} & \multicolumn{3}{|c|}{0.0553} & & \multicolumn{2}{|c|}{0.2117} \\
\hline IR Inten & \multicolumn{2}{|c|}{22.3707} & & \multicolumn{3}{|c|}{20.7859} & & \multicolumn{2}{|c|}{0.0000} \\
\hline Atom AN & $X$ & $\mathrm{Y}$ & $\mathrm{Z}$ & & $\mathrm{X}$ & $\mathrm{Y}$ & $\mathrm{Z}$ & $\mathrm{X}$ & $\mathrm{Y}$ \\
\hline 113 & 0.00 & 0.00 & -0.04 & 0.05 & -0.01 & 0.00 & 0.00 & 0.00 & -0.35 \\
\hline 13 & 0.00 & 0.00 & -0.03 & -0.10 & 0.04 & 0.00 & 0.00 & 0.00 & -0.37 \\
\hline 13 & 0.00 & 0.00 & 0.02 & 0.04 & 0.03 & 0.00 & 0.00 & 0.00 & 0.3 \\
\hline 3 & 0.00 & 0.00 & 0.99 & 0.96 & -0.09 & 0.00 & 0.00 & 0.00 & 0.00 \\
\hline 13 & 0.00 & 0.00 & -0.03 & -0.10 & 0.04 & 0.00 & 0.00 & 0.00 & 0.37 \\
\hline 13 & 0.00 & 0.00 & 0.02 & 0.04 & 0.03 & 0.00 & 0.00 & 0.00 & -0.34 \\
\hline 13 & 0.00 & 0.00 & -0.04 & 0.05 & -0.01 & 0.00 & 0.00 & 0.00 & 0.35 \\
\hline 14 & 0.00 & 0.00 & -0.07 & -0.11 & -0.04 & 0.00 & 0.00 & 0.00 & 0.35 \\
\hline 14 & 0.00 & 0.00 & -0.07 & -0.11 & -0.04 & 0.00 & 0.00 & 0.00 & -0.35 \\
\hline
\end{tabular}

$\mathrm{AU} \quad \mathrm{BU}$

Frequencies -- 115.1681

160.2405

AG

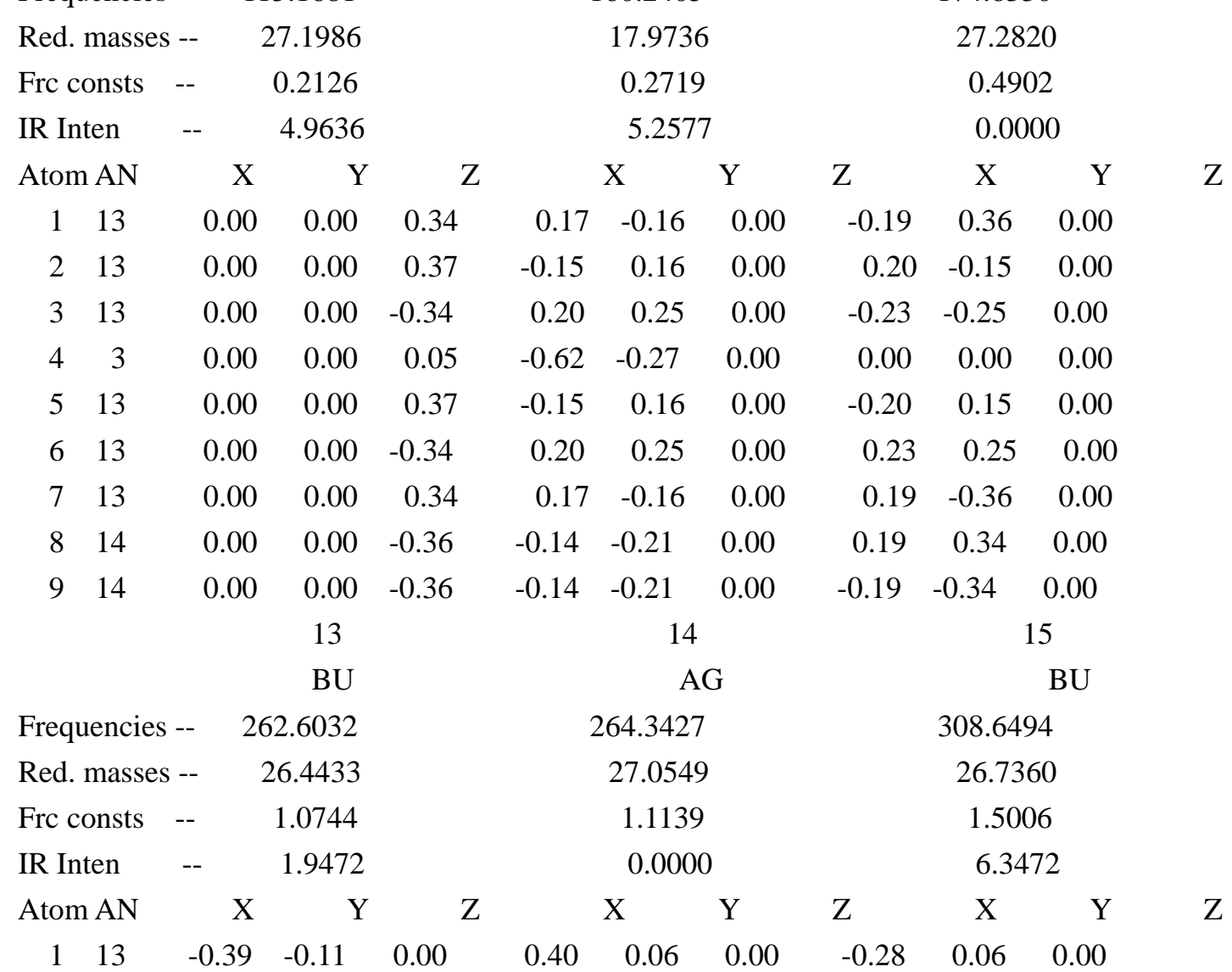




\begin{tabular}{|c|c|c|c|c|c|c|c|c|c|c|c|}
\hline 2 & 13 & -0.12 & -0.29 & 0.00 & 0.11 & 0.30 & 0.00 & -0.05 & 0.39 & 0.00 & \\
\hline 3 & 13 & 0.35 & 0.27 & 0.00 & -0.35 & -0.27 & 0.00 & 0.33 & -0.38 & 0.00 & \\
\hline 4 & 3 & 0.12 & 0.12 & 0.00 & 0.00 & 0.00 & 0.00 & -0.08 & -0.08 & 0.00 & \\
\hline 5 & 13 & -0.12 & -0.29 & 0.00 & -0.11 & -0.30 & 0.00 & -0.05 & 0.39 & 0.00 & \\
\hline 6 & 13 & 0.35 & 0.27 & 0.00 & 0.35 & 0.27 & 0.00 & 0.33 & -0.38 & 0.00 & \\
\hline 7 & 13 & -0.39 & -0.11 & 0.00 & -0.40 & -0.06 & 0.00 & -0.28 & 0.06 & 0.00 & \\
\hline 8 & 14 & 0.14 & 0.11 & 0.00 & -0.15 & -0.13 & 0.00 & 0.01 & -0.06 & 0.00 & \\
\hline 9 & 14 & 0.14 & 0.11 & 0.00 & 0.15 & 0.13 & 0.00 & 0.01 & -0.06 & 0.00 & \\
\hline & & & 16 & & \multicolumn{3}{|c|}{17} & \multicolumn{4}{|c|}{18} \\
\hline & & & AG & & \multicolumn{3}{|c|}{ BU } & \multicolumn{4}{|c|}{ AG } \\
\hline \multicolumn{2}{|c|}{ Frequencies -- } & \multicolumn{2}{|c|}{308.8452} & & \multicolumn{3}{|c|}{357.8819} & \multicolumn{4}{|c|}{367.9521} \\
\hline \multicolumn{2}{|c|}{ Red. masses - } & & 26.9873 & & \multicolumn{3}{|c|}{18.4261} & \multicolumn{4}{|c|}{27.4078} \\
\hline \multicolumn{2}{|c|}{ Frc consts } & -- & 1.5167 & & \multicolumn{3}{|c|}{1.3905} & \multicolumn{4}{|c|}{2.1863} \\
\hline \multicolumn{2}{|c|}{ IR Inten } & -- & 0.0000 & & \multicolumn{3}{|c|}{9.7049} & \multicolumn{4}{|c|}{0.0000} \\
\hline \multicolumn{2}{|c|}{ Atom AN } & $\mathrm{X}$ & $\mathrm{Y}$ & $\mathrm{Z}$ & \multicolumn{2}{|r|}{$\mathrm{X}$} & $\mathrm{Y}$ & Z & $\mathrm{X}$ & $\mathrm{Y}$ & $\mathrm{Z}$ \\
\hline 1 & 13 & -0.28 & 0.03 & 0.00 & -0.13 & 0.30 & 0.00 & -0.14 & 0.13 & 0.00 & \\
\hline 2 & 13 & -0.06 & 0.40 & 0.00 & 0.28 & -0.11 & 0.00 & 0.47 & -0.08 & 0.00 & \\
\hline 3 & 13 & 0.33 & -0.39 & 0.00 & 0.06 & 0.09 & 0.00 & 0.13 & 0.07 & 0.00 & \\
\hline 4 & 3 & 0.00 & 0.00 & 0.00 & -0.01 & -0.66 & 0.00 & 0.00 & 0.00 & 0.00 & \\
\hline 5 & 13 & 0.06 & -0.40 & 0.00 & 0.28 & -0.11 & 0.00 & -0.47 & 0.08 & 0.00 & \\
\hline 6 & 13 & -0.33 & 0.39 & 0.00 & 0.06 & 0.09 & 0.00 & -0.13 & -0.07 & 0.00 & \\
\hline 7 & 13 & 0.28 & -0.03 & 0.00 & -0.13 & 0.30 & 0.00 & 0.14 & -0.13 & 0.00 & \\
\hline 8 & 14 & 0.01 & -0.05 & 0.00 & -0.20 & -0.19 & 0.00 & -0.44 & -0.14 & 0.00 & \\
\hline \multirow[t]{3}{*}{9} & 14 & -0.01 & 0.05 & 0.00 & -0.20 & -0.19 & 0.00 & 0.44 & 0.14 & 0.00 & \\
\hline & & & 19 & & & 20 & & & & 21 & \\
\hline & & & $\mathrm{BU}$ & & & A & G & & & BU & \\
\hline Freql & lencies - & & 0.2583 & & & 415.8617 & & & 479.45 & & \\
\hline Red. & masses & & 18.3505 & & & 27.5623 & & & 10.17 & & \\
\hline Frc c & onsts - & -- & 1.5633 & & & 2.8084 & & & 1.378 & & \\
\hline IR In & & -- & 58.2881 & & & 0.000 & & & 384.05 & & \\
\hline Aton & $\mathrm{AN}$ & $X$ & $\mathrm{Y}$ & Z & & $\mathrm{X}$ & $\mathrm{Y}$ & Z & $\mathrm{X}$ & $\mathrm{Y}$ & $\mathrm{Z}$ \\
\hline 1 & 13 & -0.02 & -0.22 & 0.00 & 0.06 & -0.43 & 0.00 & -0.02 & 0.12 & 0.00 & \\
\hline 2 & 13 & 0.28 & 0.01 & 0.00 & 0.12 & 0.06 & 0.00 & -0.02 & -0.02 & 0.00 & \\
\hline 3 & 13 & 0.09 & -0.02 & 0.00 & 0.03 & -0.08 & 0.00 & 0.00 & 0.03 & 0.00 & \\
\hline 4 & 3 & -0.01 & 0.67 & 0.00 & 0.00 & 0.00 & 0.00 & 0.03 & 0.92 & 0.00 & \\
\hline 5 & 13 & 0.28 & 0.01 & 0.00 & -0.12 & -0.06 & 0.00 & -0.02 & -0.02 & 0.00 & \\
\hline 6 & 13 & 0.09 & -0.02 & 0.00 & -0.03 & 0.08 & 0.00 & 0.00 & 0.03 & 0.00 & \\
\hline 7 & 13 & -0.02 & -0.22 & 0.00 & -0.06 & 0.43 & 0.00 & -0.02 & 0.12 & 0.00 & \\
\hline 8 & 14 & -0.34 & 0.14 & 0.00 & -0.20 & 0.50 & 0.00 & 0.03 & -0.24 & 0.00 & \\
\hline 9 & 14 & -0.34 & 0.14 & 0.00 & 0.20 & -0.50 & 0.00 & 0.03 & -0.24 & 0.00 & \\
\hline
\end{tabular}




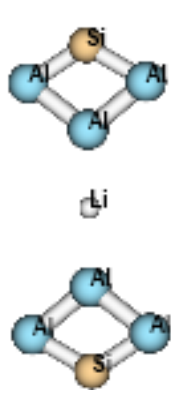

$\begin{array}{ccccc}3 & 0 & 0.000000 & 0.000000 & 0.000000 \\ 13 & 0 & 0.000000 & 1.996423 & 4.336766 \\ 13 & 0 & 0.000000 & -1.996423 & 4.336766 \\ 13 & 0 & 0.000000 & 1.996423 & -4.336766 \\ 13 & 0 & 0.000000 & -1.996423 & -4.336766 \\ 13 & 0 & 0.000000 & 0.000000 & 2.703377 \\ 14 & 0 & 0.000000 & 0.000000 & 5.615799 \\ 13 & 0 & 0.000000 & 0.000000 & -2.703377 \\ 14 & 0 & 0.000000 & 0.000000 & -5.615799\end{array}$

State $=1-A G \backslash H F=-2041.2887491$

1

B3G

Frequencies -- $\quad-18.5699$

Red. masses -- 27.0099

Frc consts -- 0.0055

IR Inten -- $\quad 0.0000$

Atom AN $\mathrm{X} \quad \mathrm{Y}$

$\begin{array}{lllll}1 & 3 & 0.00 & 0.00 & 0.00\end{array}$

213

$\begin{array}{lll}0.00 & 0.11 & 0.37\end{array}$

$\begin{array}{lll}0.00 & 0.11 & -0.37\end{array}$

$\begin{array}{lll}0.00 & -0.11 & 0.37\end{array}$

$\begin{array}{lll}0.00 & -0.11 & -0.37\end{array}$

$\begin{array}{lll}0.00 & 0.44 & 0.00\end{array}$

$\begin{array}{lll}0.00 & -0.12 & 0.00\end{array}$

$\begin{array}{lll}0.00 & -0.44 & 0.00\end{array}$

$\begin{array}{lll}0.00 & 0.12 & 0.00\end{array}$

4

B3U
2

B2U

$-14.2029$

26.9608

0.0032

0.0196

$\mathrm{X} \quad \mathrm{Y}$

$\begin{array}{lll}0.00 & -0.09 & 0.00\end{array}$

$\begin{array}{llll}0.00 & 0.03 & -0.40\end{array}$

$\begin{array}{lll}0.00 & 0.03 & 0.40\end{array}$

$\begin{array}{lll}0.00 & 0.03 & 0.40\end{array}$

$\begin{array}{lll}0.00 & 0.03 & -0.40\end{array}$

$\begin{array}{lll}0.00 & -0.32 & 0.00\end{array}$

$\begin{array}{lll}0.00 & 0.28 & 0.00\end{array}$

$\begin{array}{lll}0.00 & -0.32 & 0.00\end{array}$

$\begin{array}{lll}0.00 & 0.28 & 0.00\end{array}$

5

B2G

43.0930

27.2123

0.0298

0.0000

X Y

$0.00 \quad 0.00$

$\begin{array}{lll}0.04 & 0.00 & 0.00\end{array}$

$\begin{array}{lll}0.04 & 0.00 & 0.00\end{array}$

$\begin{array}{lll}-0.04 & 0.00 & 0.00\end{array}$

\section{3}

AU

$-7.9766$

26.9815

0.0010

0.0000

$\begin{array}{llll}\mathrm{Z} & \mathrm{X} & \mathrm{Y}\end{array}$

$\begin{array}{lll}0.00 & 0.00 & 0.00\end{array}$

$\begin{array}{lll}-0.50 & 0.00 & 0.00\end{array}$

$\begin{array}{lll}0.50 & 0.00 & 0.00\end{array}$

$\begin{array}{lll}0.50 & 0.00 & 0.00\end{array}$

$\begin{array}{lll}-0.50 & 0.00 & 0.00\end{array}$

$\begin{array}{lll}0.00 & 0.00 & 0.00\end{array}$

$\begin{array}{lll}0.00 & 0.00 & 0.00\end{array}$

$\begin{array}{lll}0.00 & 0.00 & 0.00\end{array}$

$\begin{array}{lll}0.00 & 0.00 & 0.00\end{array}$

6

AG

63.4667

27.2925

0.0648

0.0000

$\begin{array}{lllll}\mathrm{Z} & \mathrm{X} & \mathrm{Y} & \mathrm{Z}\end{array}$

$\begin{array}{lll}0.00 & 0.00 & 0.00\end{array}$

$\begin{array}{lll}0.00 & -0.03 & 0.37\end{array}$

$\begin{array}{lll}0.00 & 0.03 & 0.37\end{array}$

$\begin{array}{llll}0.00 & -0.03 & -0.37\end{array}$ 


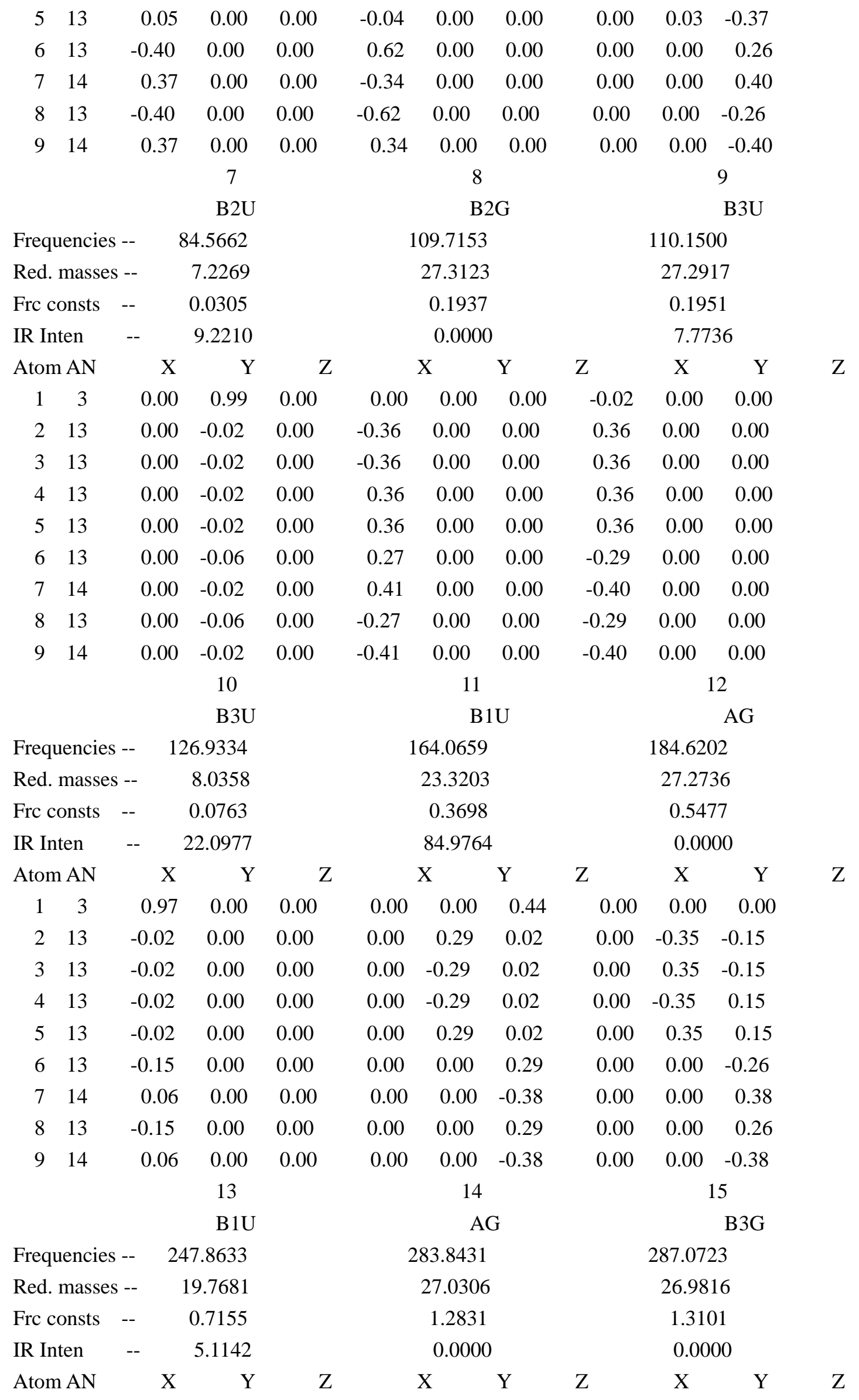




\begin{tabular}{|c|c|c|c|c|c|c|c|c|c|c|c|}
\hline 1 & 3 & 0.00 & 0.00 & -0.60 & 0.00 & 0.00 & 0.00 & 0.00 & 0.00 & 0.00 & \\
\hline 2 & 13 & 0.00 & 0.11 & 0.28 & 0.00 & 0.01 & -0.29 & 0.00 & -0.27 & -0.23 & \\
\hline 3 & 13 & 0.00 & -0.11 & 0.28 & 0.00 & -0.01 & -0.29 & 0.00 & -0.27 & 0.23 & \\
\hline 4 & 13 & 0.00 & -0.11 & 0.28 & 0.00 & 0.01 & 0.29 & 0.00 & 0.27 & -0.23 & \\
\hline 5 & 13 & 0.00 & 0.11 & 0.28 & 0.00 & -0.01 & 0.29 & 0.00 & 0.27 & 0.23 & \\
\hline 6 & 13 & 0.00 & 0.00 & -0.34 & 0.00 & 0.00 & 0.56 & 0.00 & 0.51 & 0.00 & \\
\hline 7 & 14 & 0.00 & 0.00 & -0.14 & 0.00 & 0.00 & 0.16 & 0.00 & 0.00 & 0.00 & \\
\hline 8 & 13 & 0.00 & 0.00 & -0.34 & 0.00 & 0.00 & -0.56 & 0.00 & -0.51 & 0.00 & \\
\hline \multirow[t]{3}{*}{9} & 14 & 0.00 & 0.00 & -0.14 & 0.00 & 0.00 & -0.16 & 0.00 & 0.00 & 0.00 & \\
\hline & & & 16 & & \multicolumn{3}{|c|}{17} & \multicolumn{4}{|c|}{18} \\
\hline & & \multicolumn{3}{|c|}{ B2U } & \multicolumn{3}{|c|}{ B1U } & \multicolumn{4}{|c|}{ AG } \\
\hline \multicolumn{2}{|c|}{ Frequencies -- } & \multicolumn{2}{|c|}{288.0069} & & \multicolumn{3}{|c|}{362.8002} & \multicolumn{4}{|c|}{373.3176} \\
\hline \multicolumn{2}{|c|}{ Red. masses - } & \multicolumn{2}{|c|}{26.5355} & & \multicolumn{3}{|c|}{21.4965} & \multicolumn{4}{|c|}{27.2999} \\
\hline \multicolumn{2}{|c|}{ Frc consts } & \multicolumn{2}{|c|}{1.2968} & & \multicolumn{3}{|c|}{1.6671} & \multicolumn{4}{|c|}{2.2417} \\
\hline \multicolumn{2}{|c|}{ IR Inten } & \multicolumn{2}{|r|}{3.0587} & & \multicolumn{3}{|c|}{71.8885} & \multicolumn{4}{|c|}{0.0000} \\
\hline Atom & AN & $X$ & $\mathrm{Y}$ & $\mathrm{Z}$ & & $\mathrm{X}$ & $\mathrm{Y}$ & $\mathrm{Z}$ & $\mathrm{X}$ & $\mathrm{Y}$ & $\mathrm{Z}$ \\
\hline 1 & 3 & 0.00 & -0.15 & 0.00 & 0.00 & 0.00 & 0.54 & 0.00 & 0.00 & 0.00 & \\
\hline 2 & 13 & 0.00 & 0.26 & 0.22 & 0.00 & -0.32 & 0.13 & 0.00 & 0.36 & -0.11 & \\
\hline 3 & 13 & 0.00 & 0.26 & -0.22 & 0.00 & 0.32 & 0.13 & 0.00 & -0.36 & -0.11 & \\
\hline 4 & 13 & 0.00 & 0.26 & -0.22 & 0.00 & 0.32 & 0.13 & 0.00 & 0.36 & 0.11 & \\
\hline 5 & 13 & 0.00 & 0.26 & 0.22 & 0.00 & -0.32 & 0.13 & 0.00 & -0.36 & 0.11 & \\
\hline 6 & 13 & 0.00 & -0.50 & 0.00 & 0.00 & 0.00 & 0.03 & 0.00 & 0.00 & -0.24 & \\
\hline 7 & 14 & 0.00 & -0.01 & 0.00 & 0.00 & 0.00 & -0.35 & 0.00 & 0.00 & 0.40 & \\
\hline 8 & 13 & 0.00 & -0.50 & 0.00 & 0.00 & 0.00 & 0.03 & 0.00 & 0.00 & 0.24 & \\
\hline 9 & 14 & 0.00 & -0.01 & 0.00 & 0.00 & 0.00 & -0.35 & 0.00 & 0.00 & -0.40 & \\
\hline & & & 19 & & & 20 & & & & 21 & \\
\hline & & & B3G & & & & $2 \mathrm{U}$ & & & B1U & \\
\hline Frequ & lencies - & & 7.7769 & & & 428.0512 & & & 432.19 & & \\
\hline Red. & masses & & 7.5585 & & & 27.556 & & & 9.13 & & \\
\hline Frc c & onsts & - & 2.9713 & & & 2.9748 & & & 1.00 & & \\
\hline IR In & & - & 0.0000 & & & 16.078 & & & 155.79 & & \\
\hline Atom & AN & $\mathrm{X}$ & $\mathrm{Y}$ & Z & & $\mathrm{X}$ & $\mathrm{Y}$ & Z & $\mathrm{X}$ & $\mathrm{Y}$ & $\mathrm{Z}$ \\
\hline 1 & 3 & 0.00 & 0.00 & 0.00 & 0.00 & -0.01 & 0.00 & 0.00 & 0.00 & 0.95 & \\
\hline 2 & 13 & 0.00 & -0.24 & 0.21 & 0.00 & -0.24 & 0.21 & 0.00 & 0.06 & 0.00 & \\
\hline 3 & 13 & 0.00 & -0.24 & -0.21 & 0.00 & -0.24 & -0.21 & 0.00 & -0.06 & 0.00 & \\
\hline 4 & 13 & 0.00 & 0.24 & 0.21 & 0.00 & -0.24 & -0.21 & 0.00 & -0.06 & 0.00 & \\
\hline 5 & 13 & 0.00 & 0.24 & -0.21 & 0.00 & -0.24 & 0.21 & 0.00 & 0.06 & 0.00 & \\
\hline 6 & 13 & 0.00 & -0.07 & 0.00 & 0.00 & -0.07 & 0.00 & 0.00 & 0.00 & -0.20 & \\
\hline 7 & 14 & 0.00 & 0.54 & 0.00 & 0.00 & 0.54 & 0.00 & 0.00 & 0.00 & 0.07 & \\
\hline 8 & 13 & 0.00 & 0.07 & 0.00 & 0.00 & -0.07 & 0.00 & 0.00 & 0.00 & -0.20 & \\
\hline 9 & 14 & 0.00 & -0.54 & 0.00 & 0.00 & 0.54 & 0.00 & 0.00 & 0.00 & 0.07 & \\
\hline Al3SiI & ¿iAl3Si- & $-\mathrm{c}-90-$ & Al-Si-b & $36311+g x$ & rq.frq3 & m-16.8 & 7.19 & & & & \\
\hline
\end{tabular}




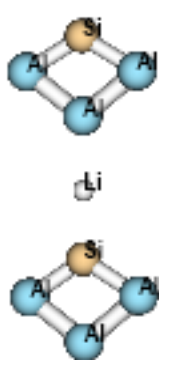

State $=1-A 1 \backslash H F=-2041.2996863$

\section{1}

B2

Frequencies -- $\quad-16.8080$

Red. masses -- 27.1099

Frc consts -- 0.0045

IR Inten $\quad$-- 0.0055

Atom AN $\mathrm{X} \quad \mathrm{Y}$

$\begin{array}{lllll}1 & 3 & 0.00 & 0.03 & 0.00\end{array}$

213

$3 \quad 13$

$$
\begin{array}{lll}
0.00 & -0.11 & -0.44
\end{array}
$$

$\begin{array}{lll}0.00 & -0.11 & 0.44\end{array}$

$\begin{array}{lll}0.00 & -0.10 & 0.00\end{array}$

$\begin{array}{lll}0.00 & 0.14 & -0.27\end{array}$

$\begin{array}{lll}0.00 & 0.14 & 0.27\end{array}$

$\begin{array}{lll}0.00 & 0.34 & 0.00\end{array}$

$\begin{array}{lll}0.00 & -0.50 & 0.00\end{array}$

$\begin{array}{lll}0.00 & 0.17 & 0.00\end{array}$

4

B1

Frequencies -- $\quad 11.9306$

Red. masses -- 23.7192

Frc consts -- 0.0020

IR Inten -- 0.0303

Atom AN $\mathrm{X} \quad \mathrm{Y}$

$\begin{array}{lllll}2 & 13 & 0.06 & 0.00 & 0.00\end{array}$

$\begin{array}{lllll}3 & 13 & 0.06 & 0.00 & 0.00\end{array}$

$\begin{array}{lllll}4 & 13 & 0.59 & 0.00 & 0.00\end{array}$

$\begin{array}{lllll}5 & 13 & -0.03 & 0.00 & 0.00\end{array}$

$\begin{array}{lllll}6 & 13 & -0.03 & 0.00 & 0.00\end{array}$

$\begin{array}{lllll}7 & 14 & -0.60 & 0.00 & 0.00\end{array}$

$\begin{array}{llllll}8 & 13 & -0.18 & 0.00 & 0.00\end{array}$

$\begin{array}{lllll}9 & 14 & 0.24 & 0.00 & 0.00\end{array}$

7

B2

Frequencies -- 96.7713

Red. masses -- 7.2492
2

B2

$-12.1924$

26.5990

0.0023

0.0099

$\mathrm{Z} \quad \mathrm{X}$ $\begin{array}{lll}0.00 & -0.17 & 0.00\end{array}$

$\begin{array}{lll}0.00 & 0.05 & -0.31\end{array}$

$\begin{array}{lll}0.00 & 0.05 & 0.31\end{array}$

$\begin{array}{lll}0.00 & 0.36 & 0.00\end{array}$

$\begin{array}{lll}0.00 & -0.03 & 0.45\end{array}$

$\begin{array}{lll}0.00 & -0.03 & -0.45\end{array}$

$\begin{array}{lll}0.00 & -0.36 & 0.00\end{array}$

$\begin{array}{lll}0.00 & -0.22 & 0.00\end{array}$

$\begin{array}{lll}0.00 & 0.25 & 0.00\end{array}$

\section{5}

B1

33.2245

23.5855

0.0153

1.7016

X Y

$\begin{array}{lll}-0.43 & 0.00 & 0.00\end{array}$

$\begin{array}{lll}-0.01 & 0.00 & 0.00\end{array}$

$\begin{array}{lll}-0.01 & 0.00 & 0.00\end{array}$

$\begin{array}{lll}-0.14 & 0.00 & 0.00\end{array}$

$\begin{array}{lll}0.09 & 0.00 & 0.00\end{array}$

$\begin{array}{lll}0.09 & 0.00 & 0.00\end{array}$

$\begin{array}{lll}0.31 & 0.00 & 0.00\end{array}$

$\begin{array}{lll}-0.69 & 0.00 & 0.00\end{array}$

$\begin{array}{lll}0.45 & 0.00 & 0.00\end{array}$

8

B1

110.2057

27.1969
3

A2

$-7.2144$

26.9815

0.0008

0.0000

$\begin{array}{lllll}Z & X & Y & Z\end{array}$

$\begin{array}{lll}0.00 & 0.00 & 0.00\end{array}$

$\begin{array}{lll}-0.50 & 0.00 & 0.00\end{array}$

$\begin{array}{lll}0.50 & 0.00 & 0.00\end{array}$

$\begin{array}{lll}0.00 & 0.00 & 0.00\end{array}$

$\begin{array}{lll}0.50 & 0.00 & 0.00\end{array}$

$\begin{array}{lll}-0.50 & 0.00 & 0.00\end{array}$

$\begin{array}{lll}0.00 & 0.00 & 0.00\end{array}$

$\begin{array}{lll}0.00 & 0.00 & 0.00\end{array}$

$\begin{array}{lll}0.00 & 0.00 & 0.00\end{array}$

\section{6}

A1

65.1500

27.1615

0.0679

1.6940

Z

X Y

Z

$\begin{array}{lll}0.00 & 0.00 & 0.04\end{array}$

$\begin{array}{lll}0.00 & 0.03 & -0.37\end{array}$

$\begin{array}{llll}0.00 & -0.03 & -0.37\end{array}$

$\begin{array}{lll}0.00 & 0.00 & 0.42\end{array}$

$\begin{array}{lll}0.00 & 0.06 & 0.36\end{array}$

$\begin{array}{lll}0.00 & -0.06 & 0.36\end{array}$

$\begin{array}{lll}0.00 & 0.00 & 0.24\end{array}$

$\begin{array}{llll}0.00 & 0.00 & -0.25\end{array}$

$\begin{array}{lll}0.00 & 0.00 & -0.40\end{array}$

\section{9}

B1

115.8544

21.0160 


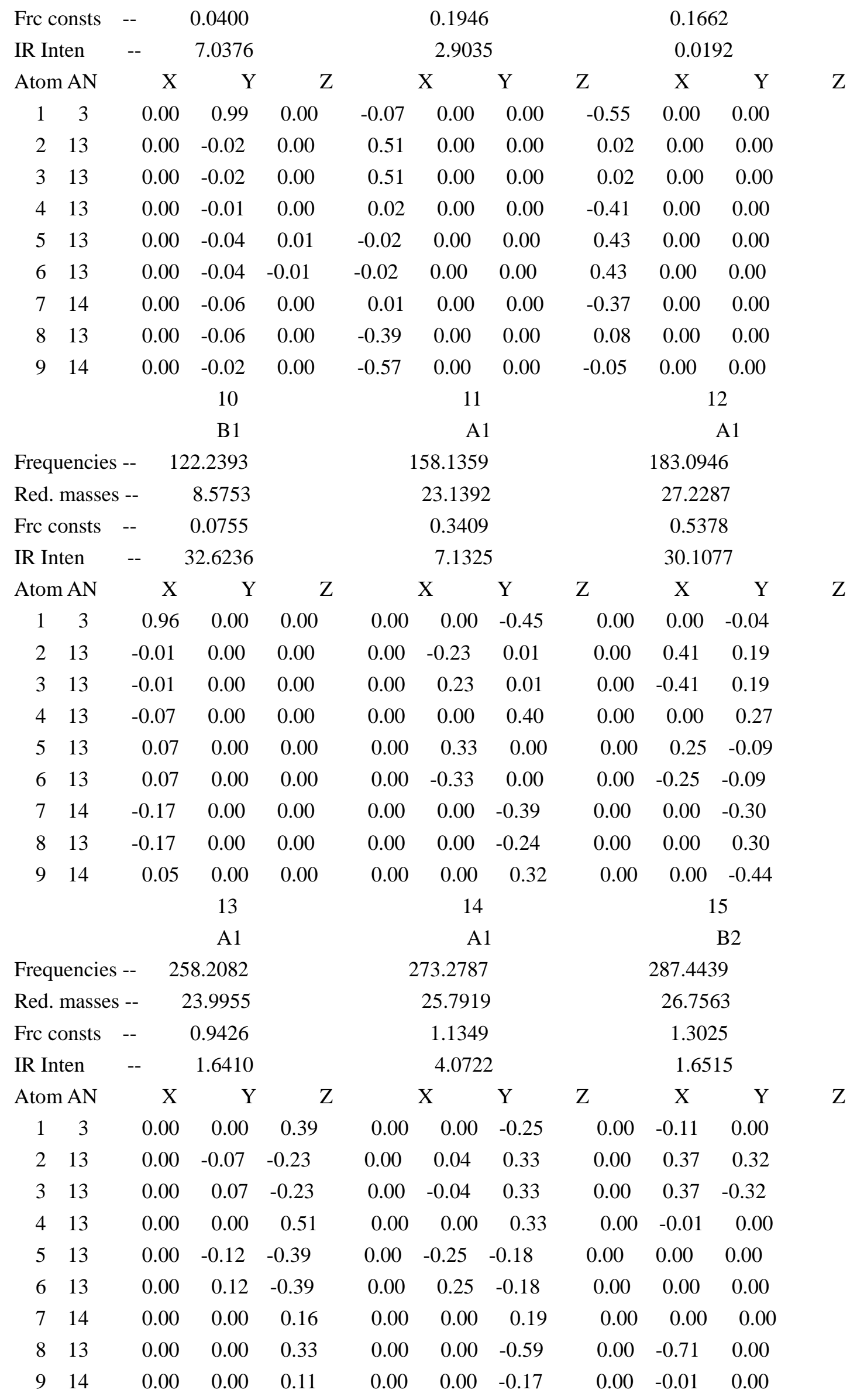


16

B2

Frequencies -- 306.8326

Red. masses -- 26.9534

Frc consts -- $\quad 1.4951$

IR Inten -- 3.6487

Atom AN $\mathrm{X} \quad \mathrm{Y}$

$\begin{array}{lllll}1 & 3 & 0.00 & -0.04 & 0.00\end{array}$

$\begin{array}{lllll}2 & 13 & 0.00 & 0.00 & 0.00\end{array}$

$\begin{array}{lllll}3 & 13 & 0.00 & 0.00 & 0.00\end{array}$

$\begin{array}{lllll}4 & 13 & 0.00 & 0.72 & 0.00\end{array}$

$\begin{array}{lllll}5 & 13 & 0.00 & -0.38 & -0.30\end{array}$

$\begin{array}{llllll}6 & 13 & 0.00 & -0.38 & 0.30\end{array}$

$\begin{array}{lllll}7 & 14 & 0.00 & 0.05 & 0.00\end{array}$

$\begin{array}{lllll}8 & 13 & 0.00 & -0.01 & 0.00\end{array}$

$\begin{array}{lllll}9 & 14 & 0.00 & 0.00 & 0.00\end{array}$

19

B2

Frequencies -- 393.6552

Red. masses -- 27.4558

Frc consts --- 2.5068

IR Inten --- 7.3525

Atom AN $\quad X \quad$ Y $\quad$ Z

$\begin{array}{lllll}1 & 3 & 0.00 & -0.06 & 0.00\end{array}$

$\begin{array}{lllll}2 & 13 & 0.00 & 0.00 & 0.00\end{array}$

$\begin{array}{lllll}3 & 13 & 0.00 & 0.00 & 0.00\end{array}$

$\begin{array}{lllll}4 & 13 & 0.00 & 0.13 & 0.00\end{array}$

$\begin{array}{lllll}5 & 13 & 0.00 & 0.33 & -0.34\end{array}$

$\begin{array}{lllll}6 & 13 & 0.00 & 0.33 & 0.34\end{array}$

$\begin{array}{lllll}7 & 14 & 0.00 & -0.74 & 0.00\end{array}$

$\begin{array}{llllll}8 & 13 & 0.00 & 0.00 & 0.00\end{array}$

$9 \quad 14$
17

A1

358.3178

18.7839

1.4209

29.7340

\begin{tabular}{ccc}
\multicolumn{2}{c}{$\mathrm{X}$} & $\mathrm{Y}$ \\
0.00 & 0.00 & 0.65 \\
0.00 & -0.25 & 0.12 \\
0.00 & 0.25 & 0.12 \\
0.00 & 0.00 & -0.12 \\
0.00 & 0.32 & -0.12 \\
0.00 & -0.32 & -0.12 \\
0.00 & 0.00 & 0.29 \\
0.00 & 0.00 & -0.05 \\
0.00 & 0.00 & -0.29
\end{tabular}

20

B2

427.4207

27.5560

2.9660

7.7320

X Y

$\begin{array}{lll}0.00 & 0.01 & 0.00\end{array}$

$\begin{array}{lll}0.00 & 0.34 & -0.30\end{array}$

$\begin{array}{lll}0.00 & 0.34 & 0.30\end{array}$

$\begin{array}{lll}0.00 & 0.00 & 0.00\end{array}$

$\begin{array}{lll}0.00 & 0.00 & 0.00\end{array}$

$\begin{array}{lll}0.00 & 0.00 & 0.00\end{array}$

$\begin{array}{lll}0.00 & 0.00 & 0.00\end{array}$

$\begin{array}{lll}0.00 & 0.10 & 0.00\end{array}$

$\begin{array}{lll}0.00 & -0.76 & 0.00\end{array}$
18

A1

375.8282

26.1670

2.1776

8.6750

$\begin{array}{lll}\mathrm{Z} & \mathrm{X} & \mathrm{Y}\end{array}$

$\begin{array}{lll}0.00 & 0.00 & -0.24\end{array}$

$\begin{array}{lll}0.00 & -0.41 & 0.11\end{array}$

$\begin{array}{lll}0.00 & 0.41 & 0.11\end{array}$

$\begin{array}{lll}0.00 & 0.00 & 0.10\end{array}$

$\begin{array}{lll}0.00 & -0.26 & 0.12\end{array}$

$\begin{array}{lll}0.00 & 0.26 & 0.12\end{array}$

$\begin{array}{lll}0.00 & 0.00 & -0.34\end{array}$

$\begin{array}{lll}0.00 & 0.00 & 0.32\end{array}$

$\begin{array}{lll}0.00 & 0.00 & -0.45\end{array}$

21

A1

463.8344

9.2843

1.1769

331.3450

$\begin{array}{lllll}Z & X & Y & Z\end{array}$

$\begin{array}{lll}0.00 & 0.00 & 0.94\end{array}$

$\begin{array}{lll}0.00 & 0.03 & 0.00\end{array}$

$\begin{array}{lll}0.00 & -0.03 & 0.00\end{array}$

$\begin{array}{lll}0.00 & 0.00 & 0.03\end{array}$

$\begin{array}{lll}0.00 & -0.07 & 0.05\end{array}$

$\begin{array}{lll}0.00 & 0.07 & 0.05\end{array}$

$\begin{array}{lll}0.00 & 0.00 & -0.27\end{array}$

$\begin{array}{lll}0.00 & 0.00 & -0.14\end{array}$

$\begin{array}{lll}0.00 & 0.00 & 0.04\end{array}$

Al3SiLiAl3Si-c-c-to-s-s-0-Al-Al-b36311+gx-frq.frq-6.1

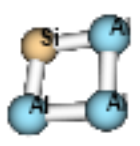

bi

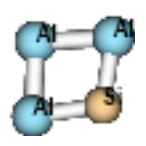

$\begin{array}{rrrrr}3 & 0 & 0.000000 & 0.000000 & 0.000000 \\ 13 & 0 & 0.000000 & 2.865921 & 0.000000 \\ 13 & 0 & 0.000000 & -2.865921 & 0.000000 \\ 13 & 0 & 2.321917 & 1.698901 & 0.000000\end{array}$




$\begin{array}{llrrc}13 & 0 & -2.321917 & -1.698901 & 0.000000 \\ 13 & 0 & -0.911285 & -5.239328 & 0.000000 \\ 14 & 0 & -2.925283 & -3.975159 & 0.000000 \\ 13 & 0 & 0.911285 & 5.239328 & 0.000000 \\ 14 & 0 & 2.925283 & 3.975159 & 0.000000\end{array}$

State $=1-A G \backslash H F=-2041.3003458$

\begin{tabular}{|c|c|c|c|c|c|c|c|c|c|c|c|}
\hline & & & & 1 & & & 2 & & & & 3 \\
\hline & & & & AU & & & B & & & & AU \\
\hline Freq & iencie & & & 5.0899 & & & 19.9535 & & & 20.141 & \\
\hline Red. & masse & & & 7.1564 & & & 20.423 & & & 21.35 & \\
\hline Frc C & onsts & -- & & 0.0006 & & & 0.0048 & & & 0.00 & \\
\hline IR In & & -- & & 0.0968 & & & 0.253 & & & 2.25 & \\
\hline Aton & $\mathrm{AN}$ & & $\mathrm{X}$ & $\mathrm{Y}$ & Z & & $\mathrm{X}$ & $\mathrm{Y}$ & Z & $\mathrm{X}$ & $\mathrm{Y}$ \\
\hline 1 & 3 & & 00 & 0.00 & 0.00 & 0.53 & -0.24 & 0.00 & 0.00 & 0.00 & 0.54 \\
\hline 2 & 13 & & 00 & 0.00 & 0.33 & 0.07 & -0.25 & 0.00 & 0.00 & 0.00 & 0.33 \\
\hline 3 & 13 & & 00 & 0.00 & 0.33 & 0.07 & -0.25 & 0.00 & 0.00 & 0.00 & 0.33 \\
\hline 4 & 13 & & 00 & 0.00 & -0.40 & 0.27 & 0.14 & 0.00 & 0.00 & 0.00 & 0.23 \\
\hline 5 & 13 & & 00 & 0.00 & -0.40 & 0.27 & 0.14 & 0.00 & 0.00 & 0.00 & 0.23 \\
\hline 6 & 13 & & 00 & 0.00 & 0.38 & -0.31 & -0.11 & 0.00 & 0.00 & 0.00 & -0.30 \\
\hline 7 & 14 & & 00 & 0.00 & -0.30 & -0.09 & 0.24 & 0.00 & 0.00 & 0.00 & -0.32 \\
\hline 8 & 13 & & 00 & 0.00 & 0.38 & -0.31 & -0.11 & 0.00 & 0.00 & 0.00 & -0.30 \\
\hline 9 & 14 & & 00 & 0.00 & -0.30 & -0.09 & 0.24 & 0.00 & 0.00 & 0.00 & -0.32 \\
\hline & & & & 4 & & & 5 & & & & 6 \\
\hline & & & & BG & & & A & & & & AG \\
\hline Freq & iencie & & & 4.3475 & & & 58.0374 & & & 66.788 & \\
\hline Red. & masse & & & 7.0751 & & & 27.006 & & & 27.27 & \\
\hline Frc C & onsts & -- & & 0.0314 & & & 0.0536 & & & 0.07 & \\
\hline IR In & & -- & & 0.0000 & & & 0.000 & & & 0.00 & \\
\hline Aton & $\mathrm{AN}$ & & $\mathrm{X}$ & $\mathrm{Y}$ & Z & & $\mathrm{X}$ & $\mathrm{Y}$ & Z & $\mathrm{X}$ & $\mathrm{Y}$ \\
\hline 1 & 3 & & 00 & 0.00 & 0.00 & 0.00 & 0.00 & 0.00 & 0.00 & 0.00 & 0.00 \\
\hline 2 & 13 & & 00 & 0.00 & -0.51 & 0.26 & -0.38 & 0.00 & -0.27 & -0.17 & 0.00 \\
\hline 3 & 13 & & 00 & 0.00 & 0.51 & -0.26 & 0.38 & 0.00 & 0.27 & 0.17 & 0.00 \\
\hline 4 & 13 & & 00 & 0.00 & -0.37 & 0.37 & -0.14 & 0.00 & -0.31 & -0.29 & 0.00 \\
\hline 5 & 13 & & 00 & 0.00 & 0.37 & -0.37 & 0.14 & 0.00 & 0.31 & 0.29 & 0.00 \\
\hline 6 & 13 & & 00 & 0.00 & -0.23 & 0.26 & 0.23 & 0.00 & 0.05 & 0.27 & 0.00 \\
\hline 7 & 14 & & 00 & 0.00 & -0.22 & 0.11 & -0.02 & 0.00 & 0.12 & 0.37 & 0.00 \\
\hline 8 & 13 & & 00 & 0.00 & 0.23 & -0.26 & -0.23 & 0.00 & -0.05 & -0.27 & 0.00 \\
\hline 9 & 14 & & 00 & 0.00 & 0.22 & -0.11 & 0.02 & 0.00 & -0.12 & -0.37 & 0.00 \\
\hline & & & & 7 & & & 8 & & & & 9 \\
\hline & & & & BU & & & A & & & & BG \\
\hline Freq & iencie & & & 7.6451 & & & 109.3330 & & & 111.250 & \\
\hline Red. & masse & & & 5.9959 & & & 25.617 & & & 27.28 & \\
\hline Frc C & onsts & -- & & 0.1092 & & & 0.1804 & & & 0.19 & \\
\hline IR In & ten & -- & & 8.2619 & & & 5.163 & & & 0.00 & \\
\hline
\end{tabular}




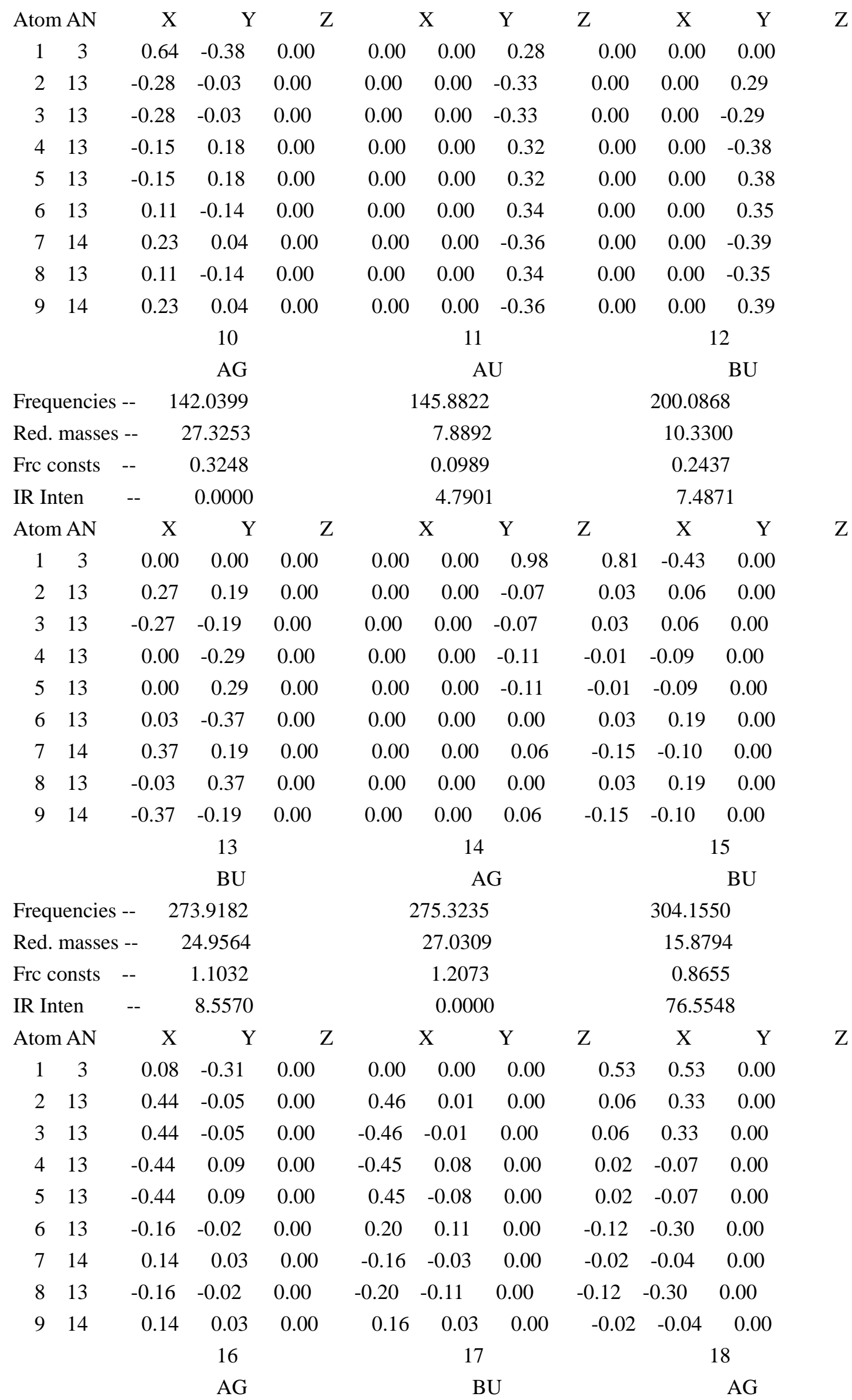




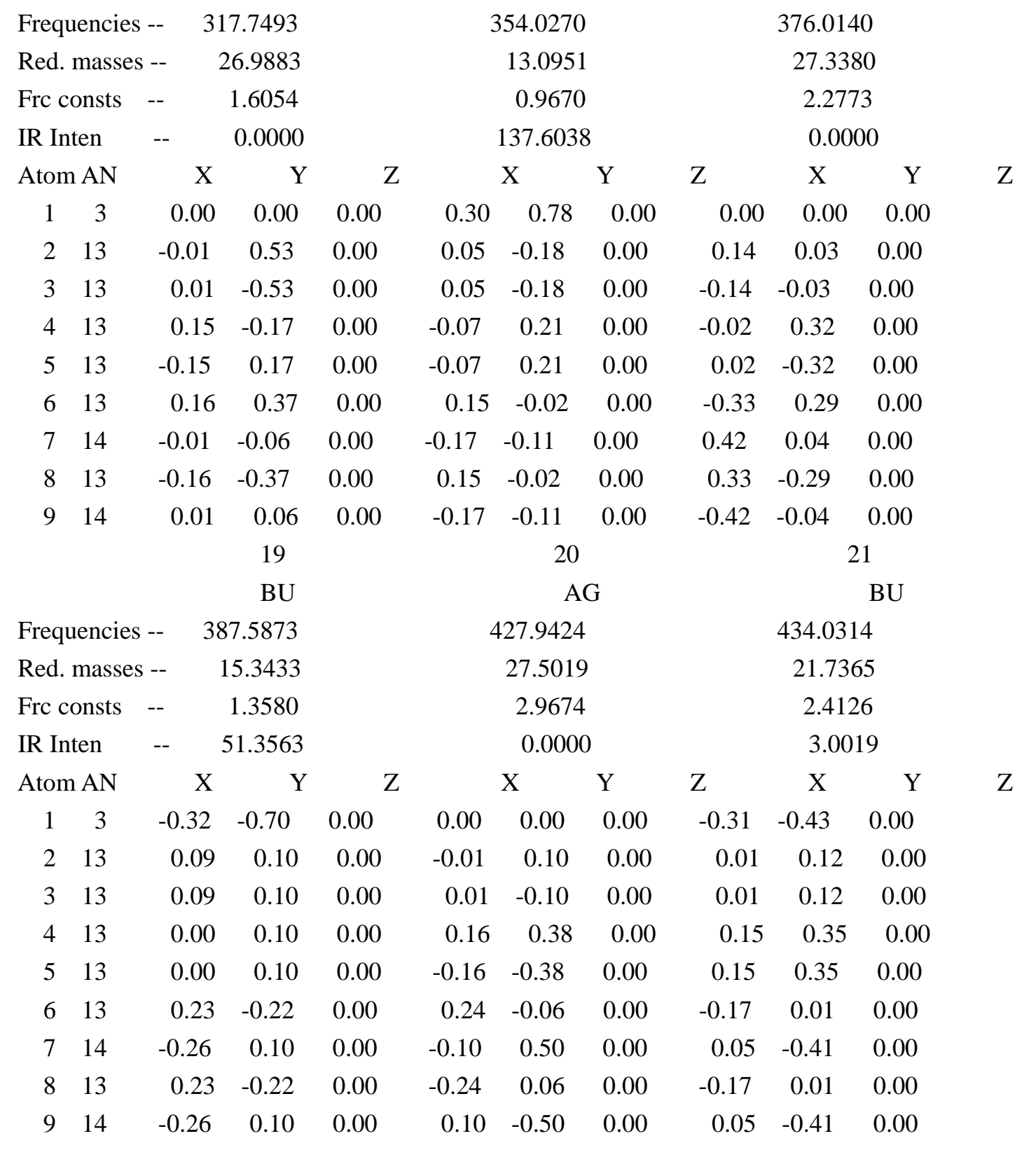
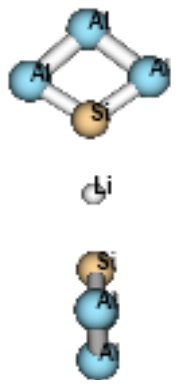

$\begin{array}{rrrrr}13 & 0 & 0.000000 & 1.955757 & 3.908237 \\ 13 & 0 & 0.000000 & 0.000000 & 5.581064 \\ 13 & 0 & 0.000000 & -1.955757 & 3.908237 \\ 13 & 0 & 0.000000 & 0.000000 & -5.581064 \\ 13 & 0 & 1.955757 & 0.000000 & -3.908237 \\ 13 & 0 & -1.955757 & 0.000000 & -3.908237\end{array}$




$\begin{array}{rrrrr}3 & 0 & 0.000000 & 0.000000 & 0.000000 \\ 14 & 0 & 0.000000 & 0.000000 & 2.536935 \\ 14 & 0 & 0.000000 & 0.000000 & -2.536935\end{array}$

\section{State $=1-A 1 \backslash H F=-2041.3101821$}

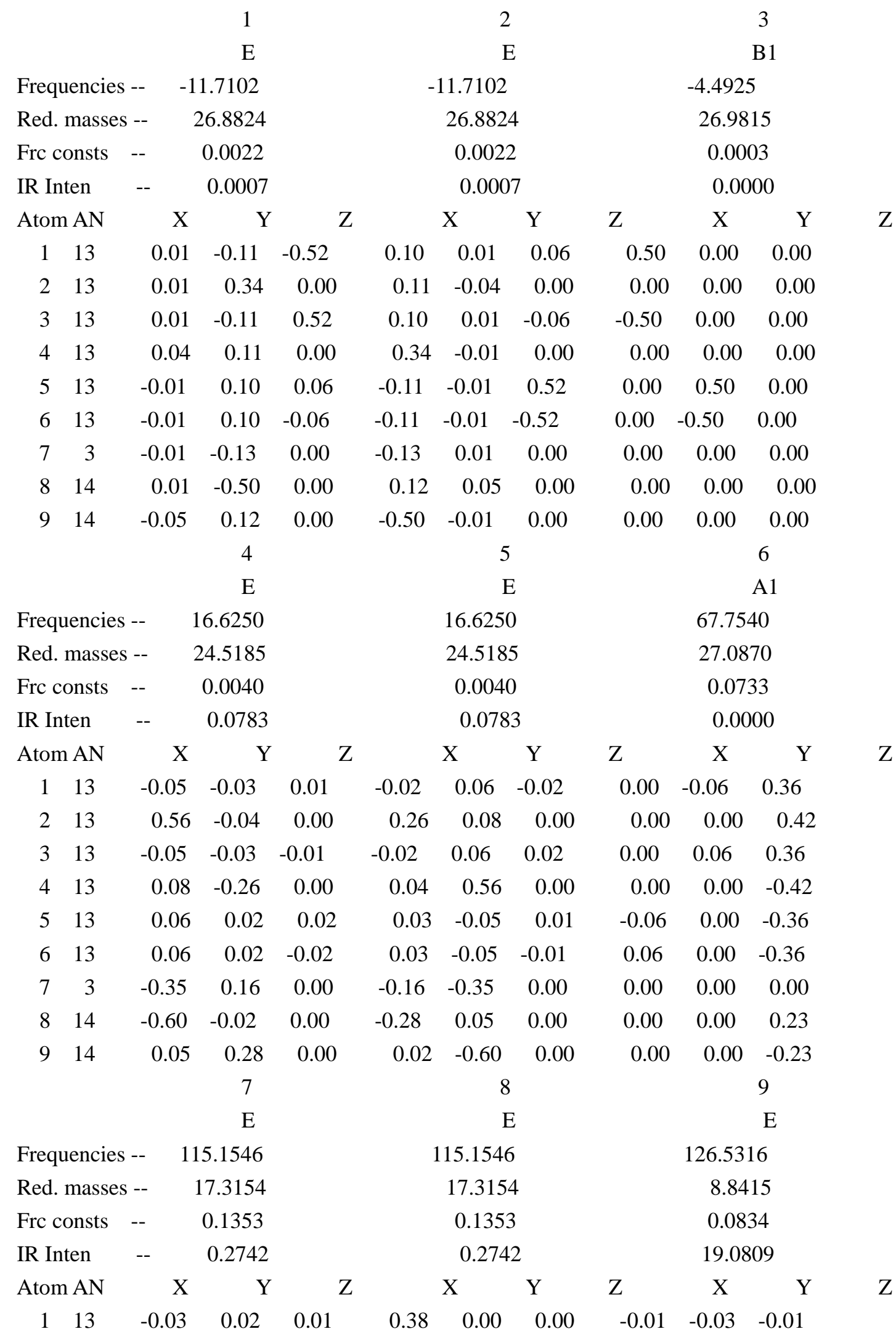




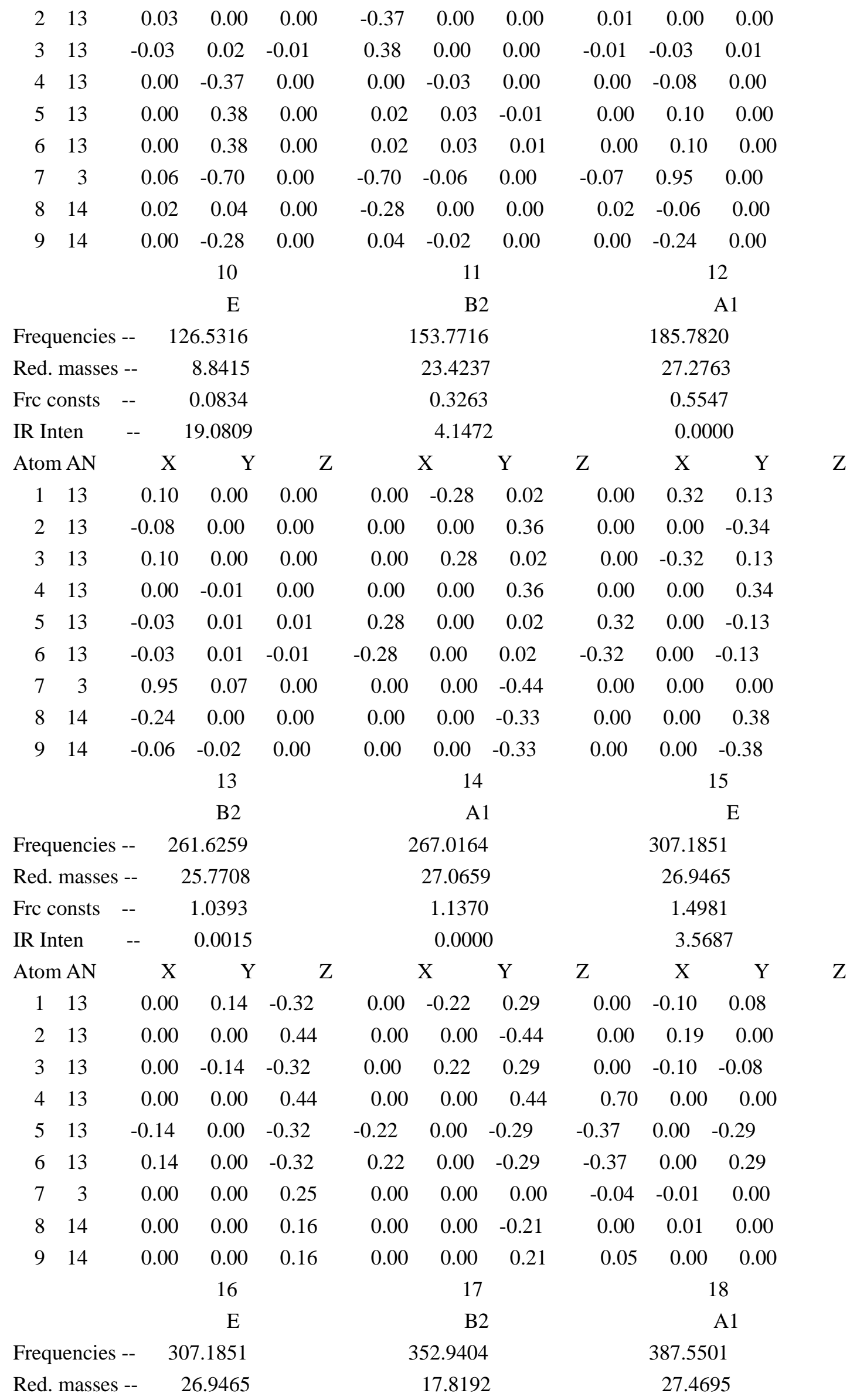




\begin{tabular}{|c|c|c|c|c|c|c|c|c|c|c|c|}
\hline \multicolumn{2}{|c|}{ Frc consts } & -- & 1.4981 & & \multicolumn{3}{|c|}{1.3078} & \multicolumn{4}{|c|}{2.4308} \\
\hline \multicolumn{2}{|c|}{ IR Inten } & -- & 3.5687 & & \multicolumn{3}{|c|}{9.4859} & \multicolumn{3}{|c|}{0.0000} & \\
\hline Atom & $\mathrm{AN}$ & $\mathrm{X}$ & $\mathrm{Y}$ & $\mathrm{Z}$ & & $\mathrm{X}$ & $\mathrm{Y}$ & Z & $\mathrm{X}$ & $\mathrm{Y}$ & $\mathrm{Z}$ \\
\hline 1 & 13 & 0.00 & -0.37 & 0.29 & 0.00 & -0.30 & -0.11 & 0.00 & -0.31 & -0.15 & \\
\hline 2 & 13 & 0.00 & 0.70 & 0.00 & 0.00 & 0.00 & -0.12 & 0.00 & 0.00 & -0.12 & \\
\hline 3 & 13 & 0.00 & -0.37 & -0.29 & 0.00 & 0.30 & -0.11 & 0.00 & 0.31 & -0.15 & \\
\hline 4 & 13 & -0.19 & 0.00 & 0.00 & 0.00 & 0.00 & -0.12 & 0.00 & 0.00 & 0.12 & \\
\hline 5 & 13 & 0.10 & 0.00 & 0.08 & 0.30 & 0.00 & -0.11 & -0.31 & 0.00 & 0.15 & \\
\hline 6 & 13 & 0.10 & 0.00 & -0.08 & -0.30 & 0.00 & -0.11 & 0.31 & 0.00 & 0.15 & \\
\hline 7 & 3 & 0.01 & -0.04 & 0.00 & 0.00 & 0.00 & 0.68 & 0.00 & 0.00 & 0.00 & \\
\hline 8 & 14 & 0.00 & 0.05 & 0.00 & 0.00 & 0.00 & 0.24 & 0.00 & 0.00 & 0.50 & \\
\hline \multirow[t]{3}{*}{9} & 14 & -0.01 & 0.00 & 0.00 & 0.00 & 0.00 & 0.24 & 0.00 & 0.00 & -0.50 & \\
\hline & & & 19 & & & 20 & & & & 21 & \\
\hline & & & E & & & $\mathrm{E}$ & & & & B2 & \\
\hline \multicolumn{3}{|c|}{ Frequencies -- } & 4.1650 & & & 394.1650 & & & 484.18 & & \\
\hline \multicolumn{2}{|c|}{ Red. masses - } & & 27.4618 & & & 27.4618 & & & 8.94 & & \\
\hline \multicolumn{2}{|c|}{ Frc consts } & -- & 2.5138 & & & 2.5138 & & & 1.23 & & \\
\hline \multicolumn{2}{|c|}{ IR Inten } & -- & 7.1923 & & & 7.192 & & & 489.61 & & \\
\hline \multicolumn{2}{|c|}{ Atom AN } & $X$ & $\mathrm{Y}$ & $\mathrm{Z}$ & & $\mathrm{X}$ & $\mathrm{Y}$ & Z & $\mathrm{X}$ & $\mathrm{Y}$ & $\mathrm{Z}$ \\
\hline 1 & 13 & 0.00 & -0.20 & -0.21 & 0.00 & 0.26 & 0.26 & 0.00 & 0.04 & 0.03 & \\
\hline 2 & 13 & 0.00 & -0.08 & 0.00 & 0.00 & 0.10 & 0.00 & 0.00 & 0.00 & 0.02 & \\
\hline 3 & 13 & 0.00 & -0.20 & 0.21 & 0.00 & 0.26 & -0.26 & 0.00 & -0.04 & 0.03 & \\
\hline 4 & 13 & 0.10 & 0.00 & 0.00 & 0.08 & 0.00 & 0.00 & 0.00 & 0.00 & 0.02 & \\
\hline 5 & 13 & 0.26 & 0.00 & -0.26 & 0.20 & 0.00 & -0.21 & -0.04 & 0.00 & 0.03 & \\
\hline 6 & 13 & 0.26 & 0.00 & 0.26 & 0.20 & 0.00 & 0.21 & 0.04 & 0.00 & 0.03 & \\
\hline 7 & 3 & -0.04 & 0.03 & 0.00 & -0.03 & -0.04 & 0.00 & 0.00 & 0.00 & 0.95 & \\
\hline 8 & 14 & 0.00 & 0.46 & 0.00 & 0.00 & -0.58 & 0.00 & 0.00 & 0.00 & -0.20 & \\
\hline 9 & 14 & -0.58 & 0.00 & 0.00 & -0.46 & 0.00 & 0.00 & 0.00 & 0.00 & -0.20 & \\
\hline
\end{tabular}

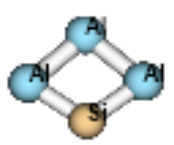

thi

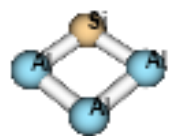

$\begin{array}{rrrrr}3 & 0 & 0.000000 & 0.000000 & 0.000000 \\ 13 & 0 & 0.000000 & 1.955734 & 3.909532 \\ 13 & 0 & 0.000000 & 0.000000 & 5.582959 \\ 13 & 0 & 0.000000 & -1.955734 & 3.909532 \\ 13 & 0 & 0.000000 & 0.000000 & -5.582959 \\ 13 & 0 & 0.000000 & 1.955734 & -3.909532 \\ 13 & 0 & 0.000000 & -1.955734 & -3.909532 \\ 14 & 0 & 0.000000 & 0.000000 & -2.538067\end{array}$


14

$0.000000 \quad 0.000000$

2.538067

State=1-AGIHF=-2041.3100499

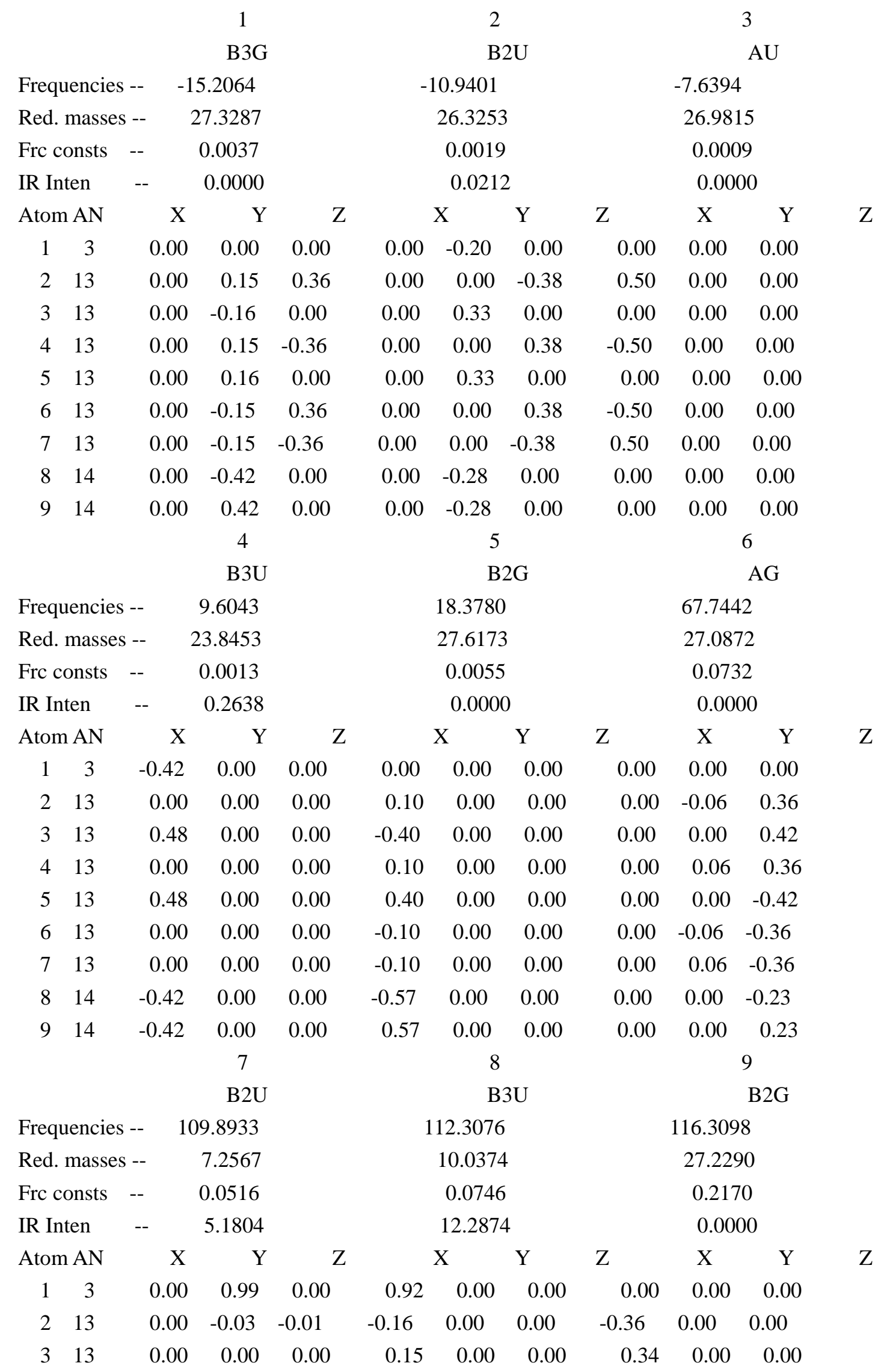




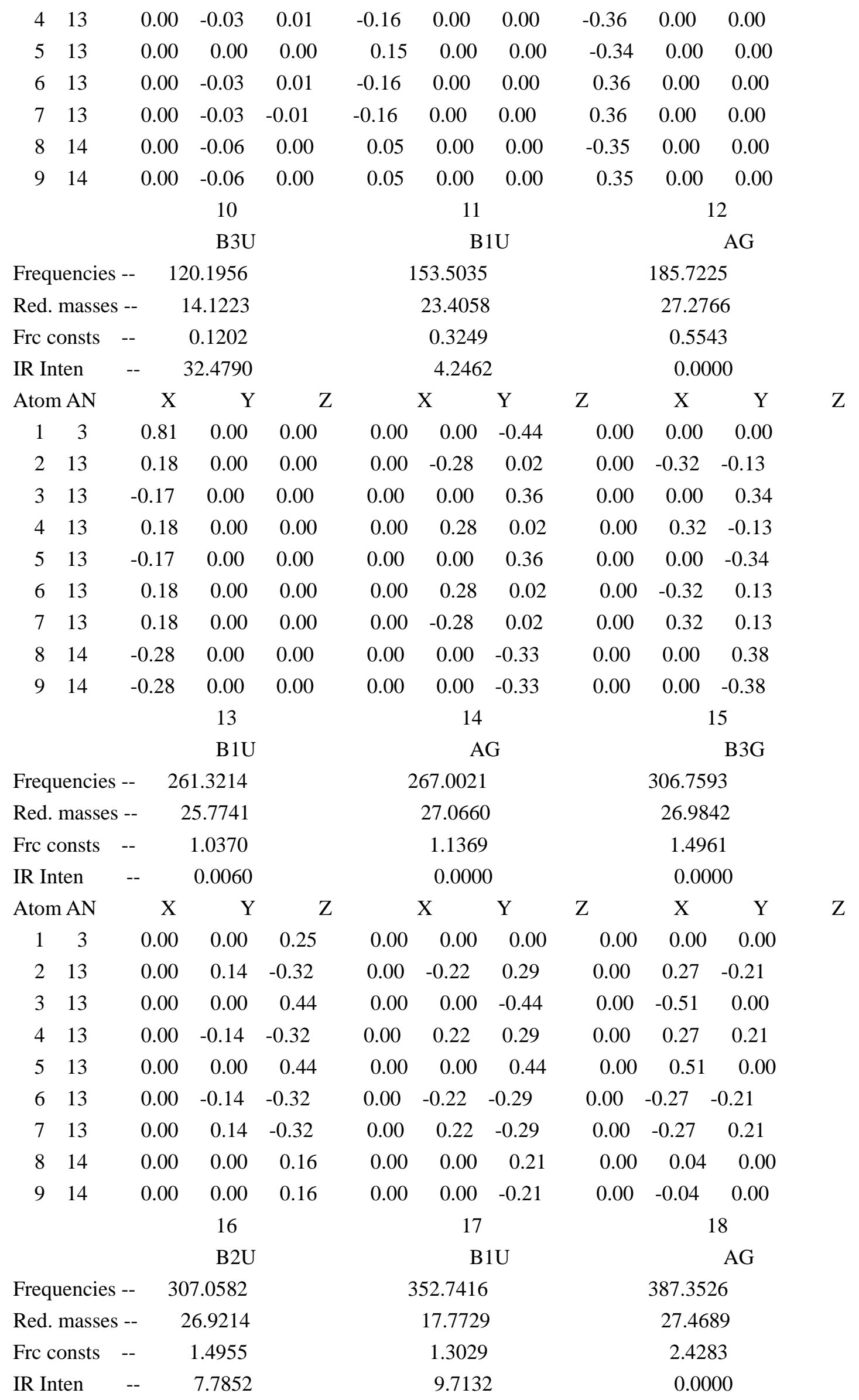




\begin{tabular}{|c|c|c|c|c|c|c|c|c|c|c|}
\hline \multicolumn{2}{|c|}{ Atom AN } & \multirow{2}{*}{$\begin{array}{r}X \\
0.00\end{array}$} & Y & Z & \multicolumn{2}{|c|}{$\mathrm{X}$} & $\mathrm{Y}$ & $\mathrm{Z}$ & $\mathrm{X}$ & $\mathrm{Y}$ \\
\hline 1 & 3 & & -0.06 & 0.00 & 0.00 & 0.00 & 0.68 & 0.00 & 0.00 & 0.00 \\
\hline 2 & 13 & 0.00 & -0.27 & 0.21 & 0.00 & -0.29 & -0.11 & 0.00 & 0.31 & 0.15 \\
\hline 3 & 13 & 0.00 & 0.51 & 0.00 & 0.00 & 0.00 & -0.12 & 0.0 & 0.00 & 0.12 \\
\hline 4 & 13 & 0.00 & -0.27 & -0.21 & 0.00 & 0.29 & -0.11 & 0.00 & -0.31 & 0.15 \\
\hline 5 & 13 & 0.00 & 0.51 & 0.00 & 0.00 & 0.00 & -0.12 & 0.0 & 0.00 & -0.12 \\
\hline 6 & 13 & 0.00 & -0.27 & -0.21 & 0.00 & 0.29 & -0.11 & 0.00 & 0.31 & -0.15 \\
\hline 7 & 13 & 0.00 & -0.27 & 0.21 & 0.00 & -0.29 & -0.11 & 0.00 & -0.31 & -0.15 \\
\hline 8 & 14 & 0.00 & 0.04 & 0.00 & 0.00 & 0.00 & 0.24 & 0.0 & $0 \quad 0.00$ & 0.49 \\
\hline \multirow[t]{3}{*}{9} & 14 & 0.00 & 0.04 & 0.00 & 0.00 & 0.00 & 0.24 & 0.0 & 0.00 & -0.49 \\
\hline & & \multirow{2}{*}{\multicolumn{3}{|c|}{$\begin{array}{l}19 \\
\text { B3G }\end{array}$}} & \multicolumn{3}{|c|}{20} & \multicolumn{3}{|c|}{21} \\
\hline & & & & & \multicolumn{3}{|c|}{$\mathrm{B} 2 \mathrm{U}$} & \multicolumn{3}{|r|}{ B1U } \\
\hline \multicolumn{2}{|c|}{ Frequencies -- } & \multicolumn{2}{|c|}{393.8544} & & \multicolumn{3}{|c|}{394.2578} & \multicolumn{3}{|c|}{483.2857} \\
\hline \multicolumn{2}{|c|}{ Red. masses } & \multicolumn{2}{|c|}{27.5224} & & \multicolumn{3}{|c|}{27.3956} & \multicolumn{3}{|c|}{8.9567} \\
\hline \multicolumn{2}{|c|}{ Frc consts } & - & 2.5154 & & \multicolumn{3}{|c|}{2.5090} & & 1.232 & \\
\hline IR Ir & & - & 0.0000 & & & 14.3211 & & & 491.28 & \\
\hline Aton & AN & X & $\mathrm{Y}$ & $\mathrm{Z}$ & & X & $\mathrm{Y}$ & Z & X & $\mathrm{Y}$ \\
\hline 1 & 3 & 0.00 & 0.00 & 0.00 & 0.00 & -0.08 & 0.00 & 0.0 & 0.00 & 0.95 \\
\hline 2 & 13 & 0.00 & -0.23 & -0.24 & 0.00 & 0.23 & 0.24 & 0.00 & 0.04 & 0.03 \\
\hline 3 & 13 & 0.00 & -0.09 & 0.00 & 0.00 & 0.09 & 0.00 & 0.0 & 0.00 & 0.02 \\
\hline 4 & 13 & 0.00 & -0.23 & 0.24 & 0.00 & 0.23 & -0.24 & 0.00 & -0.04 & 0.03 \\
\hline 5 & 13 & 0.00 & 0.09 & 0.00 & 0.00 & 0.09 & 0.00 & 0.0 & 0.00 & 0.02 \\
\hline 6 & 13 & 0.00 & 0.23 & -0.24 & 0.00 & 0.23 & -0.24 & 0.00 & -0.04 & 0.03 \\
\hline 7 & 13 & 0.00 & 0.23 & 0.24 & 0.00 & 0.23 & 0.24 & 0.0 & 0.04 & 0.03 \\
\hline 8 & 14 & 0.00 & -0.52 & 0.00 & 0.00 & -0.52 & 0.00 & 0.0 & 0.00 & -0.20 \\
\hline 9 & 14 & 0.00 & 0.52 & 0.00 & 0.00 & -0.52 & 0.00 & 0.0 & 0.00 & -0.20 \\
\hline & AI & usion & 00-b36? & $11+$ gx.fr & & & & & & \\
\hline & & 13 & & 0 & & 571468 & 2.49 & 285 & -0.260203 & \\
\hline & & 13 & & 0 & -1.8 & 324986 & $1.67 €$ & 226 & 0.327685 & \\
\hline & & 3 & & 0 & -2.1 & 44192 & -0.844 & & 1.919843 & \\
\hline & & 13 & & 0 & & 122573 & -0.012 & 372 & -1.423718 & \\
\hline & & 13 & & 0 & & 915432 & -1.761 & 513 & -0.159078 & \\
\hline & & 14 & & 0 & & 061575 & 0.03 & 087 & 1.20136 & \\
\hline & & 14 & & 0 & -0.4 & 85656 & -2.154 & 784 & 0.234929 & \\
\hline & & 13 & & 0 & -2.2 & 97433 & -0.685 & & -0.711588 & \\
\hline & & 13 & & 0 & & 464461 & 0.76 & 398 & 0.23708 & \\
\hline
\end{tabular}

State $=1-A \backslash H F=-2041.3912482$

Al3SiLiAl3Si-fusion102-b36311+gx.frq 


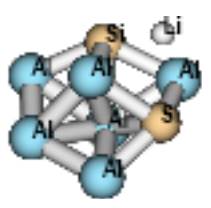

$\begin{array}{ccccc}13 & 0 & 2.471408 & 0.001101 & 0.051276 \\ 3 & 0 & -2.378377 & 0.000424 & 1.853264 \\ 13 & 0 & 0.076284 & -0.000011 & -1.274191 \\ 13 & 0 & 0.173918 & 0.000144 & 1.550983 \\ 14 & 0 & -1.065388 & -1.837489 & 0.218332 \\ 13 & 0 & -2.511638 & -0.001244 & -0.739905 \\ 13 & 0 & 1.318669 & -2.336096 & -0.242789 \\ 14 & 0 & -1.066801 & 1.836500 & 0.217818 \\ 13 & 0 & 1.316417 & 2.337073 & -0.242750\end{array}$

State $=1-A \backslash H F=-2041.3887811$

Al3SiLiAl3Si-fusion101-b36311+gx.frq

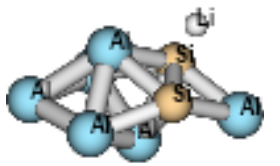

13

3

13

13

14

13

13

13

14
$-1.26321$

1.921804

0.115995

$-1.263287$

1.208892

3.203189

$-3.121397$

$-0.718557$

1.208899
$-1.984169$

0.000176

$-0.000027$

1.984145

1.279635

$-0.000047$

$-0.000049$

0.000041

$-1.279573$
$-0.288343$

2.431778

$-1.551465$

$-0.288392$

0.416636

$-0.680971$

$-0.182950$

1.533428

0.416769

State $=1-A \mid H F=-2041.3675445$

Al3SiLiAl3Si-fusion700-b36311+gx.frq

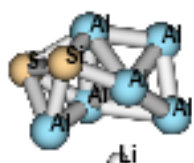

di

$\begin{array}{rrrrc}3 & 0 & -0.513023 & 2.905173 & -0.168663 \\ 13 & 0 & -2.503844 & -1.390165 & -0.010947 \\ 13 & 0 & 0.029671 & 0.444386 & -1.327371 \\ 13 & 0 & 0.057241 & -1.871839 & -0.040262 \\ 13 & 0 & -0.721080 & 0.524687 & 1.245869 \\ 13 & 0 & 1.956103 & 1.707386 & 0.061584 \\ 13 & 0 & -2.715751 & 1.245402 & -0.313869 \\ 14 & 0 & 2.200959 & -0.813218 & -1.004884 \\ 14 & 0 & 1.528230 & -0.422043 & 1.398522\end{array}$

State $=1-A \backslash H F=-2041.3666863$

Al3SiNaAl3Si-s-c-90-Si-Si-b36311+gx.frq4.4 


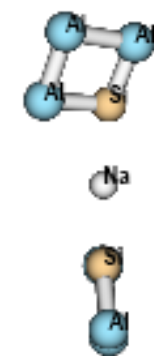

Charge $=-1$ Multiplicity $=1$

Al,0,-3.2240466469,1.3931688447,1.0779887401

Al,0,-5.6014896597,0.6405865482,0.4650785214

Na,0,0.0020536598,-0.0052920877,-0.2432454951

Al,0,3.2195153576,-1.3826932354,1.0892035168

Al,0,5.5992751907,-0.6531815355,0.4574605914

Al,0,-4.9257856845,-1.3410323771,-1.0576652617

Si,0,-2.8258172217,-0.4880690414,-0.3614106878

Si,0,2.8271840143,0.5017597447,-0.3475525718

Al,0,4.9293218001,1.332885841,-1.0627441018

\section{State $=1-A \backslash H F=-2196.085081$}

1

A
2

A

13.0626

26.7493

0.0027

0.0325

$\begin{array}{lll}\text { IR Inten } & -- & 0.0020\end{array}$

Atom AN X Y

Frequencies -- $\quad 18.8629$

Red. masses -- 27.3522

Frc consts -- 0.0057

IR Inten -- $\quad 0.7093$

Atom AN $\mathrm{X} \quad \mathrm{Y}$ $\begin{array}{llll}1 & 13 & 0.06 & -0.30\end{array}$

$\begin{array}{llll}2 & 13 & 0.03 & -0.14\end{array}$

311

41

0.00

0.00

$-0.06$

0.30

0.30

$-0.03$

0.13

0.27

$6 \quad 13$

$7 \quad 14$

$-0.04$

$-0.01$

0.08

$8 \quad 14$

0.01

$-0.0$

0.05

$-0.27$

$$
4
$$

A

113

0.28

$\begin{array}{ll}-0.07 & -0.29\end{array}$

$\begin{array}{lll}0.12 & 0.03 & 0.23\end{array}$

$\begin{array}{lll}-0.08 & 0.01 & 0.02\end{array}$

Z

0.33

0.24

$-0.14$

0.33

0.24

$-0.32$

$-0.19$

$-0.18$

$-0.31$

213

311

$\mathrm{X}^{0.0325} \mathrm{Y}$

$\begin{array}{lll}0.07 & -0.17 & 0.17\end{array}$

$\begin{array}{lll}0.03 & 0.38 & -0.34\end{array}$

$\begin{array}{lll}-0.04 & -0.29 & 0.17\end{array}$

$\begin{array}{lll}0.26 & -0.09 & 0.05\end{array}$

$\begin{array}{lll}0.13 & 0.29 & -0.03\end{array}$

$\begin{array}{lll}-0.10 & 0.22 & -0.18\end{array}$

$\begin{array}{lll}-0.05 & -0.28 & 0.29\end{array}$

$\begin{array}{lll}-0.07 & -0.21 & -0.02\end{array}$

$-0.23$

$0.12-0.10$

5

A

21.2085

27.2873

0.0072

0.0161

X Y

$\begin{array}{lll}0.33 & -0.26 & -0.02\end{array}$

$\begin{array}{lll}0.21 & 0.20 & -0.10\end{array}$

$\begin{array}{lll}0.01 & 0.02 \quad 0.11\end{array}$
3

A

13.4555

26.6709

0.0028

0.0444

Z $\quad \mathrm{Y} \quad \mathrm{Y}$

$\begin{array}{lll}0.29 & -0.03 & -0.16\end{array}$

$\begin{array}{lll}0.14 & 0.12 \quad 0.25\end{array}$

$\begin{array}{lll}-0.02 & -0.17 & -0.30\end{array}$

$\begin{array}{lll}-0.10 & -0.12 & -0.22\end{array}$

$\begin{array}{lll}-0.05 & 0.26 & 0.41\end{array}$

$\begin{array}{lll}-0.23 & 0.02 & 0.21\end{array}$

$\begin{array}{lll}-0.05 & -0.10 & -0.16\end{array}$

$\begin{array}{llll}-0.02 & -0.17 & -0.32\end{array}$

$\begin{array}{lll}0.03 & 0.17 & 0.26\end{array}$

6

A

53.6507

23.7663

0.0403

9.2737

Z $\quad \mathrm{X} \quad \mathrm{Y}$

$\begin{array}{lll}0.04 & -0.16 & 0.01\end{array}$

$\begin{array}{lll}0.01 & 0.05 & -0.07\end{array}$

$\begin{array}{lll}-0.14 & 0.90 & -0.08\end{array}$ 


$$
\begin{array}{rrrrc}
4 & 13 & 0.24 & -0.04 & 0.29 \\
5 & 13 & 0.09 & 0.01 & -0.25 \\
6 & 13 & -0.26 & 0.05 & 0.04 \\
7 & 14 & -0.07 & -0.04 & -0.43 \\
8 & 14 & -0.08 & 0.00 & 0.45 \\
9 & 13 & -0.26 & 0.04 & -0.05 \\
& & & 7 &
\end{array}
$$

A

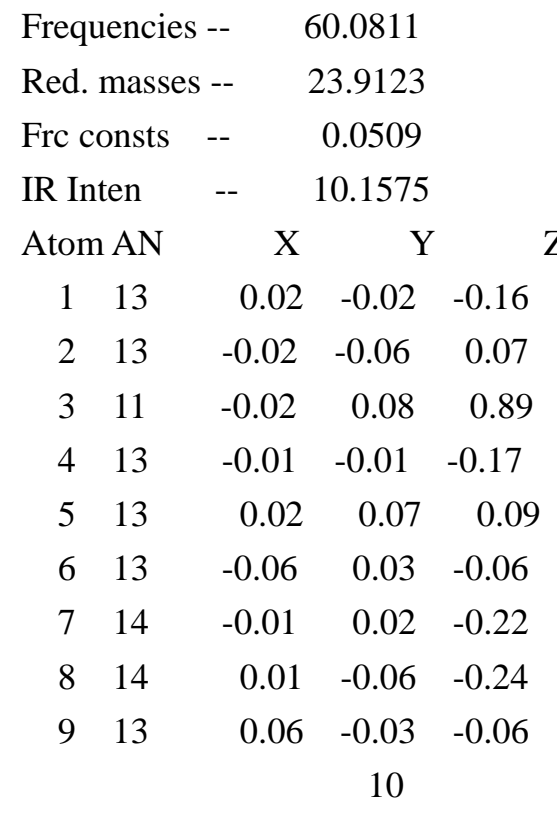

A

$$
\begin{array}{ccc}
-0.37 & 0.28 & -0.05 \\
-0.23 & -0.23 & -0.09 \\
-0.08 & 0.01 & 0.01 \\
0.06 & -0.40 & 0.08 \\
-0.05 & 0.42 & 0.04 \\
0.12 & -0.03 & 0.02 \\
\multicolumn{3}{c}{8} \\
\end{array}
$$

A

68.9975

27.1626

0.0762

\begin{tabular}{|c|c|c|c|c|c|}
\hline \multicolumn{2}{|r|}{$y$} & \multicolumn{2}{|l|}{ Y } & $X$ & $Y$ \\
\hline 0.24 & 0.14 & 0.04 & 0.01 & 0.22 & -0.29 \\
\hline 0.34 & -0.11 & -0.02 & 0.00 & -0.20 & 0.26 \\
\hline 0.00 & 0.02 & 0.07 & -0.04 & 0.02 & 0.00 \\
\hline-0.24 & -0.15 & 0.04 & 0.01 & 0.21 & 0.29 \\
\hline-0.34 & 0.12 & -0.01 & -0.01 & -0.20 & -0.26 \\
\hline 0.42 & -0.04 & -0.08 & 0.01 & 0.22 & -0.28 \\
\hline 0.27 & 0.16 & 0.03 & 0.00 & -0.23 & 0.29 \\
\hline-0.27 & -0.17 & 0.02 & 0.00 & -0.23 & -0.29 \\
\hline-0.42 & 0.04 & -0.08 & 0.01 & 0.22 & 0.28 \\
\hline & 11 & & & & 12 \\
\hline
\end{tabular}

0.0065

$$
\begin{array}{ccc}
0.06 & -0.16 & 0.02 \\
0.02 & 0.04 & 0.07 \\
-0.03 & -0.05 & 0.03 \\
0.01 & -0.21 & 0.07 \\
0.03 & -0.21 & -0.04 \\
-0.02 & -0.05 & -0.02 \\
& & 9
\end{array}
$$

A

117.4592

27.2477

0.2215

1.5946

A

A

Frequencies -- 118.5192

144.2323

171.8039

26.1407

0.3204

27.2703

0.4742

3.5261

1.9951

IR Inten -- 3.0012

$\begin{array}{lllll}\text { Atom AN } & X & Y & \text { Z }\end{array}$

$\begin{array}{lllll}1 & 13 & 0.01 & -0.21 & 0.28\end{array}$

$\begin{array}{ll}\mathrm{X} & \mathrm{Y}\end{array}$

$\begin{array}{lll}0.17 & 0.19 & 0.15\end{array}$

X $\quad \mathrm{Y}$

Z

$\begin{array}{lllll}2 & 13 & 0.03 & 0.19 & -0.26\end{array}$

$\begin{array}{lll}0.35 & -0.12 & -0.09\end{array}$

Z

$\begin{array}{lll}-0.09 & -0.20 & -0.15\end{array}$

$\begin{array}{lll}-0.50 & 0.01 & 0.00\end{array}$

$\begin{array}{lll}-0.31 & 0.13 & 0.09\end{array}$

$\begin{array}{lllll}3 & 11 & -0.01 & 0.00 & 0.05\end{array}$

$\begin{array}{rrr}-0.50 & 0.01 & 0.00 \\ 0.17 & 0.19 & -0.15\end{array}$

$\begin{array}{lll}0.00 & 0.00 & -0.06\end{array}$

$\begin{array}{lllll}4 & 13 & -0.01 & 0.21 & 0.29\end{array}$

$\begin{array}{lll}0.35 & -0.12 & 0.09\end{array}$

$\begin{array}{lll}0.09 & 0.20 & -0.15\end{array}$

$\begin{array}{llllll}5 & 13 & -0.03 & -0.19 & -0.26\end{array}$

$\begin{array}{lll}-0.05 & -0.19 & -0.15\end{array}$

$\begin{array}{lll}0.31 & -0.13 & 0.08\end{array}$

$\begin{array}{lllll}6 & 13 & 0.00 & -0.23 & 0.27\end{array}$

$\begin{array}{ccc}-0.05 & -0.19 & -0.15 \\ -0.25 & 0.11 & 0.08\end{array}$

$\begin{array}{lll}0.27 & 0.21 & 0.18\end{array}$

$\begin{array}{lllll}7 & 14 & 0.00 & 0.24 & -0.30\end{array}$

$\begin{array}{lll}-0.25 & 0.12 & -0.07\end{array}$

$\begin{array}{lll}0.37 & -0.07 & -0.09\end{array}$

$\begin{array}{ll}8 & 14\end{array}$

$\begin{array}{lll}0.00 & -0.24 & -0.30\end{array}$

$-0.25$

$-0.19 \quad 0.15$

14

A

A

264.7839

27.0494

1.1174

0.4533

$\begin{array}{lll}-0.37 & 0.07 & -0.09\end{array}$

$\begin{array}{lll}-0.27 & -0.21 & 0.18\end{array}$

15

A

266.1328

26.9257

1.1236

15.0251 


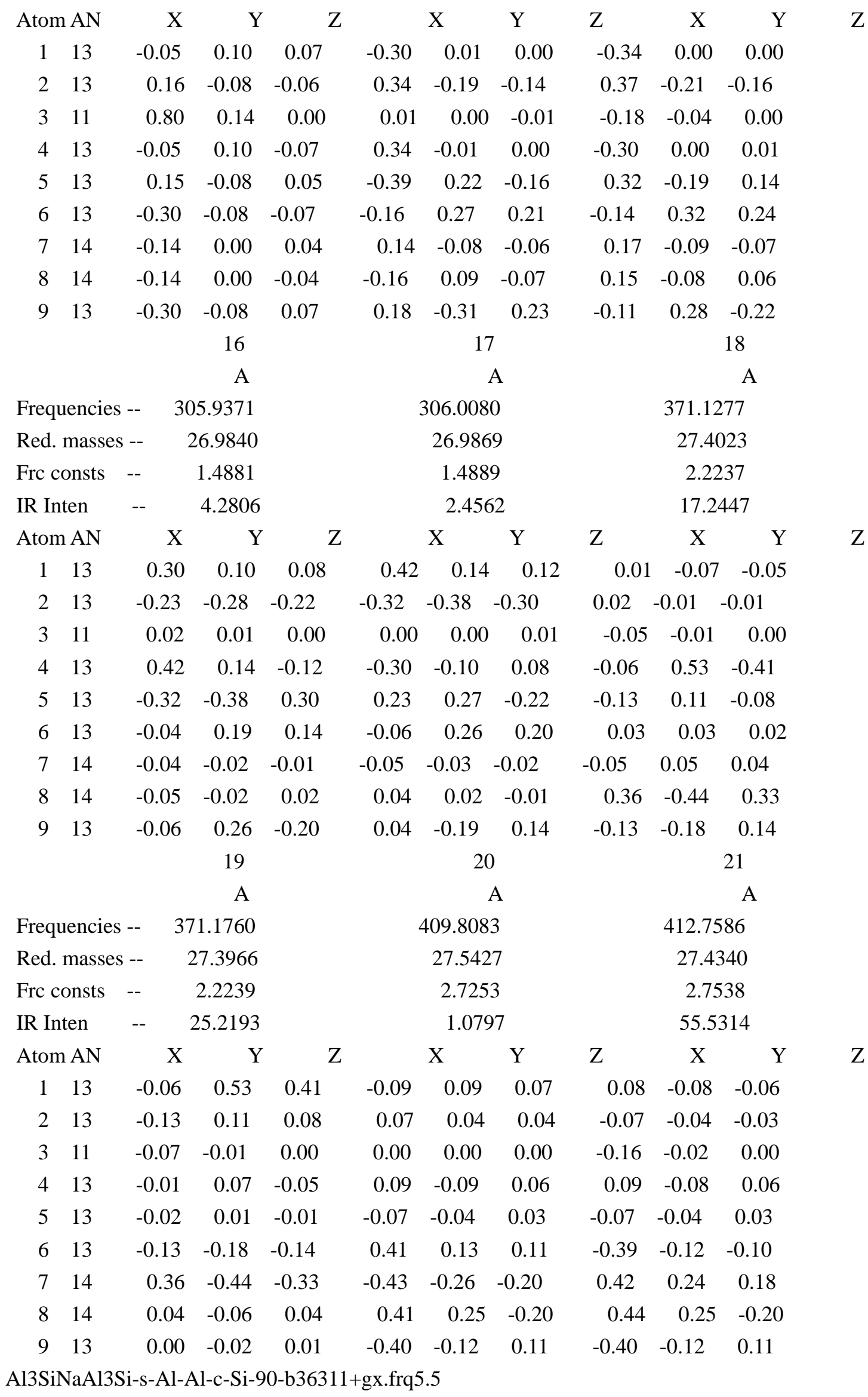




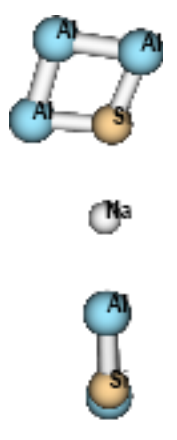

$\begin{array}{rrrrr}13 & 0 & 3.177724 & 1.642527 & 0.587695 \\ 13 & 0 & 5.587635 & 0.817798 & 0.270902 \\ 11 & 0 & 0.022319 & -0.215167 & -0.091568 \\ 13 & 0 & -2.952606 & 0.384880 & -1.092863 \\ 13 & 0 & -5.491619 & 0.554015 & -1.178899 \\ 13 & 0 & 5.001157 & -1.542597 & -0.614683 \\ 14 & 0 & 2.862919 & -0.586676 & -0.249248 \\ 14 & 0 & -5.173230 & -0.377136 & 0.986615 \\ 13 & 0 & -2.853149 & -0.636607 & 1.311242\end{array}$

\section{State $=1-A \backslash H F=-2196.0810218$}

1

A

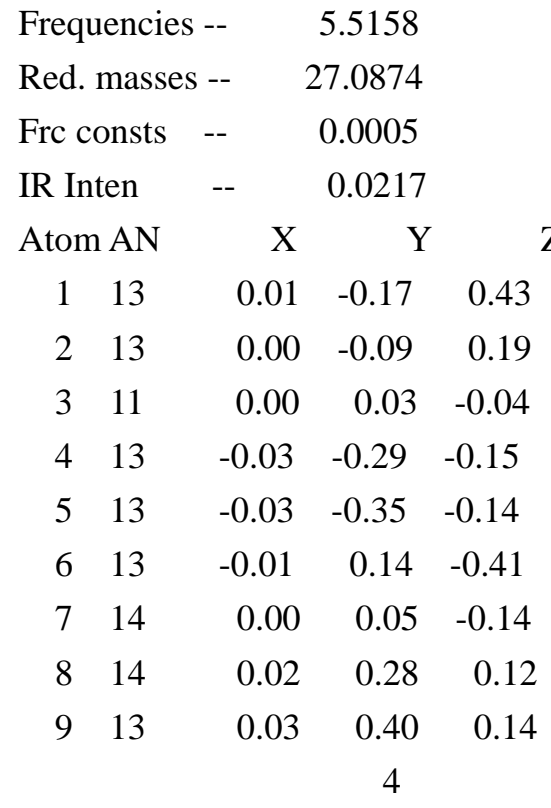

A

2

A

13.4920

26.6496

0.0029

0.1169

X Y

$\begin{array}{lll}0.00 & 0.09 & -0.24\end{array}$

$\begin{array}{lll}0.00 & -0.21 & 0.53\end{array}$

$\begin{array}{lll}0.00 & 0.14 & -0.36\end{array}$

$\begin{array}{lll}-0.13 & 0.02 & -0.06\end{array}$

$-0.14-0.07$

$\begin{array}{ll}-0.01 & -0.10\end{array}$

$\begin{array}{lll}0.00 & 0.17 & -0.45\end{array}$

$\begin{array}{lll}0.11 & -0.06 & 0.16\end{array}$

$\begin{array}{lll}0.15 & 0.03 & -0.07\end{array}$

5

A

37.3117

26.4100

0.0217

3.5194

IR Inten -- 2.4143

Atom AN $\mathrm{X} \quad \mathrm{Y}$

$\begin{array}{lllll}1 & 13 & -0.23 & -0.25 & -0.11\end{array}$
3

A

14.8585

26.7514

0.0035

0.6051

Z $\quad \mathrm{X} \quad \mathrm{Y}$

$\begin{array}{lll}0.46 & 0.25 & 0.08\end{array}$

$\begin{array}{lll}0.25 & -0.29 & -0.12\end{array}$

$\begin{array}{lll}-0.05 & 0.30 & 0.12\end{array}$

$\begin{array}{lll}-0.06 & 0.12 & 0.05\end{array}$

$\begin{array}{lll}-0.08 & -0.25 & -0.10\end{array}$

$\begin{array}{lll}-0.32 & -0.18 & -0.06\end{array}$

$\begin{array}{lll}-0.07 & 0.31 & 0.13\end{array}$

$\begin{array}{lll}-0.08 & -0.27 & -0.11\end{array}$

$\begin{array}{lll}-0.06 & 0.05 & 0.02\end{array}$

6

A

63.1082

26.2162

0.0615

1.4001

X Y

Z

$\begin{array}{lllllllllll}3 & 11 & 0.06 & 0.42 & 0.16 & 0.01 & 0.18 & -0.42 & 0.05 & -0.33 & 0.34\end{array}$ 


$\begin{array}{ccccccccccc}4 & 13 & 0.06 & 0.44 & 0.18 & -0.21 & 0.12 & -0.27 & -0.27 & 0.21 & -0.16 \\ 5 & 13 & 0.03 & -0.27 & -0.11 & -0.26 & -0.07 & 0.21 & -0.34 & -0.05 & 0.08 \\ 6 & 13 & 0.13 & -0.02 & 0.00 & 0.01 & -0.03 & 0.08 & 0.34 & 0.07 & 0.01 \\ 7 & 14 & -0.01 & -0.29 & -0.12 & 0.01 & -0.17 & 0.43 & 0.24 & -0.05 & -0.10 \\ 8 & 14 & 0.03 & -0.29 & -0.12 & 0.18 & -0.06 & 0.15 & -0.26 & -0.05 & 0.07 \\ 9 & 13 & 0.07 & 0.31 & 0.12 & 0.25 & 0.10 & -0.29 & -0.21 & 0.17 & -0.18 \\ & & & 7 & & & 8 & & & & 9\end{array}$

A

A

A

\begin{tabular}{|c|c|c|c|}
\hline Frequencies & & 64.4363 & \\
\hline Red. masses & -- & 24.3931 & \\
\hline Frc consts & -- & 0.0597 & \\
\hline IR Inten & -- & 5.0793 & \\
\hline Atom AN & $\mathrm{X}$ & $\mathrm{Y}$ & $z$ \\
\hline 113 & 0.00 & -0.10 & -0.06 \\
\hline 213 & 0.02 & 0.00 & 0.02 \\
\hline 311 & 0.07 & 0.65 & 0.47 \\
\hline $4 \quad 13$ & -0.05 & -0.35 & -0.25 \\
\hline $5 \quad 13$ & -0.04 & 0.13 & 0.10 \\
\hline $6 \quad 13$ & 0.04 & 0.00 & -0.01 \\
\hline $7 \quad 14$ & -0.01 & -0.09 & -0.07 \\
\hline $8 \quad 14$ & -0.01 & 0.08 & 0.07 \\
\hline $9 \quad 13$ & -0.01 & -0.22 & -0.20 \\
\hline & & 10 & \\
\hline
\end{tabular}

A

68.3440

111.4652

25.5916

27.2774

0.0704

0.1997

0.8103

1.2714

$\begin{array}{lllllllllllll}\text { Atom AN } & X & Y & Z & X & Y & Z & X & Y & \text { Z }\end{array}$

Frequencies -- $\quad 117.2785$

$\begin{array}{lll}-0.15 & 0.11 & 0.00\end{array}$

$\begin{array}{lll}0.00 & -0.01 & 0.00\end{array}$

$\begin{array}{llllll}-0.20 & -0.08 & 0.01 & 0.00 & 0.00 & 0.00\end{array}$

$\begin{array}{lll}-0.01 & -0.35 & 0.51\end{array}$

$\begin{array}{lll}0.00 & 0.05 & 0.03\end{array}$

$\begin{array}{lll}0.15 & 0.18 & -0.30\end{array}$

$\begin{array}{lll}0.02 & 0.37 & 0.15\end{array}$

$\begin{array}{llllll}0.11 & -0.07 & 0.12 & -0.02 & -0.45 & -0.19\end{array}$

$\begin{array}{llllll}-0.26 & -0.05 & -0.03 & 0.00 & 0.00 & 0.01\end{array}$

$\begin{array}{lllllll}-0.16 & 0.13 & -0.03 & 0.00 & 0.00 & -0.01\end{array}$

$\begin{array}{lllllll}0.24 & -0.07 & 0.10 & 0.03 & 0.51 & 0.22\end{array}$

$\begin{array}{lll}0.27 & 0.14 & -0.29\end{array}$

11

A

Red. masses -- $\quad 27.2584$

149.0735

26.5304

$\begin{array}{lrrr}\text { Frc consts } & -- & 0.2209 & 0.3474 \\ \text { IR Inten } & -- & 1.7979 & 22.3047\end{array}$

$\begin{array}{lll}-0.03 & -0.49 & -0.21\end{array}$

12

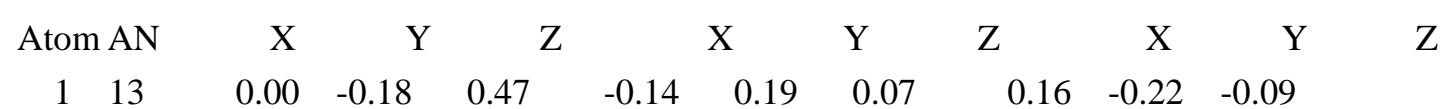

$\begin{array}{llllllllllll}2 & 13 & 0.00 & 0.16 & -0.43 & -0.29 & -0.13 & -0.04 & 0.34 & 0.15 & 0.06\end{array}$

$\begin{array}{lllllllllll}3 & 11 & 0.00 & 0.00 & 0.01 & 0.38 & 0.10 & -0.18 & -0.39 & 0.03 & -0.14\end{array}$

$\begin{array}{llllllllllll}4 & 13 & 0.01 & 0.00 & -0.01 & 0.24 & 0.04 & -0.14 & 0.28 & 0.06 & -0.17\end{array}$

$\begin{array}{lllllllllll}5 & 13 & 0.01 & 0.00 & 0.00 & 0.25 & -0.08 & 0.16 & 0.36 & -0.09 & 0.16\end{array}$

$\begin{array}{llllllllllll}6 & 13 & 0.00 & -0.18 & 0.47 & 0.09 & -0.20 & -0.08 & -0.13 & 0.24 & 0.09\end{array}$

$\begin{array}{lllllllllll}7 & 14 & 0.00 & 0.19 & -0.49 & 0.23 & 0.10 & 0.06 & -0.29 & -0.14 & -0.03\end{array}$

$\begin{array}{lllllllllll}8 & 14 & -0.01 & -0.01 & 0.00 & -0.41 & -0.08 & 0.25 & -0.21 & -0.08 & 0.21\end{array}$

$\begin{array}{lllllllllll}9 & 13 & -0.01 & 0.01 & 0.00 & -0.30 & 0.07 & -0.14 & -0.16 & 0.06 & -0.11\end{array}$

13

A

Frequencies -- $\quad 210.6721$

14

15

Red. masses -- 24.4763

265.0517

27.0283

1.1187

Frc consts -- 0.6400

5.3510

A

266.3990

26.9902

1.1286

0.3179 


$\begin{array}{ccccc}\text { Atom AN } & \text { X } & \text { Y } & \text { Z } \\ 1 & 13 & -0.01 & -0.16 & -0.06 \\ 2 & 13 & 0.20 & 0.12 & 0.04 \\ 3 & 11 & 0.80 & -0.08 & -0.04 \\ 4 & 13 & -0.01 & -0.03 & 0.07 \\ 5 & 13 & -0.12 & 0.02 & -0.03 \\ 6 & 13 & -0.37 & 0.16 & 0.06 \\ 7 & 14 & -0.26 & -0.06 & -0.02 \\ 8 & 14 & -0.07 & 0.00 & 0.00 \\ 9 & 13 & -0.02 & 0.02 & -0.03 \\ & & & 16 & \end{array}$

A

Frequencies -- 306.2942

Red. masses -- 26.9865

Frc consts -- $\quad 1.4917$

IR Inten -- 3.3537

Atom AN $\quad \mathrm{X} \quad \mathrm{Y}$

$\begin{array}{lllll}1 & 13 & 0.53 & -0.19 & -0.07\end{array}$

$\begin{array}{lllll}2 & 13 & -0.41 & 0.54 & 0.21\end{array}$

$\begin{array}{lllll}3 & 11 & 0.01 & -0.01 & 0.00\end{array}$

$\begin{array}{lllll}4 & 13 & 0.02 & 0.00 & 0.00\end{array}$

$\begin{array}{lllll}5 & 13 & -0.02 & 0.00 & 0.00\end{array}$

$\begin{array}{llllll}6 & 13 & -0.05 & -0.38 & -0.14\end{array}$

$\begin{array}{lllll}7 & 14 & -0.07 & 0.03 & 0.01\end{array}$

$\begin{array}{lllll}8 & 14 & 0.00 & 0.00 & 0.00\end{array}$

$\begin{array}{lllll}9 & 13 & 0.00 & 0.00 & 0.00\end{array}$ 19

A

Frequencies -- 375.0066

Red. masses -- $\quad 27.3321$

Frc consts -- $\quad 2.2647$

IR Inten -- 3.5363

$\begin{array}{crr}\text { Atom AN } & \mathrm{X} & \mathrm{Y}\end{array}$

$\begin{array}{lllll}1 & 13 & -0.01 & -0.04 & -0.01\end{array}$

$\begin{array}{lllll}2 & 13 & -0.01 & -0.01 & 0.00\end{array}$

$\begin{array}{lllll}3 & 11 & -0.05 & 0.00 & 0.00\end{array}$

$\begin{array}{lllll}4 & 13 & 0.13 & 0.05 & -0.13\end{array}$

$\begin{array}{llllll}5 & 13 & -0.19 & 0.24 & -0.55\end{array}$

$\begin{array}{lllll}6 & 13 & 0.01 & 0.01 & 0.00\end{array}$

$\begin{array}{lllll}7 & 14 & 0.01 & 0.04 & 0.02\end{array}$

$\begin{array}{llllll}8 & 14 & -0.30 & -0.19 & 0.48\end{array}$

$\begin{array}{lllll}9 & 13 & 0.40 & -0.10 & 0.18\end{array}$
$\begin{array}{lll}\mathrm{X} & \mathrm{Y} \\ 0.44 & 0.03 & 0.01\end{array}$

$\begin{array}{lll}-0.48 & -0.35 & -0.13\end{array}$

$\begin{array}{lll}0.07 & -0.02 & 0.00\end{array}$

$\begin{array}{lll}-0.05 & -0.06 & 0.15\end{array}$

$\begin{array}{lll}0.04 & 0.02 & -0.04\end{array}$

$\begin{array}{lll}0.20 & 0.48 & 0.18\end{array}$

$\begin{array}{lll}-0.21 & -0.14 & -0.05\end{array}$

$\begin{array}{lll}-0.03 & -0.01 & 0.04\end{array}$

$\begin{array}{lll}0.04 & 0.06 & -0.15\end{array}$

17

A

313.9360

26.9431

1.5645

2.1895

$\mathrm{X} \quad \mathrm{Y}$

$\begin{array}{lll}-0.02 & 0.02 & 0.01\end{array}$

$\begin{array}{lll}0.02 & -0.01 & 0.00\end{array}$

$\begin{array}{lll}-0.11 & 0.02 & -0.02\end{array}$

$\begin{array}{lll}0.69 & -0.14 & 0.23\end{array}$

$\begin{array}{lll}-0.58 & 0.02 & 0.04\end{array}$

$\begin{array}{lll}0.02 & -0.01 & 0.00\end{array}$

$\begin{array}{lll}0.00 & 0.00 & 0.00\end{array}$

$\begin{array}{lll}0.11 & 0.00 & -0.02\end{array}$

$-0.15$

$\begin{array}{ll}0.11 & -0.23\end{array}$

20

A

411.4742

27.4932

2.7426

28.2388

X Y

$\begin{array}{llll}-0.12 & -0.14 & -0.05\end{array}$

$\begin{array}{lll}0.10 & -0.07 & -0.03\end{array}$

$\begin{array}{lll}0.11 & -0.01 & 0.00\end{array}$

$\begin{array}{lll}0.00 & 0.00 & 0.00\end{array}$

$\begin{array}{llll}0.00 & -0.01 & 0.03\end{array}$

$\begin{array}{lll}0.57 & -0.20 & -0.08\end{array}$

$\begin{array}{lll}-0.62 & 0.40 & 0.15\end{array}$

$\begin{array}{lll}-0.03 & 0.01 & -0.03\end{array}$

$\begin{array}{lll}0.02 & 0.00 & 0.00\end{array}$
$\begin{array}{llll}\mathrm{Z} & \mathrm{X} & \mathrm{Y}\end{array}$

$\begin{array}{lll}-0.11 & 0.00 & 0.00\end{array}$

$\begin{array}{lll}0.12 & 0.09 & 0.03\end{array}$

$\begin{array}{lll}-0.09 & 0.00 & 0.03\end{array}$

$\begin{array}{lll}-0.23 & -0.22 & 0.56\end{array}$

$\begin{array}{lll}0.23 & 0.06 & -0.16\end{array}$

$\begin{array}{lll}-0.03 & -0.14 & -0.05\end{array}$

$\begin{array}{lll}0.06 & 0.04 & 0.01\end{array}$

$\begin{array}{lll}-0.10 & -0.06 & 0.15\end{array}$

$\begin{array}{lll}0.15 & 0.23 & -0.57\end{array}$

18

A

371.0632

27.4005

2.2228

21.1884

$\begin{array}{llll}Z & X & Y\end{array}$

$\begin{array}{lll}0.04 & 0.63 & 0.24\end{array}$

$\begin{array}{lll}0.13 & 0.13 & 0.05\end{array}$

$\begin{array}{llll}0.05 & -0.01 & 0.00\end{array}$

$\begin{array}{lll}0.00 & 0.00 & -0.01\end{array}$

$\begin{array}{llll}-0.01 & 0.01 & -0.03\end{array}$

$\begin{array}{llll}0.14 & -0.21 & -0.08\end{array}$

$\begin{array}{lll}-0.34 & -0.53 & -0.20\end{array}$

$\begin{array}{lll}-0.02 & -0.01 & 0.02\end{array}$

$\begin{array}{lll}0.03 & -0.01 & 0.02\end{array}$

21

A

426.8879

27.4926

2.9518

4.4005

$\begin{array}{llllll}\mathrm{Z} & \mathrm{X} & \mathrm{Y} & \mathrm{Z}\end{array}$

$\begin{array}{lll}0.00 & 0.00 & 0.00\end{array}$

$\begin{array}{lll}0.00 & 0.00 & 0.00\end{array}$

$\begin{array}{lll}0.05 & 0.00 & -0.02\end{array}$

$\begin{array}{lll}-0.12 & 0.03 & -0.05\end{array}$

$\begin{array}{lll}0.06 & 0.13 & -0.32\end{array}$

$\begin{array}{lll}0.03 & -0.01 & 0.00\end{array}$

$\begin{array}{lll}-0.03 & 0.01 & 0.01\end{array}$

$\begin{array}{lll}0.58 & -0.19 & 0.39\end{array}$

$\begin{array}{lll}-0.57 & 0.04 & -0.01\end{array}$

Al3SiNaAl3Si-s-s-90-Al-Al-b36311+gx.frq9.6 


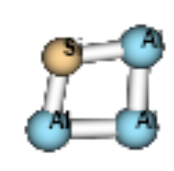

:

$\begin{array}{ccrcc}13 & 0 & -5.503986 & 0.969928 & 0.894036 \\ 13 & 0 & -2.964142 & 0.830378 & 0.800009 \\ 13 & 0 & 5.504051 & 0.969500 & -0.894394 \\ 13 & 0 & 2.964187 & 0.829948 & -0.800456 \\ 11 & 0 & 0.000002 & -0.036522 & -0.000139 \\ 13 & 0 & -2.885534 & -1.024276 & -1.040046 \\ 13 & 0 & 2.885471 & -1.023837 & 1.040475 \\ 14 & 0 & -5.204016 & -0.706250 & -0.765633 \\ 14 & 0 & 5.203971 & -0.705864 & 0.766092\end{array}$

State $=1-A \backslash H F=-2196.076951$

1

A

Frequencies -- $\quad 9.5503$

Red. masses -- 27.1140

Frc consts -- 0.0015

IR Inten $\quad--\quad 0.0079$

Atom AN $\mathrm{X} \quad \mathrm{Y}$

$\begin{array}{llll}1 & 13 & -0.02 & -0.30\end{array}$

$\begin{array}{llll}2 & 13 & -0.02 & -0.21\end{array}$

$3 \quad 13$

$4 \quad 13$

$\begin{array}{ll}0.02 & -0.30\end{array}$

$\begin{array}{llll}0.02 & -0.21 & -0.21\end{array}$

$\begin{array}{lll}0.02 & -0.21 & -0.21\end{array}$

$5 \quad 11$

$\begin{array}{lll}0.00 & 0.06 & 0.00\end{array}$

$\begin{array}{lll}0.02 & 0.30 & -0.30\end{array}$

$6 \quad 13$

$\begin{array}{lll}7 & 13\end{array}$

$\begin{array}{ll}8 & 14\end{array}$

$-0.02$

$0.30 \quad 0.30$

0.02

$\begin{array}{ll}0.17 & -0.21\end{array}$

$0.17 \quad 0.21$

$\begin{array}{ll}9 & 14\end{array}$

$-0.02$

\section{4}

A
2

A

18.6124

26.0369

0.0053

2.2176

X Y

$\begin{array}{lll}-0.14 & 0.06 & -0.31\end{array}$

$\begin{array}{lll}-0.13 & -0.10 & 0.22\end{array}$

$\begin{array}{lll}-0.14 & -0.06 & -0.31\end{array}$

$\begin{array}{lll}-0.13 & 0.09 & 0.22\end{array}$

$\begin{array}{lll}0.00 & 0.00 & 0.54\end{array}$

$\begin{array}{lll}0.15 & -0.03 & 0.16\end{array}$

$\begin{array}{lll}0.15 & 0.02 & 0.16\end{array}$

$\begin{array}{llll}0.11 & 0.10 & -0.30\end{array}$

$\begin{array}{llll}0.11 & -0.09 & -0.30\end{array}$

5

A

47.4502

26.9609

0.0358

0.5456

X Y
3

A

18.7203

26.0727

0.0054

2.2425

$\begin{array}{lllll}\mathrm{Z} & \mathrm{X} & \mathrm{Y}\end{array}$

$\begin{array}{lll}-0.16 & -0.28 & 0.03\end{array}$

$\begin{array}{lll}-0.13 & 0.24 & -0.11\end{array}$

$\begin{array}{llll}0.15 & -0.28 & -0.03\end{array}$

$\begin{array}{lll}0.13 & 0.24 & 0.12\end{array}$

$\begin{array}{lll}0.00 & 0.54 & 0.00\end{array}$

$\begin{array}{lll}0.15 & 0.14 & 0.01\end{array}$

$\begin{array}{lll}-0.15 & 0.14 & 0.00\end{array}$

$\begin{array}{lll}0.10 & -0.32 & 0.11\end{array}$

$\begin{array}{lll}-0.10 & -0.32 & -0.12\end{array}$

6

A

Frequencies -- 47.4055

Red. masses -- 26.9554

Frc consts -- 0.0357

IR Inten $\quad$-- 0.3782

Atom AN $\quad \mathrm{X} \quad \mathrm{Y} \quad \mathrm{Z}$

$\begin{array}{lllll}1 & 13 & -0.21 & 0.00 & -0.19\end{array}$

0.16

$0.19 \quad-0.01$

$\mathrm{Z}-\mathrm{X}$

61.5740

27.0616

0.0605

0.0938

$\mathrm{X} \quad \mathrm{Y}$

$\begin{array}{lll}0.34 & 0.02 & -0.02\end{array}$

$\begin{array}{lllll}2 & 13 & -0.17 & -0.07 & 0.44\end{array}$

$\begin{array}{lll}0.11 & -0.45 & 0.10\end{array}$

$\begin{array}{lll}0.31 & -0.07 & 0.05\end{array}$

$\begin{array}{lllll}3 & 13 & 0.20 & 0.01 & 0.19\end{array}$

$\begin{array}{lll}0.17 & -0.19 & 0.00\end{array}$

$\begin{array}{lll}-0.34 & 0.02 & 0.02\end{array}$ 


\begin{tabular}{ccccccccccc}
4 & 13 & 0.16 & -0.10 & -0.45 & 0.12 & 0.44 & 0.07 & -0.31 & -0.07 & -0.05 \\
5 & 11 & 0.00 & 0.15 & 0.00 & -0.02 & 0.00 & -0.15 & 0.00 & 0.22 & 0.00 \\
6 & 13 & 0.11 & 0.00 & 0.37 & -0.16 & -0.38 & 0.02 & 0.35 & -0.06 & 0.02 \\
7 & 13 & -0.10 & -0.02 & -0.37 & -0.16 & 0.37 & 0.00 & -0.35 & -0.06 & -0.02 \\
8 & 14 & 0.07 & 0.02 & -0.16 & -0.10 & 0.16 & -0.03 & 0.36 & 0.02 & 0.00 \\
9 & 14 & -0.06 & 0.03 & 0.16 & -0.11 & -0.16 & -0.02 & -0.36 & 0.02 & 0.00 \\
& & & 7 & & \multicolumn{7}{c}{8} & & & & 9
\end{tabular}

A

A

A

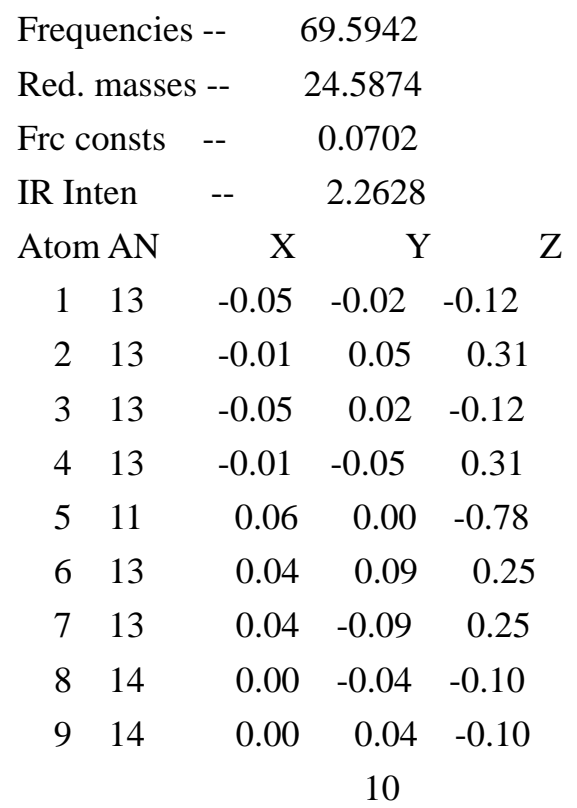

A

Frequencies -- 111.4165

Red. masses -- 27.2786

Frc consts -- 0.1995

IR Inten --- 1.5008

$\begin{array}{lllll}\text { Atom AN } & X & Y & \text { Z }\end{array}$

$\begin{array}{lllll}1 & 13 & -0.01 & -0.24 & 0.25\end{array}$

$\begin{array}{lllll}2 & 13 & 0.00 & 0.21 & -0.21\end{array}$

$\begin{array}{llllll}3 & 13 & 0.01 & -0.24 & -0.24\end{array}$

$\begin{array}{lllll}4 & 13 & 0.00 & 0.20 & 0.21\end{array}$

$\begin{array}{lllll}5 & 11 & 0.00 & 0.04 & 0.00\end{array}$

$\begin{array}{lllll}6 & 13 & 0.00 & -0.27 & 0.27\end{array}$

$\begin{array}{llllll}7 & 13 & 0.00 & -0.27 & -0.26\end{array}$

$\begin{array}{lllll}8 & 14 & 0.01 & 0.28 & -0.28\end{array}$

$\begin{array}{lllll}9 & 14 & -0.01 & 0.27 & 0.27\end{array}$

13

A

Frequencies -- 179.5158

Red. masses -- 23.5104

Frc consts -- 0.4464

IR Inten -- $\quad 107.2346$
70.7322

24.7458

0.0729

0.5320
110.7388

27.2824

0.1971

1.5469

$\begin{array}{llllll}\mathrm{Z} & \mathrm{X} & \mathrm{Y} & \mathrm{Z}\end{array}$

$\begin{array}{lll}0.00 & -0.24 & 0.24\end{array}$

$\begin{array}{lll}0.01 & 0.20 & -0.20\end{array}$

$\begin{array}{lll}0.00 & 0.25 & 0.25\end{array}$

$\begin{array}{lll}0.01 & -0.21 & -0.20\end{array}$

$\begin{array}{lll}0.00 & 0.00 & -0.04\end{array}$

$\begin{array}{lll}-0.01 & -0.26 & 0.27\end{array}$

$\begin{array}{lll}-0.01 & 0.27 & 0.27\end{array}$

$\begin{array}{lll}0.00 & 0.27 & -0.28\end{array}$

$\begin{array}{lll}0.00 & -0.28 & -0.29\end{array}$

12

A

A

151.0738

27.0383

0.3636

5.6880

36.0462

$\begin{array}{llllll}\mathrm{Z} & \mathrm{X} & \mathrm{Y} & \mathrm{Z}\end{array}$

$\begin{array}{llllll}0.28 & -0.13 & -0.12 & -0.31 & 0.13 & 0.13\end{array}$

$\begin{array}{llllll}0.25 & 0.11 & 0.10 & -0.27 & -0.11 & -0.13\end{array}$

$\begin{array}{llllll}0.28 & 0.13 & -0.12 & 0.31 & 0.13 & -0.13\end{array}$

$\begin{array}{llllll}0.26 & -0.11 & 0.10 & 0.27 & -0.11 & 0.13\end{array}$

$\begin{array}{llllll}0.15 & 0.00 & 0.27 & 0.00 & -0.26 & 0.00\end{array}$

$\begin{array}{llllll}-0.25 & 0.12 & 0.09 & 0.24 & -0.09 & -0.11\end{array}$

$\begin{array}{llllll}-0.25 & -0.12 & 0.09 & -0.24 & -0.09 & 0.11\end{array}$

$\begin{array}{llllll}-0.34 & -0.18 & -0.17 & 0.32 & 0.17 & 0.18\end{array}$

$\begin{array}{llllll}-0.34 & 0.18 & -0.17 & -0.32 & 0.17 & -0.18\end{array}$

14

A

15

A

266.1969

27.0050

1.1275

0.2051

266.2146

27.0058

1.1276

0.1964 


\begin{tabular}{|c|c|c|c|c|c|c|c|c|c|c|}
\hline \multicolumn{2}{|c|}{ Atom AN } & \multirow{2}{*}{\multicolumn{2}{|c|}{$\begin{array}{c}\mathrm{Y} \\
0.04\end{array}$}} & \multicolumn{2}{|c|}{ Z } & $\mathrm{X}$ & $\mathrm{Y}$ & Z & $\mathrm{X}$ & $\mathrm{Y}$ \\
\hline 1 & 13 & & & 0.03 & -0.01 & 0.00 & 0.00 & -0.23 & -0.12 & -0.12 \\
\hline 2 & 13 & -0.08 & -0.06 & -0.06 & 0.01 & 0.00 & 0.00 & 0.24 & 0.44 & 0.44 \\
\hline 3 & 13 & -0.19 & -0.04 & 0.03 & -0.23 & 0.12 & -0.12 & -0.01 & 0.00 & 0.00 \\
\hline 4 & 13 & -0.08 & 0.06 & -0.06 & 0.24 & -0.44 & 0.44 & 0.00 & 0.01 & -0.01 \\
\hline 5 & 11 & 0.93 & 0.00 & 0.01 & 0.05 & -0.02 & 0.02 & 0.05 & 0.02 & 0.02 \\
\hline 6 & 13 & -0.04 & 0.01 & 0.01 & 0.00 & 0.00 & -0.01 & -0.16 & -0.45 & -0.45 \\
\hline 7 & 13 & -0.04 & -0.01 & 0.01 & -0.16 & 0.45 & -0.45 & 0.00 & -0.01 & 0.01 \\
\hline 8 & 14 & -0.09 & 0.02 & 0.02 & 0.00 & 0.00 & 0.00 & 0.11 & 0.11 & 0.11 \\
\hline \multirow[t]{3}{*}{9} & 14 & -0.09 & -0.02 & 0.02 & 0.11 & -0.11 & 0.12 & -0.01 & 0.00 & 0.00 \\
\hline & & \multicolumn{3}{|c|}{16} & \multicolumn{3}{|c|}{17} & & \multicolumn{2}{|r|}{18} \\
\hline & & & A & & \multicolumn{3}{|c|}{ A } & & \multicolumn{2}{|r|}{ A } \\
\hline \multicolumn{2}{|c|}{ Frequencies -- } & \multicolumn{2}{|c|}{313.2520} & & \multicolumn{3}{|c|}{314.9998} & & \multicolumn{2}{|c|}{375.0610} \\
\hline \multicolumn{2}{|c|}{ Red. masses -- } & & 26.9873 & & \multicolumn{3}{|c|}{26.9133} & & \multicolumn{2}{|c|}{27.3411} \\
\hline \multicolumn{2}{|c|}{ Frc consts } & -- & 1.5603 & & \multicolumn{3}{|c|}{1.5734} & & 2.26 & 561 \\
\hline IR In & & -- & 2.0380 & & & 2.398 & & & 3.4 & 895 \\
\hline Atom & $\mathrm{AN}$ & $X$ & $\mathrm{Y}$ & Z & & $\mathrm{X}$ & $\mathrm{Y}$ & Z & $\mathrm{X}$ & $\mathrm{Y}$ \\
\hline 1 & 13 & -0.42 & -0.01 & -0.01 & -0.41 & -0.01 & -0.02 & -0.13 & 0.30 & 0.29 \\
\hline 2 & 13 & 0.49 & -0.14 & -0.13 & 0.49 & -0.14 & -0.13 & 0.09 & 0.07 & 0.07 \\
\hline 3 & 13 & 0.42 & -0.01 & 0.01 & -0.40 & 0.01 & -0.02 & 0.13 & 0.30 & -0.30 \\
\hline 4 & 13 & -0.49 & -0.14 & 0.13 & 0.48 & 0.14 & -0.13 & -0.09 & 0.07 & -0.07 \\
\hline 5 & 11 & 0.00 & 0.03 & 0.00 & -0.14 & 0.00 & 0.03 & 0.00 & 0.00 & 0.00 \\
\hline 6 & 13 & -0.10 & 0.13 & 0.12 & -0.10 & 0.13 & 0.13 & 0.29 & -0.11 & -0.10 \\
\hline 7 & 13 & 0.10 & 0.13 & -0.13 & -0.10 & -0.13 & 0.13 & -0.29 & -0.11 & 0.10 \\
\hline 8 & 14 & 0.07 & 0.01 & 0.01 & 0.08 & 0.01 & 0.01 & -0.22 & -0.25 & -0.26 \\
\hline 9 & 14 & -0.07 & 0.01 & -0.01 & 0.08 & -0.01 & 0.01 & 0.22 & -0.26 & 0.26 \\
\hline & & & 19 & & & 20 & & & & 21 \\
\hline & & & A & & & & A & & & A \\
\hline Frequ & Iencies & & 75.6103 & & & 427.5234 & & & 427.63 & \\
\hline Red. & masses & & 27.3238 & & & 27.504 & & & $27.4 \varepsilon$ & 358 \\
\hline Frc C & onsts & -- & 2.2713 & & & $2.961 \mathrm{C}$ & & & 2.96 & 15 \\
\hline IR In & & -- & 3.5669 & & & 1.330 & & & $9.3^{\prime}$ & 771 \\
\hline Atom & $\mathrm{AN}$ & $\mathrm{X}$ & $\mathrm{Y}$ & $\mathrm{Z}$ & & X & $\mathrm{Y}$ & Z & $X$ & $\mathrm{Y}$ \\
\hline 1 & 13 & 0.13 & -0.31 & -0.30 & 0.05 & 0.17 & 0.17 & -0.05 & -0.18 & -0.17 \\
\hline 2 & 13 & -0.10 & -0.07 & -0.07 & -0.08 & 0.03 & 0.03 & 0.09 & -0.03 & -0.03 \\
\hline 3 & 13 & 0.13 & 0.30 & -0.30 & -0.05 & 0.18 & -0.18 & -0.05 & 0.17 & -0.17 \\
\hline 4 & 13 & -0.10 & 0.07 & -0.07 & 0.08 & 0.03 & -0.03 & 0.08 & 0.03 & -0.03 \\
\hline 5 & 11 & 0.07 & 0.00 & 0.00 & 0.00 & 0.01 & 0.00 & -0.06 & 0.00 & -0.02 \\
\hline 6 & 13 & -0.29 & 0.10 & 0.10 & -0.40 & 0.02 & 0.01 & 0.41 & -0.02 & -0.01 \\
\hline 7 & 13 & -0.28 & -0.10 & 0.10 & 0.41 & 0.02 & -0.01 & 0.40 & 0.02 & -0.01 \\
\hline 8 & 14 & 0.22 & 0.26 & 0.26 & 0.40 & -0.22 & -0.21 & -0.41 & 0.22 & 0.22 \\
\hline 9 & 14 & 0.21 & -0.26 & 0.26 & -0.41 & -0.23 & 0.22 & -0.40 & -0.22 & 0.21 \\
\hline
\end{tabular}




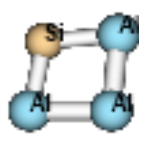

Na

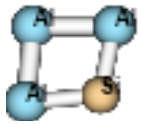

$\begin{array}{ll}11 & 0 \\ 13 & 0 \\ 13 & 0 \\ 13 & 0 \\ 13 & 0 \\ 13 & 0 \\ 14 & 0 \\ 13 & 0 \\ 14 & 0\end{array}$

$\begin{array}{rcc}0.000000 & 0.000000 & 0.000000 \\ 0.000000 & 3.196675 & 0.000000 \\ 0.000000 & -3.196675 & 0.000000 \\ 2.396773 & 2.156200 & 0.000000 \\ -2.396773 & -2.156200 & 0.000000 \\ -0.785623 & -5.618793 & 0.000000 \\ -2.866626 & -4.465506 & 0.000000 \\ 0.785623 & 5.618793 & 0.000000 \\ 2.866626 & 4.465506 & 0.000000\end{array}$

State $=1-A G \backslash H F=-2196.0766082$

1

$\mathrm{AU}$

Frequencies -- $\quad-4.4798$

Red. masses -- 27.1533

Frc consts -- 0.0003

IR Inten $\quad$-- $\quad 0.1098$

Atom AN $\quad \mathrm{X} \quad \mathrm{Y}$

$\begin{array}{lllll}1 & 11 & 0.00 & 0.00 & 0.00\end{array}$

213

$\begin{array}{lll}0.00 & 0.00 & 0.33\end{array}$

$\begin{array}{lll}0.00 & 0.00 & 0.33\end{array}$

$3 \quad 13$

$4 \quad 13$

0.00

$\begin{array}{ll}0.00 & -0.40\end{array}$

$5 \quad 13$

$\begin{array}{lll}0.00 & 0.00 & -0.40\end{array}$

$\begin{array}{lll}0.00 & 0.00 & 0.38\end{array}$

$\begin{array}{ll}6 & 13\end{array}$

$\begin{array}{ll}7 & 14\end{array}$

0.00

$\begin{array}{ll}0.00 & -0.29\end{array}$

$8 \quad 13$

$\begin{array}{lll}0.00 & 0.00 & 0.38\end{array}$

0.00

$\begin{array}{ll}0.00 & -0.29\end{array}$

4

BG

Frequencies -- $\quad 40.0803$

2

3

AU

18.9183

26.1131

0.0055

2.9303

X Y

$0.00 \quad 0.00$

0.53

0.00

0.00

0.29

$\begin{array}{lll}0.00 & 0.00 & 0.29\end{array}$

$0.00 \quad 0.00$

0.19

$0.00 \quad 0.00$

0.19

$\begin{array}{lll}0.00 & 0.00 & -0.34\end{array}$

$\begin{array}{llll}0.00 & 0.00 & -0.35\end{array}$

$\begin{array}{lll}0.00 & 0.00 & -0.34\end{array}$

$\begin{array}{llll}0.00 & 0.00 & -0.35\end{array}$

5

AG

47.8755

27.0143

0.0365

20.8701

25.8704

0.0066

1.2966

Red. masses -- 27.0916

Frc consts -- 0.0256

IR Inten -- $\quad 0.0000$

Atom AN $\mathrm{X} \quad \mathrm{Y}$

$\begin{array}{lllll}1 & 11 & 0.00 & 0.00 & 0.00\end{array}$

$\begin{array}{llllll}2 & 13 & 0.00 & 0.00 & -0.50\end{array}$

0.0000

Z

0.53

$\mathrm{X}$

$\begin{array}{lll}0.05 & -0.23 & 0.00\end{array}$

$\begin{array}{lll}0.05 & -0.23 & 0.00\end{array}$

$\begin{array}{lll}0.22 & 0.17 & 0.00\end{array}$

$\begin{array}{lll}0.22 & 0.17 & 0.00\end{array}$

$\begin{array}{lll}-0.34 & -0.10 & 0.00\end{array}$

$\begin{array}{lll}-0.15 & 0.24 & 0.00\end{array}$

$\begin{array}{lll}-0.34 & -0.10 & 0.00\end{array}$

$\begin{array}{lll}-0.15 & 0.24 & 0.00\end{array}$

6

AG

62.2465

27.2767

0.0623

X Y

0.0000

$\begin{array}{lll}\mathrm{X} & \mathrm{Y}\end{array}$

Z

$\begin{array}{lllll}3 & 13 & 0.00 & 0.00 & 0.50\end{array}$

$\begin{array}{lll}0.00 & 0.00 & 0.00\end{array}$

$\begin{array}{lll}0.00 & 0.00 & 0.00\end{array}$

$\begin{array}{lll}0.29 & -0.34 & 0.00\end{array}$

$\begin{array}{lll}-0.20 & -0.24 & 0.00\end{array}$

$4 \quad 13$

$\begin{array}{llll}0.00 & 0.00 & -0.37\end{array}$

$\begin{array}{lll}-0.29 & 0.34 & 0.00\end{array}$

$\begin{array}{lll}0.20 & 0.24 & 0.00\end{array}$

$\begin{array}{lll}0.42 & -0.03 & 0.00\end{array}$

$\begin{array}{lll}-0.22 & -0.33 & 0.00\end{array}$ 


\begin{tabular}{|c|c|c|c|c|c|c|c|c|c|c|c|}
\hline 5 & 13 & 0.00 & 0.00 & 0.37 & -0.42 & 0.03 & 0.00 & 0.22 & 0.33 & 0.00 & \\
\hline 6 & 13 & 0.00 & 0.00 & -0.23 & 0.25 & 0.20 & 0.00 & 0.08 & 0.30 & 0.00 & \\
\hline 7 & 14 & 0.00 & 0.00 & -0.24 & 0.09 & -0.10 & 0.00 & 0.12 & 0.36 & 0.00 & \\
\hline 8 & 13 & 0.00 & 0.00 & 0.23 & -0.25 & -0.20 & 0.00 & -0.08 & -0.30 & 0.00 & \\
\hline 9 & 14 & 0.00 & 0.00 & 0.24 & -0.09 & 0.10 & 0.00 & -0.12 & -0.36 & 0.00 & \\
\hline & & & 7 & & \multicolumn{4}{|c|}{8} & \multicolumn{2}{|r|}{9} & \\
\hline & & & $\mathrm{BU}$ & & \multicolumn{4}{|c|}{$\mathrm{AU}$} & \multicolumn{3}{|c|}{ BG } \\
\hline \multicolumn{2}{|c|}{ Frequencies - } & \multicolumn{2}{|c|}{72.4113} & & \multicolumn{3}{|c|}{77.6031} & & \multicolumn{2}{|c|}{111.5185} & \\
\hline \multicolumn{2}{|c|}{ Red. masses -- } & \multicolumn{2}{|c|}{24.7874} & & \multicolumn{3}{|c|}{24.2472} & & \multicolumn{2}{|c|}{ 27.2856 } & \\
\hline \multicolumn{2}{|c|}{ Frc consts } & -- & 0.0766 & & \multicolumn{3}{|c|}{0.0860} & & \multicolumn{2}{|c|}{0.1999} & \\
\hline \multicolumn{2}{|c|}{ IR Inten } & -- & 3.0179 & & \multicolumn{3}{|c|}{5.3572} & & \multicolumn{2}{|c|}{0.0000} & \\
\hline \multicolumn{2}{|c|}{ Atom AN } & $\mathrm{X}$ & $\mathrm{Y}$ & Z & & $\mathrm{X}$ & $\mathrm{Y}$ & Z & $\mathrm{X}$ & $\mathrm{Y}$ & Z \\
\hline 1 & 11 & 0.67 & -0.34 & 0.00 & 0.00 & 0.00 & 0.83 & 0.00 & 0.00 & 0.00 & \\
\hline 2 & 13 & -0.28 & 0.12 & 0.00 & 0.00 & 0.00 & -0.32 & 0.00 & 0.00 & 0.30 & \\
\hline 3 & 13 & -0.28 & 0.12 & 0.00 & 0.00 & 0.00 & -0.32 & 0.00 & 0.00 & -0.30 & \\
\hline 4 & 13 & -0.29 & 0.07 & 0.00 & 0.00 & 0.00 & -0.20 & 0.00 & 0.00 & -0.37 & \\
\hline 5 & 13 & -0.29 & 0.07 & 0.00 & 0.00 & 0.00 & -0.20 & 0.00 & 0.00 & 0.37 & \\
\hline 6 & 13 & 0.16 & 0.01 & 0.00 & 0.00 & 0.00 & 0.11 & 0.00 & 0.00 & 0.35 & \\
\hline 7 & 14 & 0.12 & -0.05 & 0.00 & 0.00 & 0.00 & 0.05 & 0.00 & 0.00 & -0.39 & \\
\hline 8 & 13 & 0.16 & 0.01 & 0.00 & 0.00 & 0.00 & 0.11 & 0.00 & 0.00 & -0.35 & \\
\hline 9 & 14 & 0.12 & -0.05 & 0.00 & 0.00 & 0.00 & 0.05 & 0.00 & 0.00 & 0.39 & \\
\hline & & & 10 & & & 11 & & & & 12 & \\
\hline & & & $\mathrm{AU}$ & & & A & & & & $\mathrm{BU}$ & \\
\hline Freq & iencies - & & 1.6308 & & & 145.6907 & & & 153.80 & & \\
\hline Red. & masses - & & 7.2528 & & & 27.320 & & & 26.73 & & \\
\hline Frc $c$ & onsts - & -- & 0.2001 & & & 0.3417 & & & 0.37 & & \\
\hline IR Ir & & -- & 2.4858 & & & 0.000 & & & 38.73 & & \\
\hline Aton & AN & X & $\mathrm{Y}$ & Z & & $\mathrm{X}$ & $\mathrm{Y}$ & $\mathrm{Z}$ & X & $\mathrm{Y}$ & Z \\
\hline 1 & 11 & 0.00 & 0.00 & -0.09 & 0.00 & 0.00 & 0.00 & -0.38 & -0.02 & 0.00 & \\
\hline 2 & 13 & 0.00 & 0.00 & -0.28 & 0.27 & 0.19 & 0.00 & -0.22 & -0.18 & 0.00 & \\
\hline 3 & 13 & 0.00 & 0.00 & -0.28 & -0.27 & -0.19 & 0.00 & -0.22 & -0.18 & 0.00 & \\
\hline 4 & 13 & 0.00 & 0.00 & 0.38 & 0.05 & -0.29 & 0.00 & -0.04 & 0.27 & 0.00 & \\
\hline 5 & 13 & 0.00 & 0.00 & 0.38 & -0.05 & 0.29 & 0.00 & -0.04 & 0.27 & 0.00 & \\
\hline 6 & 13 & 0.00 & 0.00 & 0.34 & 0.06 & -0.36 & 0.00 & 0.06 & -0.32 & 0.00 & \\
\hline 7 & 14 & 0.00 & 0.00 & -0.39 & 0.36 & 0.20 & 0.00 & 0.34 & 0.22 & 0.00 & \\
\hline 8 & 13 & 0.00 & 0.00 & 0.34 & -0.06 & 0.36 & 0.00 & 0.06 & -0.32 & 0.00 & \\
\hline 9 & 14 & 0.00 & 0.00 & -0.39 & -0.36 & -0.20 & 0.00 & 0.34 & 0.22 & 0.00 & \\
\hline & & & 13 & & & 14 & & & & 15 & \\
\hline & & & $\mathrm{BU}$ & & & B & & & & AG & \\
\hline Freq & iencies - & & 8.0079 & & & 266.3749 & & & 266.84 & & \\
\hline Red. & masses - & & 3.5154 & & & 26.990 & & & 27.02 & & \\
\hline Frc $c$ & onsts - & -- & 0.4390 & & & 1.128 & & & 1.13 & & \\
\hline IR In & & -- & 5.6626 & & & 0.548 & & & 0.00 & & \\
\hline Aton & $\mathrm{AN}$ & $\mathrm{X}$ & $\mathrm{Y}$ & Z & & $X$ & $\mathrm{Y}$ & $\mathrm{Z}$ & $X$ & $\mathrm{Y}$ & \\
\hline
\end{tabular}




$\begin{array}{ccccc}1 & 11 & 0.35 & 0.87 & 0.00 \\ 2 & 13 & -0.11 & -0.04 & 0.00 \\ 3 & 13 & -0.11 & -0.04 & 0.00 \\ 4 & 13 & -0.01 & -0.04 & 0.00 \\ 5 & 13 & -0.01 & -0.04 & 0.00 \\ 6 & 13 & -0.02 & -0.20 & 0.00 \\ 7 & 14 & 0.00 & -0.09 & 0.00 \\ 8 & 13 & -0.02 & -0.20 & 0.00 \\ 9 & 14 & 0.00 & -0.09 & 0.00 \\ & & & 16 & \end{array}$

AG

Frequencies -- $\quad 312.7291$

Red. masses -- 26.9908

Frc consts -- 1.5553

IR Inten -- 0.0000

Atom AN $\mathrm{X} \quad \mathrm{Y}$ $\begin{array}{lll}0.06 & 0.05 & 0.00\end{array}$

$\begin{array}{lll}0.47 & 0.00 & 0.00\end{array}$

$\begin{array}{lll}0.47 & 0.00 & 0.00\end{array}$

$\begin{array}{lll}-0.46 & 0.06 & 0.00\end{array}$

$\begin{array}{lll}-0.46 & 0.06 & 0.00\end{array}$

$\begin{array}{lll}-0.18 & -0.11 \quad 0.00\end{array}$

$\begin{array}{lll}0.13 & 0.03 & 0.00\end{array}$

$\begin{array}{lll}-0.18 & -0.11 & 0.00\end{array}$

$\begin{array}{lll}0.13 & 0.03 & 0.00\end{array}$

17

BU

314.7227

26.9135

1.5706

3.7951

X Y

111

$$
\text { Z }
$$

213

$\begin{array}{lll}0.00 & 0.00 & 0.00 \\ 0.00 & 0.53 & 0.00\end{array}$

$-0.01$

$-0.14$

0.00

$\begin{array}{lll}0.00 & 0.52 & 0.00\end{array}$

$\begin{array}{lll}0.00 & 0.52 & 0.00\end{array}$

$\begin{array}{lll}0.13 & -0.16 & 0.00\end{array}$

$\begin{array}{lll}0.13 & -0.16 & 0.00\end{array}$

$\begin{array}{lll}-0.17 & -0.37 & 0.00\end{array}$

$\begin{array}{lll}0.04 & 0.07 & 0.00\end{array}$

$\begin{array}{lll}-0.17 & -0.37 & 0.00\end{array}$

$\begin{array}{lll}0.04 & 0.07 & 0.00\end{array}$

20

AG

426.8173

27.5056

2.9523

0.0000

\section{BU}

Frequencies -- $\quad 374.8637$

Red. masses -- 27.3254

Frc consts -- $\quad 2.2624$

IR Inten --- 6.6973

$\begin{array}{lll}0.00 & 0.00 & 0.00\end{array}$

$\begin{array}{lll}0.47 & 0.00 & 0.00\end{array}$

$\begin{array}{lll}-0.47 & 0.00 & 0.00\end{array}$

$\begin{array}{lll}-0.46 & 0.07 & 0.00\end{array}$

$\begin{array}{lll}0.46 & -0.07 & 0.00\end{array}$

$\begin{array}{lll}0.18 & 0.10 & 0.00\end{array}$

$\begin{array}{lll}-0.14 & -0.03 & 0.00\end{array}$

$\begin{array}{lll}-0.18 & -0.10 & 0.00\end{array}$

$\begin{array}{lll}0.14 & 0.03 & 0.00\end{array}$

18

AG

374.6848

27.3406

2.2615

0.0000

$\begin{array}{llll}Z & X & Y\end{array}$

Z

$\begin{array}{lll}0.00 & 0.00 & 0.00\end{array}$

$\begin{array}{lll}0.13 & 0.05 & 0.00\end{array}$

$\begin{array}{lll}-0.13 & -0.05 & 0.00\end{array}$

$\begin{array}{lll}-0.03 & 0.32 & 0.00\end{array}$

$\begin{array}{lll}0.03 & -0.32 & 0.00\end{array}$

$\begin{array}{lll}-0.35 & 0.28 & 0.00\end{array}$

$\begin{array}{lll}0.42 & 0.07 & 0.00\end{array}$

$\begin{array}{lll}0.35 & -0.28 & 0.00\end{array}$

$\begin{array}{lll}-0.42 & -0.07 & 0.00\end{array}$

$\begin{array}{llll}\text { Atom AN } & \mathrm{X} & \mathrm{Y} & \mathrm{Z}\end{array}$

$\begin{array}{lllll}1 & 11 & -0.03 & -0.06 & 0.00\end{array}$

$\mathrm{X} \quad \mathrm{Y}$

$\begin{array}{lll}0.00 & 0.00 & 0.00\end{array}$

$-0.04$

21

BU

427.0547

27.4848

2.9533

10.8546

$\begin{array}{lllllllllll}2 & 13 & 0.13 & 0.05 & 0.00 & -0.01 & 0.09 & 0.00 & -0.01 & 0.10 & 0.00\end{array}$

$\begin{array}{lllllllllll}3 & 13 & 0.13 & 0.05 & 0.00 & 0.01 & -0.09 & 0.00 & -0.01 & 0.10 & 0.00\end{array}$

$\begin{array}{lllllllllll}4 & 13 & -0.03 & 0.31 & 0.00 & 0.13 & 0.38 & 0.00 & 0.13 & 0.39 & 0.00\end{array}$

$\begin{array}{lllllllllll}5 & 13 & -0.03 & 0.31 & 0.00 & -0.13 & -0.38 & 0.00 & 0.13 & 0.39 & 0.00\end{array}$

$\begin{array}{lllllllllll}6 & 13 & 0.35 & -0.28 & 0.00 & 0.25 & -0.05 & 0.00 & -0.24 & 0.05 & 0.00\end{array}$

$\begin{array}{lllllllllll}7 & 14 & -0.42 & -0.06 & 0.00 & -0.14 & 0.49 & 0.00 & 0.13 & -0.49 & 0.00\end{array}$

$\begin{array}{lllllllllll}8 & 13 & 0.35 & -0.28 & 0.00 & -0.25 & 0.05 & 0.00 & -0.24 & 0.05 & 0.00\end{array}$

$\begin{array}{lllllllllll}9 & 14 & -0.42 & -0.06 & 0.00 & 0.14 & -0.49 & 0.00 & 0.13 & -0.49 & 0.00\end{array}$

Al3SiNaAl3Si-s-s-to-c-c-0-C2h-b36311+gx.frq-4.9 


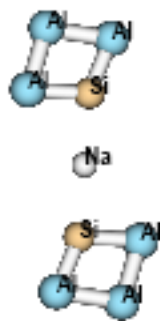

$\begin{array}{lrrrc}13 & 0 & -0.728649 & 5.129195 & 0.000000 \\ 13 & 0 & 2.401836 & 2.864883 & 0.000000 \\ 13 & 0 & 1.849598 & 5.372614 & 0.000000 \\ 11 & 0 & 0.000000 & 0.000000 & 0.000000 \\ 13 & 0 & -2.401836 & -2.864883 & 0.000000 \\ 13 & 0 & -1.849598 & -5.372614 & 0.000000 \\ 13 & 0 & 0.728649 & -5.129195 & 0.000000 \\ 14 & 0 & 0.000000 & 2.872928 & 0.000000 \\ 14 & 0 & 0.000000 & -2.872928 & 0.000000\end{array}$

State=1-AG $\backslash H F=-2196.0850662$

\section{1}

AU

Frequencies -- $\quad-4.9298$

Red. masses -- $\quad 26.9777$

Frc consts -- $\quad 0.0004$

IR Inten $\quad$-- $\quad 0.1339$

Atom AN $\mathrm{X} \quad \mathrm{Y}$

$\begin{array}{lllll}1 & 13 & 0.00 & 0.00 & 0.46\end{array}$

$\begin{array}{lllll}2 & 13 & 0.00 & 0.00 & -0.44\end{array}$

$\begin{array}{lllll}3 & 13 & 0.00 & 0.00 & 0.23\end{array}$

$\begin{array}{lllll}4 & 11 & 0.00 & 0.00 & -0.14\end{array}$

$\begin{array}{lllll}5 & 13 & 0.00 & 0.00 & -0.44\end{array}$

$\begin{array}{lllll}6 & 13 & 0.00 & 0.00 & 0.23\end{array}$

$\begin{array}{lllll}7 & 13 & 0.00 & 0.00 & 0.46\end{array}$

$\begin{array}{llllll}8 & 14 & 0.00 & 0.00 & -0.19\end{array}$

$\begin{array}{lllll}9 & 14 & 0.00 & 0.00 & -0.19\end{array}$

4

BG
2

$\mathrm{AU}$

3.7352

26.9505

0.0002

0.0168

X Y

$\begin{array}{lll}0.00 & 0.00 & -0.21\end{array}$

$\begin{array}{lll}0.00 & 0.00 & 0.24\end{array}$

$\begin{array}{lll}0.00 & 0.00 & 0.47\end{array}$

$\begin{array}{lll}0.00 & 0.00 & -0.28\end{array}$

$\begin{array}{lll}0.00 & 0.00 & 0.24\end{array}$

$\begin{array}{lll}0.00 & 0.00 & 0.47\end{array}$

$\begin{array}{lll}0.00 & 0.00 & -0.21\end{array}$

$\begin{array}{llll}0.00 & 0.00 & -0.37\end{array}$

0.00

0.00

\section{5}

AG

20.4946

27.2581

0.0067

0.0000

$\mathrm{X} \quad \mathrm{Y}$

$-0.05$

0.37

$-0.09$

$\begin{array}{ll}-0.16 & 0.00\end{array}$

$0.35 \quad 0.00$

$\begin{array}{ll}0.26 & 0.00\end{array}$

$\begin{array}{lll}0.00 & 0.00 & 0.00\end{array}$

$\begin{array}{lll}-0.37 & -0.35 & 0.00\end{array}$

\section{3}

BU

14.0059

26.8020

0.0031

0.2985

$\begin{array}{lllll}Z & X & Y & \text { Z }\end{array}$

$\begin{array}{lll}0.24 & 0.29 & 0.00\end{array}$

$\begin{array}{lll}-0.21 & -0.30 & 0.00\end{array}$

$\begin{array}{lll}0.29 & -0.19 & 0.00\end{array}$

$\begin{array}{lll}-0.25 & 0.13 & 0.00\end{array}$

$\begin{array}{lll}-0.21 & -0.30 & 0.00\end{array}$

$\begin{array}{lll}0.29 & -0.19 & 0.00\end{array}$

$\begin{array}{lll}0.24 & 0.29 & 0.00\end{array}$

$\begin{array}{lll}-0.21 & 0.14 & 0.00\end{array}$

$\begin{array}{lll}-0.21 & 0.14 & 0.00\end{array}$

6

BU

48.2959

23.6080

0.0324

8.3659

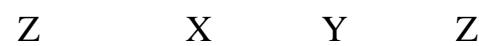

$\begin{array}{lll}-0.02 & 0.05 & 0.00\end{array}$

$\begin{array}{lll}-0.18 & -0.04 & 0.00\end{array}$

$\begin{array}{lll}-0.01 & -0.02 & 0.00\end{array}$

$\begin{array}{lll}0.93 & -0.03 & 0.00\end{array}$

$\begin{array}{lll}-0.18 & -0.04 & 0.00\end{array}$ 


$\begin{array}{ccccccccccc}6 & 13 & 0.00 & 0.00 & 0.38 & 0.09 & -0.26 & 0.00 & -0.01 & -0.02 & 0.00 \\ 7 & 13 & 0.00 & 0.00 & 0.06 & 0.05 & 0.16 & 0.00 & -0.02 & 0.05 & 0.00 \\ 8 & 14 & 0.00 & 0.00 & 0.53 & 0.37 & -0.03 & 0.00 & -0.18 & 0.01 & 0.00 \\ 9 & 14 & 0.00 & 0.00 & -0.53 & -0.37 & 0.03 & 0.00 & -0.18 & 0.01 & 0.00\end{array}$

AU AG

BG

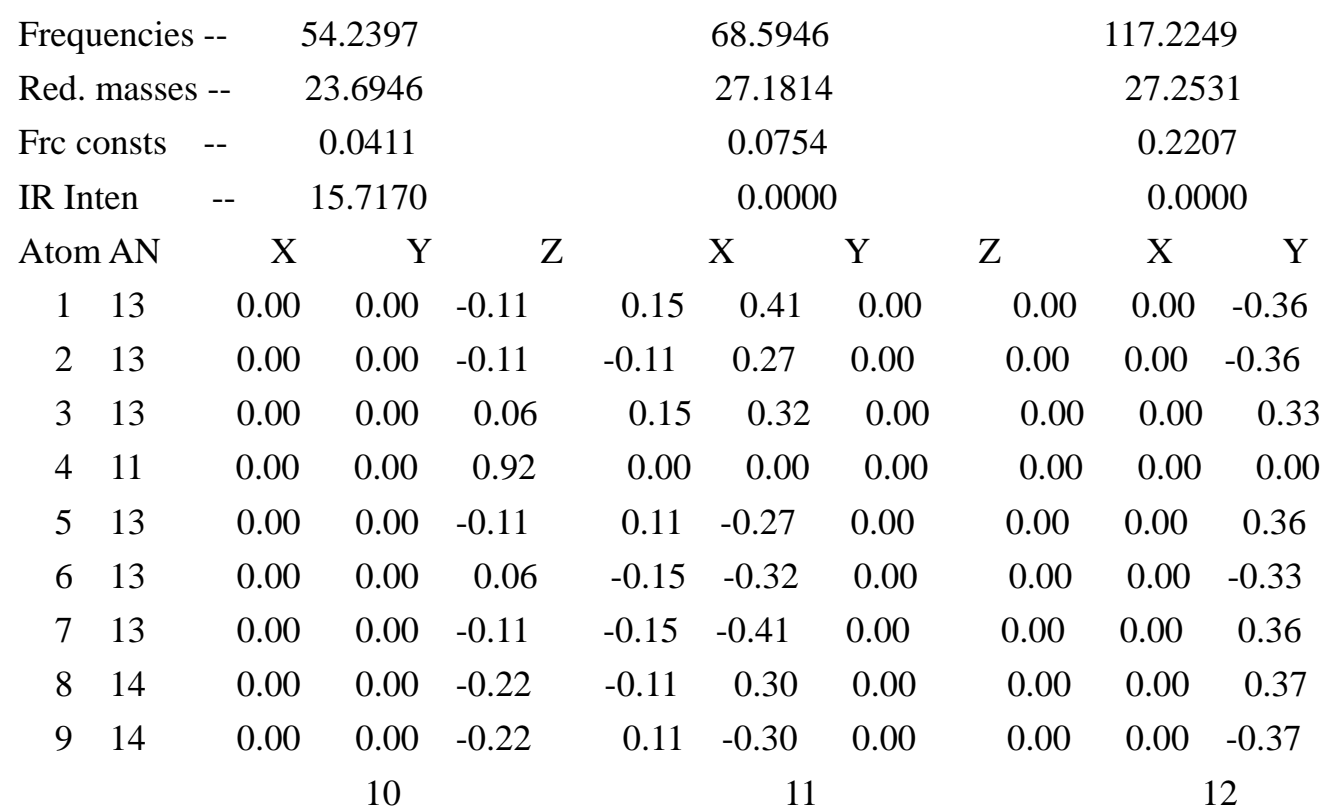

AU BU

Frequencies -- $\quad 117.8386$

144.2680

AG

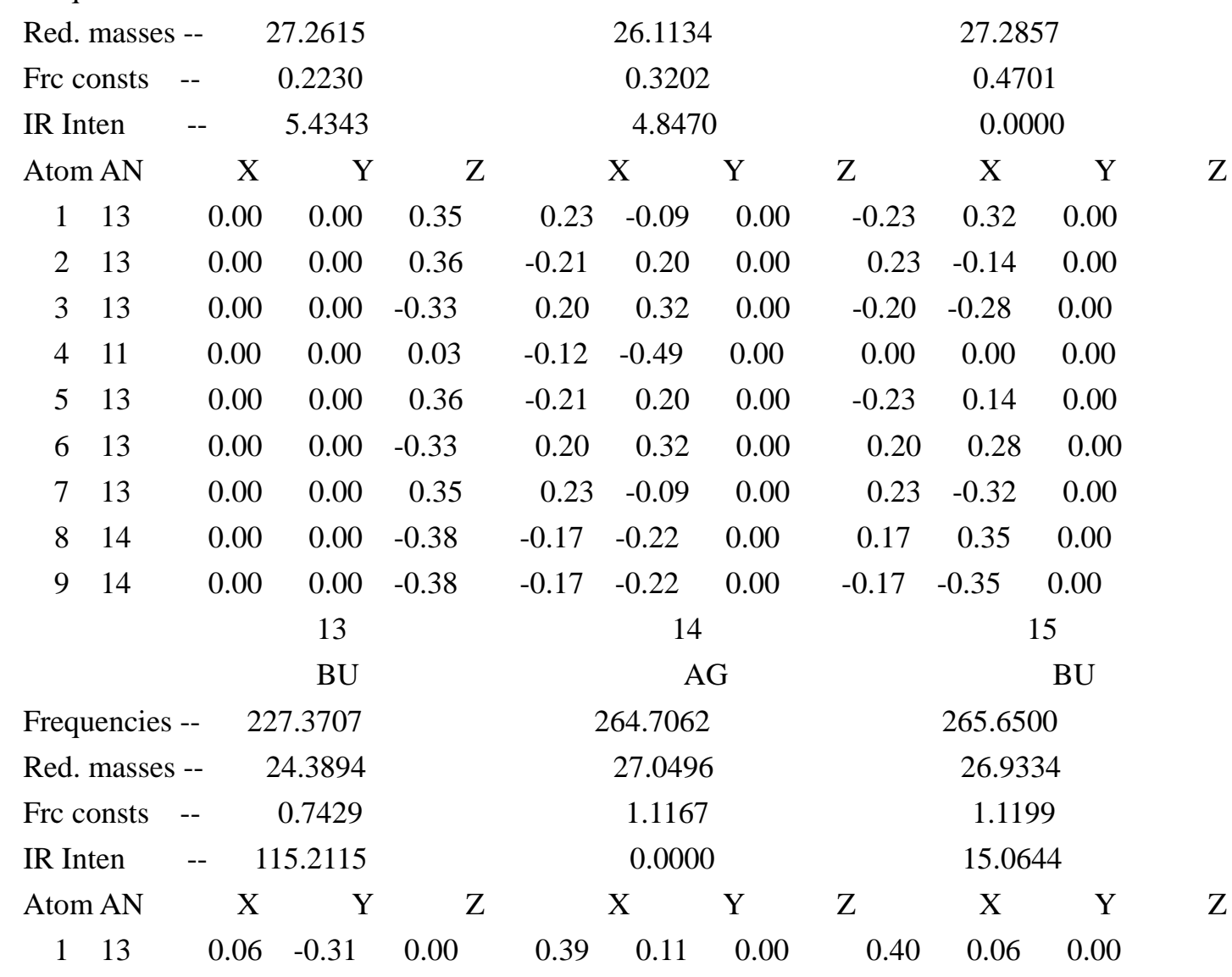




\begin{tabular}{|c|c|c|c|c|c|c|c|c|c|c|}
\hline 2 & 13 & -0.12 & -0.03 & 0.00 & 0.07 & 0.31 & 0.00 & 0.05 & 0.31 & 0.00 \\
\hline 3 & 13 & 0.12 & 0.13 & 0.00 & -0.32 & -0.31 & 0.00 & -0.31 & -0.30 & 0.00 \\
\hline 4 & 11 & 0.00 & 0.81 & 0.00 & 0.00 & 0.00 & 0.00 & -0.01 & 0.18 & 0.00 \\
\hline 5 & 13 & -0.12 & -0.03 & 0.00 & -0.07 & -0.31 & 0.00 & 0.05 & 0.31 & 0.00 \\
\hline 6 & 13 & 0.12 & 0.13 & 0.00 & 0.32 & 0.31 & 0.00 & -0.31 & -0.30 & 0.00 \\
\hline 7 & 13 & 0.06 & -0.31 & 0.00 & -0.39 & -0.11 & 0.00 & 0.40 & 0.06 & 0.00 \\
\hline 8 & 14 & -0.05 & -0.14 & 0.00 & -0.13 & -0.13 & 0.00 & -0.13 & -0.14 & 0.00 \\
\hline \multirow[t]{3}{*}{9} & 14 & -0.05 & -0.14 & 0.00 & 0.13 & 0.13 & 0.00 & -0.13 & -0.14 & 0.00 \\
\hline & & & 16 & & \multicolumn{3}{|c|}{17} & \multicolumn{3}{|c|}{18} \\
\hline & & & BU & & \multicolumn{3}{|c|}{ AG } & \multicolumn{3}{|r|}{ AG } \\
\hline \multicolumn{2}{|c|}{ Frequencies -- } & \multicolumn{2}{|c|}{305.7217} & & \multicolumn{3}{|c|}{305.7705} & \multicolumn{3}{|c|}{371.0313} \\
\hline \multicolumn{2}{|c|}{ Red. masses - } & \multicolumn{2}{|c|}{26.9838} & & \multicolumn{3}{|c|}{26.9876} & \multicolumn{3}{|c|}{27.4105} \\
\hline \multicolumn{2}{|c|}{ Frc consts } & -- & 1.4860 & & \multicolumn{3}{|c|}{1.4866} & \multicolumn{3}{|c|}{2.2232} \\
\hline \multicolumn{2}{|c|}{ IR Inten } & -- & 7.0234 & & \multicolumn{3}{|c|}{0.0000} & \multicolumn{3}{|c|}{0.0000} \\
\hline \multicolumn{2}{|c|}{ Atom AN } & $\mathrm{X}$ & $\mathrm{Y}$ & Z & \multicolumn{2}{|r|}{$\mathrm{X}$} & $\mathrm{Y}$ & \multirow{2}{*}{$\begin{array}{l}\mathrm{Z} \\
-0.15\end{array}$} & $\mathrm{X}$ & $\mathrm{Y}$ \\
\hline 1 & 13 & -0.28 & 0.00 & 0.00 & -0.29 & 0.00 & 0.00 & & 0.13 & 0.00 \\
\hline 2 & 13 & -0.09 & 0.39 & 0.00 & -0.09 & 0.39 & 0.00 & 0.48 & -0.04 & 0.00 \\
\hline 3 & 13 & 0.37 & -0.35 & 0.00 & 0.37 & -0.35 & 0.00 & 0.11 & 0.07 & 0.00 \\
\hline 4 & 11 & -0.01 & 0.03 & 0.00 & 0.00 & 0.00 & 0.00 & 0.00 & 0.00 & 0.00 \\
\hline 5 & 13 & -0.09 & 0.39 & 0.00 & 0.09 & -0.39 & 0.00 & -0.48 & 0.04 & 0.00 \\
\hline 6 & 13 & 0.37 & -0.35 & 0.00 & -0.37 & 0.35 & 0.00 & -0.11 & -0.07 & 0.00 \\
\hline 7 & 13 & -0.28 & 0.00 & 0.00 & 0.29 & 0.00 & 0.00 & 0.15 & -0.13 & 0.00 \\
\hline 8 & 14 & 0.02 & -0.05 & 0.00 & 0.02 & -0.05 & 0.00 & -0.42 & -0.19 & 0.00 \\
\hline 9 & 14 & 0.02 & -0.05 & 0.00 & -0.02 & 0.05 & 0.00 & 0.42 & 0.19 & 0.00 \\
\hline & & & 19 & & & 20 & & & & 21 \\
\hline & & & $\mathrm{BU}$ & & & A & G & & & $\mathrm{BU}$ \\
\hline Freq & uencies & & 71.1354 & & & 409.8495 & & & 412.850 & \\
\hline Red. & masses & & 27.3876 & & & 27.543 & & & 27.43 & \\
\hline Frc & onsts & -- & 2.2226 & & & 2.7259 & & & 2.755 & \\
\hline IR II & ten & -- & 41.8090 & & & 0.000 & & & 56.28 & \\
\hline Aton & $\mathrm{AN}$ & $\mathrm{X}$ & $\mathrm{Y}$ & Z & & $\mathrm{X}$ & $\mathrm{Y}$ & Z & $\mathrm{X}$ & $\mathrm{Y}$ \\
\hline 1 & 13 & -0.14 & 0.11 & 0.00 & 0.10 & -0.43 & 0.00 & -0.09 & 0.42 & 0.00 \\
\hline 2 & 13 & 0.48 & -0.04 & 0.00 & 0.12 & 0.07 & 0.00 & -0.11 & -0.07 & 0.00 \\
\hline 3 & 13 & 0.11 & 0.07 & 0.00 & 0.04 & -0.08 & 0.00 & -0.04 & 0.08 & 0.00 \\
\hline 4 & 11 & 0.00 & 0.08 & 0.00 & 0.00 & 0.00 & 0.00 & 0.01 & 0.16 & 0.00 \\
\hline 5 & 13 & 0.48 & -0.04 & 0.00 & -0.12 & -0.07 & 0.00 & -0.11 & -0.07 & 0.00 \\
\hline 6 & 13 & 0.11 & 0.07 & 0.00 & -0.04 & 0.08 & 0.00 & -0.04 & 0.08 & 0.00 \\
\hline 7 & 13 & -0.14 & 0.11 & 0.00 & -0.10 & 0.43 & 0.00 & -0.09 & 0.42 & 0.00 \\
\hline 8 & 14 & -0.43 & -0.18 & 0.00 & -0.25 & 0.47 & 0.00 & 0.23 & -0.48 & 0.00 \\
\hline 9 & 14 & -0.43 & -0.18 & 0.00 & 0.25 & -0.47 & 0.00 & 0.23 & -0.48 & 0.00 \\
\hline
\end{tabular}




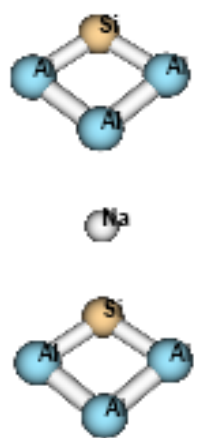

11

13

$\begin{array}{rrr}0.000000 & 0.000000 & -0.148665 \\ 0.000000 & 1.985534 & 4.507585 \\ 0.000000 & -1.985534 & 4.507585 \\ 0.000000 & 0.000000 & -6.055391 \\ 0.000000 & 1.950730 & -4.366754 \\ 0.000000 & -1.950730 & -4.366754 \\ 0.000000 & 0.000000 & -2.991374 \\ 0.000000 & 0.000000 & 2.865596 \\ 0.000000 & 0.000000 & 5.808591\end{array}$

State $=1-A 1 \backslash H F=-2196.075953$

B2

B2

A2

\begin{tabular}{|c|c|c|c|}
\hline \multicolumn{2}{|c|}{ Freauencies -- } & -18.7916 & \\
\hline \multicolumn{2}{|c|}{ Red. masses -- } & \multicolumn{2}{|c|}{27.0755} \\
\hline Frc consts & \multicolumn{3}{|c|}{0.0056} \\
\hline IR Inten & \multicolumn{3}{|c|}{0.0390} \\
\hline Atom AN & $\mathrm{X}$ & $\mathrm{Y}$ & 2 \\
\hline $\begin{array}{ll}1 & 11\end{array}$ & 0.00 & 0.04 & 0.00 \\
\hline 213 & 0.00 & -0.09 & -0.51 \\
\hline 313 & 0.00 & -0.09 & 0.51 \\
\hline $4 \quad 13$ & 0.00 & 0.00 & 0.00 \\
\hline $5 \quad 13$ & 0.00 & 0.11 & -0.12 \\
\hline $6 \quad 13$ & 0.00 & 0.11 & 0.12 \\
\hline $7 \quad 14$ & 0.00 & 0.20 & 0.00 \\
\hline $8 \quad 13$ & 0.00 & -0.55 & 0.00 \\
\hline $9 \quad 14$ & 0.00 & 0.24 & 0.00 \\
\hline & & & \\
\hline
\end{tabular}

$-11.5410$

$-3.7663$

27.1535

26.9815

0.0021

0.0002

0.0003

0.0000

B1

\begin{tabular}{ccc}
\multicolumn{2}{c}{$\mathrm{X}$} & $\mathrm{Y}$ \\
0.00 & -0.12 & 0.00 \\
0.00 & 0.08 & -0.17 \\
0.00 & 0.08 & 0.17 \\
0.00 & 0.39 & 0.00 \\
0.00 & -0.07 & 0.51 \\
0.00 & -0.07 & -0.51 \\
0.00 & -0.44 & 0.00 \\
0.00 & -0.06 & 0.00 \\
0.00 & 0.19 & 0.00 \\
& 5 &
\end{tabular}

B1

$\begin{array}{llll}\mathrm{Z} & \mathrm{X} & \mathrm{Y}\end{array}$

Z

\begin{tabular}{|c|c|c|c|c|c|c|c|c|c|}
\hline Frequencies & & 8.5984 & & \multicolumn{3}{|c|}{26.2282} & & \multicolumn{2}{|c|}{45.3760} \\
\hline Red. masses & & 26.8645 & & \multicolumn{3}{|c|}{26.7559} & & \multicolumn{2}{|c|}{23.4005} \\
\hline Frc consts & -- & 0.0012 & & \multicolumn{3}{|c|}{0.0108} & & \multicolumn{2}{|c|}{0.0284} \\
\hline IR Inten & -- & 0.0003 & & \multicolumn{3}{|c|}{2.3557} & & \multicolumn{2}{|c|}{6.1283} \\
\hline Atom AN & $\mathrm{X}$ & $\mathrm{Y}$ & $\mathrm{Z}$ & & $\mathrm{X}$ & $\mathrm{Y}$ & $\mathrm{Z}$ & $\mathrm{X}$ & $\mathrm{Y}$ \\
\hline $\begin{array}{ll}1 & 11\end{array}$ & -0.37 & 0.00 & 0.00 & -0.38 & 0.00 & 0.00 & 0.00 & 0.95 & 0.00 \\
\hline 13 & 0.09 & 0.00 & 0.00 & 0.02 & 0.00 & 0.00 & 0.00 & -0.08 & 0.0 \\
\hline 13 & 0.09 & 0.00 & 0.00 & 0.02 & 0.00 & 0.00 & 0.00 & -0.08 & -0.01 \\
\hline
\end{tabular}




$\begin{array}{rcccccccccc}4 & 13 & 0.64 & 0.00 & 0.00 & -0.16 & 0.00 & 0.00 & 0.00 & -0.06 & 0.00 \\ 5 & 13 & -0.01 & 0.00 & 0.00 & 0.12 & 0.00 & 0.00 & 0.00 & -0.12 & 0.05 \\ 6 & 13 & -0.01 & 0.00 & 0.00 & 0.12 & 0.00 & 0.00 & 0.00 & -0.12 & -0.05 \\ 7 & 14 & -0.59 & 0.00 & 0.00 & 0.38 & 0.00 & 0.00 & 0.00 & -0.17 & 0.00 \\ 8 & 13 & -0.14 & 0.00 & 0.00 & -0.66 & 0.00 & 0.00 & 0.00 & -0.10 & 0.00 \\ 9 & 14 & 0.26 & 0.00 & 0.00 & 0.47 & 0.00 & 0.00 & 0.00 & -0.08 & 0.00 \\ & & & 7 & & & 8 & & & & 9\end{array}$

A1 B1

B1

\begin{tabular}{|c|c|c|c|c|c|c|c|c|c|c|c|}
\hline \multicolumn{3}{|c|}{ Frequencies -- } & \multicolumn{2}{|c|}{60.0025} & & \multicolumn{3}{|c|}{62.3516} & \multicolumn{3}{|c|}{111.4350} \\
\hline \multicolumn{3}{|c|}{ Red. masses -- } & \multicolumn{2}{|c|}{27.1982} & & \multicolumn{3}{|c|}{24.2594} & \multicolumn{3}{|c|}{27.3063} \\
\hline \multicolumn{2}{|c|}{ Frc consts } & -- & \multicolumn{2}{|r|}{0.0577} & & \multicolumn{3}{|c|}{0.0556} & \multicolumn{3}{|c|}{0.1998} \\
\hline \multicolumn{2}{|c|}{ IR Inten } & -- & & 1.6163 & & \multicolumn{3}{|c|}{15.8739} & \multicolumn{3}{|c|}{3.4706} \\
\hline \multicolumn{2}{|c|}{ Atom AN } & & $\mathrm{X}$ & $\mathrm{Y}$ & Z & \multicolumn{2}{|r|}{$\mathrm{X}$} & $\mathrm{Y}$ & \multirow{2}{*}{$\begin{array}{l}\mathrm{Z} \\
-0.01\end{array}$} & \multirow{2}{*}{$\begin{array}{c}X \\
0.00\end{array}$} & \multirow{2}{*}{$\begin{array}{r}\mathrm{Y} \\
0.00\end{array}$} \\
\hline 1 & 11 & & 0.00 & 0.00 & 0.05 & 0.84 & 0.00 & 0.00 & & & \\
\hline 2 & 13 & & 0.00 & 0.03 & -0.37 & -0.07 & 0.00 & 0.00 & 0.51 & 0.00 & 0.00 \\
\hline 3 & 13 & & 0.00 & -0.03 & -0.37 & -0.07 & 0.00 & 0.00 & 0.51 & 0.00 & 0.00 \\
\hline 4 & 13 & & 0.00 & 0.00 & 0.40 & 0.07 & 0.00 & 0.00 & 0.02 & 0.00 & 0.00 \\
\hline 5 & 13 & & 0.00 & 0.05 & 0.36 & -0.09 & 0.00 & 0.00 & -0.02 & 0.00 & 0.00 \\
\hline 6 & 13 & & 0.00 & -0.05 & 0.36 & -0.09 & 0.00 & 0.00 & -0.02 & 0.00 & 0.00 \\
\hline 7 & 14 & & 0.00 & 0.00 & 0.26 & -0.19 & 0.00 & 0.00 & 0.01 & 0.00 & 0.00 \\
\hline 8 & 13 & & 0.00 & 0.00 & -0.27 & -0.45 & 0.00 & 0.00 & -0.40 & 0.00 & 0.00 \\
\hline \multirow{3}{*}{\multicolumn{2}{|c|}{14}} & & 0.00 & 0.00 & -0.40 & 0.18 & 0.00 & 0.00 & -0.57 & 0.00 & 0.00 \\
\hline & & \multicolumn{4}{|c|}{10} & \multicolumn{3}{|c|}{11} & \multicolumn{3}{|c|}{12} \\
\hline & & \multicolumn{4}{|c|}{ B1 } & \multicolumn{3}{|c|}{ A1 } & \multicolumn{3}{|c|}{ A1 } \\
\hline \multicolumn{3}{|c|}{ Frequencies -- } & \multicolumn{2}{|c|}{117.4451} & & \multicolumn{3}{|c|}{139.7296} & \multicolumn{3}{|c|}{179.5030} \\
\hline \multicolumn{3}{|c|}{ Red. masses -- } & \multicolumn{2}{|c|}{27.2421} & & & 25.649 & & & 27.27 & \\
\hline Frc c & onsts & -- & & 0.2214 & & & 0.2951 & & & 0.51 & \\
\hline IR In & & -- & & 3.7333 & & & 31.414 & & & 36.39 & \\
\hline Aton & AN & & $\mathrm{X}$ & $\mathrm{Y}$ & $\mathrm{Z}$ & & $\mathrm{X}$ & Y & Z & $\mathrm{X}$ & $\mathrm{Y}$ \\
\hline
\end{tabular}

\begin{tabular}{|c|c|c|c|c|c|c|c|c|c|}
\hline \multicolumn{2}{|c|}{ Atom AN } & $\mathrm{X}$ & $\mathrm{Y}$ & $\mathrm{Z}$ & \multicolumn{2}{|c|}{ X } & Y & Z & \multirow{2}{*}{$\begin{array}{c}X \\
0.00\end{array}$} \\
\hline 1 & 11 & 0.02 & 0.00 & 0.00 & 0.00 & 0.00 & 0.62 & 0.00 & \\
\hline 2 & 13 & 0.01 & 0.00 & 0.00 & 0.00 & 0.17 & -0.08 & 0.00 & 0.41 \\
\hline 3 & 13 & 0.01 & 0.00 & 0.00 & 0.00 & -0.17 & -0.08 & 0.00 & -0.41 \\
\hline 4 & 13 & -0.47 & 0.00 & 0.00 & 0.00 & 0.00 & -0.40 & 0.00 & 0.00 \\
\hline 5 & 13 & 0.51 & 0.00 & 0.00 & 0.00 & -0.28 & -0.08 & 0.00 & 0.24 \\
\hline 6 & 13 & 0.51 & 0.00 & 0.00 & 0.00 & 0.28 & -0.08 & 0.00 & -0.24 \\
\hline 7 & 14 & -0.51 & 0.00 & 0.00 & 0.00 & 0.00 & 0.30 & 0.00 & 0.00 \\
\hline 8 & 13 & -0.03 & 0.00 & 0.00 & 0.00 & 0.00 & 0.19 & 0.00 & 0.00 \\
\hline \multirow[t]{3}{*}{9} & 14 & -0.02 & 0.00 & 0.00 & 0.00 & 0.00 & -0.30 & 0.00 & 0.00 \\
\hline & & & 13 & & & 14 & & & 15 \\
\hline & & & A1 & & & A & & & $\mathrm{A} 1$ \\
\hline \multicolumn{2}{|c|}{ Frequencies -- } & & 218.8144 & & \multicolumn{3}{|c|}{265.8398} & & 287.0421 \\
\hline \multicolumn{3}{|c|}{ Red. masses -- } & 25.2332 & & \multicolumn{3}{|c|}{26.9876} & & 26.5490 \\
\hline \multicolumn{2}{|c|}{ Frc consts } & \multicolumn{2}{|r|}{0.7118} & & \multicolumn{3}{|c|}{1.1237} & & 1.2888 \\
\hline \multicolumn{2}{|c|}{ IR Inten } & \multicolumn{2}{|r|}{39.5558} & & \multicolumn{3}{|c|}{7.5160} & & 31.8346 \\
\hline
\end{tabular}




\begin{tabular}{|c|c|c|c|c|c|c|c|c|c|c|c|}
\hline \multicolumn{2}{|c|}{ Atom AN } & $\mathrm{X}$ & $\mathrm{Y}$ & $\mathrm{Z}$ & \multicolumn{2}{|r|}{$\mathrm{X}$} & $\mathrm{Y}$ & Z & $\mathrm{X}$ & $\mathrm{Y}$ & Z \\
\hline 1 & 11 & 0.00 & 0.00 & 0.68 & 0.00 & 0.00 & -0.14 & 0.00 & 0.00 & 0.35 & \\
\hline 2 & 13 & 0.00 & -0.19 & -0.21 & 0.00 & 0.03 & 0.10 & 0.00 & -0.02 & 0.37 & \\
\hline 3 & 13 & 0.00 & 0.19 & -0.21 & 0.00 & -0.03 & 0.10 & 0.00 & 0.02 & 0.37 & \\
\hline 4 & 13 & 0.00 & 0.00 & 0.30 & 0.00 & 0.00 & 0.60 & 0.00 & 0.00 & -0.08 & \\
\hline 5 & 13 & 0.00 & 0.22 & -0.23 & 0.00 & -0.32 & -0.38 & 0.00 & 0.11 & 0.02 & \\
\hline 6 & 13 & 0.00 & -0.22 & -0.23 & 0.00 & 0.32 & -0.38 & 0.00 & -0.11 & 0.02 & \\
\hline 7 & 14 & 0.00 & 0.00 & -0.22 & 0.00 & 0.00 & 0.28 & 0.00 & 0.00 & -0.05 & \\
\hline 8 & 13 & 0.00 & 0.00 & 0.06 & 0.00 & 0.00 & -0.15 & 0.00 & 0.00 & -0.72 & \\
\hline \multirow[t]{3}{*}{9} & 14 & 0.00 & 0.00 & 0.17 & 0.00 & 0.00 & -0.05 & 0.00 & 0.00 & -0.21 & \\
\hline & & & 16 & & & 17 & & & & 18 & \\
\hline & & & B2 & & & B2 & & & & A1 & \\
\hline \multicolumn{2}{|c|}{ Frequencies -- } & \multicolumn{2}{|c|}{291.0301} & & \multicolumn{3}{|c|}{303.3953} & & \multicolumn{2}{|c|}{371.8200} & \\
\hline \multicolumn{2}{|c|}{ Red. masses -- } & \multicolumn{2}{|c|}{26.9793} & & \multicolumn{3}{|c|}{26.9838} & & \multicolumn{2}{|c|}{27.2865} & \\
\hline \multicolumn{2}{|c|}{ Frc consts } & - & 1.3463 & & \multicolumn{3}{|c|}{1.4634} & & \multicolumn{2}{|c|}{2.2226} & \\
\hline \multicolumn{2}{|c|}{ IR Inten } & - & 1.4667 & & \multicolumn{3}{|c|}{3.7865} & & \multicolumn{2}{|c|}{6.7139} & \\
\hline \multicolumn{2}{|c|}{ Atom AN } & $\mathrm{X}$ & $\mathrm{Y}$ & Z & \multicolumn{2}{|r|}{$\mathrm{X}$} & $\mathrm{Y}$ & $\mathrm{Z}$ & $\mathrm{X}$ & $\mathrm{Y}$ & Z \\
\hline 1 & 11 & 0.00 & -0.02 & 0.00 & 0.00 & -0.01 & 0.00 & 0.00 & 0.00 & -0.06 & \\
\hline 2 & 13 & 0.00 & 0.38 & 0.32 & 0.00 & 0.00 & 0.00 & 0.00 & -0.51 & 0.16 & \\
\hline 3 & 13 & 0.00 & 0.38 & -0.32 & 0.00 & 0.00 & 0.00 & 0.00 & 0.51 & 0.16 & \\
\hline 4 & 13 & 0.00 & 0.00 & 0.00 & 0.00 & 0.72 & 0.00 & 0.00 & 0.00 & 0.02 & \\
\hline 5 & 13 & 0.00 & 0.00 & 0.00 & 0.00 & -0.38 & -0.30 & 0.00 & -0.05 & 0.02 & \\
\hline 6 & 13 & 0.00 & 0.00 & 0.00 & 0.00 & -0.38 & 0.30 & 0.00 & 0.05 & 0.02 & \\
\hline 7 & 14 & 0.00 & 0.00 & 0.00 & 0.00 & 0.05 & 0.00 & 0.00 & 0.00 & -0.07 & \\
\hline 8 & 13 & 0.00 & -0.72 & 0.00 & 0.00 & 0.00 & 0.00 & 0.00 & 0.00 & 0.32 & \\
\hline 9 & 14 & 0.00 & -0.01 & 0.00 & 0.00 & 0.00 & 0.00 & 0.00 & 0.00 & -0.56 & \\
\hline & & & 19 & & & 20 & & & & 21 & \\
\hline & & & A1 & & & $\mathrm{B} 2$ & & & & B2 & \\
\hline Freq & lencies - & & 7.0650 & & & 395.8625 & & & 423.64 & & \\
\hline Red. & masses . & & 7.3446 & & & 27.5239 & & & 27.55 & & \\
\hline Fre C & onsts & - & 2.4137 & & & 2.5413 & & & 2.913 & & \\
\hline IR In & & -- & 51.8245 & & & 7.3910 & & & 7.39 & & \\
\hline Aton & $\mathrm{AN}$ & $\mathrm{X}$ & $\mathrm{Y}$ & Z & & $\mathrm{X}$ & $\mathrm{Y}$ & $\mathrm{Z}$ & $\mathrm{X}$ & $\mathrm{Y}$ & Z \\
\hline 1 & 11 & 0.00 & 0.00 & -0.17 & 0.00 & -0.01 & 0.00 & 0.00 & 0.00 & 0.00 & \\
\hline 2 & 13 & 0.00 & -0.04 & 0.01 & 0.00 & 0.00 & 0.00 & 0.00 & 0.34 & -0.30 & \\
\hline 3 & 13 & 0.00 & 0.04 & 0.01 & 0.00 & 0.00 & 0.00 & 0.00 & 0.34 & 0.30 & \\
\hline 4 & 13 & 0.00 & 0.00 & -0.16 & 0.00 & 0.12 & 0.00 & 0.00 & 0.00 & 0.00 & \\
\hline 5 & 13 & 0.00 & 0.43 & -0.21 & 0.00 & 0.33 & -0.34 & 0.00 & 0.00 & 0.00 & \\
\hline 6 & 13 & 0.00 & -0.43 & -0.21 & 0.00 & 0.33 & 0.34 & 0.00 & 0.00 & 0.00 & \\
\hline 7 & 14 & 0.00 & 0.00 & 0.69 & 0.00 & -0.74 & 0.00 & 0.00 & 0.00 & 0.00 & \\
\hline 8 & 13 & 0.00 & 0.00 & 0.06 & 0.00 & 0.00 & 0.00 & 0.00 & 0.11 & 0.00 & \\
\hline 9 & 14 & 0.00 & 0.00 & -0.06 & 0.00 & 0.00 & 0.00 & 0.00 & -0.76 & 0.00 & \\
\hline
\end{tabular}




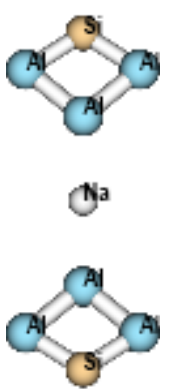

$\begin{array}{rrrrr}11 & 0 & 0.000000 & 0.000000 & 0.000000 \\ 13 & 0 & 0.000000 & 1.988968 & 4.639634 \\ 13 & 0 & 0.000000 & -1.988968 & 4.639634 \\ 13 & 0 & 0.000000 & 1.988968 & -4.639634 \\ 13 & 0 & 0.000000 & -1.988968 & -4.639634 \\ 13 & 0 & 0.000000 & 0.000000 & 3.002478 \\ 14 & 0 & 0.000000 & 0.000000 & 5.935564 \\ 13 & 0 & 0.000000 & 0.000000 & -3.002478 \\ 14 & 0 & 0.000000 & 0.000000 & -5.935564\end{array}$

State $=1-A G \backslash H F=-2196.0671887$

1

B3G
2

B2U

$-16.9608$

27.1288

0.0046

0.0835

IR Inten -- $\quad 0.0000$

Atom AN $\quad \mathrm{X} \quad \mathrm{Y}$

$\begin{array}{lllll}1 & 11 & 0.00 & 0.00 & 0.00\end{array}$

$\begin{array}{lllll}2 & 13 & 0.00 & 0.11 & 0.37\end{array}$

$\begin{array}{lllll}3 & 13 & 0.00 & 0.11 & -0.37\end{array}$

$\begin{array}{llllll}4 & 13 & 0.00 & -0.11 & 0.37\end{array}$

$\begin{array}{llllll}5 & 13 & 0.00 & -0.11 & -0.37\end{array}$

$\begin{array}{lllll}6 & 13 & 0.00 & 0.43 & 0.00\end{array}$

$\begin{array}{llllll}7 & 14 & 0.00 & -0.13 & 0.00\end{array}$

$\begin{array}{lllll}8 & 13 & 0.00 & -0.43 & 0.00\end{array}$

$9 \quad 14$

$\begin{array}{ccc}X \quad Y & \end{array}$

$\begin{array}{lll}0.00 & 0.03 & 0.00\end{array}$

$\begin{array}{lll}0.00 & 0.02 & -0.40\end{array}$

$\begin{array}{lll}0.00 & 0.02 & 0.40\end{array}$

$\begin{array}{lll}0.00 & 0.02 & 0.40\end{array}$

$\begin{array}{lll}0.00 & 0.02 & -0.40\end{array}$

$\begin{array}{lll}0.00 & -0.33 & 0.00\end{array}$

$\begin{array}{lll}0.00 & 0.27 & 0.00\end{array}$

$\begin{array}{lll}0.00 & -0.33 & 0.00\end{array}$

$\begin{array}{lll}0.00 & 0.27 & 0.00\end{array}$ $\begin{array}{lll}0.00 & 0.13 & 0.00\end{array}$

4

B3U

Frequencies -- $\quad 16.4747$

Red. masses -- 26.0683

Frc consts -- 0.0042

IR Inten -- 1.6551

$\begin{array}{llll}\text { Atom AN } & \mathrm{X} & \mathrm{Y} & \mathrm{Z}\end{array}$

$\begin{array}{lllll}1 & 11 & -0.56 & 0.00 & 0.00\end{array}$

$\begin{array}{lllll}2 & 13 & 0.09 & 0.00 & 0.00\end{array}$

5

B2G

33.8016

27.2220

0.0183

0.0000

$\mathrm{X} \quad \mathrm{Y}$

$\begin{array}{lll}0.00 & 0.00 & 0.00\end{array}$

$\begin{array}{lll}0.03 & 0.00 & 0.00\end{array}$

$\begin{array}{lll}0.03 & 0.00 & 0.00\end{array}$

$\begin{array}{lllll}3 & 13 & 0.09 & 0.00 & 0.00\end{array}$

$\begin{array}{lll}0.09 & 0.00 & 0.00\end{array}$

$4 \quad 13$

\begin{abstract}
0.09
\end{abstract}

\begin{abstract}
$\begin{array}{lll}-0.03 & 0.00 & 0.00\end{array}$
\end{abstract}

\section{3}

AU

$-3.8010$

26.9815

0.0002

0.0000

$\begin{array}{llll}\mathrm{Z} & \mathrm{X} & \mathrm{Y}\end{array}$

Z

$\begin{array}{lll}0.00 & 0.00 & 0.00\end{array}$

$\begin{array}{lll}-0.50 & 0.00 & 0.00\end{array}$

$\begin{array}{lll}0.50 & 0.00 & 0.00\end{array}$

$\begin{array}{lll}0.50 & 0.00 & 0.00\end{array}$

$\begin{array}{lll}-0.50 & 0.00 & 0.00\end{array}$

$\begin{array}{lll}0.00 & 0.00 & 0.00\end{array}$

$\begin{array}{lll}0.00 & 0.00 & 0.00\end{array}$

$\begin{array}{lll}0.00 & 0.00 & 0.00\end{array}$

$\begin{array}{lll}0.00 & 0.00 & 0.00\end{array}$

6

B2U

39.1145

23.3442

0.0210

6.8710

$\begin{array}{lllll}Z & X & Y & \text { Z }\end{array}$

$\begin{array}{lll}0.00 & 0.96 & 0.00\end{array}$

$\begin{array}{lll}0.00 & -0.09 & 0.02\end{array}$

$\begin{array}{lll}0.00 & -0.09 & -0.02\end{array}$

$\begin{array}{llll}0.00 & -0.09 & -0.02\end{array}$ 


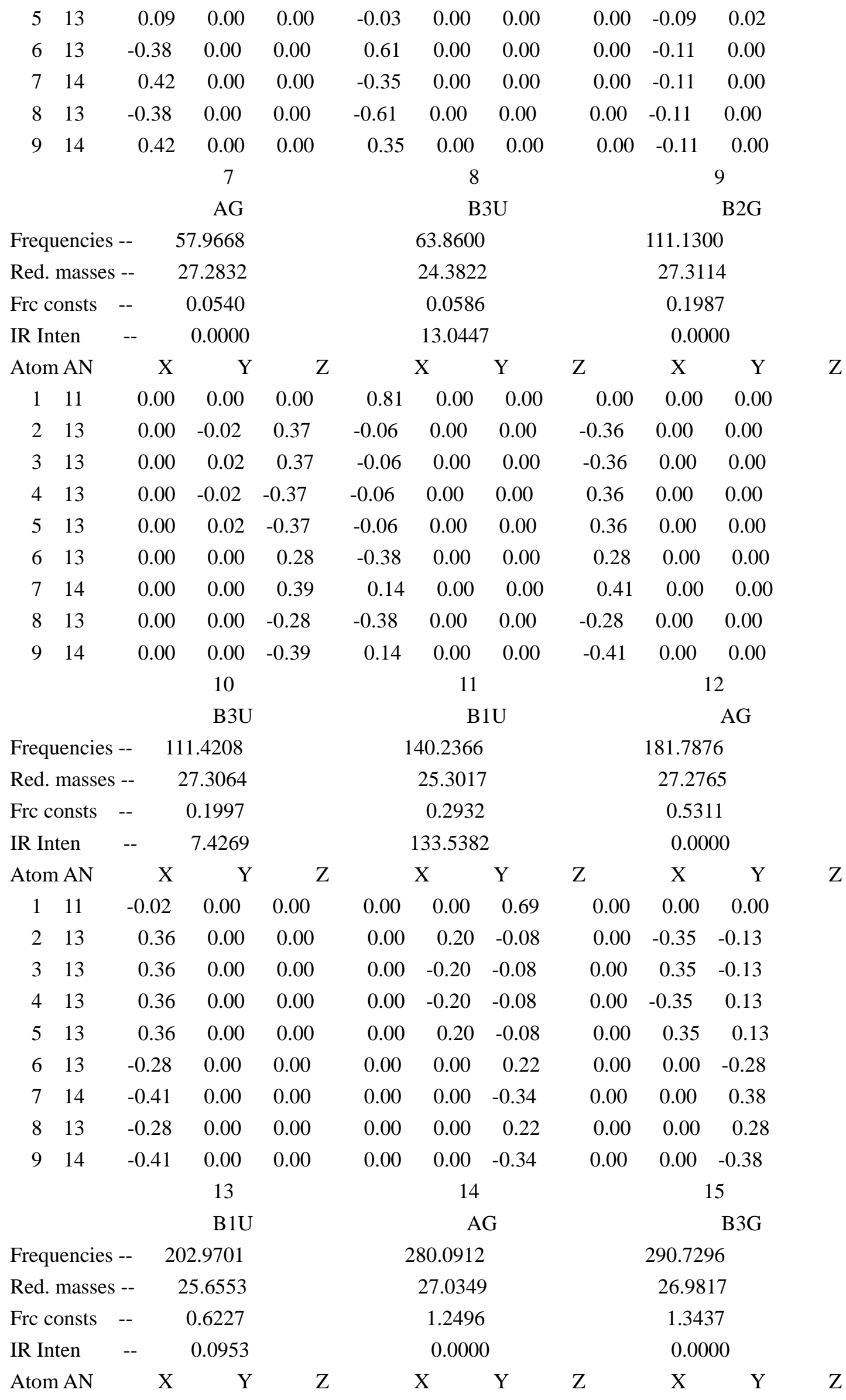




\begin{tabular}{|c|c|c|c|c|c|c|c|c|c|c|c|}
\hline 1 & 11 & 0.00 & 0.00 & 0.60 & 0.00 & 0.00 & 0.00 & 0.00 & 0.00 & 0.00 & \\
\hline 2 & 13 & 0.00 & -0.27 & -0.23 & 0.00 & -0.02 & -0.30 & 0.00 & -0.27 & -0.22 & \\
\hline 3 & 13 & 0.00 & 0.27 & -0.23 & 0.00 & 0.02 & -0.30 & 0.00 & -0.27 & 0.22 & \\
\hline 4 & 13 & 0.00 & 0.27 & -0.23 & 0.00 & -0.02 & 0.30 & 0.00 & 0.27 & -0.22 & \\
\hline 5 & 13 & 0.00 & -0.27 & -0.23 & 0.00 & 0.02 & 0.30 & 0.00 & 0.27 & 0.22 & \\
\hline 6 & 13 & 0.00 & 0.00 & -0.07 & 0.00 & 0.00 & 0.55 & 0.00 & 0.51 & 0.00 & \\
\hline 7 & 14 & 0.00 & 0.00 & 0.25 & 0.00 & 0.00 & 0.16 & 0.00 & 0.01 & 0.00 & \\
\hline 8 & 13 & 0.00 & 0.00 & -0.07 & 0.00 & 0.00 & -0.55 & 0.00 & -0.51 & 0.00 & \\
\hline \multirow[t]{3}{*}{9} & 14 & 0.00 & 0.00 & 0.25 & 0.00 & 0.00 & -0.16 & 0.00 & -0.01 & 0.00 & \\
\hline & & & 16 & & \multicolumn{3}{|c|}{17} & \multicolumn{4}{|c|}{18} \\
\hline & & \multicolumn{3}{|c|}{ B2U } & \multicolumn{3}{|c|}{ B1U } & \multicolumn{4}{|c|}{ AG } \\
\hline \multicolumn{2}{|c|}{ Frequencies -- } & \multicolumn{2}{|c|}{291.0325} & & \multicolumn{3}{|c|}{292.4954} & \multicolumn{4}{|c|}{371.4687} \\
\hline \multicolumn{2}{|c|}{ Red. masses -- } & \multicolumn{2}{|c|}{26.9767} & & \multicolumn{3}{|c|}{26.4198} & \multicolumn{4}{|c|}{27.3019} \\
\hline \multicolumn{2}{|c|}{ Frc consts } & \multicolumn{2}{|r|}{1.3462} & & \multicolumn{3}{|c|}{1.3317} & \multicolumn{4}{|c|}{2.2197} \\
\hline \multicolumn{2}{|c|}{ IR Inten } & - & 2.7758 & & \multicolumn{3}{|c|}{41.0533} & \multicolumn{4}{|c|}{0.0000} \\
\hline \multicolumn{2}{|c|}{ Atom AN } & $\mathrm{X}$ & $\mathrm{Y}$ & $\mathrm{Z}$ & \multicolumn{2}{|r|}{$\mathrm{X}$} & $\mathrm{Y}$ & Z & $\mathrm{X}$ & Y & $\mathrm{Z}$ \\
\hline 1 & 11 & 0.00 & -0.04 & 0.00 & 0.00 & 0.00 & 0.39 & 0.00 & 0.00 & 0.00 & \\
\hline 2 & 13 & 0.00 & 0.27 & 0.22 & 0.00 & -0.04 & 0.26 & 0.00 & 0.36 & -0.11 & \\
\hline 3 & 13 & 0.00 & 0.27 & -0.22 & 0.00 & 0.04 & 0.26 & 0.00 & -0.36 & -0.11 & \\
\hline 4 & 13 & 0.00 & 0.27 & -0.22 & 0.00 & 0.04 & 0.26 & 0.00 & 0.36 & 0.11 & \\
\hline 5 & 13 & 0.00 & 0.27 & 0.22 & 0.00 & -0.04 & 0.26 & 0.00 & -0.36 & 0.11 & \\
\hline 6 & 13 & 0.00 & -0.51 & 0.00 & 0.00 & 0.00 & -0.52 & 0.00 & 0.00 & -0.22 & \\
\hline 7 & 14 & 0.00 & -0.01 & 0.00 & 0.00 & 0.00 & -0.15 & 0.00 & 0.00 & 0.40 & \\
\hline 8 & 13 & 0.00 & -0.51 & 0.00 & 0.00 & 0.00 & -0.52 & 0.00 & 0.00 & 0.22 & \\
\hline 9 & 14 & 0.00 & -0.01 & 0.00 & 0.00 & 0.00 & -0.15 & 0.00 & 0.00 & -0.40 & \\
\hline & & & 19 & & & 20 & & & & 21 & \\
\hline & & & B1U & & & & $3 G$ & & & B2U & \\
\hline Freq & uencies - & & 2.6139 & & & 423.8146 & & & 424.03 & & \\
\hline Red. & masses - & & 7.2433 & & & $27.554 \mathrm{~S}$ & & & 27.55 & & \\
\hline Fre C & onsts - & - & 2.2286 & & & 2.9161 & & & 2.91 & & \\
\hline IR In & ten & - & 6.5448 & & & 0.000 & & & $15.2 \mathrm{C}$ & 999 & \\
\hline Aton & $\mathrm{n}$ AN & $\mathrm{X}$ & $\mathrm{Y}$ & $\mathrm{Z}$ & & $X$ & $\mathrm{Y}$ & $\mathrm{Z}$ & $X$ & $\mathrm{Y}$ & $\mathrm{Z}$ \\
\hline 1 & 11 & 0.00 & 0.00 & -0.11 & 0.00 & 0.00 & 0.00 & 0.00 & 0.00 & 0.00 & \\
\hline 2 & 13 & 0.00 & -0.36 & 0.10 & 0.00 & -0.24 & 0.21 & 0.00 & -0.24 & 0.21 & \\
\hline 3 & 13 & 0.00 & 0.36 & 0.10 & 0.00 & -0.24 & -0.21 & 0.00 & -0.24 & -0.21 & \\
\hline 4 & 13 & 0.00 & 0.36 & 0.10 & 0.00 & 0.24 & 0.21 & 0.00 & -0.24 & -0.21 & \\
\hline 5 & 13 & 0.00 & -0.36 & 0.10 & 0.00 & 0.24 & -0.21 & 0.00 & -0.24 & 0.21 & \\
\hline 6 & 13 & 0.00 & 0.00 & 0.24 & 0.00 & -0.07 & 0.00 & 0.00 & -0.07 & 0.00 & \\
\hline 7 & 14 & 0.00 & 0.00 & -0.39 & 0.00 & 0.54 & 0.00 & 0.00 & 0.54 & 0.00 & \\
\hline 8 & 13 & 0.00 & 0.00 & 0.24 & 0.00 & 0.07 & 0.00 & 0.00 & -0.07 & 0.00 & \\
\hline 9 & 14 & 0.00 & 0.00 & -0.39 & 0.00 & -0.54 & 0.00 & 0.00 & 0.54 & 0.00 & \\
\hline
\end{tabular}




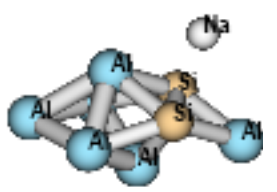

$\begin{array}{llrrr}13 & 0 & 1.475910 & -0.254826 & -1.977607 \wedge \mathrm{M} \\ 11 & 0 & -2.344693 & 2.253935 & -0.000186^{\wedge} \mathrm{M} \\ 13 & 0 & 0.347736 & -1.732321 & 0.000138^{\wedge} \mathrm{M} \\ 13 & 0 & 1.475896 & -0.254515 & 1.977658^{\wedge} \mathrm{M} \\ 14 & 0 & -1.097373 & 0.013346 & 1.273640^{\wedge} \mathrm{M} \\ 13 & 0 & -2.866358 & -1.409829 & 0.000101^{\wedge} \mathrm{M} \\ 13 & 0 & 3.288606 & 0.278821 & -0.000011^{\wedge} \mathrm{M} \\ 13 & 0 & 0.625743 & 1.436966 & -0.000110^{\wedge} \mathrm{M} \\ 14 & 0 & -1.097363 & 0.013145 & -1.273651^{\wedge} \mathrm{M}\end{array}$

|State=1-A|HF=-2196.1391016

Al3SiNaAl3Si-fusion10000-b36311+gx.frq

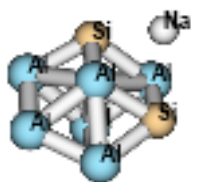

$\begin{array}{llrcc}13 & 0 & -2.763603 & -0.002349 & -0.724674 \wedge \mathrm{M} \\ 11 & 0 & 2.867737 & -0.001515 & -1.478807 \wedge \mathrm{M} \\ 13 & 0 & -0.682197 & 0.001192 & 1.621449 \wedge \mathrm{M} \\ 13 & 0 & -0.041397 & -0.001956 & -1.089224 \wedge \mathrm{M} \\ 14 & 0 & 0.998335 & -1.987022 & 0.060042^{\wedge} \mathrm{M} \\ 13 & 0 & 1.876276 & 0.004020 & 1.334233^{\wedge} \mathrm{M} \\ 13 & 0 & -1.479779 & -2.177702 & -0.006627 \wedge \mathrm{M} \\ 14 & 0 & 0.995086 & 1.988536 & 0.054461^{\wedge} \mathrm{M} \\ 13 & 0 & -1.482607 & 2.176447 & -0.007169 \wedge \mathrm{M}\end{array}$

|State=1-A|HF=-2196.1586209

Al3SiNaAl3Si-fusion100-b36311+gx.frq

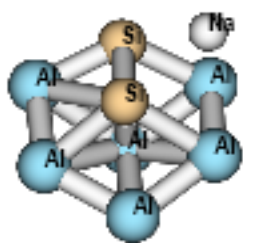

13

13

11

13

13

14

14

13

13

13

3

13

1

3

3

4

4

13

$\begin{array}{rrrr}0 & 1.433016 & 2.307578 & -0.080903 \\ 0 & -1.140035 & 2.073104 & -0.144910 \\ 0 & -2.736771 & -0.182329 & 1.633637 \\ 0 & 0.590891 & 0.037903 & -1.472453 \\ 0 & 1.675030 & -2.161063 & -0.047053 \\ 0 & 0.022341 & -0.021597 & 1.093614 \\ 0 & -0.767259 & -1.966355 & -0.225400 \\ 0 & -2.061813 & -0.108696 & -1.274745 \\ 0 & 2.620861 & 0.146322 & 0.702756\end{array}$




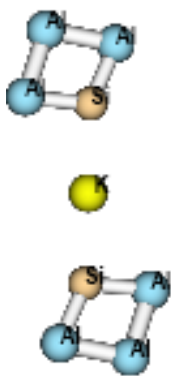

$\begin{array}{rrrrc}13 & 0 & -0.854843 & 5.496910 & 0.000000 \\ 13 & 0 & 2.390106 & 3.446985 & 0.000000 \\ 13 & 0 & 1.697754 & 5.925052 & 0.000000 \\ 19 & 0 & 0.000000 & 0.000000 & 0.000000 \\ 13 & 0 & -2.390106 & -3.446985 & 0.000000 \\ 13 & 0 & -1.697754 & -5.925052 & 0.000000 \\ 13 & 0 & 0.854843 & -5.496910 & 0.000000 \\ 14 & 0 & 0.000000 & 3.281601 & 0.000000 \\ 14 & 0 & 0.000000 & -3.281601 & 0.000000\end{array}$

State $=1-A G \backslash H F=-2633.724323$

\section{1}

AU
2

AU

9.0390

28.7554

0.0014

0.3233

X Y

$\begin{array}{lll}0.00 & 0.00 & 0.31\end{array}$

$\begin{array}{lll}0.00 & 0.00 & -0.20\end{array}$

$\begin{array}{lll}0.00 & 0.00 & 0.46\end{array}$

$\begin{array}{lll}0.00 & 0.00 & -0.37\end{array}$

0.00

0.00

0.00

0.00

0.00

0.00

$0.00-0.20$

$0.00 \quad 0.46$

$0.00 \quad 0.31$

$0.00 \quad-0.29$

$\begin{array}{ll}0.00 & -0.29\end{array}$

\section{4}

BG

5

AG

Frequencies -- 11.7453

Red. masses -- 27.5256

14.7239

27.2604

0.0035

Frc consts -- 0.0022

IR Inten -- 0.0000

Atom AN $\quad \mathrm{X} \quad \mathrm{Y} \quad \mathrm{Z}$

$\begin{array}{lllll}1 & 13 & 0.00 & 0.00 & -0.06\end{array}$

$\begin{array}{lllll}2 & 13 & 0.00 & 0.00 & 0.26\end{array}$

0.0000

$\mathrm{Z}$

0.24

$\begin{array}{lll}-0.17 & -0.32 & 0.00\end{array}$

$\begin{array}{lll}0.32 & -0.19 & 0.00\end{array}$

$\begin{array}{lll}-0.23 & 0.10 & 0.00\end{array}$

$\begin{array}{lll}-0.17 & -0.32 & 0.00\end{array}$

$\begin{array}{lll}0.32 & -0.19 & 0.00\end{array}$

$\begin{array}{lll}0.24 & 0.30 & 0.00\end{array}$

$\begin{array}{lll}-0.21 & 0.13 & 0.00\end{array}$

$-0.21$

$0.13 \quad 0.00$

6

BU

25.9656

35.5059

0.0141

9.6152

X Y

X Y

Z 


$\begin{array}{rrrrr}3 & 13 & 0.00 & 0.00 & -0.39 \\ 4 & 19 & 0.00 & 0.00 & 0.00 \\ 5 & 13 & 0.00 & 0.00 & -0.26 \\ 6 & 13 & 0.00 & 0.00 & 0.39 \\ 7 & 13 & 0.00 & 0.00 & 0.06 \\ 8 & 14 & 0.00 & 0.00 & 0.52 \\ 9 & 14 & 0.00 & 0.00 & -0.52 \\ & & & 7 & \end{array}$

AU

Frequencies -- 36.9613

Red. masses -- 34.0558

Frc consts -- 0.0274

IR Inten $\quad$-- 17.2461

$\begin{array}{ccccc}\text { Atom AN } & \mathrm{X} & \mathrm{Y} & \mathrm{Z} \\ 1 & 13 & 0.00 & 0.00 & -0.09 \\ 2 & 13 & 0.00 & 0.00 & -0.22 \\ 3 & 13 & 0.00 & 0.00 & 0.15 \\ 4 & 19 & 0.00 & 0.00 & 0.75 \\ 5 & 13 & 0.00 & 0.00 & -0.22 \\ 6 & 13 & 0.00 & 0.00 & 0.15 \\ 7 & 13 & 0.00 & 0.00 & -0.09 \\ 8 & 14 & 0.00 & 0.00 & -0.37 \\ 9 & 14 & 0.00 & 0.00 & -0.37 \\ & & & 10 & \end{array}$

$\mathrm{AU}$

Frequencies -- $\quad 119.2591$

Red. masses -- $\quad 27.2753$

Frc consts -- $\quad 0.2286$

IR Inten --- 5.3734

$\begin{array}{ccccc}\text { Atom AN } & \mathrm{X} & \mathrm{Y} & \mathrm{Z} \\ 1 & 13 & 0.00 & 0.00 & 0.35 \\ 2 & 13 & 0.00 & 0.00 & 0.35 \\ 3 & 13 & 0.00 & 0.00 & -0.32 \\ 4 & 19 & 0.00 & 0.00 & 0.02 \\ 5 & 13 & 0.00 & 0.00 & 0.35 \\ 6 & 13 & 0.00 & 0.00 & -0.32 \\ 7 & 13 & 0.00 & 0.00 & 0.35 \\ 8 & 14 & 0.00 & 0.00 & -0.38 \\ 9 & 14 & 0.00 & 0.00 & -0.38\end{array}$

13

BU

Frequencies -- 179.5342

Red. masses -- 29.4790

Frc consts -- 0.5598

\begin{tabular}{cccccc}
-0.12 & 0.24 & 0.00 & -0.04 & -0.04 & 0.00 \\
0.00 & 0.00 & 0.00 & 0.84 & -0.01 & 0.00 \\
-0.34 & -0.36 & 0.00 & -0.24 & -0.08 & 0.00 \\
0.12 & -0.24 & 0.00 & -0.04 & -0.04 & 0.00 \\
0.05 & 0.19 & 0.00 & -0.06 & 0.10 & 0.00 \\
0.37 & -0.03 & 0.00 & -0.26 & 0.03 & 0.00 \\
-0.37 & 0.03 & 0.00 & -0.26 & 0.03 & 0.00 \\
& 8 & & & \multicolumn{2}{c}{9}
\end{tabular}

AG

59.0738

27.1977

0.0559

0.0000

\begin{tabular}{ccc}
\multicolumn{2}{c}{$\mathrm{X}$} & $\mathrm{Y}$ \\
0.12 & 0.42 & 0.00 \\
-0.08 & 0.27 & 0.00 \\
0.13 & 0.33 & 0.00 \\
0.00 & 0.00 & 0.00 \\
0.08 & -0.27 & 0.00 \\
-0.13 & -0.33 & 0.00 \\
-0.12 & -0.42 & 0.00 \\
-0.09 & 0.32 & 0.00 \\
0.09 & -0.32 & 0.00 \\
& 11 &
\end{tabular}

BU

123.6814

32.9242

0.2967

23.4337

$\mathrm{X} \quad \mathrm{Y}$

$\begin{array}{lll}-0.19 & -0.08 & 0.00\end{array}$

$\begin{array}{lll}0.16 & -0.20 & 0.00\end{array}$

$\begin{array}{lll}-0.16 & -0.31 & 0.00\end{array}$

$\begin{array}{lll}0.07 & 0.70 & 0.00\end{array}$

$\begin{array}{lll}0.16 & -0.20 & 0.00\end{array}$

$\begin{array}{lll}-0.16 & -0.31 & 0.00\end{array}$

$\begin{array}{lll}-0.19 & -0.08 & 0.00\end{array}$

$\begin{array}{lll}0.13 & 0.08 & 0.00\end{array}$

$\begin{array}{lll}0.13 & 0.08 & 0.00\end{array}$

14

AG

265.9993

27.0433

1.1274
BG

118.4244

27.2546

0.2252

0.0000

$\begin{array}{llll}Z & X & Y\end{array}$

$0.00-0.36$

$\begin{array}{lll}0.00 & 0.00 & -0.36\end{array}$

$\begin{array}{lll}0.00 & 0.00 & 0.33\end{array}$

$\begin{array}{lll}0.00 & 0.00 & 0.00\end{array}$

$\begin{array}{lll}0.00 & 0.00 & 0.36\end{array}$

$\begin{array}{llll}0.00 & 0.00 & -0.33\end{array}$

$\begin{array}{lll}0.00 & 0.00 & 0.36\end{array}$

$\begin{array}{lll}0.00 & 0.00 & 0.37\end{array}$

$\begin{array}{llll}0.00 & 0.00 & -0.37\end{array}$

12

AG

166.0108

27.2831

0.4430

0.0000

$\begin{array}{llll}Z & X & Y\end{array}$

$\begin{array}{lll}-0.25 & 0.27 & 0.00\end{array}$

$\begin{array}{lll}0.25 & -0.14 & 0.00\end{array}$

$\begin{array}{llll}-0.19 & -0.31 & 0.00\end{array}$

$\begin{array}{lll}0.00 & 0.00 & 0.00\end{array}$

$\begin{array}{lll}-0.25 & 0.14 & 0.00\end{array}$

$\begin{array}{lll}0.19 & 0.31 & 0.00\end{array}$

$\begin{array}{llll}0.25 & -0.27 & 0.00\end{array}$

$\begin{array}{lll}0.17 & 0.35 & 0.00\end{array}$

$\begin{array}{lll}-0.17 & -0.35 & 0.00\end{array}$

15

$\mathrm{BU}$

265.9996

27.0612

1.1281 


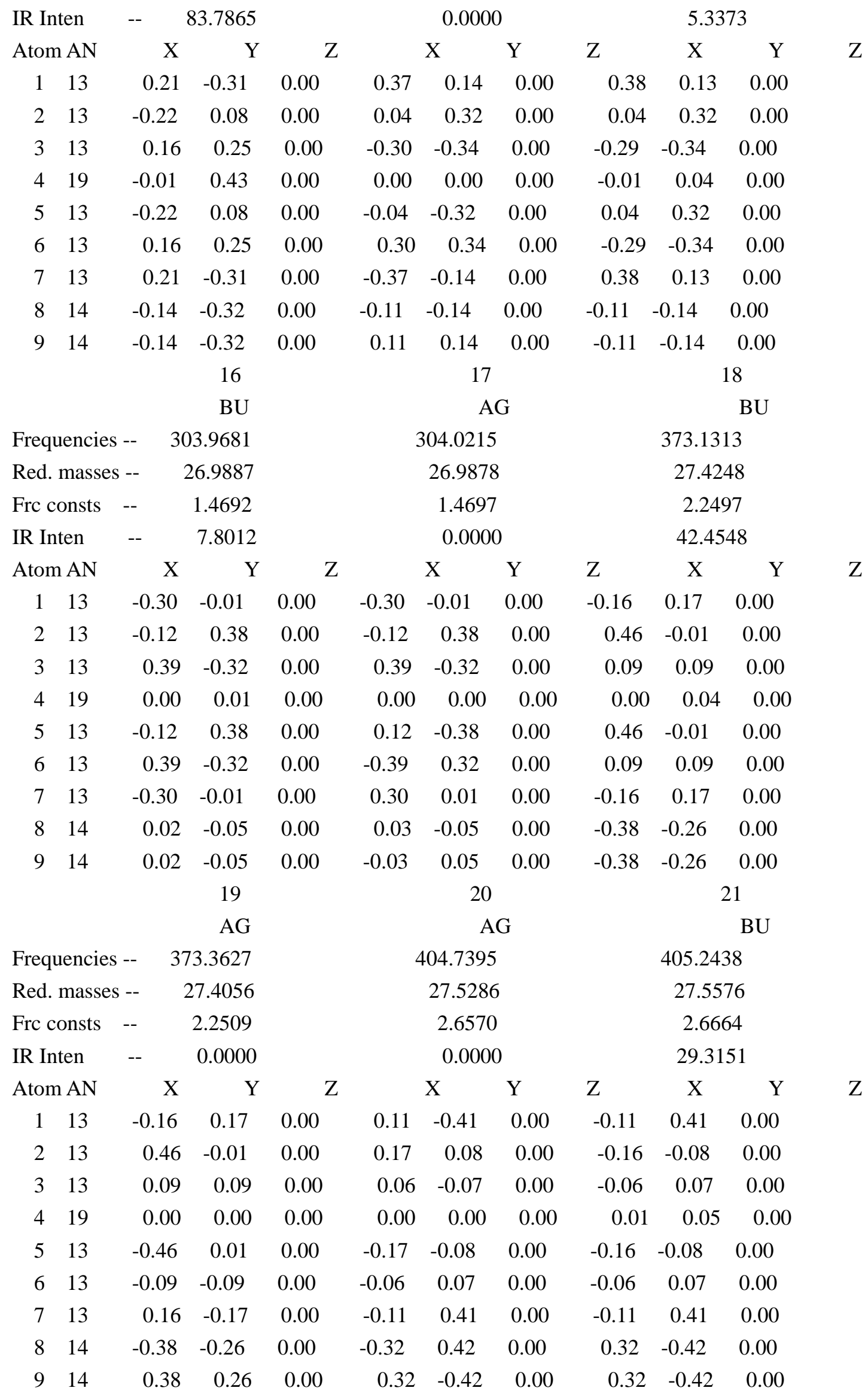

Al3SiKAl3Si-s-Al-Al-c-Si-90-b36311+gx.frq3.8 


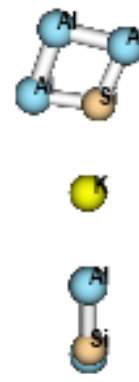

13

13

$\begin{array}{rrrr}0 & 3.685974 & 1.720930 & 0.274922 \\ 0 & 6.084017 & 0.814424 & 0.082511 \\ 0 & 0.041898 & -0.141057 & -0.009114 \\ 0 & -3.395219 & 0.180843 & -1.134215 \\ 0 & -5.941384 & 0.262571 & -1.303267 \\ 0 & 5.407640 & -1.649858 & -0.319146 \\ 0 & 3.284241 & -0.610628 & -0.108559 \\ 0 & -5.662080 & -0.164721 & 1.019188 \\ 0 & -3.341515 & -0.287757 & 1.431838\end{array}$

State $=1-A \mid H F=-2633.7202661$

1

A

\begin{tabular}{|c|c|c|c|c|c|c|c|c|c|c|}
\hline & \multicolumn{3}{|c|}{1} & \multicolumn{4}{|c|}{2} & \multicolumn{3}{|c|}{3} \\
\hline & & $\mathrm{A}$ & & \multicolumn{3}{|c|}{ A } & \multicolumn{4}{|c|}{ A } \\
\hline \multicolumn{2}{|c|}{ Frequencies -- } & \multicolumn{2}{|l|}{3.7956} & \multicolumn{3}{|c|}{7.6326} & \multicolumn{4}{|c|}{13.5636} \\
\hline \multicolumn{2}{|c|}{ Red. masses -- } & \multicolumn{2}{|l|}{27.1270} & \multicolumn{3}{|c|}{28.2785} & \multicolumn{4}{|c|}{28.9119} \\
\hline Frc consts & -- & \multicolumn{2}{|l|}{0.0002} & \multicolumn{3}{|c|}{0.0010} & \multicolumn{4}{|c|}{0.0031} \\
\hline IR Inten & -- & \multicolumn{2}{|l|}{0.0199} & \multicolumn{3}{|c|}{0.1651} & \multicolumn{4}{|c|}{1.9052} \\
\hline Atom AN & $\mathrm{X}$ & $\mathrm{Y}$ & $\mathrm{Z}$ & & $\mathrm{X}$ & $\mathrm{Y}$ & Z & $\mathrm{X}$ & $\mathrm{Y}$ & $\mathrm{Z}$ \\
\hline 113 & 0.01 & -0.08 & 0.43 & -0.04 & 0.02 & -0.28 & 0.42 & 0.19 & 0.01 & \\
\hline 213 & 0.00 & -0.05 & 0.24 & -0.01 & -0.07 & 0.60 & 0.22 & -0.36 & -0.02 & \\
\hline 319 & 0.00 & 0.02 & -0.04 & 0.00 & 0.03 & -0.29 & -0.03 & 0.38 & 0.03 & \\
\hline $4 \quad 13$ & -0.02 & -0.32 & -0.07 & -0.09 & 0.02 & -0.01 & -0.06 & 0.13 & 0.02 & \\
\hline $5 \quad 13$ & -0.02 & -0.38 & -0.05 & -0.11 & 0.00 & 0.19 & -0.07 & -0.30 & -0.03 & \\
\hline 613 & -0.01 & 0.06 & -0.42 & 0.04 & -0.03 & 0.30 & -0.32 & -0.21 & -0.01 & \\
\hline $7 \quad 14$ & 0.00 & 0.02 & -0.19 & 0.00 & 0.05 & -0.51 & -0.08 & 0.28 & 0.02 & \\
\hline $8 \quad 14$ & 0.02 & 0.29 & 0.06 & 0.08 & -0.03 & 0.17 & -0.04 & -0.33 & -0.04 & \\
\hline \multirow[t]{3}{*}{13} & 0.02 & 0.42 & 0.06 & 0.12 & -0.01 & -0.02 & -0.03 & 0.04 & 0.00 & \\
\hline & \multicolumn{3}{|c|}{4} & \multicolumn{3}{|c|}{5} & \multicolumn{4}{|c|}{6} \\
\hline & \multicolumn{3}{|c|}{ A } & \multicolumn{3}{|c|}{ A } & \multicolumn{4}{|c|}{ A } \\
\hline Frequencies -- & \multicolumn{3}{|c|}{23.2904} & \multicolumn{3}{|c|}{25.1811} & \multicolumn{4}{|c|}{42.0932} \\
\hline Red. masses & & 29.2047 & & & 30.813 & & & 31.30 & 047 & \\
\hline Frc consts & -- & 0.0093 & & & 0.011 & & & 0.03 & 327 & \\
\hline IR Inten & -- & 3.6120 & & & 6.988 & & & 4.9 & 726 & \\
\hline Atom AN & $X$ & $\mathrm{Y}$ & $\mathrm{Z}$ & & $\mathrm{X}$ & $\mathrm{Y}$ & Z & $\mathrm{X}$ & $\mathrm{Y}$ & $\mathrm{Z}$ \\
\hline 113 & -0.31 & -0.31 & -0.10 & 0.07 & 0.11 & -0.26 & -0.08 & -0.11 & -0.01 & \\
\hline 213 & -0.17 & 0.08 & 0.02 & 0.04 & -0.05 & 0.15 & -0.05 & -0.02 & -0.01 & \\
\hline 319 & 0.06 & 0.36 & 0.17 & -0.01 & -0.15 & 0.52 & 0.04 & 0.59 & 0.10 & \\
\hline $4 \quad 13$ & 0.10 & 0.38 & 0.10 & 0.17 & -0.11 & 0.17 & 0.01 & -0.57 & -0.11 & \\
\hline
\end{tabular}




\begin{tabular}{ccccccccccc}
5 & 13 & 0.09 & -0.25 & -0.09 & 0.21 & 0.08 & -0.22 & 0.03 & 0.21 & 0.04 \\
6 & 13 & 0.18 & -0.01 & -0.02 & -0.04 & 0.02 & -0.11 & 0.00 & -0.03 & 0.00 \\
7 & 14 & 0.00 & -0.37 & -0.13 & -0.01 & 0.16 & -0.46 & -0.04 & -0.12 & -0.01 \\
8 & 14 & 0.02 & -0.28 & -0.09 & -0.17 & 0.08 & -0.18 & 0.04 & 0.21 & 0.04 \\
9 & 13 & 0.02 & 0.26 & 0.08 & -0.24 & -0.09 & 0.18 & 0.03 & -0.42 & -0.08 \\
& & & 7 & & \multicolumn{7}{c}{8} & & & & 9
\end{tabular}

A

Frequencies -- $\quad 50.8109$

Red. masses -- 29.8448

Frc consts -- $\quad 0.0454$

IR Inten --- 1.7221

Atom AN $\quad \mathrm{X} \quad \mathrm{Y} \quad \mathrm{Z}$

$\begin{array}{rrrrr}1 & 13 & 0.07 & -0.02 & -0.03 \\ 2 & 13 & 0.09 & 0.02 & 0.02 \\ 3 & 19 & 0.04 & -0.08 & 0.48 \\ 4 & 13 & -0.25 & 0.10 & -0.48 \\ 5 & 13 & -0.33 & -0.02 & 0.20 \\ 6 & 13 & 0.12 & 0.02 & -0.01 \\ 7 & 14 & 0.09 & -0.02 & -0.05 \\ 8 & 14 & 0.03 & -0.03 & 0.15 \\ 9 & 13 & 0.13 & 0.08 & -0.47\end{array}$
10

A

Frequencies -- $\quad 117.7770$

Red. masses -- $\quad 27.2586$

Frc consts -- $\quad 0.2228$

IR Inten -- 1.9261

$\begin{array}{rrr}\text { Atom AN } & \mathrm{X} & \mathrm{Y}\end{array}$

$\begin{array}{lllll}1 & 13 & 0.01 & -0.08 & 0.50\end{array}$

$\begin{array}{lllll}2 & 13 & 0.00 & 0.08 & -0.46\end{array}$

$\begin{array}{lllll}3 & 19 & -0.01 & 0.00 & 0.00\end{array}$

$\begin{array}{lllll}4 & 13 & 0.01 & 0.00 & 0.00\end{array}$

$\begin{array}{lllll}5 & 13 & 0.01 & 0.00 & 0.00\end{array}$

$\begin{array}{llllll}6 & 13 & 0.01 & -0.08 & 0.49\end{array}$

$\begin{array}{lllll}7 & 14 & -0.01 & 0.09 & -0.52\end{array}$

$\begin{array}{lllll}8 & 14 & 0.00 & 0.00 & 0.00\end{array}$

$\begin{array}{lllll}9 & 13 & 0.00 & 0.00 & 0.00\end{array}$ 13

A

Frequencies -- 173.0791

Red. masses -- 28.6413

Frc consts -- 0.5055

IR Inten -- 50.0695
A

57.2006

27.5190

0.0530

$$
1.2078
$$

X Y

$\begin{array}{lll}0.24 & -0.12 & -0.01\end{array}$

$\begin{array}{lll}0.32 & 0.09 & 0.00\end{array}$

$\begin{array}{lll}0.05 & 0.08 & -0.12\end{array}$

$\begin{array}{lll}-0.28 & -0.02 & 0.14\end{array}$

$\begin{array}{lll}-0.28 & 0.02 & -0.05\end{array}$

$\begin{array}{lll}0.42 & 0.06 & 0.01\end{array}$

$\begin{array}{lll}0.30 & -0.13 & 0.00\end{array}$

$\begin{array}{lll}-0.39 & 0.02 & -0.03\end{array}$

$\begin{array}{lll}-0.40 & -0.02 & 0.13\end{array}$

11

A

124.9952

34.0809

0.3137

48.3621

X Y

$\begin{array}{lll}-0.21 & 0.21 & 0.05\end{array}$

$\begin{array}{lll}-0.37 & -0.13 & -0.02\end{array}$

$\begin{array}{lll}0.76 & -0.02 & -0.03\end{array}$

$\begin{array}{lll}-0.05 & 0.00 & 0.00\end{array}$

$\begin{array}{lll}-0.10 & 0.00 & 0.02\end{array}$

$\begin{array}{lll}-0.10 & -0.20 & -0.02\end{array}$

$\begin{array}{lll}0.11 & 0.14 & 0.01\end{array}$

$\begin{array}{lll}-0.21 & 0.00 & 0.04\end{array}$

$\begin{array}{lll}-0.17 & 0.01 & -0.03\end{array}$

14

A

265.2420

27.0202

1.1200

0.8655
A

112.0798

27.2839

0.2019

1.3829

$\begin{array}{llllll}\mathrm{Z} & \mathrm{X} & \mathrm{Y} & \mathrm{Z}\end{array}$

$\begin{array}{lll}0.00 & 0.00 & 0.00\end{array}$

$\begin{array}{lll}0.00 & 0.00 & 0.00\end{array}$

$\begin{array}{ccc}0.00 & -0.01 & 0.00\end{array}$

$\begin{array}{lll}-0.01 & -0.42 & -0.07\end{array}$

$\begin{array}{lll}0.01 & 0.49 & 0.09\end{array}$

$\begin{array}{lll}0.00 & 0.00 & 0.00\end{array}$

$\begin{array}{lll}0.00 & 0.00 & 0.00\end{array}$

$\begin{array}{lll}-0.01 & -0.54 & -0.10\end{array}$

$\begin{array}{lll}0.01 & 0.51 \quad 0.10\end{array}$

12

A

148.9749

27.4531

0.3590

11.2229

$\begin{array}{llllll}Z & X & Y & \text { Z }\end{array}$

$\begin{array}{lll}0.03 & -0.03 & -0.01\end{array}$

$\begin{array}{lll}0.05 & 0.02 & 0.01\end{array}$

$\begin{array}{lll}-0.06 & 0.02 & -0.09\end{array}$

$\begin{array}{lll}0.39 & 0.04 & -0.27\end{array}$

$\begin{array}{lll}0.44 & -0.06 & 0.27\end{array}$

$\begin{array}{lll}-0.01 & 0.04 & 0.00\end{array}$

$\begin{array}{lll}-0.04 & -0.02 & 0.01\end{array}$

$\begin{array}{lll}-0.44 & -0.06 & 0.36\end{array}$

$\begin{array}{lll}-0.31 & 0.06 & -0.25\end{array}$

15

A

266.9631

27.0499

1.1358

2.1307

$\begin{array}{lll}\mathrm{X} & \mathrm{Y} & \mathrm{Z}\end{array}$ 


$\begin{array}{ccccc}1 & 13 & 0.12 & -0.33 & -0.06 \\ 2 & 13 & 0.40 & 0.20 & 0.03 \\ 3 & 19 & 0.34 & -0.05 & -0.01 \\ 4 & 13 & -0.03 & 0.00 & 0.03 \\ 5 & 13 & -0.06 & 0.00 & -0.02 \\ 6 & 13 & -0.38 & 0.36 & 0.07 \\ 7 & 14 & -0.50 & -0.15 & -0.02 \\ 8 & 14 & -0.01 & 0.00 & -0.02 \\ 9 & 13 & 0.00 & 0.00 & 0.01 \\ & & & 16 & \end{array}$

A

Frequencies -- 304.9762

Red. masses -- 26.9887

Frc consts -- 1.4790

IR Inten -- 3.9615

$\begin{array}{ccc}0.01 & 0.00 & 0.00 \\ -0.01 & -0.01 & 0.00 \\ -0.01 & 0.00 & 0.01 \\ -0.25 & -0.11 & 0.62 \\ 0.22 & 0.03 & -0.17 \\ 0.01 & 0.01 & 0.00 \\ 0.00 & 0.00 & 0.00 \\ -0.12 & -0.02 & 0.15 \\ 0.16 & 0.11 & -0.62\end{array}$

17

A

307.1793

27.0066

$$
1.5014
$$

2.4007 \begin{tabular}{ccc}
0.46 & 0.02 & 0.00 \\
-0.52 & -0.37 & -0.05 \\
0.02 & -0.01 & 0.00 \\
0.01 & 0.00 & -0.01 \\
-0.01 & 0.00 & 0.00 \\
0.24 & 0.51 & 0.08 \\
-0.21 & -0.14 & -0.02 \\
0.00 & 0.00 & 0.00 \\
0.00 & 0.00 & 0.01 \\
\multicolumn{3}{c}{18}
\end{tabular}

A

\begin{tabular}{|c|c|c|c|c|c|c|c|c|c|c|}
\hline \multicolumn{2}{|c|}{ Atom AN } & \multicolumn{2}{|c|}{$\mathrm{Y}$} & $\mathrm{Z}$ & \multicolumn{2}{|r|}{$\mathrm{X}$} & $\mathrm{Y}$ & $\mathrm{Z}$ & $\mathrm{X}$ & $\mathrm{Y}$ \\
\hline 1 & 13 & 0.51 & -0.22 & -0.04 & -0.01 & 0.01 & 0.00 & 0.05 & 0.65 & 0.11 \\
\hline 2 & 13 & -0.39 & 0.58 & 0.10 & 0.01 & -0.01 & 0.00 & 0.13 & 0.12 & 0.02 \\
\hline 3 & 19 & 0.00 & 0.00 & 0.00 & -0.04 & 0.00 & -0.01 & 0.02 & 0.00 & 0.00 \\
\hline 4 & 13 & 0.02 & 0.00 & 0.01 & 0.68 & -0.06 & 0.27 & 0.01 & 0.00 & -0.01 \\
\hline 5 & 13 & -0.02 & 0.00 & 0.00 & -0.60 & 0.01 & 0.02 & -0.01 & 0.01 & -0.03 \\
\hline 6 & 13 & -0.06 & -0.41 & -0.07 & 0.01 & 0.01 & 0.00 & 0.20 & -0.25 & -0.04 \\
\hline 7 & 14 & -0.07 & 0.04 & 0.01 & 0.00 & 0.00 & 0.00 & -0.41 & -0.50 & -0.08 \\
\hline 8 & 14 & 0.00 & 0.00 & 0.00 & 0.09 & 0.00 & -0.01 & -0.02 & 0.00 & 0.03 \\
\hline \multirow[t]{3}{*}{9} & 13 & 0.00 & 0.00 & -0.01 & -0.13 & 0.05 & -0.26 & 0.03 & 0.00 & 0.01 \\
\hline & & & 19 & & \multicolumn{3}{|c|}{20} & & \multicolumn{2}{|c|}{21} \\
\hline & & & A & & \multicolumn{3}{|c|}{ A } & & \multicolumn{2}{|r|}{ A } \\
\hline \multicolumn{2}{|c|}{ Frequencies -- } & \multicolumn{2}{|c|}{374.7029} & & \multicolumn{3}{|c|}{405.7158} & & \multicolumn{2}{|c|}{425.0439} \\
\hline \multicolumn{2}{|c|}{ Red. masses -- } & \multicolumn{2}{|c|}{27.3495} & & \multicolumn{3}{|c|}{27.5463} & & \multicolumn{2}{|c|}{27.5137} \\
\hline \multicolumn{2}{|c|}{ Frc consts } & \multicolumn{2}{|c|}{2.2624} & & \multicolumn{3}{|c|}{2.6715} & & \multicolumn{2}{|c|}{2.9286} \\
\hline \multicolumn{2}{|c|}{ IR Inten } & -- & 3.8367 & & \multicolumn{3}{|c|}{15.5341} & & \multicolumn{2}{|c|}{5.2778} \\
\hline \multicolumn{2}{|c|}{ Atom AN } & $\mathrm{X}$ & $\mathrm{Y}$ & Z & & $\mathrm{X}$ & $\mathrm{Y}$ & $\mathrm{Z}$ & $\mathrm{X}$ & $\mathrm{Y}$ \\
\hline 1 & 13 & 0.00 & 0.04 & 0.01 & -0.14 & -0.21 & -0.03 & 0.00 & 0.00 & 0.00 \\
\hline 2 & 13 & 0.01 & 0.01 & 0.00 & 0.09 & -0.09 & -0.02 & 0.00 & 0.00 & 0.00 \\
\hline 3 & 19 & 0.02 & 0.00 & 0.00 & 0.04 & 0.00 & 0.00 & 0.02 & 0.00 & -0.01 \\
\hline 4 & 13 & -0.13 & -0.02 & 0.14 & 0.00 & 0.00 & 0.00 & -0.11 & 0.01 & -0.06 \\
\hline 5 & 13 & 0.18 & -0.11 & 0.58 & 0.00 & 0.00 & 0.01 & 0.06 & 0.06 & -0.36 \\
\hline 6 & 13 & 0.01 & -0.01 & 0.00 & 0.57 & -0.20 & -0.04 & 0.01 & 0.00 & 0.00 \\
\hline 7 & 14 & -0.02 & -0.03 & -0.01 & -0.55 & 0.49 & 0.09 & -0.01 & 0.00 & 0.00 \\
\hline 8 & 14 & 0.33 & 0.08 & -0.49 & 0.00 & 0.00 & -0.01 & 0.57 & -0.09 & 0.45 \\
\hline 9 & 13 & -0.42 & 0.05 & -0.20 & 0.00 & 0.00 & 0.00 & -0.56 & 0.02 & -0.04 \\
\hline
\end{tabular}

Al3SiKAl3Si-s-s-90-Al-Al-b36311+gx.frq7.3 
o

의

$\begin{array}{rrrrc}13 & 0 & 5.982036 & -1.027691 & 0.822615 \\ 13 & 0 & 3.434749 & -0.894156 & 0.723904 \\ 13 & 0 & -5.982383 & -0.871706 & -0.985592 \\ 13 & 0 & -3.435060 & -0.756107 & -0.867050 \\ 19 & 0 & -0.000016 & -0.007503 & -0.001409 \\ 13 & 0 & 3.363212 & 1.102543 & -0.955033 \\ 13 & 0 & -3.362840 & 0.922824 & 1.129615 \\ 14 & 0 & 5.685510 & 0.778271 & -0.695091 \\ 14 & 0 & -5.685223 & 0.647326 & 0.819147\end{array}$

State $=1-A \backslash H F=-2633.7160786$

A

A

A

15.6479

15.6842

30.8014

30.8120

Red. masses -- $\quad 27.1468$

0.0044

0.0045

Frc consts -- 0.0008

4.3607

4.3978

$\begin{array}{llrr}\text { IR Inten } & -- & & 0.0220 \\ \text { Atom AN } & & \mathrm{X} & \mathrm{Y}\end{array}$

X Y

X Y

Z

$\begin{array}{lllllllllll}1 & 13 & 0.01 & 0.26 & 0.28 & 0.11 & -0.09 & -0.35 & -0.16 & -0.31 & 0.00\end{array}$

$\begin{array}{lllll}2 & 13 & 0.01 & 0.20 & 0.24\end{array}$

$\begin{array}{llllll}0.10 & 0.10 & 0.19 & -0.14 & 0.15 & 0.07\end{array}$

$\begin{array}{lllll}3 & 13 & -0.01 & 0.31 & -0.24\end{array}$

$\begin{array}{llllll}0.16 & 0.01 & -0.32 & 0.11 & -0.33 & 0.07\end{array}$

$\begin{array}{lllll}4 & 13 & -0.01 & 0.24 & -0.19\end{array}$

$\begin{array}{lll}0.14 & -0.06 & 0.14\end{array}$

$\begin{array}{lll}0.09 & 0.20 & -0.11\end{array}$

$\begin{array}{lllll}5 & 19 & 0.00 & -0.02 & 0.01\end{array}$

$\begin{array}{lll}0.00 & 0.06 & 0.54\end{array}$

$0.00 \quad 0.54 \quad-0.06$

$\begin{array}{lllll}6 & 13 & -0.01 & -0.27 & -0.32\end{array}$

$\begin{array}{lll}-0.12 & 0.04 & 0.13\end{array}$

$\begin{array}{lll}0.18 & 0.10 & -0.01\end{array}$

$\begin{array}{lllll}7 & 13 & 0.01 & -0.32 & 0.28\end{array}$

$-0.18-0.01$

0.11

$\begin{array}{lll}-0.12 & 0.11 & -0.02\end{array}$

$\begin{array}{lllll}8 & 14 & -0.01 & -0.17 & -0.23\end{array}$

$\begin{array}{lll}-0.09 & -0.12 & -0.35\end{array}$

$\begin{array}{lll}0.13 & -0.31 & -0.06\end{array}$

95

$\begin{array}{lll}0.01 & -0.20 & 0.19\end{array}$

$\begin{array}{lll}-0.13 & 0.05 & -0.30\end{array}$

$\begin{array}{lll}-0.08 & -0.36 & 0.13\end{array}$

\section{A}

5

A

A

Frequencies -- $\quad 37.3579$

37.3693

51.5909

Red. masses -- $\quad 27.8205$

27.8007

28.9755

Frc consts - - 0.0229

0.0229

0.0454

IR Inten $\quad$-- 1.3064

1.2816

0.8227

\begin{tabular}{cccccccccccc} 
Atom AN & \multicolumn{1}{c}{$\mathrm{X}$} & \multicolumn{2}{c}{$\mathrm{Y}$} & $\mathrm{Z}$ & \multicolumn{2}{c}{$\mathrm{X}$} & $\mathrm{Y}$ & $\mathrm{Z}$ & $\mathrm{X}$ & $\mathrm{Y}$ \\
1 & 13 & -0.15 & -0.18 & -0.02 & 0.10 & -0.07 & -0.20 & 0.36 & 0.10 & -0.04 \\
2 & 13 & -0.12 & 0.36 & 0.13 & 0.09 & 0.24 & 0.48 & 0.31 & -0.26 & 0.08 \\
3 & 13 & -0.09 & 0.20 & -0.07 & -0.16 & -0.02 & 0.17 & -0.35 & 0.09 & 0.05 \\
4 & 13 & -0.07 & -0.48 & 0.24 & -0.13 & 0.13 & -0.36 & -0.30 & -0.23 & -0.12
\end{tabular}




\begin{tabular}{ccccccccccc}
5 & 19 & 0.01 & 0.08 & -0.24 & 0.00 & -0.23 & -0.08 & 0.00 & 0.40 & 0.03 \\
6 & 13 & 0.15 & 0.31 & 0.06 & -0.08 & 0.15 & 0.37 & 0.13 & -0.24 & 0.11 \\
7 & 13 & 0.10 & -0.37 & 0.15 & 0.14 & 0.06 & -0.30 & -0.13 & -0.21 & -0.14 \\
8 & 14 & 0.10 & -0.16 & -0.05 & -0.05 & -0.10 & -0.20 & 0.18 & 0.08 & -0.03 \\
9 & 14 & 0.07 & 0.20 & -0.09 & 0.09 & -0.05 & 0.16 & -0.18 & 0.07 & 0.04 \\
& & & 7 & & \multicolumn{2}{c}{8} & & & & 9
\end{tabular}

A

Frequencies -- 53.1733

Red. masses -- $\quad 30.4973$

Frc consts --- 0.0508

IR Inten -- 1.7903

Atom AN $\mathrm{X} \quad \mathrm{Y}$

$\begin{array}{lllll}1 & 13 & 0.16 & 0.08 & -0.12\end{array}$

$\begin{array}{lllll}2 & 13 & 0.11 & -0.18 & 0.31\end{array}$

$3 \quad 13$

$\begin{array}{lll}0.16 & -0.06 & -0.14\end{array}$

$\begin{array}{lll}0.11 & 0.14 & 0.34\end{array}$

$4 \quad 13$

$\begin{array}{ll}5 & 19\end{array}$

$\begin{array}{lll}-0.04 & 0.03 & -0.54\end{array}$

$\begin{array}{ll}6 & 13\end{array}$

$\begin{array}{lll}-0.14 & -0.20 & 0.28\end{array}$

$\begin{array}{lllll}7 & 13 & -0.15 & 0.16 & 0.32\end{array}$

$\begin{array}{llllll}8 & 14 & -0.08 & 0.06 & -0.10\end{array}$

$\begin{array}{llllll}9 & 14 & -0.09 & -0.04 & -0.11\end{array}$

10

A

Frequencies -- 112.0788

Red. masses -- $\quad 27.2825$

Frc consts -- 0.2019

IR Inten -- 1.4427

Atom AN $\quad \mathrm{X} \quad \mathrm{Y}$

$\begin{array}{lllll}1 & 13 & 0.00 & 0.23 & 0.28\end{array}$

$\begin{array}{llllll}2 & 13 & 0.00 & -0.20 & -0.24\end{array}$

$\begin{array}{lllll}3 & 13 & 0.00 & 0.26 & -0.22\end{array}$

$\begin{array}{llllll}4 & 13 & 0.00 & -0.22 & 0.19\end{array}$

$\begin{array}{llllll}5 & 19 & 0.00 & -0.01 & 0.00\end{array}$

$\begin{array}{lllll}6 & 13 & 0.00 & 0.24 & 0.29\end{array}$

$\begin{array}{llllll}7 & 13 & 0.00 & 0.27 & -0.23\end{array}$

$\begin{array}{llllll}8 & 14 & 0.00 & -0.26 & -0.31\end{array}$

$\begin{array}{ll}9 & 14\end{array}$

$\begin{array}{lll}0.00 & -0.29 & 0.24\end{array}$

13

A

Frequencies -- $\quad 149.3712$

Red. masses -- 27.6159

Frc consts -- 0.3630

IR Inten -- $\quad 9.9416$

Atom AN X Y
$\mathrm{A}$

55.9693

28.6067

0.0528

0.5460

$\mathrm{X} \quad \mathrm{Y}$

$\begin{array}{lll}0.17 & -0.09 & 0.04\end{array}$

$\begin{array}{lll}0.19 & 0.22 & -0.10\end{array}$

$\begin{array}{lll}-0.18 & -0.08 & -0.05\end{array}$

$\begin{array}{lll}-0.19 & 0.19 & 0.13\end{array}$

$\begin{array}{llll}0.00 & -0.34 & -0.02\end{array}$

$\begin{array}{lll}0.37 & 0.20 & -0.11\end{array}$

$\begin{array}{lll}-0.37 & 0.17 & 0.14\end{array}$

$\begin{array}{lll}0.34 & -0.07 & 0.03\end{array}$

$\begin{array}{lll}-0.34 & -0.06 & -0.03\end{array}$

11

A

125.0666

36.1842

0.3335

95.6387

X Y

$\begin{array}{lll}-0.12 & -0.02 & 0.01 \\ -0.06 & 0.00 & 0.00\end{array}$

$\begin{array}{lll}-0.06 & 0.00 & 0.00\end{array}$

$\begin{array}{lll}-0.12 & 0.01 & 0.02\end{array}$

$\begin{array}{lll}-0.06 & 0.00 & 0.00\end{array}$

$\begin{array}{lll}0.87 & 0.00 & -0.05\end{array}$

$\begin{array}{lll}-0.20 & 0.03 & -0.02\end{array}$

$\begin{array}{lll}-0.20 & -0.02 & -0.02\end{array}$

$\begin{array}{lll}-0.25 & -0.04 & 0.03\end{array}$

$\begin{array}{lll}-0.25 & 0.03 & 0.04\end{array}$

14

A

264.9368

27.0203

1.1174

1.5636

X Y
A

111.7945

27.2831

0.2009

1.4460

$\begin{array}{llll}\mathrm{Z} & \mathrm{X} & \mathrm{Y}\end{array}$

$\begin{array}{lll}0.00 & 0.22 & 0.26\end{array}$

$\begin{array}{lll}0.00 & -0.19 & -0.22\end{array}$

$\begin{array}{llll}0.00 & -0.28 & 0.23\end{array}$

$\begin{array}{lll}0.00 & 0.24 & -0.20\end{array}$

$\begin{array}{llll}0.00 & 0.00 & -0.01\end{array}$

$\begin{array}{lll}0.00 & 0.23 & 0.27\end{array}$

$\begin{array}{lll}0.00 & -0.29 & 0.24\end{array}$

$\begin{array}{llll}0.00 & -0.24 & -0.29\end{array}$

$\begin{array}{lll}0.00 & 0.31 & -0.26\end{array}$

12

A

149.2330

27.4321

0.3599

5.6098

$\begin{array}{lllll}Z & X & Y & \text { Z }\end{array}$

$\begin{array}{lll}-0.31 & 0.15 & -0.13\end{array}$

$\begin{array}{lll}-0.27 & -0.14 & 0.13\end{array}$

$\begin{array}{lll}0.30 & 0.13 & 0.15\end{array}$

$\begin{array}{lll}0.27 & -0.12 & -0.15\end{array}$

$\begin{array}{lll}0.00 & -0.10 & -0.01\end{array}$

$\begin{array}{lll}0.23 & -0.14 & 0.12\end{array}$

$\begin{array}{lll}-0.23 & -0.11 & -0.14\end{array}$

$\begin{array}{lll}0.32 & 0.20 & -0.17\end{array}$

$\begin{array}{lll}-0.32 & 0.16 & 0.20\end{array}$

15

A

265.0906

27.0194

1.1187

0.1101

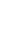




$\begin{array}{rrrrc}1 & 13 & 0.33 & -0.15 & 0.12 \\ 2 & 13 & 0.28 & 0.15 & -0.12 \\ 3 & 13 & 0.33 & 0.13 & 0.15 \\ 4 & 13 & 0.28 & -0.13 & -0.15 \\ 5 & 19 & -0.14 & 0.01 & -0.09 \\ 6 & 13 & -0.21 & 0.14 & -0.11 \\ 7 & 13 & -0.21 & -0.12 & -0.13 \\ 8 & 14 & -0.29 & -0.19 & 0.16 \\ 9 & 14 & -0.29 & 0.16 & 0.20 \\ & & & 16 & \end{array}$

A

Frequencies -- 307.1885

Red. masses -- 26.9904

Frc consts -- 1.5006

IR Inten -- 2.2510

Atom AN $\mathrm{X} \quad \mathrm{Y}$

$\begin{array}{lllll}1 & 13 & -0.42 & -0.01 & 0.01\end{array}$

$\begin{array}{lllll}2 & 13 & 0.48 & -0.15 & 0.12\end{array}$

$\begin{array}{llllll}3 & 13 & 0.43 & -0.01 & -0.01\end{array}$

$\begin{array}{lllll}4 & 13 & -0.49 & -0.12 & -0.15\end{array}$

$\begin{array}{lllll}5 & 19 & 0.00 & 0.01 & 0.00\end{array}$

$\begin{array}{llllll}6 & 13 & -0.09 & 0.14 & -0.12\end{array}$

$\begin{array}{ll}7 & 13\end{array}$

$\begin{array}{ll}8 & 14\end{array}$

$9 \quad 14$ $\begin{array}{lll}-0.15 & -0.09 & 0.08\end{array}$

$\begin{array}{lll}0.17 & 0.34 & -0.29\end{array}$

$\begin{array}{lll}-0.16 & 0.08 & 0.10\end{array}$

$\begin{array}{lll}0.18 & -0.29 & -0.35\end{array}$

$\begin{array}{lll}0.01 & 0.00 & -0.01\end{array}$

$\begin{array}{lll}-0.11 & -0.34 & 0.28\end{array}$

$\begin{array}{lll}-0.11 & 0.29 & 0.35\end{array}$

$\begin{array}{lll}0.08 & 0.08 & -0.07\end{array}$

$\begin{array}{lll}0.08 & -0.07 & -0.08\end{array}$

17

A

307.6164

27.0226

1.5066

2.4336

$$
\begin{array}{ccc}
-0.16 & -0.09 & 0.08 \\
0.18 & 0.35 & -0.29 \\
0.15 & -0.08 & -0.09 \\
-0.17 & 0.28 & 0.34 \\
0.00 & 0.01 & 0.00 \\
-0.11 & -0.35 & 0.29 \\
0.11 & -0.28 & -0.34 \\
0.08 & 0.08 & -0.07 \\
-0.08 & 0.07 & 0.08 \\
& \multicolumn{2}{c}{18}
\end{array}
$$

A

374.8459

27.3435

2.2637

3.6951

$\begin{array}{lll}\mathrm{X} & \mathrm{Y} & \mathrm{Z}\end{array}$

$\begin{array}{llll}Z & X & Y\end{array}$

$\begin{array}{cccccc}-0.42 & -0.01 & 0.01 & -0.13 & 0.32 & -0.27 \\ 0.49 & -0.15 & 0.12 & 0.09 & 0.08 & -0.06\end{array}$

$\begin{array}{llllll}-0.42 & 0.01 & 0.01 & 0.13 & 0.27 & 0.32\end{array}$

$\begin{array}{llllll}0.48 & 0.12 & 0.14 & -0.09 & 0.06 & 0.08\end{array}$

$\begin{array}{llllll}-0.05 & 0.00 & -0.01 & 0.00 & 0.00 & 0.00\end{array}$

$\begin{array}{llllll}-0.09 & 0.14 & -0.12 & 0.30 & -0.11 & 0.09\end{array}$

$\begin{array}{llllll}-0.09 & -0.12 & -0.14 & -0.30 & -0.09 & -0.11\end{array}$

$\begin{array}{lll}0.07 & 0.01 & -0.01\end{array}$

$\begin{array}{lll}-0.24 & -0.27 & 0.23\end{array}$

$\begin{array}{lll}0.23 & -0.23 & -0.27\end{array}$

20

A

425.9722

27.5151

2.9416

9.3637

21

A

426.0409

27.5119

2.9422

1.6390

IR Inten -- $\quad 4.2584$

$\begin{array}{llllll}\text { Atom AN } & \text { X } & \text { Y } & \text { Z } & \text { X } & \text { Y }\end{array}$

$\begin{array}{llllllll}1 & 13 & -0.13 & 0.32 & -0.27 & -0.04 & -0.21 & 0.18\end{array}$

$\begin{array}{llllllll}2 & 13 & 0.09 & 0.07 & -0.06 & 0.08 & -0.03 & 0.03\end{array}$

Z

X $\quad \mathrm{Y}$

Z

$\begin{array}{lllllllllll}3 & 13 & -0.13 & -0.27 & -0.32 & -0.04 & 0.15 & 0.18 & -0.04 & 0.18 & 0.21\end{array}$

$\begin{array}{lllllllllll}4 & 13 & 0.09 & -0.06 & -0.08 & 0.07 & 0.02 & 0.03 & 0.08 & 0.03 & 0.03\end{array}$

$\begin{array}{lllllllllll}5 & 19 & -0.03 & 0.00 & 0.00 & -0.02 & 0.00 & 0.01 & 0.00 & 0.01 & 0.00\end{array}$

$\begin{array}{lllllllllll}6 & 13 & 0.29 & -0.11 & 0.09 & 0.43 & -0.02 & 0.02 & -0.36 & 0.02 & -0.01\end{array}$

$\begin{array}{lllllllllll}7 & 13 & 0.30 & 0.09 & 0.11 & 0.36 & 0.02 & 0.02 & 0.43 & 0.02 & 0.02\end{array}$

$\begin{array}{llllllllllll}8 & 14 & -0.23 & -0.27 & 0.23 & -0.44 & 0.27 & -0.22 & 0.37 & -0.23 & 0.19\end{array}$

$\begin{array}{lllllllllll}9 & 14 & -0.23 & 0.23 & 0.28 & -0.37 & -0.19 & -0.22 & -0.44 & -0.23 & -0.26\end{array}$

Al3SiKAl3Si-c-c-0-C2h-b36311+gx.frq3m-23.2 


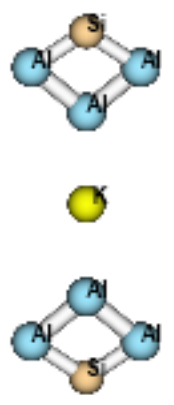

$\begin{array}{ccccc}19 & 0 & 0.000000 & 0.000000 & 0.000000 \\ 13 & 0 & 0.000000 & 1.972318 & 5.081069 \\ 13 & 0 & 0.000000 & -1.972318 & 5.081069 \\ 13 & 0 & 0.000000 & 1.972318 & -5.081069 \\ 13 & 0 & 0.000000 & -1.972318 & -5.081069 \\ 13 & 0 & 0.000000 & 0.000000 & 3.430127 \\ 14 & 0 & 0.000000 & 0.000000 & 6.407674 \\ 13 & 0 & 0.000000 & 0.000000 & -3.430127 \\ 14 & 0 & 0.000000 & 0.000000 & -6.407674\end{array}$

State $=1-A G \backslash H F=-2633.7068616$

B2U

B3G

\begin{tabular}{|c|c|c|c|}
\hline \multicolumn{2}{|c|}{ Frequencies -- } & \multicolumn{2}{|c|}{-23.2418} \\
\hline Red. masses & & 27.8639 & \\
\hline Frc consts & -- & 0.0089 & \\
\hline IR Inten & -- & 1.3081 & \\
\hline Atom AN & $\mathrm{X}$ & $\mathrm{Y}$ & 2 \\
\hline 119 & 0.00 & 0.26 & 0.00 \\
\hline 213 & 0.00 & -0.03 & -0.38 \\
\hline 313 & 0.00 & -0.03 & 0.38 \\
\hline $4 \quad 13$ & 0.00 & -0.03 & 0.38 \\
\hline $5 \quad 13$ & 0.00 & -0.03 & -0.38 \\
\hline $6 \quad 13$ & 0.00 & -0.36 & 0.00 \\
\hline $7 \quad 14$ & 0.00 & 0.23 & 0.00 \\
\hline 813 & 0.00 & -0.36 & 0.00 \\
\hline $9 \quad 14$ & 0.00 & 0.23 & 0.00 \\
\hline
\end{tabular}

B3U

Frequencies -- 12.5713

Red. masses -- 30.8843

Frc consts -- 0.0029

IR Inten $\quad$-- 3.5028

Atom AN $\mathrm{X} \quad \mathrm{Y}$

$\begin{array}{lllll}1 & 19 & 0.54 & 0.00 & 0.00\end{array}$

$\begin{array}{lllll}2 & 13 & -0.12 & 0.00 & 0.00\end{array}$

$\begin{array}{lllll}3 & 13 & -0.12 & 0.00 & 0.00\end{array}$
$-22.4300$

27.0269

0.0080

0.0000

$\mathrm{X} \quad \mathrm{Y}$

$\begin{array}{lll}0.00 & 0.00 & 0.00\end{array}$

$\begin{array}{lll}0.00 & 0.10 & 0.37\end{array}$

$\begin{array}{lll}0.00 & 0.10 & -0.37\end{array}$

$\begin{array}{lll}0.00 & -0.10 & 0.37\end{array}$

$\begin{array}{lll}0.00 & -0.10 & -0.37\end{array}$

$\begin{array}{lll}0.00 & 0.43 & 0.00\end{array}$

$\begin{array}{lll}0.00 & -0.15 & 0.00\end{array}$

$\begin{array}{lll}0.00 & -0.43 & 0.00\end{array}$

0.00

$0.15 \quad 0.00$

5

B2U

20.1041

35.3319

0.0084

8.6274

X Y

$\begin{array}{lrr}0.00 & 0.83 & 0.00\end{array}$

$\begin{array}{lll}0.00 & -0.15 & 0.15\end{array}$

$\begin{array}{lll}0.00 & -0.15 & -0.15\end{array}$
AU

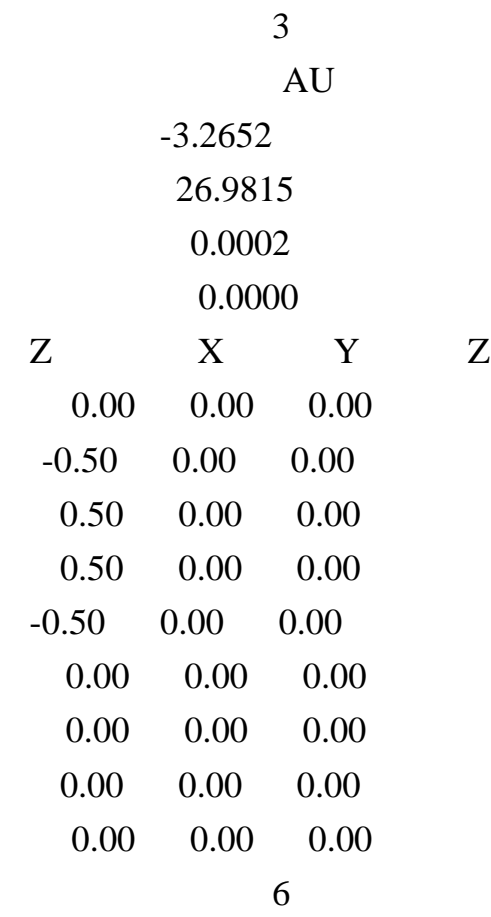

B2G

\subsection{5}

27.2398

0.0100

0.0000

$\begin{array}{llll}\mathrm{Z} & \mathrm{X} & \mathrm{Y}\end{array}$

$\begin{array}{lll}0.00 & 0.00 & 0.00\end{array}$

$\begin{array}{lll}0.03 & 0.00 & 0.00\end{array}$

$\begin{array}{lll}0.03 & 0.00 & 0.00\end{array}$ 


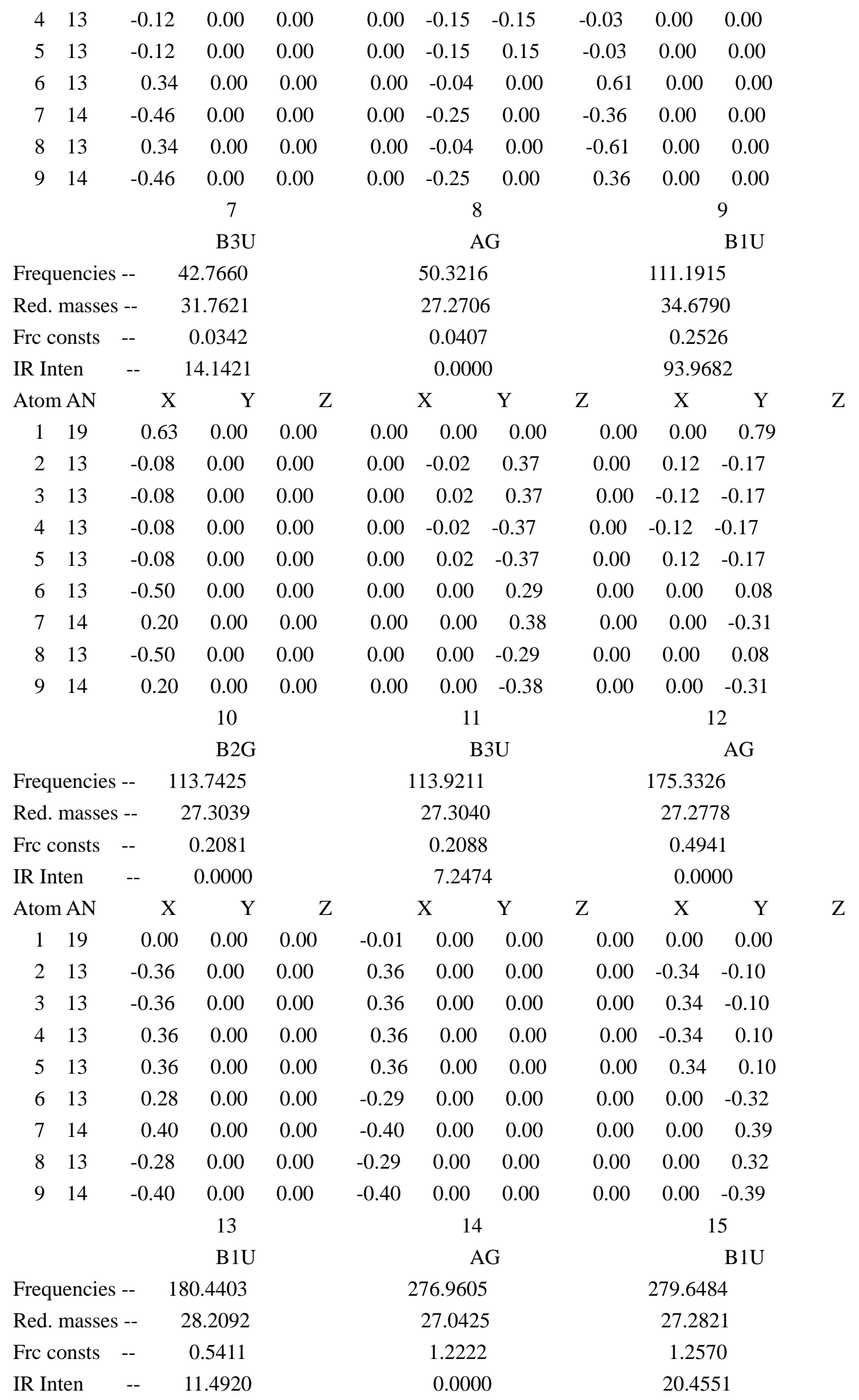




\begin{tabular}{|c|c|c|c|c|c|c|c|c|c|c|c|}
\hline \multicolumn{2}{|c|}{ Atom AN } & $\mathrm{X}$ & $\mathrm{Y}$ & $\mathrm{Z}$ & \multicolumn{2}{|r|}{$\mathrm{X}$} & $\mathrm{Y}$ & $\mathrm{Z}$ & $X$ & $\mathrm{Y}$ & Z \\
\hline 1 & 19 & 0.00 & 0.00 & -0.29 & 0.00 & 0.00 & 0.00 & 0.00 & 0.00 & 0.14 & \\
\hline 2 & 13 & 0.00 & 0.33 & 0.15 & 0.00 & -0.05 & -0.31 & 0.00 & 0.03 & 0.30 & \\
\hline 3 & 13 & 0.00 & -0.33 & 0.15 & 0.00 & 0.05 & -0.31 & 0.00 & -0.03 & 0.30 & \\
\hline 4 & 13 & 0.00 & -0.33 & 0.15 & 0.00 & -0.05 & 0.31 & 0.00 & -0.03 & 0.30 & \\
\hline 5 & 13 & 0.00 & 0.33 & 0.15 & 0.00 & 0.05 & 0.31 & 0.00 & 0.03 & 0.30 & \\
\hline 6 & 13 & 0.00 & 0.00 & 0.27 & 0.00 & 0.00 & 0.52 & 0.00 & 0.00 & -0.53 & \\
\hline 7 & 14 & 0.00 & 0.00 & -0.35 & 0.00 & 0.00 & 0.17 & 0.00 & 0.00 & -0.17 & \\
\hline 8 & 13 & 0.00 & 0.00 & 0.27 & 0.00 & 0.00 & -0.52 & 0.00 & 0.00 & -0.53 & \\
\hline \multirow[t]{3}{*}{9} & 14 & 0.00 & 0.00 & -0.35 & 0.00 & 0.00 & -0.17 & 0.00 & 0.00 & -0.17 & \\
\hline & & & 16 & & & 17 & & & & 18 & \\
\hline & & & B3G & & & & $2 \mathrm{U}$ & & & AG & \\
\hline \multicolumn{2}{|c|}{ Frequencies -- } & \multicolumn{2}{|c|}{296.9017} & & \multicolumn{3}{|c|}{297.0300} & & \multicolumn{2}{|c|}{369.8881} & \\
\hline \multicolumn{2}{|c|}{ Red. masses -- } & \multicolumn{2}{|c|}{26.9822} & & \multicolumn{3}{|c|}{26.9844} & & \multicolumn{2}{|c|}{27.3056} & \\
\hline \multicolumn{2}{|c|}{ Frc consts } & -- & 1.4014 & & \multicolumn{3}{|c|}{1.4027} & & \multicolumn{2}{|c|}{2.2011} & \\
\hline \multicolumn{2}{|c|}{ IR Inten } & -- & 0.0000 & & \multicolumn{3}{|c|}{2.8278} & & \multicolumn{2}{|c|}{0.0000} & \\
\hline \multicolumn{2}{|c|}{ Atom AN } & $\mathrm{X}$ & $\mathrm{Y}$ & $\mathrm{Z}$ & \multicolumn{2}{|r|}{$\mathrm{X}$} & $\mathrm{Y}$ & $\mathrm{Z}$ & $\mathrm{X}$ & $\mathrm{Y}$ & Z \\
\hline 1 & 19 & 0.00 & 0.00 & 0.00 & 0.00 & -0.01 & 0.00 & 0.00 & 0.00 & 0.00 & \\
\hline 2 & 13 & 0.00 & -0.27 & -0.22 & 0.00 & 0.27 & 0.22 & 0.00 & 0.37 & -0.12 & \\
\hline 3 & 13 & 0.00 & -0.27 & 0.22 & 0.00 & 0.27 & -0.22 & 0.00 & -0.37 & -0.12 & \\
\hline 4 & 13 & 0.00 & 0.27 & -0.22 & 0.00 & 0.27 & -0.22 & 0.00 & 0.37 & 0.12 & \\
\hline 5 & 13 & 0.00 & 0.27 & 0.22 & 0.00 & 0.27 & 0.22 & 0.00 & -0.37 & 0.12 & \\
\hline 6 & 13 & 0.00 & 0.51 & 0.00 & 0.00 & -0.51 & 0.00 & 0.00 & 0.00 & -0.20 & \\
\hline 7 & 14 & 0.00 & 0.02 & 0.00 & 0.00 & -0.02 & 0.00 & 0.00 & 0.00 & 0.40 & \\
\hline 8 & 13 & 0.00 & -0.51 & 0.00 & 0.00 & -0.51 & 0.00 & 0.00 & 0.00 & 0.20 & \\
\hline 9 & 14 & 0.00 & -0.02 & 0.00 & 0.00 & -0.02 & 0.00 & 0.00 & 0.00 & -0.40 & \\
\hline & & & 19 & & & 20 & & & & 21 & \\
\hline & & & B1U & & & & $3 G$ & & & B2U & \\
\hline Freq & uencies & & 0.0518 & & & 418.9646 & & & 419.13 & & \\
\hline Red. & masses & & 27.3146 & & & $27.547 \mathrm{~S}$ & & & 27.54 & & \\
\hline Frc c & onsts & -- & 2.2038 & & & 2.8490 & & & 2.85 & & \\
\hline IR In & ten & -- & 9.0353 & & & 0.000 & & & 13.85 & & \\
\hline Aton & $\mathrm{AN}$ & $\mathrm{X}$ & $\mathrm{Y}$ & Z & & $\mathrm{X}$ & $\mathrm{Y}$ & $\mathrm{Z}$ & $\mathrm{X}$ & $\mathrm{Y}$ & Z \\
\hline 1 & 19 & 0.00 & 0.00 & -0.03 & 0.00 & 0.00 & 0.00 & 0.00 & 0.00 & 0.00 & \\
\hline 2 & 13 & 0.00 & -0.37 & 0.11 & 0.00 & -0.24 & 0.22 & 0.00 & -0.24 & 0.22 & \\
\hline 3 & 13 & 0.00 & 0.37 & 0.11 & 0.00 & -0.24 & -0.22 & 0.00 & -0.24 & -0.22 & \\
\hline 4 & 13 & 0.00 & 0.37 & 0.11 & 0.00 & 0.24 & 0.22 & 0.00 & -0.24 & -0.22 & \\
\hline 5 & 13 & 0.00 & -0.37 & 0.11 & 0.00 & 0.24 & -0.22 & 0.00 & -0.24 & 0.22 & \\
\hline 6 & 13 & 0.00 & 0.00 & 0.21 & 0.00 & -0.08 & 0.00 & 0.00 & -0.08 & 0.00 & \\
\hline 7 & 14 & 0.00 & 0.00 & -0.40 & 0.00 & 0.53 & 0.00 & 0.00 & 0.53 & 0.00 & \\
\hline 8 & 13 & 0.00 & 0.00 & 0.21 & 0.00 & 0.08 & 0.00 & 0.00 & -0.08 & 0.00 & \\
\hline 9 & 14 & 0.00 & 0.00 & -0.40 & 0.00 & -0.53 & 0.00 & 0.00 & 0.53 & 0.00 & \\
\hline
\end{tabular}


O

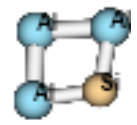

$\begin{array}{ccccc}19 & 0 & 0.000000 & 0.000000 & 0.000000 \\ 13 & 0 & 0.000000 & 3.617128 & 0.000000 \\ 13 & 0 & 0.000000 & -3.617128 & 0.000000 \\ 13 & 0 & 2.455317 & 2.735785 & 0.000000 \\ 13 & 0 & -2.455317 & -2.735785 & 0.000000 \\ 13 & 0 & -0.633680 & -6.089655 & 0.000000 \\ 14 & 0 & -2.783207 & -5.072627 & 0.000000 \\ 13 & 0 & 0.633680 & 6.089655 & 0.000000 \\ 14 & 0 & 2.783207 & 5.072627 & 0.000000\end{array}$

State $=1-A G \mid H F=-2633.7158605$

1

$\mathrm{AU}$

Frequencies -- $\quad-2.8198$

Red. masses -- $\quad 27.1520$

Frc consts -- 0.0001

IR Inten $\quad$-- $\quad 0.1365$

Atom AN $\quad \mathrm{X} \quad \mathrm{Y}$

$\begin{array}{lllll}1 & 19 & 0.00 & 0.00 & 0.01\end{array}$

213

$\begin{array}{lll}0.00 & 0.00 & 0.33\end{array}$

$\begin{array}{lll}0.00 & 0.00 & 0.33\end{array}$

313

$4 \quad 13$

0.00

$0.00-0.41$

513

$\begin{array}{lll}0.00 & 0.00 & -0.41\end{array}$

$\begin{array}{lll}0.00 & 0.00 & 0.37\end{array}$

$\begin{array}{lll}0.00 & 0.00 & -0.29\end{array}$

$\begin{array}{lll}0.00 & 0.00 & 0.37\end{array}$

0.00

$\begin{array}{ll}0.00 & -0.29\end{array}$

4

BG

Frequencies -- 32.9656

Red. masses -- 27.1133

Frc consts --- 0.0174

IR Inten $\quad$-- $\quad 0.0000$

2

3

AU

$\mathrm{BU}$

15.6532

17.1639

30.6559

31.0351

0.0044

0.0054

3.5832

4.8307

$\mathrm{X} \quad \mathrm{Y}$

Z $\begin{array}{llllll}0.00 & 0.00 & 0.53 & 0.54 & -0.18 & 0.00\end{array}$

$\begin{array}{llllll}0.00 & 0.00 & 0.24 & 0.02 & -0.20 & 0.00\end{array}$

$\begin{array}{llllll}0.00 & 0.00 & 0.24 & 0.02 & -0.20 & 0.00\end{array}$

$\begin{array}{lll}0.00 & 0.00 & 0.14\end{array}$

$\begin{array}{lll}0.16 & 0.19 & 0.00\end{array}$

$\begin{array}{lll}0.00 & 0.00 & 0.14\end{array}$

$\begin{array}{lll}0.16 & 0.19 & 0.00\end{array}$

$\begin{array}{llllll}0.00 & 0.00 & -0.37 & -0.36 & -0.10 & 0.00\end{array}$

$\begin{array}{lll}0.00 & 0.00 & -0.38\end{array}$

$\begin{array}{lll}-0.20 & 0.24 & 0.00\end{array}$

$\begin{array}{llllll}0.00 & 0.00 & -0.37 & -0.36 & -0.10 & 0.00\end{array}$

0.00

$0.00 \quad-0.38$

$-0.20$

$0.24 \quad 0.00$

5

AG

6

$\mathrm{AU}$

$43.4050 \quad 49.9957$

$27.0265 \quad 31.7986$

$0.0300 \quad 0.0468$

$0.0000 \quad 6.3763$

$\begin{array}{llllllllll}\text { Atom AN } & \text { X } & \text { Y } & \text { Z } & \text { X } & \text { Y } & \text { Z } & \text { X } & \text { Y } & \text { Z }\end{array}$

$\begin{array}{lllllllllll}1 & 19 & 0.00 & 0.00 & 0.00 & 0.00 & 0.00 & 0.00 & 0.00 & 0.00 & 0.63\end{array}$

$\begin{array}{lllllllllll}2 & 13 & 0.00 & 0.00 & -0.50 & 0.30 & -0.33 & 0.00 & 0.00 & 0.00 & -0.42\end{array}$

$\begin{array}{lllllllllll}3 & 13 & 0.00 & 0.00 & 0.50 & -0.30 & 0.33 & 0.00 & 0.00 & 0.00 & -0.42\end{array}$

$\begin{array}{lllllllllll}4 & 13 & 0.00 & 0.00 & -0.36 & 0.42 & 0.04 & 0.00 & 0.00 & 0.00 & -0.31\end{array}$ 


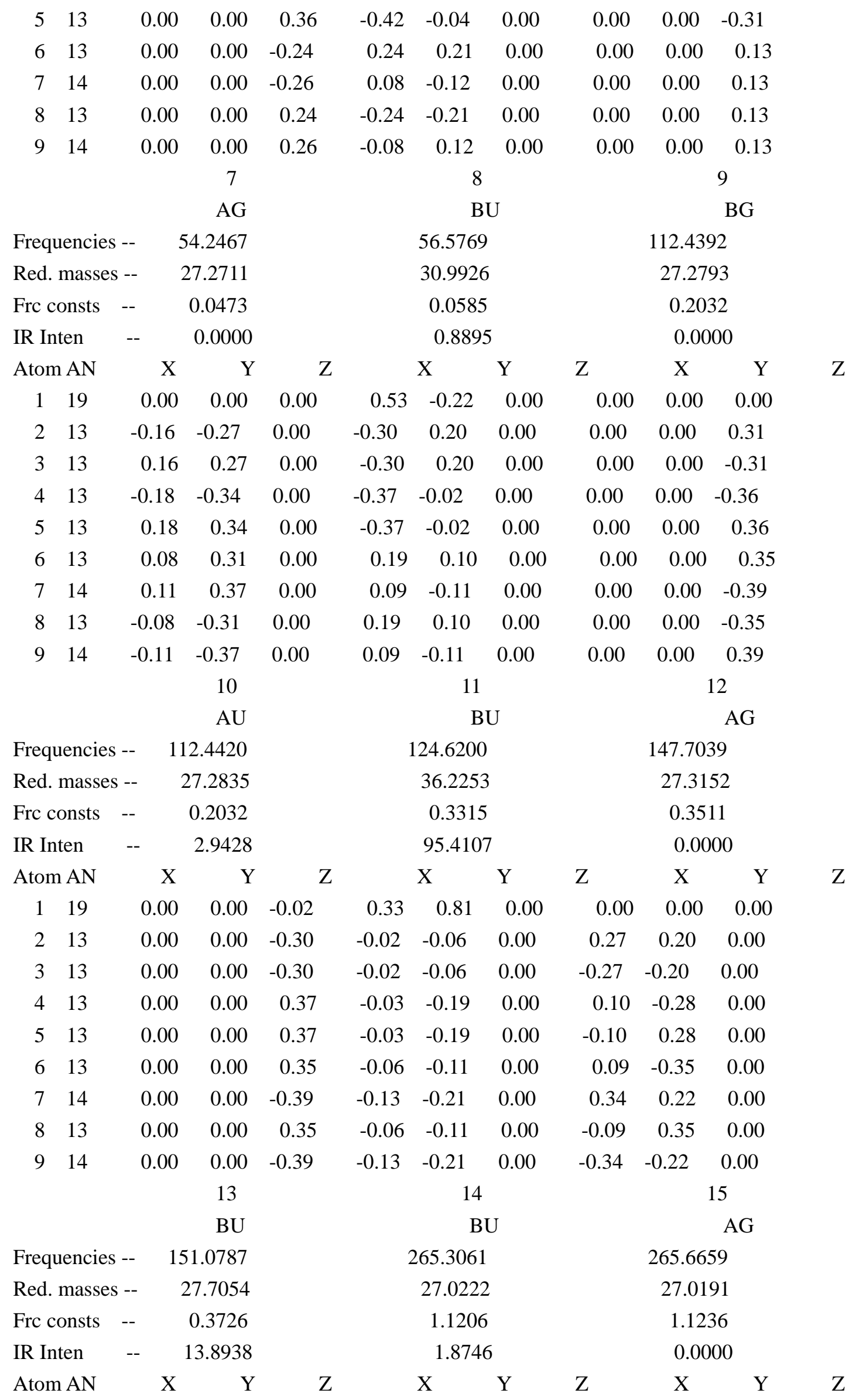




\begin{tabular}{|c|c|c|c|c|c|c|c|c|c|c|c|}
\hline 1 & 19 & -0.08 & 0.17 & 0.00 & 0.02 & 0.01 & 0.00 & 0.00 & 0.00 & 0.00 & \\
\hline 2 & 13 & -0.27 & -0.21 & 0.00 & 0.48 & 0.03 & 0.00 & 0.48 & 0.03 & 0.00 & \\
\hline 3 & 13 & -0.27 & -0.21 & 0.00 & 0.48 & 0.03 & 0.00 & -0.48 & -0.03 & 0.00 & \\
\hline 4 & 13 & -0.10 & 0.25 & 0.00 & -0.46 & 0.03 & 0.00 & -0.46 & 0.03 & 0.00 & \\
\hline 5 & 13 & -0.10 & 0.25 & 0.00 & -0.46 & 0.03 & 0.00 & 0.46 & -0.03 & 0.00 & \\
\hline 6 & 13 & 0.08 & -0.37 & 0.00 & -0.17 & -0.11 & 0.00 & 0.17 & 0.11 & 0.00 & \\
\hline 7 & 14 & 0.33 & 0.20 & 0.00 & 0.13 & 0.05 & 0.00 & -0.13 & -0.05 & 0.00 & \\
\hline 8 & 13 & 0.08 & -0.37 & 0.00 & -0.17 & -0.11 & 0.00 & -0.17 & -0.11 & 0.00 & \\
\hline 9 & 14 & 0.33 & 0.20 & 0.00 & 0.13 & 0.05 & 0.00 & 0.13 & 0.05 & 0.00 & \\
\hline & & & 16 & & \multicolumn{3}{|c|}{17} & \multicolumn{4}{|c|}{18} \\
\hline & & & AG & & \multicolumn{3}{|c|}{$\mathrm{BU}$} & \multicolumn{4}{|c|}{ AG } \\
\hline \multicolumn{2}{|c|}{ Frequencies -- } & 30 & 7.5932 & & \multicolumn{3}{|c|}{308.1476} & \multicolumn{4}{|c|}{374.6644} \\
\hline \multicolumn{2}{|c|}{ Red. masses -- } & & 26.9894 & & \multicolumn{3}{|c|}{27.0241} & \multicolumn{4}{|c|}{27.3423} \\
\hline \multicolumn{2}{|c|}{ Frc consts } & -- & 1.5045 & & \multicolumn{3}{|c|}{1.5119} & \multicolumn{4}{|c|}{2.2614} \\
\hline \multicolumn{2}{|c|}{ IR Inten } & -- & 0.0000 & & \multicolumn{3}{|c|}{4.3654} & \multicolumn{4}{|c|}{0.0000} \\
\hline \multicolumn{2}{|c|}{ Atom AN } & $X$ & $\mathrm{Y}$ & Z & & $X$ & $\mathrm{Y}$ & Z & $\mathrm{X}$ & Y & $\mathrm{Z}$ \\
\hline 1 & 19 & 0.00 & 0.00 & 0.00 & 0.00 & -0.05 & 0.00 & 0.00 & 0.00 & 0.00 & \\
\hline 2 & 13 & -0.03 & 0.52 & 0.00 & -0.03 & 0.52 & 0.00 & 0.12 & 0.05 & 0.00 & \\
\hline 3 & 13 & 0.03 & -0.52 & 0.00 & -0.03 & 0.52 & 0.00 & -0.12 & -0.05 & 0.00 & \\
\hline 4 & 13 & 0.15 & -0.15 & 0.00 & 0.15 & -0.15 & 0.00 & -0.05 & 0.33 & 0.00 & \\
\hline 5 & 13 & -0.15 & 0.15 & 0.00 & 0.15 & -0.15 & 0.00 & 0.05 & -0.33 & 0.00 & \\
\hline 6 & 13 & 0.14 & 0.40 & 0.00 & -0.15 & -0.40 & 0.00 & -0.36 & 0.25 & 0.00 & \\
\hline 7 & 14 & -0.03 & -0.06 & 0.00 & 0.03 & 0.06 & 0.00 & 0.41 & 0.11 & 0.00 & \\
\hline 8 & 13 & -0.14 & -0.40 & 0.00 & -0.15 & -0.40 & 0.00 & 0.36 & -0.25 & 0.00 & \\
\hline \multirow[t]{3}{*}{9} & 14 & 0.03 & 0.06 & 0.00 & 0.03 & 0.06 & 0.00 & -0.41 & -0.11 & 0.00 & \\
\hline & & & 19 & & \multicolumn{3}{|c|}{20} & \multicolumn{4}{|c|}{21} \\
\hline & & & $\mathrm{BU}$ & & \multicolumn{3}{|c|}{$\mathrm{BU}$} & \multicolumn{4}{|c|}{ AG } \\
\hline \multicolumn{2}{|c|}{ Frequencies -- } & 37 & 74.8047 & & & 425.4373 & & & 425.467 & & \\
\hline Red. & masses & & 27.3522 & & & 27.5157 & & & 27.512 & & \\
\hline Frc c & onsts & -- & 2.2639 & & & 2.9343 & & & 2.934 & & \\
\hline IR In & ten & -- & 7.5740 & & & 11.0039 & & & $0.00 c$ & & \\
\hline Atom & AN & $\mathrm{X}$ & $\mathrm{Y}$ & Z & & $\mathrm{X}$ & $\mathrm{Y}$ & Z & $\mathrm{X}$ & $\mathrm{Y}$ & $\mathrm{Z}$ \\
\hline 1 & 19 & -0.01 & -0.03 & 0.00 & -0.01 & -0.02 & 0.00 & 0.00 & 0.00 & 0.00 & \\
\hline 2 & 13 & 0.12 & 0.06 & 0.00 & -0.02 & 0.09 & 0.00 & -0.02 & 0.08 & 0.00 & \\
\hline 3 & 13 & 0.12 & 0.06 & 0.00 & -0.02 & 0.09 & 0.00 & 0.02 & -0.08 & 0.00 & \\
\hline 4 & 13 & -0.04 & 0.33 & 0.00 & 0.10 & 0.38 & 0.00 & 0.10 & 0.38 & 0.00 & \\
\hline 5 & 13 & -0.04 & 0.33 & 0.00 & 0.10 & 0.38 & 0.00 & -0.10 & -0.38 & 0.00 & \\
\hline 6 & 13 & 0.36 & -0.25 & 0.00 & -0.26 & 0.04 & 0.00 & 0.26 & -0.04 & 0.00 & \\
\hline 7 & 14 & -0.41 & -0.11 & 0.00 & 0.18 & -0.48 & 0.00 & -0.18 & 0.48 & 0.00 & \\
\hline 8 & 13 & 0.36 & -0.25 & 0.00 & -0.26 & 0.04 & 0.00 & -0.26 & 0.04 & 0.00 & \\
\hline 9 & 14 & -0.41 & -0.11 & 0.00 & 0.18 & -0.48 & 0.00 & 0.18 & -0.48 & 0.00 & \\
\hline
\end{tabular}




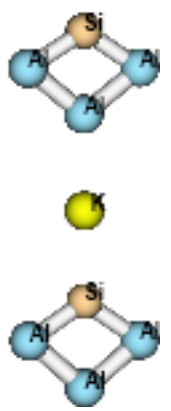

$\begin{array}{rrrrr}19 & 0 & 0.000000 & 0.000000 & 0.158148 \\ 13 & 0 & 0.000000 & 1.967947 & -4.951194 \\ 13 & 0 & 0.000000 & -1.967947 & -4.951194 \\ 13 & 0 & 0.000000 & 0.000000 & 6.500769 \\ 13 & 0 & 0.000000 & 1.935818 & 4.788144 \\ 13 & 0 & 0.000000 & -1.935818 & 4.788144 \\ 14 & 0 & 0.000000 & 0.000000 & 3.395378 \\ 13 & 0 & 0.000000 & 0.000000 & -3.294197 \\ 14 & 0 & 0.000000 & 0.000000 & -6.284732\end{array}$

State $=1-A 1 \backslash H F=-2633.7155456$

B2

B2

A2

Frequencies -- $\quad-22.3694$

$-12.2860$

$-3.1033$

Red. masses -- 27.3548

27.2421

26.9815

Frc consts -- 0.0081

0.0024

0.0002

IR Inten $\quad--\quad 0.4826$

0.0822

0.0000

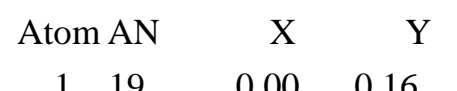

Z

$\mathrm{X} \quad \mathrm{Y}$

119

$\begin{array}{lll}0.00 & 0.16 & 0.00\end{array}$

$\begin{array}{ccc}0.00 & -0.02 & 0.00\end{array}$

$\begin{array}{llll}\mathrm{Z} & \mathrm{X} & \mathrm{Y}\end{array}$

Z

$\begin{array}{lllll}2 & 13 & 0.00 & -0.09 & 0.53\end{array}$

$\begin{array}{lll}0.00 & -0.08 & -0.06\end{array}$

$\begin{array}{lll}0.00 & 0.00 & 0.00\end{array}$

$\begin{array}{llllll}3 & 13 & 0.00 & -0.09 & -0.53\end{array}$

$\begin{array}{llll}0.00 & -0.08 & 0.06\end{array}$

$\begin{array}{lll}-0.50 & 0.00 & 0.00\end{array}$

$\begin{array}{lllll}4 & 13 & 0.00 & 0.03 & 0.00\end{array}$

$\begin{array}{lll}0.00 & -0.38 & 0.00\end{array}$

$\begin{array}{lll}0.50 & 0.00 & 0.00\end{array}$

$\begin{array}{lllll}5 & 13 & 0.00 & 0.06 & 0.03\end{array}$

$\begin{array}{lll}0.00 & 0.10 & 0.53\end{array}$

$\begin{array}{lll}0.00 & 0.00 & 0.00\end{array}$

$\begin{array}{lllll}6 & 13 & 0.00 & 0.06 & -0.03\end{array}$

$\begin{array}{lll}0.00 & 0.10 & -0.53\end{array}$

$\begin{array}{lll}0.50 & 0.00 & 0.00\end{array}$

$\begin{array}{lllll}7 & 14 & 0.00 & 0.08 & 0.00\end{array}$

$\begin{array}{lll}0.00 & 0.49 & 0.00\end{array}$

$\begin{array}{lll}-0.50 & 0.00 & 0.00\end{array}$

$8 \quad 13$

$\begin{array}{lll}0.00 & -0.56 & 0.00\end{array}$

$\begin{array}{lll}0.00 & -0.02 & 0.00\end{array}$

$\begin{array}{lll}0.00 & 0.00 & 0.00\end{array}$

$\begin{array}{ll}9 & 14\end{array}$

$\begin{array}{lll}0.00 & 0.26 & 0.00\end{array}$

$\begin{array}{lll}0.00 & -0.12 & 0.00\end{array}$

$\begin{array}{lll}0.00 & 0.00 & 0.00\end{array}$

$\begin{array}{lll}0.00 & 0.00 & 0.00\end{array}$

4

5

6

B1

B1

B2

\begin{tabular}{|c|c|c|c|c|c|c|c|c|c|}
\hline Frequencies & & 8.7528 & & \multicolumn{3}{|c|}{18.6653} & & \multicolumn{2}{|c|}{21.6594} \\
\hline Red. masses & & 29.2226 & & \multicolumn{3}{|c|}{28.5573} & & \multicolumn{2}{|c|}{36.0668} \\
\hline Frc consts & -- & 0.0013 & & \multicolumn{3}{|c|}{0.0059} & & \multicolumn{2}{|c|}{0.0100} \\
\hline IR Inten & -- & 0.5504 & & \multicolumn{3}{|c|}{3.1320} & & \multicolumn{2}{|c|}{8.5964} \\
\hline Atom AN & $\mathrm{X}$ & $\mathrm{Y}$ & Z & & $\mathrm{X}$ & $\mathrm{Y}$ & $\mathrm{Z}$ & $\mathrm{X}$ & $\mathrm{Y}$ \\
\hline 119 & -0.40 & 0.00 & 0.00 & -0.31 & 0.00 & 0.00 & 0.00 & 0.87 & 0.00 \\
\hline 13 & 0.11 & 0.00 & 0.00 & 0.03 & 0.00 & 0.00 & 0.00 & -0.13 & -0.13 \\
\hline 13 & 0.11 & 0.00 & 0.00 & 0.03 & 0.00 & 0.00 & 0.00 & -0.13 & 0.13 \\
\hline
\end{tabular}




$\begin{array}{rrrrrrrrrrr}4 & 13 & 0.67 & 0.00 & 0.00 & -0.23 & 0.00 & 0.00 & 0.00 & -0.19 & 0.00 \\ 5 & 13 & 0.02 & 0.00 & 0.00 & 0.14 & 0.00 & 0.00 & 0.00 & -0.18 & 0.02 \\ 6 & 13 & 0.02 & 0.00 & 0.00 & 0.14 & 0.00 & 0.00 & 0.00 & -0.18 & -0.02 \\ 7 & 14 & -0.53 & 0.00 & 0.00 & 0.46 & 0.00 & 0.00 & 0.00 & -0.17 & 0.00 \\ 8 & 13 & -0.12 & 0.00 & 0.00 & -0.61 & 0.00 & 0.00 & 0.00 & -0.04 & 0.00 \\ 9 & 14 & 0.29 & 0.00 & 0.00 & 0.47 & 0.00 & 0.00 & 0.00 & -0.22 & 0.00 \\ & & & 7 & & & 8 & & & & 9\end{array}$

B1 A1

B1

\begin{tabular}{|c|c|c|c|}
\hline \multicolumn{2}{|c|}{ Frequencies -- } & 40.8868 & \\
\hline \multicolumn{2}{|c|}{ Red. masses -- } & 2.1631 & \\
\hline Frc consts & -- & .0317 & \\
\hline IR Inten & -- & 6.0794 & \\
\hline Atom AN & $\mathrm{X}$ & $\mathrm{Y}$ & Z \\
\hline $1 \quad 19$ & 0.65 & 0.00 & 0.00 \\
\hline 213 & -0.09 & 0.00 & 0.00 \\
\hline 313 & -0.09 & 0.00 & 0.00 \\
\hline $4 \quad 13$ & 0.13 & 0.00 & 0.00 \\
\hline 513 & -0.12 & 0.00 & 0.00 \\
\hline $6 \quad 13$ & -0.12 & 0.00 & 0.00 \\
\hline $7 \quad 14$ & -0.30 & 0.00 & 0.00 \\
\hline 813 & -0.61 & 0.00 & 0.00 \\
\hline $9 \quad 14$ & 0.26 & 0.00 & 0.00 \\
\hline & & 10 & \\
\hline
\end{tabular}

A1

52.2273

27.2592

0.0438

114.0556

27.3015

1.4061

0.2093

3.3089

\begin{tabular}{cccccc}
\multicolumn{2}{c}{$\mathrm{X}$} & $\mathrm{Y}$ & $\mathrm{Z}$ & $\mathrm{X}$ & $\mathrm{Y}$ \\
0.00 & 0.00 & 0.06 & -0.01 & 0.00 & 0.00 \\
0.00 & -0.02 & -0.38 & 0.51 & 0.00 & 0.00 \\
0.00 & 0.02 & -0.38 & 0.51 & 0.00 & 0.00 \\
0.00 & 0.00 & 0.38 & 0.02 & 0.00 & 0.00 \\
0.00 & -0.03 & 0.35 & -0.02 & 0.00 & 0.00 \\
0.00 & 0.03 & 0.35 & -0.02 & 0.00 & 0.00 \\
0.00 & 0.00 & 0.28 & 0.01 & 0.00 & 0.00 \\
0.00 & 0.00 & -0.29 & -0.41 & 0.00 & 0.00 \\
0.00 & 0.00 & -0.40 & -0.57 & 0.00 & 0.00 \\
& 11 & & & \multicolumn{2}{c}{12}
\end{tabular}

B1

A1

Frequencies -- 115.7595

118.8825

172.3618

27.2582

27.2834

0.2270

0.4776

Frc consts -- 0.2656

3.7423

$\begin{array}{llr}\text { IR Inten } & -- & 45.4976 \\ \text { Atom AN } & & \text { X }\end{array}$

$\begin{array}{ccccc}\text { Atom } & \text { AN } & \text { X } & \text { Y } & \text { Z } \\ 1 & 19 & 0.00 & 0.00 & 0.74 \\ 2 & 13 & 0.00 & -0.12 & -0.13 \\ 3 & 13 & 0.00 & 0.12 & -0.13 \\ 4 & 13 & 0.00 & 0.00 & -0.39 \\ 5 & 13 & 0.00 & 0.19 & -0.18 \\ 6 & 13 & 0.00 & -0.19 & -0.18 \\ 7 & 14 & 0.00 & 0.00 & 0.13 \\ 8 & 13 & 0.00 & 0.00 & 0.10 \\ 9 & 14 & 0.00 & 0.00 & -0.27\end{array}$

A1

\begin{tabular}{ccc}
\multicolumn{2}{c}{$\mathrm{X}$} & $\mathrm{Y}$ \\
0.01 & 0.00 & 0.00 \\
0.01 & 0.00 & 0.00 \\
0.01 & 0.00 & 0.00 \\
-0.46 & 0.00 & 0.00 \\
0.50 & 0.00 & 0.00 \\
0.50 & 0.00 & 0.00 \\
-0.53 & 0.00 & 0.00 \\
-0.02 & 0.00 & 0.00 \\
-0.02 & 0.00 & 0.00 \\
\multicolumn{3}{c}{14}
\end{tabular}

38.6044

A1

Frequencies -- $\quad 184.2507$

263.7710

27.0809

1.1101

4.2125

\begin{tabular}{ccc}
$Z$ & $X$ & $Y$ \\
0.00 & 0.00 & 0.02 \\
0.00 & -0.39 & 0.11 \\
0.00 & 0.39 & 0.11 \\
0.00 & 0.00 & 0.30 \\
0.00 & -0.27 & -0.06 \\
0.00 & 0.27 & -0.06 \\
0.00 & 0.00 & -0.33 \\
0.00 & 0.00 & 0.37 \\
0.00 & 0.00 & -0.44 \\
\multicolumn{3}{c}{15}
\end{tabular}

A1

277.5196

27.1613

1.2325

11.3942 


\begin{tabular}{|c|c|c|c|c|c|c|c|c|c|c|}
\hline \multicolumn{2}{|c|}{ Atom AN } & $\mathrm{X}$ & $\mathrm{Y}$ & $\mathrm{Z}$ & \multicolumn{2}{|c|}{$\mathrm{X}$} & Y & Z & $\mathrm{X}$ & $\mathrm{Y}$ \\
\hline 1 & 19 & 0.00 & 0.00 & 0.37 & 0.00 & 0.00 & -0.05 & 0.00 & 0.00 & 0.10 \\
\hline 2 & 13 & 0.00 & 0.28 & -0.13 & 0.00 & -0.01 & 0.02 & 0.00 & -0.07 & 0.43 \\
\hline 3 & 13 & 0.00 & -0.28 & -0.13 & 0.00 & 0.01 & 0.02 & 0.00 & 0.07 & 0.43 \\
\hline 4 & 13 & 0.00 & 0.00 & 0.35 & 0.00 & 0.00 & 0.64 & 0.00 & 0.00 & -0.02 \\
\hline 5 & 13 & 0.00 & -0.33 & -0.14 & 0.00 & 0.30 & -0.42 & 0.00 & -0.02 & 0.01 \\
\hline 6 & 13 & 0.00 & 0.33 & -0.14 & 0.00 & -0.30 & -0.42 & 0.00 & 0.02 & 0.01 \\
\hline 7 & 14 & 0.00 & 0.00 & -0.40 & 0.00 & 0.00 & 0.26 & 0.00 & 0.00 & -0.01 \\
\hline 8 & 13 & 0.00 & 0.00 & -0.21 & 0.00 & 0.00 & -0.03 & 0.00 & 0.00 & -0.74 \\
\hline \multirow[t]{3}{*}{9} & 14 & 0.00 & 0.00 & 0.28 & 0.00 & 0.00 & -0.01 & 0.00 & 0.00 & -0.24 \\
\hline & & & 16 & & \multicolumn{3}{|c|}{17} & \multicolumn{3}{|c|}{18} \\
\hline & & & B2 & & \multicolumn{3}{|c|}{ B2 } & & \multicolumn{2}{|c|}{ A1 } \\
\hline \multicolumn{2}{|c|}{ Frequencies -- } & \multicolumn{2}{|c|}{297.0542} & & \multicolumn{3}{|c|}{300.3067} & & \multicolumn{2}{|c|}{369.7872} \\
\hline \multicolumn{2}{|c|}{ Red. masses -- } & \multicolumn{2}{|c|}{26.9834} & & \multicolumn{3}{|c|}{26.9851} & & \multicolumn{2}{|c|}{27.3110} \\
\hline \multicolumn{2}{|c|}{ Frc consts } & \multicolumn{2}{|c|}{1.4029} & & \multicolumn{3}{|c|}{1.4339} & & \multicolumn{2}{|c|}{2.2004} \\
\hline \multicolumn{2}{|c|}{ IR Inten } & - & 1.5218 & & & 3.8588 & & & 6.01 & \\
\hline Atom & AN & X & $\mathrm{Y}$ & Z & & $\mathrm{X}$ & $\mathrm{Y}$ & $\mathrm{Z}$ & $\mathrm{X}$ & $\mathrm{Y}$ \\
\hline 1 & 19 & 0.00 & -0.01 & 0.00 & 0.00 & 0.00 & 0.00 & 0.00 & 0.00 & -0.02 \\
\hline 2 & 13 & 0.00 & 0.38 & -0.31 & 0.00 & 0.00 & 0.00 & 0.00 & 0.52 & 0.17 \\
\hline 3 & 13 & 0.00 & 0.38 & 0.31 & 0.00 & 0.00 & 0.00 & 0.00 & -0.52 & 0.17 \\
\hline 4 & 13 & 0.00 & 0.00 & 0.00 & 0.00 & 0.72 & 0.00 & 0.00 & 0.00 & 0.01 \\
\hline 5 & 13 & 0.00 & 0.00 & 0.00 & 0.00 & -0.39 & 0.30 & 0.00 & 0.02 & 0.01 \\
\hline 6 & 13 & 0.00 & 0.00 & 0.00 & 0.00 & -0.39 & -0.30 & 0.00 & -0.02 & 0.01 \\
\hline 7 & 14 & 0.00 & 0.00 & 0.00 & 0.00 & 0.06 & 0.00 & 0.00 & 0.00 & -0.03 \\
\hline 8 & 13 & 0.00 & -0.72 & 0.00 & 0.00 & 0.00 & 0.00 & 0.00 & 0.00 & 0.28 \\
\hline 9 & 14 & 0.00 & -0.03 & 0.00 & 0.00 & 0.00 & 0.00 & 0.00 & 0.00 & -0.57 \\
\hline & & & 19 & & & 20 & & & & 1 \\
\hline & & & $\mathrm{A} 1$ & & & B2 & & & & $\mathrm{B} 2$ \\
\hline Frequ & encies & & 1.5164 & & & 98.9013 & & & 418.318 & \\
\hline Red. & masses & & 7.4664 & & & 27.5234 & & & 27.546 & \\
\hline Frc C & onsts & - & 2.3555 & & & 2.5804 & & & 2.840 & \\
\hline IR In & & - & 5.7856 & & & 7.6446 & & & 6.712 & \\
\hline Atom & AN & $\mathrm{X}$ & $\mathrm{Y}$ & Z & & $\mathrm{X}$ & $\mathrm{Y}$ & Z & $\mathrm{X}$ & $\mathrm{Y}$ \\
\hline 1 & 19 & 0.00 & 0.00 & 0.06 & 0.00 & -0.01 & 0.00 & 0.00 & 0.00 & 0.00 \\
\hline 2 & 13 & 0.00 & -0.02 & -0.01 & 0.00 & 0.00 & 0.00 & 0.00 & 0.33 & 0.31 \\
\hline 3 & 13 & 0.00 & 0.02 & -0.01 & 0.00 & 0.00 & 0.00 & 0.00 & 0.33 & -0.31 \\
\hline 4 & 13 & 0.00 & 0.00 & 0.16 & 0.00 & 0.13 & 0.00 & 0.00 & 0.00 & 0.00 \\
\hline 5 & 13 & 0.00 & 0.46 & 0.23 & 0.00 & 0.32 & 0.34 & 0.00 & 0.00 & 0.00 \\
\hline 6 & 13 & 0.00 & -0.46 & 0.23 & 0.00 & 0.32 & -0.34 & 0.00 & 0.00 & 0.00 \\
\hline 7 & 14 & 0.00 & 0.00 & -0.67 & 0.00 & -0.74 & 0.00 & 0.00 & 0.00 & 0.00 \\
\hline 8 & 13 & 0.00 & 0.00 & -0.02 & 0.00 & 0.00 & 0.00 & 0.00 & 0.11 & 0.00 \\
\hline 9 & 14 & 0.00 & 0.00 & 0.03 & 0.00 & 0.00 & 0.00 & 0.00 & -0.75 & 0.00 \\
\hline
\end{tabular}




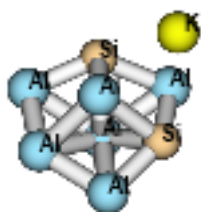

$\begin{array}{rrrrr}13 & 0 & 2.650828 & 0.043249 & -0.996981 \\ 19 & 0 & -3.300293 & 0.002800 & -1.010914 \\ 13 & 0 & 1.120811 & -0.001476 & 1.299812 \\ 13 & 0 & -0.057326 & 0.001796 & -1.305868 \\ 14 & 0 & -0.517640 & 1.844983 & 0.480674 \\ 13 & 0 & -1.552028 & -0.050670 & 1.768928 \\ 13 & 0 & 1.820745 & 2.351948 & -0.121994 \\ 14 & 0 & -0.460482 & -1.885115 & 0.444845 \\ 13 & 0 & 1.893836 & -2.305719 & -0.163121\end{array}$

State $=1-A \backslash H F=-2633.7995536$

Al3SiKAl3Si-fusion106-b36311+gx.frq

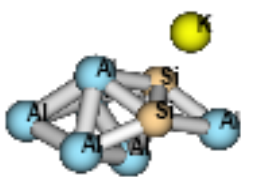

13
19
13
13
14
13
13
13
14$$
0.055888-1.965395
$$$$
\begin{array}{lll}
3.048139 & -1.586900 & -0.000029
\end{array}
$$$$
\begin{array}{lll}
-0.977948 & 1.748217 & 0.000026
\end{array}
$$$$
\begin{array}{lll}
-1.748355 & 0.055827 & 1.965405
\end{array}
$$$$
\begin{array}{lll}
0.814394 & 0.315325 & 1.262561
\end{array}
$$$$
\begin{array}{lll}
2.192486 & 2.113630 & 0.000036
\end{array}
$$$$
\begin{array}{lll}
-3.392715 & -0.888874 & -0.000008
\end{array}
$$$$
\begin{array}{lll}
-0.534159 & -1.444579 & -0.000029
\end{array}
$$$$
\begin{array}{lll}
0.814399 & 0.315366 & -1.262554
\end{array}
$$

State $=1-A \backslash H F=-2633.7793968$

Al3SiKAl3Si-fusion110-b36311+gx.frq

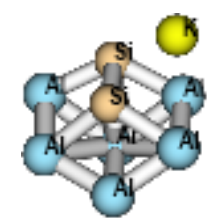

13

13

19

13

13

14

14

13

13

0
0

0.814399

0.315366

$-1.262554$ 


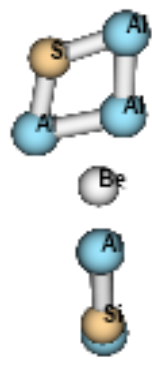

13

13

13

13

4

13

13

14

14

State $=1-A \backslash H F=-2048.409137$
A

$\begin{array}{llr}\text { Frequencies -- } & 21.9643 \\ \text { Red. masses -- } & 22.5136 \\ \text { Frc consts } & -- & 0.0064 \\ \text { IR Inten } & -- & 0.1188\end{array}$

$\begin{array}{rcc}4.648547 & -0.872502 & -0.902451 \\ 2.148885 & -0.660308 & -0.760364 \\ -4.648551 & 0.872500 & -0.902450 \\ -2.148885 & 0.660295 & -0.760370 \\ 0.000000 & -0.000006 & -0.233473 \\ 1.735081 & 1.230787 & 0.986943 \\ -1.735080 & -1.230782 & 0.986954 \\ 4.015771 & 0.772148 & 0.660939 \\ -4.015769 & -0.772137 & 0.660953\end{array}$

2

3

A

A

22.1946

22.7333

33.5942

26.8062

0.0066

0.0178

0.0916

X Y

$\mathrm{Z} \quad \mathrm{X}$

Atom AN $\mathrm{X} \quad \mathrm{Y}$

113

$\begin{array}{lll}0.17 & 0.32 & -0.08\end{array}$

$0.11-0.11$

0.36

$-0.03$

$\begin{array}{ll}-0.19 & 0.18\end{array}$

213

$\begin{array}{lll}0.12 & -0.27 & 0.11\end{array}$

$\begin{array}{lll}0.08 & 0.07 & -0.23\end{array}$

$\begin{array}{lll}-0.02 & -0.21 & 0.29\end{array}$

313

$\begin{array}{lll}0.17 & 0.32 & 0.08\end{array}$

$\begin{array}{lll}-0.11 & 0.11 & 0.36\end{array}$

$\begin{array}{lll}0.03 & 0.19 & 0.18\end{array}$

$4 \quad 13$

$\begin{array}{lll}0.12 & -0.27 & -0.11\end{array}$

$\begin{array}{lll}-0.08 & -0.07 & -0.23\end{array}$

0.02

$0.21 \quad 0.29$

$\begin{array}{lllll}5 & 4 & 0.03 & -0.50 & 0.00\end{array}$

$\begin{array}{lll}0.00 & 0.00 & -0.49\end{array}$

$\begin{array}{lll}0.00 & 0.00 & 0.14\end{array}$

$\begin{array}{ll}6 & 13\end{array}$

$\begin{array}{lll}-0.18 & -0.19 & -0.04\end{array}$

$\begin{array}{lll}-0.20 & 0.02 & -0.25\end{array}$

0.05

$\begin{array}{ll}0.35 & -0.29\end{array}$

$\begin{array}{lllll}7 & 13 & -0.18 & -0.19 & 0.04\end{array}$

$\begin{array}{lll}0.20 & -0.02 & -0.25\end{array}$

$\begin{array}{lll}-0.05 & -0.35 & -0.29\end{array}$

$8 \quad 14$

$-0.11$

$0.21-0.08$

$-0.15-0.05$

0.19

0.03

$0.20-0.20$

$\begin{array}{ll}9 & 14\end{array}$
$\begin{array}{lll}-0.11 & 0.21 & 0.07\end{array}$
4

A

$\begin{array}{lll}0.15 & 0.05 & 0.19\end{array}$ 5

A

82.4931

26.6949

0.1070

2.3039

IR Inten -- 2.7306

$\begin{array}{llll}\text { Atom AN } & X & Y & \text { Z }\end{array}$

$\begin{array}{lllll}1 & 13 & -0.07 & 0.20 & -0.27\end{array}$

$\mathrm{X} \quad \mathrm{Y}$

$\begin{array}{lll}-0.03 & -0.28 & 0.22\end{array}$

$\mathrm{Z}$

0.15

$\begin{array}{lll}-0.03 & -0.20 & -0.20\end{array}$

$\begin{array}{lllll}2 & 13 & -0.06 & -0.21 & 0.30\end{array}$

$\begin{array}{lll}0.01 & 0.33 & -0.21\end{array}$

$\begin{array}{llll}0.08 & 0.06 & -0.40\end{array}$

$\begin{array}{lllll}3 & 13 & -0.07 & 0.20 & 0.27\end{array}$

$\begin{array}{lll}0.03 & 0.28 & 0.22\end{array}$
6

A

104.9186

26.3457

0.1709

1.5174

$\begin{array}{ll}\mathrm{X} & \mathrm{Y}\end{array}$

Z 


$$
\begin{array}{rrrrr}
4 & 13 & -0.06 & -0.21 & -0.30 \\
5 & 4 & -0.01 & 0.18 & 0.00 \\
6 & 13 & 0.08 & 0.25 & -0.16 \\
7 & 13 & 0.08 & 0.25 & 0.16 \\
8 & 14 & 0.04 & -0.26 & 0.26 \\
9 & 14 & 0.04 & -0.26 & -0.26
\end{array}
$$

A

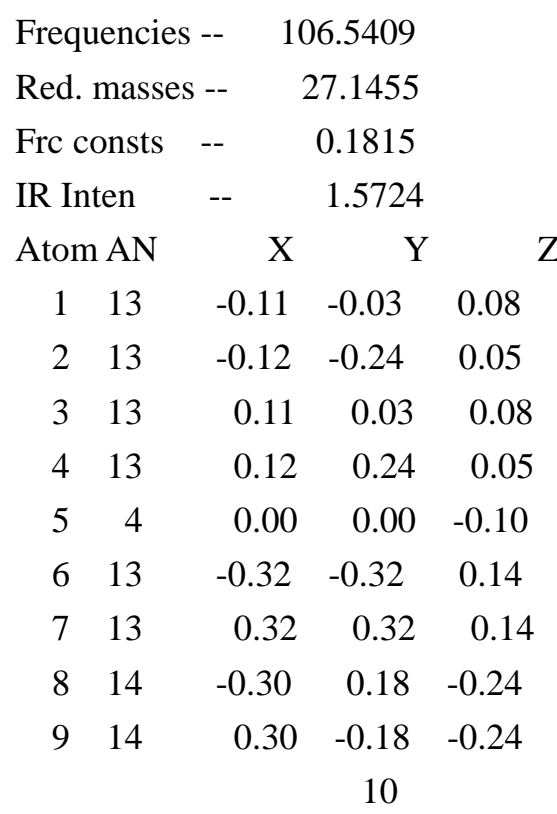

A

Frequencies -- 162.4125

Red. masses -- $\quad 25.9020$

Frc consts -- 0.4026

IR Inten $\quad--\quad 3.3551$

Atom AN $\quad \mathrm{X} \quad \mathrm{Y} \quad \mathrm{Z}$

$$
\begin{aligned}
& \begin{array}{lll}
-0.01 & -0.33 & -0.21
\end{array} \\
& \begin{array}{lll}
0.00 & 0.00 & 0.18
\end{array} \\
& \begin{array}{lll}
0.14 & -0.05 & 0.22
\end{array} \\
& \begin{array}{lll}
-0.14 & 0.05 & 0.22
\end{array} \\
& \begin{array}{llll}
0.14 & 0.23 & -0.24
\end{array} \\
& \begin{array}{lll}
-0.14 & -0.23 & -0.24
\end{array}
\end{aligned}
$$

\begin{tabular}{|c|c|c|c|c|c|c|c|c|c|c|}
\hline 1 & 13 & 0.39 & -0.12 & -0.10 & 0.20 & 0.09 & 0.10 & -0.22 & -0.10 & -0.10 \\
\hline 2 & 13 & 0.24 & 0.14 & 0.20 & -0.14 & -0.14 & -0.21 & 0.16 & 0.24 & 0.16 \\
\hline 3 & 13 & -0.39 & 0.12 & -0.10 & -0.20 & -0.09 & 0.10 & -0.22 & -0.10 & 0.10 \\
\hline 4 & 13 & -0.25 & -0.14 & 0.20 & 0.14 & 0.14 & -0.21 & 0.16 & 0.24 & -0.16 \\
\hline 5 & 4 & 0.00 & 0.00 & -0.27 & 0.00 & 0.00 & 0.77 & -0.02 & -0.71 & 0.00 \\
\hline 6 & 13 & -0.15 & 0.13 & 0.17 & 0.07 & 0.12 & 0.05 & -0.09 & -0.09 & -0.14 \\
\hline 7 & 13 & 0.15 & -0.13 & 0.17 & -0.07 & -0.12 & 0.06 & -0.09 & -0.09 & 0.14 \\
\hline 8 & 14 & -0.14 & -0.18 & -0.22 & -0.15 & -0.11 & -0.07 & 0.15 & 0.07 & 0.11 \\
\hline \multirow[t]{3}{*}{9} & 14 & 0.14 & 0.18 & -0.22 & 0.15 & 0.11 & -0.07 & 0.15 & 0.07 & -0.11 \\
\hline & & & 13 & & & 1 & 4 & & & 15 \\
\hline & & & A & & & & A & & & A \\
\hline \multicolumn{2}{|c|}{ Frequencies -- } & \multicolumn{2}{|c|}{295.4526} & & \multicolumn{3}{|c|}{297.7778} & \multicolumn{3}{|c|}{340.6122} \\
\hline \multicolumn{2}{|c|}{ Red. masses -- } & \multicolumn{2}{|c|}{18.3324} & & \multicolumn{3}{|c|}{16.0602} & \multicolumn{3}{|c|}{15.1466} \\
\hline \multicolumn{2}{|c|}{ Frc consts } & \multicolumn{3}{|c|}{0.9429} & \multicolumn{3}{|c|}{0.8390} & \multicolumn{3}{|c|}{1.0353} \\
\hline \multicolumn{2}{|c|}{ IR Inten } & \multicolumn{2}{|r|}{0.3910} & & \multicolumn{3}{|c|}{0.3114} & \multicolumn{3}{|c|}{11.3029} \\
\hline
\end{tabular}

A

116.3088

26.7423

0.2131

0.0019

$$
\begin{array}{ccc}
0.08 & 0.06 & 0.40 \\
0.02 & -0.21 & 0.00 \\
-0.12 & 0.05 & -0.42 \\
-0.12 & 0.05 & 0.42 \\
-0.11 & -0.15 & 0.20 \\
-0.11 & -0.15 & -0.20 \\
& & 9
\end{array}
$$

A

153.3439

26.2683

0.3639

37.1068 


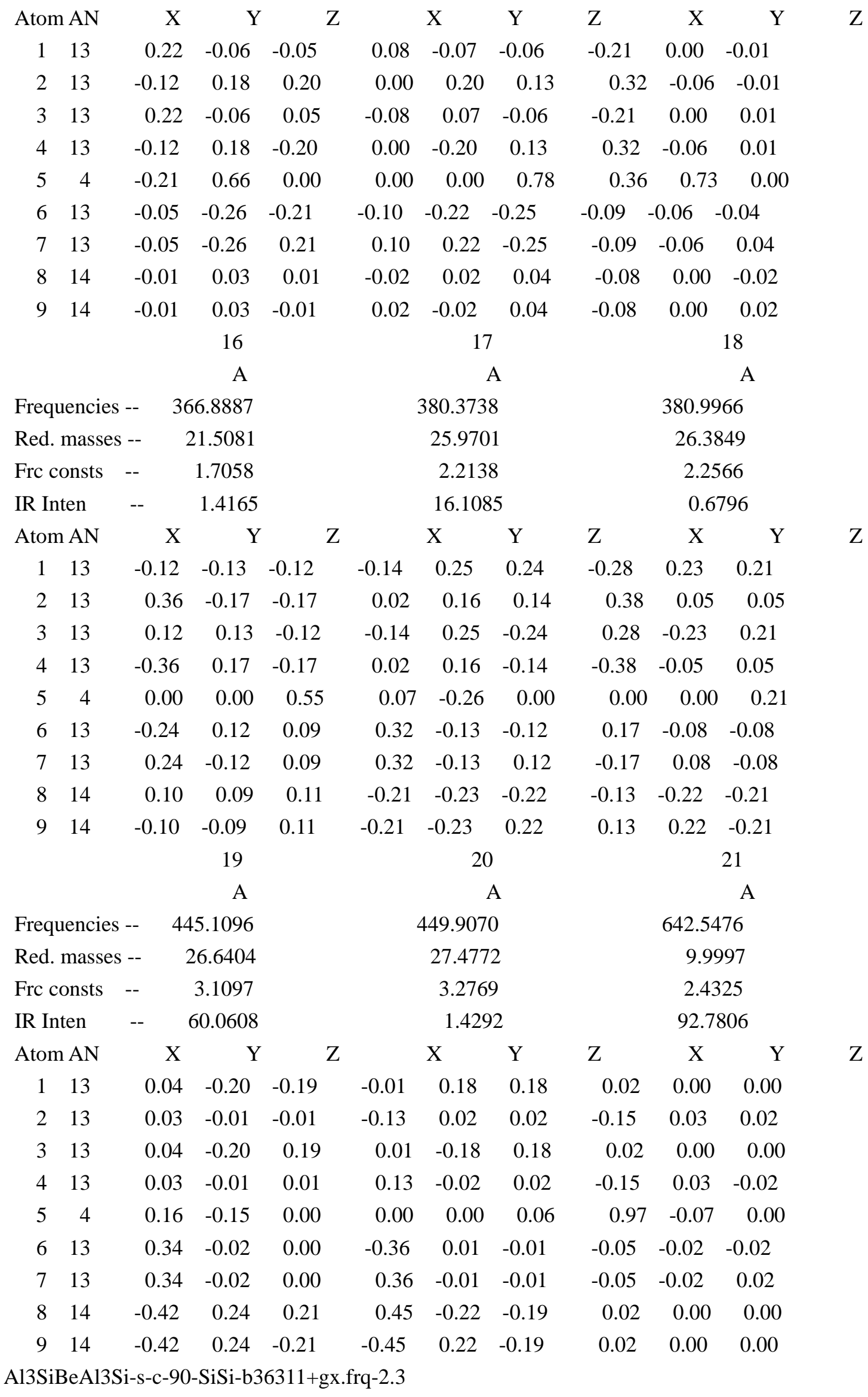




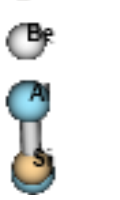

Charge $=0$ Multiplicity $=1$

Al,0,-3.2666136333,-2.0231757225,-0.3027604784

Al,0,-4.8406663237,-0.0201324241,0.0795072608

Be,0,0.1230684536,0.0314186935,-0.2070387922

Al,0,2.2832563133,0.1430506141,-1.0411649781

Al,0,4.7774337281,0.1732283359,-1.2792153879

Al,0,-3.2843732947,2.0248210669,0.2643654163

Si,0,-1.9908300713,0.0189527635,-0.1053449321

Si,0,4.1573182256,-0.1320276924,0.9850132328

Al,0,1.8743156024,-0.203591635,1.5253656588

State $=1-A \backslash H F=-2048.4155804$

1

A

\begin{tabular}{|c|c|c|c|}
\hline Frequencies & S -- & 2.2994 & \\
\hline Red. masses & $S--$ & 26.3627 & \\
\hline Frc consts & -- & 0.0001 & \\
\hline IR Inten & -- & 0.0101 & \\
\hline Atom AN & $X$ & $\mathrm{Y}$ & Z \\
\hline 113 & 0.50 & 0.09 & 0.10 \\
\hline $2 \quad 13$ & 0.00 & -0.31 & 0.00 \\
\hline 4 & 0.00 & 0.21 & 0.00 \\
\hline $4 \quad 13$ & 0.00 & -0.05 & 0.00 \\
\hline 13 & 0.00 & -0.33 & 0.00 \\
\hline 13 & -0.49 & 0.09 & -0.10 \\
\hline 14 & 0.00 & 0.43 & 0.01 \\
\hline 14 & 0.00 & -0.14 & 0.00 \\
\hline $9 \quad 13$ & 0.00 & 0.13 & 0.00 \\
\hline
\end{tabular}

A

Frequencies -- $\quad 41.5114$

Red. masses -- 23.2124

Frc consts -- $\quad 0.0236$

IR Inten -- $\quad 0.1774$

Atom AN $\quad \mathrm{X} \quad \mathrm{Y} \quad \mathrm{Z}$

$\begin{array}{lllll}1 & 13 & -0.09 & -0.11 & -0.01\end{array}$

$\begin{array}{lllll}2 & 13 & 0.00 & -0.02 & 0.00\end{array}$

$\begin{array}{lllll}3 & 4 & 0.00 & 0.46 & 0.01\end{array}$

$\begin{array}{lllll}4 & 13 & 0.00 & 0.64 & 0.01\end{array}$
2

A

17.7832

26.9266

0.0050

0.0313

X Y $-0.14-0.06$

$-0.01$

0.04

$\begin{array}{llll}0.00 & -0.10 & -0.04\end{array}$

$\begin{array}{lll}-0.01 & -0.29 & -0.02\end{array}$

$\begin{array}{lll}-0.01 & -0.32 & 0.01\end{array}$

$\begin{array}{llll}0.13 & -0.04 & -0.44\end{array}$

$\begin{array}{lll}0.00 & -0.12 & -0.04\end{array}$

$\begin{array}{lll}0.01 & 0.36 & 0.02\end{array}$

0.02

$0.46-0.01$

5

A

75.3917

24.0439

0.0805

2.5312

X Y

$-0.08$

0.01

0.15

$-0.06$

$0.00 \quad-0.26$

$-0.03$

$0.01-0.43$

$-0.10$

$0.01 \quad-0.29$

$\begin{array}{ccc}Z & X & Y \\ -0.03 & 0.00 & 0.01 \\ -0.06 & -0.01 & 0.54 \\ -0.01 & 0.01 & -0.44 \\ -0.12 & 0.02 & -0.13 \\ -0.16 & 0.02 & 0.23 \\ -0.04 & 0.00 & 0.08 \\ -0.01 & 0.02 & -0.50 \\ 0.18 & -0.03 & 0.13 \\ 0.25 & -0.03 & -0.20 \\ & & 6\end{array}$

A

97.6837

27.0056

0.1518

3.8730

$\begin{array}{ccc}Z & X & Y \\ 0.02 & 0.03 & 0.00 \\ 0.00 & 0.00 & 0.00 \\ 0.00 & -0.14 & 0.00 \\ 0.00 & 0.32 & 0.01\end{array}$




$\begin{array}{rrrrrrrrrrr}5 & 13 & 0.00 & -0.37 & -0.01 & -0.17 & 0.00 & 0.17 & 0.00 & -0.44 & -0.01 \\ 6 & 13 & 0.09 & -0.11 & 0.01 & -0.08 & -0.02 & 0.15 & -0.02 & 0.03 & 0.00 \\ 7 & 14 & 0.00 & -0.20 & 0.00 & -0.07 & -0.01 & 0.50 & 0.00 & 0.04 & 0.00 \\ 8 & 14 & 0.00 & -0.26 & -0.01 & 0.25 & 0.00 & 0.04 & 0.00 & 0.61 & 0.01 \\ 9 & 13 & 0.00 & 0.29 & 0.01 & 0.31 & 0.01 & -0.34 & 0.00 & -0.56 & -0.01 \\ & & & 7 & & & 8 & & & & 9\end{array}$

A

A

A

Frequencies -- 103.8011

Red. masses -- 26.8275

106.7376

153.3109

27.1850

24.6195

0.1825

0.3409

Frc consts $\quad--\quad 0.1703$

2.7076

14.5256

\begin{tabular}{rrrcc}
\multicolumn{2}{l}{ IR Inten } & \multicolumn{3}{c}{1.6844} \\
Atom AN & $\mathrm{X}$ & $\mathrm{Y}$ & $\mathrm{Z}$ \\
1 & 13 & 0.35 & -0.14 & 0.07 \\
2 & 13 & 0.49 & 0.00 & -0.04 \\
3 & 4 & -0.07 & 0.00 & -0.10 \\
4 & 13 & -0.29 & 0.00 & -0.11 \\
5 & 13 & -0.39 & 0.00 & 0.10 \\
6 & 13 & 0.35 & 0.14 & 0.08 \\
7 & 14 & 0.08 & 0.00 & 0.00 \\
8 & 14 & -0.30 & 0.00 & 0.09 \\
9 & 13 & -0.26 & 0.01 & -0.16
\end{tabular}

A

Frequencies -- 199.4985

Red. masses -- 25.6973

Frc consts -- $\quad 0.6026$

IR Inten -- 3.0510

Atom AN $\mathrm{X} \quad \mathrm{Y}$

$\begin{array}{rrrcc}1 & 13 & -0.23 & -0.39 & -0.02 \\ 2 & 13 & 0.49 & 0.00 & 0.03 \\ 3 & 4 & -0.26 & 0.00 & 0.12 \\ 4 & 13 & 0.09 & 0.00 & -0.11 \\ 5 & 13 & 0.27 & 0.00 & 0.03 \\ 6 & 13 & -0.24 & 0.39 & -0.01 \\ 7 & 14 & -0.40 & 0.00 & -0.02 \\ 8 & 14 & 0.08 & 0.00 & 0.05 \\ 9 & 13 & 0.04 & 0.00 & 0.01 \\ & & & 13 & \end{array}$

A

Frequencies -- $\quad 256.5488$

Red. masses -- 25.8394

Frc consts -- $\quad 1.0020$

IR Inten -- 3.2628

Atom AN $\mathrm{X} \quad \mathrm{Y}$

$\mathrm{Z}$

$$
\begin{array}{r}
-0.06 \\
-0.02 \\
0.01 \\
0.04 \\
0.06 \\
-0.06 \\
0.02 \\
0.01 \\
0.00
\end{array}
$$$$
\mathrm{X}
$$$$
\begin{array}{lll}
-0.06 & 0.00 & 0.50
\end{array}
$$$$
\begin{array}{lll}
-0.02 & 0.01 & -0.50
\end{array}
$$$$
\begin{array}{lll}
0.01 & 0.00 & 0.04
\end{array}
$$$$
\begin{array}{lll}
0.04 & 0.00 & 0.01
\end{array}
$$$$
\begin{array}{lll}
0.06 & 0.00 & -0.02
\end{array}
$$$$
\begin{array}{lll}
-0.06 & -0.02 & 0.50
\end{array}
$$$$
\begin{array}{lll}
0.02 & 0.01 & -0.49
\end{array}
$$$$
\begin{array}{lll}
0.01 & 0.00 & -0.01
\end{array}
$$$$
\begin{array}{lll}
0.00 & 0.00 & 0.02
\end{array}
$$$$
11
$$

A

$$
210.6431
$$

10.1101

0.2643

0.1954

X Y

$\begin{array}{lll}0.00 & -0.04 & 0.00\end{array}$

$\begin{array}{lll}0.00 & 0.04 & 0.00\end{array}$

$\begin{array}{lll}0.00 & 0.97 & 0.02\end{array}$

$\begin{array}{lll}0.00 & -0.18 & 0.00\end{array}$

$\begin{array}{lll}0.00 & 0.01 & 0.00\end{array}$

$\begin{array}{lll}0.00 & -0.04 & 0.00\end{array}$

$\begin{array}{llll}0.00 & -0.07 & 0.00\end{array}$

$\begin{array}{lll}0.00 & 0.07 & 0.00\end{array}$

$\begin{array}{llll}0.00 & -0.12 & 0.00\end{array}$

14

A

288.0575

17.4022

0.8508

1.3622
$\begin{array}{lcr}Z & X & Y \\ -0.03 & 0.09 & -0.02\end{array}$

$\begin{array}{lll}-0.11 & 0.00 & 0.02\end{array}$

$\begin{array}{lll}0.12 & -0.01 & 0.36\end{array}$

$\begin{array}{lll}0.27 & 0.01 & -0.38\end{array}$

$\begin{array}{lll}0.31 & -0.01 & 0.25\end{array}$

$\begin{array}{llll}-0.03 & -0.09 & -0.02\end{array}$

$\begin{array}{lll}0.09 & 0.00 & -0.01\end{array}$

$\begin{array}{lll}-0.27 & -0.01 & 0.39\end{array}$

$\begin{array}{lll}-0.27 & 0.01 & -0.37\end{array}$

12

A

237.9383

15.8863

0.5299

11.8896

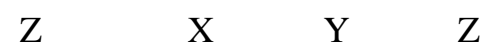

$\begin{array}{llll}0.02 & -0.01 & -0.01\end{array}$

$\begin{array}{lll}-0.03 & 0.00 & 0.02\end{array}$

$\begin{array}{lll}-0.04 & -0.01 & 0.79\end{array}$

$\begin{array}{lll}0.13 & 0.01 & -0.33\end{array}$

$\begin{array}{lll}-0.31 & 0.00 & 0.16\end{array}$

$\begin{array}{lll}0.02 & 0.01 & -0.01\end{array}$

$\begin{array}{lll}-0.01 & 0.00 & -0.05\end{array}$

$\begin{array}{lll}0.23 & 0.00 & -0.20\end{array}$

$\begin{array}{lll}-0.05 & 0.00 & 0.17\end{array}$

15

A

310.6876

26.5036

1.5073

2.2186

$\begin{array}{llll}\mathrm{X} & \mathrm{Y} & \mathrm{Z}\end{array}$ 


$\begin{array}{rrrcc}1 & 13 & -0.38 & 0.30 & -0.02 \\ 2 & 13 & 0.55 & 0.00 & 0.03 \\ 3 & 4 & 0.25 & 0.00 & 0.08 \\ 4 & 13 & 0.03 & 0.00 & 0.08 \\ 5 & 13 & -0.20 & 0.00 & -0.03 \\ 6 & 13 & -0.38 & -0.31 & -0.02 \\ 7 & 14 & 0.32 & 0.00 & 0.01 \\ 8 & 14 & -0.02 & 0.00 & -0.02 \\ 9 & 13 & 0.00 & 0.00 & -0.05 \\ & & & 16 & \end{array}$

A

$$
\begin{array}{ccc}
0.01 & -0.08 & -0.01 \\
-0.06 & 0.00 & 0.01 \\
0.01 & -0.01 & 0.73 \\
-0.01 & -0.01 & 0.37 \\
-0.13 & 0.00 & -0.16 \\
0.01 & 0.08 & -0.01 \\
-0.04 & 0.00 & -0.02 \\
0.06 & 0.00 & 0.07 \\
0.15 & 0.01 & -0.49 \\
\multicolumn{3}{c}{17} \\
\hline
\end{array}
$$

A

368.3161

27.3329

$$
2.1846
$$$$
11.4563
$$

$\begin{array}{lll}\text { Frc consts } & -- & 1.4327 \\ \text { IR Inten } & -- & 1.4182\end{array}$

\begin{tabular}{ccccc} 
Atom AN & X & \multicolumn{2}{c}{$\mathrm{Y}$} & $\mathrm{Z}$ \\
1 & 13 & -0.11 & -0.32 & -0.01 \\
2 & 13 & -0.15 & 0.00 & -0.01 \\
3 & 4 & 0.40 & 0.01 & -0.43 \\
4 & 13 & 0.44 & 0.00 & 0.13 \\
5 & 13 & -0.29 & 0.00 & 0.06 \\
6 & 13 & -0.11 & 0.32 & 0.00 \\
7 & 14 & 0.27 & 0.00 & 0.02 \\
8 & 14 & -0.04 & 0.00 & -0.03 \\
9 & 13 & -0.15 & 0.00 & -0.03 \\
& & & 19 &
\end{tabular}

Frequencies -- 393.5467

Red. masses -- 25.0423

Frc consts -- $\quad 2.2852$

IR Inten -- 17.2437
A

$\begin{array}{ll}\mathrm{X} & \mathrm{Y}\end{array}$

$\begin{array}{lll}0.31 & 0.35 & 0.02\end{array}$

$\begin{array}{lll}0.00 & 0.12 & 0.00\end{array}$

$\begin{array}{llll}0.00 & -0.10 & -0.01\end{array}$

$\begin{array}{lll}0.01 & 0.00 & 0.01\end{array}$

$\begin{array}{lll}0.00 & 0.00 & 0.01\end{array}$

$\begin{array}{lll}-0.31 & 0.34 & -0.01\end{array}$

$\begin{array}{lll}-0.01 & -0.74 & -0.01\end{array}$

$\begin{array}{lll}0.01 & 0.00 & -0.01\end{array}$

$\begin{array}{lll}-0.01 & 0.00 & -0.01\end{array}$

20

A

445.7688

27.3292

3.1996

33.6748

$\begin{array}{ll}\mathrm{X} & \mathrm{Y}\end{array}$

$\begin{array}{llllllll}1 & 13 & 0.12 & 0.23 & 0.01 & -0.02 & -0.03 & 0.00\end{array}$

213

$\begin{array}{lll}0.11 & 0.00 & 0.00\end{array}$

$\begin{array}{lll}-0.20 & 0.01 & -0.29\end{array}$

$\begin{array}{lll}0.54 & 0.00 & 0.06\end{array}$

$\begin{array}{lll}-0.30 & 0.00 & -0.20\end{array}$

$\begin{array}{lll}0.12 & -0.23 & 0.00\end{array}$

$\begin{array}{lll}-0.50 & 0.01 & -0.02\end{array}$

$\begin{array}{lll}0.01 & 0.00 & 0.22\end{array}$

$\begin{array}{lll}-0.01 & 0.00 & 0.02\end{array}$

$\begin{array}{lll}-0.02 & 0.00 & 0.00\end{array}$

$\begin{array}{lll}-0.04 & 0.00 & -0.10\end{array}$

$\begin{array}{lll}-0.18 & 0.00 & -0.04\end{array}$

$\begin{array}{lll}0.01 & 0.01 & -0.34\end{array}$

$\begin{array}{lll}-0.02 & 0.03 & 0.00\end{array}$

$\begin{array}{lll}0.12 & 0.00 & 0.01\end{array}$

$\begin{array}{lll}0.62 & -0.01 & 0.39\end{array}$

$\begin{array}{lll}-0.53 & 0.00 & 0.00\end{array}$

$$
\begin{array}{ccc}
0.29 & -0.37 & 0.01 \\
0.00 & 0.73 & 0.01 \\
0.00 & -0.16 & 0.00 \\
0.00 & 0.02 & 0.00 \\
0.00 & 0.00 & 0.00 \\
-0.29 & -0.37 & -0.02 \\
0.00 & 0.03 & 0.00 \\
0.00 & -0.01 & 0.00 \\
0.00 & 0.01 & 0.00 \\
\multicolumn{3}{c}{18}
\end{array}
$$

A

371.4943

26.1372

2.1253

5.6872

$\begin{array}{lllll}Z & X & Y & \text { Z }\end{array}$

$\begin{array}{lll}0.04 & 0.11 & 0.01\end{array}$

$\begin{array}{lll}0.07 & 0.00 & 0.00\end{array}$

$\begin{array}{lll}-0.13 & 0.01 & -0.22\end{array}$

$\begin{array}{lll}0.06 & -0.01 & 0.32\end{array}$

$\begin{array}{lll}0.16 & -0.01 & 0.48\end{array}$

$\begin{array}{lll}0.05 & -0.12 & 0.00\end{array}$

$\begin{array}{lll}-0.17 & 0.02 & -0.01\end{array}$

$\begin{array}{lll}0.25 & 0.01 & -0.44\end{array}$

$\begin{array}{lll}-0.42 & 0.01 & -0.27\end{array}$

21

A

732.0497

10.2710

3.2430

439.1130

$\begin{array}{lll}\mathrm{Z} & \mathrm{X} & \mathrm{Y}\end{array}$

Z

$\begin{array}{lll}0.01 & 0.01 & 0.00\end{array}$

$\begin{array}{lll}0.01 & 0.00 & 0.00\end{array}$

$\begin{array}{lll}0.96 & 0.00 & 0.04\end{array}$

$\begin{array}{lll}-0.11 & 0.00 & -0.02\end{array}$

$\begin{array}{lll}0.01 & 0.00 & 0.00\end{array}$

$\begin{array}{lll}0.01 & -0.01 & 0.00\end{array}$

$\begin{array}{lll}-0.23 & 0.00 & -0.01\end{array}$

$\begin{array}{lll}0.01 & 0.00 & 0.00\end{array}$

$\begin{array}{lll}-0.03 & 0.00 & 0.02\end{array}$

Al3SiBeAl3Si-c-c-0-C2h-Al-Al-b36311+gx.frq3m-15.1 


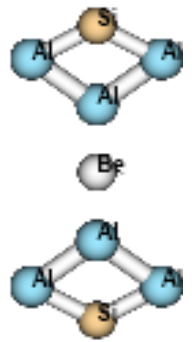

$\begin{array}{rrrrr}4 & 0 & 0.000000 & 0.000000 & 0.000000 \\ 13 & 0 & 0.000000 & 2.087851 & 3.808351 \\ 13 & 0 & 0.000000 & -2.087851 & 3.808351 \\ 13 & 0 & 0.000000 & 2.087851 & -3.808351 \\ 13 & 0 & 0.000000 & -2.087851 & -3.808351 \\ 13 & 0 & 0.000000 & 0.000000 & 2.286971 \\ 14 & 0 & 0.000000 & 0.000000 & 4.939661 \\ 13 & 0 & 0.000000 & 0.000000 & -2.286971 \\ 14 & 0 & 0.000000 & 0.000000 & -4.939661\end{array}$

State $=1-A G \mid H F=-2048.3838816$

1

B3G
2

$\mathrm{AU}$

$-9.8180$

26.9815

0.0015

0.0000

IR Inten - - $\quad 0.0000$

Atom AN $\mathrm{X} \quad \mathrm{Y}$

14 $\begin{array}{lll}0.00 & 0.00 & 0.00\end{array}$

0.00

$0.12 \quad 0.36$

$\begin{array}{lll}0.00 & 0.12 & -0.36\end{array}$

$\begin{array}{lll}0.00 & -0.12 & 0.36\end{array}$

$\begin{array}{lll}0.00 & -0.12 & -0.36\end{array}$

$\begin{array}{lll}0.00 & 0.45 & 0.00\end{array}$

$\begin{array}{lll}0.00 & -0.08 & 0.00\end{array}$

$\begin{array}{lll}0.00 & -0.45 & 0.00\end{array}$

$\begin{array}{lll}0.00 & 0.08 & 0.00\end{array}$

4

B3U

5

.

-3.8117
25.7717
0.0002
0.0093
$X$

B2U

$\begin{array}{lll}0.00 & 0.00 & 0.00\end{array}$

0.00

$\mathrm{X} \quad \mathrm{Y}$

$\begin{array}{lll}-0.50 & 0.00 & 0.00\end{array}$

$\begin{array}{lll}0.00 & -0.04 & 0.40\end{array}$

$\begin{array}{lll}0.50 & 0.00 & 0.00\end{array}$

$\begin{array}{lll}0.00 & -0.04 & -0.40\end{array}$

$\begin{array}{lll}0.50 & 0.00 & 0.00\end{array}$

$\begin{array}{llll}0.00 & -0.04 & -0.40\end{array}$

$\begin{array}{lll}-0.50 & 0.00 & 0.00\end{array}$

$\begin{array}{llll}0.00 & -0.04 & 0.40\end{array}$

$\begin{array}{lll}0.00 & 0.00 & 0.00\end{array}$

$\begin{array}{lll}0.00 & 0.29 & 0.00\end{array}$

$\begin{array}{lll}0.00 & 0.00 & 0.00\end{array}$

$\begin{array}{lll}0.00 & -0.25 & 0.00\end{array}$

$\begin{array}{lll}0.00 & 0.00 & 0.00\end{array}$

$\begin{array}{lll}0.00 & 0.29 & 0.00\end{array}$

$\begin{array}{lll}0.00 & 0.00 & 0.00\end{array}$

B2G

49.9631

27.1528

0.0399

$\begin{array}{lll}0.00 & -0.25 & 0.00\end{array}$

6

AG

Frequencies -- $\quad 22.4261$

Red. masses -- 21.4430

Frc consts --- 0.0064

IR Inten $\quad$-- $\quad 0.0184$

Atom AN $\mathrm{X} \quad \mathrm{Y}$

$\begin{array}{lllll}2 & 13 & 0.07 & 0.00 & 0.00\end{array}$

$\begin{array}{lllll}3 & 13 & 0.07 & 0.00 & 0.00\end{array}$

$\begin{array}{lllll}4 & 13 & 0.07 & 0.00 & 0.00\end{array}$
0.0000

X Y

$\begin{array}{lll}0.00 & 0.00 & 0.00\end{array}$

$\begin{array}{lll}0.00 & 0.00 & 0.00\end{array}$

$\begin{array}{lll}0.00 & 0.00 & 0.00\end{array}$

$\begin{array}{lll}0.00 & 0.00 & 0.00\end{array}$
98.7979

27.2707

0.1568

0.0000

$\begin{array}{llll}\mathrm{Z} & \mathrm{X} & \mathrm{Y}\end{array}$

$\begin{array}{lll}0.00 & 0.00 & 0.00\end{array}$

$\begin{array}{lll}0.00 & -0.03 & 0.40\end{array}$

$\begin{array}{lll}0.00 & 0.03 & 0.40\end{array}$

$\begin{array}{lll}0.00 & -0.03 & -0.40\end{array}$ 


\begin{tabular}{ccccccccccc}
5 & 13 & 0.07 & 0.00 & 0.00 & 0.00 & 0.00 & 0.00 & 0.00 & 0.03 & -0.40 \\
6 & 13 & -0.44 & 0.00 & 0.00 & 0.64 & 0.00 & 0.00 & 0.00 & 0.00 & 0.20 \\
7 & 14 & 0.37 & 0.00 & 0.00 & -0.29 & 0.00 & 0.00 & 0.00 & 0.00 & 0.38 \\
8 & 13 & -0.44 & 0.00 & 0.00 & -0.64 & 0.00 & 0.00 & 0.00 & 0.00 & -0.20 \\
9 & 14 & 0.37 & 0.00 & 0.00 & 0.29 & 0.00 & 0.00 & 0.00 & 0.00 & -0.38 \\
& & & 7 & & & 8 & & & \multicolumn{2}{c}{9}
\end{tabular}

B2G

B3U

Frequencies -- 102.2471

Red. masses -- 27.3689

Frc consts -- 0.1686

IR Inten $\quad--\quad 0.0000$

Atom AN $\mathrm{X} \quad \mathrm{Y}$

$\begin{array}{llll}1 & 4 & 0.00 & 0.00\end{array}$

$\begin{array}{lllll}2 & 13 & -0.36 & 0.00 & 0.00\end{array}$

$\begin{array}{lllll}3 & 13 & -0.36 & 0.00 & 0.00\end{array}$

$\begin{array}{lllll}4 & 13 & 0.36 & 0.00 & 0.00\end{array}$

$\begin{array}{lllll}5 & 13 & 0.36 & 0.00 & 0.00\end{array}$

$\begin{array}{lllll}6 & 13 & 0.21 & 0.00 & 0.00\end{array}$

$\begin{array}{lllll}7 & 14 & 0.44 & 0.00 & 0.00\end{array}$

$\begin{array}{lllll}8 & 13 & -0.21 & 0.00 & 0.00\end{array}$

$\begin{array}{lllll}9 & 14 & -0.44 & 0.00 & 0.00\end{array}$

10

B2U

Frequencies -- 162.5447

Red. masses -- 9.6976

Frc consts -- 0.1510

IR Inten $\quad$-- 2.0245

Atom AN $\quad \mathrm{X} \quad \mathrm{Y}$

$\begin{array}{lllll}1 & 4 & 0.00 & 0.98 & 0.00\end{array}$

$\begin{array}{llllll}2 & 13 & 0.00 & -0.01 & -0.01\end{array}$

$\begin{array}{lllll}3 & 13 & 0.00 & -0.01 & 0.01\end{array}$

$\begin{array}{llllll}4 & 13 & 0.00 & -0.01 & 0.01\end{array}$

$\begin{array}{lllll}5 & 13 & 0.00 & -0.01 & -0.01\end{array}$

$\begin{array}{llllll}6 & 13 & 0.00 & -0.14 & 0.00\end{array}$

$\begin{array}{llllll}7 & 14 & 0.00 & -0.01 & 0.00\end{array}$

$\begin{array}{llllll}8 & 13 & 0.00 & -0.14 & 0.00\end{array}$

$\begin{array}{lllll}9 & 14 & 0.00 & -0.01 & 0.00\end{array}$

13

B1U

Frequencies -- 252.1573

Red. masses -- 22.0775

Frc consts --- 0.8271

IR Inten -- 15.4674

Atom AN $\mathrm{X} \quad \mathrm{Y}$
103.2115

27.3487

0.1716

10.9444

$\mathrm{X}$

$\begin{array}{lll}-0.01 & 0.00 & 0.00\end{array}$

$\begin{array}{lll}0.35 & 0.00 & 0.00\end{array}$

$\begin{array}{lll}0.35 & 0.00 & 0.00\end{array}$

$\begin{array}{lll}0.35 & 0.00 & 0.00\end{array}$

$\begin{array}{lll}0.35 & 0.00 & 0.00\end{array}$

$\begin{array}{lll}-0.26 & 0.00 & 0.00\end{array}$

$\begin{array}{lll}-0.43 & 0.00 & 0.00\end{array}$

$\begin{array}{lll}-0.26 & 0.00 & 0.00\end{array}$

$\begin{array}{lll}-0.43 & 0.00 & 0.00\end{array}$

11

B1U

209.0045

26.6977

0.6871

34.4235

$\mathrm{X}$

$\begin{array}{llll}0.00 & 0.00 & -0.19\end{array}$

$\begin{array}{lll}0.00 & -0.36 & -0.12\end{array}$

$\begin{array}{lll}0.00 & 0.36 & -0.12\end{array}$

$\begin{array}{lll}0.00 & 0.36 & -0.12\end{array}$

$\begin{array}{llll}0.00 & -0.36 & -0.12\end{array}$

$\begin{array}{lll}0.00 & 0.00 & -0.16\end{array}$

$\begin{array}{lll}0.00 & 0.00 & 0.41\end{array}$

$\begin{array}{llll}0.00 & 0.00 & -0.16\end{array}$

$\begin{array}{llll}0.00 & 0.00 & 0.41\end{array}$

14

B3G

262.6619

26.9829

1.0968

0.0000
B3U

154.4690

10.2086

0.1435

9.8648

$\begin{array}{llll}\mathrm{Z} & \mathrm{X} & \mathrm{Y}\end{array}$

$\begin{array}{lll}0.97 & 0.00 & 0.00\end{array}$

$\begin{array}{lll}-0.02 & 0.00 & 0.00\end{array}$

$\begin{array}{lll}-0.02 & 0.00 & 0.00\end{array}$

$\begin{array}{lll}-0.02 & 0.00 & 0.00\end{array}$

$\begin{array}{lll}-0.02 & 0.00 & 0.00\end{array}$

$\begin{array}{lll}-0.17 & 0.00 & 0.00\end{array}$

$\begin{array}{lll}0.06 & 0.00 & 0.00\end{array}$

$\begin{array}{lll}-0.17 & 0.00 & 0.00\end{array}$

$\begin{array}{lll}0.06 & 0.00 & 0.00\end{array}$

12

AG

213.6965

27.2949

0.7344

0.0000

$\begin{array}{llll}Z & X & Y\end{array}$

Z

$\begin{array}{lll}0.00 & 0.00 & 0.00\end{array}$

$\begin{array}{lll}0.00 & -0.35 & -0.21\end{array}$

$\begin{array}{lll}0.00 & 0.35 & -0.21\end{array}$

$\begin{array}{lll}0.00 & -0.35 & 0.21\end{array}$

$\begin{array}{lll}0.00 & 0.35 & 0.21\end{array}$

$\begin{array}{lll}0.00 & 0.00 & -0.06\end{array}$

$\begin{array}{lll}0.00 & 0.00 & 0.40\end{array}$

$\begin{array}{lll}0.00 & 0.00 & 0.06\end{array}$

$\begin{array}{lll}0.00 & 0.00 & -0.40\end{array}$

15

B2U

\subsection{1}

24.9849

1.0492

0.6283

$x^{2}$ 


\begin{tabular}{|c|c|c|c|c|c|c|c|c|c|c|c|}
\hline 1 & 4 & 0.00 & 0.00 & -0.52 & 0.00 & 0.00 & 0.00 & 0.00 & -0.33 & 0.00 & \\
\hline 2 & 13 & 0.00 & -0.03 & 0.30 & 0.00 & -0.26 & -0.23 & 0.00 & 0.25 & 0.22 & \\
\hline 3 & 13 & 0.00 & 0.03 & 0.30 & 0.00 & -0.26 & 0.23 & 0.00 & 0.25 & -0.22 & \\
\hline 4 & 13 & 0.00 & 0.03 & 0.30 & 0.00 & 0.26 & -0.23 & 0.00 & 0.25 & -0.22 & \\
\hline 5 & 13 & 0.00 & -0.03 & 0.30 & 0.00 & 0.26 & 0.23 & 0.00 & 0.25 & 0.22 & \\
\hline 6 & 13 & 0.00 & 0.00 & -0.42 & 0.00 & 0.51 & 0.00 & 0.00 & -0.47 & 0.00 & \\
\hline 7 & 14 & 0.00 & 0.00 & -0.08 & 0.00 & -0.03 & 0.00 & 0.00 & 0.02 & 0.00 & \\
\hline 8 & 13 & 0.00 & 0.00 & -0.42 & 0.00 & -0.51 & 0.00 & 0.00 & -0.47 & 0.00 & \\
\hline 9 & 14 & 0.00 & 0.00 & -0.08 & 0.00 & 0.03 & 0.00 & 0.00 & 0.02 & 0.00 & \\
\hline & & & 16 & & \multicolumn{3}{|c|}{17} & \multicolumn{4}{|c|}{18} \\
\hline & & & AG & & \multicolumn{3}{|c|}{ B1U } & \multicolumn{4}{|c|}{ AG } \\
\hline \multicolumn{2}{|c|}{ Frequencies -- } & \multicolumn{2}{|c|}{323.4446} & & \multicolumn{3}{|c|}{367.2606} & \multicolumn{4}{|c|}{406.2150} \\
\hline \multicolumn{2}{|c|}{ Red. masses -- } & \multicolumn{2}{|c|}{27.1578} & & \multicolumn{3}{|c|}{25.5341} & \multicolumn{4}{|c|}{27.1718} \\
\hline \multicolumn{2}{|c|}{ Frc consts } & \multicolumn{2}{|r|}{1.6740} & & \multicolumn{3}{|c|}{2.0292} & \multicolumn{4}{|c|}{2.6417} \\
\hline \multicolumn{2}{|c|}{ IR Inten } & - & 0.0000 & & \multicolumn{3}{|c|}{21.6330} & \multicolumn{4}{|c|}{0.0000} \\
\hline \multicolumn{2}{|c|}{ Atom AN } & X & $\mathrm{Y}$ & $\mathrm{Z}$ & & $\mathrm{X}$ & $\mathrm{Y}$ & Z & $\mathrm{X}$ & $\mathrm{Y}$ & Z \\
\hline 1 & 4 & 0.00 & 0.00 & 0.00 & 0.00 & 0.00 & 0.32 & 0.00 & 0.00 & 0.00 & \\
\hline 2 & 13 & 0.00 & 0.28 & -0.22 & 0.00 & -0.33 & 0.09 & 0.00 & -0.23 & 0.00 & \\
\hline 3 & 13 & 0.00 & -0.28 & -0.22 & 0.00 & 0.33 & 0.09 & 0.00 & 0.23 & 0.00 & \\
\hline 4 & 13 & 0.00 & 0.28 & 0.22 & 0.00 & 0.33 & 0.09 & 0.00 & -0.23 & 0.00 & \\
\hline 5 & 13 & 0.00 & -0.28 & 0.22 & 0.00 & -0.33 & 0.09 & 0.00 & 0.23 & 0.00 & \\
\hline 6 & 13 & 0.00 & 0.00 & 0.40 & 0.00 & 0.00 & 0.20 & 0.00 & 0.00 & 0.55 & \\
\hline 7 & 14 & 0.00 & 0.00 & 0.30 & 0.00 & 0.00 & -0.42 & 0.00 & 0.00 & -0.31 & \\
\hline 8 & 13 & 0.00 & 0.00 & -0.40 & 0.00 & 0.00 & 0.20 & 0.00 & 0.00 & -0.55 & \\
\hline \multirow[t]{3}{*}{9} & 14 & 0.00 & 0.00 & -0.30 & 0.00 & 0.00 & -0.42 & 0.00 & 0.00 & 0.31 & \\
\hline & & & 19 & & \multicolumn{3}{|c|}{20} & \multicolumn{4}{|c|}{21} \\
\hline & & & B3G & & & & $2 \mathrm{U}$ & & & B1U & \\
\hline Freql & lencies - & & 6.4324 & & & 436.6957 & & & 706.265 & & \\
\hline Red. & masses & & 27.5858 & & & 27.5682 & & & 10.38 & & \\
\hline Frc c & onsts & - & 3.0958 & & & 3.0975 & & & 3.051 & & \\
\hline IR In & ten & - & 0.0000 & & & 21.102 & & & 234.55 & & \\
\hline Aton & $\mathrm{AN}$ & $\mathrm{X}$ & $\mathrm{Y}$ & Z & & $\mathrm{X}$ & $\mathrm{Y}$ & Z & X & $\mathrm{Y}$ & $\mathrm{Z}$ \\
\hline 1 & 4 & 0.00 & 0.00 & 0.00 & 0.00 & -0.03 & 0.00 & 0.00 & 0.00 & 0.96 & \\
\hline 2 & 13 & 0.00 & -0.25 & 0.18 & 0.00 & -0.25 & 0.18 & 0.00 & 0.01 & 0.01 & \\
\hline 3 & 13 & 0.00 & -0.25 & -0.18 & 0.00 & -0.25 & -0.18 & 0.00 & -0.01 & 0.01 & \\
\hline 4 & 13 & 0.00 & 0.25 & 0.18 & 0.00 & -0.25 & -0.18 & 0.00 & -0.01 & 0.01 & \\
\hline 5 & 13 & 0.00 & 0.25 & -0.18 & 0.00 & -0.25 & 0.18 & 0.00 & 0.01 & 0.01 & \\
\hline 6 & 13 & 0.00 & -0.07 & 0.00 & 0.00 & -0.06 & 0.00 & 0.00 & 0.00 & -0.19 & \\
\hline 7 & 14 & 0.00 & 0.55 & 0.00 & 0.00 & 0.55 & 0.00 & 0.00 & 0.00 & 0.02 & \\
\hline 8 & 13 & 0.00 & 0.07 & 0.00 & 0.00 & -0.06 & 0.00 & 0.00 & 0.00 & -0.19 & \\
\hline 9 & 14 & 0.00 & -0.55 & 0.00 & 0.00 & 0.55 & 0.00 & 0.00 & 0.00 & 0.02 & \\
\hline
\end{tabular}




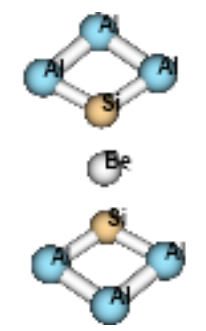

$\begin{array}{rrrrc}13 & 0 & -2.042333 & 3.399355 & 0.000000 \\ 13 & 0 & 2.043030 & 3.398312 & 0.000000 \\ 13 & 0 & 0.000768 & 4.974377 & 0.000000 \\ 4 & 0 & 0.000000 & 0.000000 & 0.000000 \\ 13 & 0 & -2.043030 & -3.398312 & 0.000000 \\ 13 & 0 & -0.000768 & -4.974377 & 0.000000 \\ 13 & 0 & 2.042333 & -3.399355 & 0.000000 \\ 14 & 0 & 0.000000 & 2.117008 & 0.000000 \\ 14 & 0 & 0.000000 & -2.117008 & 0.000000\end{array}$

State $=1-A G \backslash H F=-2048.4241279$

AG

BG

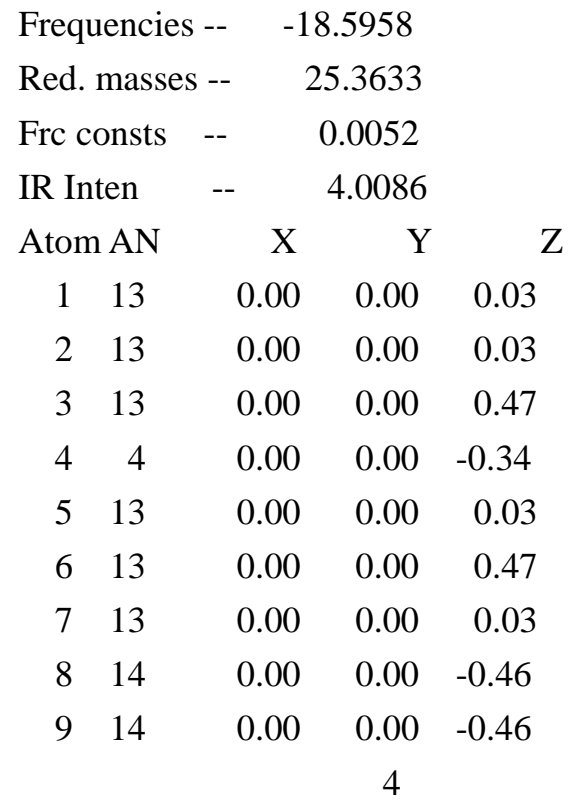

$\mathrm{AU}$

Frequencies -- $\quad-7.7124$

Red. masses -- 26.9815

Frc consts -- 0.0009

IR Inten -- $\quad 0.0001$

\begin{tabular}{rrrrr}
\multicolumn{2}{c}{ Atom AN } & $\mathrm{X}$ & $\mathrm{Y}$ & $\mathrm{Z}$ \\
1 & 13 & 0.00 & 0.00 & -0.50 \\
2 & 13 & 0.00 & 0.00 & 0.50 \\
3 & 13 & 0.00 & 0.00 & 0.00 \\
4 & 4 & 0.00 & 0.00 & 0.00 \\
5 & 13 & 0.00 & 0.00 & 0.50
\end{tabular}

$-12.4726$

27.3362

0.0025

0.0000

$\mathrm{X} \quad \mathrm{Y}$

$\begin{array}{lll}0.17 & -0.35 & 0.00\end{array}$

$\begin{array}{lll}0.17 & 0.35 & 0.00\end{array}$

$\begin{array}{lll}-0.13 & 0.00 & 0.00\end{array}$

$\begin{array}{lll}0.00 & 0.00 & 0.00\end{array}$

$\begin{array}{lll}-0.17 & -0.35 & 0.00\end{array}$

$\begin{array}{lll}0.13 & 0.00 & 0.00\end{array}$

$\begin{array}{lll}-0.17 & 0.35 & 0.00\end{array}$

$\begin{array}{lll}0.42 & 0.00 & 0.00\end{array}$

$\begin{array}{lll}-0.42 & 0.00 & 0.00\end{array}$

\section{5}

$\mathrm{BU}$

6.8598

25.8470

0.0007

$$
0.0304
$$

X Y

$\begin{array}{lll}0.00 & 0.39 & 0.00\end{array}$

$\begin{array}{lll}0.00 & -0.39 & 0.00\end{array}$

$\begin{array}{lll}0.31 & 0.00 & 0.00\end{array}$

$\begin{array}{lll}-0.27 & 0.00 & 0.00\end{array}$

$\begin{array}{lll}0.00 & -0.39 & 0.00\end{array}$
$-9.8915$

27.7211

0.0016

0.0000

Z

$\mathrm{X} \quad \mathrm{Y}$

Z

$\begin{array}{lll}0.00 & 0.00 & 0.06\end{array}$

$\begin{array}{lll}0.00 & 0.00 & 0.06\end{array}$

$\begin{array}{lll}0.00 & 0.00 & -0.35\end{array}$

$\begin{array}{lll}0.00 & 0.00 & 0.00\end{array}$

$\begin{array}{lll}0.00 & 0.00 & -0.06\end{array}$

$\begin{array}{lll}0.00 & 0.00 & 0.35\end{array}$

$\begin{array}{lll}0.00 & 0.00 & -0.06\end{array}$

$\begin{array}{lll}0.00 & 0.00 & 0.61\end{array}$

$\begin{array}{lll}0.00 & 0.00 & -0.61\end{array}$

6

AG

97.0049

27.0133

0.1498

0.0000

$\begin{array}{llll}\mathrm{Z} & \mathrm{X} & \mathrm{Y}\end{array}$

Z 


\begin{tabular}{ccccccccccc}
6 & 13 & 0.00 & 0.00 & 0.00 & 0.31 & 0.00 & 0.00 & 0.00 & -0.46 & 0.00 \\
7 & 13 & 0.00 & 0.00 & -0.50 & 0.00 & 0.39 & 0.00 & -0.11 & -0.36 & 0.00 \\
8 & 14 & 0.00 & 0.00 & 0.00 & -0.26 & 0.00 & 0.00 & 0.00 & 0.13 & 0.00 \\
9 & 14 & 0.00 & 0.00 & 0.00 & -0.26 & 0.00 & 0.00 & 0.00 & -0.13 & 0.00 \\
& & & 7 & & & 8 & & & \multicolumn{2}{c}{9}
\end{tabular}

BG $\quad$ AU $\quad$ AU

$\begin{array}{llll}\text { Frequencies -- } & 107.5800 & 109.9088 & 144.1977\end{array}$

$\begin{array}{lll}\text { Red. masses -- } & 27.1376 & 27.1913\end{array}$

$\begin{array}{llll}\text { Frc consts } & -- & 0.1850 & 0.1935\end{array}$

$\begin{array}{lllll}\text { IR Inten } \quad-- & 0.0000 & 7.2996 & 25.7762\end{array}$

\begin{tabular}{rrrrrrrrrrrr} 
Atom AN & $\mathrm{X}$ & $\mathrm{Y}$ & \multicolumn{2}{c}{$\mathrm{Z}$} & \multicolumn{2}{c}{$\mathrm{X}$} & $\mathrm{Y}$ & $\mathrm{Z}$ & $\mathrm{X}$ & $\mathrm{Y}$ \\
1 & 13 & 0.00 & 0.00 & -0.37 & 0.00 & 0.00 & 0.36 & 0.00 & 0.00 & -0.04 \\
2 & 13 & 0.00 & 0.00 & -0.37 & 0.00 & 0.00 & 0.36 & 0.00 & 0.00 & -0.04 \\
3 & 13 & 0.00 & 0.00 & 0.38 & 0.00 & 0.00 & -0.38 & 0.00 & 0.00 & 0.02 \\
4 & 4 & 0.00 & 0.00 & 0.00 & 0.00 & 0.00 & 0.00 & 0.00 & 0.00 & 0.99 \\
5 & 13 & 0.00 & 0.00 & 0.37 & 0.00 & 0.00 & 0.36 & 0.00 & 0.00 & -0.04 \\
6 & 13 & 0.00 & 0.00 & -0.38 & 0.00 & 0.00 & -0.38 & 0.00 & 0.00 & 0.02 \\
7 & 13 & 0.00 & 0.00 & 0.37 & 0.00 & 0.00 & 0.36 & 0.00 & 0.00 & -0.04 \\
8 & 14 & 0.00 & 0.00 & 0.28 & 0.00 & 0.00 & -0.32 & 0.00 & 0.00 & -0.10 \\
9 & 14 & 0.00 & 0.00 & -0.28 & 0.00 & 0.00 & -0.32 & 0.00 & 0.00 & -0.10
\end{tabular}

$10 \quad 11 \quad 12$

BU BU $\quad$ BG

Frequencies -- 169.1683

191.8744

218.9370

$\begin{array}{lrrr}\text { Red. masses -- } & 24.7346 & 9.6121 & 27.0577 \\ \text { Frc consts -- } & 0.4171 & 0.2085 & 0.7642\end{array}$

\begin{tabular}{lrrrrrrrr} 
IR Inten & -- & \multicolumn{9}{c}{$\begin{array}{c}7.2447 \\
\text { Atom AN }\end{array}$} & $\mathrm{X}$ & $\mathrm{Y}$ & $\mathrm{Z}$ & $\mathrm{X}$ & $\mathrm{Y}$ & $\mathrm{Z}$ & $\mathrm{X}$ & $\mathrm{Y}$
\end{tabular}

$\begin{array}{lllllllllll}1 & 13 & 0.30 & 0.01 & 0.00 & -0.06 & 0.01 & 0.00 & -0.23 & 0.30 & 0.00\end{array}$

$\begin{array}{lllllllllll}2 & 13 & -0.30 & 0.01 & 0.00 & -0.06 & -0.01 & 0.00 & 0.23 & 0.30 & 0.00\end{array}$

$\begin{array}{lllllllllll}3 & 13 & 0.00 & 0.39 & 0.00 & 0.04 & 0.00 & 0.00 & 0.00 & -0.42 & 0.00\end{array}$

$\begin{array}{lllllllllll}4 & 4 & 0.00 & -0.37 & 0.00 & 0.98 & 0.00 & 0.00 & 0.00 & 0.00 & 0.00\end{array}$

$\begin{array}{lllllllllll}5 & 13 & -0.30 & 0.01 & 0.00 & -0.06 & -0.01 & 0.00 & -0.23 & -0.30 & 0.00\end{array}$

$\begin{array}{lllllllllll}6 & 13 & 0.00 & 0.39 & 0.00 & 0.04 & 0.00 & 0.00 & 0.00 & 0.42 & 0.00\end{array}$

$\begin{array}{lllllllllll}7 & 13 & 0.30 & 0.01 & 0.00 & -0.06 & 0.01 & 0.00 & 0.23 & -0.30 & 0.00\end{array}$

$\begin{array}{llllllllllll}8 & 14 & 0.00 & -0.33 & 0.00 & -0.09 & 0.00 & 0.00 & 0.00 & 0.20 & 0.00\end{array}$

$\begin{array}{lllllllllll}9 & 14 & 0.00 & -0.33 & 0.00 & -0.09 & 0.00 & 0.00 & 0.00 & -0.20 & 0.00\end{array}$

$13 \quad 14 \quad 15$

BU AG AG

Frequencies -- 249.4593

Red. masses -- 26.1492

$\begin{array}{llccc}\text { Frc consts } & -- & 0.9588 & 1.2131 & 1.5204 \\ \text { IR Inten } & -- & 5.2864 & 0.0000 & 0.0000\end{array}$

$275.7033 \quad 309.2556$

$\begin{array}{lllllllllll}\text { Atom AN } & \text { X } & \text { Y } & \text { Z } & \text { X } & \text { Y } & \text { Z } & \text { X } & \text { Y } & \text { Z }\end{array}$

$\begin{array}{lllllllllll}1 & 13 & 0.14 & 0.33 & 0.00 & 0.38 & 0.14 & 0.00 & 0.25 & 0.22 & 0.00\end{array}$ 


\begin{tabular}{|c|c|c|c|c|c|c|c|c|c|c|c|}
\hline 2 & 13 & -0.14 & 0.33 & 0.00 & -0.38 & 0.14 & 0.00 & 0.25 & -0.22 & 0.00 & \\
\hline 3 & 13 & 0.00 & -0.43 & 0.00 & 0.00 & -0.34 & 0.00 & -0.53 & 0.00 & 0.00 & \\
\hline 4 & 4 & 0.00 & -0.22 & 0.00 & 0.00 & 0.00 & 0.00 & 0.00 & 0.00 & 0.00 & \\
\hline 5 & 13 & -0.14 & 0.33 & 0.00 & 0.38 & -0.14 & 0.00 & -0.25 & 0.22 & 0.00 & \\
\hline 6 & 13 & 0.00 & -0.43 & 0.00 & 0.00 & 0.34 & 0.00 & 0.53 & 0.00 & 0.00 & \\
\hline 7 & 13 & 0.14 & 0.33 & 0.00 & -0.38 & -0.14 & 0.00 & -0.25 & -0.22 & 0.00 & \\
\hline 8 & 14 & 0.00 & -0.18 & 0.00 & 0.00 & -0.23 & 0.00 & 0.00 & 0.00 & 0.00 & \\
\hline 9 & 14 & 0.00 & -0.18 & 0.00 & 0.00 & 0.23 & 0.00 & 0.00 & 0.00 & 0.00 & \\
\hline & & & 16 & & \multicolumn{3}{|c|}{17} & \multicolumn{4}{|c|}{18} \\
\hline & & & $\mathrm{BU}$ & & \multicolumn{3}{|c|}{ BU } & \multicolumn{4}{|c|}{ AG } \\
\hline \multicolumn{2}{|c|}{ Frequencies -- } & \multicolumn{2}{|c|}{312.5611} & & \multicolumn{3}{|c|}{350.9317} & \multicolumn{4}{|c|}{368.8334} \\
\hline \multicolumn{2}{|c|}{ Red. masses - } & & 25.7818 & & \multicolumn{3}{|c|}{23.9724} & \multicolumn{4}{|c|}{27.5290} \\
\hline \multicolumn{2}{|c|}{ Frc consts } & -- & 1.4840 & & \multicolumn{3}{|c|}{1.7394} & \multicolumn{4}{|c|}{2.2065} \\
\hline \multicolumn{2}{|c|}{ IR Inten } & -- & 7.3218 & & \multicolumn{3}{|c|}{15.4989} & \multicolumn{4}{|c|}{0.0000} \\
\hline \multicolumn{2}{|c|}{ Atom AN } & $X$ & $\mathrm{Y}$ & Z & \multicolumn{2}{|r|}{$\mathrm{X}$} & Y & Z & \multicolumn{2}{|c|}{$\mathrm{X}$} & $\mathrm{Z}$ \\
\hline 1 & 13 & -0.24 & -0.21 & 0.00 & -0.34 & 0.13 & 0.00 & -0.25 & 0.22 & 0.00 & \\
\hline 2 & 13 & -0.24 & 0.21 & 0.00 & 0.34 & 0.13 & 0.00 & -0.25 & -0.22 & 0.00 & \\
\hline 3 & 13 & 0.51 & 0.00 & 0.00 & 0.00 & 0.15 & 0.00 & -0.07 & 0.00 & 0.00 & \\
\hline 4 & 4 & -0.26 & 0.00 & 0.00 & 0.00 & -0.42 & 0.00 & 0.00 & 0.00 & 0.00 & \\
\hline 5 & 13 & -0.24 & 0.21 & 0.00 & 0.34 & 0.13 & 0.00 & 0.25 & 0.22 & 0.00 & \\
\hline 6 & 13 & 0.51 & 0.00 & 0.00 & 0.00 & 0.15 & 0.00 & 0.07 & 0.00 & 0.00 & \\
\hline 7 & 13 & -0.24 & -0.21 & 0.00 & -0.34 & 0.13 & 0.00 & 0.25 & -0.22 & 0.00 & \\
\hline 8 & 14 & 0.02 & 0.00 & 0.00 & 0.00 & -0.34 & 0.00 & 0.52 & 0.00 & 0.00 & \\
\hline \multirow[t]{3}{*}{9} & 14 & 0.02 & 0.00 & 0.00 & 0.00 & -0.34 & 0.00 & -0.52 & 0.00 & 0.00 & \\
\hline & & & 19 & & & 20 & & & 2 & 1 & \\
\hline & & & $\mathrm{BU}$ & & & A & & & & $\mathrm{BU}$ & \\
\hline Freql & lencies - & & 0.6673 & & & 31.9430 & & & 815.657 & & \\
\hline Red. & masses & & 27.2430 & & & 27.7509 & & & 10.22 & & \\
\hline Frc c & onsts - & -- & 2.2053 & & & 3.0506 & & & $4.00 €$ & & \\
\hline IR In & & -- & 20.7522 & & & 0.000 & & & 906.28 & & \\
\hline Atom & $\mathrm{AN}$ & $\mathrm{X}$ & $\mathrm{Y}$ & $\mathrm{Z}$ & & $\mathrm{X}$ & Y & Z & X & $\mathrm{Y}$ & Z \\
\hline 1 & 13 & 0.25 & -0.22 & 0.00 & 0.20 & -0.12 & 0.00 & 0.00 & 0.01 & 0.00 & \\
\hline 2 & 13 & 0.25 & 0.22 & 0.00 & -0.20 & -0.12 & 0.00 & 0.00 & 0.01 & 0.00 & \\
\hline 3 & 13 & 0.07 & 0.00 & 0.00 & 0.00 & -0.09 & 0.00 & 0.00 & 0.00 & 0.00 & \\
\hline 4 & 4 & -0.13 & 0.00 & 0.00 & 0.00 & 0.00 & 0.00 & 0.00 & 0.97 & 0.00 & \\
\hline 5 & 13 & 0.25 & 0.22 & 0.00 & 0.20 & 0.12 & 0.00 & 0.00 & 0.01 & 0.00 & \\
\hline 6 & 13 & 0.07 & 0.00 & 0.00 & 0.00 & 0.09 & 0.00 & 0.00 & 0.00 & 0.00 & \\
\hline 7 & 13 & 0.25 & -0.22 & 0.00 & -0.20 & 0.12 & 0.00 & 0.00 & 0.01 & 0.00 & \\
\hline 8 & 14 & -0.52 & 0.00 & 0.00 & 0.00 & 0.62 & 0.00 & 0.00 & -0.18 & 0.00 & \\
\hline 9 & 14 & -0.52 & 0.00 & 0.00 & 0.00 & -0.62 & 0.00 & 0.00 & -0.18 & 0.00 & \\
\hline
\end{tabular}




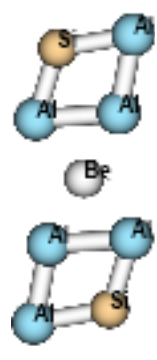

$\begin{array}{rrrrc}4 & 0 & 0.000000 & 0.000000 & 0.000000 \\ 13 & 0 & 0.000000 & 2.351150 & 0.000000 \\ 13 & 0 & 0.000000 & -2.351150 & 0.000000 \\ 13 & 0 & 2.208288 & 0.993224 & 0.000000 \\ 13 & 0 & -2.208288 & -0.993224 & 0.000000 \\ 13 & 0 & -0.742301 & -4.760882 & 0.000000 \\ 14 & 0 & -2.644294 & -3.299146 & 0.000000 \\ 13 & 0 & 0.742301 & 4.760882 & 0.000000 \\ 14 & 0 & 2.644294 & 3.299146 & 0.000000\end{array}$

State $=1-A G \backslash H F=-2048.403811$

1

AU

Frequencies -- $\quad-34.5801$

Red. masses -- 26.7053

Frc consts --- 0.0188

IR Inten $\quad$-- 0.5055

Atom AN $\mathrm{X} \quad \mathrm{Y}$

$\begin{array}{llll}1 & 4 & 0.00 & 0.00\end{array}$

$\begin{array}{llll}2 & 13 & 0.00 & 0.00\end{array}$

$\begin{array}{llll}3 & 13 & 0.00 & 0.00\end{array}$

$4 \quad 13$

$5 \quad 13$

0.00

0.00

613

0.00

0.00

0.00

$8 \quad 13$

0.00

0.00

\section{$\begin{array}{ll}0.00 & -0.32\end{array}$}

$0.00 \quad-0.32$

$0.00 \quad 0.35$

$0.00 \quad-0.38$

$0.00 \quad 0.35$

$0.00 \quad-0.38$

4

BG

Frequencies -- $\quad 42.8927$

Red. masses -- $\quad 27.0274$

Frc consts -- 0.0293

IR Inten $\quad$-- $\quad 0.0000$

Atom AN $\quad \mathrm{X} \quad \mathrm{Y}$

$\begin{array}{lllll}1 & 4 & 0.00 & 0.00 & 0.00\end{array}$

$\begin{array}{lllll}2 & 13 & 0.00 & 0.00 & 0.59\end{array}$

2

3

$\mathrm{AU}$

12.2410

22.9163

0.0020

0.4270

$\mathrm{X}$

0.00

0.00

0.00

0.00

0.48

0.45

$\begin{array}{lll}0.00 & 0.00 & 0.31\end{array}$

$\begin{array}{lll}0.00 & 0.00 & 0.26\end{array}$

$\begin{array}{lll}0.00 & 0.00 & 0.26\end{array}$

$\begin{array}{lll}0.00 & 0.00 & -0.39\end{array}$

$\begin{array}{llll}0.00 & 0.00 & -0.25\end{array}$

$\begin{array}{lll}0.00 & 0.00 & -0.39\end{array}$

$\begin{array}{lll}0.00 & 0.00 & -0.25\end{array}$

5

AU

102.3096

27.1606

0.1675

7.5519

24.2042

22.2986

0.0077

1.0947

$\mathrm{X} \quad \mathrm{Y}$

$\begin{array}{lll}0.45 & -0.26 & 0.00\end{array}$

$\begin{array}{lll}0.06 & -0.23 & 0.00\end{array}$

$\begin{array}{lll}0.06 & -0.23 & 0.00\end{array}$

$\begin{array}{lll}0.29 & 0.16 & 0.00\end{array}$

$\begin{array}{lll}0.29 & 0.16 & 0.00\end{array}$

$\begin{array}{lll}-0.35 & -0.13 & 0.00\end{array}$

$\begin{array}{lll}-0.07 & 0.23 & 0.00\end{array}$

$\begin{array}{lll}-0.35 & -0.13 & 0.00\end{array}$

$\begin{array}{lll}-0.07 & 0.23 & 0.00\end{array}$

6

AG

109.1909

27.3653

0.1922

0.0000

X Y

$\begin{array}{lll}0.00 & 0.00 & 0.07\end{array}$

Z

$\begin{array}{ll}\mathrm{X} & \mathrm{Y}\end{array}$

Z

$\begin{array}{llllll}3 & 13 & 0.00 & 0.00 & -0.59\end{array}$

0.00

$\begin{array}{ll}0.00 & -0.33\end{array}$

$\begin{array}{lll}0.00 & 0.00 & 0.00\end{array}$

$\begin{array}{lll}0.00 & 0.00 & -0.33\end{array}$

$\begin{array}{lll}-0.26 & -0.11 & 0.00\end{array}$

$4 \quad 13$

$\begin{array}{lll}0.00 & 0.00 & 0.27\end{array}$

$\begin{array}{lll}0.00 & 0.00 & 0.42\end{array}$

$\begin{array}{lll}0.26 & 0.11 & 0.00\end{array}$

$\begin{array}{lll}-0.32 & -0.31 & 0.00\end{array}$ 


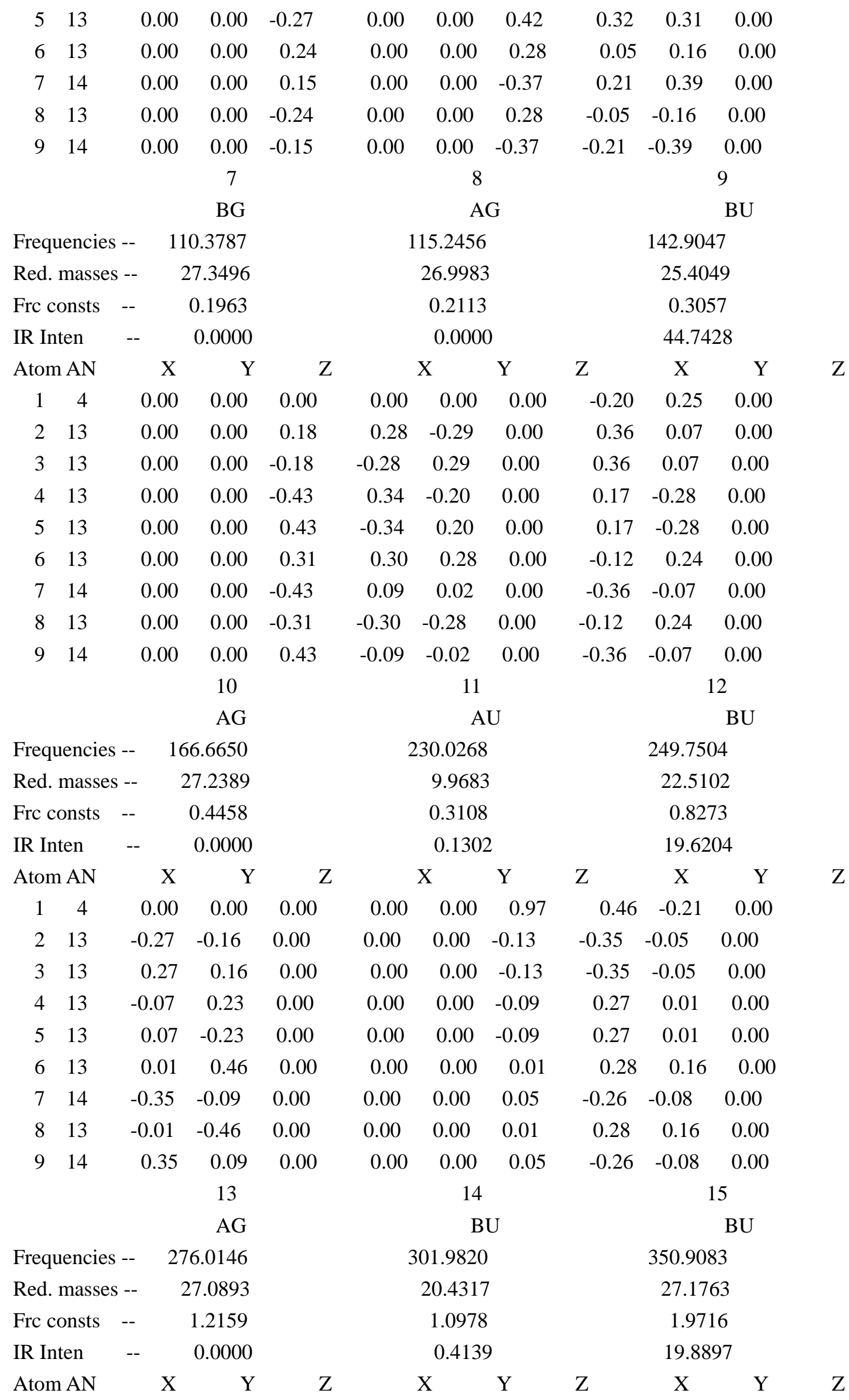




$\begin{array}{rrrrrrccccc}1 & 4 & 0.00 & 0.00 & 0.00 & -0.30 & 0.53 & 0.00 & 0.02 & 0.06 & 0.00 \\ 2 & 13 & -0.40 & -0.05 & 0.00 & -0.14 & 0.31 & 0.00 & 0.25 & -0.04 & 0.00 \\ 3 & 13 & 0.40 & 0.05 & 0.00 & -0.14 & 0.31 & 0.00 & 0.25 & -0.04 & 0.00 \\ 4 & 13 & 0.46 & 0.00 & 0.00 & 0.25 & -0.09 & 0.00 & -0.17 & 0.32 & 0.00 \\ 5 & 13 & -0.46 & 0.00 & 0.00 & 0.25 & -0.09 & 0.00 & -0.17 & 0.32 & 0.00 \\ 6 & 13 & -0.27 & 0.00 & 0.00 & -0.03 & -0.35 & 0.00 & 0.28 & -0.31 & 0.00 \\ 7 & 14 & 0.23 & 0.00 & 0.00 & -0.03 & 0.04 & 0.00 & -0.36 & 0.02 & 0.00 \\ 8 & 13 & 0.27 & 0.00 & 0.00 & -0.03 & -0.35 & 0.00 & 0.28 & -0.31 & 0.00 \\ 9 & 14 & -0.23 & 0.00 & 0.00 & -0.03 & 0.04 & 0.00 & -0.36 & 0.02 & 0.00\end{array}$

AG

Frequencies -- 354.1013

AG

$\begin{array}{lrrrr}\text { Red. masses -- } & 27.0143 & & 27.2624 \\ \text { Frc consts } & -- & 1.9957 & & 2.1301 \\ \text { IR Inten } & -- & 0.0000 & & 0.0000 \\ \text { Atom AN } & & \mathrm{X} & \mathrm{Y} & \mathrm{X}\end{array}$

\begin{tabular}{rrrrrrrrrrrr} 
Atom AN & \multicolumn{1}{c}{$\mathrm{X}$} & \multicolumn{2}{c}{$\mathrm{Y}$} & \multicolumn{2}{c}{$\mathrm{Z}$} & \multicolumn{2}{c}{$\mathrm{X}$} & $\mathrm{Y}$ & $\mathrm{Z}$ & $\mathrm{X}$ & $\mathrm{Y}$ \\
1 & 4 & 0.00 & 0.00 & 0.00 & 0.00 & 0.00 & 0.00 & 0.83 & -0.08 & 0.00 \\
2 & 13 & 0.05 & 0.58 & 0.00 & 0.26 & -0.14 & 0.00 & 0.05 & 0.23 & 0.00 \\
3 & 13 & -0.05 & -0.58 & 0.00 & -0.26 & 0.14 & 0.00 & 0.05 & 0.23 & 0.00 \\
4 & 13 & 0.05 & -0.11 & 0.00 & -0.16 & 0.34 & 0.00 & -0.06 & 0.08 & 0.00 \\
5 & 13 & -0.05 & 0.11 & 0.00 & 0.16 & -0.34 & 0.00 & -0.06 & 0.08 & 0.00 \\
6 & 13 & 0.03 & 0.36 & 0.00 & -0.29 & 0.21 & 0.00 & -0.14 & -0.03 & 0.00 \\
7 & 14 & 0.11 & -0.06 & 0.00 & 0.38 & 0.02 & 0.00 & 0.01 & -0.26 & 0.00 \\
8 & 13 & -0.03 & -0.36 & 0.00 & 0.29 & -0.21 & 0.00 & -0.14 & -0.03 & 0.00 \\
9 & 14 & -0.11 & 0.06 & 0.00 & -0.38 & -0.02 & 0.00 & 0.01 & -0.26 & 0.00
\end{tabular}

AG

BU

Frequencies -- $\quad 435.3678$

443.9086

18.4370

603.2734

Red. masses -- 27.5086

2.1406

390.3149

14.6137

1.3117

7.0455

Frc consts -- 3.0721

$\begin{array}{lllll}\text { IR Inten } \quad-- & 0.0000 & 26.1767 & 100.1486\end{array}$

10.0606

2.1573

$\begin{array}{lllllllllll}\text { Atom AN } & \text { X } & \text { Y } & \text { Z } & \text { X } & \text { Y } & \text { Z } & \text { X } & \text { Y } & \text { Z }\end{array}$

$\begin{array}{lllllllllll}1 & 4 & 0.00 & 0.00 & 0.00 & -0.58 & 0.38 & 0.00 & 0.41 & 0.88 & 0.00\end{array}$

$\begin{array}{lllllllllll}2 & 13 & 0.01 & 0.14 & 0.00 & -0.01 & -0.11 & 0.00 & -0.03 & -0.14 & 0.00\end{array}$

$\begin{array}{lllllllllll}3 & 13 & -0.01 & -0.14 & 0.00 & -0.01 & -0.11 & 0.00 & -0.03 & -0.14 & 0.00\end{array}$

$\begin{array}{lllllllllll}4 & 13 & 0.18 & 0.38 & 0.00 & 0.15 & 0.31 & 0.00 & -0.06 & -0.05 & 0.00\end{array}$

$\begin{array}{lllllllllll}5 & 13 & -0.18 & -0.38 & 0.00 & 0.15 & 0.31 & 0.00 & -0.06 & -0.05 & 0.00\end{array}$

$\begin{array}{lllllllllll}6 & 13 & 0.20 & -0.05 & 0.00 & -0.09 & 0.08 & 0.00 & 0.01 & 0.01 & 0.00\end{array}$

$\begin{array}{lllllllllll}7 & 14 & -0.06 & 0.51 & 0.00 & 0.05 & -0.33 & 0.00 & 0.01 & 0.03 & 0.00\end{array}$

$\begin{array}{lllllllllll}8 & 13 & -0.20 & 0.05 & 0.00 & -0.09 & 0.08 & 0.00 & 0.01 & 0.01 & 0.00\end{array}$

$\begin{array}{lllllllllll}9 & 14 & 0.06 & -0.51 & 0.00 & 0.05 & -0.33 & 0.00 & 0.01 & 0.03 & 0.00\end{array}$

Al3SiBeAl3Si-c-Al-c-Si-0-C2v-b36311+gx.frq3m-19.6 


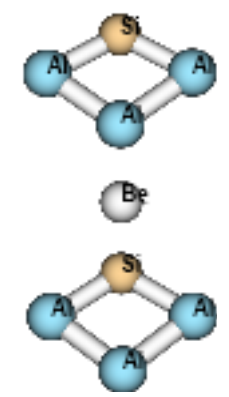

$\begin{array}{rrrrr}4 & 0 & 0.000000 & 0.000000 & -0.141643 \\ 13 & 0 & 0.000000 & 2.087301 & 3.667913 \\ 13 & 0 & 0.000000 & -2.087301 & 3.667913 \\ 13 & 0 & 0.000000 & 0.000000 & -5.109533 \\ 13 & 0 & 0.000000 & 2.048815 & -3.528881 \\ 13 & 0 & 0.000000 & -2.048815 & -3.528881 \\ 14 & 0 & 0.000000 & 0.000000 & -2.254799 \\ 13 & 0 & 0.000000 & 0.000000 & 2.139301 \\ 14 & 0 & 0.000000 & 0.000000 & 4.795140\end{array}$

State $=1-A 1 \backslash H F=-2048.4051022$

\section{1}

B1

Frequencies -- $\quad-19.6275$

Red. masses -- 26.1241

Frc consts -- 0.0059

Atom AN $\mathrm{X} \quad \mathrm{Y}$

$\begin{array}{llll}1 & 4 & -0.28 & 0.00\end{array}$

$\begin{array}{lllll}2 & 13 & 0.07 & 0.00 & 0.00\end{array}$

$\begin{array}{lllll}3 & 13 & 0.07 & 0.00 & 0.00\end{array}$

$\begin{array}{lllll}4 & 13 & 0.59 & 0.00 & 0.00\end{array}$

$\begin{array}{lllll}5 & 13 & 0.00 & 0.00 & 0.00\end{array}$

$\begin{array}{lllll}6 & 13 & 0.00 & 0.00 & 0.00\end{array}$

$\begin{array}{lllll}7 & 14 & -0.73 & 0.00 & 0.00\end{array}$

$\begin{array}{lllll}8 & 13 & -0.07 & 0.00 & 0.00\end{array}$

$9 \quad 14$
IR Inten $\quad$-- 2.3162

2

B2

$-12.2092$

26.9931

0.0024

0.0006

$\mathrm{X} \quad \mathrm{Y}$

$\begin{array}{lll}0.00 & -0.11 & 0.00\end{array}$

0.00

0.00

0.00

0.13

$0.13 \quad-0.16$

$0.25 \quad 0.00$

$\begin{array}{lll}0.00 & -0.15 & 0.49\end{array}$

$\begin{array}{lll}0.00 & -0.15 & -0.49\end{array}$

$\begin{array}{lll}0.00 & -0.48 & 0.00\end{array}$

$\begin{array}{lll}0.00 & 0.30 & 0.00\end{array}$

$\begin{array}{llll}0.00 & 0.04 & 0.00\end{array}$

5

B1

39.0753

23.1428

0.0208

0.7181

IR Inten -- $\quad 0.0033$

Atom AN $\quad \mathrm{X} \quad \mathrm{Y}$

$\begin{array}{llll}1 & 4 & 0.00 & 0.25\end{array}$

213

$3 \quad 13$

$\begin{array}{lll}0.00 & 0.25 & 0.00 \\ 0.00 & 0.01 & 0.51\end{array}$

Z $\quad$ X $\quad$ Y

$\begin{array}{lll}-0.48 & 0.00 & 0.00\end{array}$

Z

$\begin{array}{lll}\mathrm{Z} & \mathrm{X} & \mathrm{Y}\end{array}$

$-5.5206$

26.9815

0.0005

0.0000

$\begin{array}{lll}0.00 & 0.00 & 0.00\end{array}$

$\begin{array}{lll}-0.50 & 0.00 & 0.00\end{array}$

$\begin{array}{lll}0.50 & 0.00 & 0.00\end{array}$

$\begin{array}{lll}0.00 & 0.00 & 0.00\end{array}$

$\begin{array}{lll}0.50 & 0.00 & 0.00\end{array}$

$\begin{array}{lll}-0.50 & 0.00 & 0.00\end{array}$

$\begin{array}{lll}0.00 & 0.00 & 0.00\end{array}$

$\begin{array}{lll}0.00 & 0.00 & 0.00\end{array}$

$\begin{array}{lll}0.00 & 0.00 & 0.00\end{array}$

6

A1

97.4518

27.1252

0.1518

1.6472

$\mathrm{X}$

Z 


$\begin{array}{rrrrrrrrrrr}4 & 13 & 0.00 & -0.23 & 0.00 & 0.00 & 0.00 & 0.00 & 0.00 & 0.00 & -0.47 \\ 5 & 13 & 0.00 & -0.07 & -0.21 & 0.06 & 0.00 & 0.00 & 0.00 & -0.12 & -0.37 \\ 6 & 13 & 0.00 & -0.07 & 0.21 & 0.06 & 0.00 & 0.00 & 0.00 & 0.12 & -0.37 \\ 7 & 14 & 0.00 & 0.07 & 0.00 & 0.23 & 0.00 & 0.00 & 0.00 & 0.00 & -0.12 \\ 8 & 13 & 0.00 & 0.45 & 0.00 & -0.72 & 0.00 & 0.00 & 0.00 & 0.00 & 0.20 \\ 9 & 14 & 0.00 & -0.26 & 0.00 & 0.44 & 0.00 & 0.00 & 0.00 & 0.00 & 0.37 \\ & & & 7 & & & 8 & & & & 9\end{array}$

B1 B1

B1

Frequencies -- 101.7991

108.4963

151.2744

27.1575

10.0610

Red. masses -- 27.3236

0.1884

0.1357

$\begin{array}{lll}\text { Frc consts } & -- & 0.1668 \\ \text { IR Inten } & -- & 4.1104\end{array}$

4.4596

14.7449

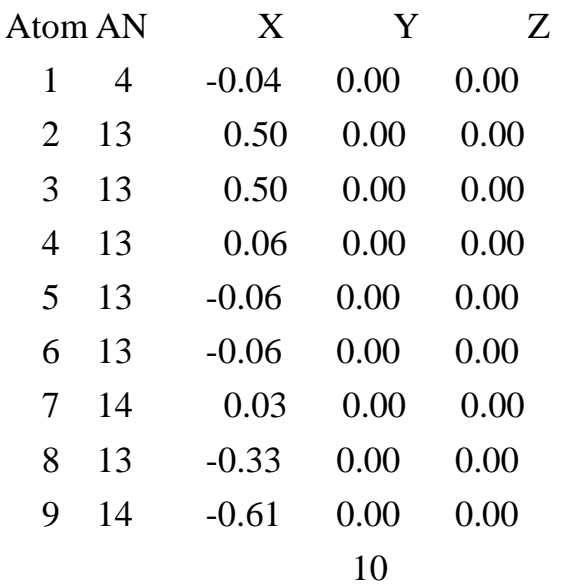

B2

$\begin{array}{ll}\mathrm{X} & \mathrm{Y}\end{array}$

$\begin{array}{lll}0.01 & 0.00 & 0.00\end{array}$

Z

0.97

X Y

Z

$\begin{array}{lll}0.04 & 0.00 & 0.00\end{array}$

$\begin{array}{lll}-0.02 & 0.00 & 0.00\end{array}$

$\begin{array}{lll}0.04 & 0.00 & 0.00\end{array}$

$\begin{array}{lll}-0.02 & 0.00 & 0.00\end{array}$

$\begin{array}{lll}-0.54 & 0.00 & 0.00\end{array}$

$\begin{array}{lll}0.02 & 0.00 & 0.00\end{array}$

$\begin{array}{lll}0.51 & 0.00 & 0.00\end{array}$

$\begin{array}{lll}-0.04 & 0.00 & 0.00\end{array}$

$\begin{array}{lll}0.51 & 0.00 & 0.00\end{array}$

$\begin{array}{lll}-0.04 & 0.00 & 0.00\end{array}$

$-0.42 \quad 0.00$
-0.09

$\begin{array}{ll}-0.09 & 0.00\end{array}$

0.00

$\begin{array}{lll}-0.08 & 0.00 & 0.00\end{array}$

$-0.05$

$0.00 \quad 0.00$

11

A1

$\begin{array}{lll}-0.21 & 0.00 & 0.00\end{array}$

$\begin{array}{lll}0.06 & 0.00 & 0.00\end{array}$

12

A1

Frequencies -- 174.7493

187.6789

210.9086

24.8786

27.2569

0.5163

0.7144

Frc consts -- 0.1744

0.0493

12.2910

$\begin{array}{lllr}\text { IR Inten } & -- & & 0.7353 \\ \text { Atom AN } & & \text { X } & \text { Y }\end{array}$

$\begin{array}{ccccc}\text { Atom } & \text { AN } & \text { X } & \text { Y } & \text { Z } \\ 1 & 4 & 0.00 & 0.98 & 0.00 \\ 2 & 13 & 0.00 & 0.00 & 0.00 \\ 3 & 13 & 0.00 & 0.00 & 0.00 \\ 4 & 13 & 0.00 & 0.03 & 0.00 \\ 5 & 13 & 0.00 & -0.05 & 0.01 \\ 6 & 13 & 0.00 & -0.05 & -0.01 \\ 7 & 14 & 0.00 & -0.09 & 0.00 \\ 8 & 13 & 0.00 & -0.15 & 0.00 \\ 9 & 14 & 0.00 & -0.01 & 0.00 \\ & & & 13 & \end{array}$

A1

Frequencies -- $\quad 246.4401$

Red. masses -- 25.5703

Frc consts -- $\quad 0.9150$

\begin{tabular}{lcc}
\multicolumn{2}{c}{$\mathrm{X}$} & $\mathrm{Y}$ \\
0.00 & 0.00 & -0.36 \\
0.00 & -0.15 & 0.13 \\
0.00 & 0.15 & 0.13 \\
0.00 & 0.00 & 0.46 \\
0.00 & 0.38 & -0.12 \\
0.00 & -0.38 & -0.12 \\
0.00 & 0.00 & -0.42 \\
0.00 & 0.00 & -0.18 \\
0.00 & 0.00 & 0.24 \\
& 14 &
\end{tabular}

B2

Z

$\begin{array}{ll}\mathrm{X} & \mathrm{Y}\end{array}$

$\begin{array}{lll}0.00 & 0.00 & -0.03\end{array}$

$\begin{array}{lll}0.00 & 0.48 & 0.31\end{array}$

$\begin{array}{lll}0.00 & -0.48 & 0.31\end{array}$

$\begin{array}{lll}0.00 & 0.00 & 0.15\end{array}$

$\begin{array}{lll}0.00 & 0.09 & -0.09\end{array}$

$\begin{array}{lll}0.00 & -0.09 & -0.09\end{array}$

$\begin{array}{lll}0.00 & 0.00 & -0.09\end{array}$

$\begin{array}{lll}0.00 & 0.00 & 0.07\end{array}$

$\begin{array}{lll}0.00 & 0.00 & -0.53\end{array}$

15

261.3997

A1

25.5942

302.7030

25.7448

1.0304

1.3899

0.4613

2.5424 


\begin{tabular}{|c|c|c|c|c|c|c|c|c|c|c|c|}
\hline \multicolumn{2}{|c|}{ Atom AN } & $\mathrm{X}$ & $\mathrm{Y}$ & $\mathrm{Z}$ & \multicolumn{2}{|c|}{$X$} & $\mathrm{Y}$ & $\mathrm{Z}$ & \multirow{2}{*}{$\begin{array}{c}X \\
0.00\end{array}$} & \multirow{2}{*}{$\begin{array}{c}\mathrm{Y} \\
0.27\end{array}$} & Z \\
\hline 1 & 4 & 0.00 & 0.00 & 0.29 & 0.00 & -0.28 & 0.00 & 0.00 & & & \\
\hline 2 & 13 & 0.00 & 0.00 & -0.17 & 0.00 & 0.36 & 0.32 & 0.00 & 0.21 & -0.28 & \\
\hline 3 & 13 & 0.00 & 0.00 & -0.17 & 0.00 & 0.36 & -0.32 & 0.00 & -0.21 & -0.28 & \\
\hline 4 & 13 & 0.00 & 0.00 & 0.56 & 0.00 & -0.03 & 0.00 & 0.00 & 0.00 & -0.21 & \\
\hline 5 & 13 & 0.00 & -0.20 & -0.42 & 0.00 & 0.01 & 0.01 & 0.00 & 0.39 & -0.04 & \\
\hline 6 & 13 & 0.00 & 0.20 & -0.42 & 0.00 & 0.01 & -0.01 & 0.00 & -0.39 & -0.04 & \\
\hline 7 & 14 & 0.00 & 0.00 & 0.24 & 0.00 & 0.02 & 0.00 & 0.00 & 0.00 & -0.02 & \\
\hline 8 & 13 & 0.00 & 0.00 & 0.22 & 0.00 & -0.67 & 0.00 & 0.00 & 0.00 & 0.55 & \\
\hline \multirow[t]{3}{*}{9} & 14 & 0.00 & 0.00 & 0.04 & 0.00 & 0.04 & 0.00 & 0.00 & 0.00 & 0.21 & \\
\hline & & & 16 & & \multicolumn{3}{|c|}{17} & \multicolumn{3}{|c|}{18} & \\
\hline & & & B2 & & \multicolumn{3}{|c|}{$\mathrm{A} 1$} & \multicolumn{3}{|c|}{ B2 } & \\
\hline \multicolumn{2}{|c|}{ Frequencies -- } & \multicolumn{2}{|c|}{306.1527} & & \multicolumn{3}{|c|}{361.9604} & \multicolumn{3}{|c|}{370.1683} & \\
\hline \multicolumn{2}{|c|}{ Red. masses -- } & \multicolumn{2}{|c|}{26.4611} & & \multicolumn{3}{|c|}{25.1208} & \multicolumn{3}{|c|}{27.3914} & \\
\hline \multicolumn{2}{|c|}{ Frc consts } & - & 1.4613 & & \multicolumn{3}{|c|}{1.9391} & \multicolumn{3}{|c|}{2.2114} & \\
\hline \multicolumn{2}{|c|}{ IR Inten } & - & 2.9691 & & & $0.004 \mathrm{C}$ & & & 11.34 & & \\
\hline Ator & $\mathrm{AN}$ & X & $\mathrm{Y}$ & Z & & X & $\mathrm{Y}$ & $\mathrm{Z}$ & X & $\mathrm{Y}$ & 7 \\
\hline 1 & 4 & 0.00 & -0.17 & 0.00 & 0.00 & 0.00 & 0.35 & 0.00 & -0.09 & 0.00 & \\
\hline 2 & 13 & 0.00 & 0.01 & 0.01 & 0.00 & -0.38 & 0.13 & 0.00 & 0.00 & 0.00 & \\
\hline 3 & 13 & 0.00 & 0.01 & -0.01 & 0.00 & 0.38 & 0.13 & 0.00 & 0.00 & 0.00 & \\
\hline 4 & 13 & 0.00 & 0.73 & 0.00 & 0.00 & 0.00 & -0.12 & 0.00 & 0.10 & 0.00 & \\
\hline 5 & 13 & 0.00 & -0.35 & -0.30 & 0.00 & 0.28 & -0.12 & 0.00 & 0.35 & -0.30 & \\
\hline 6 & 13 & 0.00 & -0.35 & 0.30 & 0.00 & -0.28 & -0.12 & 0.00 & 0.35 & 0.30 & \\
\hline 7 & 14 & 0.00 & 0.01 & 0.00 & 0.00 & 0.00 & 0.36 & 0.00 & -0.74 & 0.00 & \\
\hline 8 & 13 & 0.00 & -0.01 & 0.00 & 0.00 & 0.00 & 0.11 & 0.00 & -0.01 & 0.00 & \\
\hline 9 & 14 & 0.00 & 0.00 & 0.00 & 0.00 & 0.00 & -0.47 & 0.00 & 0.00 & 0.00 & \\
\hline & & & 19 & & & 20 & & & & 21 & \\
\hline & & & A1 & & & B2 & & & & A1 & \\
\hline Freq & lencies - & & 4.5489 & & & 40.0131 & & & 771.36 & & \\
\hline Red. & masses & & 7.2242 & & & 27.5775 & & & 10.31 & & \\
\hline Frc & onsts & - & 2.7565 & & & 3.1458 & & & 3.617 & & \\
\hline IR I & & - & 57.6148 & & & 10.7683 & & & 517.40 & & \\
\hline Ator & $\mathrm{AN}$ & X & $\mathrm{Y}$ & Z & & X & $\mathrm{Y}$ & $\mathrm{Z}$ & $\mathrm{X}$ & $\mathrm{Y}$ & Z \\
\hline 1 & 4 & 0.00 & 0.00 & -0.10 & 0.00 & 0.02 & 0.00 & 0.00 & 0.00 & 0.96 & \\
\hline 2 & 13 & 0.00 & -0.22 & -0.01 & 0.00 & 0.36 & -0.25 & 0.00 & 0.01 & 0.01 & \\
\hline 3 & 13 & 0.00 & 0.22 & -0.01 & 0.00 & 0.36 & 0.25 & 0.00 & -0.01 & 0.01 & \\
\hline 4 & 13 & 0.00 & 0.00 & 0.09 & 0.00 & 0.00 & 0.00 & 0.00 & 0.00 & 0.01 & \\
\hline 5 & 13 & 0.00 & -0.20 & 0.12 & 0.00 & 0.00 & 0.00 & 0.00 & -0.01 & 0.01 & \\
\hline 6 & 13 & 0.00 & 0.20 & 0.12 & 0.00 & 0.00 & 0.00 & 0.00 & 0.01 & 0.01 & \\
\hline 7 & 14 & 0.00 & 0.00 & -0.56 & 0.00 & 0.00 & 0.00 & 0.00 & 0.00 & -0.21 & \\
\hline 8 & 13 & 0.00 & 0.00 & 0.61 & 0.00 & 0.09 & 0.00 & 0.00 & 0.00 & -0.15 & \\
\hline 9 & 14 & 0.00 & 0.00 & -0.30 & 0.00 & -0.78 & 0.00 & 0.00 & 0.00 & 0.01 & \\
\hline
\end{tabular}




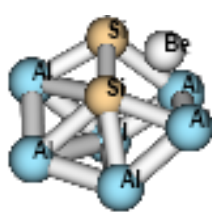

$\begin{array}{rrrrr}13 & 0 & 0.131434 & 2.489437 & 0.217057 \\ 13 & 0 & 2.283442 & 1.191493 & -0.544873 \\ 4 & 0 & 1.750646 & -1.078822 & -1.248440 \\ 13 & 0 & -0.306999 & -0.106822 & 1.532886 \\ 13 & 0 & -2.247283 & -1.325707 & -0.043087 \\ 14 & 0 & -0.036035 & 0.129230 & -1.044591 \\ 14 & 0 & 0.028896 & -2.238259 & -0.493845 \\ 13 & 0 & 1.946614 & -1.067623 & 1.001643 \\ 13 & 0 & -2.338181 & 1.422430 & -0.122713\end{array}$

State $=1-A \backslash H F=-2048.4962359$

Al3SiBeAl3Si-fusion106-b36311+gx.frq

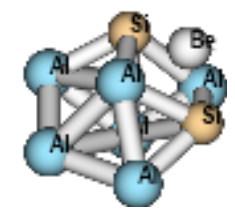

$\begin{array}{rrrrc}13 & 0 & -2.697280 & 0.000033 & 0.107121 \\ 4 & 0 & 2.008313 & -0.000028 & -1.249336 \\ 13 & 0 & -0.121408 & 0.000000 & 1.317589 \\ 13 & 0 & -0.255377 & 0.000005 & -1.357623 \\ 14 & 0 & 1.189434 & -1.880328 & -0.444228 \\ 13 & 0 & 2.347562 & -0.000035 & 1.000277 \\ 13 & 0 & -1.226713 & -2.300599 & 0.136921 \\ 14 & 0 & 1.189493 & 1.880299 & -0.444229 \\ 13 & 0 & -1.226648 & 2.300636 & 0.136926\end{array}$

State $=1-A \backslash H F=-2048.4983729$

Al3SiBeAl3Si-fusion107-b36311+gx.frq

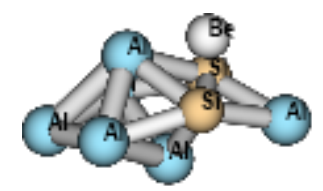

13
4
13
13
14
13
13
13
14

$\begin{array}{ccc}-1.375085 & -2.053945 & -0.142856^{\wedge} \mathrm{M} \\ 1.533521 & 0.000098 & 1.904560^{\wedge} \mathrm{M} \\ 0.063928 & -0.000025 & -1.464131^{\wedge} \mathrm{M} \\ -1.375181 & 2.053924 & -0.142870^{\wedge} \mathrm{M} \\ 1.243438 & 1.374027 & 0.299646^{\wedge} \mathrm{M} \\ 3.326592 & -0.000014 & -0.636964^{\wedge} \mathrm{M} \\ -3.077193 & -0.000055 & -0.398521^{\wedge} \mathrm{M} \\ -0.713119 & 0.000031 & 1.553814 \wedge \mathrm{M} \\ 1.243467 & -1.373978 & 0.299756\end{array}$

|State=1-A|HF=-2048.4641529 
Al3SiMgAl3Si-c-c-90-Si-Si-b36311+gx.frq6.

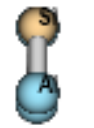

Charge $=0$ Multiplicity $=1$

Al,0,2.7474236516,1.6629721348,0.7840310425

Al,0,5.0874500196,0.7307507231,0.3620753167

Mg,0,0.0138905602,-0.3844509185,0.1524300603

Al,0,-2.6732672705,0.6460074546,-1.7812470155

Al,0,-5.0311743011,0.3691853576,-0.84022738

Al,0,4.5778724518,-1.631780058,-0.618878053

Si,0,2.5039416545,-0.5673968618,-0.1694866131

Si,0,-2.4749490722,-0.2143158024,0.4913114065

Al,0,-4.5700376939,-0.5699350294,1.5491842356

State $=1-A \backslash H F=-2233.7952034$

1

A
2

A

10.9293

26.9053

0.0019

Frc consts -- 0.0006

IR Inten -- 0.0009

Atom AN $\mathrm{X} \quad \mathrm{Y}$

113

$\begin{array}{lll}0.14 & -0.37 & 0.28\end{array}$

$2 \quad 13$

$0.08 \quad-0.14$

$0.00-0.00$

0.22

$-0.15$

$3 \quad 12$

$-0.13$

0.37

0.28

$\begin{array}{lll}5 & 13 & -0.08\end{array}$

0.14

0.22

$\begin{array}{ll}6 & 13\end{array}$

$-0.08$

$0.29 \quad-0.26$

$\begin{array}{ll}7 & 14\end{array}$

$\begin{array}{ll}8 & 14\end{array}$

$-0.01$

0.03

$-0.17$

$\begin{array}{lll}0.01 & -0.03 & -0.16\end{array}$

$9 \quad 13$

0.07

\section{4}

A

Frequencies -- $\quad 18.4687$

Red. masses -- $\quad 27.3848$

Frc consts --- 0.0055

IR Inten $\quad$-- 1.6815

Atom AN $\quad \mathrm{X} \quad \mathrm{Y} \quad \mathrm{Z}$

$\begin{array}{lllll}1 & 13 & 0.24 & -0.06 & -0.29\end{array}$

213

$0.12 \quad-0.02$

0.19

\begin{tabular}{|c|c|c|}
\hline \multicolumn{2}{|r|}{$X$} & $\mathrm{Y}$ \\
\hline 0.31 & 0.03 & -0.25 \\
\hline 0.19 & -0.03 & 0.30 \\
\hline 0.00 & 0.00 & -0.25 \\
\hline-0.30 & -0.03 & -0.24 \\
\hline-0.19 & 0.03 & 0.29 \\
\hline-0.16 & -0.13 & 0.30 \\
\hline 0.00 & -0.03 & -0.25 \\
\hline 0.00 & 0.03 & -0.23 \\
\hline 0.15 & 0.13 & 0.30 \\
\hline
\end{tabular}

A

23.2507

27.2602

0.0087

$$
0.4152
$$

$\mathrm{X}$

0.25

0.15

$-0.23-0.05$

Z 0.21

$\begin{array}{lll}0.08 & 0.39 & -0.15\end{array}$

$\begin{array}{lll}-0.05 & -0.33 & 0.00\end{array}$

$\begin{array}{lll}0.20 & -0.15 & -0.03\end{array}$

$\begin{array}{lll}0.07 & 0.38 & 0.14\end{array}$

$\begin{array}{lll}-0.20 & 0.22 & -0.06\end{array}$

$\begin{array}{lll}-0.05 & -0.30 & 0.14\end{array}$

$\begin{array}{lll}-0.06 & -0.29 & -0.14\end{array}$

$\begin{array}{ccc}-0.19 & 0.23 \quad 0.06 \\ & 6\end{array}$

A

86.2368

24.7235

0.1083

9.9648

$\begin{array}{lllll}Z & X & Y & \text { Z }\end{array}$

$\begin{array}{lll}0.03 & -0.18 & 0.01\end{array}$

$\begin{array}{lll}-0.01 & 0.09 & -0.09\end{array}$ 


$\begin{array}{rrrrr}3 & 12 & -0.08 & 0.07 & 0.00 \\ 4 & 13 & 0.26 & -0.07 & 0.29 \\ 5 & 13 & 0.13 & -0.02 & -0.21 \\ 6 & 13 & -0.25 & 0.03 & 0.05 \\ 7 & 14 & -0.08 & 0.03 & -0.46 \\ 8 & 14 & -0.08 & 0.03 & 0.46 \\ 9 & 13 & -0.26 & 0.03 & -0.05 \\ & & & 7 & \end{array}$

A

\begin{tabular}{|c|c|c|c|c|}
\hline \multicolumn{3}{|c|}{ Frequencies -- } & \multicolumn{2}{|c|}{87.5088} \\
\hline \multicolumn{3}{|c|}{ Red. masses -- } & \multicolumn{2}{|c|}{25.1615} \\
\hline \multicolumn{2}{|c|}{ Frc consts } & -- & \multicolumn{2}{|c|}{0.1135} \\
\hline \multicolumn{2}{|c|}{ IR Inten } & -- & \multicolumn{2}{|c|}{7.8029} \\
\hline \multicolumn{2}{|c|}{ Atom AN } & $\mathrm{X}$ & \multicolumn{2}{|c|}{$\mathrm{Y}$} \\
\hline 1 & 13 & -0.09 & -0.06 & -0.17 \\
\hline 2 & 13 & -0.14 & -0.03 & 0.09 \\
\hline 3 & 12 & -0.01 & 0.05 & 0.81 \\
\hline 4 & 13 & 0.09 & 0.04 & -0.18 \\
\hline 5 & 13 & 0.14 & 0.05 & 0.10 \\
\hline 6 & 13 & -0.17 & 0.05 & -0.02 \\
\hline 7 & 14 & -0.07 & 0.01 & -0.25 \\
\hline 8 & 14 & 0.07 & -0.03 & -0.25 \\
\hline 9 & 13 & 0.17 & -0.06 & -0.02 \\
\hline
\end{tabular}

A

$$
\begin{aligned}
& \begin{array}{llllll}
0.00 & 0.01 & 0.24 & -0.12 & 0.88 & -0.05
\end{array} \\
& \begin{array}{llllll}
-0.25 & 0.23 & -0.05 & 0.03 & -0.18 & 0.01
\end{array} \\
& \begin{array}{llllll}
-0.14 & -0.25 & -0.19 & -0.01 & 0.08 & 0.09
\end{array} \\
& \begin{array}{llllll}
-0.06 & 0.01 & -0.01 & 0.00 & -0.08 & 0.07
\end{array} \\
& \begin{array}{llllll}
0.06 & -0.45 & 0.14 & 0.04 & -0.22 & 0.06
\end{array} \\
& \begin{array}{llllll}
-0.06 & 0.46 & 0.14 & 0.04 & -0.21 & -0.03
\end{array} \\
& \begin{array}{llllll}
0.06 & -0.02 & -0.01 & -0.01 & -0.08 & -0.06
\end{array} \\
& 8
\end{aligned}
$$

A

98.4309

26.6171

0.1519

0.0275

$\mathrm{X} \quad \mathrm{Y}$

$\begin{array}{lll}0.23 & 0.19 & -0.02\end{array}$

$\begin{array}{lll}0.35 & -0.17 & 0.01\end{array}$

$\begin{array}{lll}0.00 & 0.02 & 0.38\end{array}$

$\begin{array}{lll}-0.23 & -0.20 & -0.02\end{array}$

$\begin{array}{lll}-0.35 & 0.18 & 0.01\end{array}$

$\begin{array}{lll}0.33 & 0.00 & -0.18\end{array}$

$\begin{array}{lll}0.15 & 0.11 & 0.02\end{array}$

$\begin{array}{lll}-0.15 & -0.12 & 0.02\end{array}$

$\begin{array}{lll}-0.33 & 0.00 & -0.18\end{array}$

11

A

158.1062

26.7515

0.3940

2.5387

\begin{tabular}{ccccc} 
IR Inten & - & \multicolumn{3}{c}{3.1342} \\
Atom AN & $\mathrm{X}$ & $\mathrm{Y}$ & $\mathrm{Z}$ \\
1 & 13 & 0.06 & -0.21 & 0.27 \\
2 & 13 & 0.08 & 0.20 & -0.26 \\
3 & 12 & 0.00 & 0.00 & 0.13 \\
4 & 13 & -0.07 & 0.22 & 0.27 \\
5 & 13 & -0.08 & -0.20 & -0.26 \\
6 & 13 & 0.07 & -0.26 & 0.20 \\
7 & 14 & 0.01 & 0.27 & -0.26 \\
8 & 14 & -0.01 & -0.28 & -0.25 \\
9 & 13 & -0.07 & 0.26 & 0.20 \\
& & & 13 &
\end{tabular}

A

Frequencies -- $\quad 248.4520$

Red. masses -- 26.9231

Frc consts -- 0.9792

$\begin{array}{llllll}\mathrm{X} & \mathrm{Y} & \mathrm{Z} & \mathrm{X} & \mathrm{Y}\end{array}$

$\begin{array}{lll}0.15 & 0.19 & 0.18\end{array}$

$\begin{array}{lll}0.04 & -0.18 & -0.17\end{array}$

$\begin{array}{lll}0.39 & -0.12 & -0.13\end{array}$

$\begin{array}{lll}-0.32 & 0.13 & 0.13\end{array}$

$\begin{array}{lll}-0.36 & 0.08 & 0.00\end{array}$

$\begin{array}{lll}0.00 & 0.00 & -0.05\end{array}$

$\begin{array}{lll}0.15 & 0.19 & -0.18\end{array}$

$\begin{array}{lll}-0.04 & 0.18 & -0.17\end{array}$

$\begin{array}{lll}0.39 & -0.12 & 0.13\end{array}$

$\begin{array}{lll}0.32 & -0.13 & 0.14\end{array}$

$\begin{array}{lll}-0.10 & -0.18 & -0.17\end{array}$

$\begin{array}{lll}0.42 & 0.13 & 0.12\end{array}$

$\begin{array}{lll}-0.27 & 0.07 & 0.10\end{array}$

$\begin{array}{lll}0.30 & 0.00 & -0.06\end{array}$

$\begin{array}{lll}-0.30 & -0.01 & -0.06\end{array}$

$\begin{array}{lll}-0.10 & -0.18 & 0.18\end{array}$

14

A

256.5021

27.1049

1.0507 $\begin{array}{lll}-0.27 & 0.07 & -0.10\end{array}$ $\begin{array}{lll}-0.42 & -0.13 & 0.12\end{array}$

15

A

311.0529

26.7235

1.5234 


\begin{tabular}{rrrrrrccccccc} 
IR Inten & - & \multicolumn{1}{c}{4.7171} & \multicolumn{9}{c}{1.7374} & \multicolumn{3}{c}{4.8420} & \\
Atom AN & $\mathrm{X}$ & $\mathrm{Y}$ & \multicolumn{2}{c}{$\mathrm{Z}$} & \multicolumn{2}{c}{$\mathrm{X}$} & $\mathrm{Y}$ & $\mathrm{Z}$ & $\mathrm{X}$ & $\mathrm{Y}$ & $\mathrm{Z}$ \\
1 & 13 & -0.31 & 0.05 & 0.05 & 0.28 & 0.02 & 0.00 & -0.31 & -0.03 & -0.02 & \\
2 & 13 & 0.31 & -0.20 & -0.20 & -0.25 & 0.19 & 0.19 & 0.23 & 0.29 & 0.27 & \\
3 & 12 & 0.20 & 0.02 & 0.00 & 0.00 & 0.00 & 0.03 & 0.30 & 0.03 & 0.00 & \\
4 & 13 & -0.30 & 0.05 & -0.06 & -0.29 & -0.02 & 0.00 & -0.30 & -0.03 & 0.02 & \\
5 & 13 & 0.31 & -0.20 & 0.21 & 0.25 & -0.19 & 0.20 & 0.22 & 0.28 & -0.26 & \\
6 & 13 & -0.24 & 0.24 & 0.24 & 0.02 & -0.33 & -0.32 & -0.14 & -0.27 & -0.25 & \\
7 & 14 & 0.13 & -0.10 & -0.07 & -0.21 & 0.09 & 0.11 & 0.08 & 0.00 & 0.01 \\
8 & 14 & 0.13 & -0.10 & 0.07 & 0.21 & -0.09 & 0.11 & 0.08 & 0.00 & -0.01 \\
9 & 13 & -0.24 & 0.24 & -0.24 & -0.02 & 0.33 & -0.33 & -0.14 & -0.26 & 0.25 &
\end{tabular}

A A

Frequencies -- $\quad 316.2570$

A

\begin{tabular}{|c|c|c|c|c|}
\hline \multicolumn{3}{|c|}{ Red. masses -- } & \multicolumn{2}{|c|}{26.9875} \\
\hline \multicolumn{2}{|c|}{ Frc consts } & -- & 1.5903 & \\
\hline \multicolumn{2}{|c|}{ IR Inten } & -- & 2.0170 & \\
\hline \multicolumn{2}{|c|}{ Atom AN } & $\mathrm{X}$ & \multicolumn{2}{|c|}{$\mathrm{Y}$} \\
\hline 1 & 13 & -0.39 & -0.12 & -0.10 \\
\hline 2 & 13 & 0.32 & 0.29 & 0.27 \\
\hline 3 & 12 & 0.00 & 0.00 & -0.03 \\
\hline 4 & 13 & 0.40 & 0.12 & -0.10 \\
\hline 5 & 13 & -0.32 & -0.29 & 0.28 \\
\hline 6 & 13 & 0.06 & -0.15 & -0.15 \\
\hline 7 & 14 & 0.06 & 0.00 & -0.01 \\
\hline 8 & 14 & -0.07 & 0.00 & -0.01 \\
\hline 9 & 13 & -0.05 & 0.16 & -0.16 \\
\hline
\end{tabular}

A
Frequencies -- 356.1842

Red. masses -- 27.3141

Frc consts -- 2.0417

IR Inten $\quad$-- 40.1457
330.3831

\section{A}

\subsection{4}

1.6854

6.7569

\begin{tabular}{ccc}
\multicolumn{1}{c}{$\mathrm{X}$} & $\mathrm{Y}$ \\
0.23 & 0.22 & 0.20 \\
-0.24 & -0.10 & -0.08 \\
0.51 & 0.10 & 0.00 \\
0.24 & 0.21 & -0.20 \\
-0.24 & -0.10 & 0.08 \\
-0.33 & -0.12 & -0.11 \\
0.11 & -0.04 & -0.01 \\
0.11 & -0.04 & 0.01 \\
-0.33 & -0.12 & 0.11 \\
\multicolumn{3}{c}{20} \\
\multicolumn{3}{c}{$\mathrm{A}$}
\end{tabular}

A

424.1612

27.6496

2.9309

0.0472
353.7892

27.4574

2.0249

12.7167

$$
\begin{array}{ccc}
Z & X & Y \\
0.04 & -0.33 & -0.32 \\
0.09 & -0.09 & -0.09 \\
-0.01 & 0.00 & 0.02 \\
-0.05 & 0.35 & -0.34 \\
-0.09 & 0.09 & -0.10 \\
-0.04 & 0.07 & 0.06 \\
-0.12 & 0.33 & 0.32 \\
0.13 & -0.34 & 0.35 \\
0.05 & -0.07 & 0.07 \\
& \multicolumn{2}{c}{21}
\end{array}
$$

A

480.4582

26.2554

3.5709

355.4130

$\begin{array}{llllllllllll}1 & 13 & -0.08 & 0.30 & 0.29 & -0.04 & -0.02 & -0.02 & -0.01 & -0.01 & -0.01 \\ 2 & 13 & -0.05 & 0.10 & 0.10 & 0.07 & 0.02 & 0.01 & 0.04 & 0.00 & 0.00 \\ 3 & 12 & -0.22 & -0.05 & 0.00 & 0.00 & 0.00 & -0.01 & 0.63 & 0.09 & 0.00 \\ 4 & 13 & -0.08 & 0.28 & -0.27 & 0.04 & 0.02 & -0.02 & -0.01 & -0.01 & 0.01 \\ 5 & 13 & -0.04 & 0.10 & -0.09 & -0.07 & -0.02 & 0.01 & 0.04 & 0.00 & 0.00 \\ 6 & 13 & 0.19 & 0.00 & -0.01 & 0.36 & 0.12 & 0.11 & 0.19 & 0.06 & 0.05 \\ 7 & 14 & 0.04 & -0.36 & -0.35 & -0.55 & -0.14 & -0.10 & -0.49 & -0.09 & -0.04 \\ 8 & 14 & 0.03 & -0.34 & 0.34 & 0.55 & 0.14 & -0.10 & -0.49 & -0.09 & 0.04 \\ 9 & 13 & 0.19 & 0.00 & 0.01 & -0.36 & -0.12 & 0.11 & 0.19 & 0.06 & -0.06\end{array}$

Al3SiMgAl3Si-c-c-90-SiSi-b36311+gx.frq6.1 


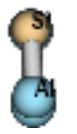

Charge $=0$ Multiplicity $=1$

Al,0,2.7473728488,1.6632024793,0.7831988932

Al,0,5.087341134,0.7313300432,0.3601346695

Mg,0,0.0138103839,-0.3846400951,0.1534355312

Al,0,-2.6725420303,0.6475532503,-1.7803875433

Al,0,-5.0308226019,0.369111743,-0.8408767911

Al,0,4.57777451,-1.6321910878,-0.6184650599

Si,0,2.5038811995,-0.56818397,-0.1679947327

Si,0,-2.4750589168,-0.2138497164,0.4918757585

Al,0,-4.5706065271,-0.5712956465,1.5482722747

State $=1-A \mid H F=-2233.7952034$

1

A

\begin{tabular}{|c|c|c|c|}
\hline \multicolumn{2}{|c|}{ Frequencies -- } & \multicolumn{2}{|l|}{6.0995} \\
\hline Red. masses & & 26.9745 & \\
\hline Frc consts & -- & 0.0006 & \\
\hline IR Inten & -- & 0.0012 & \\
\hline Atom AN & $\mathrm{X}$ & $\mathrm{Y}$ & \\
\hline 113 & 0.14 & -0.37 & 0.27 \\
\hline 213 & 0.08 & -0.14 & 0.22 \\
\hline 312 & 0.00 & 0.00 & -0.15 \\
\hline $4 \quad 13$ & -0.13 & 0.38 & 0.28 \\
\hline $5 \quad 13$ & -0.08 & 0.14 & 0.21 \\
\hline $6 \quad 13$ & -0.08 & 0.28 & -0.26 \\
\hline $7 \quad 14$ & -0.01 & 0.03 & -0.17 \\
\hline $8 \quad 14$ & 0.01 & -0.03 & -0.16 \\
\hline $9 \quad 13$ & 0.07 & -0.29 & -0.26 \\
\hline
\end{tabular}

A

Frequencies -- $\quad 18.4142$

Red. masses -- 27.3835

Frc consts -- 0.0055

IR Inten $\quad$-- $\quad 1.6720$

Atom AN $\quad \mathrm{X} \quad \mathrm{Y}$

$\begin{array}{lllll}1 & 13 & 0.24 & -0.06 & -0.29\end{array}$

$\begin{array}{lllll}2 & 13 & 0.11 & -0.02 & 0.19\end{array}$

$\begin{array}{lllll}3 & 12 & -0.08 & 0.06 & 0.00\end{array}$
2

A

10.9462

26.9050

0.0019

0.2001

X Y

$\begin{array}{lll}0.31 & 0.03 & -0.25\end{array}$

$\begin{array}{lll}0.19 & -0.03 & 0.30\end{array}$

$\begin{array}{lll}0.00 & 0.00 & -0.25\end{array}$

$\begin{array}{lll}-0.30 & -0.04 & -0.24\end{array}$

$\begin{array}{lll}-0.19 & 0.03 & 0.29\end{array}$

$\begin{array}{lll}-0.16 & -0.12 & 0.30\end{array}$

$\begin{array}{lll}0.00 & -0.03 & -0.25\end{array}$

$\begin{array}{lll}0.00 & 0.03 & -0.23\end{array}$

0.15

0.1

5

A

23.1921

27.2613

0.0086

0.4191

$\mathrm{X} \quad \mathrm{Y}$

$\begin{array}{lll}0.25 & -0.23 & -0.06\end{array}$

$\begin{array}{lll}0.15 & 0.24 & -0.18\end{array}$

$\begin{array}{lll}0.00 & 0.01 & 0.24\end{array}$
3

A

12.7918

26.8650

0.0026

0.4389

Z X

$-0.16 \quad 0.03$

$\begin{array}{lll}0.08 & 0.39 & -0.15\end{array}$

$\begin{array}{lll}-0.05 & -0.34 & 0.00\end{array}$

$\begin{array}{lll}0.19 & -0.14 & -0.02\end{array}$

$\begin{array}{lll}0.07 & 0.38 & 0.14\end{array}$

$\begin{array}{lll}-0.19 & 0.23 & -0.06\end{array}$

$\begin{array}{lll}-0.05 & -0.31 & 0.15\end{array}$

$\begin{array}{lll}-0.06 & -0.29 & -0.14\end{array}$

$\begin{array}{lll}-0.19 & 0.22 & 0.06\end{array}$

6

A

86.2033

24.7265

0.1083

9.9617

$\begin{array}{llll}\mathrm{Z} & \mathrm{X} & \mathrm{Y}\end{array}$

$\begin{array}{lll}0.04 & -0.17 & 0.03\end{array}$

$\begin{array}{lll}0.00 & 0.09 & -0.10\end{array}$

$\begin{array}{lll}-0.12 & 0.87 & -0.13\end{array}$ 


$$
\begin{array}{rrrrr}
4 & 13 & 0.26 & -0.07 & 0.29 \\
5 & 13 & 0.13 & -0.02 & -0.20 \\
6 & 13 & -0.25 & 0.03 & 0.05 \\
7 & 14 & -0.08 & 0.03 & -0.46 \\
8 & 14 & -0.08 & 0.02 & 0.46 \\
9 & 13 & -0.26 & 0.03 & -0.05 \\
& & & 7 &
\end{array}
$$

\begin{tabular}{|c|c|c|c|}
\hline Frequencies & & 87.5060 & \\
\hline Red. masse & $S--$ & 25.1561 & \\
\hline Frc consts & -- & 0.1135 & \\
\hline IR Inten & -- & 7.8429 & \\
\hline Atom AN & $\mathrm{X}$ & $\mathrm{Y}$ & \\
\hline 113 & -0.09 & -0.08 & -0.17 \\
\hline 213 & -0.14 & -0.03 & 0.08 \\
\hline 312 & -0.02 & 0.14 & 0.80 \\
\hline $4 \quad 13$ & 0.09 & 0.02 & -0.18 \\
\hline $\begin{array}{ll}5 & 13\end{array}$ & 0.14 & 0.05 & 0.11 \\
\hline $\begin{array}{ll}6 & 13\end{array}$ & -0.17 & 0.04 & -0.01 \\
\hline $\begin{array}{ll}7 & 14\end{array}$ & -0.06 & -0.01 & -0.24 \\
\hline 814 & 0.08 & -0.05 & -0.26 \\
\hline $9 \quad 13$ & 0.17 & -0.07 & -0.03 \\
\hline & & 10 & \\
\hline
\end{tabular}

A

A

Frequencies -- 113.3022

Red. masses -- $\quad 27.2131$

Frc consts -- 0.2058

IR Inten -- $\quad 3.1371$

$\begin{array}{ccccc}\text { Atom AN } & \mathrm{X} & \mathrm{Y} & \mathrm{Z} \\ 1 & 13 & 0.07 & -0.21 & 0.27 \\ 2 & 13 & 0.08 & 0.20 & -0.26 \\ 3 & 12 & 0.00 & 0.00 & 0.13 \\ 4 & 13 & -0.07 & 0.22 & 0.27 \\ 5 & 13 & -0.08 & -0.20 & -0.26 \\ 6 & 13 & 0.07 & -0.26 & 0.20 \\ 7 & 14 & 0.01 & 0.27 & -0.26 \\ 8 & 14 & -0.01 & -0.28 & -0.25 \\ 9 & 13 & -0.07 & 0.26 & 0.20 \\ & & & 13 & \end{array}$

A

$$
\begin{array}{ccc}
-0.25 & 0.23 & -0.05 \\
-0.14 & -0.26 & -0.19 \\
-0.06 & 0.01 & -0.01 \\
0.06 & -0.45 & 0.13 \\
-0.07 & 0.46 & 0.14 \\
0.06 & -0.02 & -0.01 \\
\multicolumn{4}{c}{8} &
\end{array}
$$

A

98.4311

26.6180

0.1519

0.0277

\begin{tabular}{ccc}
$Z$ & $X$ & $Y$ \\
0.23 & 0.19 & -0.02 \\
0.35 & -0.17 & 0.00 \\
0.00 & 0.02 & 0.38 \\
-0.23 & -0.20 & -0.02 \\
-0.35 & 0.18 & 0.01 \\
0.34 & 0.00 & -0.18 \\
0.15 & 0.11 & 0.02 \\
-0.15 & -0.12 & 0.02 \\
-0.33 & 0.00 & -0.18 \\
\multicolumn{3}{c}{11}
\end{tabular}

A

158.1037

26.7516

0.3940

2.5347

$$
\begin{array}{ccc}
0.02 & -0.18 & 0.02 \\
-0.02 & 0.08 & 0.08 \\
0.01 & -0.09 & 0.07 \\
0.04 & -0.22 & 0.08 \\
0.03 & -0.21 & 0.00 \\
-0.02 & -0.07 & -0.06 \\
& & 9
\end{array}
$$

A
111.7838

27.1933

0.2002

4.2185

\begin{tabular}{|c|c|c|c|}
\hline Frequencies - & 248.4389 & 256.4882 & 311.0503 \\
\hline Red. masses & 26.9231 & 27.1049 & 26.7232 \\
\hline Frc consts & 0.9791 & 1.0506 & 1.5233 \\
\hline IR Inten & 4.7217 & 1.7475 & 4.8448 \\
\hline
\end{tabular}

$\begin{array}{llllll}Z & X & Y & Z\end{array}$

\begin{tabular}{ccc}
$Z$ & $X$ & $Y$ \\
0.00 & 0.23 & -0.29 \\
0.00 & -0.24 & 0.25 \\
-0.04 & 0.12 & 0.00 \\
0.00 & 0.23 & 0.28 \\
0.00 & -0.24 & -0.25 \\
0.00 & 0.23 & -0.24 \\
0.02 & -0.27 & 0.24 \\
0.02 & -0.27 & -0.24 \\
0.00 & 0.24 & 0.24 \\
& \multicolumn{2}{c}{12}
\end{tabular}

A

201.9486

27.1567

0.6525

2.9621

$\begin{array}{cccccccc}X & Y & Z & X & Y & Z\end{array}$

$\begin{array}{llllll}0.15 & 0.19 & 0.18 & 0.04 & -0.18 & -0.17\end{array}$

$\begin{array}{llllll}0.39 & -0.12 & -0.13 & -0.32 & 0.13 & 0.13\end{array}$

$\begin{array}{llllll}-0.36 & 0.08 & 0.00 & 0.00 & 0.00 & -0.05\end{array}$

$\begin{array}{llllll}0.15 & 0.19 & -0.18 & -0.04 & 0.18 & -0.17\end{array}$

$\begin{array}{llllll}0.39 & -0.12 & 0.13 & 0.32 & -0.13 & 0.14\end{array}$

$\begin{array}{llllll}-0.10 & -0.18 & -0.17 & 0.42 & 0.13 & 0.12\end{array}$

$\begin{array}{llllll}-0.27 & 0.07 & 0.10 & 0.30 & 0.00 & -0.06\end{array}$

$\begin{array}{llllll}-0.27 & 0.07 & -0.10 & -0.30 & -0.01 & -0.06\end{array}$

$\begin{array}{llllll}-0.10 & -0.18 & 0.18 & -0.42 & -0.12 & 0.12\end{array}$

14

A

15

Frequencies -- 248.4389

256.4882

A

Red. masses -- 26.9231

1.0506

4.8448 


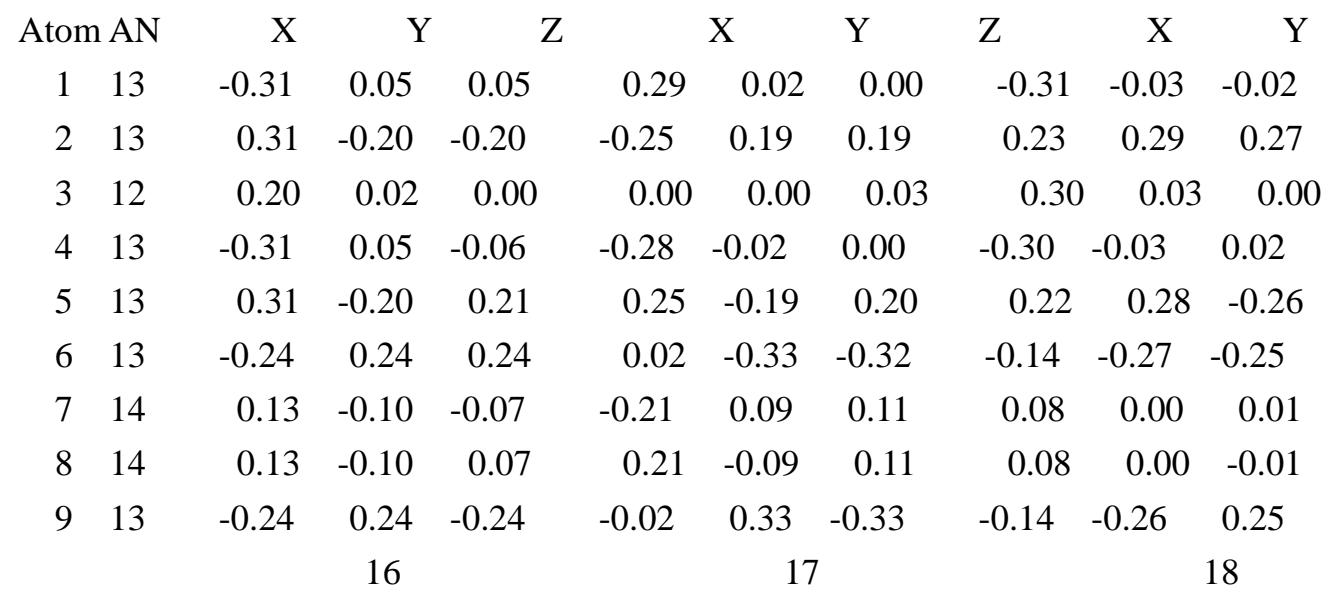

A

Frequencies -- 316.2619

Red. masses -- 26.9875

Frc consts -- 1.5904

IR Inten $\quad$-- 2.0171

$\begin{array}{rrrcc}\text { Atom } & \text { AN } & \mathrm{X} & \mathrm{Y} & \mathrm{Z} \\ 1 & 13 & -0.39 & -0.12 & -0.10 \\ 2 & 13 & 0.32 & 0.29 & 0.27 \\ 3 & 12 & -0.01 & 0.00 & -0.03 \\ 4 & 13 & 0.40 & 0.12 & -0.10 \\ 5 & 13 & -0.32 & -0.29 & 0.28 \\ 6 & 13 & 0.06 & -0.15 & -0.15 \\ 7 & 14 & 0.06 & 0.00 & -0.01 \\ 8 & 14 & -0.07 & 0.00 & -0.01 \\ 9 & 13 & -0.05 & 0.16 & -0.16 \\ & & & 19 & \end{array}$

A

Frequencies -- 356.1623

Red. masses -- $\quad 27.3139$

Frc consts -- 2.0414

IR Inten $\quad$-- 40.1172

Atom AN $\mathrm{X} \quad \mathrm{Y}$

$\begin{array}{llll}1 & 13 & -0.08 & 0.30\end{array}$

$\begin{array}{lllll}2 & 13 & -0.05 & 0.10 & 0.10\end{array}$

$\begin{array}{lllll}3 & 12 & -0.22 & -0.05 & 0.00\end{array}$

$\begin{array}{llllll}4 & 13 & -0.08 & 0.28 & -0.27\end{array}$

$\begin{array}{llllll}5 & 13 & -0.04 & 0.10 & -0.09\end{array}$

$\begin{array}{llllll}6 & 13 & 0.19 & 0.00 & -0.01\end{array}$

$\begin{array}{llllll}7 & 14 & 0.04 & -0.37 & -0.36\end{array}$

$\begin{array}{llllll}8 & 14 & 0.03 & -0.34 & 0.34\end{array}$

$9 \quad 13$

$\begin{array}{lll}0.19 & 0.00 & 0.01\end{array}$
A

330.3765

26.2078

1.6854

6.7344

X $\quad \mathrm{Y}$

$\begin{array}{lll}0.23 & 0.22 & 0.20\end{array}$

$\begin{array}{lll}-0.24 & -0.10 & -0.08\end{array}$

$\begin{array}{lll}0.51 & 0.10 & 0.00\end{array}$

$\begin{array}{lll}0.24 & 0.21 & -0.20\end{array}$

$\begin{array}{lll}-0.24 & -0.10 & 0.08\end{array}$

$\begin{array}{lll}-0.33 & -0.12 & -0.11\end{array}$

$\begin{array}{lll}0.11 & -0.04 & -0.01\end{array}$

$\begin{array}{llll}0.11 & -0.04 & 0.01\end{array}$

$\begin{array}{lll}-0.33 & -0.12 & 0.11\end{array}$

20

A

424.1521

27.6496

2.9308

0.0498

$\begin{array}{ll}\mathrm{X} & \mathrm{Y}\end{array}$

$\begin{array}{lll}-0.04 & -0.02 & -0.02\end{array}$

$\begin{array}{lll}0.07 & 0.02 & 0.01\end{array}$

$\begin{array}{lll}0.00 & 0.00 & -0.01\end{array}$

$\begin{array}{lll}0.04 & 0.02 & -0.02\end{array}$

$\begin{array}{lll}-0.07 & -0.02 & 0.01\end{array}$

$\begin{array}{lll}0.36 & 0.12 & 0.11\end{array}$

$\begin{array}{lll}-0.55 & -0.14 & -0.10\end{array}$

$\begin{array}{lll}0.55 & 0.14 & -0.10\end{array}$

$\begin{array}{lll}-0.36 & -0.12 \quad 0.11\end{array}$
A

353.7653

27.4574

2.0246

12.7296

$\begin{array}{llllll}\mathrm{Z} & \mathrm{X} & \mathrm{Y} & \mathrm{Z}\end{array}$

$\begin{array}{lll}0.04 & -0.33 & -0.32\end{array}$

$\begin{array}{llll}0.09 & -0.09 & -0.09\end{array}$

$\begin{array}{lll}-0.01 & 0.00 & 0.02\end{array}$

$\begin{array}{lll}-0.05 & 0.35 & -0.34\end{array}$

$\begin{array}{lll}-0.09 & 0.09 & -0.10\end{array}$

$\begin{array}{lll}-0.04 & 0.07 & 0.06\end{array}$

$\begin{array}{lll}-0.12 & 0.33 & 0.32\end{array}$

$\begin{array}{lll}0.13 & -0.34 & 0.35\end{array}$

$\begin{array}{llll}0.05 & -0.07 & 0.07\end{array}$

21

A

480.4534

26.2555

3.5709

355.3499

$\begin{array}{lllll}Z & X & Y & \text { Z }\end{array}$

$\begin{array}{lll}-0.01 & -0.01 & -0.01\end{array}$

$\begin{array}{lll}0.04 & 0.00 & 0.00\end{array}$

$\begin{array}{lll}0.63 & 0.09 & 0.00\end{array}$

$\begin{array}{lll}-0.01 & -0.01 & 0.01\end{array}$

$\begin{array}{lll}0.04 & 0.00 & 0.00\end{array}$

$\begin{array}{lll}0.19 & 0.06 & 0.05\end{array}$

$\begin{array}{lll}-0.49 & -0.09 & -0.04\end{array}$

$\begin{array}{lll}-0.49 & -0.09 & 0.04\end{array}$

$\begin{array}{lll}0.19 & 0.06 & -0.06\end{array}$

Al3SiMgAl3Si-s-Al-Al-c-Si-90-b36311+gx.frq4.4 


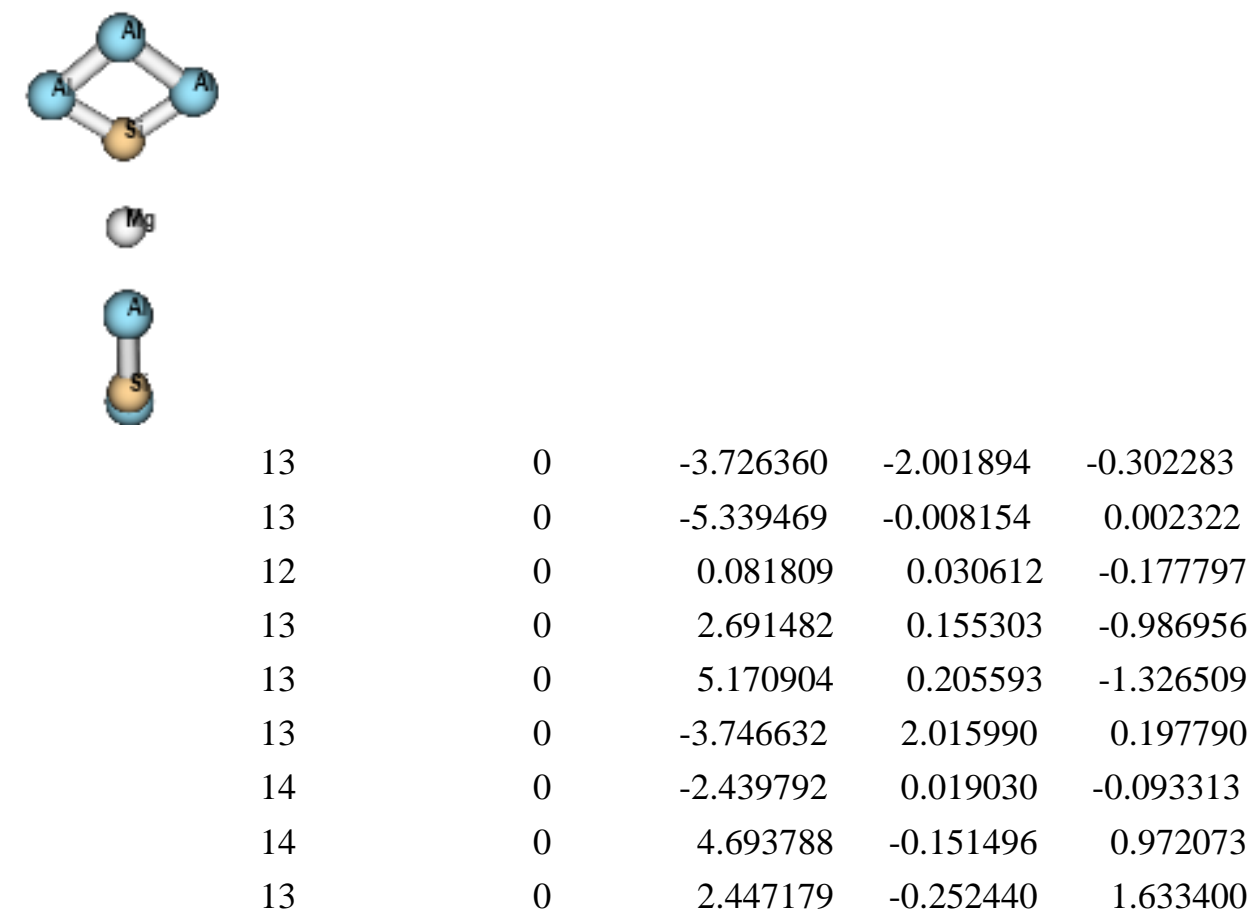

State $=1-A \backslash H F=-2233.786651$

$$
2
$$

A

6.4946

27.0754

0.0007

0.0562

X Y $\begin{array}{lrr}\text { Frequencies -- } & 4.3588 \\ \text { Red. masses -- } & 27.0184 \\ \text { Frc consts } & -- & 0.0003 \\ \text { IR Inten } & -- & 0.0333 \\ \text { Atom AN } & & \text { X }\end{array}$

$\begin{array}{lllll}1 & 13 & 0.49 & 0.09 & -0.11\end{array}$

$\begin{array}{llllll}2 & 13 & 0.00 & -0.34 & -0.04\end{array}$

$\begin{array}{lllll}3 & 12 & 0.00 & 0.22 & 0.03\end{array}$

$\begin{array}{llllll}4 & 13 & 0.00 & -0.06 & -0.01\end{array}$

$\begin{array}{llllll}5 & 13 & 0.00 & -0.33 & -0.04\end{array}$

$\begin{array}{lllll}6 & 13 & -0.49 & 0.05 & 0.13\end{array}$

$\begin{array}{lllll}7 & 14 & 0.00 & 0.40 & 0.05\end{array}$

$\begin{array}{lllll}8 & 14 & 0.00 & -0.14 & -0.02\end{array}$

$9 \quad 13$

$-0.01$

$0.12 \quad 0.02$

4

A

Frequencies -- $\quad 36.2164$

Red. masses -- $\quad 26.5570$

Frc consts - - $\quad 0.0205$

$\begin{array}{lll}0.15 & -0.02 & 0.46\end{array}$

$\begin{array}{lll}0.00 & -0.07 & 0.01\end{array}$

$\begin{array}{lll}0.00 & 0.10 & 0.00\end{array}$

$\begin{array}{lll}0.00 & 0.27 & 0.04\end{array}$

$\begin{array}{lll}0.01 & 0.31 & 0.06\end{array}$

$\begin{array}{lll}-0.15 & 0.09 & -0.45\end{array}$

$\begin{array}{lll}0.00 & 0.13 & 0.00\end{array}$

$\begin{array}{lll}-0.01 & -0.33 & -0.04\end{array}$

$\begin{array}{lll}-0.01 & -0.46 & -0.08\end{array}$

5

A

56.2814

26.7512

0.0499

5.2104

IR Inten -- 0.5103

Atom AN $\quad \mathrm{X} \quad \mathrm{Y} \quad \mathrm{Z}$

$\begin{array}{rrrrrrrrrrr}1 & 13 & -0.13 & -0.14 & -0.01 & -0.05 & 0.03 & -0.14 & 0.37 & -0.10 & -0.04 \\ 2 & 13 & 0.00 & -0.01 & 0.00 & -0.04 & -0.03 & 0.20 & 0.47 & 0.00 & 0.00 \\ 3 & 12 & 0.00 & 0.44 & 0.07 & -0.02 & -0.07 & 0.43 & -0.03 & -0.02 & 0.12\end{array}$

$\mathrm{X}$

3

A

10.7361

26.8677

0.0018

0.3993

Z $\quad \mathrm{X}$

$\begin{array}{lll}0.01 & -0.01 & 0.03\end{array}$

$\begin{array}{lll}0.04 & -0.07 & 0.60\end{array}$

$\begin{array}{lll}0.01 & 0.05 & -0.39\end{array}$

$\begin{array}{lll}0.10 & 0.00 & -0.07\end{array}$

$\begin{array}{lll}0.14 & -0.04 & 0.21\end{array}$

$\begin{array}{lll}0.03 & -0.01 & 0.06\end{array}$

$\begin{array}{lll}0.00 & 0.06 & -0.54\end{array}$

$\begin{array}{lll}-0.13 & -0.01 & 0.16\end{array}$

$\begin{array}{lll}-0.21 & 0.03 & -0.10\end{array}$

6

A 


$\begin{array}{ccccc}4 & 13 & 0.00 & 0.57 & 0.09 \\ 5 & 13 & 0.00 & -0.35 & -0.05 \\ 6 & 13 & 0.13 & -0.14 & -0.03 \\ 7 & 14 & 0.00 & -0.25 & -0.04 \\ 8 & 14 & 0.00 & -0.34 & -0.05 \\ 9 & 13 & 0.00 & 0.28 & 0.04 \\ & & & 7 & \end{array}$

A

Frequencies -- $\quad 97.4439$

Red. masses -- 26.6824

Frc consts -- 0.1493

IR Inten -- $\quad 6.3515$

$\begin{array}{ccccc}\text { Atom AN } & \text { X } & \text { Y } & \text { Z } \\ 1 & 13 & 0.03 & 0.05 & 0.01 \\ 2 & 13 & 0.00 & 0.00 & 0.00 \\ 3 & 12 & 0.00 & -0.41 & -0.07 \\ 4 & 13 & 0.00 & 0.53 & 0.08 \\ 5 & 13 & 0.00 & -0.45 & -0.07 \\ 6 & 13 & -0.03 & 0.05 & 0.01 \\ 7 & 14 & 0.00 & 0.08 & 0.01 \\ 8 & 14 & 0.00 & 0.45 & 0.07 \\ 9 & 13 & 0.00 & -0.35 & -0.05 \\ & & & 10 & \end{array}$

A

Frequencies -- 111.0802

Red. masses -- 27.1448

Frc consts -- 0.1973

$\begin{array}{llrr}\text { IR Inten } & -- & 2.6673 \\ \text { Atom AN } & \mathrm{X} & \mathrm{Y}\end{array}$

$\begin{array}{llll}\text { Atom AN } & X & \text { Y } & \text { Z }\end{array}$

$\begin{array}{lllll}1 & 13 & 0.02 & -0.06 & 0.50\end{array}$

$\begin{array}{llllll}2 & 13 & -0.02 & 0.06 & -0.51\end{array}$

$\begin{array}{lllll}3 & 12 & 0.00 & 0.02 & -0.10\end{array}$

$\begin{array}{llllll}4 & 13 & 0.01 & -0.01 & 0.06\end{array}$

$\begin{array}{llllll}5 & 13 & -0.02 & 0.00 & -0.04\end{array}$

$\begin{array}{llllll}6 & 13 & 0.02 & -0.06 & 0.50\end{array}$

$\begin{array}{lllll}7 & 14 & -0.01 & 0.05 & -0.44\end{array}$

$\begin{array}{llllll}8 & 14 & 0.00 & 0.01 & -0.03\end{array}$

$9 \quad 13$

$\begin{array}{lll}0.01 & -0.01 & 0.06\end{array}$

$$
13
$$

A

Frequencies -- 246.3838

Red. masses -- 26.8132

Frc consts -- 0.9590

IR Inten -- $\quad$-- 6.7125

\begin{tabular}{ccc}
-0.11 & -0.04 & 0.28 \\
-0.20 & 0.03 & -0.21 \\
-0.06 & 0.02 & -0.14 \\
-0.06 & 0.07 & -0.52 \\
0.22 & 0.02 & -0.12 \\
0.32 & -0.05 & 0.30 \\
\multicolumn{4}{c}{8} \\
\end{tabular}

A

101.3338

25.5757

0.1547

1.4091

X Y

$\begin{array}{lll}0.00 & -0.01 & 0.04\end{array}$

$\begin{array}{lll}0.00 & 0.00 & -0.03\end{array}$

$\begin{array}{llll}0.01 & -0.11 & 0.69\end{array}$

$\begin{array}{lll}-0.06 & 0.07 & -0.45\end{array}$

$\begin{array}{lll}0.07 & -0.03 & 0.20\end{array}$

$\begin{array}{lll}0.00 & 0.00 & 0.04\end{array}$

$\begin{array}{lll}-0.01 & 0.02 & -0.16\end{array}$

$\begin{array}{lll}0.02 & -0.02 & 0.17\end{array}$

$\begin{array}{lll}-0.05 & 0.06 & -0.43\end{array}$

11

A

166.7336

26.8116

0.4392

27.5585

X Y

$\begin{array}{lll}0.00 & 0.23 & 0.02\end{array}$

$\begin{array}{lll}-0.30 & 0.00 & 0.02\end{array}$

$\begin{array}{lll}0.32 & -0.04 & 0.27\end{array}$

$\begin{array}{lll}0.19 & 0.00 & 0.01\end{array}$

$\begin{array}{lll}0.28 & 0.02 & -0.13\end{array}$

$\begin{array}{lll}0.01 & -0.23 & -0.04\end{array}$

$\begin{array}{lll}0.26 & 0.01 & -0.04\end{array}$

$\begin{array}{lll}-0.44 & 0.05 & -0.31\end{array}$

$\begin{array}{lll}-0.28 & -0.04 & 0.23\end{array}$

14

A

250.3921

26.8320

0.9912

5.7940

$$
\begin{array}{ccc}
-0.30 & 0.00 & 0.04 \\
-0.38 & 0.01 & -0.06 \\
0.37 & 0.11 & -0.01 \\
0.15 & 0.00 & -0.04 \\
-0.34 & 0.01 & -0.07 \\
-0.29 & -0.01 & 0.08 \\
& \multicolumn{2}{c}{9}
\end{array}
$$

A

109.1176

25.5242

0.1791

0.3646

$\begin{array}{llllll}Z & X & Y & \text { Z }\end{array}$

$\begin{array}{lll}-0.04 & -0.06 & -0.01\end{array}$

$\begin{array}{lll}0.00 & 0.01 & 0.01\end{array}$

$\begin{array}{lll}0.00 & 0.73 & 0.11\end{array}$

$\begin{array}{lll}0.00 & -0.23 & -0.04\end{array}$

$\begin{array}{lll}0.00 & -0.15 & -0.02\end{array}$

$\begin{array}{lll}0.04 & -0.06 & -0.02\end{array}$

$\begin{array}{lll}0.00 & -0.11 & -0.01\end{array}$

$\begin{array}{lll}0.00 & 0.39 & 0.06\end{array}$

$\begin{array}{lll}0.00 & -0.44 & -0.07\end{array}$

12

A

188.9061

26.9525

0.5667

5.9200

$\begin{array}{lllll}Z & X & Y & \text { Z }\end{array}$

$\begin{array}{lll}-0.13 & -0.31 & -0.04\end{array}$

$\begin{array}{lll}0.40 & 0.00 & -0.01\end{array}$

$\begin{array}{lll}-0.22 & -0.03 & 0.17\end{array}$

$\begin{array}{lll}0.20 & -0.01 & 0.06\end{array}$

$\begin{array}{lll}0.49 & 0.01 & -0.10\end{array}$

$\begin{array}{lll}-0.13 & 0.31 & 0.04\end{array}$

$\begin{array}{lll}-0.36 & 0.00 & 0.00\end{array}$

$\begin{array}{lll}-0.17 & 0.03 & -0.22\end{array}$

$\begin{array}{lll}-0.09 & -0.02 & 0.12\end{array}$

15

A

305.4099

26.1744

1.4384

22.9484 


$\begin{array}{ccccc}\text { Atom AN } & \text { X } & \text { Y } & \text { Z } \\ 1 & 13 & 0.30 & -0.08 & -0.02 \\ 2 & 13 & -0.38 & 0.00 & 0.01 \\ 3 & 12 & -0.23 & -0.02 & 0.10 \\ 4 & 13 & 0.13 & -0.08 & 0.51 \\ 5 & 13 & 0.12 & 0.03 & -0.22 \\ 6 & 13 & 0.30 & 0.08 & 0.00 \\ 7 & 14 & -0.12 & 0.00 & 0.00 \\ 8 & 14 & 0.06 & -0.01 & 0.07 \\ 9 & 13 & -0.20 & 0.07 & -0.44 \\ & & & 16 & \end{array}$

A

Frequencies -- $\quad 308.4579$

Red. masses -- 26.9775

Frc consts --- 1.5123

IR Inten -- 2.7225

Atom AN $\quad \mathrm{X} \quad \mathrm{Y} \quad \mathrm{Z}$

$\begin{array}{lllll}1 & 13 & 0.31 & -0.37 & -0.06\end{array}$

$\begin{array}{lllll}2 & 13 & -0.01 & 0.73 & 0.09\end{array}$

$\begin{array}{lllll}3 & 12 & 0.01 & -0.04 & 0.00\end{array}$

$\begin{array}{lllll}4 & 13 & 0.00 & 0.01 & 0.00\end{array}$

$\begin{array}{llllll}5 & 13 & 0.00 & 0.00 & 0.00\end{array}$

$\begin{array}{llllll}6 & 13 & -0.30 & -0.36 & -0.03\end{array}$

$\begin{array}{lllll}7 & 14 & 0.00 & 0.02 & 0.00\end{array}$

$\begin{array}{lllll}8 & 14 & 0.00 & 0.00 & 0.00\end{array}$

$\begin{array}{lllll}9 & 13 & 0.00 & 0.00 & 0.00\end{array}$

19

A

Frequencies -- 379.6650

Red. masses -- $\quad 27.1799$

Frc consts -- 2.3083

IR Inten --- 0.9667

Atom AN $\quad \mathrm{X} \quad \mathrm{Y} \quad \mathrm{Z}$

$\begin{array}{lllll}1 & 13 & 0.06 & 0.14 & 0.01 \\ 2 & 13 & 0.05 & 0.00 & 0.00\end{array}$

$\begin{array}{llllll}3 & 12 & -0.14 & 0.00 & 0.03\end{array}$

$\begin{array}{lllll}4 & 13 & 0.47 & -0.01 & 0.09\end{array}$

$\begin{array}{llllll}5 & 13 & -0.36 & -0.07 & 0.47\end{array}$

$\begin{array}{llllll}6 & 13 & 0.07 & -0.14 & -0.02\end{array}$

$\begin{array}{llllll}7 & 14 & -0.23 & 0.01 & 0.01\end{array}$

$\begin{array}{llllll}8 & 14 & -0.17 & 0.07 & -0.42\end{array}$

$\begin{array}{lllll}9 & 13 & 0.23 & 0.02 & -0.16\end{array}$
$\begin{array}{ccc}X & Y \\ -0.34 & 0.15 & 0.03\end{array}$

$\begin{array}{ccc}-0.34 & 0.15 & 0.03 \\ 0.44 & 0.00 & -0.01\end{array}$

$\begin{array}{lll}0.25 & -0.01 & 0.06\end{array}$

$\begin{array}{lll}0.16 & -0.06 & 0.39\end{array}$

$\begin{array}{lll}-0.17 & 0.03 & -0.17\end{array}$

$\begin{array}{lll}-0.34 & -0.15 & -0.01\end{array}$

$\begin{array}{lll}0.18 & 0.00 & 0.00\end{array}$

$\begin{array}{lll}0.03 & -0.02 & 0.10\end{array}$

$\begin{array}{lll}-0.20 & 0.06 & -0.38\end{array}$

17

A

350.2564

26.8774

1.9427

5.6341

X Y

$\begin{array}{lll}0.07 & 0.21 & 0.02\end{array}$

$\begin{array}{lll}0.09 & 0.00 & 0.00\end{array}$

$\begin{array}{lll}-0.29 & -0.01 & 0.06\end{array}$

$\begin{array}{lll}0.48 & 0.04 & -0.28\end{array}$

$\begin{array}{lll}-0.20 & 0.05 & -0.31\end{array}$

$\begin{array}{llll}0.08 & -0.20 & -0.03\end{array}$

$\begin{array}{lll}-0.18 & 0.00 & 0.01\end{array}$

$\begin{array}{lll}0.25 & -0.04 & 0.24\end{array}$

$\begin{array}{lll}-0.34 & -0.04 & 0.29\end{array}$

20

A

439.9151

27.1311

3.0935

188.2483

$\mathrm{X} \quad \mathrm{Y}$

$\begin{array}{lll}-0.10 & -0.15 & -0.02\end{array}$

$\begin{array}{lll}-0.06 & 0.00 & 0.00\end{array}$

$\begin{array}{lll}-0.37 & -0.01 & 0.04\end{array}$

$\begin{array}{lll}0.01 & 0.00 & 0.02\end{array}$

$\begin{array}{lll}-0.02 & -0.04 & 0.27\end{array}$

$\begin{array}{lll}-0.10 & 0.15 & 0.02\end{array}$

$\begin{array}{lll}0.51 & 0.00 & -0.02\end{array}$

$\begin{array}{lll}0.42 & 0.06 & -0.36\end{array}$

$\begin{array}{lll}-0.37 & -0.01 & 0.06\end{array}$

$$
\begin{array}{ccc}
Z & X & Y \\
0.04 & 0.44 & 0.05 \\
0.23 & 0.01 & -0.01 \\
-0.52 & 0.00 & -0.01 \\
-0.34 & -0.01 & 0.09 \\
0.36 & -0.01 & 0.04 \\
0.04 & -0.45 & -0.06 \\
0.02 & 0.00 & 0.00 \\
-0.01 & 0.01 & -0.05 \\
0.11 & 0.01 & -0.05 \\
& \multicolumn{2}{c}{18}
\end{array}
$$

A

374.7068

27.5323

2.2776

10.2708

$\begin{array}{llllll}Z & X & Y & \text { Z }\end{array}$

$\begin{array}{lll}0.31 & 0.35 & 0.03\end{array}$

$\begin{array}{lll}0.00 & 0.10 & 0.01\end{array}$

$\begin{array}{lll}0.00 & -0.02 & 0.00\end{array}$

$\begin{array}{lll}0.00 & 0.00 & 0.00\end{array}$

$\begin{array}{lll}0.00 & 0.00 & 0.01\end{array}$

$\begin{array}{lll}-0.31 & 0.34 & 0.05\end{array}$

$\begin{array}{lll}0.00 & -0.74 & -0.09\end{array}$

$\begin{array}{lll}0.00 & 0.00 & -0.01\end{array}$

$\begin{array}{lll}0.00 & 0.00 & 0.00\end{array}$

21

A

455.2143

26.7703

3.2684

83.0362

$\begin{array}{llll}\mathrm{Z} & \mathrm{X} & \mathrm{Y}\end{array}$

$\begin{array}{lll}0.08 & 0.12 & 0.01\end{array}$

$\begin{array}{lll}0.05 & 0.00 & 0.00\end{array}$

$\begin{array}{lll}0.47 & 0.00 & -0.01\end{array}$

$\begin{array}{lll}-0.26 & -0.01 & 0.06\end{array}$

$\begin{array}{lll}0.08 & -0.03 & 0.19\end{array}$

$\begin{array}{lll}0.09 & -0.12 & -0.02\end{array}$

$\begin{array}{lll}-0.48 & 0.00 & 0.02\end{array}$

$\begin{array}{lll}0.41 & 0.04 & -0.26\end{array}$

$\begin{array}{lll}-0.38 & 0.00 & 0.03\end{array}$

Al3SiMgAl3Si-s-s-90-Al-Al-b36311+gx.frq18.7 


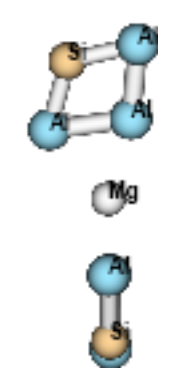

13

$\begin{array}{ccc}-5.090967 & 0.941651 & -0.949655 \\ -2.609520 & 0.645463 & -0.768326 \\ 5.090977 & -0.941659 & -0.949619 \\ 2.609526 & -0.645501 & -0.768288 \\ 0.000001 & -0.000013 & -0.235356 \\ -2.369723 & -1.234010 & 1.095951 \\ 2.369712 & 1.234033 & 1.095926 \\ -4.615672 & -0.714983 & 0.678448 \\ 4.615666 & 0.715015 & 0.678439\end{array}$

State $=1-A \backslash H F=-2233.7786906$

A

A

A

Frequencies -- 18.7098

22.8881

23.2820

26.3702

26.4594

Red. masses -- $\quad 27.0610$

0.0081

0.0085

Frc consts -- 0.0056

0.5286

0.6091

$\begin{array}{llrr}\text { IR Inten } & -- & & 0.0063 \\ \text { Atom AN } & & X & Y\end{array}$

$\begin{array}{rrrrr}\text { Atom AN } & \mathrm{X} & \mathrm{Y} & \mathrm{Z} \\ 1 & 13 & -0.01 & 0.26 & 0.34 \\ 2 & 13 & -0.01 & 0.14 & 0.15 \\ 3 & 13 & 0.01 & -0.26 & 0.34 \\ 4 & 13 & 0.01 & -0.14 & 0.15 \\ 5 & 12 & 0.00 & 0.00 & -0.06 \\ 6 & 13 & 0.02 & -0.34 & -0.34 \\ 7 & 13 & -0.02 & 0.34 & -0.34 \\ 8 & 14 & 0.02 & -0.18 & -0.12 \\ 9 & 14 & -0.02 & 0.18 & -0.12 \\ & & & 4 & \end{array}$

A

$\mathrm{X} \quad \mathrm{Y}$

$\mathrm{X} \quad \mathrm{Y}$

Z

$\begin{array}{lll}-0.16 & -0.33 & -0.07\end{array}$

Z

$\begin{array}{lll}0.10 & -0.02 & -0.26\end{array}$

$\begin{array}{lll}-0.11 & 0.23 & 0.11\end{array}$

$\begin{array}{lll}0.08 & 0.14 & 0.26\end{array}$

$\begin{array}{lll}-0.16 & -0.33 & 0.07\end{array}$

$\begin{array}{lll}-0.10 & 0.02 & -0.26\end{array}$

$\begin{array}{lll}-0.11 & 0.23 & -0.11\end{array}$

$\begin{array}{lll}-0.08 & -0.14 & 0.26\end{array}$

$\begin{array}{lll}-0.01 & 0.52 & 0.00\end{array}$

$\begin{array}{lll}0.00 & 0.00 & 0.51\end{array}$

$\begin{array}{lll}0.18 & 0.16 & 0.01\end{array}$

$\begin{array}{lll}-0.21 & -0.07 & 0.09\end{array}$

0.18

$0.16 \quad-0.01$

$\begin{array}{lll}0.21 & 0.07 & 0.09\end{array}$

$\begin{array}{llll}0.10 & -0.28 & -0.09\end{array}$

$\begin{array}{lll}-0.15 & -0.14 & -0.31\end{array}$

$\begin{array}{lll}0.10 & -0.28 & 0.09\end{array}$

$\begin{array}{lll}0.15 & 0.14 & -0.31\end{array}$

5

A

72.3253

26.8722

0.0828

1.1002
6

A

95.4065

27.0628

0.1451

2.6644

\begin{tabular}{rrrrrrccccccc} 
IR Inten & -- & \multicolumn{2}{c}{0.8583} & \multicolumn{4}{c}{1.1002} & & \multicolumn{2}{c}{2.6644} \\
Atom AN & $\mathrm{X}$ & \multicolumn{2}{c}{$\mathrm{Y}$} & $\mathrm{Z}$ & \multicolumn{2}{c}{$\mathrm{X}$} & $\mathrm{Y}$ & $\mathrm{Z}$ & $\mathrm{X}$ & $\mathrm{Y}$ \\
1 & 13 & -0.07 & -0.19 & -0.02 & -0.14 & 0.03 & 0.21 & 0.05 & 0.26 & 0.15 \\
2 & 13 & 0.01 & 0.50 & 0.18 & -0.10 & -0.18 & -0.50 & -0.01 & -0.27 & -0.03 \\
3 & 13 & 0.07 & 0.19 & -0.02 & -0.14 & 0.03 & -0.21 & 0.05 & 0.26 & -0.15 \\
4 & 13 & -0.01 & -0.50 & 0.18 & -0.10 & -0.18 & 0.50 & -0.01 & -0.27 & 0.03
\end{tabular}




$$
\begin{array}{rrrrr}
5 & 12 & 0.00 & 0.00 & -0.18 \\
6 & 13 & 0.23 & 0.26 & -0.06 \\
7 & 13 & -0.23 & -0.26 & -0.06 \\
8 & 14 & 0.17 & -0.10 & -0.01 \\
9 & 14 & -0.17 & 0.10 & -0.01 \\
& & & 7 &
\end{array}
$$

A

Frequencies -- $\quad 96.4523$

Red. masses -- $\quad 27.1319$

Frc consts -- 0.1487

IR Inten --- 1.8067

Atom AN $\quad \mathrm{X} \quad \mathrm{Y}$

$\begin{array}{rrrcc}1 & 13 & 0.32 & 0.14 & 0.10 \\ 2 & 13 & 0.25 & -0.11 & -0.14 \\ 3 & 13 & -0.32 & -0.14 & 0.10 \\ 4 & 13 & -0.25 & 0.11 & -0.14 \\ 5 & 12 & 0.00 & 0.00 & 0.21 \\ 6 & 13 & 0.28 & 0.10 & 0.14 \\ 7 & 13 & -0.28 & -0.10 & 0.14 \\ 8 & 14 & 0.31 & -0.12 & -0.19 \\ 9 & 14 & -0.31 & 0.12 & -0.19 \\ & & & 10 & \end{array}$

A

$$
\begin{array}{ccc}
-0.02 & 0.22 & 0.00 \\
0.15 & 0.06 & -0.27 \\
0.15 & 0.06 & 0.27 \\
0.10 & -0.01 & 0.09 \\
0.10 & -0.01 & -0.09 \\
\multicolumn{3}{c}{8} \\
\multicolumn{4}{c}{8}
\end{array}
$$

A

97.5323

27.1029

0.1519

1.0982

$\mathrm{Z}$

\begin{tabular}{ccc}
\multicolumn{2}{c}{$\mathrm{X}$} & $\mathrm{Y}$ \\
-0.22 & 0.10 & 0.25
\end{tabular}

$\begin{array}{lll}-0.14 & 0.02 & -0.25\end{array}$

$\begin{array}{lll}0.22 & -0.10 & 0.25\end{array}$

$\begin{array}{lll}0.14 & -0.02 & -0.25\end{array}$

$\begin{array}{lll}0.00 & 0.00 & 0.22\end{array}$

$\begin{array}{lll}-0.11 & 0.35 & 0.05\end{array}$

$\begin{array}{lll}0.11 & -0.35 & 0.05\end{array}$

$\begin{array}{lll}-0.18 & -0.28 & -0.14\end{array}$

$\begin{array}{lll}0.18 & 0.28 & -0.14\end{array}$

11

A

166.7433

26.7837

0.4388

68.2797

$$
\begin{array}{ccc}
0.01 & 0.26 & 0.00 \\
-0.03 & 0.12 & 0.36 \\
-0.03 & 0.12 & -0.36 \\
-0.02 & -0.22 & -0.31 \\
-0.02 & -0.22 & 0.31
\end{array}
$$

A

108.2592

25.8226

0.1783

3.4744

Frequencies -- 109.3528

Red. masses -- 25.7280

Frc consts -- 0.1813

IR Inten --- 0.4554

$\begin{array}{lrrr}\text { Atom AN } & X & \text { Y } & \text { Z }\end{array}$

X $\quad$ Y

$\begin{array}{ccc}\text { X } & \text { Y } \\ 0.00 & -0.02 & 0.15\end{array}$

$\begin{array}{lll}0.07 & 0.23 & -0.13\end{array}$

$\begin{array}{lll}0.00 & -0.02 & -0.15\end{array}$

$\begin{array}{lll}0.07 & 0.23 & 0.13\end{array}$

$\begin{array}{lll}0.01 & -0.66 & 0.00\end{array}$

$\begin{array}{lll}0.00 & 0.34 & -0.01\end{array}$

$\begin{array}{lll}0.00 & 0.34 & 0.01\end{array}$

$\begin{array}{lll}-0.06 & -0.25 & -0.07\end{array}$

$\begin{array}{lll}-0.06 & -0.25 & 0.07\end{array}$

$\begin{array}{rrrrr}1 & 13 & 0.04 & -0.15 & 0.00 \\ 2 & 13 & 0.09 & 0.12 & -0.22\end{array}$

$\begin{array}{lll}0.30 & -0.08 & 0.06\end{array}$

$\begin{array}{lll}0.18 & -0.03 & -0.02\end{array}$

$\begin{array}{lll}0.30 & -0.08 & -0.06\end{array}$

$\begin{array}{lll}0.18 & -0.03 & 0.02\end{array}$

12

A

$\begin{array}{lllll}3 & 13 & -0.04 & 0.15 & 0.00\end{array}$

$\begin{array}{ccc}0.22 & 0.38 & 0.00\end{array}$

177.8405

26.9414

0.5020

4.3088

$\begin{array}{rrrrr}4 & 13 & -0.09 & -0.12 & -0.22\end{array}$

$\begin{array}{lll}\mathrm{Z} & \mathrm{X} & \mathrm{Y}\end{array}$

Z

$\begin{array}{lllll}5 & 12 & 0.00 & 0.00 & 0.68\end{array}$

$\begin{array}{lll}-0.22 & 0.11 & -0.14 \\ -0.22 & 0.11 & 0.14\end{array}$

$\begin{array}{lll}-0.22 & 0.11 & 0.14\end{array}$

$\begin{array}{lll}-0.35 & -0.16 & 0.21\end{array}$

$\begin{array}{lll}0.42 & -0.09 & 0.07\end{array}$

$\begin{array}{lll}0.22 & 0.04 & -0.01\end{array}$

$\begin{array}{lll}-0.42 & 0.09 & 0.07\end{array}$

$\begin{array}{lll}-0.22 & -0.04 & -0.01\end{array}$

$\begin{array}{llllll}6 & 13 & 0.03 & -0.02 & -0.33\end{array}$

$\begin{array}{lllll}7 & 13 & -0.03 & 0.02 & -0.33\end{array}$

$\begin{array}{lll}-0.35 & -0.16 & -0.21\end{array}$

$\begin{array}{lll}0.00 & 0.00 & -0.34\end{array}$

$\begin{array}{lll}-0.17 & 0.13 & -0.10\end{array}$

$\begin{array}{lll}0.17 & -0.13 & -0.10\end{array}$

$\begin{array}{lllll}8 & 14 & 0.01 & 0.09 & 0.24\end{array}$

$\begin{array}{llllll}9 & 14 & -0.01 & -0.09 & 0.24\end{array}$

13

A

14

A

$\begin{array}{lll}-0.28 & -0.19 & 0.19\end{array}$

$\begin{array}{lll}0.28 & 0.19 & 0.19\end{array}$

Frequencies -- $\quad 246.8307$

Red. masses -- 26.7819

250.2447

Frc consts -- 0.9614

26.9638

0.9949

(5.5040

25.5040

1.1093

0.3333

$\begin{array}{lrrr}\text { IR Inten } & -- & & 5.8817 \\ \text { Atom AN } & & \text { X } & \text { Y }\end{array}$

Z $\quad \mathrm{X}^{0.3333} \mathrm{Y}$




\begin{tabular}{llcccccccccc}
1 & 13 & 0.11 & -0.14 & 0.13 & 0.03 & 0.14 & -0.14 & -0.40 & -0.02 & 0.04 \\
2 & 13 & 0.11 & 0.32 & -0.33 & -0.15 & -0.32 & 0.32 & 0.24 & 0.02 & -0.03 \\
3 & 13 & 0.11 & -0.14 & -0.13 & -0.03 & -0.14 & -0.14 & -0.40 & -0.02 & -0.04 \\
4 & 13 & 0.11 & 0.32 & 0.33 & 0.15 & 0.32 & 0.32 & 0.24 & 0.02 & 0.03 \\
5 & 12 & -0.23 & 0.13 & 0.00 & 0.00 & 0.00 & 0.11 & 0.71 & 0.01 & 0.00 \\
6 & 13 & -0.17 & -0.28 & 0.28 & 0.20 & 0.29 & -0.30 & -0.13 & -0.06 & 0.07 \\
7 & 13 & -0.17 & -0.28 & -0.28 & -0.20 & -0.29 & -0.30 & -0.13 & -0.06 & -0.07 \\
8 & 14 & 0.05 & 0.04 & -0.04 & -0.05 & -0.06 & 0.07 & -0.02 & 0.06 & -0.05 \\
9 & 14 & 0.05 & 0.04 & 0.04 & 0.05 & 0.06 & 0.07 & -0.02 & 0.06 & 0.05 \\
& & & 16 & & - & 17 & & & \multicolumn{4}{c}{18}
\end{tabular}

A

Frequencies -- $\quad 339.2121$

Red. masses -- $\quad 27.0227$

Frc consts -- 1.8320

IR Inten -- 1.3082

$\begin{array}{llll}\text { Atom AN } & X & Y & \text { Z }\end{array}$

$\begin{array}{lllll}1 & 13 & -0.29 & -0.09 & 0.11\end{array}$

$\begin{array}{lllll}2 & 13 & 0.49 & -0.16 & 0.13\end{array}$

$\begin{array}{lllll}3 & 13 & 0.29 & 0.09 & 0.11\end{array}$

$\begin{array}{lllll}4 & 13 & -0.49 & 0.16 & 0.13\end{array}$

$\begin{array}{lllll}5 & 12 & 0.00 & 0.00 & -0.07\end{array}$

$\begin{array}{lllll}6 & 13 & -0.21 & 0.14 & -0.13\end{array}$

$\begin{array}{llllll}7 & 13 & 0.21 & -0.14 & -0.13\end{array}$

$\begin{array}{lllll}8 & 14 & 0.12 & 0.08 & -0.08\end{array}$

$9 \quad 14$ $\begin{array}{lll}-0.12 & -0.08 & -0.08\end{array}$

19
A

Frequencies -- 394.1034

Red. masses -- 26.4507

Frc consts -- $\quad 2.4205$

IR Inten -- 62.8716

Atom AN $\quad \mathrm{X} \quad \mathrm{Y} \quad \mathrm{Z}$

$\begin{array}{lllll}1 & 13 & -0.24 & 0.21 & -0.20\end{array}$

$\begin{array}{lllll}2 & 13 & 0.40 & -0.01 & -0.01\end{array}$

$\begin{array}{lllll}3 & 13 & -0.24 & 0.21 & 0.20\end{array}$

$\begin{array}{lllll}4 & 13 & 0.40 & -0.01 & 0.01\end{array}$

$\begin{array}{lllll}5 & 12 & -0.48 & 0.06 & 0.00\end{array}$

$\begin{array}{lllll}6 & 13 & 0.06 & -0.02 & 0.02\end{array}$

$\begin{array}{lllll}7 & 13 & 0.06 & -0.02 & -0.02\end{array}$

$\begin{array}{lllll}8 & 14 & -0.01 & -0.20 & 0.20\end{array}$

$\begin{array}{lllll}9 & 14 & -0.01 & -0.20 & -0.20\end{array}$
A

358.7248

26.8281

2.0341

32.9232

1

0.04

$\begin{array}{lll}-0.24 & 0.16 & -0.15\end{array}$

$\begin{array}{lll}0.04 & 0.21 & 0.21\end{array}$

$\begin{array}{lll}-0.24 & 0.16 & 0.15\end{array}$

$\begin{array}{lll}0.34 & -0.06 & 0.00\end{array}$

$\begin{array}{lll}0.29 & -0.17 & 0.16\end{array}$

$\begin{array}{lll}0.29 & -0.17 & -0.16\end{array}$

$\begin{array}{lll}-0.23 & -0.16 & 0.17\end{array}$

$\begin{array}{lll}-0.23 & -0.16 & -0.17\end{array}$

20

A

449.1855

27.5432

3.2743

2.5434
A

376.7428

27.2489

2.2787

1.1992

$\begin{array}{lll}\mathrm{X} & \mathrm{Y} & \mathrm{Z}\end{array}$

$\begin{array}{lll}0.25 & -0.27 & 0.26\end{array}$

$\begin{array}{lll}-0.28 & -0.06 & 0.07\end{array}$

$\begin{array}{lll}-0.25 & 0.27 & 0.26\end{array}$

$\begin{array}{lll}0.28 & 0.06 & 0.07\end{array}$

$\begin{array}{lll}0.00 & 0.00 & 0.02\end{array}$

$\begin{array}{lll}-0.22 & 0.11 & -0.10\end{array}$

$\begin{array}{lll}0.22 & -0.11 & -0.10\end{array}$

$\begin{array}{lll}0.17 & 0.23 & -0.23\end{array}$

$\begin{array}{lll}-0.17 & -0.23 & -0.23\end{array}$

21

A

451.1930

27.3971

3.2861

12.2195

Al3SiMgAl3Si-c-c-0-C2h-Al-Al-b36311+gx.frq4m-100.3 $\begin{array}{rrrrrrr}0.03 & 0.17 & -0.17 & -0.05 & -0.15 & 0.15\end{array}$

$\begin{array}{llllll}-0.12 & 0.03 & -0.02 & 0.17 & -0.03 & 0.03\end{array}$

$\begin{array}{lll}-0.03 & -0.17 & -0.17\end{array}$

$\begin{array}{lll}-0.05 & -0.15 & -0.15\end{array}$

$\begin{array}{llll}0.12 & -0.03 & -0.02\end{array}$

$\begin{array}{lll}0.17 & -0.03 & -0.03\end{array}$

$\begin{array}{lll}0.00 & 0.00 & -0.02\end{array}$

$\begin{array}{lll}-0.18 & -0.01 & 0.00\end{array}$

$\begin{array}{llll}-0.38 & 0.04 & -0.02\end{array}$

$\begin{array}{lll}0.38 & -0.04 & 0.02\end{array}$

$\begin{array}{llll}0.38 & -0.04 & -0.02\end{array}$

$\begin{array}{llll}0.38 & -0.04 & -0.02\end{array}$

$\begin{array}{llll}0.42 & -0.24 & 0.22\end{array}$

$\begin{array}{lll}-0.41 & 0.22 & -0.20\end{array}$

$\begin{array}{lll}-0.41 & 0.22 & 0.20\end{array}$ 


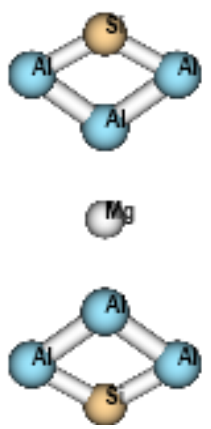

$\begin{array}{ccccc}12 & 0 & 0.000000 & 0.000000 & 0.000000 \\ 13 & 0 & 0.000000 & 2.076400 & 4.203264 \\ 13 & 0 & 0.000000 & -2.076400 & 4.203264 \\ 13 & 0 & 0.000000 & 2.076400 & -4.203264 \\ 13 & 0 & 0.000000 & -2.076400 & -4.203264 \\ 13 & 0 & 0.000000 & 0.000000 & 2.681166 \\ 14 & 0 & 0.000000 & 0.000000 & 5.359985 \\ 13 & 0 & 0.000000 & 0.000000 & -2.681166 \\ 14 & 0 & 0.000000 & 0.000000 & -5.359985\end{array}$

State $=1-A G \backslash H F=-2233.7663057$

B3U

B3G

$-18.8596$

27.0007

0.0057

0.0000

IR Inten $\quad--\quad 16.4763$

Atom AN $\mathrm{X} \quad \mathrm{Y}$

$\begin{array}{llll}1 & 12 & 0.68 & 0.00\end{array}$

$\begin{array}{lllll}2 & 13 & 0.01 & 0.00 & 0.00\end{array}$

$\begin{array}{lllll}3 & 13 & 0.01 & 0.00 & 0.00\end{array}$

$\begin{array}{lllll}4 & 13 & 0.01 & 0.00 & 0.00\end{array}$

$\begin{array}{lllll}5 & 13 & 0.01 & 0.00 & 0.00\end{array}$

$\begin{array}{lllll}6 & 13 & -0.49 & 0.00 & 0.00\end{array}$

$\begin{array}{lllll}7 & 14 & 0.17 & 0.00 & 0.00\end{array}$

$8 \quad 13$

$\begin{array}{lll}-0.49 & 0.00 & 0.00\end{array}$

$9 \quad 14$ $\begin{array}{lll}0.17 & 0.00 & 0.00\end{array}$

4

AU

$\begin{array}{lll}\mathrm{X} & \mathrm{Y}\end{array}$

$\begin{array}{lll}0.00 & 0.00 & 0.00\end{array}$

$\begin{array}{lll}0.00 & 0.11 & 0.37\end{array}$

$\begin{array}{lll}0.00 & 0.11 & -0.37\end{array}$

$\begin{array}{lll}0.00 & -0.11 & 0.37\end{array}$

$\begin{array}{lll}0.00 & -0.11 & -0.37\end{array}$

$\begin{array}{lll}0.00 & 0.44 & 0.00\end{array}$

$\begin{array}{lll}0.00 & -0.10 & 0.00\end{array}$

$\begin{array}{lll}0.00 & -0.44 & 0.00\end{array}$

0.00

$$
0.10 \quad 0.00
$$

5

B2G
B2U

$-9.9732$

26.9922

0.0016

0.0884

$\mathrm{Z}$

$\begin{array}{lll}0.00 & 0.21 & 0.00\end{array}$

$\begin{array}{lll}0.00 & -0.05 & 0.40\end{array}$

$\begin{array}{lll}0.00 & -0.05 & -0.40\end{array}$

$\begin{array}{lll}0.00 & -0.05 & -0.40\end{array}$

$\begin{array}{lll}0.00 & -0.05 & 0.40\end{array}$

$\begin{array}{lll}0.00 & 0.28 & 0.00\end{array}$

$\begin{array}{lll}0.00 & -0.27 & 0.00\end{array}$

$\begin{array}{lll}0.00 & 0.28 & 0.00\end{array}$

$\begin{array}{lll}0.00 & -0.27 & 0.00\end{array}$

6

B3U

23.7381

24.6879

27.1669

25.7954

0.0090

0.0093

0.0000

1.7381

IR Inten $\quad$-- $\quad 0.0000$

$\begin{array}{llll}\text { Atom AN } & \mathrm{X} & \mathrm{Y} & \mathrm{Z}\end{array}$

$\begin{array}{lllll}1 & 12 & 0.00 & 0.00 & 0.00\end{array}$

$\mathrm{X} \quad \mathrm{Y}$

$\begin{array}{lll}0.00 & 0.00 & 0.00\end{array}$

$\mathrm{Z} \quad \mathrm{X}$

0.70

0.00

$\mathrm{Y}$

Z

$\begin{array}{lllllllllll}2 & 13 & -0.50 & 0.00 & 0.00 & 0.00 & 0.00 & 0.00 & -0.11 & 0.00 & 0.00\end{array}$

$\begin{array}{lllllllllll}3 & 13 & 0.50 & 0.00 & 0.00 & 0.00 & 0.00 & 0.00 & -0.11 & 0.00 & 0.00\end{array}$ 


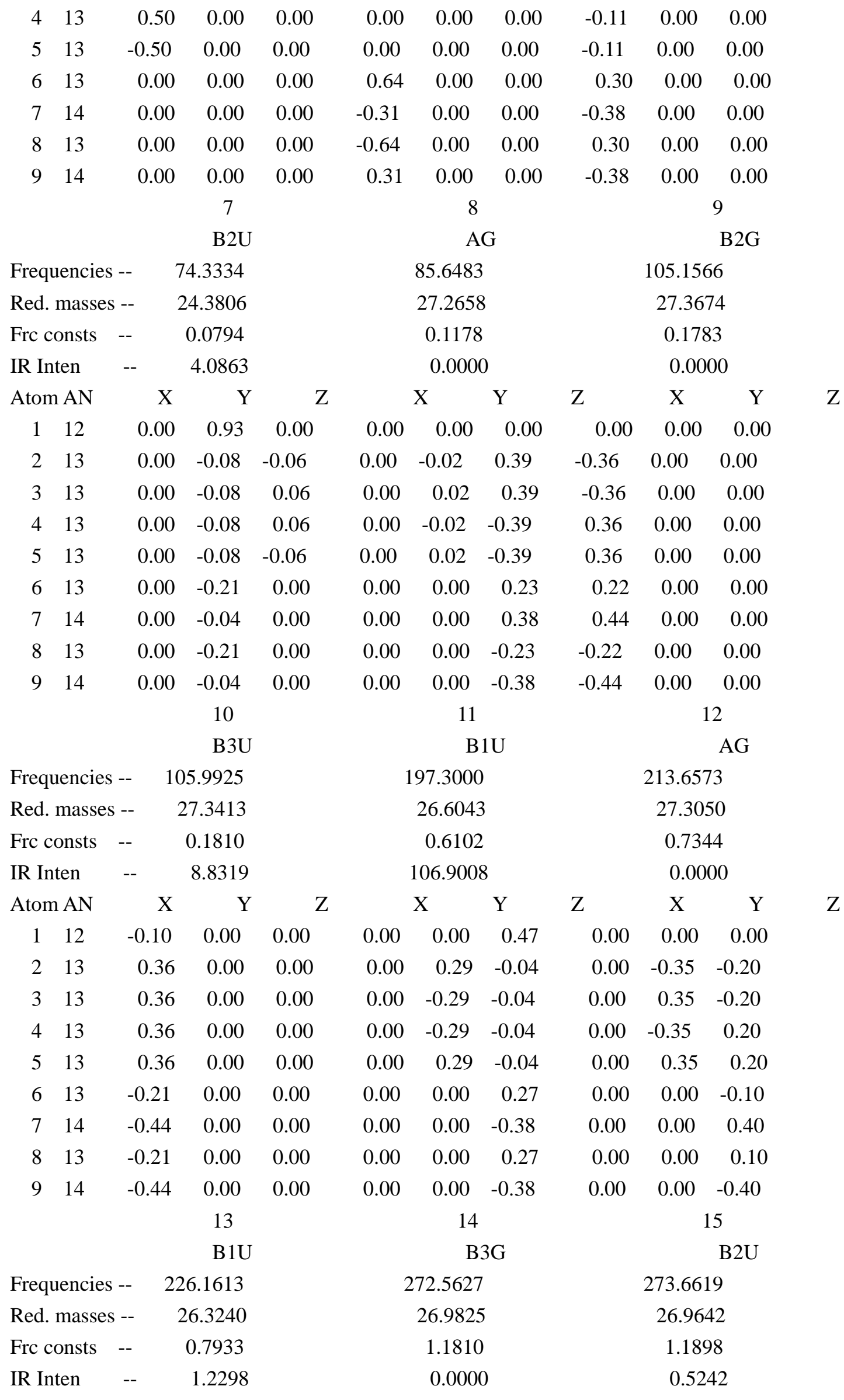




\begin{tabular}{|c|c|c|c|c|c|c|c|c|c|c|}
\hline \multicolumn{2}{|c|}{ Atom AN } & $\mathrm{X}$ & $\mathrm{Y}$ & $\mathrm{Z}$ & \multicolumn{2}{|r|}{$\mathrm{X}$} & $\mathrm{Y}$ & Z & \multirow{2}{*}{$\begin{array}{c}\mathrm{X} \\
-0.08\end{array}$} & \multirow{2}{*}{$\begin{array}{c}\mathrm{Y} \\
0.00\end{array}$} \\
\hline 1 & 12 & 0.00 & 0.00 & 0.50 & 0.00 & 0.00 & 0.00 & 0.00 & & \\
\hline 2 & 13 & 0.00 & -0.18 & -0.33 & 0.00 & -0.26 & -0.23 & 0.00 & 0.26 & 0.23 \\
\hline 3 & 13 & 0.00 & 0.18 & -0.33 & 0.00 & -0.26 & 0.23 & 0.00 & 0.26 & -0.23 \\
\hline 4 & 13 & 0.00 & 0.18 & -0.33 & 0.00 & 0.26 & -0.23 & 0.00 & 0.26 & -0.23 \\
\hline 5 & 13 & 0.00 & -0.18 & -0.33 & 0.00 & 0.26 & 0.23 & 0.00 & 0.26 & 0.23 \\
\hline 6 & 13 & 0.00 & 0.00 & 0.22 & 0.00 & 0.51 & 0.00 & 0.00 & -0.51 & 0.00 \\
\hline 7 & 14 & 0.00 & 0.00 & 0.21 & 0.00 & -0.02 & 0.00 & 0.00 & 0.02 & 0.00 \\
\hline 8 & 13 & 0.00 & 0.00 & 0.22 & 0.00 & -0.51 & 0.00 & 0.00 & -0.51 & 0.00 \\
\hline \multirow[t]{3}{*}{9} & 14 & 0.00 & 0.00 & 0.21 & 0.00 & 0.02 & 0.00 & 0.00 & 0.02 & 0.00 \\
\hline & & \multicolumn{3}{|c|}{16} & \multicolumn{3}{|c|}{17} & \multicolumn{3}{|c|}{18} \\
\hline & & & AG & & \multicolumn{3}{|c|}{ B1U } & \multicolumn{3}{|c|}{ AG } \\
\hline \multicolumn{2}{|c|}{ Frequencies -- } & \multicolumn{2}{|c|}{308.4306} & & \multicolumn{3}{|c|}{347.6208} & \multicolumn{3}{|c|}{389.7730} \\
\hline \multicolumn{2}{|c|}{ Red. masses -- } & \multicolumn{2}{|c|}{27.0931} & & \multicolumn{3}{|c|}{26.7679} & \multicolumn{3}{|c|}{27.2318} \\
\hline \multicolumn{2}{|c|}{ Frc consts } & - & 1.5185 & & \multicolumn{3}{|c|}{1.9058} & \multicolumn{3}{|c|}{2.4375} \\
\hline \multicolumn{2}{|c|}{ IR Inten } & - & 0.0000 & & & 39.2581 & & & 0.00 & \\
\hline Atom & AN & $\mathrm{X}$ & $\mathrm{Y}$ & $\mathrm{Z}$ & & $\mathrm{X}$ & $\mathrm{Y}$ & Z & $\mathrm{X}$ & $\mathrm{Y}$ \\
\hline 1 & 12 & 0.00 & 0.00 & 0.00 & 0.00 & 0.00 & 0.41 & 0.00 & 0.00 & 0.00 \\
\hline 2 & 13 & 0.00 & 0.21 & -0.25 & 0.00 & -0.33 & 0.16 & 0.00 & 0.29 & -0.02 \\
\hline 3 & 13 & 0.00 & -0.21 & -0.25 & 0.00 & 0.33 & 0.16 & 0.00 & -0.29 & -0.02 \\
\hline 4 & 13 & 0.00 & 0.21 & 0.25 & 0.00 & 0.33 & 0.16 & 0.00 & 0.29 & 0.02 \\
\hline 5 & 13 & 0.00 & -0.21 & 0.25 & 0.00 & -0.33 & 0.16 & 0.00 & -0.29 & 0.02 \\
\hline 6 & 13 & 0.00 & 0.00 & 0.48 & 0.00 & 0.00 & -0.10 & 0.00 & 0.00 & -0.46 \\
\hline 7 & 14 & 0.00 & 0.00 & 0.24 & 0.00 & 0.00 & -0.38 & 0.00 & 0.00 & 0.35 \\
\hline 8 & 13 & 0.00 & 0.00 & -0.48 & 0.00 & 0.00 & -0.10 & 0.00 & 0.00 & 0.46 \\
\hline 9 & 14 & 0.00 & 0.00 & -0.24 & 0.00 & 0.00 & -0.38 & 0.00 & 0.00 & -0.35 \\
\hline & & & 19 & & & 20 & & & & 21 \\
\hline & & & B3G & & & & $1 \mathrm{U}$ & & & B2U \\
\hline Frequ & encies - & & 2.4380 & & & 32.5593 & & & 432.753 & \\
\hline Red. & masses - & & 7.5811 & & & 25.9924 & & & 27.580 & \\
\hline Frc C & onsts & - & 3.0388 & & & 2.8654 & & & 3.043 & \\
\hline IR In & en & - & 0.0000 & & & 32.0235 & & & 20.39 & \\
\hline Atom & AN & X & $\mathrm{Y}$ & Z & & $\mathrm{X}$ & $\mathrm{Y}$ & $\mathrm{Z}$ & $\mathrm{X}$ & $\mathrm{Y}$ \\
\hline 1 & 12 & 0.00 & 0.00 & 0.00 & 0.00 & 0.00 & 0.59 & 0.00 & -0.02 & 0.00 \\
\hline 2 & 13 & 0.00 & -0.25 & 0.19 & 0.00 & 0.14 & 0.02 & 0.00 & -0.25 & 0.19 \\
\hline 3 & 13 & 0.00 & -0.25 & -0.19 & 0.00 & -0.14 & 0.02 & 0.00 & -0.25 & -0.19 \\
\hline 4 & 13 & 0.00 & 0.25 & 0.19 & 0.00 & -0.14 & 0.02 & 0.00 & -0.25 & -0.19 \\
\hline 5 & 13 & 0.00 & 0.25 & -0.19 & 0.00 & 0.14 & 0.02 & 0.00 & -0.25 & 0.19 \\
\hline 6 & 13 & 0.00 & -0.06 & 0.00 & 0.00 & 0.00 & -0.50 & 0.00 & -0.06 & 0.00 \\
\hline 7 & 14 & 0.00 & 0.55 & 0.00 & 0.00 & 0.00 & 0.18 & 0.00 & 0.55 & 0.00 \\
\hline 8 & 13 & 0.00 & 0.06 & 0.00 & 0.00 & 0.00 & -0.50 & 0.00 & -0.06 & 0.00 \\
\hline 9 & 14 & 0.00 & -0.55 & 0.00 & 0.00 & 0.00 & 0.18 & 0.00 & 0.55 & 0.00 \\
\hline
\end{tabular}




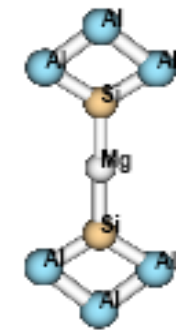

$\begin{array}{rrrrc}13 & 0 & -2.025219 & 3.814582 & 0.000000 \\ 13 & 0 & 2.027865 & 3.810685 & 0.000000 \\ 13 & 0 & 0.002921 & 5.415698 & 0.000000 \\ 12 & 0 & 0.000000 & 0.000000 & 0.000000 \\ 13 & 0 & -2.027865 & -3.810685 & 0.000000 \\ 13 & 0 & -0.002921 & -5.415698 & 0.000000 \\ 13 & 0 & 2.025219 & -3.814582 & 0.000000 \\ 14 & 0 & 0.000000 & 2.518176 & 0.000000 \\ 14 & 0 & 0.000000 & -2.518176 & 0.000000\end{array}$

State $=1-A G \backslash H F=-2233.7950298$

1

AU

Frequencies -- -104.0426

Red. masses -- 26.0272

Frc consts -- $\quad 0.1660$

IR Inten $\quad$-- $\quad 34.2477$

Atom AN $\quad \mathrm{X} \quad \mathrm{Y}$

$\begin{array}{lllll}1 & 13 & 0.00 & 0.00 & -0.01\end{array}$

$\begin{array}{lllll}2 & 13 & 0.00 & 0.00 & -0.01\end{array}$

$\begin{array}{lllll}3 & 13 & 0.00 & 0.00 & 0.21\end{array}$

$\begin{array}{lllll}4 & 12 & 0.00 & 0.00 & 0.68\end{array}$

$\begin{array}{lllll}5 & 13 & 0.00 & 0.00 & -0.01\end{array}$

$\begin{array}{lllll}6 & 13 & 0.00 & 0.00 & 0.21\end{array}$

$\begin{array}{lllll}7 & 13 & 0.00 & 0.00 & -0.01\end{array}$

$\begin{array}{llllll}8 & 14 & 0.00 & 0.00 & -0.47\end{array}$

$\begin{array}{llllll}9 & 14 & 0.00 & 0.00 & -0.47\end{array}$

4

BU

Frequencies -- $\quad 6.1920$

Red. masses -- 26.8945

Frc consts -- 0.0006

IR Inten - - $\quad 0.0385$

Atom AN $\mathrm{X} \quad \mathrm{Y}$

$\begin{array}{lllll}1 & 13 & 0.02 & 0.38 & 0.00\end{array}$

$$
2
$$

BG

$-26.4795$

27.6852

0.0114

0.0000

$\mathrm{X}$

$\begin{array}{lll}0.00 & 0.00 & 0.06\end{array}$

$\begin{array}{lll}0.00 & 0.00 & 0.06\end{array}$

$\begin{array}{lll}0.00 & 0.00 & -0.37\end{array}$

$\begin{array}{lll}0.00 & 0.00 & 0.00\end{array}$

$\begin{array}{llll}0.00 & 0.00 & -0.06\end{array}$

$\begin{array}{lll}0.00 & 0.00 & 0.37\end{array}$

$\begin{array}{lll}0.00 & 0.00 & -0.06\end{array}$

$\begin{array}{lll}0.00 & 0.00 & 0.59\end{array}$

$\begin{array}{lll}0.00 & 0.00 & -0.59\end{array}$

\section{5}

AG

8.3289

27.3182

0.0011

0.0000

$\mathrm{X}$

$\begin{array}{lll}0.16 & -0.36 & 0.00\end{array}$

$\begin{array}{lll}0.16 & 0.36 & 0.00\end{array}$

$\begin{array}{lll}-0.15 & 0.00 & 0.00\end{array}$

$\begin{array}{lll}0.00 & 0.00 & 0.00\end{array}$

$\begin{array}{lll}-0.16 & -0.36 & 0.00\end{array}$

Z

$\begin{array}{lll}0.00 & 0.00 & -0.50\end{array}$

$\begin{array}{lll}0.00 & 0.00 & 0.50\end{array}$

$\begin{array}{lll}0.00 & 0.00 & 0.00\end{array}$

$\begin{array}{lll}0.00 & 0.00 & 0.00\end{array}$

$\begin{array}{lll}0.00 & 0.00 & 0.50\end{array}$

$\begin{array}{lll}0.00 & 0.00 & 0.00\end{array}$

$\begin{array}{lll}0.00 & 0.00 & -0.50\end{array}$

$\begin{array}{lll}0.00 & 0.00 & 0.00\end{array}$

$\begin{array}{lll}0.00 & 0.00 & 0.00\end{array}$

\section{6}

AU

26.6783

25.6569

0.0108

0.8837

$\begin{array}{lll}\mathrm{X} & \mathrm{Y} & \mathrm{Z}\end{array}$

$\begin{array}{lllll}2 & 13 & 0.02 & -0.38 & 0.00\end{array}$

$\begin{array}{lll}0.33 & 0.00 & 0.00\end{array}$

$\begin{array}{rrr}-0.26 & 0.00 & 0.00 \\ 0.02 & -0.38 & 0.00\end{array}$ 


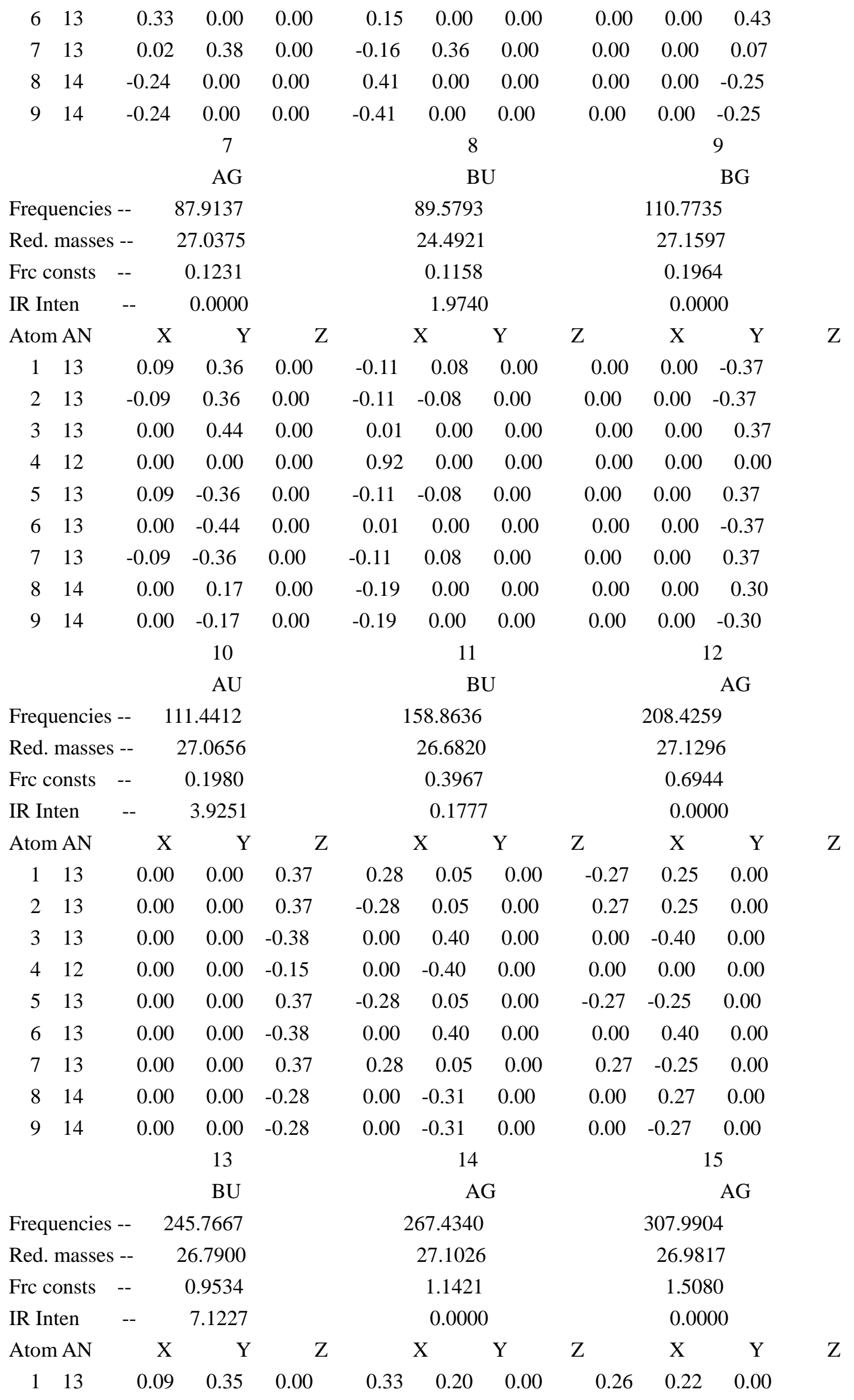




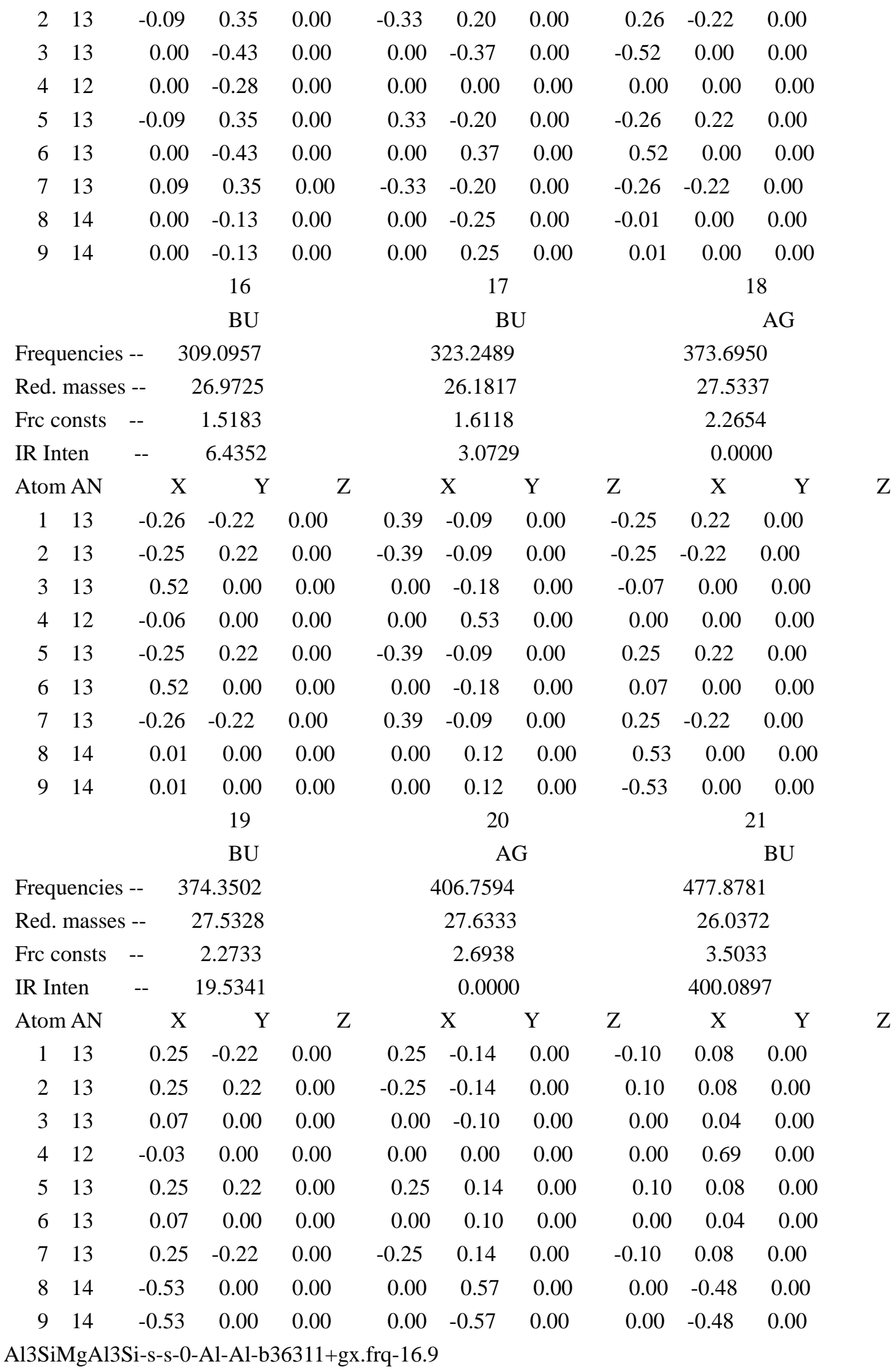




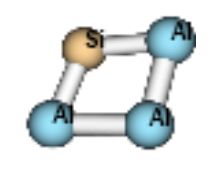

c.

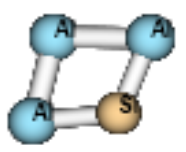

$\begin{array}{lcccc}12 & 0 & 0.000000 & 0.000000 & 0.000000 \\ 13 & 0 & 0.000000 & 2.750387 & 0.000000 \\ 13 & 0 & 0.000000 & -2.750387 & 0.000000 \\ 13 & 0 & 2.443975 & 1.728626 & 0.000000 \\ 13 & 0 & -2.443975 & -1.728626 & 0.000000 \\ 13 & 0 & -0.419456 & -5.219557 & 0.000000 \\ 14 & 0 & -2.504416 & -4.073929 & 0.000000 \\ 13 & 0 & 0.419456 & 5.219557 & 0.000000 \\ 14 & 0 & 2.504416 & 4.073929 & 0.000000\end{array}$

State $=1-A G \mid H F=-2233.7768278$

2

3

AU

$\mathrm{BU}$

Frequencies -- $\quad-16.8984$

20.8060

29.1394

26.4537

26.2424

0.0067

0.0131

Frc consts -- 0.0046

0.6604

IR Inten -- 0.2073

Atom AN $\quad$ X $\quad$ Y

Z

$\begin{array}{lll}\mathrm{X} & \mathrm{Y}\end{array}$

$\begin{array}{lllll}1 & 12 & 0.00 & 0.00 & 0.13\end{array}$

$\begin{array}{lll}0.00 & 0.00 & 0.49\end{array}$

0.3026

$\begin{array}{lllll}2 & 13 & 0.00 & 0.00 & 0.32\end{array}$

$\begin{array}{lll}0.00 & 0.00 & 0.29\end{array}$

$\begin{array}{lll}0.50 & -0.21 & 0.00\end{array}$

$\begin{array}{lllll}3 & 13 & 0.00 & 0.00 & 0.32\end{array}$

$\begin{array}{lll}0.00 & 0.00 & 0.29\end{array}$

$\begin{array}{lll}0.06 & -0.20 & 0.00\end{array}$

$\begin{array}{lllll}4 & 13 & 0.00 & 0.00 & -0.42\end{array}$

$\begin{array}{lll}0.00 & 0.00 & 0.21\end{array}$

$\begin{array}{lll}0.06 & -0.20 & 0.00\end{array}$

$\begin{array}{llllll}5 & 13 & 0.00 & 0.00 & -0.42\end{array}$

$\begin{array}{lll}0.00 & 0.00 & 0.21\end{array}$

$\begin{array}{lll}0.23 & 0.21 & 0.00\end{array}$

$\begin{array}{lllll}6 & 13 & 0.00 & 0.00 & 0.35\end{array}$

$\begin{array}{lll}0.00 & 0.00 & -0.39\end{array}$

$\begin{array}{lll}0.23 & 0.21 & 0.00\end{array}$

$\begin{array}{llllll}7 & 14 & 0.00 & 0.00 & -0.30\end{array}$

$\begin{array}{llll}0.00 & 0.00 & -0.31\end{array}$

$\begin{array}{lll}-0.35 & -0.14 & 0.00\end{array}$

$\begin{array}{lllll}8 & 13 & 0.00 & 0.00 & 0.35\end{array}$

$\begin{array}{lll}0.00 & 0.00 & -0.39\end{array}$

$\begin{array}{lll}-0.15 & 0.22 & 0.00\end{array}$

$\begin{array}{ll}9 & 14\end{array}$

$\begin{array}{lll}0.00 & 0.00 & -0.30\end{array}$

$\begin{array}{llll}0.00 & 0.00 & -0.31\end{array}$

5

AG

$\begin{array}{lll}-0.35 & -0.14 & 0.00\end{array}$

$\begin{array}{lll}-0.15 & 0.22 & 0.00\end{array}$

BG

Frequencies -- $\quad 50.4420$

69.1365

AG

Red. masses -- 27.0659

27.0739

96.7284

0.0762

27.2546

Frc consts -- 0.0406

0.0000

0.1502

IR Inten $\quad--\quad 0.0000$

\begin{tabular}{lll}
\multicolumn{2}{c}{$\mathrm{X}$} & \multicolumn{2}{c}{$\mathrm{Y}$} \\
0.00 & 0.00 & 0.00
\end{tabular}

$\begin{array}{llll}\text { Atom AN } & \mathrm{X} & \mathrm{Y} & \mathrm{Z}\end{array}$

$\begin{array}{lllll}1 & 12 & 0.00 & 0.00 & 0.00\end{array}$

Z

0.0000

$\begin{array}{lllll}2 & 13 & 0.00 & 0.00 & 0.58\end{array}$

$\begin{array}{lll}0.38 & -0.13 & 0.00\end{array}$

0.00

$\mathrm{X}$

Z

$3 \quad 13$

$\begin{array}{lll}0.00 & 0.00 & -0.58\end{array}$

$-0.38$

$0.13 \quad 0.00$

$\begin{array}{lll}0.01 & -0.30 & 0.00\end{array}$

$\begin{array}{lll}-0.01 & 0.30 & 0.00\end{array}$ 


$\begin{array}{ccccccccccc}4 & 13 & 0.00 & 0.00 & 0.26 & 0.48 & 0.15 & 0.00 & 0.04 & -0.33 & 0.00 \\ 5 & 13 & 0.00 & 0.00 & -0.26 & -0.48 & -0.15 & 0.00 & -0.04 & 0.33 & 0.00 \\ 6 & 13 & 0.00 & 0.00 & 0.23 & 0.18 & 0.06 & 0.00 & 0.20 & 0.35 & 0.00 \\ 7 & 14 & 0.00 & 0.00 & 0.21 & 0.02 & -0.21 & 0.00 & 0.20 & 0.31 & 0.00 \\ 8 & 13 & 0.00 & 0.00 & -0.23 & -0.18 & -0.06 & 0.00 & -0.20 & -0.35 & 0.00 \\ 9 & 14 & 0.00 & 0.00 & -0.21 & -0.02 & 0.21 & 0.00 & -0.20 & -0.31 & 0.00 \\ & & & 7 & & & 8 & & & & 9\end{array}$

$\mathrm{AU}$

BG

Frequencies -- $\quad 99.3544$

102.9497

107.5064

Red. masses -- 27.0750

27.3534

25.7966

Frc consts -- 0.1575

0.1708

0.1757

IR Inten $\quad$-- $\quad 9.9940$

0.0000

6.6817

$\begin{array}{llllllllllll}\text { Atom AN } & \mathrm{X} & \mathrm{Y} & \mathrm{Z} & \mathrm{X} & \mathrm{Y} & \mathrm{Z} & \mathrm{X} & \mathrm{Y} & \mathrm{Z}\end{array}$

$\begin{array}{lllllllllll}1 & 12 & 0.00 & 0.00 & 0.24 & 0.00 & 0.00 & 0.00 & 0.61 & -0.22 & 0.00\end{array}$

$\begin{array}{lllllllllll}2 & 13 & 0.00 & 0.00 & -0.37 & 0.00 & 0.00 & 0.21 & -0.34 & 0.02 & 0.00\end{array}$

$\begin{array}{lllllllllll}3 & 13 & 0.00 & 0.00 & -0.37 & 0.00 & 0.00 & -0.21 & -0.34 & 0.02 & 0.00\end{array}$

$\begin{array}{llllllllllll}4 & 13 & 0.00 & 0.00 & 0.31 & 0.00 & 0.00 & -0.40 & -0.30 & 0.11 & 0.00\end{array}$

$\begin{array}{lllllllllll}5 & 13 & 0.00 & 0.00 & 0.31 & 0.00 & 0.00 & 0.40 & -0.30 & 0.11 & 0.00\end{array}$

$\begin{array}{lllllllllll}6 & 13 & 0.00 & 0.00 & 0.33 & 0.00 & 0.00 & 0.33 & 0.18 & -0.04 & 0.00\end{array}$

$\begin{array}{llllllllllll}7 & 14 & 0.00 & 0.00 & -0.37 & 0.00 & 0.00 & -0.43 & 0.19 & 0.01 & 0.00\end{array}$

$\begin{array}{lllllllllll}8 & 13 & 0.00 & 0.00 & 0.33 & 0.00 & 0.00 & -0.33 & 0.18 & -0.04 & 0.00\end{array}$

$\begin{array}{lllllllllll}9 & 14 & 0.00 & 0.00 & -0.37 & 0.00 & 0.00 & 0.43 & 0.19 & 0.01 & 0.00\end{array}$

$10 \quad 11 \quad 12$

AU AG

Frequencies -- 121.9890

172.5395

BU

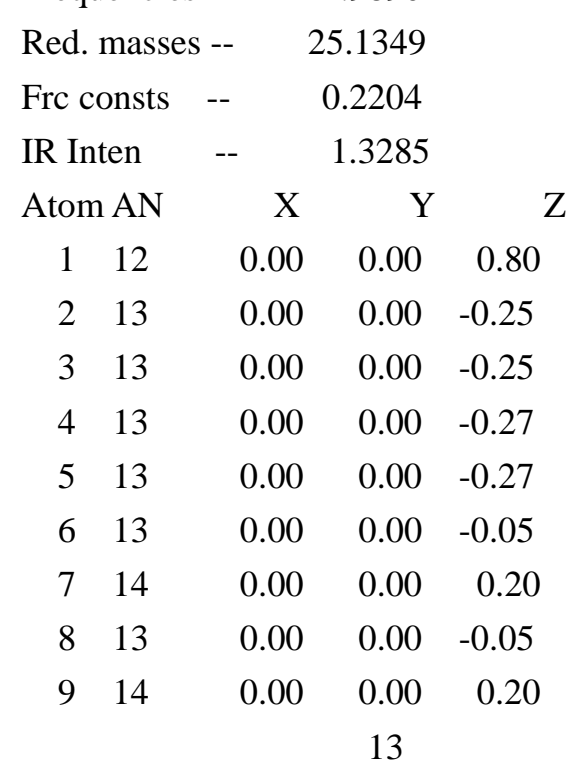

$\mathrm{BU}$

27.2990

176.5115

26.4863

0.4788

0.4862

0.0000

75.6494

$\begin{array}{cccccc}X & Y & Z & X & Y\end{array}$

Z

$\begin{array}{llllll}0.00 & 0.00 & 0.00 & 0.53 & 0.08 & 0.00\end{array}$

$\begin{array}{llllll}-0.18 & -0.21 & 0.00 & 0.01 & 0.16 & 0.00\end{array}$

$\begin{array}{lllllll}0.18 & 0.21 & 0.00 & 0.01 & 0.16 & 0.00\end{array}$

$\begin{array}{llllll}-0.11 & 0.23 & 0.00 & 0.10 & -0.24 & 0.00\end{array}$

$\begin{array}{lll}0.11 & -0.23 & 0.00\end{array}$

$\begin{array}{lll}0.10 & -0.24 & 0.00\end{array}$

$\begin{array}{llllll}-0.01 & 0.44 & 0.00 & 0.01 & 0.30 & 0.00\end{array}$

$\begin{array}{llllll}-0.35 & -0.19 & 0.00 & -0.34 & -0.25 & 0.00\end{array}$

$\begin{array}{llllll}0.01 & -0.44 & 0.00 & 0.01 & 0.30 & 0.00\end{array}$

$\begin{array}{lll}0.35 & 0.19 & 0.00\end{array}$

14

AG

$\begin{array}{lll}-0.34 & -0.25 & 0.00\end{array}$

15

Frequencies -- $\quad 250.5485$

251.2847

27.0211

1.0053

0.0000

BU

Red. masses -- 26.7355

Frc consts -- $\quad 0.9888$

IR Inten $\quad$-- $\quad 7.8411$

0.0000

270.1684
25.4336
1.0938
66.0097




\begin{tabular}{|c|c|c|c|c|c|c|c|c|c|c|c|}
\hline \multicolumn{2}{|c|}{ Atom AN } & $\mathrm{X}$ & $\mathrm{Y}$ & $\mathrm{Z}$ & \multicolumn{2}{|r|}{$X$} & $\mathrm{Y}$ & $\mathrm{Z}$ & $\mathrm{X}$ & $\mathrm{Y}$ & Z \\
\hline 1 & 12 & 0.09 & -0.28 & 0.00 & 0.00 & 0.00 & 0.00 & 0.25 & 0.68 & 0.00 & \\
\hline 2 & 13 & 0.46 & -0.03 & 0.00 & 0.48 & 0.01 & 0.00 & 0.11 & 0.19 & 0.00 & \\
\hline 3 & 13 & 0.46 & -0.03 & 0.00 & -0.48 & -0.01 & 0.00 & 0.11 & 0.19 & 0.00 & \\
\hline 4 & 13 & -0.43 & -0.04 & 0.00 & -0.45 & -0.05 & 0.00 & -0.14 & -0.09 & 0.00 & \\
\hline 5 & 13 & -0.43 & -0.04 & 0.00 & 0.45 & 0.05 & 0.00 & -0.14 & -0.09 & 0.00 & \\
\hline 6 & 13 & -0.15 & 0.17 & 0.00 & 0.21 & -0.01 & 0.00 & -0.16 & -0.36 & 0.00 & \\
\hline 7 & 14 & 0.07 & 0.03 & 0.00 & -0.13 & -0.04 & 0.00 & 0.08 & -0.04 & 0.00 & \\
\hline 8 & 13 & -0.15 & 0.17 & 0.00 & -0.21 & 0.01 & 0.00 & -0.16 & -0.36 & 0.00 & \\
\hline \multirow[t]{3}{*}{9} & 14 & 0.07 & 0.03 & 0.00 & 0.13 & 0.04 & 0.00 & 0.08 & $\begin{array}{ll}3 & -0.04\end{array}$ & 0.00 & \\
\hline & & & 16 & & \multicolumn{3}{|c|}{17} & \multicolumn{3}{|c|}{18} & \\
\hline & & & AG & & \multicolumn{3}{|c|}{$\mathrm{BU}$} & \multicolumn{3}{|r|}{ AG } & \\
\hline \multicolumn{2}{|c|}{ Frequencies - } & \multicolumn{2}{|c|}{339.9609} & & \multicolumn{3}{|c|}{355.7172} & \multicolumn{3}{|c|}{374.2943} & \\
\hline \multicolumn{2}{|c|}{ Red. masses -- } & & 27.0396 & & \multicolumn{3}{|c|}{26.9122} & \multicolumn{3}{|c|}{27.2483} & \\
\hline \multicolumn{2}{|c|}{ Frc consts } & -- & 1.8412 & & \multicolumn{3}{|c|}{2.0064} & \multicolumn{3}{|c|}{2.2491} & \\
\hline \multicolumn{2}{|c|}{ IR Inten } & -- & 0.0000 & & \multicolumn{3}{|c|}{32.2234} & & 0.00 & & \\
\hline Ator & $\mathrm{AN}$ & X & $\mathrm{Y}$ & Z & & $\mathrm{X}$ & $\mathrm{Y}$ & $\mathrm{Z}$ & $\mathrm{X}$ & $\mathrm{Y}$ & Z \\
\hline 1 & 12 & 0.00 & 0.00 & 0.00 & 0.02 & 0.31 & 0.00 & 0.00 & 0.00 & 0.00 & \\
\hline 2 & 13 & -0.06 & 0.53 & 0.00 & 0.14 & -0.28 & 0.00 & 0.18 & 0.24 & 0.00 & \\
\hline 3 & 13 & 0.06 & -0.53 & 0.00 & 0.14 & -0.28 & 0.00 & -0.18 & -0.24 & 0.00 & \\
\hline 4 & 13 & 0.13 & -0.26 & 0.00 & -0.15 & 0.35 & 0.00 & -0.09 & 0.25 & 0.00 & \\
\hline 5 & 13 & -0.13 & 0.26 & 0.00 & -0.15 & 0.35 & 0.00 & 0.09 & -0.25 & 0.00 & \\
\hline 6 & 13 & 0.22 & 0.24 & 0.00 & 0.31 & -0.06 & 0.00 & -0.29 & 0.35 & 0.00 & \\
\hline 7 & 14 & -0.14 & -0.09 & 0.00 & -0.30 & -0.14 & 0.00 & 0.36 & 0.06 & 0.00 & \\
\hline 8 & 13 & -0.22 & -0.24 & 0.00 & 0.31 & -0.06 & 0.00 & 0.29 & -0.35 & 0.00 & \\
\hline 9 & 14 & 0.14 & 0.09 & 0.00 & -0.30 & -0.14 & 0.00 & -0.36 & -0.06 & 0.00 & \\
\hline & & & 19 & & & 20 & & & & 21 & \\
\hline & & & $\mathrm{BU}$ & & & A & & & & $\mathrm{BU}$ & \\
\hline Freq & uencies & & 90.3728 & & & 442.9821 & & & 445.22 & & \\
\hline Red. & masses & s -- & 26.4628 & & & 27.5387 & & & 27.38 & & \\
\hline Frc & onsts & -- & 2.3760 & & & 3.1840 & & & 3.198 & & \\
\hline IR II & ten & -- & 62.3872 & & & 0.000 & & & 10.05 & & \\
\hline Ator & $\mathrm{AN}$ & $X$ & $\mathrm{Y}$ & Z & & $\mathrm{X}$ & $\mathrm{Y}$ & $\mathrm{Z}$ & $\mathrm{X}$ & $\mathrm{Y}$ & \\
\hline 1 & 12 & 0.06 & 0.47 & 0.00 & 0.00 & 0.00 & 0.00 & -0.07 & -0.17 & 0.00 & \\
\hline 2 & 13 & -0.12 & -0.40 & 0.00 & 0.00 & 0.13 & 0.00 & 0.01 & 0.19 & 0.00 & \\
\hline 3 & 13 & -0.12 & -0.40 & 0.00 & 0.00 & -0.13 & 0.00 & 0.01 & 0.19 & 0.00 & \\
\hline 4 & 13 & 0.01 & -0.04 & 0.00 & 0.07 & 0.37 & 0.00 & 0.08 & 0.38 & 0.00 & \\
\hline 5 & 13 & 0.01 & -0.04 & 0.00 & -0.07 & -0.37 & 0.00 & 0.08 & 0.38 & 0.00 & \\
\hline 6 & 13 & -0.19 & 0.32 & 0.00 & 0.24 & -0.04 & 0.00 & -0.21 & 0.01 & 0.00 & \\
\hline 7 & 14 & 0.26 & -0.09 & 0.00 & -0.17 & 0.50 & 0.00 & 0.15 & -0.48 & 0.00 & \\
\hline 8 & 13 & -0.19 & 0.32 & 0.00 & -0.24 & 0.04 & 0.00 & -0.21 & 0.01 & 0.00 & \\
\hline 9 & 14 & 0.26 & -0.09 & 0.00 & 0.17 & -0.50 & 0.00 & 0.15 & -0.48 & 0.00 & \\
\hline
\end{tabular}




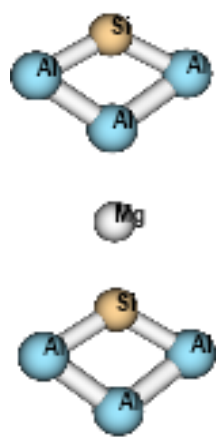

12

13

$\begin{array}{ccc}0.000000 & 0.000000 & -0.117727 \\ 0.000000 & 2.077326 & 4.079709 \\ 0.000000 & -2.077326 & 4.079709 \\ 0.000000 & 0.000000 & -5.538171 \\ 0.000000 & 2.027312 & -3.931961 \\ 0.000000 & -2.027312 & -3.931961 \\ 0.000000 & 0.000000 & -2.638057 \\ 0.000000 & 0.000000 & 2.556422 \\ 0.000000 & 0.000000 & 5.233343\end{array}$

State $=1-A 1 \backslash H F=-2233.7809723$

B1

B2

B1

Frequencies -- $\quad-96.4531$

Red. masses -- $\quad 25.8740$

$-13.7653$

$-7.7946$

27.0526

27.3322

Frc consts --- 0.1418

0.0030

0.0010

IR Inten -- 24.3250

0.0039

0.2027

$\begin{array}{lrr}\text { Atom AN } & \text { X } & \text { Y }\end{array}$

$\mathrm{Z}$

$\begin{array}{lllll}1 & 12 & 0.67 & 0.00 & 0.00\end{array}$

$\begin{array}{ccc}\mathrm{X} & \mathrm{Y} \\ 0.00 & -0.08 & 0.00\end{array}$

Z $\quad \mathrm{X}$

Z

$\begin{array}{lllll}2 & 13 & 0.01 & 0.00 & 0.00\end{array}$

$\begin{array}{lll}0.00 & -0.07 & -0.52\end{array}$

0.16

$0.00 \quad 0.00$

$\begin{array}{lllll}3 & 13 & 0.01 & 0.00 & 0.00\end{array}$

$\begin{array}{lll}0.00 & -0.07 & 0.52\end{array}$

$\begin{array}{lll}-0.03 & 0.00 & 0.00\end{array}$

$\begin{array}{lllll}4 & 13 & 0.21 & 0.00 & 0.00\end{array}$

$\begin{array}{lll}0.00 & 0.04 & 0.00\end{array}$

$\begin{array}{lll}-0.03 & 0.00 & 0.00\end{array}$

$\begin{array}{lllll}5 & 13 & -0.02 & 0.00 & 0.00\end{array}$

$\begin{array}{lll}0.00 & 0.13 & -0.10\end{array}$

$\begin{array}{lll}-0.48 & 0.00 & 0.00\end{array}$

$\begin{array}{lllll}6 & 13 & -0.02 & 0.00 & 0.00\end{array}$

$\begin{array}{lll}0.00 & 0.13 & 0.10\end{array}$

$\begin{array}{lll}0.05 & 0.00 & 0.00\end{array}$

$\begin{array}{lllll}7 & 14 & -0.47 & 0.00 & 0.00\end{array}$

$\begin{array}{lll}0.00 & 0.21 & 0.00\end{array}$

$\begin{array}{lll}0.05 & 0.00 & 0.00\end{array}$

$\begin{array}{lllll}8 & 13 & -0.49 & 0.00 & 0.00\end{array}$

$\begin{array}{lll}0.00 & -0.53 & 0.00\end{array}$

$\begin{array}{lll}0.62 & 0.00 & 0.00\end{array}$

$\begin{array}{ll}9 & 14\end{array}$

$\begin{array}{lll}0.18 & 0.00 & 0.00\end{array}$

$\begin{array}{lll}0.00 & 0.22 & 0.00\end{array}$

$\begin{array}{lll}-0.56 & 0.00 & 0.00\end{array}$

4

A2

5

$\begin{array}{lll}0.20 & 0.00 & 0.00\end{array}$

B2

6

6.2548

B1

Frequencies -- $\quad-0.4218$

Red. masses -- 26.9815

27.0235

26.4763

0.0006

25.7539

Frc consts -- 0.0000

0.0671

IR Inten -- $\quad 0.0000$

$\begin{array}{llll}\text { Atom AN } & \mathrm{X} & \mathrm{Y} & \mathrm{Z}\end{array}$

$\begin{array}{lllll}1 & 12 & 0.00 & 0.00 & 0.00\end{array}$

$\mathrm{X} \quad \mathrm{Y}$

$\begin{array}{lllll}2 & 13 & -0.49 & 0.00 & 0.00\end{array}$

$\begin{array}{lll}0.00 & -0.23 & 0.00\end{array}$

Z $\quad \mathrm{X}$

0.0106

1.7235

$\begin{array}{lllll}3 & 13 & 0.49 & 0.00 & 0.00\end{array}$

$\begin{array}{lll}0.00 & 0.10 & -0.15\end{array}$

$\begin{array}{lll}0.69 & 0.00 & 0.00\end{array}$

$\begin{array}{lll}-0.11 & 0.00 & 0.00\end{array}$

0.00

0.10

0.15

$\begin{array}{lll}-0.11 & 0.00 & 0.00\end{array}$ 


$\begin{array}{rrrrrrrrrrc}4 & 13 & 0.00 & 0.00 & 0.00 & 0.00 & 0.37 & 0.00 & -0.34 & 0.00 & 0.00 \\ 5 & 13 & 0.51 & 0.00 & 0.00 & 0.00 & -0.06 & 0.52 & -0.08 & 0.00 & 0.00 \\ 6 & 13 & -0.51 & 0.00 & 0.00 & 0.00 & -0.06 & -0.52 & -0.08 & 0.00 & 0.00 \\ 7 & 14 & 0.00 & 0.00 & 0.00 & 0.00 & -0.41 & 0.00 & 0.11 & 0.00 & 0.00 \\ 8 & 13 & 0.00 & 0.00 & 0.00 & 0.00 & 0.00 & 0.00 & 0.43 & 0.00 & 0.00 \\ 9 & 14 & 0.00 & 0.00 & 0.00 & 0.00 & 0.19 & 0.00 & -0.43 & 0.00 & 0.00 \\ & & & 7 & & & 8 & & & & 9\end{array}$

B2 A1

B1

\begin{tabular}{|c|c|c|c|}
\hline \multicolumn{2}{|c|}{ Frequencies -- } & \multicolumn{2}{|c|}{81.3884} \\
\hline Red. masses & & 24.4540 & \\
\hline Frc consts & -- & 0.0954 & \\
\hline IR Inten & -- & 2.9181 & \\
\hline Atom AN & $\mathrm{X}$ & $\mathrm{Y}$ & 2 \\
\hline 112 & 0.00 & 0.93 & 0.00 \\
\hline 213 & 0.00 & -0.07 & -0.06 \\
\hline 313 & 0.00 & -0.07 & 0.06 \\
\hline $4 \quad 13$ & 0.00 & 0.00 & 0.00 \\
\hline $5 \quad 13$ & 0.00 & -0.12 & 0.09 \\
\hline $6 \quad 13$ & 0.00 & -0.12 & -0.09 \\
\hline $\begin{array}{ll}7 & 14\end{array}$ & 0.00 & -0.20 & 0.00 \\
\hline 813 & 0.00 & -0.19 & 0.00 \\
\hline $9 \quad 14$ & 0.00 & -0.04 & 0.00 \\
\hline & & 10 & \\
\hline
\end{tabular}

B1
86.9055

27.1479

0.1208

1.0458

$\begin{array}{llllll}\mathrm{X} & \mathrm{Y} & \mathrm{Z} & \mathrm{X} & \mathrm{Y}\end{array}$

$\begin{array}{llllll}0.00 & 0.00 & 0.00 & -0.06 & 0.00 & 0.00\end{array}$

$\begin{array}{llllll}0.00 & -0.02 & 0.38 & 0.51 & 0.00 & 0.00\end{array}$

$\begin{array}{llllll}0.00 & 0.02 & 0.38 & 0.51 & 0.00 & 0.00\end{array}$

$\begin{array}{llllll}0.00 & 0.00 & -0.45 & 0.04 & 0.00 & 0.00\end{array}$

$\begin{array}{llllll}0.00 & -0.09 & -0.37 & -0.04 & 0.00 & 0.00\end{array}$

$\begin{array}{llllll}0.00 & 0.09 & -0.37 & -0.04 & 0.00 & 0.00\end{array}$

$\begin{array}{llllll}0.00 & 0.00 & -0.17 & 0.03 & 0.00 & 0.00\end{array}$

$\begin{array}{llllll}0.00 & 0.00 & 0.22 & -0.30 & 0.00 & 0.00\end{array}$

$\begin{array}{llllll}0.00 & 0.00 & 0.37 & -0.62 & 0.00 & 0.00\end{array}$

11

A1

171.5822

26.6540

0.4623

6.6191

X Y

$\begin{array}{lll}0.00 & 0.00 & -0.43\end{array}$

$\begin{array}{lll}0.00 & -0.11 & 0.16\end{array}$

$\begin{array}{lll}0.00 & 0.11 & 0.16\end{array}$

$\begin{array}{lll}0.00 & 0.00 & 0.48\end{array}$

$\begin{array}{lll}0.00 & 0.36 & -0.03\end{array}$

$\begin{array}{lll}0.00 & -0.36 & -0.03\end{array}$

$\begin{array}{lll}0.00 & 0.00 & -0.42\end{array}$

$\begin{array}{lll}0.00 & 0.00 & -0.14\end{array}$

$\begin{array}{lll}0.00 & 0.00 & 0.22\end{array}$

14

B2

271.5577

26.9726

1.1719

0.3000
12

A1

211.7859

27.2885

0.7211

28.5087

\begin{tabular}{ccc}
$Z$ & $\mathrm{X}$ & $\mathrm{Y}$ \\
0.00 & 0.00 & 0.01 \\
0.00 & 0.49 & 0.27 \\
0.00 & -0.49 & 0.27 \\
0.00 & 0.00 & 0.15 \\
0.00 & 0.09 & -0.09 \\
0.00 & -0.09 & -0.09 \\
0.00 & 0.00 & -0.09 \\
0.00 & 0.00 & 0.14 \\
0.00 & 0.00 & -0.55 \\
\multicolumn{3}{c}{15}
\end{tabular}

A1

283.6429

26.7780

1.2693

9.0058 


\begin{tabular}{|c|c|c|c|c|c|c|c|c|c|c|}
\hline \multicolumn{2}{|c|}{ Atom AN } & $\mathrm{X}$ & $\mathrm{Y}$ & $\mathrm{Z}$ & \multicolumn{2}{|c|}{$\mathrm{X}$} & Y & Z & $\mathrm{X}$ & $\mathrm{Y}$ \\
\hline 1 & 12 & 0.00 & 0.00 & 0.36 & 0.00 & -0.06 & 0.00 & 0.00 & 0.00 & 0.29 \\
\hline 2 & 13 & 0.00 & -0.03 & -0.20 & 0.00 & 0.37 & 0.32 & 0.00 & 0.11 & -0.25 \\
\hline 3 & 13 & 0.00 & 0.03 & -0.20 & 0.00 & 0.37 & -0.32 & 0.00 & -0.11 & -0.25 \\
\hline 4 & 13 & 0.00 & 0.00 & 0.55 & 0.00 & -0.01 & 0.00 & 0.00 & 0.00 & -0.31 \\
\hline 5 & 13 & 0.00 & -0.05 & -0.45 & 0.00 & 0.00 & 0.00 & 0.00 & 0.44 & 0.07 \\
\hline 6 & 13 & 0.00 & 0.05 & -0.45 & 0.00 & 0.00 & 0.00 & 0.00 & -0.44 & 0.07 \\
\hline 7 & 14 & 0.00 & 0.00 & 0.12 & 0.00 & 0.00 & 0.00 & 0.00 & 0.00 & -0.19 \\
\hline 8 & 13 & 0.00 & 0.00 & 0.22 & 0.00 & -0.72 & 0.00 & 0.00 & 0.00 & 0.46 \\
\hline \multirow[t]{3}{*}{9} & 14 & 0.00 & 0.00 & 0.08 & 0.00 & 0.03 & 0.00 & 0.00 & 0.00 & 0.14 \\
\hline & & \multicolumn{3}{|c|}{16} & \multicolumn{3}{|c|}{17} & \multicolumn{3}{|c|}{18} \\
\hline & & & B2 & & \multicolumn{3}{|c|}{ A1 } & \multicolumn{3}{|c|}{ B2 } \\
\hline \multicolumn{2}{|c|}{ Frequencies -- } & \multicolumn{2}{|c|}{307.1062} & & \multicolumn{3}{|c|}{339.6374} & \multicolumn{3}{|c|}{374.1479} \\
\hline \multicolumn{2}{|c|}{ Red. masses -- } & \multicolumn{2}{|c|}{26.9774} & & \multicolumn{3}{|c|}{26.6613} & \multicolumn{3}{|c|}{27.5339} \\
\hline \multicolumn{2}{|c|}{ Frc consts } & - & 1.4991 & & \multicolumn{3}{|c|}{1.8120} & \multicolumn{3}{|c|}{2.2709} \\
\hline \multicolumn{2}{|c|}{ IR Inten } & - & 2.9422 & & & 8.5325 & & & 10.07 & \\
\hline Atom & $\mathrm{AN}$ & $X$ & $\mathrm{Y}$ & $\mathrm{Z}$ & & $\mathrm{X}$ & $\mathrm{Y}$ & Z & $\mathrm{X}$ & $\mathrm{Y}$ \\
\hline 1 & 12 & 0.00 & -0.04 & 0.00 & 0.00 & 0.00 & 0.42 & 0.00 & -0.02 & 0.00 \\
\hline 2 & 13 & 0.00 & 0.00 & 0.00 & 0.00 & -0.36 & 0.21 & 0.00 & 0.00 & 0.00 \\
\hline 3 & 13 & 0.00 & 0.00 & 0.00 & 0.00 & 0.36 & 0.21 & 0.00 & 0.00 & 0.00 \\
\hline 4 & 13 & 0.00 & 0.74 & 0.00 & 0.00 & 0.00 & -0.13 & 0.00 & 0.10 & 0.00 \\
\hline 5 & 13 & 0.00 & -0.36 & -0.31 & 0.00 & 0.29 & -0.10 & 0.00 & 0.35 & -0.31 \\
\hline 6 & 13 & 0.00 & -0.36 & 0.31 & 0.00 & -0.29 & -0.10 & 0.00 & 0.35 & 0.31 \\
\hline 7 & 14 & 0.00 & 0.02 & 0.00 & 0.00 & 0.00 & 0.20 & 0.00 & -0.75 & 0.00 \\
\hline 8 & 13 & 0.00 & 0.00 & 0.00 & 0.00 & 0.00 & -0.25 & 0.00 & 0.00 & 0.00 \\
\hline 9 & 14 & 0.00 & 0.00 & 0.00 & 0.00 & 0.00 & -0.41 & 0.00 & 0.00 & 0.00 \\
\hline & & & 19 & & & 20 & & & & 21 \\
\hline & & & A1 & & & B2 & & & & A1 \\
\hline Frequ & Iencies & & 4.3881 & & & 33.7044 & & & 459.130 & \\
\hline Red. & masses & & 7.2961 & & & 27.5814 & & & 26.128 & \\
\hline Frc C & onsts & - & 2.5015 & & & 3.0567 & & & 3.245 & \\
\hline IR In & & - & 6.7664 & & & 10.1215 & & & 203.17 & \\
\hline Atom & $\mathrm{AN}$ & X & $\mathrm{Y}$ & Z & & X & $\mathrm{Y}$ & $\mathrm{Z}$ & $\mathrm{X}$ & $\mathrm{Y}$ \\
\hline 1 & 12 & 0.00 & 0.00 & -0.08 & 0.00 & 0.01 & 0.00 & 0.00 & 0.00 & 0.64 \\
\hline 2 & 13 & 0.00 & -0.32 & 0.02 & 0.00 & 0.35 & -0.26 & 0.00 & 0.08 & 0.02 \\
\hline 3 & 13 & 0.00 & 0.32 & 0.02 & 0.00 & 0.35 & 0.26 & 0.00 & -0.08 & 0.02 \\
\hline 4 & 13 & 0.00 & 0.00 & 0.08 & 0.00 & 0.00 & 0.00 & 0.00 & 0.00 & 0.06 \\
\hline 5 & 13 & 0.00 & -0.21 & 0.11 & 0.00 & 0.00 & 0.00 & 0.00 & -0.15 & 0.10 \\
\hline 6 & 13 & 0.00 & 0.21 & 0.11 & 0.00 & 0.00 & 0.00 & 0.00 & 0.15 & 0.10 \\
\hline 7 & 14 & 0.00 & 0.00 & -0.42 & 0.00 & 0.00 & 0.00 & 0.00 & 0.00 & -0.60 \\
\hline 8 & 13 & 0.00 & 0.00 & 0.58 & 0.00 & 0.09 & 0.00 & 0.00 & 0.00 & -0.37 \\
\hline 9 & 14 & 0.00 & 0.00 & -0.40 & 0.00 & -0.78 & 0.00 & 0.00 & 0.00 & 0.11 \\
\hline
\end{tabular}




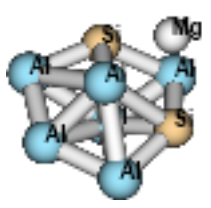

$\begin{array}{rrrrr}13 & 0 & -2.657570 & 0.000068 & 0.207085 \\ 12 & 0 & 2.356668 & -0.000002 & 1.521705 \\ 13 & 0 & -0.379867 & 0.000000 & -1.331860 \\ 13 & 0 & -0.237437 & 0.000007 & 1.396767 \\ 14 & 0 & 0.891407 & 1.893801 & -0.192099 \\ 13 & 0 & 2.251431 & -0.000071 & -1.189547 \\ 13 & 0 & -1.535834 & 2.357446 & -0.036700 \\ 14 & 0 & 0.891316 & -1.893855 & -0.192062 \\ 13 & 0 & -1.535962 & -2.357389 & -0.036683\end{array}$

State $=1-A \backslash H F=-2233.8622851$

Al3SiMgAl3Si-fusion108-b36311+gx.frq

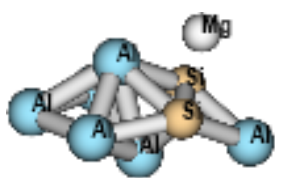

$\begin{array}{ccccc}13 & 0 & 1.494327 & 2.061154 & -0.198306 \\ 12 & 0 & -1.837377 & -0.000079 & 2.179198 \\ 13 & 0 & 0.226036 & 0.000062 & -1.567740 \\ 13 & 0 & 1.494239 & -2.061140 & -0.198416 \\ 14 & 0 & -1.168471 & -1.343108 & 0.008888 \\ 13 & 0 & -3.032472 & 0.000063 & -1.331311 \\ 13 & 0 & 3.305608 & -0.000048 & -0.111824 \\ 13 & 0 & 0.724997 & -0.000021 & 1.376783 \\ 14 & 0 & -1.168459 & 1.343111 & 0.008984\end{array}$

State $=1-A \backslash H F=-2233.8320211$

Al3SiMgAl3Si-fusion110-b36311+gx.frq

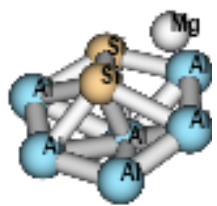

$\begin{array}{lcccc}13 & 0 & 0.813516 & 2.606549 & -0.124167 \\ 13 & 0 & -1.558115 & 1.695226 & -0.470802 \\ 12 & 0 & -2.379717 & -0.056670 & 1.635945 \\ 13 & 0 & 0.368199 & 0.038926 & -1.468034 \\ 13 & 0 & 2.207010 & -1.807150 & 0.079987 \\ 14 & 0 & 0.150455 & -0.034000 & 1.097610 \\ 14 & 0 & -0.208486 & -2.174516 & -0.104404 \\ 13 & 0 & -2.112580 & -0.891562 & -0.997065 \\ 13 & 0 & 2.541127 & 0.788725 & 0.400373\end{array}$

State $=1-A \mid H F=-2233.8653281$ 
Al3SiCaAl3Si-s-s-90-SiSi-b36311+gx.frq8.8

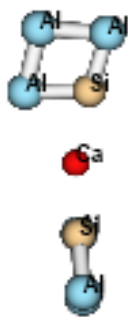

Charge $=0$ Multiplicity $=1$

Al,0,2.8814126943,-1.0385082823,1.2990729287

Al,0,5.3250038536,-0.6138013055,0.7540367514

Ca,0,-0.0002259003,-0.0076008988,-0.0932019221

Al,0,-2.8743125904,1.224057262,1.1421411042

Al,0,-5.320520683,0.7331631826,0.67023836

Al,0,4.9903509321,0.9450413074,-1.2718400882

Si,0,2.7700732267,0.4429292939,-0.6236918133

Si,0,-2.7734747675,-0.5226741868,-0.54397884

Al,0,-4.99696386,-1.1058368205,-1.1076606939

State $=1-A \backslash H F=-2711.3087926$

1

A

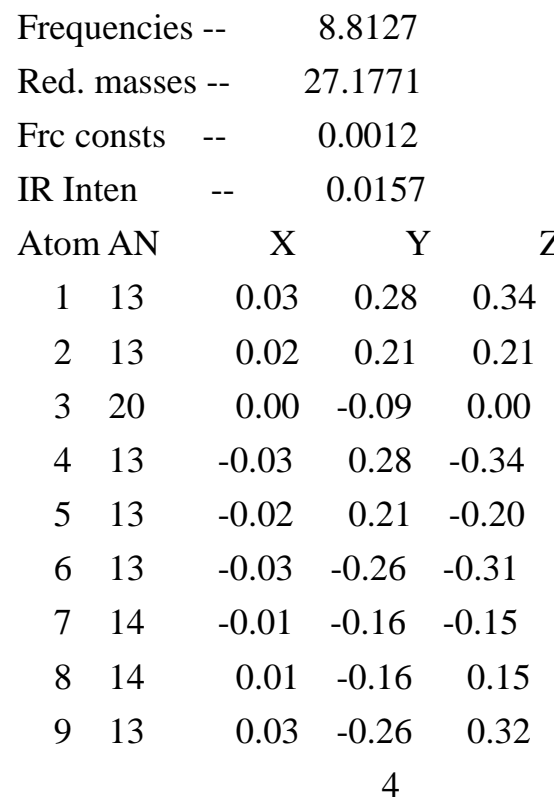

A

Frequencies -- $\quad 36.4130$

Red. masses -- $\quad 29.1278$

Frc consts --- 0.0228

IR Inten -- $\quad 6.0670$

Atom AN $\mathrm{X} \quad \mathrm{Y}$

113

0.13

$-0.30$

213

$0.08 \quad 0.23$

$-0.01 \quad 0.01$
2

A

13.6127

30.0085

0.0033

0.3188

$\mathrm{X}$

$-0.16$

$-0.09$

0.01

$-0.15$

$-0.08$

0.17

0.08

0.07

0.15

$-0.03$

$\mathrm{Y}$
$03-0.12$

$0.10 \quad 0.40$

$\begin{array}{ll}0.02 & -0.47\end{array}$

$0.04-0.12$

$-0.14 \quad 0.41$

$0.04 \quad 0.28$

$-0.09-0.20$

$0.11-0.21$

$-0.06 \quad 0.28$

5

A

36.5854

28.6724

0.0226

3.9040

X Y

$\begin{array}{lll}-0.19 & -0.06 & -0.30\end{array}$

$\begin{array}{lll}-0.12 & 0.07 & 0.25\end{array}$

0.00

$0.31-0.01$
3

A

14.7728

29.5834

0.0038

0.1990

Z $\quad \mathrm{Y} \quad \mathrm{Y}$

$\begin{array}{lll}-0.17 & 0.16 & 0.11\end{array}$

$\begin{array}{lll}-0.11 & -0.37 & -0.13\end{array}$

$\begin{array}{lll}0.00 & 0.44 & 0.02\end{array}$

$\begin{array}{lll}0.19 & 0.16 & -0.10\end{array}$

$\begin{array}{lll}0.12 & -0.36 & 0.09\end{array}$

$\begin{array}{lll}0.14 & -0.31 & -0.11\end{array}$

$\begin{array}{lll}0.06 & 0.19 \quad 0.12\end{array}$

$\begin{array}{lll}-0.07 & 0.18 & -0.10\end{array}$

$\begin{array}{lll}-0.16 & -0.31 & 0.08\end{array}$

6

A

68.0949

30.6133

0.0836

21.8950

$\begin{array}{lllll}Z & X & Y & \text { Z }\end{array}$

$\begin{array}{lll}-0.17 & 0.24 & -0.27\end{array}$

$\begin{array}{lll}-0.07 & -0.08 & 0.12\end{array}$

$\begin{array}{lll}0.07 & 0.00 & 0.50\end{array}$ 


$$
\begin{array}{rrrrr}
4 & 13 & 0.14 & 0.29 & -0.12 \\
5 & 13 & 0.09 & -0.22 & 0.10 \\
6 & 13 & -0.14 & 0.07 & -0.02 \\
7 & 14 & -0.06 & -0.40 & -0.24 \\
8 & 14 & -0.06 & 0.39 & -0.21 \\
9 & 13 & -0.15 & -0.07 & -0.02 \\
& & & 7 &
\end{array}
$$

A

Frequencies -- $\quad 70.3528$

Red. masses -- $\quad 31.1301$

Frc consts -- 0.0908

IR Inten -- $\quad 5.1581$

Atom AN $\quad \mathrm{X} \quad \mathrm{Y}$

$\begin{array}{lllll}1 & 13 & 0.25 & -0.27 & 0.16\end{array}$

213

$\begin{array}{lll}0.19 & 0.10 & -0.03\end{array}$

$\begin{array}{lll}0.00 & 0.55 & 0.01\end{array}$

$\begin{array}{lll}-0.26 & -0.28 & -0.17\end{array}$

$\begin{array}{lll}-0.19 & 0.10 & 0.03\end{array}$

$\begin{array}{rrr}-0.03 & 0.07 & -0.07 \\ 0.11 & -0.29 & 0.16\end{array}$

$\begin{array}{lll}-0.11 & -0.30 & -0.16\end{array}$

$\begin{array}{lll}0.03 & 0.07 & 0.07\end{array}$

10

A

Frequencies -- $\quad 114.2005$

Red. masses -- 27.2602

Frc consts -- 0.2095

IR Inten -- 1.9925

$\begin{array}{lllll}\text { Atom AN } & X & Y & \text { Z }\end{array}$

$\begin{array}{lllll}1 & 13 & 0.01 & 0.24 & 0.27\end{array}$

213

$\begin{array}{lll}0.01 & -0.22 & -0.24\end{array}$

$\begin{array}{lll}0.00 & 0.01 & 0.00\end{array}$

$\begin{array}{lll}-0.01 & 0.24 & -0.27\end{array}$

$\begin{array}{lll}-0.01 & -0.22 & 0.24\end{array}$

$\begin{array}{lllllllllll}6 & 13 & 0.01 & 0.23 & 0.27 & -0.16 & -0.14 & 0.12 & 0.42 & 0.14 & -0.12\end{array}$

$\begin{array}{rrrrrrrrrrr}7 & 14 & 0.00 & -0.25 & -0.28 & -0.23 & 0.06 & -0.03 & 0.34 & -0.03 & 0.03\end{array}$

$\begin{array}{llllllllllll}8 & 14 & 0.00 & -0.25 & 0.28 & -0.23 & -0.06 & -0.04 & -0.34 & -0.03 & -0.03\end{array}$

$\begin{array}{lllllllllll}9 & 13 & -0.01 & 0.23 & -0.26 & -0.16 & 0.14 & 0.12 & -0.42 & 0.14 & 0.12\end{array}$

$$
\begin{array}{ccc}
0.18 & -0.08 & 0.30 \\
0.12 & 0.08 & -0.25 \\
0.12 & -0.05 & 0.07 \\
0.03 & -0.16 & -0.43 \\
-0.03 & -0.19 & 0.44 \\
-0.11 & -0.05 & -0.07 \\
\multicolumn{3}{c}{8}
\end{array}
$$

A

94.7917

27.1959

0.1440

1.3347

$$
\begin{array}{ccc}
-0.16 & -0.24 & -0.27 \\
-0.06 & 0.08 & 0.12 \\
0.18 & -0.10 & 0.08 \\
0.00 & 0.24 & -0.29 \\
0.00 & -0.23 & -0.29 \\
0.18 & 0.10 & 0.08 \\
& & 9
\end{array}
$$

\section{9}

A

113.3559

27.2549

0.2063

2.8069

$\begin{array}{lllllll}0.24 & 0.44 & 0.15 & 0.14 & 0.29 & 0.14 & 0.13\end{array}$

13

A

14

A

Frequencies -- 254.4386

Red. masses -- 33.6127

Frc consts -- 1.2821

IR Inten $\quad$-- 200.4059
272.0570

27.1095

1.1822

2.1233
15

A

272.7140

27.2759

1.1952

7.8404 


\begin{tabular}{|c|c|c|c|c|c|c|c|c|c|c|c|}
\hline \multicolumn{2}{|c|}{ Atom AN } & \multicolumn{2}{|l|}{$\mathrm{X}$} & \multicolumn{2}{|c|}{$\mathrm{Z}$} & $\mathrm{X}$ & $\mathrm{Y}$ & $\mathrm{Z}$ & $X$ & $\mathrm{Y}$ & Z \\
\hline 1 & 13 & -0.10 & 0.07 & -0.06 & 0.28 & -0.07 & 0.06 & -0.31 & 0.07 & -0.06 & \\
\hline 2 & 13 & 0.10 & -0.11 & 0.09 & -0.20 & 0.22 & -0.20 & 0.23 & -0.25 & 0.22 & \\
\hline 3 & 20 & 0.71 & 0.00 & -0.08 & -0.01 & 0.05 & 0.00 & -0.11 & 0.00 & 0.06 & \\
\hline 4 & 13 & -0.10 & -0.07 & -0.06 & -0.32 & -0.08 & -0.07 & -0.26 & -0.06 & -0.05 & \\
\hline 5 & 13 & 0.10 & 0.11 & 0.10 & 0.23 & 0.26 & 0.23 & 0.19 & 0.21 & 0.19 & \\
\hline 6 & 13 & -0.43 & -0.03 & 0.03 & 0.05 & -0.31 & 0.27 & 0.01 & 0.37 & -0.33 & \\
\hline 7 & 14 & -0.09 & 0.02 & -0.01 & -0.13 & 0.11 & -0.10 & 0.16 & -0.14 & 0.12 & \\
\hline 8 & 14 & -0.09 & -0.02 & -0.01 & 0.15 & 0.13 & 0.12 & 0.13 & 0.12 & 0.10 & \\
\hline \multirow[t]{3}{*}{9} & 13 & -0.43 & 0.03 & 0.03 & -0.04 & -0.36 & -0.32 & 0.02 & -0.32 & -0.28 & \\
\hline & & & 16 & & & 17 & & & & 18 & \\
\hline & & & A & & & & A & & & A & \\
\hline \multicolumn{2}{|c|}{ Frequencies -- } & & 331.0529 & & & \multicolumn{2}{|c|}{331.1318} & & \multicolumn{2}{|c|}{355.6175} & \\
\hline \multicolumn{2}{|c|}{ Red. masses - } & & 27.0122 & & & \multicolumn{2}{|c|}{27.0076} & & \multicolumn{2}{|c|}{27.4213} & \\
\hline \multicolumn{2}{|c|}{ Frc consts } & -- & 1.7442 & & & \multicolumn{2}{|c|}{1.7448} & & \multicolumn{2}{|c|}{2.0432} & \\
\hline \multicolumn{2}{|c|}{ IR Inten } & -- & 3.4697 & & & \multicolumn{2}{|c|}{3.1057} & & \multicolumn{2}{|c|}{29.3208} & \\
\hline Aton & AN & $\mathrm{X}$ & $\mathrm{Y}$ & Z & & $\mathrm{X}$ & $\mathrm{Y}$ & Z & X & $\mathrm{Y}$ & Z \\
\hline 1 & 13 & 0.58 & 0.11 & -0.10 & -0.21 & -0.04 & 0.04 & 0.01 & 0.35 & -0.31 & \\
\hline 2 & 13 & -0.47 & -0.34 & 0.31 & 0.17 & 0.12 & -0.11 & -0.10 & 0.11 & -0.10 & \\
\hline 3 & 20 & 0.02 & 0.01 & -0.02 & 0.01 & -0.02 & -0.01 & -0.02 & 0.00 & 0.01 & \\
\hline 4 & 13 & 0.21 & -0.04 & -0.03 & 0.58 & -0.11 & -0.10 & 0.01 & -0.36 & -0.32 & \\
\hline 5 & 13 & -0.17 & 0.12 & 0.11 & -0.47 & 0.34 & 0.31 & -0.10 & -0.11 & -0.10 & \\
\hline 6 & 13 & -0.01 & 0.21 & -0.19 & 0.00 & -0.08 & 0.07 & -0.03 & -0.11 & 0.10 & \\
\hline 7 & 14 & -0.12 & -0.01 & 0.01 & 0.04 & 0.01 & -0.01 & 0.13 & -0.33 & 0.29 & \\
\hline 8 & 14 & -0.04 & 0.00 & 0.00 & -0.12 & 0.01 & 0.01 & 0.13 & 0.34 & 0.30 & \\
\hline 9 & 13 & -0.01 & -0.08 & -0.07 & -0.01 & -0.21 & -0.19 & -0.03 & 0.11 & 0.10 & \\
\hline & & & 19 & & & 20 & & & & 21 & \\
\hline & & & A & & & & A & & & A & \\
\hline Freq & uencies & & 56.6183 & & & 415.111 & & & 422.53 & & \\
\hline Red. & masses & & 27.4175 & & & 27.599 & & & 28.17 & 746 & \\
\hline Frc $c$ & onsts & -- & 2.0544 & & & 2.802 & & & 2.96 & 637 & \\
\hline IR In & ten & -- & 13.4144 & & & 0.014 & & & 206.9 & 523 & \\
\hline Aton & $\mathrm{AN}$ & X & $\mathrm{Y}$ & Z & & $\mathrm{X}$ & $\mathrm{Y}$ & Z & X & $\mathrm{Y}$ & Z \\
\hline 1 & 13 & 0.01 & 0.36 & -0.32 & -0.07 & 0.02 & -0.02 & 0.07 & -0.02 & 0.02 & \\
\hline 2 & 13 & -0.10 & 0.11 & -0.10 & 0.07 & 0.04 & -0.04 & -0.07 & -0.04 & 0.03 & \\
\hline 3 & 20 & 0.00 & -0.01 & 0.00 & 0.00 & 0.02 & 0.00 & -0.21 & 0.00 & 0.02 & \\
\hline 4 & 13 & -0.01 & 0.35 & 0.31 & 0.07 & 0.02 & 0.02 & 0.07 & 0.02 & 0.02 & \\
\hline 5 & 13 & 0.09 & 0.11 & 0.10 & -0.07 & 0.04 & 0.04 & -0.07 & 0.04 & 0.03 & \\
\hline 6 & 13 & -0.03 & -0.12 & 0.10 & 0.41 & 0.07 & -0.06 & -0.38 & -0.06 & 0.06 & \\
\hline 7 & 14 & 0.13 & -0.34 & 0.30 & -0.52 & -0.14 & 0.13 & 0.53 & 0.13 & -0.12 & \\
\hline 8 & 14 & -0.13 & -0.33 & -0.29 & 0.52 & -0.14 & -0.13 & 0.53 & -0.13 & -0.12 & \\
\hline 9 & 13 & 0.03 & -0.11 & -0.10 & -0.41 & 0.07 & 0.06 & -0.39 & 0.06 & 0.06 & \\
\hline
\end{tabular}




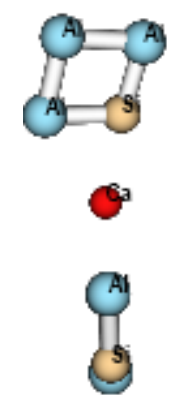

$\begin{array}{rrrrc}13 & 0 & 2.905512 & 1.642743 & -0.217090 \\ 13 & 0 & 5.354494 & 0.973131 & -0.180395 \\ 20 & 0 & 0.051892 & -0.111448 & -0.019210 \\ 13 & 0 & -2.884815 & -0.172658 & -1.021279 \\ 13 & 0 & -5.381995 & -0.164010 & -1.372381 \\ 13 & 0 & 5.049730 & -1.568625 & 0.120499 \\ 14 & 0 & 2.822193 & -0.768660 & 0.070871 \\ 14 & 0 & -5.009695 & 0.123996 & 0.951377 \\ 13 & 0 & -2.766988 & 0.155131 & 1.599316\end{array}$

State $=1-A \backslash H F=-2711.2989129$

A

A

A

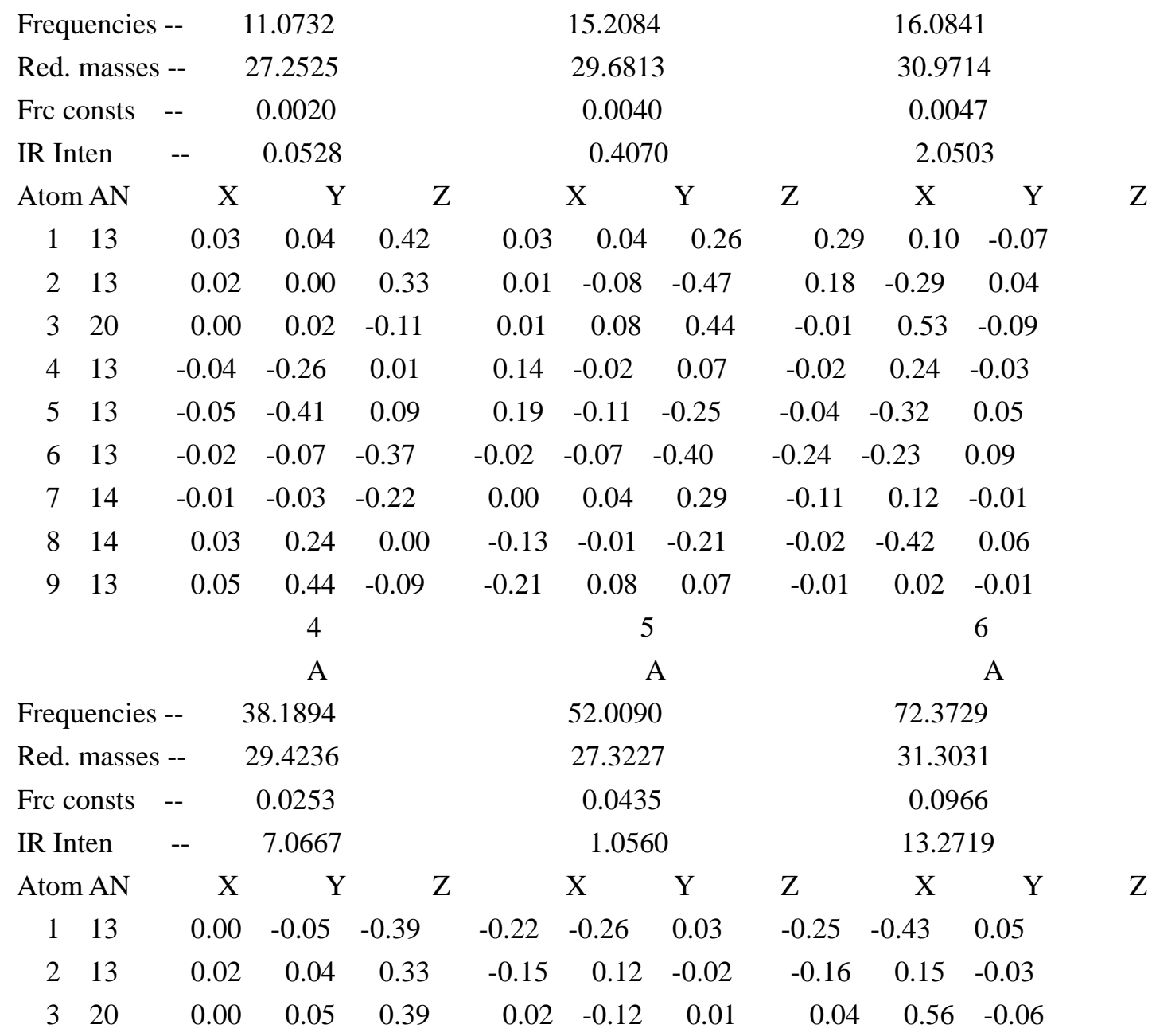




$\begin{array}{rrrrc}4 & 13 & 0.11 & 0.01 & 0.17 \\ 5 & 13 & 0.16 & -0.04 & -0.14 \\ 6 & 13 & 0.02 & 0.00 & 0.01 \\ 7 & 14 & 0.01 & -0.08 & -0.62 \\ 8 & 14 & -0.12 & 0.00 & -0.10 \\ 9 & 13 & -0.20 & 0.05 & 0.18 \\ & & & 7 & \end{array}$

A
Frequencies -- 91.3585

Red. masses -- $\quad 28.8122$

Frc consts -- $\quad 0.1417$

IR Inten --- 2.2730

$$
\begin{array}{ccc}
0.04 & 0.67 & -0.09 \\
0.03 & -0.25 & 0.03 \\
0.17 & 0.09 & 0.00 \\
0.03 & -0.28 & 0.04 \\
0.03 & -0.24 & 0.03 \\
0.04 & 0.35 & -0.05
\end{array}
$$

A

93.2971

27.9402

0.1433

1.8536

$$
\begin{array}{ccc}
0.07 & -0.30 & 0.03 \\
0.08 & 0.12 & -0.02 \\
0.12 & 0.15 & -0.01 \\
-0.08 & -0.44 & 0.05 \\
0.08 & 0.05 & -0.01 \\
0.07 & -0.11 & 0.01 \\
& \multicolumn{2}{c}{9}
\end{array}
$$

A

\begin{tabular}{cccccccccccc} 
Atom AN & \multicolumn{1}{c}{$\mathrm{X}$} & \multicolumn{2}{c}{$\mathrm{Y}$} & $\mathrm{Z}$ & \multicolumn{2}{c}{$\mathrm{X}$} & $\mathrm{Y}$ & $\mathrm{Z}$ & $\mathrm{X}$ & $\mathrm{Y}$ & $\mathrm{Z}$ \\
1 & 13 & 0.16 & -0.09 & -0.01 & 0.27 & -0.16 & 0.06 & -0.01 & 0.02 & -0.02 & \\
2 & 13 & 0.22 & 0.07 & 0.01 & 0.37 & 0.11 & -0.05 & -0.01 & -0.01 & 0.02 & \\
3 & 20 & 0.03 & 0.06 & 0.36 & 0.01 & 0.00 & -0.25 & 0.00 & -0.06 & 0.01 & \\
4 & 13 & -0.15 & -0.06 & -0.47 & -0.25 & 0.03 & 0.30 & 0.00 & -0.34 & 0.04 & \\
5 & 13 & -0.29 & 0.04 & 0.24 & -0.23 & 0.01 & -0.13 & 0.01 & 0.46 & -0.06 & \\
6 & 13 & 0.19 & 0.05 & -0.01 & 0.31 & 0.10 & 0.01 & -0.01 & -0.02 & -0.02 & \\
7 & 14 & 0.12 & -0.07 & -0.02 & 0.19 & -0.12 & 0.04 & 0.00 & 0.02 & 0.01 & \\
8 & 14 & -0.22 & 0.03 & 0.22 & -0.32 & -0.02 & -0.08 & -0.01 & -0.59 & 0.08 & \\
9 & 13 & -0.07 & -0.05 & -0.48 & -0.36 & 0.05 & 0.24 & 0.02 & 0.55 & -0.07 &
\end{tabular}

A

A

155.3903

28.8703

0.4107

31.7148
99.7750

27.3804

0.1606

1.9782

Frequencies -- 113.7506

Red. masses -- 27.2573

Frc consts -- $\quad 0.2078$

\begin{tabular}{|c|c|c|c|c|c|}
\hline & $\mathrm{y}$ & $\mathrm{Y}$ & Z & $X$ & $y$ \\
\hline-0.16 & 0.18 & -0.02 & 0.13 & -0.18 & 0.02 \\
\hline-0.37 & -0.18 & 0.03 & 0.37 & 0.20 & -0.03 \\
\hline 0.28 & 0.05 & -0.19 & -0.20 & -0.11 & -0.13 \\
\hline 0.18 & -0.01 & 0.02 & 0.27 & 0.00 & -0.05 \\
\hline 0.29 & 0.01 & 0.11 & 0.51 & 0.01 & 0.13 \\
\hline 0.19 & -0.17 & 0.01 & -0.30 & 0.18 & -0.02 \\
\hline 0.23 & 0.06 & 0.02 & -0.30 & -0.05 & 0.02 \\
\hline-0.47 & 0.05 & 0.26 & -0.23 & 0.03 & 0.23 \\
\hline-0.30 & -0.02 & -0.15 & -0.13 & 0.00 & -0.12 \\
\hline & 14 & & & & 15 \\
\hline
\end{tabular}

\begin{tabular}{lllr} 
IR Inten & -- & & 2.2355 \\
\hline & & X & Y
\end{tabular}

$\begin{array}{rrrcc}\text { Atom } & \text { AN } & \mathrm{X} & \mathrm{Y} & \mathrm{Z} \\ 1 & 13 & 0.00 & 0.07 & 0.50 \\ 2 & 13 & -0.03 & -0.06 & -0.46 \\ 3 & 20 & 0.00 & 0.00 & 0.01 \\ 4 & 13 & 0.01 & -0.02 & -0.01 \\ 5 & 13 & 0.02 & 0.01 & 0.00 \\ 6 & 13 & 0.01 & 0.05 & 0.50 \\ 7 & 14 & -0.01 & -0.05 & -0.52 \\ 8 & 14 & 0.00 & -0.02 & 0.01 \\ 9 & 13 & 0.00 & 0.02 & -0.01\end{array}$

A

Frequencies -- 238.3435

Red. masses -- 32.5759

Frc consts -- 1.0903

IR Inten -- 147.3331

A

166.2640

28.0769

0.4573

4.5990
A

253.9417

27.7382

1.0539

24.9327

\section{A}

272.2682

27.1591

1.1862

3.7102 


\begin{tabular}{|c|c|c|c|c|c|c|c|c|c|c|}
\hline \multicolumn{2}{|c|}{ Atom AN } & \multicolumn{2}{|l|}{$\mathrm{X}$} & \multicolumn{2}{|c|}{ Z } & $\mathrm{X}$ & Y & Z & $\mathrm{X}$ & $\mathrm{Y}$ \\
\hline 1 & 13 & -0.08 & -0.11 & 0.01 & 0.04 & 0.04 & -0.01 & 0.42 & 0.13 & -0.02 \\
\hline 2 & 13 & 0.12 & 0.15 & -0.02 & -0.04 & -0.05 & 0.01 & -0.29 & -0.45 & 0.06 \\
\hline 3 & 20 & 0.65 & -0.07 & 0.04 & -0.23 & 0.02 & 0.05 & 0.06 & -0.06 & 0.00 \\
\hline 4 & 13 & -0.03 & 0.04 & 0.29 & -0.22 & 0.08 & 0.60 & 0.02 & 0.00 & -0.01 \\
\hline 5 & 13 & -0.25 & -0.01 & -0.12 & 0.15 & -0.03 & -0.21 & -0.03 & 0.00 & 0.00 \\
\hline 6 & 13 & -0.50 & 0.09 & 0.00 & 0.15 & -0.01 & 0.00 & 0.02 & 0.64 & -0.07 \\
\hline 7 & 14 & -0.18 & -0.03 & -0.01 & 0.03 & 0.00 & 0.00 & -0.20 & -0.23 & 0.03 \\
\hline 8 & 14 & -0.09 & 0.00 & 0.01 & -0.03 & 0.02 & 0.12 & 0.00 & 0.00 & -0.01 \\
\hline \multirow[t]{3}{*}{9} & 13 & 0.05 & -0.03 & -0.22 & 0.27 & -0.08 & -0.59 & -0.01 & 0.00 & 0.01 \\
\hline & & & 16 & & \multicolumn{3}{|c|}{17} & & \multicolumn{2}{|r|}{18} \\
\hline & & & A & & \multicolumn{3}{|c|}{$\mathrm{A}$} & & \multicolumn{2}{|c|}{ A } \\
\hline \multicolumn{2}{|c|}{ Frequencies -- } & \multicolumn{2}{|c|}{331.0792} & & \multicolumn{3}{|c|}{331.7957} & & \multicolumn{2}{|c|}{356.2290} \\
\hline \multicolumn{2}{|c|}{ Red. masses -- } & & 27.1137 & & \multicolumn{3}{|c|}{27.2007} & & \multicolumn{2}{|c|}{27.4223} \\
\hline \multicolumn{2}{|c|}{ Frc consts } & -- & 1.7511 & & \multicolumn{3}{|c|}{1.7643} & & \multicolumn{2}{|c|}{2.0503} \\
\hline IR In & & -- & 10.0429 & & & 5.702 & & & 22.12 & 253 \\
\hline Atom & $\mathrm{AN}$ & X & $\mathrm{Y}$ & Z & & $\mathrm{X}$ & $\mathrm{Y}$ & Z & X & $\mathrm{Y}$ \\
\hline 1 & 13 & 0.45 & -0.10 & 0.00 & -0.43 & 0.11 & -0.01 & -0.03 & 0.67 & -0.08 \\
\hline 2 & 13 & -0.36 & 0.35 & -0.03 & 0.35 & -0.33 & 0.03 & 0.14 & 0.21 & -0.03 \\
\hline 3 & 20 & -0.09 & -0.02 & -0.03 & -0.12 & 0.02 & -0.03 & 0.02 & -0.01 & 0.00 \\
\hline 4 & 13 & 0.51 & 0.01 & 0.17 & 0.54 & 0.01 & 0.18 & -0.01 & 0.00 & 0.00 \\
\hline 5 & 13 & -0.33 & 0.02 & 0.09 & -0.34 & 0.02 & 0.10 & 0.01 & 0.00 & 0.00 \\
\hline 6 & 13 & 0.04 & -0.24 & 0.03 & 0.05 & 0.19 & -0.02 & 0.05 & -0.21 & 0.02 \\
\hline 7 & 14 & -0.11 & 0.01 & 0.00 & 0.07 & 0.00 & 0.00 & -0.18 & -0.63 & 0.08 \\
\hline 8 & 14 & 0.08 & -0.01 & -0.06 & 0.08 & -0.01 & -0.07 & 0.00 & 0.00 & 0.00 \\
\hline 9 & 13 & -0.15 & -0.02 & -0.15 & -0.16 & -0.02 & -0.16 & 0.01 & 0.00 & 0.01 \\
\hline & & & 19 & & & 20 & & & & 21 \\
\hline & & & A & & & & A & & & A \\
\hline Frequ & Iencies & & 75.0329 & & & 419.243 & & & 456.92 & \\
\hline Red. & masses & & 27.3466 & & & 27.913 & & & 27.54 & \\
\hline Frc C & onsts & -- & 2.2662 & & & 2.890 & & & 3.38 & \\
\hline IR In & & -- & 6.5384 & & & 107.408 & & & 4.87 & 718 \\
\hline Atom & $\mathrm{AN}$ & $\mathrm{X}$ & $\mathrm{Y}$ & Z & & $\mathrm{X}$ & $\mathrm{Y}$ & $\mathrm{Z}$ & $\mathrm{X}$ & $\mathrm{Y}$ \\
\hline 1 & 13 & 0.00 & 0.00 & 0.00 & -0.09 & -0.04 & 0.01 & 0.00 & 0.00 & 0.00 \\
\hline 2 & 13 & -0.01 & 0.01 & 0.00 & 0.10 & -0.07 & 0.01 & 0.00 & 0.00 & 0.00 \\
\hline 3 & 20 & 0.06 & 0.00 & 0.00 & 0.15 & -0.02 & 0.01 & 0.04 & 0.00 & -0.01 \\
\hline 4 & 13 & -0.25 & 0.02 & 0.15 & -0.03 & 0.00 & 0.00 & -0.12 & 0.00 & -0.04 \\
\hline 5 & 13 & 0.25 & 0.07 & 0.59 & 0.01 & 0.00 & 0.03 & 0.04 & -0.04 & -0.31 \\
\hline 6 & 13 & -0.04 & 0.01 & 0.00 & 0.56 & -0.12 & 0.00 & 0.02 & 0.00 & 0.00 \\
\hline 7 & 14 & 0.03 & -0.01 & 0.00 & -0.75 & 0.25 & -0.02 & -0.03 & 0.01 & 0.00 \\
\hline 8 & 14 & 0.26 & -0.07 & -0.50 & -0.02 & 0.00 & -0.04 & 0.58 & 0.04 & 0.45 \\
\hline 9 & 13 & -0.36 & -0.02 & -0.23 & 0.02 & 0.00 & 0.01 & -0.57 & 0.00 & -0.10 \\
\hline
\end{tabular}




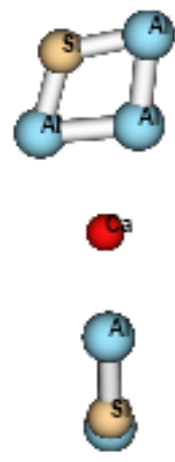

$\begin{array}{llrcc}13 & 0 & 5.440755 & -0.944372 & -0.984595 \\ 13 & 0 & 2.940517 & -0.692203 & -0.760903 \\ 13 & 0 & -5.440772 & 0.944234 & -0.984701 \\ 13 & 0 & -2.940529 & 0.692124 & -0.760974 \\ 20 & 0 & 0.000000 & 0.000018 & -0.087381 \\ 13 & 0 & 2.797936 & 1.177380 & 1.099509 \\ 13 & 0 & -2.797919 & -1.177244 & 1.099651 \\ 14 & 0 & 5.043727 & 0.714169 & 0.662238 \\ 14 & 0 & -5.043717 & -0.714119 & 0.662319\end{array}$

State $=1-A \backslash H F=-2711.2885911$

1

A
2

A

16.3826

31.0401

0.0049

2.6968

IR Inten -- 0.0037

Atom AN $\mathrm{X} \quad \mathrm{Y}$

$\begin{array}{llll}1 & 13 & 0.03 & -0.26\end{array}$

$\begin{array}{llll}2 & 13 & 0.02 & -0.17\end{array}$

$\begin{array}{llll}3 & 13 & -0.03 & 0.26\end{array}$

$\begin{array}{llll}4 & 13 & -0.02 & 0.17\end{array}$

$5 \quad 20$

$\begin{array}{lllll}6 & 13 & -0.04 & 0.31 & -0.32\end{array}$

$\begin{array}{llllll}7 & 13 & 0.04 & -0.31 & -0.32\end{array}$

$\begin{array}{lllll}8 & 14 & -0.02 & 0.18 & -0.12\end{array}$

$\begin{array}{llllll}9 & 14 & 0.02 & -0.18 & -0.12\end{array}$

4

A

Frequencies -- $\quad 54.2468$

Red. masses -- 27.9205

Frc consts -- 0.0484

IR Inten $\quad$-- 2.5277

Atom AN $\mathrm{X} \quad \mathrm{Y}$

$\begin{array}{lllll}1 & 13 & -0.09 & -0.19 & 0.08\end{array}$

$\begin{array}{lllll}2 & 13 & -0.06 & 0.45 & -0.27\end{array}$
$0.35 \quad-0.16$

0.17

0.35

0.17

0.09

$-0.12$

$-0.16$

$-0.12$

$-0.01$

0.18

0.18

0.11

0.10

$\mathrm{X}$

$\begin{array}{ll}0.16 & -0.06\end{array}$

$\begin{array}{ll}-0.34 & -0.04\end{array}$

$\begin{array}{ll}0.16 & 0.06\end{array}$

$\begin{array}{ll}0.54 & 0.00\end{array}$

$0.11 \quad 0.01$

$0.11-0.01$

$\begin{array}{ll}0.32 & 0.08\end{array}$

$-0.32 \quad-0.08$

5

A

54.4711

27.9165

0.0488

2.5397

$\mathrm{X}$

$\begin{array}{lll}0.10 & -0.08 & 0.19\end{array}$

$\begin{array}{lll}0.07 & 0.27 & -0.46\end{array}$
3

A

16.5605

31.0091

0.0050

2.8392

$\begin{array}{lllll}Z & X & Y & Z\end{array}$

$\begin{array}{lll}-0.14 & -0.02 & -0.26\end{array}$

$\begin{array}{lll}-0.10 & -0.11 & 0.21\end{array}$

$\begin{array}{lll}0.14 & 0.02 & -0.26\end{array}$

$\begin{array}{lll}0.11 & 0.11 & 0.21\end{array}$

$\begin{array}{lll}0.00 & 0.00 & 0.53\end{array}$

$\begin{array}{lll}0.19 & 0.09 & 0.03\end{array}$

$\begin{array}{lll}-0.19 & -0.09 & 0.03\end{array}$

$\begin{array}{lll}0.12 & 0.13 & -0.36\end{array}$

$\begin{array}{lll}-0.12 & -0.13 & -0.36\end{array}$

\section{6}

A

$$
91.0571
$$

27.5338

0.1345

0.0062

$\begin{array}{llllll}\mathrm{Z} & \mathrm{X} & \mathrm{Y} & \mathrm{Z}\end{array}$

$\begin{array}{lll}0.37 & 0.04 & 0.09\end{array}$

$\begin{array}{lll}0.28 & -0.07 & -0.14\end{array}$ 


\begin{tabular}{ccccccccccc}
3 & 13 & 0.09 & 0.19 & 0.08 & 0.10 & -0.08 & -0.19 & -0.37 & -0.04 & 0.09 \\
4 & 13 & 0.05 & -0.46 & -0.27 & 0.07 & 0.27 & 0.45 & -0.28 & 0.07 & -0.14 \\
5 & 20 & 0.00 & 0.00 & 0.26 & 0.01 & -0.26 & 0.00 & 0.00 & 0.00 & 0.15 \\
6 & 13 & 0.11 & 0.28 & -0.08 & -0.10 & 0.08 & -0.28 & 0.28 & -0.14 & -0.12 \\
7 & 13 & -0.11 & -0.28 & -0.09 & -0.10 & 0.08 & 0.28 & -0.28 & 0.14 & -0.12 \\
8 & 14 & 0.07 & -0.15 & 0.09 & -0.06 & -0.08 & 0.15 & 0.35 & 0.08 & 0.06 \\
9 & 14 & -0.06 & 0.15 & 0.09 & -0.06 & -0.08 & -0.15 & -0.35 & -0.08 & 0.06 \\
& & & 7 & & \multicolumn{7}{c}{8} & & & 9
\end{tabular}

A

\begin{tabular}{|c|c|c|c|}
\hline \multicolumn{2}{|c|}{ Frequencies -- } & \multicolumn{2}{|l|}{93.2268} \\
\hline \multicolumn{2}{|c|}{ Red. masses -- } & 29.4110 & \\
\hline Frc consts & -- & 0.1506 & \\
\hline IR Inten & -- & 1.3301 & \\
\hline Atom AN & $\mathrm{X}$ & $\mathrm{Y}$ & \\
\hline 113 & 0.09 & 0.22 & 0.04 \\
\hline 213 & 0.00 & -0.37 & -0.16 \\
\hline 313 & 0.09 & 0.22 & -0.04 \\
\hline $4 \quad 13$ & 0.00 & -0.37 & 0.16 \\
\hline $5 \quad 20$ & -0.03 & 0.42 & 0.00 \\
\hline $6 \quad 13$ & -0.08 & -0.20 & -0.32 \\
\hline 13 & -0.08 & -0.20 & 0.32 \\
\hline $8 \quad 14$ & 0.02 & 0.03 & 0.19 \\
\hline $9 \quad 14$ & 0.02 & 0.03 & -0.19 \\
\hline & & 10 & \\
\hline
\end{tabular}

A
A

93.9350

29.2873

0.1523

0.6143
A

99.0626

27.6679

0.1600

2.1115

Frequencies -- $\quad 100.1151$

156.7936

28.5987

0.4142

Z

$\mathrm{X} \quad \mathrm{Y}$

$\begin{array}{lll}-0.01 & 0.18 & -0.26\end{array}$

$\begin{array}{llllll}-0.13 & -0.16 & -0.34 & 0.01 & -0.07 & 0.22\end{array}$

$\begin{array}{lll}0.09 & -0.05 & 0.18\end{array}$

$\begin{array}{lll}-0.01 & 0.18 & 0.26\end{array}$

$\begin{array}{lll}0.13 & 0.16 & -0.34\end{array}$

$\begin{array}{lll}0.01 & -0.07 & -0.22\end{array}$

$\begin{array}{lll}0.00 & 0.00 & 0.41\end{array}$

$\begin{array}{lll}0.01 & -0.16 & 0.00\end{array}$

$\begin{array}{lll}-0.24 & -0.27 & -0.18\end{array}$

$\begin{array}{lll}0.02 & 0.35 & -0.20\end{array}$

$\begin{array}{lll}0.24 & 0.27 & -0.18\end{array}$

$\begin{array}{lll}0.02 & 0.35 & 0.20\end{array}$

$\begin{array}{lll}-0.17 & 0.14 & 0.03\end{array}$

$\begin{array}{lll}-0.02 & -0.32 & 0.26\end{array}$

$\begin{array}{lll}0.17 & -0.14 & 0.03\end{array}$

$\begin{array}{lll}-0.02 & -0.32 & -0.26\end{array}$

11

A

61.4376

12

A

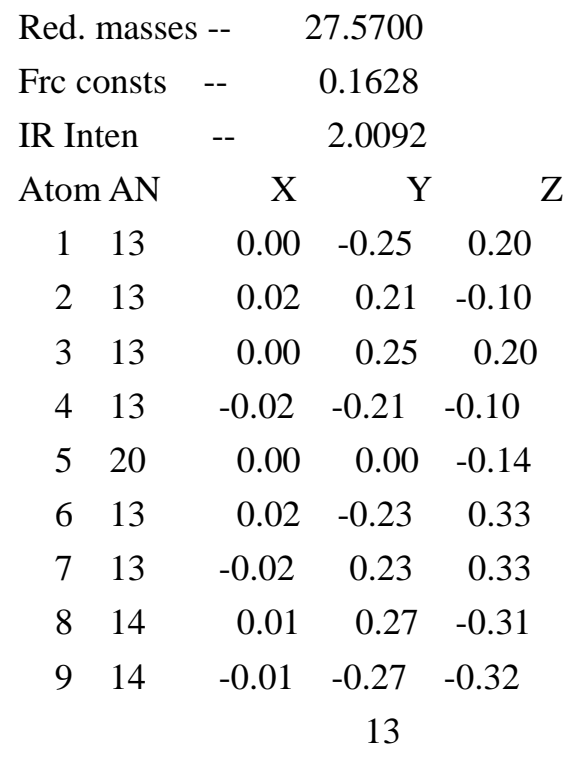

$\begin{array}{ccccc}\text { Atom AN } & \mathrm{X} & \mathrm{Y} & \mathrm{Z} \\ 1 & 13 & 0.00 & -0.25 & 0.20 \\ 2 & 13 & 0.02 & 0.21 & -0.10 \\ 3 & 13 & 0.00 & 0.25 & 0.20 \\ 4 & 13 & -0.02 & -0.21 & -0.10 \\ 5 & 20 & 0.00 & 0.00 & -0.14 \\ 6 & 13 & 0.02 & -0.23 & 0.33 \\ 7 & 13 & -0.02 & 0.23 & 0.33 \\ 8 & 14 & 0.01 & 0.27 & -0.31 \\ 9 & 14 & -0.01 & -0.27 & -0.32 \\ & & & 13 & \end{array}$

A

Frequencies -- $\quad 213.8279$

Red. masses -- 34.7297

Frc consts -- 0.9356

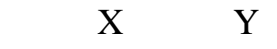

$\begin{array}{lll}0.32 & -0.07 & -0.07\end{array}$

$\begin{array}{lll}0.19 & -0.02 & 0.00\end{array}$

$\begin{array}{lll}0.32 & -0.07 & 0.07\end{array}$

$\begin{array}{lll}0.19 & -0.02 & 0.00\end{array}$

$\begin{array}{lll}0.19 & 0.23 & 0.00\end{array}$

$\begin{array}{lll}-0.25 & 0.09 & 0.11\end{array}$

$\begin{array}{lll}-0.25 & 0.09 & -0.11\end{array}$

$\begin{array}{lll}-0.40 & -0.17 & -0.18\end{array}$

$\begin{array}{lll}-0.40 & -0.17 & 0.18\end{array}$

14

A

253.0789

27.0466

1.0206
163.0521

27.9400

0.4377

5.3849

$\begin{array}{lllll}Z & X & Y & \text { Z }\end{array}$

$\begin{array}{lll}-0.42 & 0.08 & 0.08\end{array}$

$\begin{array}{lll}-0.23 & -0.03 & -0.01\end{array}$

$\begin{array}{lll}0.42 & -0.08 & 0.08\end{array}$

$\begin{array}{lll}0.23 & 0.03 & -0.01\end{array}$

$\begin{array}{lll}0.00 & 0.00 & -0.22\end{array}$

$\begin{array}{lll}0.20 & -0.11 & -0.09\end{array}$

$\begin{array}{lll}-0.20 & 0.11 & -0.09\end{array}$

$\begin{array}{lll}0.33 & 0.18 & 0.18\end{array}$

$\begin{array}{lll}-0.33 & -0.18 & 0.18\end{array}$

15

A

253.7068

27.3384

1.0368 


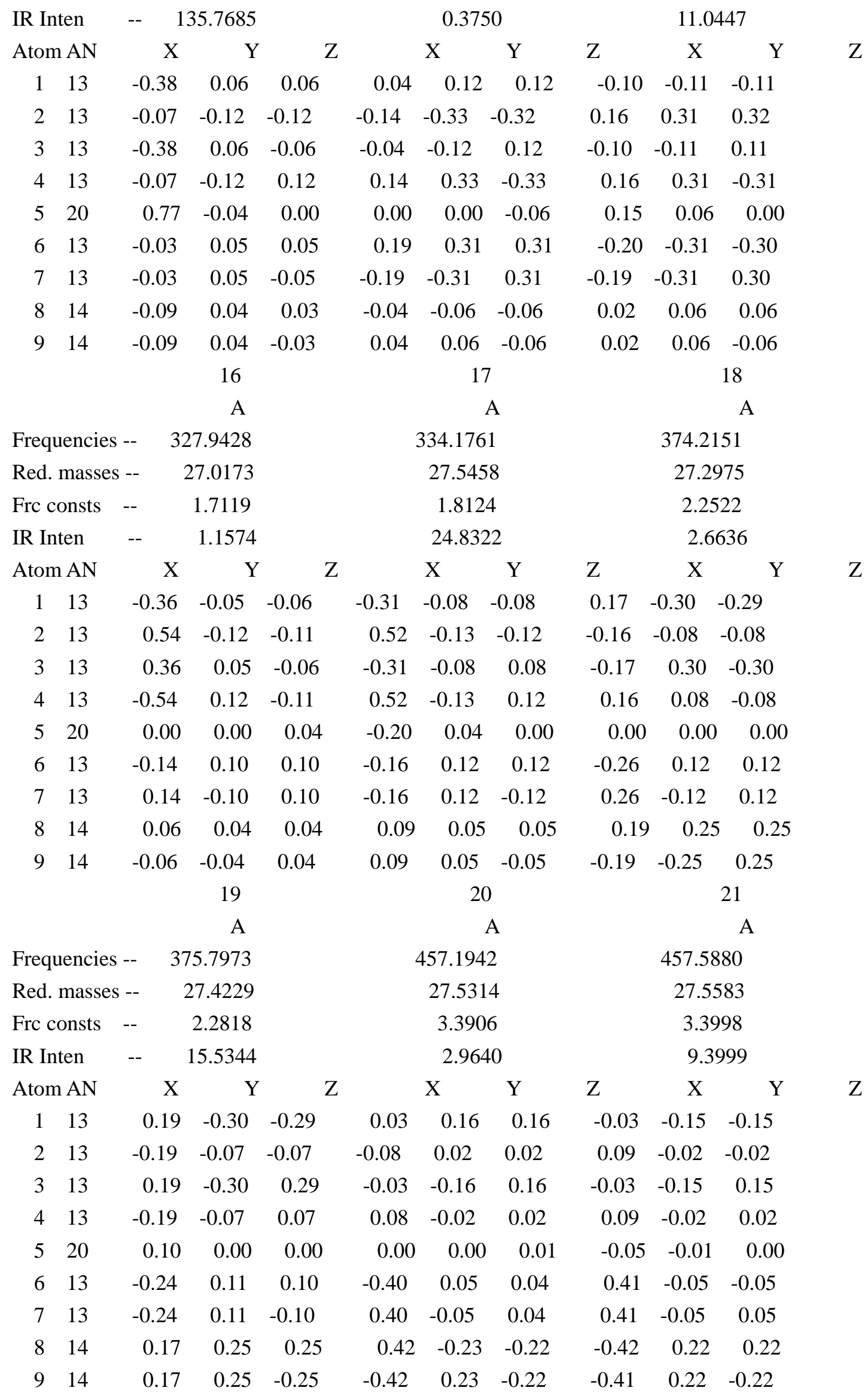

Al3SiCaAl3Si-s-s-0-C2h-Si-b36311+gx.frq-8.1 


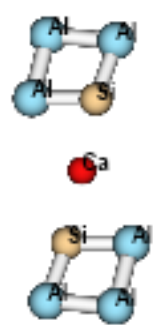

$\begin{array}{llrrc}13 & 0 & -0.310298 & 5.199945 & 0.000000 \\ 13 & 0 & 2.387242 & 2.403694 & 0.000000 \\ 13 & 0 & 2.256119 & 4.940724 & 0.000000 \\ 20 & 0 & 0.000000 & 0.000000 & 0.000000 \\ 13 & 0 & -2.387242 & -2.403694 & 0.000000 \\ 13 & 0 & -2.256119 & -4.940724 & 0.000000 \\ 13 & 0 & 0.310298 & -5.199945 & 0.000000 \\ 14 & 0 & 0.000000 & 2.852042 & 0.000000 \\ 14 & 0 & 0.000000 & -2.852042 & 0.000000\end{array}$

State=1-AGlHF=-2711.3084494

1

AU

\begin{tabular}{|c|c|c|c|}
\hline Frequencies & & 8.0883 & \\
\hline Red. masses & -- & 27.1462 & \\
\hline Frc consts & -- & 0.0010 & \\
\hline IR Inten & -- & 0.1994 & \\
\hline Atom AN & $\mathrm{X}$ & $\mathrm{Y}$ & Z \\
\hline 113 & 0.00 & 0.00 & 0.49 \\
\hline 213 & 0.00 & 0.00 & -0.44 \\
\hline 313 & 0.00 & 0.00 & -0.17 \\
\hline $4 \quad 20$ & 0.00 & 0.00 & -0.09 \\
\hline $\begin{array}{ll}5 & 13\end{array}$ & 0.00 & 0.00 & -0.44 \\
\hline $\begin{array}{ll}6 & 13\end{array}$ & 0.00 & 0.00 & -0.17 \\
\hline $\begin{array}{ll}7 & 13\end{array}$ & 0.00 & 0.00 & 0.49 \\
\hline $8 \quad 14$ & 0.00 & 0.00 & 0.18 \\
\hline $\begin{array}{ll}9 & 14\end{array}$ & 0.00 & 0.00 & 0.18 \\
\hline & & 4 & \\
\hline
\end{tabular}

BG

$$
2
$$

AU

13.1453

30.2668

0.0031

0.2555

$\mathrm{X}$

0.00

0.00

0.00

0.00

0.00

0.00

$\begin{array}{lll}0.00 & 0.51\end{array}$

$\begin{array}{lll}0.00 & 0.00 & 0.19\end{array}$

0.00

0.00

$0.00 \quad-0.29$

$0.00 \quad-0.29$

5

AU

48.9713

32.8015

0.0463

19.5761

IR Inten -- $\quad 0.0000$

$\mathrm{X}$

$\begin{array}{rrr}\mathrm{X} & \mathrm{Y} & \mathrm{Z} \\ 0.00 & 0.00 & -0.10 \\ 0.00 & 0.00 & 0.32 \\ 0.00 & 0.00 & -0.35 \\ 0.00 & 0.00 & 0.00 \\ 0.00 & 0.00 & -0.32\end{array}$

0.00

$\mathrm{Y}$

$\mathrm{Z}$

$\begin{array}{lll}-0.13 & -0.12 & 0.00\end{array}$

113

0.00

$\begin{array}{ll}0.00 & 0.03\end{array}$

213

0.00

$0.00 \quad-0.30$

$\begin{array}{lll}0.44 & 0.24 & 0.00\end{array}$

$3 \quad 13$

$\begin{array}{ll}0.00 & 0.20\end{array}$

$\begin{array}{lll}-0.07 & 0.25 & 0.00\end{array}$

$\begin{array}{rrrrr}4 & 20 & 0.00 & 0.00 & 0.00 \\ 5 & 13 & 0.00 & 0.00 & -0.32\end{array}$

$\begin{array}{lll}0.00 & 0.00 & 0.65\end{array}$

$\begin{array}{lll}0.00 & 0.00 & 0.00\end{array}$

$\begin{array}{llll}0.00 & 0.00 & -0.30\end{array}$

$\begin{array}{lll}-0.44 & -0.24 & 0.00\end{array}$ 


\begin{tabular}{ccccccccccc}
6 & 13 & 0.00 & 0.00 & 0.35 & 0.00 & 0.00 & 0.20 & 0.07 & -0.25 & 0.00 \\
7 & 13 & 0.00 & 0.00 & 0.10 & 0.00 & 0.00 & 0.03 & 0.13 & 0.12 & 0.00 \\
8 & 14 & 0.00 & 0.00 & 0.51 & 0.00 & 0.00 & -0.40 & 0.39 & -0.06 & 0.00 \\
9 & 14 & 0.00 & 0.00 & -0.51 & 0.00 & 0.00 & -0.40 & -0.39 & 0.06 & 0.00 \\
& & & 7 & & \multicolumn{3}{c}{8} & & & \multicolumn{2}{c}{9}
\end{tabular}

BU AG

AU

\begin{tabular}{|c|c|c|c|c|c|c|c|c|c|}
\hline \multicolumn{2}{|c|}{ Frequencies -- } & 67.1590 & & \multicolumn{3}{|c|}{94.2443} & \multicolumn{3}{|c|}{114.2987} \\
\hline Red. masses - & & 31.3770 & & \multicolumn{3}{|c|}{27.1055} & \multicolumn{3}{|c|}{27.2622} \\
\hline Frc consts - & -- & \multicolumn{2}{|l|}{0.0834} & \multicolumn{3}{|c|}{0.1418} & \multicolumn{3}{|c|}{0.2098} \\
\hline IR Inten & -- & \multicolumn{2}{|l|}{29.2711} & \multicolumn{3}{|c|}{0.0000} & \multicolumn{3}{|c|}{6.2260} \\
\hline Atom AN & $\mathrm{X}$ & $\mathrm{Y}$ & Z & & $\mathrm{X}$ & $\mathrm{Y}$ & $\mathrm{Z}$ & $\mathrm{X}$ & $\mathrm{Y}$ \\
\hline $1 \quad 13$ & 0.18 & 0.12 & 0.00 & 0.19 & 0.31 & 0.00 & 0.00 & 0.00 & 0.36 \\
\hline 13 & -0.37 & -0.06 & 0.00 & -0.10 & 0.33 & 0.00 & 0.00 & 0.00 & 0.35 \\
\hline 313 & 0.13 & -0.09 & 0.00 & 0.21 & 0.37 & 0.00 & 0.00 & 0.00 & -0.33 \\
\hline 20 & 0.56 & -0.06 & 0.00 & 0.00 & 0.00 & 0.00 & 0.00 & 0.00 & 0.01 \\
\hline 13 & -0.37 & -0.06 & 0.00 & 0.10 & -0.33 & 0.00 & 0.00 & 0.00 & 0.35 \\
\hline $6 \quad 13$ & 0.13 & -0.09 & 0.00 & -0.21 & -0.37 & 0.00 & 0.00 & 0.00 & -0.33 \\
\hline 13 & 0.18 & 0.12 & 0.00 & -0.19 & -0.31 & 0.00 & 0.00 & 0.00 & 0.36 \\
\hline 14 & -0.35 & 0.06 & 0.00 & -0.08 & 0.24 & 0.00 & 0.00 & 0.00 & -0.37 \\
\hline \multirow[t]{3}{*}{14} & -0.35 & 0.06 & 0.00 & 0.08 & -0.24 & 0.00 & 0.00 & 0.00 & -0.37 \\
\hline & \multicolumn{3}{|c|}{10} & \multicolumn{3}{|c|}{11} & \multicolumn{3}{|c|}{12} \\
\hline & \multicolumn{3}{|c|}{ BG } & \multicolumn{3}{|c|}{$\mathrm{BU}$} & \multicolumn{3}{|c|}{ AG } \\
\hline Frequencies -- & \multicolumn{2}{|c|}{114.4736} & & \multicolumn{3}{|c|}{151.0362} & \multicolumn{3}{|c|}{181.5423} \\
\hline Red. masses -- & \multicolumn{2}{|c|}{27.2485} & & & 29.056 & & & 27.23 & \\
\hline Frc consts - & -- & 0.2104 & & & 0.390 & & & 0.528 & \\
\hline IR Inten & -- & 0.0000 & & & 10.490 & & & 0.00 & \\
\hline Atom AN & $\mathrm{X}$ & $\mathrm{Y}$ & Z & & $\mathrm{X}$ & $\mathrm{Y}$ & $\mathrm{Z}$ & $\mathrm{X}$ & $\mathrm{Y}$ \\
\hline 113 & 0.00 & 0.00 & -0.36 & 0.15 & -0.18 & 0.00 & -0.09 & 0.45 & 0.00 \\
\hline 13 & 0.00 & 0.00 & -0.36 & -0.15 & 0.24 & 0.00 & 0.18 & -0.10 & 0.00 \\
\hline 13 & 0.00 & 0.00 & 0.33 & 0.30 & 0.38 & 0.00 & -0.26 & -0.24 & 0.00 \\
\hline 20 & 0.00 & 0.00 & 0.00 & -0.24 & -0.31 & 0.00 & 0.00 & 0.00 & 0.00 \\
\hline 13 & 0.00 & 0.00 & 0.36 & -0.15 & 0.24 & 0.00 & -0.18 & 0.10 & 0.00 \\
\hline 13 & 0.00 & 0.00 & -0.33 & 0.30 & 0.38 & 0.00 & 0.26 & 0.24 & 0.00 \\
\hline 13 & 0.00 & 0.00 & 0.36 & 0.15 & -0.18 & 0.00 & 0.09 & -0.45 & 0.00 \\
\hline 14 & 0.00 & 0.00 & 0.37 & -0.12 & -0.20 & 0.00 & 0.14 & 0.33 & 0.00 \\
\hline 14 & 0.00 & 0.00 & -0.37 & -0.12 & -0.20 & 0.00 & -0.14 & -0.33 & 0.00 \\
\hline & & 13 & & & 14 & & & & 15 \\
\hline & & $\mathrm{BU}$ & & & & & & & $\mathrm{BU}$ \\
\hline Frequencies - & & 54.6175 & & & 270.689 & & & 273.09 & \\
\hline Red. masses - & & 32.8073 & & & 27.073 & & & 27.73 & \\
\hline Frc consts - & -- & 1.2531 & & & 1.168 & & & 1.218 & \\
\hline IR Inten & -- & 167.7000 & & & 0.000 & & & 26.61 & \\
\hline Atom AN & $\mathrm{X}$ & $\mathrm{Y}$ & Z & & $\mathrm{X}$ & $\mathrm{Y}$ & $\mathrm{Z}$ & $X$ & $\mathrm{Y}$ \\
\hline 113 & -0.13 & -0.41 & 0.00 & 0.45 & -0.06 & 0.00 & 0.44 & -0.18 & 0.00 \\
\hline
\end{tabular}




\begin{tabular}{|c|c|c|c|c|c|c|c|c|c|c|c|}
\hline 2 & 13 & -0.14 & -0.12 & 0.00 & 0.15 & 0.27 & 0.00 & 0.12 & 0.25 & 0.00 & \\
\hline 3 & 13 & 0.22 & 0.10 & 0.00 & -0.36 & -0.14 & 0.00 & -0.33 & -0.12 & 0.00 & \\
\hline 4 & 20 & 0.07 & 0.67 & 0.00 & 0.00 & 0.00 & 0.00 & -0.05 & 0.22 & 0.00 & \\
\hline 5 & 13 & -0.14 & -0.12 & 0.00 & -0.15 & -0.27 & 0.00 & 0.12 & 0.25 & 0.00 & \\
\hline 6 & 13 & 0.22 & 0.10 & 0.00 & 0.36 & 0.14 & 0.00 & -0.33 & -0.12 & 0.00 & \\
\hline 7 & 13 & -0.13 & -0.41 & 0.00 & -0.45 & 0.06 & 0.00 & 0.44 & -0.18 & 0.00 & \\
\hline 8 & 14 & 0.00 & -0.07 & 0.00 & -0.19 & -0.10 & 0.00 & -0.20 & -0.11 & 0.00 & \\
\hline 9 & 14 & 0.00 & -0.07 & 0.00 & 0.19 & 0.10 & 0.00 & -0.20 & -0.11 & 0.00 & \\
\hline & & & 16 & & \multicolumn{3}{|c|}{17} & \multicolumn{4}{|c|}{18} \\
\hline & & & AG & & \multicolumn{3}{|c|}{$\mathrm{BU}$} & \multicolumn{4}{|c|}{$\mathrm{BU}$} \\
\hline \multicolumn{2}{|c|}{ Frequencies -- } & \multicolumn{2}{|c|}{330.2970} & & \multicolumn{3}{|c|}{330.4834} & \multicolumn{4}{|c|}{356.3280} \\
\hline \multicolumn{2}{|c|}{ Red. masses } & & 26.9968 & & \multicolumn{3}{|c|}{27.0267} & \multicolumn{4}{|c|}{27.4325} \\
\hline \multicolumn{2}{|c|}{ Frc consts } & -- & 1.7353 & & \multicolumn{3}{|c|}{1.7392} & \multicolumn{4}{|c|}{2.0522} \\
\hline \multicolumn{2}{|c|}{ IR Inten } & -- & 0.0000 & & \multicolumn{3}{|c|}{6.1885} & \multicolumn{4}{|c|}{45.9332} \\
\hline \multicolumn{2}{|c|}{ Atom AN } & $\mathrm{X}$ & $\mathrm{Y}$ & Z & \multicolumn{2}{|r|}{$\mathrm{X}$} & $\mathrm{Y}$ & Z & $\mathrm{X}$ & $\mathrm{Y}$ & $\mathrm{Z}$ \\
\hline 1 & 13 & -0.22 & 0.05 & 0.00 & -0.21 & 0.03 & 0.00 & -0.14 & 0.06 & 0.00 & \\
\hline 2 & 13 & -0.01 & 0.44 & 0.00 & -0.01 & 0.45 & 0.00 & 0.46 & -0.11 & 0.00 & \\
\hline 3 & 13 & 0.26 & -0.42 & 0.00 & 0.26 & -0.42 & 0.00 & 0.17 & 0.06 & 0.00 & \\
\hline 4 & 20 & 0.00 & 0.00 & 0.00 & -0.03 & 0.04 & 0.00 & -0.01 & 0.03 & 0.00 & \\
\hline 5 & 13 & 0.01 & -0.44 & 0.00 & -0.01 & 0.45 & 0.00 & 0.46 & -0.11 & 0.00 & \\
\hline 6 & 13 & -0.26 & 0.42 & 0.00 & 0.26 & -0.42 & 0.00 & 0.17 & 0.06 & 0.00 & \\
\hline 7 & 13 & 0.22 & -0.05 & 0.00 & -0.21 & 0.03 & 0.00 & -0.14 & 0.06 & 0.00 & \\
\hline 8 & 14 & -0.01 & -0.09 & 0.00 & -0.01 & -0.08 & 0.00 & -0.47 & -0.02 & 0.00 & \\
\hline 9 & 14 & 0.01 & 0.09 & 0.00 & -0.01 & -0.08 & 0.00 & -0.47 & -0.02 & 0.00 & \\
\hline & & & 19 & & & 20 & & & & 21 & \\
\hline & & & AG & & & $\mathrm{AC}$ & & & & $\mathrm{BU}$ & \\
\hline Freql & lencies - & & 7.3315 & & & 414.0790 & & & 422.115 & & \\
\hline Red. & masses & & 27.4216 & & & 27.5964 & & & 28.20 & & \\
\hline Frc c & onsts - & -- & 2.0629 & & & 2.7878 & & & 2.961 & & \\
\hline IR In & & -- & 0.0000 & & & 0.0000 & & & 206.36 & & \\
\hline Atom & $\mathrm{AN}$ & $X$ & $\mathrm{Y}$ & Z & & X & $\mathrm{Y}$ & Z & X & $\mathrm{Y}$ & $\mathrm{Z}$ \\
\hline 1 & 13 & -0.14 & 0.06 & 0.00 & 0.00 & -0.42 & 0.00 & 0.00 & 0.39 & 0.00 & \\
\hline 2 & 13 & 0.46 & -0.12 & 0.00 & 0.04 & 0.06 & 0.00 & -0.04 & -0.06 & 0.00 & \\
\hline 3 & 13 & 0.17 & 0.06 & 0.00 & 0.03 & -0.08 & 0.00 & -0.03 & 0.08 & 0.00 & \\
\hline 4 & 20 & 0.00 & 0.00 & 0.00 & 0.00 & 0.00 & 0.00 & 0.02 & 0.22 & 0.00 & \\
\hline 5 & 13 & -0.46 & 0.12 & 0.00 & -0.04 & -0.06 & 0.00 & -0.04 & -0.06 & 0.00 & \\
\hline 6 & 13 & -0.17 & -0.06 & 0.00 & -0.03 & 0.08 & 0.00 & -0.03 & 0.08 & 0.00 & \\
\hline 7 & 13 & 0.14 & -0.06 & 0.00 & 0.00 & 0.42 & 0.00 & 0.00 & 0.39 & 0.00 & \\
\hline 8 & 14 & -0.47 & -0.03 & 0.00 & -0.06 & 0.55 & 0.00 & 0.06 & -0.56 & 0.00 & \\
\hline 9 & 14 & 0.47 & 0.03 & 0.00 & 0.06 & -0.55 & 0.00 & 0.06 & -0.56 & 0.00 & \\
\hline
\end{tabular}




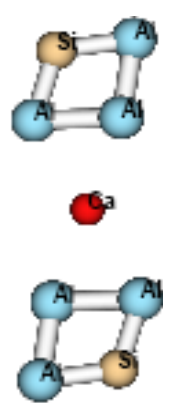

$\begin{array}{lcrcc}20 & 0 & 0.000000 & 0.000000 & 0.000000 \\ 13 & 0 & 0.000000 & 3.097443 & 0.000000 \\ 13 & 0 & 0.000000 & -3.097443 & 0.000000 \\ 13 & 0 & 2.457451 & 2.149484 & 0.000000 \\ 13 & 0 & -2.457451 & -2.149484 & 0.000000 \\ 13 & 0 & -0.445775 & -5.581312 & 0.000000 \\ 14 & 0 & -2.549509 & -4.483520 & 0.000000 \\ 13 & 0 & 0.445775 & 5.581312 & 0.000000 \\ 14 & 0 & 2.549509 & 4.483520 & 0.000000\end{array}$

State $=1-A G \backslash H F=-2711.2878676$

AU

$\mathrm{AU}$

BU

\begin{tabular}{|c|c|c|c|}
\hline \multicolumn{2}{|c|}{ Frequencies -- } & \multicolumn{2}{|c|}{-8.1859} \\
\hline Red. masses & & 7.1414 & \\
\hline Frc consts & -- & 0.0011 & \\
\hline IR Inten & -- & 0.1481 & \\
\hline Atom AN & $\mathrm{X}$ & $\mathrm{Y}$ & \\
\hline 120 & 0.00 & 0.00 & 0.04 \\
\hline 213 & 0.00 & 0.00 & 0.31 \\
\hline 313 & 0.00 & 0.00 & 0.31 \\
\hline $4 \quad 13$ & 0.00 & 0.00 & -0.44 \\
\hline $5 \quad 13$ & 0.00 & 0.00 & -0.44 \\
\hline $6 \quad 13$ & 0.00 & 0.00 & 0.38 \\
\hline $\begin{array}{ll}7 & 14\end{array}$ & 0.00 & 0.00 & -0.27 \\
\hline $8 \quad 13$ & 0.00 & 0.00 & 0.38 \\
\hline $9 \quad 14$ & 0.00 & 0.00 & -0.27 \\
\hline & & 4 & \\
\hline
\end{tabular}

BG

Frequencies -- $\quad 48.2777$

17.6191

19.3031

31.3402

30.8809

0.0057

0.0068

$\mathrm{X} Y .1251$

1.9989

$\begin{array}{lll}0.00 & 0.00 & 0.56\end{array}$

$\begin{array}{llll}\mathrm{Z} & \mathrm{X} & \mathrm{Y}\end{array}$

Z

Red. masses -- 27.0985

$\begin{array}{llllll}0.00 & 0.00 & 0.23 & 0.02 & -0.19 & 0.00\end{array}$

$\begin{array}{lll}0.00 & 0.00 & 0.23\end{array}$

$\begin{array}{lll}0.02 & -0.19 & 0.00\end{array}$

$\begin{array}{lll}0.00 & 0.00 & 0.11\end{array}$

$\begin{array}{lll}0.18 & 0.21 & 0.00\end{array}$

$\begin{array}{lll}0.00 & 0.00 & 0.11\end{array}$

$\begin{array}{lll}0.18 & 0.21 & 0.00\end{array}$

$\begin{array}{lll}0.00 & 0.00 & -0.37\end{array}$

$\begin{array}{lll}-0.38 & -0.12 & 0.00\end{array}$

$\begin{array}{lll}0.00 & 0.00 & -0.38\end{array}$

$\begin{array}{lll}-0.19 & 0.23 & 0.00\end{array}$

$\begin{array}{lll}0.00 & 0.00 & -0.37\end{array}$

$\begin{array}{lll}-0.38 & -0.12 & 0.00\end{array}$

$\begin{array}{llll}0.00 & 0.00 & -0.38\end{array}$

5

$\mathrm{AU}$

$\begin{array}{lll}-0.19 & 0.23 & 0.00\end{array}$

6

AG

$\begin{array}{ll}71.9303 & 78.2686\end{array}$

$31.4544 \quad 27.0333$

$0.0959 \quad 0.0976$

$\begin{array}{lll}\text { Frc consts } & -- & 0.0372 \\ \text { IR Inten } & - & 0.0000\end{array}$

9.6472

\begin{tabular}{ccccccccccc} 
Atom AN & $\mathrm{X}$ & $\mathrm{Y}$ & $\mathrm{Z}$ & \multicolumn{2}{c}{$\mathrm{X}$} & \multicolumn{1}{l}{$\mathrm{Y}$} & $\mathrm{Z}$ & $\mathrm{X}$ & $\mathrm{Y}$ \\
1 & 20 & 0.00 & 0.00 & 0.00 & 0.00 & 0.00 & 0.59 & 0.00 & 0.00 & 0.00
\end{tabular}

$\begin{array}{lllllllllll}2 & 13 & 0.00 & 0.00 & -0.55 & 0.00 & 0.00 & -0.48 & 0.39 & -0.19 & 0.00\end{array}$

$\begin{array}{lllllllllll}3 & 13 & 0.00 & 0.00 & 0.55 & 0.00 & 0.00 & -0.48 & -0.39 & 0.19 & 0.00\end{array}$ 


\begin{tabular}{|c|c|c|c|c|c|c|c|c|c|c|}
\hline 4 & 13 & 0.00 & 0.00 & -0.30 & 0.00 & 0.00 & -0.23 & 0.47 & 0.06 & 0.00 \\
\hline 5 & 13 & 0.00 & 0.00 & 0.30 & 0.00 & 0.00 & -0.23 & -0.47 & -0.06 & 0.00 \\
\hline 6 & 13 & 0.00 & 0.00 & -0.22 & 0.00 & 0.00 & 0.16 & 0.23 & 0.10 & 0.00 \\
\hline 7 & 14 & 0.00 & 0.00 & -0.24 & 0.00 & 0.00 & 0.11 & 0.10 & -0.13 & 0.00 \\
\hline 8 & 13 & 0.00 & 0.00 & 0.22 & 0.00 & 0.00 & 0.16 & -0.23 & -0.10 & 0.00 \\
\hline 9 & 14 & 0.00 & 0.00 & 0.24 & 0.00 & 0.00 & 0.11 & -0.10 & 0.13 & 0.00 \\
\hline & & & 7 & & \multicolumn{4}{|c|}{8} & \multicolumn{2}{|r|}{9} \\
\hline & & & AG & & \multicolumn{4}{|c|}{$\mathrm{BU}$} & \multicolumn{2}{|r|}{$\mathrm{AU}$} \\
\hline Frequ & iencies - & & 91.4528 & & \multicolumn{3}{|c|}{97.8832} & & \multicolumn{2}{|c|}{100.7687} \\
\hline Red. & masses & & 27.2885 & & \multicolumn{3}{|c|}{30.1449} & & \multicolumn{2}{|c|}{ 27.3971 } \\
\hline Frc c & onsts - & -- & 0.1345 & & \multicolumn{3}{|c|}{0.1702} & & \multicolumn{2}{|c|}{0.1639} \\
\hline IR In & & -- & 0.0000 & & \multicolumn{3}{|c|}{2.2183} & & \multicolumn{2}{|c|}{4.8358} \\
\hline \multicolumn{2}{|c|}{ Atom AN } & $X$ & $\mathrm{Y}$ & Z & \multicolumn{2}{|r|}{$\mathrm{X}$} & \multicolumn{2}{|l|}{ Y } & \multicolumn{2}{|c|}{ X } \\
\hline 1 & 20 & 0.00 & 0.00 & 0.00 & 0.45 & -0.19 & 0.00 & 0.00 & 0.00 & -0.07 \\
\hline 2 & 13 & -0.09 & -0.28 & 0.00 & -0.38 & 0.08 & 0.00 & 0.00 & 0.00 & -0.24 \\
\hline 3 & 13 & 0.09 & 0.28 & 0.00 & -0.38 & 0.08 & 0.00 & 0.00 & 0.00 & -0.24 \\
\hline 4 & 13 & -0.07 & -0.35 & 0.00 & -0.37 & 0.08 & 0.00 & 0.00 & 0.00 & 0.39 \\
\hline 5 & 13 & 0.07 & 0.35 & 0.00 & -0.37 & 0.08 & 0.00 & 0.00 & 0.00 & 0.39 \\
\hline 6 & 13 & 0.13 & 0.34 & 0.00 & 0.21 & -0.02 & 0.00 & 0.00 & 0.00 & 0.34 \\
\hline 7 & 14 & 0.16 & 0.36 & 0.00 & 0.20 & 0.00 & 0.00 & 0.00 & 0.00 & -0.42 \\
\hline 8 & 13 & -0.13 & -0.34 & 0.00 & 0.21 & -0.02 & 0.00 & 0.00 & 0.00 & 0.34 \\
\hline \multirow[t]{3}{*}{9} & 14 & -0.16 & -0.36 & 0.00 & 0.20 & 0.00 & 0.00 & 0.00 & 0.00 & -0.42 \\
\hline & & & 10 & & \multicolumn{3}{|c|}{11} & & \multicolumn{2}{|c|}{12} \\
\hline & & & BG & & \multicolumn{3}{|c|}{ AG } & & \multicolumn{2}{|c|}{$\mathrm{BU}$} \\
\hline Freql & encies - & & 1.2617 & & & 158.3253 & & & 162.39 & \\
\hline Red. & masses & -- & 27.3199 & & & 27.3323 & & & 29.32 & \\
\hline Frc c & onsts & -- & 0.1651 & & & 0.4037 & & & 0.45 & \\
\hline IR In & & -- & 0.0000 & & & 0.0000 & & & 70.01 & \\
\hline Aton & AN & $X$ & $\mathrm{Y}$ & Z & & $\mathrm{X}$ & $\mathrm{Y}$ & Z & $\mathrm{X}$ & $\mathrm{Y}$ \\
\hline 1 & 20 & 0.00 & 0.00 & 0.00 & 0.00 & 0.00 & 0.00 & -0.37 & -0.10 & 0.00 \\
\hline 2 & 13 & 0.00 & 0.00 & 0.26 & 0.14 & 0.22 & 0.00 & -0.03 & -0.18 & 0.00 \\
\hline 3 & 13 & 0.00 & 0.00 & -0.26 & -0.14 & -0.22 & 0.00 & -0.03 & -0.18 & 0.00 \\
\hline 4 & 13 & 0.00 & 0.00 & -0.38 & 0.08 & -0.24 & 0.00 & -0.05 & 0.27 & 0.00 \\
\hline 5 & 13 & 0.00 & 0.00 & 0.38 & -0.08 & 0.24 & 0.00 & -0.05 & 0.27 & 0.00 \\
\hline 6 & 13 & 0.00 & 0.00 & 0.34 & 0.00 & -0.44 & 0.00 & -0.01 & -0.33 & 0.00 \\
\hline 7 & 14 & 0.00 & 0.00 & -0.41 & 0.35 & 0.23 & 0.00 & 0.35 & 0.30 & 0.00 \\
\hline 8 & 13 & 0.00 & 0.00 & -0.34 & 0.00 & 0.44 & 0.00 & -0.01 & -0.33 & 0.00 \\
\hline 9 & 14 & 0.00 & 0.00 & 0.41 & -0.35 & -0.23 & 0.00 & 0.35 & 0.30 & 0.00 \\
\hline & & & 13 & & & 14 & & & & 5 \\
\hline & & & $\mathrm{BU}$ & & & B & & & & AG \\
\hline Freql & encies - & & 13.5692 & & & 255.7553 & & & 255.92 & \\
\hline Red. & masses & -- & 34.8132 & & & 27.2776 & & & 27.00 & \\
\hline Frc c & onsts & -- & 0.9356 & & & 1.0512 & & & 1.04 & \\
\hline IR In & & -- & 31.0200 & & & 6.856 & & & 0.00 & \\
\hline
\end{tabular}




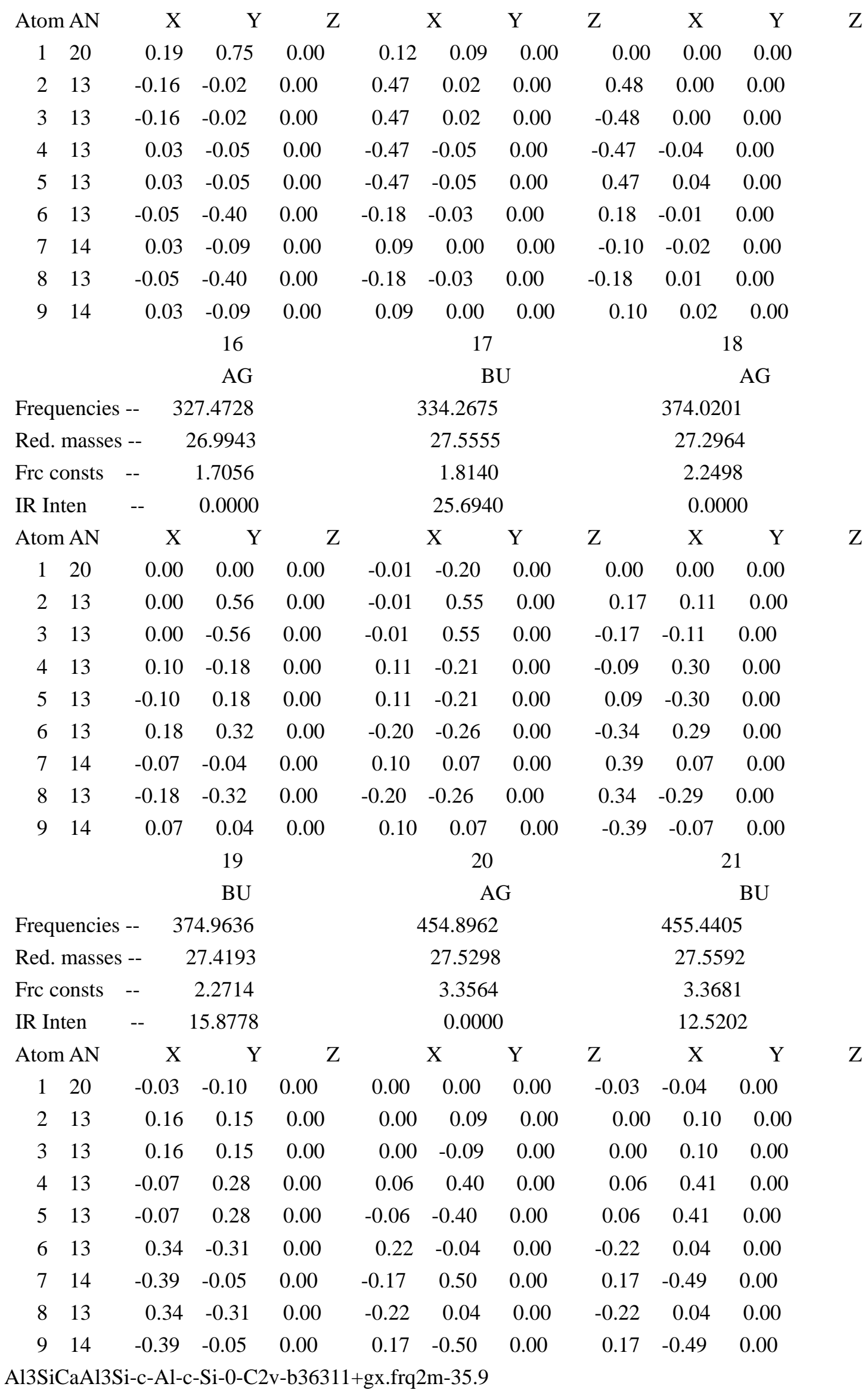




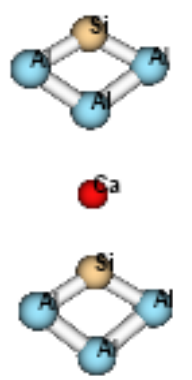

$\begin{array}{ll}20 & 0 \\ 13 & 0 \\ 13 & 0 \\ 13 & 0 \\ 13 & 0 \\ 13 & 0 \\ 14 & 0 \\ 13 & 0 \\ 14 & 0\end{array}$

$\begin{array}{rrr}0.000000 & 0.000000 & 0.133386 \\ 0.000000 & 2.056239 & -4.422551 \\ 0.000000 & -2.056239 & -4.422551 \\ 0.000000 & 0.000000 & 5.907407 \\ 0.000000 & 2.003947 & 4.255133 \\ 0.000000 & -2.003947 & 4.255133 \\ 0.000000 & 0.000000 & 2.938722 \\ 0.000000 & 0.000000 & -2.890298 \\ 0.000000 & 0.000000 & -5.619954\end{array}$

State $=1-A 1 \backslash H F=-2711.2859128$

B2

B2

A2

\begin{tabular}{|c|c|c|c|}
\hline \multicolumn{2}{|c|}{ Frequencies -- } & \multicolumn{2}{|c|}{-35.8995} \\
\hline Red. masses & \multicolumn{3}{|c|}{28.4922} \\
\hline Frc consts & \multicolumn{3}{|c|}{0.0216} \\
\hline IR Inten & \multicolumn{3}{|c|}{2.0695} \\
\hline Atom AN & $\mathrm{X}$ & $\mathrm{Y}$ & 7 \\
\hline 120 & 0.00 & 0.34 & 0.00 \\
\hline 213 & 0.00 & -0.12 & 0.51 \\
\hline 313 & 0.00 & -0.12 & -0.51 \\
\hline $4 \quad 13$ & 0.00 & 0.03 & 0.00 \\
\hline $5 \quad 13$ & 0.00 & 0.02 & 0.00 \\
\hline $6 \quad 13$ & 0.00 & 0.02 & 0.00 \\
\hline $\begin{array}{ll}7 & 14\end{array}$ & 0.00 & 0.02 & 0.00 \\
\hline $8 \quad 13$ & 0.00 & -0.56 & 0.00 \\
\hline \multirow[t]{2}{*}{$9 \quad 14$} & 0.00 & 0.18 & 0.00 \\
\hline & & 4 & \\
\hline
\end{tabular}

$-16.7933$

5.8410

27.3257

26.9815

0.0045

0.0005

0.1675

0.0000

Atom AN $\quad \mathrm{X} \quad \mathrm{Y}$

B1

$\begin{array}{lll}X & Y\end{array}$

$\begin{array}{lll}0.00 & -0.08 & 0.00\end{array}$

Z

$\mathrm{X}$

$\mathrm{Y}$

Z

$$
\begin{array}{llllll}
0.00 & -0.08 & -0.01 & -0.49 & 0.00 & 0.00
\end{array}
$$

$\begin{array}{lll}\mathrm{X} & 0.00 & 0.00\end{array}$

$\begin{array}{lll}0.00 & -0.08 & 0.01\end{array}$

$\begin{array}{lll}0.00 & -0.33 & 0.00\end{array}$

$\begin{array}{lll}0.49 & 0.00 & 0.00\end{array}$

$\begin{array}{lll}0.00 & 0.13 & 0.53\end{array}$

$\begin{array}{lll}0.00 & 0.00 & 0.00\end{array}$

0.00

$\begin{array}{lll}0.00 & 0.50 & 0.00\end{array}$

$\begin{array}{lll}0.51 & 0.00 & 0.00\end{array}$

$\begin{array}{lll}0.00 & -0.07 & 0.00\end{array}$

$\begin{array}{lll}-0.51 & 0.00 & 0.00\end{array}$

$\begin{array}{lll}0.00 & -0.07 & 0.00\end{array}$

$\begin{array}{lll}0.00 & 0.00 & 0.00\end{array}$

$\begin{array}{lll}0.00 & -0.09 & 0.00\end{array}$

$\begin{array}{lll}0.00 & 0.00 & 0.00\end{array}$

$\begin{array}{lll}0.00 & 0.00 & 0.00\end{array}$

\section{5}

6

B2

\begin{tabular}{|c|c|c|c|c|c|c|c|c|c|}
\hline Frequencies & -- & 10.4977 & & \multicolumn{3}{|c|}{18.0008} & & \multicolumn{2}{|c|}{29.1283} \\
\hline Red. masses & & 30.6490 & & \multicolumn{3}{|c|}{34.6741} & & \multicolumn{2}{|c|}{28.8160} \\
\hline Frc consts & -- & 0.0020 & & \multicolumn{3}{|c|}{0.0066} & & \multicolumn{2}{|c|}{0.0144} \\
\hline IR Inten & -- & 0.8604 & & \multicolumn{3}{|c|}{6.5312} & & \multicolumn{2}{|c|}{5.8184} \\
\hline Atom AN & $\mathrm{X}$ & $\mathrm{Y}$ & $\mathrm{Z}$ & & $\mathrm{X}$ & $\mathrm{Y}$ & Z & $\mathrm{X}$ & $\mathrm{Y}$ \\
\hline 120 & 0.51 & 0.00 & 0.00 & 0.00 & 0.77 & 0.00 & -0.32 & 0.00 & 0.00 \\
\hline 13 & -0.14 & 0.00 & 0.00 & 0.00 & -0.11 & -0.28 & 0.04 & 0.00 & 0.00 \\
\hline 13 & -0.14 & 0.00 & 0.00 & 0.00 & -0.11 & 0.28 & 0.04 & 0.00 & 0.00 \\
\hline 13 & -0.60 & 0.00 & 0.00 & 0.00 & -0.23 & 0.00 & -0.35 & 0.00 & 0.00 \\
\hline
\end{tabular}

B1 


$$
\begin{array}{ccccc}
5 & 13 & -0.07 & 0.00 & 0.00 \\
6 & 13 & -0.07 & 0.00 & 0.00 \\
7 & 14 & 0.42 & 0.00 & 0.00 \\
8 & 13 & 0.18 & 0.00 & 0.00 \\
9 & 14 & -0.35 & 0.00 & 0.00 \\
& & & 7 &
\end{array}
$$

\begin{tabular}{|c|c|c|c|}
\hline \multicolumn{2}{|c|}{ Frequencies -- } & \multicolumn{2}{|c|}{51.6707} \\
\hline Red. masses & & 0.7011 & \\
\hline Frc consts & -- & 0.0483 & \\
\hline IR Inten & -- & 2.7566 & \\
\hline Atom AN & $\mathrm{X}$ & $\mathrm{Y}$ & \\
\hline 120 & 0.53 & 0.00 & 0.00 \\
\hline 213 & -0.01 & 0.00 & 0.00 \\
\hline 313 & -0.01 & 0.00 & 0.00 \\
\hline $4 \quad 13$ & 0.11 & 0.00 & 0.00 \\
\hline $5 \quad 13$ & -0.08 & 0.00 & 0.00 \\
\hline $6 \quad 13$ & -0.08 & 0.00 & 0.00 \\
\hline $\begin{array}{ll}7 & 14\end{array}$ & -0.25 & 0.00 & 0.00 \\
\hline $8 \quad 13$ & -0.75 & 0.00 & 0.00 \\
\hline $9 \quad 14$ & 0.28 & 0.00 & 0.00 \\
\hline
\end{tabular}

B1

B1

Frequencies -- 114.6391

Red. masses -- $\quad 27.2268$

Frc consts -- 0.2108

IR Inten -- 4.9999

$\begin{array}{rrrrr}\text { Atom AN } & \mathrm{X} & \mathrm{Y} & \mathrm{Z} \\ 1 & 20 & -0.01 & 0.00 & 0.00 \\ 2 & 13 & 0.01 & 0.00 & 0.00 \\ 3 & 13 & 0.01 & 0.00 & 0.00 \\ 4 & 13 & -0.48 & 0.00 & 0.00 \\ 5 & 13 & 0.51 & 0.00 & 0.00 \\ 6 & 13 & 0.51 & 0.00 & 0.00 \\ 7 & 14 & -0.50 & 0.00 & 0.00 \\ 8 & 13 & -0.02 & 0.00 & 0.00 \\ 9 & 14 & -0.02 & 0.00 & 0.00 \\ & & & 13 & \end{array}$

A1

Frequencies -- $\quad 224.0499$

Red. masses -- 28.8356

Frc consts --- 0.8528

IR Inten $\quad$-- 24.1164

$$
\begin{array}{ccc}
0.00 & -0.18 & 0.08 \\
0.00 & -0.18 & -0.08 \\
0.00 & -0.14 & 0.00 \\
0.00 & 0.09 & 0.00 \\
0.00 & -0.27 & 0.00 \\
\multicolumn{4}{c}{8} &
\end{array}
$$

\section{8}

A1

80.4554

27.1721

$$
0.1036
$$$$
1.7366
$$$$
\text { X Y }
$$

$\begin{array}{lll}0.00 & 0.00 & -0.03\end{array}$

$\begin{array}{lll}0.00 & 0.02 & 0.39\end{array}$

$\begin{array}{lll}0.00 & -0.02 & 0.39\end{array}$

$\begin{array}{lll}0.00 & 0.00 & -0.43\end{array}$

$\begin{array}{llll}0.00 & 0.08 & -0.36\end{array}$

$\begin{array}{lll}0.00 & -0.08 & -0.36\end{array}$

$\begin{array}{lll}0.00 & 0.00 & -0.20\end{array}$

$\begin{array}{lll}0.00 & 0.00 & 0.24\end{array}$

$\begin{array}{lll}0.00 & 0.00 & 0.38\end{array}$

\section{1}

A1

152.7656

30.5311

0.4198

20.2401

$$
\mathrm{X}
$$

\begin{tabular}{lll}
\multicolumn{2}{c}{$\mathrm{X}$} & $\mathrm{Y}$ \\
0.00 & 0.00 & 0.51
\end{tabular}

$\begin{array}{lll}0.00 & -0.10 & -0.18\end{array}$

$\begin{array}{lll}0.00 & 0.10 & -0.18\end{array}$

$\begin{array}{llll}0.00 & 0.00 & -0.47\end{array}$

$\begin{array}{lll}0.00 & 0.33 & -0.07\end{array}$

$\begin{array}{lll}0.00 & -0.33 & -0.07\end{array}$

$\begin{array}{lll}0.00 & 0.00 & 0.36\end{array}$

$\begin{array}{lll}0.00 & 0.00 & 0.12\end{array}$

$\begin{array}{llll}0.00 & 0.00 & -0.24\end{array}$

14

A1

268.9617

27.7967

1.1847

16.1501 \begin{tabular}{ccc}
0.13 & 0.00 & 0.00 \\
0.13 & 0.00 & 0.00 \\
0.61 & 0.00 & 0.00 \\
-0.50 & 0.00 & 0.00 \\
0.33 & 0.00 & 0.00 \\
\multicolumn{2}{c}{9}
\end{tabular}

B1

109.9216

27.3956

0.1950

4.0857

$\begin{array}{lll}\mathrm{Z} & \mathrm{X} & \mathrm{Y}\end{array}$

$\begin{array}{lll}-0.04 & 0.00 & 0.00\end{array}$

$\begin{array}{lll}0.51 & 0.00 & 0.00\end{array}$

$\begin{array}{lll}0.51 & 0.00 & 0.00\end{array}$

$\begin{array}{lll}0.02 & 0.00 & 0.00\end{array}$

$\begin{array}{lll}-0.02 & 0.00 & 0.00\end{array}$

$\begin{array}{lll}-0.02 & 0.00 & 0.00\end{array}$

$\begin{array}{lll}0.01 & 0.00 & 0.00\end{array}$

$\begin{array}{lll}-0.30 & 0.00 & 0.00\end{array}$

$\begin{array}{lll}-0.62 & 0.00 & 0.00\end{array}$

\section{2}

A1

205.6565

27.3295

0.6810

44.0425

$\begin{array}{lllll}Z & X & Y & \text { Z }\end{array}$

$\begin{array}{lll}0.00 & 0.00 & 0.06\end{array}$

$\begin{array}{lll}0.00 & -0.46 & 0.22\end{array}$

$\begin{array}{lll}0.00 & 0.46 & 0.22\end{array}$

$\begin{array}{lll}0.00 & 0.00 & 0.22\end{array}$

$\begin{array}{lll}0.00 & -0.13 & -0.13\end{array}$

$\begin{array}{lll}0.00 & 0.13 & -0.13\end{array}$

$\begin{array}{lll}0.00 & 0.00 & -0.14\end{array}$

$\begin{array}{lll}0.00 & 0.00 & 0.23\end{array}$

$\begin{array}{llll}0.00 & 0.00 & -0.54\end{array}$

15

B2

\subsection{8}

26.9869

1.3489

0.1060

Atom AN $\mathrm{X} \quad \mathrm{Y}$

Z

X Y

Z

X $\quad \mathrm{Y}$ 


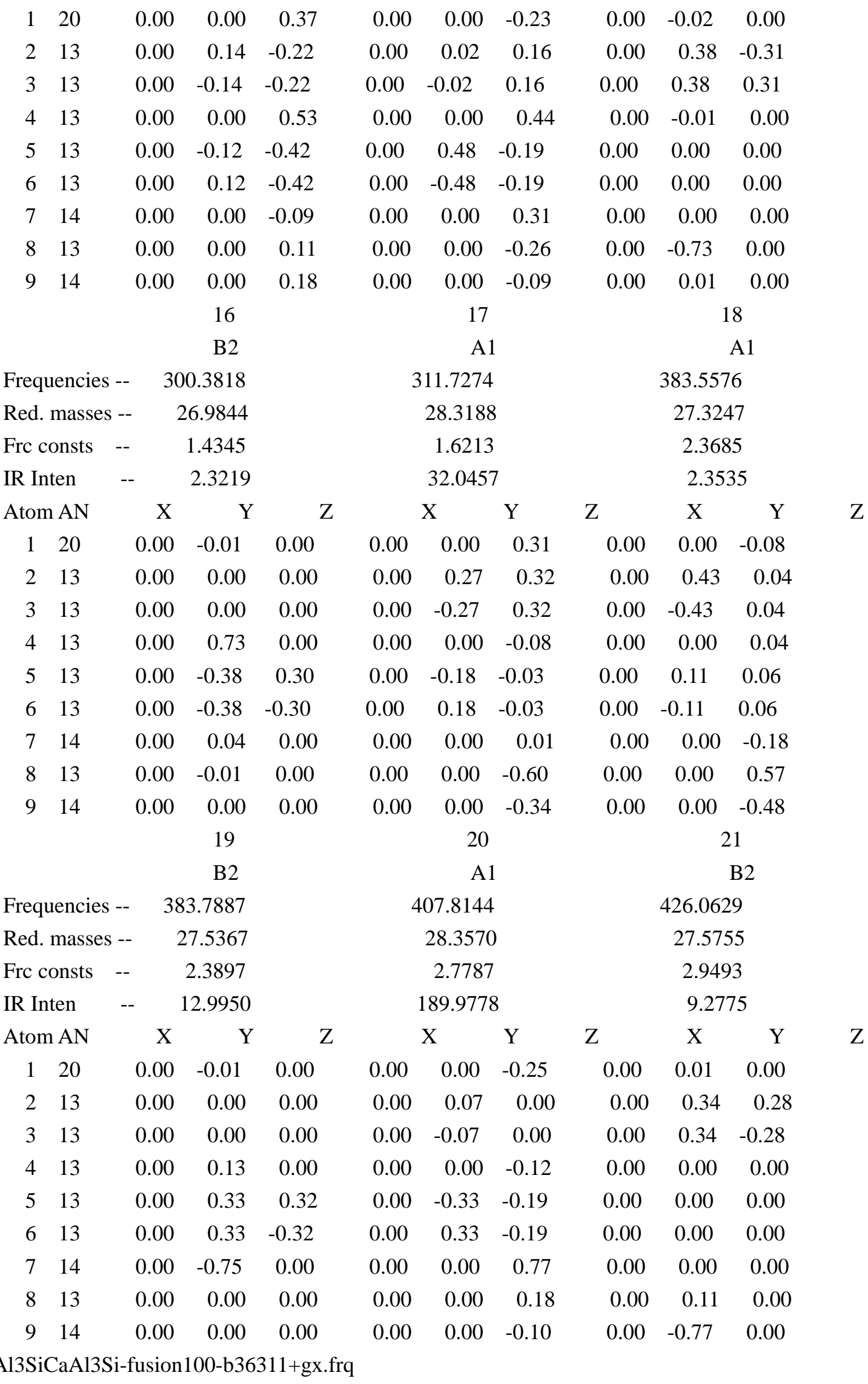




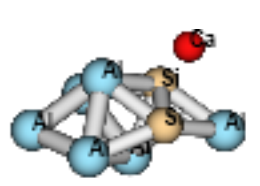

$\begin{array}{llrcc}13 & 0 & -1.699295 & 0.051701 & -2.024750^{\wedge} \mathrm{M} \\ 20 & 0 & 2.509025 & -1.584477 & -0.000256^{\wedge} \mathrm{M} \\ 13 & 0 & -0.780181 & 1.703036 & 0.000283^{\wedge} \mathrm{M} \\ 13 & 0 & -1.699283 & 0.051010 & 2.024769^{\wedge} \mathrm{M} \\ 14 & 0 & 0.879396 & 0.258252 & 1.299038^{\wedge} \mathrm{M} \\ 13 & 0 & 2.425464 & 1.967793 & 0.000318^{\wedge} \mathrm{M} \\ 13 & 0 & -3.422388 & -0.443836 & -0.000069^{\wedge} \mathrm{M} \\ 13 & 0 & -0.578439 & -1.448741 & -0.000248 \wedge \mathrm{M} \\ 14 & 0 & 0.879396 & 0.258678 & -1.298953^{\wedge} \mathrm{M}\end{array}$

|State=1-A $\mid \mathrm{HF}=-2711.357793$

Al3SiCaAl3Si-fusion106-b36311+gx.frq

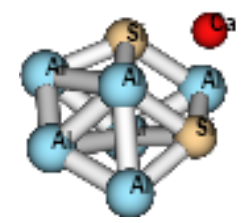

$\begin{array}{llrcc}13 & 0 & -2.797510 & 0.000011 & -0.66232 \wedge^{\wedge} \mathrm{M} \\ 20 & 0 & 2.769677 & 0.000001 & -0.966091^{\wedge} \mathrm{M} \\ 13 & 0 & -0.910724 & 0.000000 & 1.381977 \wedge \mathrm{M} \\ 13 & 0 & -0.157764 & -0.000001 & -1.260049^{\wedge} \mathrm{M} \\ 14 & 0 & 0.686080 & -1.882487 & 0.223735^{\wedge} \mathrm{M} \\ 13 & 0 & 1.612659 & -0.000008 & 1.694303^{\wedge} \mathrm{M} \\ 13 & 0 & -1.742720 & -2.318926 & -0.074756^{\wedge} \mathrm{M} \\ 14 & 0 & 0.686093 & 1.882479 & 0.223739^{\wedge} \mathrm{M} \\ 13 & 0 & -1.742708 & 2.318932 & -0.074750^{\wedge} \mathrm{M}\end{array}$

|State $=1-\mathrm{A} \mid \mathrm{HF}=-2711.3845758$

Al3SiCaAl3Si-c-c-0-Al-Al-C2v-b36311+gx.frq

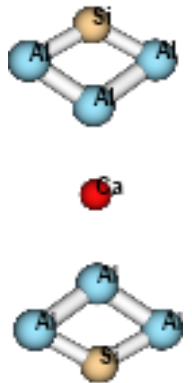

Charge $=0$ Multiplicity $=1$

Ca, 0,0.,0.,0.

$\mathrm{Al}, 0,0 ., 2.0580477101,4.5433980447$

$\mathrm{Al}, 0,0 .,-2.0580477101,4.5433980447$

$\mathrm{Al}, 0,0 ., 2.0580477101,-4.5433980447$

Al,0,0.,-2.0580477101,-4.5433980447

Al,0,0.,0.,3.0148819972

Si,0,0.,0.,5.739324028 
Al,0,0.,0.,-3.0148819972

Si,0,0.,0.,-5.739324028

State $=1-A G \mid H F=-2711.2668671$

Al3SiCaAl3Si-fusion109-b36311+gx.frq

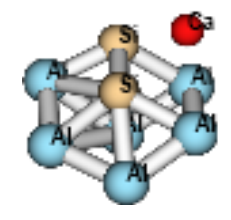

$\begin{array}{rrrrc}13 & 0 & -1.733638 & 2.216053 & -0.100035 \\ 13 & 0 & 0.855784 & 2.212651 & 0.125679 \\ 20 & 0 & 2.697669 & -0.170107 & -1.039850 \\ 13 & 0 & -0.983223 & -0.002514 & 1.521193 \\ 13 & 0 & -1.692581 & -2.249867 & -0.053738 \\ 14 & 0 & -0.127043 & 0.017355 & -0.950116 \\ 14 & 0 & 0.705872 & -1.896558 & 0.330659 \\ 13 & 0 & 1.574806 & 0.135667 & 1.652466 \\ 13 & 0 & -2.794762 & -0.026531 & -0.878689\end{array}$

State $=1-A \backslash H F=-2711.3898925$

CpLiAl3Si-f-c-Si-b36311+gx.frq

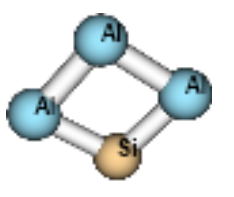

过

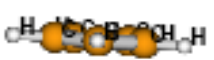

$\begin{array}{rcrrr}6 & 0 & 3.560769 & -1.146505 & -0.406203 \\ 6 & 0 & 3.512496 & 0.023611 & -1.205053 \\ 6 & 0 & 3.546003 & -0.748763 & 0.954208 \\ 1 & 0 & 3.595847 & -2.166139 & -0.771344 \\ 6 & 0 & 3.468276 & 1.144336 & -0.338184 \\ 1 & 0 & 3.505365 & 0.055596 & -2.288171 \\ 6 & 0 & 3.488898 & 0.667149 & 0.996266 \\ 1 & 0 & 3.568289 & -1.411050 & 1.811604 \\ 1 & 0 & 3.420700 & 2.183194 & -0.642531 \\ 1 & 0 & 3.459707 & 1.277249 & 1.891314 \\ 13 & 0 & -4.046743 & 0.286499 & 0.001681 \\ 13 & 0 & -2.682509 & -1.901025 & 0.000085 \\ 13 & 0 & -2.052961 & 1.913609 & -0.000493 \\ 3 & 0 & 1.666683 & -0.086051 & -0.000632 \\ 14 & 0 & -0.988560 & -0.229124 & -0.001553\end{array}$

State $=1-A \backslash H F=-1218.0467874$

CpLiAl3Si-f-f-b36311+gx.frq 


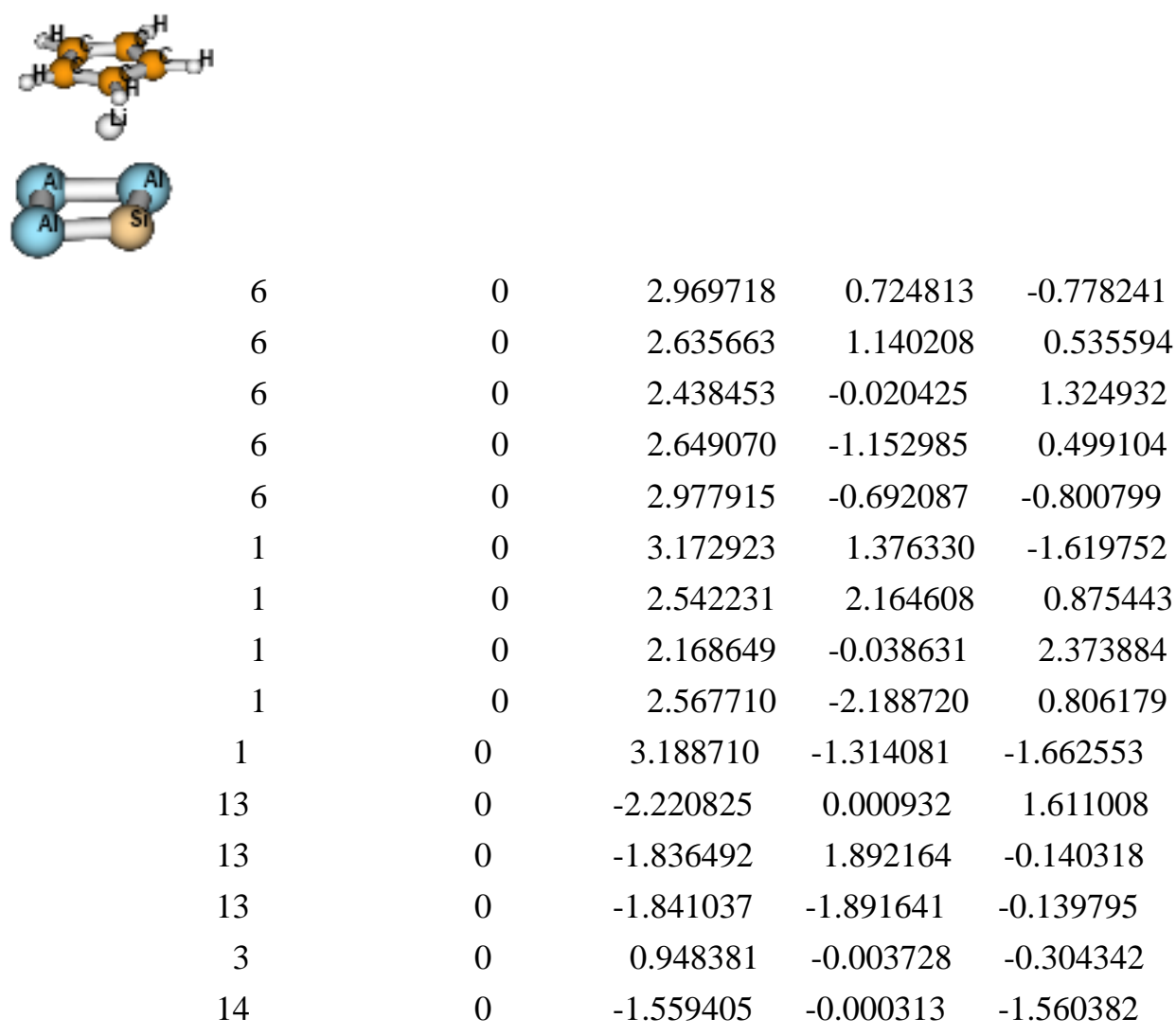

IState=1-A $\backslash H F=-1218.045827$

CpLiAl3Si-f-s-Al-Al-b36311+gx.frq

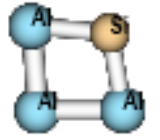

bi

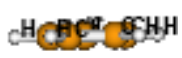

$\begin{array}{rrrrc}13 & 0 & -1.033102 & 1.375355 & -0.008440 \\ 13 & 0 & -3.761505 & -1.214666 & 0.005220 \\ 6 & 0 & 3.504281 & 1.011840 & -0.531831 \\ 6 & 0 & 3.480468 & 0.776514 & 0.865673 \\ 6 & 0 & 3.442687 & -0.242945 & -1.188543 \\ 1 & 0 & 3.555117 & 1.981099 & -1.013252 \\ 6 & 0 & 3.404177 & -0.623741 & 1.072814 \\ 1 & 0 & 3.509677 & 1.534562 & 1.639222 \\ 6 & 0 & 3.381088 & -1.253812 & -0.196668 \\ 1 & 0 & 3.439862 & -0.401724 & -2.260408 \\ 1 & 0 & 3.365709 & -1.124842 & 2.032689 \\ 1 & 0 & 3.318633 & -2.320124 & -0.377188 \\ 3 & 0 & 1.603806 & 0.031060 & -0.011028 \\ 13 & 0 & -1.199411 & -1.214014 & -0.010240 \\ 14 & 0 & -3.382456 & 1.137425 & 0.004166\end{array}$

State $=1-A \backslash H F=-1218.0430708$ 


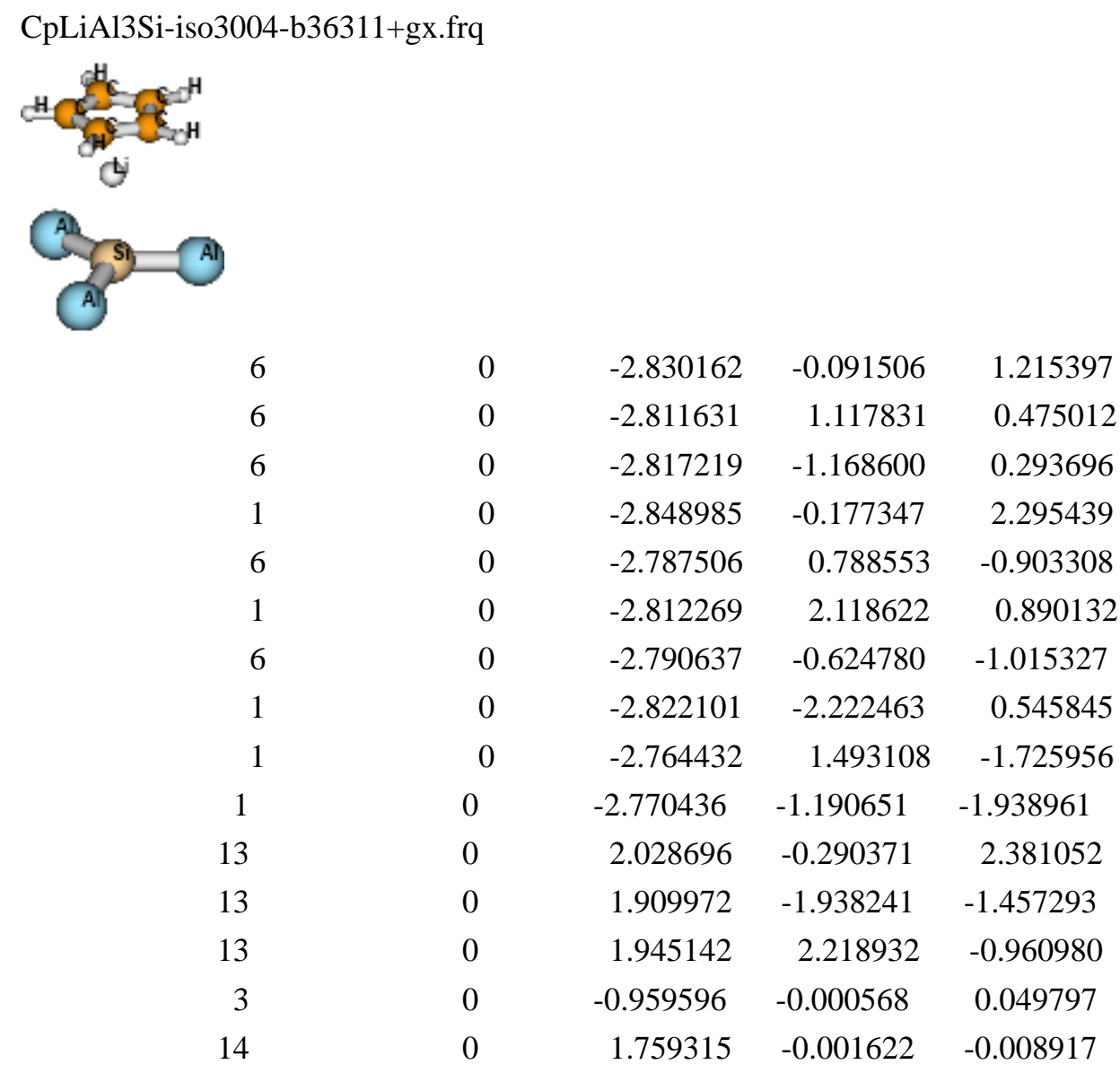

State $=1-A \backslash H F=-1218.036643$

CpLiAl3Si-iso123-b36311+gx.frq

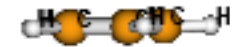

bi

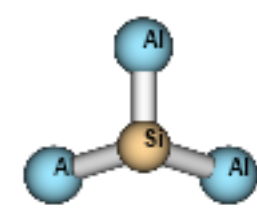

$\begin{array}{rcccc}6 & 0 & -4.062139 & 0.988809 & -0.593551 \\ 6 & 0 & -4.117337 & -0.351223 & -1.052537 \\ 6 & 0 & -3.920440 & 0.963450 & 0.816741 \\ 1 & 0 & -4.118032 & 1.876653 & -1.212136 \\ 6 & 0 & -4.009393 & -1.205010 & 0.074031 \\ 1 & 0 & -4.223214 & -0.667326 & -2.083510 \\ 6 & 0 & -3.887656 & -0.392366 & 1.229158 \\ 1 & 0 & -3.848126 & 1.828333 & 1.465360 \\ 1 & 0 & -4.018249 & -2.288332 & 0.055434 \\ 1 & 0 & -3.786323 & -0.745727 & 2.248403 \\ 13 & 0 & 3.757056 & -2.016718 & 0.412259 \\ 3 & 0 & -2.171546 & -0.013163 & -0.088920 \\ 13 & 0 & 0.614704 & -0.019332 & -0.324230\end{array}$




$\begin{array}{ccccc}13 & 0 & 3.717419 & 2.036581 & 0.405954 \\ 14 & 0 & 2.952218 & 0.000502 & -0.676546\end{array}$

State $=1-A \backslash H F=-1218.0350825$

CpLiAl3Si-iso3003-b36311+gx.frq

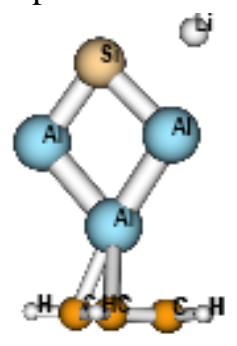

$\begin{array}{rcrcc}6 & 0 & 2.609156 & -1.140963 & -0.004180 \\ 6 & 0 & 2.663822 & -0.298068 & -1.148647 \\ 6 & 0 & 2.801236 & 1.042033 & -0.698055 \\ 6 & 0 & 2.799730 & 1.036743 & 0.706485 \\ 6 & 0 & 2.661554 & -0.306762 & 1.146776 \\ 1 & 0 & 2.513362 & -2.217499 & -0.008326 \\ 1 & 0 & 2.631221 & -0.627572 & -2.178638 \\ 1 & 0 & 2.810493 & 1.920771 & -1.328590 \\ 1 & 0 & 2.807862 & 1.910708 & 1.343642 \\ 1 & 0 & 2.627258 & -0.644056 & 2.174182 \\ 3 & 0 & -4.071406 & 2.103742 & 0.000722 \\ 13 & 0 & 0.543625 & -0.118703 & -0.000952 \\ 13 & 0 & -1.584609 & 1.398983 & -0.000821 \\ 14 & 0 & -3.438989 & -0.224596 & 0.000631 \\ 13 & 0 & -1.593102 & -1.703906 & -0.000345\end{array}$

State $=1-A \backslash H F=-1218.0110666$

CpLiAl3Si-iso3002-b36311+gx.frq

bi

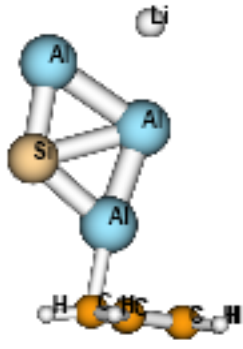

$\begin{array}{ccccc}6 & 0 & -2.330355 & -1.123676 & -0.008268 \\ 6 & 0 & -2.685580 & -0.352839 & 1.153371 \\ 6 & 0 & -3.237096 & 0.860097 & 0.715755 \\ 6 & 0 & -3.245047 & 0.869064 & -0.696513 \\ 6 & 0 & -2.698420 & -0.338021 & -1.155570 \\ 1 & 0 & -2.085563 & -2.178395 & -0.016337 \\ 1 & 0 & -2.554521 & -0.670501 & 2.179735 \\ 1 & 0 & -3.532052 & 1.683678 & 1.354302 \\ 1 & 0 & -3.546804 & 1.700866 & -1.321080 \\ 1 & 0 & -2.577151 & -0.641727 & -2.187356\end{array}$




$\begin{array}{rrrrc}3 & 0 & 3.998092 & 2.327035 & 0.001127 \\ 13 & 0 & 3.776809 & -0.292073 & 0.002549 \\ 13 & 0 & -0.426096 & -0.102609 & -0.004337 \\ 13 & 0 & 1.589410 & 1.353514 & -0.002594 \\ 14 & 0 & 1.661371 & -1.344828 & -0.000595\end{array}$

State $=1-A \mid H F=-1218.0093691$

CpLiAl3Si-iso3001-b36311+gx.frq

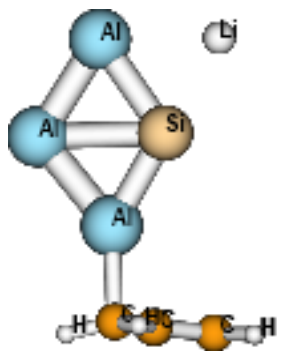

$\begin{array}{ccccc}6 & 0 & -2.383140 & -1.062410 & -0.026139 \\ 6 & 0 & -2.729310 & -0.289324 & 1.152143 \\ 6 & 0 & -3.186113 & 0.953063 & 0.735235 \\ 6 & 0 & -3.191227 & 0.985593 & -0.688362 \\ 6 & 0 & -2.737235 & -0.236138 & -1.164735 \\ 1 & 0 & -2.380237 & -2.148191 & -0.051017 \\ 1 & 0 & -2.617860 & -0.626583 & 2.175176 \\ 1 & 0 & -3.439048 & 1.783716 & 1.383702 \\ 1 & 0 & -3.448102 & 1.845226 & -1.296246 \\ 1 & 0 & -2.630539 & -0.525139 & -2.202920 \\ 3 & 0 & 3.117011 & 2.759836 & 0.003312 \\ 13 & 0 & 2.007798 & -1.624716 & -0.000014 \\ 13 & 0 & 3.763542 & 0.226776 & 0.005996 \\ 14 & 0 & 1.389172 & 1.072367 & -0.002594 \\ 13 & 0 & -0.303763 & -0.581013 & -0.008380\end{array}$

State $=1-A \backslash H F=-1218.0095269$

CpLiAl3Si-iso3000-b36311+gx.frq

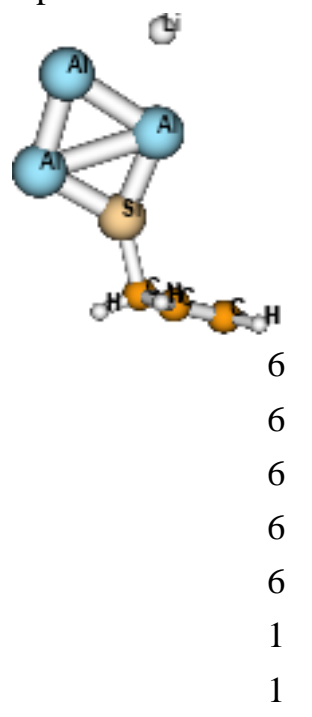

6

6

6

6

6

1

1

$\begin{array}{lrrr}0 & 2.062699 & -0.891922 & 0.000494 \\ 0 & 2.706084 & -0.247255 & -1.166263 \\ 0 & 3.540787 & 0.736628 & -0.725292 \\ 0 & 3.540437 & 0.737585 & 0.724813 \\ 0 & 2.705777 & -0.245932 & 1.166680 \\ 0 & 2.010193 & -1.982605 & 0.001109 \\ 0 & 2.479829 & -0.491498 & -2.196406\end{array}$




$\begin{array}{ccccc}1 & 0 & 4.095336 & 1.429903 & -1.348559 \\ 1 & 0 & 4.094700 & 1.431665 & 1.347436 \\ 1 & 0 & 2.479206 & -0.488967 & 2.197038 \\ 3 & 0 & -3.718235 & 2.680627 & -0.000350 \\ 13 & 0 & -1.902485 & -1.677299 & 0.000191 \\ 13 & 0 & -1.430141 & 1.383081 & -0.000293 \\ 13 & 0 & -3.803141 & 0.058464 & 0.000011 \\ 14 & 0 & 0.101835 & -0.386442 & -0.000070\end{array}$

State $=1-A \mid H F=-1217.9751419$

CpLiAl3Si-iso2000-b36311+gx.frq

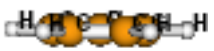

ఉ)

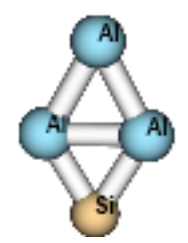

$\begin{array}{rcccc}6 & 0 & -4.142210 & -1.144691 & 0.373834 \\ 6 & 0 & -4.131970 & 0.002199 & 1.207246 \\ 6 & 0 & -4.145382 & -0.706461 & -0.974183 \\ 1 & 0 & -4.144659 & -2.175169 & 0.708610 \\ 6 & 0 & -4.129599 & 1.149257 & 0.374057 \\ 1 & 0 & -4.127282 & 0.002078 & 2.290756 \\ 6 & 0 & -4.137607 & 0.711420 & -0.974024 \\ 1 & 0 & -4.151832 & -1.343320 & -1.850767 \\ 1 & 0 & -4.120351 & 2.179604 & 0.709068 \\ 1 & 0 & -4.136816 & 1.348494 & -1.850462 \\ 13 & 0 & 2.916624 & -1.266682 & -0.000544 \\ 13 & 0 & 2.909371 & 1.264808 & -0.000524 \\ 3 & 0 & -2.304394 & -0.007664 & -0.002705 \\ 14 & 0 & 4.945256 & 0.004868 & 0.001417 \\ 13 & 0 & 0.518708 & -0.007911 & -0.003587\end{array}$

State $=1-A \backslash H F=-1218.0103754$

CpLiAl3Si-iso1000-b36311+gx.frq

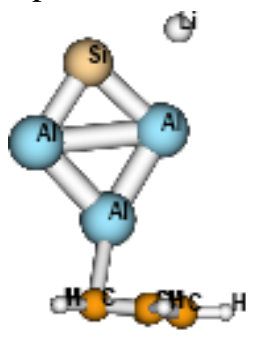

$\begin{array}{rrrrr}6 & 0 & -2.851811 & 0.416240 & -1.141103 \\ 6 & 0 & -2.534916 & -0.900843 & -0.716739 \\ 6 & 0 & -3.051423 & 1.216073 & -0.000415\end{array}$




$\begin{array}{ccccc}1 & 0 & -2.886617 & 0.751966 & -2.169583 \\ 6 & 0 & -2.533920 & -0.899303 & 0.719711 \\ 1 & 0 & -2.411010 & -1.770066 & -1.349103 \\ 6 & 0 & -2.850242 & 0.418674 & 1.141685 \\ 1 & 0 & -3.228054 & 2.283811 & -0.001433 \\ 1 & 0 & -2.409066 & -1.767146 & 1.353779 \\ 1 & 0 & -2.883746 & 0.756601 & 2.169487 \\ 3 & 0 & 3.982693 & 2.231424 & 0.003484 \\ 13 & 0 & 1.536401 & 1.404601 & -0.001960 \\ 13 & 0 & 1.711528 & -1.689534 & -0.000110 \\ 14 & 0 & 3.479185 & -0.126386 & 0.000495 \\ 13 & 0 & -0.471337 & -0.229304 & -0.000958\end{array}$

State $=1-A \backslash H F=-1218.0111696$

CpLiAl3Si-iso1001-b36311+gx.frq

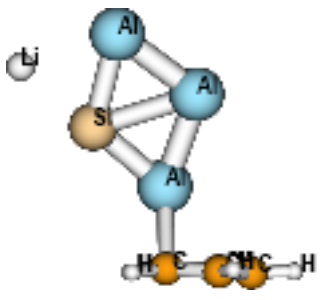

$\begin{array}{rcrrc}6 & 0 & 2.983104 & 0.200814 & -1.142086 \\ 6 & 0 & 3.367706 & 0.934363 & -0.006335 \\ 6 & 0 & 2.352382 & -0.994939 & -0.713989 \\ 1 & 0 & 3.094425 & 0.514535 & -2.172455 \\ 6 & 0 & 2.985135 & 0.216800 & 1.140658 \\ 1 & 0 & 3.795786 & 1.929223 & -0.013733 \\ 6 & 0 & 2.354443 & -0.984562 & 0.731136 \\ 1 & 0 & 2.067548 & -1.833337 & -1.336935 \\ 1 & 0 & 3.097637 & 0.545806 & 2.166125 \\ 1 & 0 & 2.067715 & -1.812571 & 1.366970 \\ 3 & 0 & -3.972865 & -2.114942 & 0.002928 \\ 13 & 0 & 0.398587 & -0.107029 & -0.004927 \\ 13 & 0 & -3.709238 & 0.477658 & 0.002331 \\ 13 & 0 & -1.427750 & 1.650685 & -0.002096 \\ 14 & 0 & -1.775851 & -1.107912 & -0.001004\end{array}$

State $=1-A \mid H F=-1218.0131236$

CpLiAl3Si-iso1002-b36311+gx.frq

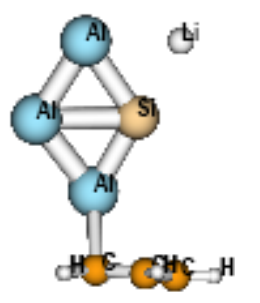




$\begin{array}{ccccc}6 & 0 & -3.098856 & 1.226909 & 0.002287 \\ 6 & 0 & -2.499427 & -0.863687 & 0.722619 \\ 1 & 0 & -2.913871 & 0.773510 & 2.170266 \\ 6 & 0 & -2.871648 & 0.441228 & -1.140589 \\ 1 & 0 & -3.317473 & 2.287644 & 0.004455 \\ 6 & 0 & -2.498792 & -0.860659 & -0.726182 \\ 1 & 0 & -2.412295 & -1.741902 & 1.350347 \\ 1 & 0 & -2.911789 & 0.782714 & -2.167317 \\ 1 & 0 & -2.410913 & -1.736285 & -1.357423 \\ 3 & 0 & 3.197905 & 2.692730 & 0.001031 \\ 13 & 0 & 1.889691 & -1.638134 & 0.000157 \\ 13 & 0 & -0.379163 & -0.496702 & -0.000832 \\ 14 & 0 & 1.389078 & 1.089788 & -0.000126 \\ 13 & 0 & 3.718100 & 0.136184 & 0.000428\end{array}$

State $=1-A \backslash H F=-1218.0098743$

CpLiAl3Si-iso2001-b36311+gx.frq

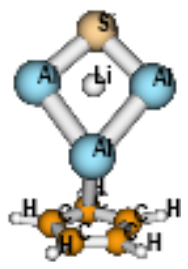

$\begin{array}{rcrcc}6 & 0 & 2.376017 & 1.097325 & -0.674466 \\ 6 & 0 & 2.025440 & -0.149352 & -1.277651 \\ 6 & 0 & 2.487604 & -1.187762 & -0.415333 \\ 6 & 0 & 3.074764 & -0.585375 & 0.713639 \\ 6 & 0 & 3.008481 & 0.822916 & 0.552852 \\ 1 & 0 & 2.146812 & 2.076257 & -1.071635 \\ 1 & 0 & 1.573003 & -0.282668 & -2.251167 \\ 1 & 0 & 2.349377 & -2.247684 & -0.577188 \\ 1 & 0 & 3.465252 & -1.107318 & 1.577767 \\ 1 & 0 & 3.334319 & 1.560513 & 1.274614 \\ 3 & 0 & -1.632071 & -0.006923 & 2.032823 \\ 13 & 0 & 0.471614 & -0.012177 & 0.406115 \\ 13 & 0 & -1.718865 & 1.734818 & 0.021448 \\ 13 & 0 & -1.734083 & -1.730373 & 0.007800 \\ 14 & 0 & -3.360646 & 0.009690 & -0.293201\end{array}$

State $=1-A \backslash H F=-1218.0043123$

Cp2Li2Al3Si-f-f-c-f-Si-b3631+gx.frq 


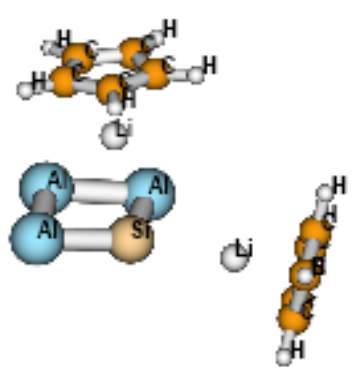

$\begin{array}{rrrrr}13 & 0 & 1.731152 & -2.280460 & -1.634311 \\ 13 & 0 & 1.993840 & -2.617475 & .939900 \\ 13 & 0 & -.153364 & -1.323065 & 1.594702 \\ 6 & 0 & -4.091199 & .660564 & 1.164403 \\ 6 & 0 & -3.748665 & 1.738422 & .304871 \\ 6 & 0 & -4.646568 & -.376714 & .367899 \\ 1 & 0 & -3.948734 & .632959 & 2.239779 \\ 6 & 0 & -4.091337 & 1.367425 & -1.022136 \\ 1 & 0 & -3.295057 & 2.676378 & .608034 \\ 6 & 0 & -4.646134 & .059874 & -.983218 \\ 1 & 0 & -5.000568 & -1.337463 & .727559 \\ 1 & 0 & -3.946464 & 1.972513 & -1.911197 \\ 1 & 0 & -4.999585 & -.508562 & -1.837415 \\ 6 & 0 & 3.739876 & 1.307418 & .744974 \\ 6 & 0 & 3.836127 & 1.455735 & -.664514 \\ 6 & 0 & 2.695607 & 2.153202 & 1.204876 \\ 1 & 0 & 4.352888 & .658326 & 1.361244 \\ 6 & 0 & 2.852188 & 2.394697 & -1.075240 \\ 1 & 0 & 4.535058 & .938679 & -1.313489 \\ 6 & 0 & 2.147146 & 2.825461 & .079785 \\ 1 & 0 & 2.370937 & 2.263323 & 2.234179 \\ 1 & 0 & 2.665505 & 2.718742 & -2.093720 \\ 1 & 0 & 1.327777 & 3.536386 & .098580 \\ 3 & 0 & -2.571753 & -.025005 & -.269332 \\ 3 & 0 & 1.847711 & .680413 & -.173994 \\ 14 & -.185932 & -1.154357 & -.801254\end{array}$

State $=1-A \backslash H F=-1419.0573213$

Cp3Li3Al3Si-extend3-b3631+gx.frq

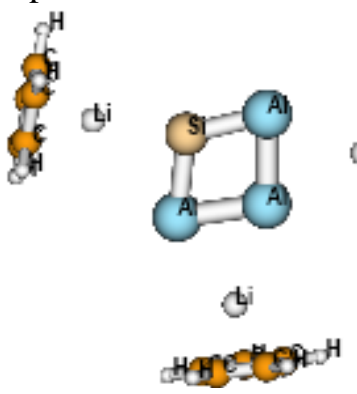

13

de

13

0

$\begin{array}{ccc}1.279023 & 0.990246 & -0.086680 \\ -1.069581 & -0.025335 & -0.069352\end{array}$ 


\begin{tabular}{|c|c|c|c|c|}
\hline 3 & 0 & -0.938092 & 3.001863 & -0.021013 \\
\hline 3 & 0 & -3.332864 & -2.129692 & -0.025123 \\
\hline 6 & 0 & -0.556524 & 5.108348 & -0.390112 \\
\hline 6 & 0 & -1.655525 & 4.687863 & -1.186306 \\
\hline 6 & 0 & -0.910875 & 4.932936 & 0.974995 \\
\hline 1 & 0 & 0.387278 & 5.496125 & -0.758940 \\
\hline 6 & 0 & -2.688317 & 4.251827 & -0.313822 \\
\hline 1 & 0 & -1.698233 & 4.697058 & -2.270440 \\
\hline 6 & 0 & -2.228099 & 4.403614 & 1.021985 \\
\hline 1 & 0 & -0.284710 & 5.163182 & 1.830644 \\
\hline 1 & 0 & -3.657456 & 3.867949 & -0.614231 \\
\hline 1 & 0 & -2.785097 & 4.156370 & 1.919587 \\
\hline 6 & 0 & -4.897502 & -3.013671 & 1.193025 \\
\hline 6 & 0 & -5.278590 & -1.694581 & 0.829617 \\
\hline 6 & 0 & -4.752086 & -3.772982 & 0.000720 \\
\hline 1 & 0 & -4.743336 & -3.377679 & 2.203432 \\
\hline 6 & 0 & -5.368779 & -1.638357 & -0.587569 \\
\hline 1 & 0 & -5.464673 & -0.872473 & 1.512486 \\
\hline 6 & 0 & -5.044141 & -2.922747 & -1.099760 \\
\hline 1 & 0 & -4.469961 & -4.818916 & -0.058589 \\
\hline 1 & 0 & -5.637603 & -0.766369 & -1.174234 \\
\hline 1 & 0 & -5.022548 & -3.205072 & -2.147167 \\
\hline 14 & 0 & 1.630956 & -1.366445 & -0.131723 \\
\hline 13 & 0 & -0.426322 & -2.514466 & -0.120419 \\
\hline 3 & 0 & 4.290654 & -1.083372 & -0.011220 \\
\hline 6 & 0 & 5.992804 & -0.083057 & 0.928682 \\
\hline 6 & 0 & 6.100702 & -1.482749 & 1.145355 \\
\hline 6 & 0 & 6.036506 & 0.148697 & -0.472333 \\
\hline 1 & 0 & 5.892590 & 0.675763 & 1.697860 \\
\hline 6 & 0 & 6.211601 & -2.115942 & -0.121566 \\
\hline 1 & 0 & 6.095954 & -1.981180 & 2.109228 \\
\hline 6 & 0 & 6.172019 & -1.107670 & -1.121405 \\
\hline 1 & 0 & 5.977097 & 1.115859 & -0.960789 \\
\hline 1 & 0 & 6.306967 & -3.182868 & -0.295005 \\
\hline 1 & 0 & 6.231524 & -1.268866 & -2.192833 \\
\hline $\mathrm{te}=1-\mathrm{A} \backslash \mathrm{HF}=-1$ & & & & \\
\hline & & & & \\
\hline
\end{tabular}




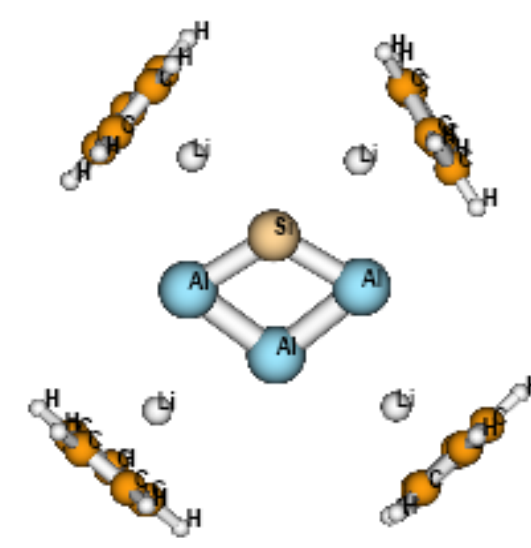

\begin{tabular}{|c|c|c|c|c|}
\hline 13 & 0 & 0.045228 & 2.094079 & -0.047060 \\
\hline 13 & 0 & -1.548444 & 0.070174 & -0.010250 \\
\hline 3 & 0 & -2.811240 & 2.874853 & -0.029916 \\
\hline 3 & 0 & -2.815431 & -2.716764 & 0.025483 \\
\hline 6 & 0 & -3.414016 & 4.905143 & -0.487251 \\
\hline 6 & 0 & -4.209807 & 3.999935 & -1.239484 \\
\hline 6 & 0 & -3.643328 & 4.650021 & 0.892011 \\
\hline 1 & 0 & -2.748788 & 5.659493 & -0.893922 \\
\hline 6 & 0 & -4.930627 & 3.185248 & -0.325652 \\
\hline 1 & 0 & -4.258602 & 3.941014 & -2.321714 \\
\hline 6 & 0 & -4.580280 & 3.587061 & 0.991685 \\
\hline 1 & 0 & -3.183107 & 5.175206 & 1.722330 \\
\hline 1 & 0 & -5.625105 & 2.393897 & -0.586547 \\
\hline 1 & 0 & -4.962638 & 3.157166 & 1.911447 \\
\hline 6 & 0 & -3.768608 & -4.305887 & 1.148605 \\
\hline 6 & 0 & -4.723974 & -3.295152 & 0.860940 \\
\hline 6 & 0 & -3.295916 & -4.827555 & -0.086081 \\
\hline 1 & 0 & -3.455168 & -4.624797 & 2.137090 \\
\hline 6 & 0 & -4.842330 & -3.191864 & -0.551772 \\
\hline 1 & 0 & -5.268485 & -2.704649 & 1.590114 \\
\hline 6 & 0 & -3.960144 & -4.139086 & -1.136822 \\
\hline 1 & 0 & -2.559435 & -5.615124 & -0.205171 \\
\hline 1 & 0 & -5.492757 & -2.509104 & -1.087890 \\
\hline 1 & 0 & -3.818202 & -4.307462 & -2.199156 \\
\hline 4 & 0 & 1.298514 & 0.067989 & -0.014805 \\
\hline 3 & 0 & 0.041700 & -1.952805 & 0.020972 \\
\hline 3 & 0 & 3.204706 & 1.954865 & 0.034595 \\
\hline 6 & 0 & 3.882838 & 3.732355 & 1.098708 \\
\hline 6 & 0 & 4.962879 & 2.821753 & 0.967317 \\
\hline 6 & 0 & 3.463117 & 4.108264 & -0.207296 \\
\hline 1 & 0 & 3.452683 & 4.081910 & 2.031328 \\
\hline 6 & 0 & 5.213332 & 2.634202 & -0.420361 \\
\hline 1 & 0 & 5.502607 & 2.350117 & 1.781745 \\
\hline 6 & 0 & 4.287364 & 3.429146 & -1.145125 \\
\hline
\end{tabular}




$\begin{array}{rrrrr}1 & 0 & 2.670175 & 4.809438 & -0.445229 \\ 1 & 0 & 5.978554 & 1.995731 & -0.849177 \\ 1 & 0 & 4.219105 & 3.504003 & -2.225240 \\ 3 & 0 & 3.045572 & -1.963343 & -0.042740 \\ 6 & 0 & 4.640055 & -3.159807 & -0.893975 \\ 6 & 0 & 4.819119 & -3.069556 & 0.516024 \\ 6 & 0 & 3.413713 & -3.826085 & -1.138712 \\ 1 & 0 & 5.324005 & -2.784481 & -1.647788 \\ 6 & 0 & 3.702016 & -3.678844 & 1.140515 \\ 1 & 0 & 5.663480 & -2.613979 & 1.022364 \\ 6 & 0 & 2.828485 & -4.146292 & 0.119194 \\ 1 & 0 & 2.994657 & -4.054929 & -2.112669 \\ 1 & 0 & 3.540667 & -3.773612 & 2.208915 \\ 1 & 0 & 1.912718 & -4.707356 & 0.271763\end{array}$

State $=1-\mathrm{A} \backslash \mathrm{HF}=-1821.2836695$ 